\title{
ALGEBRAIC AND GEOMETRIC SURGERY
}

\author{
by Andrew Ranicki
}

Oxford Mathematical Monograph (OUP), 2002

This electronic version (March 2014) incorporates the errata which were included in the second printing (2003) as well as the errata found subsequently, and some additional comments. Note that the pagination of the electronic version is somewhat different from the printed version. The list of errata is maintained on http://www.maths.ed.ac.uk/ aar/books/surgerr.pdf 
For Frank Auerbach 


\section{CONTENTS}

Preface

1 The surgery classification of manifolds 1

2 Manifolds 14

2.1 Differentiable manifolds 14

2.2 Surgery 16

2.3 Morse theory 18

2.4 Handles 22

3 Homotopy and homology 29

$\begin{array}{lll}3.1 & \text { Homotopy } & 29\end{array}$

3.2 Homology 32

4 Poincaré duality 48

4.1 Poincaré duality 48

4.2 The homotopy and homology effects of surgery 53

$\begin{array}{lll}4.3 & \text { Surfaces } & 61\end{array}$

4.4 Rings with involution $\quad 66$

$\begin{array}{lll}4.5 & \text { Universal Poincaré duality } & 71\end{array}$

5 Bundles $\quad 85$

5.1 Fibre bundles and fibrations $\quad 85$

$\begin{array}{lll}5.2 & \text { Vector bundles } & 89\end{array}$ 
$\begin{array}{lll}5.3 & \text { The tangent and normal bundles } & 105\end{array}$

$\begin{array}{lll}5.4 & \text { Surgery and bundles } & 112\end{array}$

5.5 The Hopf invariant and the $J$-homomorphism 118

6 Cobordism theory 124

6.1 Cobordism and transversality 124

$\begin{array}{llr}6.2 & \text { Framed cobordism } & 129\end{array}$

$\begin{array}{lll}\text { 6.3 Unoriented and oriented cobordism } & 133\end{array}$

$\begin{array}{lll}6.4 & \text { Signature } & 135\end{array}$

7 Embeddings, immersions and singularities 143

7.1 The Whitney Immersion and Embedding Theorems 143

$\begin{array}{lll}7.2 & \text { Algebraic and geometric intersections } & 149\end{array}$

$\begin{array}{lll}7.3 & \text { The Whitney trick } & 156\end{array}$

7.4 The Smale-Hirsch classification of immersions 161

$\begin{array}{lll}7.5 & \text { Singularities } & 167\end{array}$

8 Whitehead torsion $\quad 170$

$\begin{array}{lll}8.1 & \text { The Whitehead group } & 170\end{array}$

$\begin{array}{ll}\text { 8.2 The } h \text { - and } s \text {-Cobordism Theorems } & 175\end{array}$

$\begin{array}{lll}8.3 & \text { Lens spaces } & 185\end{array}$

$9 \quad$ Poincaré complexes and spherical fibrations 193

9.1 Geometric Poincaré complexes 194

$\begin{array}{lll}9.2 & \text { Spherical fibrations } & 198\end{array}$

9.3 The Spivak normal fibration 205 
9.4 Browder-Novikov theory

10 Surgery on maps

10.1 Surgery on normal maps

10.2 The regular homotopy groups

10.3 Kernels 


\section{PREFACE}

Surgery theory is the standard method for the classification of high-dimensional manifolds, where high means $\geqslant 5$. The theory is not intrinsically difficult, but the wide variety of algebraic and geometric techniques required makes heavy demands on beginners. Where to start?

This book aims to be an entry point to surgery theory for a reader who already has some background in topology. Familiarity with a book such as Bredon [10] or Hatcher [31] is helpful but not essential. The prerequisites from algebraic and geometric topology are presented, along with the purely algebraic ingredients. Enough machinery is developed to prove the main result of surgery theory: the surgery exact sequence computing the structure set of a differentiable manifold $M$ of dimension $\geqslant 5$ in terms of the topological $K$-theory of vector bundles over $M$ and the algebraic $L$-theory of quadratic forms over the fundamental group ring $\mathbb{Z}\left[\pi_{1}(M)\right]$. The surgery exact sequence is stated in Chapter 1 , and finally proved in Chapter 13. Along the way, there are basic treatments of Morse theory, embeddings and immersions, handlebodies, homotopy, homology, cohomology, Steenrod squares, Poincaré duality, vector bundles, cobordism, transversality, Whitehead torsion, the $h$ - and $s$-Cobordism Theorems, algebraic and geometric intersections of submanifolds, the Whitney trick, Poincaré complexes, spherical fibrations, quadratic forms and formations, exotic spheres, as well as the surgery obstruction groups $L_{*}(\mathbb{Z}[\pi])$.

This text introduces surgery, concentrating on the basic mechanics and working out some fundamental concrete examples. It is definitely not an encyclopedia of surgery theory and its applications. Many results and applications are not covered, including such important items as Novikov's theorem on the topological invariance of the rational Pontrjagin classes, surgery on piecewise linear and topological manifolds, the algebraic calculations of the $L$-groups for finite groups, the geometric calculations of the $L$-groups for infinite groups, the Novikov and Borel conjectures, surgery on submanifolds, splitting theorems, controlled topology, knots and links, group actions, stratified sets, the connections between surgery and index theory, .... . In other words, there is a vast research literature on surgery theory, to which this book is only an introduction.

The books of Browder [14], Novikov [65] and Wall [92] are by pioneers of surgery theory, and are recommended to any serious student of the subject. However, note that [14] only deals with the simply-connected case, that only a relatively small part of [65] deals with surgery, and that the monumental [92] is 
notoriously difficult for beginners, probably even with the commentary I had the privilege to add to the second edition. The papers collected in Ferry, Ranicki and Rosenberg [24], Cappell, Ranicki and Rosenberg [17] and Farrell and Lück [23] give a flavour of current research and include many surveys of topics in surgery theory, including the history. In addition, the books of Kosinski [42], Madsen and Milgram [45], Ranicki [70], [71], [74] and Weinberger [94] provide accounts of various aspects of surgery theory.

On the afternoon of my first day as a graduate student in Cambridge, in October, 1970 my official supervisor Frank Adams suggested that I work on surgery theory. This is still surprising to me, since he was a heavy duty homotopy theorist. In the morning he had indeed proposed three topics in homotopy theory, but I was distinctly unenthusiastic. Then at tea-time he said that I might look at the recent work of Novikov [64] on surgery theory and hamiltonian physics, draining the physics out to see what mathematics was left over. Novikov himself had not been permitted by the Soviet authorities to attend the Nice ICM in September, but Frank had attended the lecture delivered on Novikov's behalf by Mishchenko. The mathematics and the circumstances of the lecture definitely sparked my interest. However, as he was not himself a surgeon, Frank suggested that I actually work with Andrew Casson. Andrew explained that he did not have a Ph.D. himself and was therefore not formally qualified to be a supervisor of a Ph.D. student, though he would be willing to answer questions. He went on to say that in any case this was the wrong time to start work on high-dimensional surgery theory! There had just been major breakthroughs in the field, and what was left to do was going to be hard. This brought out a stubborn streak in me, and I have been working on high-dimensional surgery theory ever since.

It is worth remarking here that surgery theory started in 1963 with the classification by Kervaire and Milnor [38] of the exotic spheres, which are the differentiable manifolds which are homeomorphic but not diffeomorphic to the standard sphere. Students are still advised to read this classic paper, exactly as I was advised to do by Andrew Casson in 1970.

This book grew out of a joint lecture course with Jim Milgram at Göttingen in 1987. I am grateful to the Leverhulme Trust for the more recent (2001/2002) Fellowship during which I completed the book. I am grateful to Markus Banagl, Jeremy Brookman (who deserves special thanks for designing many of the diagrams), Diarmuid Crowley, Jonathan Kelner, Dirk Schuetz, Des Sheiham, Joerg Sixt, Chris Stark, Ida Thompson and Shmuel Weinberger for various suggestions.

Any comments on the book subsequent to publication will be posted on the website

$$
\text { http://www.maths.ed.ac.uk/aar/books }
$$




\section{THE SURGERY CLASSIFICATION OF MANIFOLDS}

Chapter 1 is an introduction to the surgery method of classifying manifolds.

Manifolds are understood to be differentiable, compact and closed, unless otherwise specified.

A classification of manifolds up to diffeomorphism requires the construction of a complete set of algebraic invariants such that:

(i) the invariants of a manifold are computable,

(ii) two manifolds are diffeomorphic if and only if they have the same invariants,

(iii) there is given a list of non-diffeomorphic manifolds realizing every possible set of invariants.

One could also seek a homotopy classification of manifolds, asking for a complete set of invariants for distinguishing the homotopy types of manifolds. Diffeomorphic manifolds are homotopy equivalent.

The most important invariant of a manifold $M^{m}$ is its dimension, the number $m \geqslant 0$ such that $M$ is locally diffeomorphic to the Euclidean space $\mathbb{R}^{m}$. If $m \neq n$ then $\mathbb{R}^{m}$ is not diffeomorphic to $\mathbb{R}^{n}$, so that an $m$-dimensional manifold $M^{m}$ cannot be diffeomorphic to an $n$-dimensional manifold $N^{n}$. The homology and cohomology of an orientable $m$-dimensional manifold $M$ are related by the Poincaré duality isomorphisms

$$
H^{*}(M) \cong H_{m-*}(M) .
$$

Any $m$-dimensional manifold $M$ has $\mathbb{Z}_{2}$-coefficient Poincaré duality

$$
H^{*}\left(M ; \mathbb{Z}_{2}\right) \cong H_{m-*}\left(M ; \mathbb{Z}_{2}\right)
$$

with

$$
H_{m}\left(M ; \mathbb{Z}_{2}\right)=\mathbb{Z}_{2}, H_{n}\left(M ; \mathbb{Z}_{2}\right)=0 \text { for } n>m .
$$

The dimension of a manifold $M$ is thus characterised homologically as the largest integer $m \geqslant 0$ with $H_{m}\left(M ; \mathbb{Z}_{2}\right) \neq 0$. Homology is homotopy invariant, so that the dimension is also a homotopy invariant : if $m \neq n$ an $m$-dimensional manifold $M^{m}$ cannot be homotopy equivalent to an $n$-dimensional manifold $N^{n}$. 
There is a complete diffeomorphism classification of $m$-dimensional manifolds only in the dimensions $m=0,1,2$, where it coincides with the homotopy classification. For $m \geqslant 3$ there exist $m$-dimensional manifolds which are homotopy equivalent but not diffeomorphic, so that the diffeomorphism and homotopy classifications must necessarily differ. For $m=3$ complete classifications are theoretically possible, but have not been achieved in practice - the Poincaré conjecture that every 3-dimensional manifold homotopy equivalent to $S^{3}$ is actually diffeomorphic to $S^{3}$ remains unsolved!

For $m \geqslant 4$ group-theoretic decision problems prevent a complete classification of $m$-dimensional manifolds, by the following argument. Every manifold $M$ can be triangulated by a finite simplicial complex, so that the fundamental group $\pi_{1}(M)$ is finitely presented. Homotopy equivalent manifolds have isomorphic fundamental groups. Every finitely presented group arises as the fundamental group $\pi_{1}(M)$ of an $m$-dimensional manifold $M$. It is not possible to have a complete set of invariants for distinguishing the isomorphism class of a group from a finite presentation. Group-theoretic considerations thus make the following questions unanswerable in general :

(a) Is $M$ homotopy equivalent to $M^{\prime}$ ?

(b) Is $M$ diffeomorphic to $M^{\prime}$ ?

since already the question

(c) Is $\pi_{1}(M)$ isomorphic to $\pi_{1}\left(M^{\prime}\right)$ ?

is unanswerable in general.

The surgery method of classifying manifolds seeks to answer a different question :

Given a homotopy equivalence of m-dimensional manifolds $f: M \rightarrow M^{\prime}$ is $f$ homotopic to a diffeomorphism?

Every homotopy equivalence of 2-dimensional manifolds (= surfaces) is homotopic to a diffeomorphism, by the 19th century classification of surfaces which is recalled in Chapter 3.

A homotopy equivalence of 3-dimensional manifolds is not in general homotopic to a diffeomorphism. The first examples of such homotopy equivalences appeared in the classification of the 3-dimensional lens spaces in the 1930's : the Reidemeister torsion of a lens space is a diffeomorphism invariant which is not homotopy invariant. Algebraic $K$-theory invariants such as Reidemeister and 
Whitehead torsion are significant in the classification of manifolds with finite fundamental group, and in deciding if ' $h$-cobordant' manifolds are diffeomorphic (via the $s$-Cobordism Theorem, stated in 1.11 below), but they are too special to decide if an arbitrary homotopy equivalence of manifolds is homotopic to a diffeomorphism. Chapter 8 deals with the main applications of Whitehead torsion to the topology of manifolds.

In 1956, Milnor [49] constructed an exotic sphere, a differentiable manifold $\Sigma^{7}$ with a homotopy equivalence (in fact a homeomorphism) $\Sigma^{7} \rightarrow S^{7}$ which is not homotopic to a diffeomorphism. The subsequent classification by Kervaire and Milnor [38] for $m \geqslant 5$ of pairs

$$
\text { ( } m \text {-dimensional manifold } \Sigma^{m} \text {, homotopy equivalence } \Sigma^{m} \rightarrow S^{m} \text { ) }
$$

was the first triumph of surgery theory. It remains the best introduction to surgery, particularly as it deals with simply-connected manifolds $M$ (i.e. those with $\left.\pi_{1}(M)=\{1\}\right)$ and so avoids the fundamental group. The surgery classification of homotopy spheres is outlined in Section 13.3.

Definition 1.1 An $(m+1)$-dimensional cobordism $\left(W ; M, M^{\prime}\right)$ is an $(m+$ 1)-dimensional manifold $W^{m+1}$ with boundary the disjoint union of closed $m$ dimensional manifolds $M, M^{\prime}$

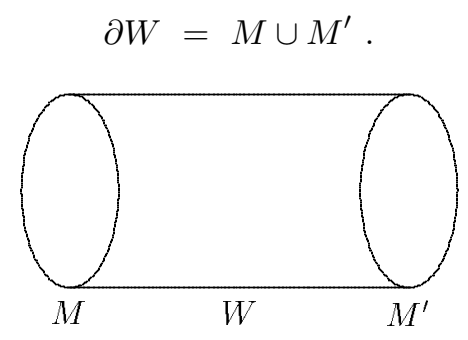

The cobordism classes of manifolds are groups, with addition by disjoint union. The computation of the cobordism groups was a major achievement of topology in the 1950's - Chapter 6 is an introduction to cobordism theory. The cobordism classification of manifolds is very crude: for example, the 0 - and 2dimensional cobordism groups have order two, and the 1- and 3-dimensional cobordism groups are trivial. Surgery theory applies the methods of cobordism theory to the rather more delicate classification of the homotopy types of manifolds.

What is surgery? 
Definition 1.2 A surgery on an $m$-dimensional manifold $M^{m}$ is the procedure of constructing a new $m$-dimensional manifold

$$
M^{\prime m}=\operatorname{cl} .\left(M \backslash S^{n} \times D^{m-n}\right) \cup_{S^{n} \times S^{m-n-1}} D^{n+1} \times S^{m-n-1}
$$

by cutting out $S^{n} \times D^{m-n} \subset M$ and replacing it by $D^{n+1} \times S^{m-n-1}$. The surgery removes $S^{n} \times D^{m-n} \subset M$ and kills the homotopy class $S^{n} \rightarrow M$ in $\pi_{n}(M)$.

Terminology: given a subset $Y \subseteq X$ of a space $X$ write cl.( $Y)$ for the closure of $Y$ in $X$, the intersection of all the closed subsets $Z \subseteq X$ with $Y \subseteq Z$.

At first sight, it might seem surprising that surgery can be used to answer such a delicate question as whether a homotopy equivalence of manifolds is homotopic to a diffeomorphism, since an individual surgery has such a drastic effect on the homotopy type of a manifold:

Example 1.3 (i) View the $m$-sphere $S^{m}$ as

$$
S^{m}=\partial\left(D^{n+1} \times D^{m-n}\right)=S^{n} \times D^{m-n} \cup D^{n+1} \times S^{m-n-1} .
$$

The surgery on $S^{m}$ removing $S^{n} \times D^{m-n} \subset S^{m}$ converts the $m$-sphere $S^{m}$ into the product of spheres

$$
D^{n+1} \times S^{m-n-1} \cup D^{n+1} \times S^{m-n-1}=S^{n+1} \times S^{m-n-1} .
$$

(ii) For $m=1, n=0$ the surgery of (i) converts the circle $S^{1}$ into the disjoint union $S^{0} \times S^{1}=S^{1} \cup S^{1}$ of two circles.

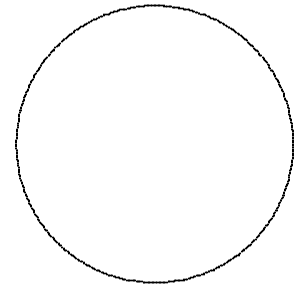

$S^{1}$

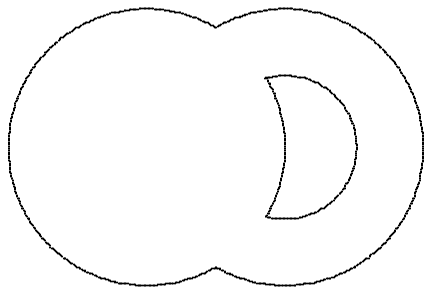

$S^{1} \cup S^{1}$

(iii) For $m=2, n=0$ the surgery of (i) converts the 2-sphere $S^{2}$ into the torus $S^{1} \times S^{1}$.

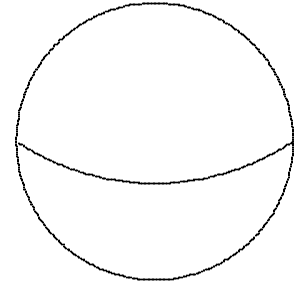

$S^{2}$

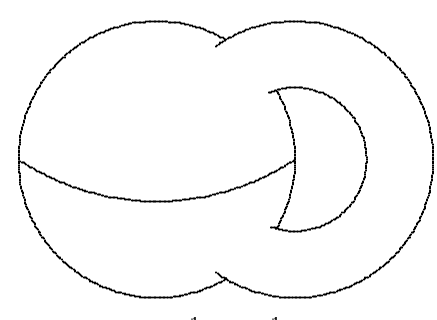

$S^{1} \times S^{1}$ 
(iv) For $m=n$ the surgery of (i) converts the $m$-sphere $S^{m}$ into the empty set $\emptyset$.

There is an intimate connection between surgery and cobordism. A surgery on a manifold $M$ determines a cobordism $\left(W ; M, M^{\prime}\right)$ :

Definition 1.4 The trace of the surgery removing $S^{n} \times D^{m-n} \subset M^{m}$ is the cobordism $\left(W ; M, M^{\prime}\right)$ obtained by attaching $D^{n+1} \times D^{m-n}$ to $M \times I$ at

$$
S^{n} \times D^{m-n} \times\{1\} \subset M \times\{1\} .
$$

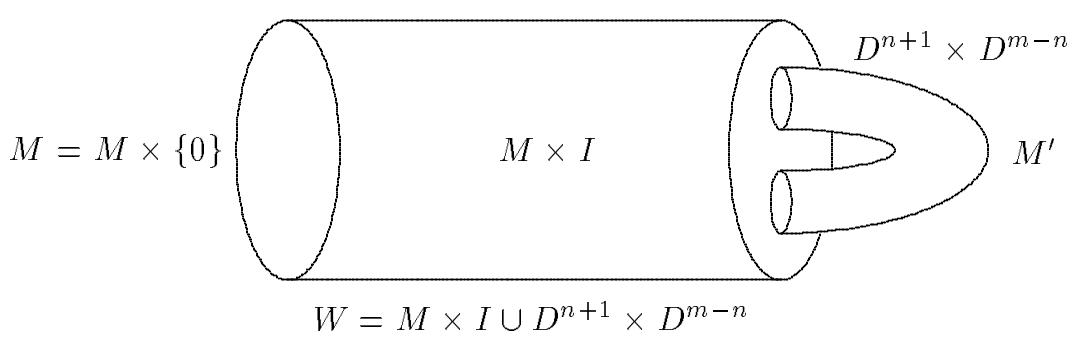

Here is a more symmetric picture of the trace $\left(W ; M, M^{\prime}\right)$ :

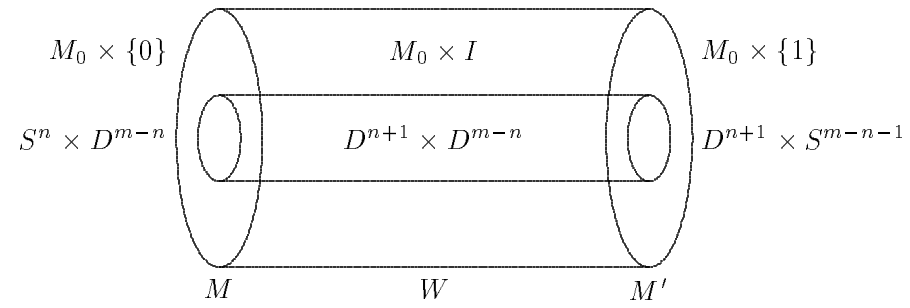

The $m$-dimensional manifold with boundary

$$
\left(M_{0}, \partial M_{0}\right)=\left(\operatorname{cl} .\left(M^{m} \backslash S^{n} \times D^{m-n}\right), S^{n} \times S^{m-n-1}\right)
$$

is obtained from $M$ by cutting out the interior of $S^{n} \times D^{m-n} \subset M$, with

$$
\begin{aligned}
& M=M_{0} \cup_{\partial M_{0}} S^{n} \times D^{m-n}, \\
& M^{\prime}=M_{0} \cup_{\partial M_{0}} D^{n+1} \times S^{m-n-1}, \\
& W=\left(M_{0} \times I\right) \cup\left(D^{n+1} \times D^{m-n}\right), \\
& \left(M_{0} \times I\right) \cap\left(D^{n+1} \times D^{m-n}\right)=S^{n} \times S^{m-n-1} \times I .
\end{aligned}
$$

Note that $M$ is obtained from $M^{\prime}$ by the opposite surgery removing $D^{n+1} \times$ $S^{m-n-1} \subset M^{\prime}$. 
In fact, two $m$-dimensional manifolds $M^{m}, M^{\prime m}$ are cobordant if and only if $M^{\prime}$ can be obtained from $M$ by a finite sequence of surgeries.

Definition 1.5 A bordism of maps $f: M^{m} \rightarrow X, f^{\prime}: M^{\prime m} \rightarrow X$ from $m$ dimensional manifolds to a space $X$ is a cobordism $\left(W ; M, M^{\prime}\right)$ together with a map

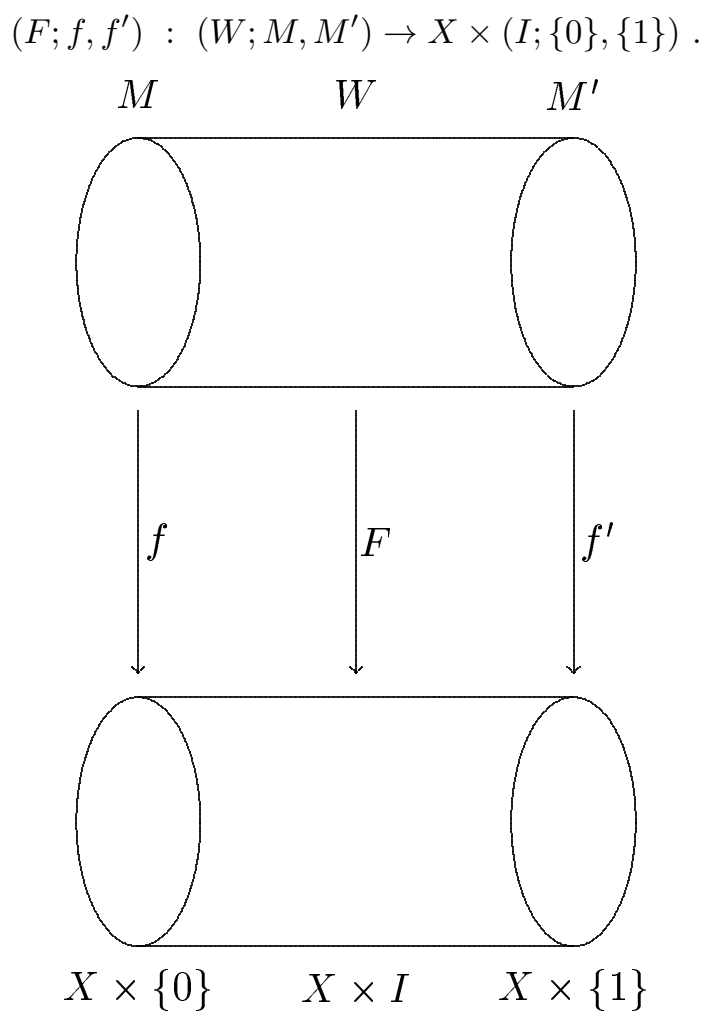

Example 1.6 A homotopy $h: f \simeq f^{\prime}: M^{m} \rightarrow X$ can be regarded as a bordism

$$
\left(F ; f, f^{\prime}\right):\left(W ; M, M^{\prime}\right)=M \times(I ;\{0\},\{1\}) \rightarrow X \times(I ;\{0\},\{1\}),
$$

with

$$
F: M \times I \rightarrow X \times I ;(x, t) \mapsto(h(x, t), t)
$$

Diffeomorphic manifolds are cobordant. Homotopy equivalent closed manifolds are cobordant, but in general only by a nonorientable cobordism. It is possible to decide if two manifolds are cobordant, but it is not possible to decide if cobordant manifolds are homotopy equivalent, or if homotopy equivalent manifolds are diffeomorphic. Given that cobordism is considerably weaker than 
diffeomorphism and that cobordism drastically alters homotopy types, it may appear surprising that cobordism is a sufficiently powerful tool to distinguish manifolds within a homotopy type. However, surgery theory provides a systematic procedure for deciding if a map of $m$-dimensional manifolds $f: M \rightarrow M^{\prime}$ satisfying certain bundle-theoretic conditions is bordant to a homotopy equivalence, and if the bordism can be chosen to be a homotopy (as in 1.6), at least in dimensions $m \geqslant 5$. This works because surgery makes it comparatively easy to construct cobordisms with prescribed homotopy types. The applications of cobordism theory to the surgery classification of high-dimensional manifolds depend on the following fundamental result:

Whitney Embedding Theorem 1.7 ([97], [99], 1944)

If $f: N^{n} \rightarrow M^{m}$ is a map of manifolds such that

$$
\begin{array}{ll}
\text { either } & 2 n+1 \leqslant m \\
\text { or } \quad & m=2 n \geqslant 6 \text { and } \pi_{1}(M)=\{1\}
\end{array}
$$

then $f$ is homotopic to an embedding $N^{n} \hookrightarrow M^{m}$.

The proof of 1.7 will be outlined in Chapter 7 .

Definition 1.8 (i) An $h$-cobordism is a cobordism $\left(W^{m+1} ; M^{m}, M^{\prime m}\right)$ such that the inclusions $M \hookrightarrow W, M^{\prime} \hookrightarrow W$ are homotopy equivalences.

(ii) An $h$-cobordism $\left(W ; M, M^{\prime}\right)$ is trivial if there exists a diffeomorphism

$$
\left(W ; M, M^{\prime}\right) \cong M \times(I ;\{0\},\{1\})
$$

which is the identity on $M$, in which case the composite homotopy equivalence $M \simeq W \simeq M^{\prime}$ is homotopic to a diffeomorphism.

The $h$-Cobordism Theorem was the crucial first step in the homotopy classification of high-dimensional manifolds :

h-Cobordism Theorem 1.9 (Smale [83], 1962)

A simply-connected $(m+1)$-dimensional h-cobordism $\left(W^{m+1} ; M, M^{\prime}\right)$ with $m \geqslant 5$ is trivial.

Thus for $m \geqslant 5$ simply-connected $m$-dimensional manifolds $M, M^{\prime}$ are diffeomorphic if and only if they are $h$-cobordant.

The $h$-Cobordism Theorem was subsequently generalised to non-simply-connected manifolds, using Whitehead torsion (which is described in Chapter 8). The Whitehead group $W h(\pi)$ of a group $\pi$ is an abelian group which measures the 
extent to which Gaussian elimination fails for invertible matrices with entries in the group ring $\mathbb{Z}[\pi]$. The Whitehead torsion of a homotopy equivalence $f$ : $M^{m} \rightarrow M^{\prime m}$ of manifolds (or more generally of finite $C W$ complexes) is an element $\tau(f) \in W h\left(\pi_{1}(M)\right)$. A homotopy equivalence $f$ is simple if $\tau(f)=0$.

Definition 1.10 An $s$-cobordism is a cobordism $\left(W^{m+1} ; M^{m}, M^{\prime m}\right)$ such that the inclusions $M \hookrightarrow W, M^{\prime} \hookrightarrow W$ are simple homotopy equivalences.

A diffeomorphism $f: M^{m} \rightarrow M^{\prime m}$ of $m$-dimensional manifolds determines an $(m+1)$-dimensional $s$-cobordism $\left(W ; M, M^{\prime}\right)$ with

$$
W=\left(M \times I \cup M^{\prime}\right) /\{(x, 1) \sim f(x) \mid x \in M\}
$$

the mapping cylinder, such that there is defined a diffeomorphism

$$
\left(W ; M, M^{\prime}\right) \cong M \times(I ;\{0\},\{1\}) .
$$

The $s$-Cobordism Theorem is the non-simply-connected version of the $h$ Cobordism Theorem :

$s$-Cobordism Theorem 1.11 (Barden-Mazur-Stallings, 1964)

An $(m+1)$-dimensional $h$-cobordism $\left(W^{m+1} ; M, M^{\prime}\right)$ with $m \geqslant 5$ is trivial if and only if it is an s-cobordism.

It follows that for $m \geqslant 5 h$-cobordant $m$-dimensional manifolds $M, M^{\prime}$ are diffeomorphic if and only if they are $s$-cobordant. The proofs of the $h$ - and $s$ Cobordism Theorems will be outlined in Chapter 8 .

The Whitehead group of the trivial group is trivial, $W h(\{1\})=0$, and the $h$ Cobordism Theorem is just the simply-connected special case of the $s$-Cobordism Theorem. The condition $m \geqslant 5$ in the $h$ - and $s$-Cobordism Theorems is due to the use of the Whitney Embedding Theorem (1.7) in their proof. It is known that the $h$ - and $s$-Cobordism Theorems for $(m+1)$-dimensional cobordisms are true for $m=0,1$, and are false for $m=4$ (Donaldson [21]), $m=3$ (Cappell and Shaneson [18]). It is not known if they are true for $m=2$, on account of the classical 3-dimensional Poincaré conjecture.

Milnor [58] used the lens spaces to construct $h$-cobordisms $\left(W^{m+1} ; M, M^{\prime}\right)$ of non-simply-connected manifolds which are not diffeomorphic.

One way to prove that manifolds are diffeomorphic is to first decide if they are cobordant, and then to decide if some cobordism can be modified by successive surgeries on the interior to be an $s$-cobordism. 
The tangent bundle of an $m$-dimensional manifold $M^{m}$ is classified by the homotopy class of a map

$$
\tau_{M}: M \rightarrow B O(m)
$$

(See Chapter 5 for some basic information on bundles, including the classifying space $B O(m))$. If $f: M \rightarrow M^{\prime}$ is a homotopy equivalence of $m$-dimensional manifolds which is homotopic to a diffeomorphism there exists a homotopy $f^{*} \tau_{M^{\prime}} \simeq \tau_{M}: M \rightarrow B O(m)$. The tubular neighbourhood of an embedding $M^{m} \hookrightarrow S^{m+k}$ ( $k$ large) is a $k$-plane bundle $\nu_{M}: M \rightarrow B O(k)$ which is a stable inverse of $\tau_{M}$. The stable normal bundle of $M$ is classified by a map

$$
\nu_{M}: M \rightarrow B O=\varliminf_{k} B O(k)
$$

By the result of Mazur [47] for $m \geqslant 5$ a homotopy equivalence of $m$-dimensional manifolds $f: M \rightarrow M^{\prime}$ is covered by a stable bundle map $b: \nu_{M} \rightarrow \nu_{M^{\prime}}$ if and only if $f \times 1: M \times \mathbb{R}^{k} \rightarrow M^{\prime} \times \mathbb{R}^{k}$ is homotopic to a diffeomorphism for some $k \geqslant 0$. It is possible to extend $f$ to a homotopy equivalence of $h$-cobordisms

$$
(F ; f, 1):\left(W ; M, M^{\prime}\right) \rightarrow M^{\prime} \times(I ;\{0\},\{1\})
$$

if and only if $f \times 1: M \times \mathbb{R} \rightarrow M^{\prime} \times \mathbb{R}$ is homotopic to a diffeomorphism.

The surgery theory developed by Browder, Novikov, Sullivan and Wall in the 1960's provides a systematic solution to the problem of deciding if a homotopy equivalence $f: M \rightarrow M^{\prime}$ of $m$-dimensional manifolds is homotopic to a diffeomorphism, with obstructions taking values in the topological $K$-theory of vector bundles and the algebraic $L$-theory of quadratic forms. The obstruction theory was obtained as the relative version of the systematic solution to the problem of deciding if a space $X$ with $m$-dimensional Poincaré duality $H^{*}(X) \cong H_{m-*}(X)$ is homotopy equivalent to an $m$-dimensional manifold. The theory thus deals both with the existence and the uniqueness of manifold structures in homotopy types.

Definition 1.12 An $m$-dimensional geometric Poincaré complex is a finite $C W$ complex $X$ with a fundamental homology class $[X] \in H_{m}(X)$ (using twisted coefficients in the nonorientable case) such that the cap products are isomorphisms

$$
[X] \cap-: H^{*}(X ; \Lambda) \rightarrow H_{m-*}(X ; \Lambda)
$$

for every $\mathbb{Z}\left[\pi_{1}(X)\right]$-module $\Lambda$.

Example 1.13 An $m$-dimensional manifold is an $m$-dimensional geometric Poincaré complex. 
The property of being a geometric Poincaré complex is homotopy invariant, unlike the property of being a manifold. Thus any finite $C W$ complex homotopy equivalent to a manifold is a geometric Poincaré complex. In order for a space to have a fighting chance of being homotopy equivalent to an $m$-dimensional manifold it must at least be homotopy equivalent to an $m$-dimensional geometric Poincaré complex. Geometric Poincaré complexes which are not homotopy equivalent to a manifolds may be obtained by glueing together $m$-dimensional manifolds with boundary $(M, \partial M),\left(M^{\prime}, \partial M^{\prime}\right)$ using a homotopy equivalence $\partial M \simeq \partial M^{\prime}$ which is not homotopic to a diffeomorphism.

Definition 1.14 Let $X$ be an $m$-dimensional geometric Poincaré complex.

(i) A manifold structure $(M, f)$ on $X$ is an $m$-dimensional manifold $M$ together with a homotopy equivalence $f: M \rightarrow X$.

(ii) The manifold structure set $\mathscr{S}(X)$ of $X$ is the set of equivalence classes of manifold structures $(M, f)$, subject to the equivalence relation :

$$
\begin{aligned}
(M, f) \sim\left(M^{\prime}, f^{\prime}\right) & \text { if there exists a bordism } \\
& \left(F ; f, f^{\prime}\right):\left(W ; M, M^{\prime}\right) \rightarrow X \times(I ;\{0\},\{1\}) \\
& \text { with } F \text { a homotopy equivalence, } \\
& \text { so that }\left(W ; M, M^{\prime}\right) \text { is an } h \text {-cobordism. }
\end{aligned}
$$

Surgery theory asks: is $\mathscr{S}(X)$ non-empty? And if so, then how large is it? In any case, it is clear from the definition that $\mathscr{S}(X)$ is a homotopy invariant of $X$, i.e. that a homotopy equivalence $X \rightarrow Y$ induces a bijection $\mathscr{S}(X) \rightarrow$ $\mathscr{S}(Y)$. Surgery theory reduces $\mathscr{S}(X)$ to more familiar homotopy invariant objects associated to $X$. A homotopy equivalence $f: M^{m} \rightarrow N^{m}$ of $m$-dimensional manifolds determines an element $(M, f) \in \mathscr{S}(N)$, such that $f$ is $h$-cobordant to $1: N \rightarrow N$ if and only if

$$
(M, f)=(N, 1) \in \mathscr{S}(N) .
$$

In particular, if $f$ is homotopic to a diffeomorphism then $f$ is $h$-cobordant to $1: N \rightarrow N$, and $(M, f)=(N, 1) \in \mathscr{S}(N)$.

The determination of $\mathscr{S}(X)$ is closely related to the bundle properties of manifolds and geometric Poincaré complexes.

A finite $C W$ complex $X$ is an $m$-dimensional geometric Poincaré complex if and only if a regular neighbourhood $(Y, \partial Y) \subset S^{m+k}$ of an embedding $X \hookrightarrow$ $S^{m+k}$ is such that

$$
\text { mapping fibre }(\partial Y \rightarrow Y) \simeq S^{k-1} \text {. }
$$

A regular neighbourhood is the $P L$ analogue of a tubular neighbourhood. The $(k-1)$-spherical fibration 


$$
S^{k-1} \rightarrow \partial Y \rightarrow Y \simeq X
$$

is the Spivak normal fibration of a geometric Poincaré complex $X$, with a classifying map

$$
\nu_{X}: X \rightarrow B G=\underset{k}{\lim _{\vec{m}}} B G(k) .
$$

(See Section 9.2 for an exposition of fibrations). The Spivak normal fibration is the homotopy theoretic analogue of the stable normal bundle $\nu_{M}=-\tau_{M}$ of a manifold $M$.

The classifying spaces $B O, B G$ for stable bundles and spherical fibrations are related by a fibration sequence

$$
G / O \rightarrow B O \rightarrow B G \rightarrow B(G / O),
$$

with $G / O$ the classifying space for stable bundles with a fibre homotopy trivialisation. The homotopy class of the composite map

$$
t\left(\nu_{X}\right): X \stackrel{\nu_{X}}{\longrightarrow} B G \longrightarrow B(G / O)
$$

is the primary obstruction to $X$ being homotopy equivalent to an $m$-dimensional manifold. There exists a null-homotopy $t\left(\nu_{X}\right) \simeq\{*\}$ if and only if the Spivak normal fibration $\nu_{X}$ admits a vector bundle reduction $\tilde{\nu}_{X}: X \rightarrow B O$. Surgery theory offers a two-stage programme for deciding if a geometric Poincaré complex $X$ is homotopy equivalent to a manifold, involving the concept of a normal map :

Definition 1.15 A degree 1 normal map from an $m$-dimensional manifold $M^{m}$ to an $m$-dimensional geometric Poincaré complex $X$

$$
(f, b): M^{m} \rightarrow X
$$

is a map $f: M \rightarrow X$ such that

$$
f_{*}[M]=[X] \in H_{m}(X),
$$

together with a stable bundle map $b: \nu_{M} \rightarrow \eta$ over $f$, from the stable normal bundle $\nu_{M}: M \rightarrow B O$ to a stable bundle $\eta: X \rightarrow B O$.

The two stages of the obstruction theory for deciding if an $m$-dimensional geometric Poincaré complex $X$ is homotopy equivalent to an $m$-dimensional manifold are :

(i) Does $X$ admit a degree 1 normal map $(f, b): M^{m} \rightarrow X$ ? This is the case precisely when the map $t\left(\nu_{X}\right): X \rightarrow B(G / O)$ is null-homotopic.

(iii) If the answer to (i) is yes, is there a degree 1 normal map $(f, b): M^{m} \rightarrow X$ which is bordant to a homotopy equivalence $\left(f^{\prime}, b^{\prime}\right): M^{\prime m} \rightarrow X$ ? 
The extent to which a degree 1 normal map $(f, b): M^{m} \rightarrow X$ of connected $M, X$ fails to be a homotopy equivalence is measured by the relative homotopy groups $\pi_{n+1}(f)(n \geqslant 0)$ of pairs of elements

$$
\left(\operatorname{map} g: S^{n} \rightarrow M, \text { null-homotopy } h: f g \simeq *: S^{n} \rightarrow X\right) \text {. }
$$

By J.H.C. Whitehead's Theorem, $f$ is a homotopy equivalence if and only if $\pi_{*}(f)=0$. Let $m=2 n$ or $2 n+1$. It turns out that it is always possible to 'kill' $\pi_{i}(f)$ for $i \leqslant n$, meaning that there is a bordant degree 1 normal map $\left(f^{\prime}, b^{\prime}\right): M^{\prime} \rightarrow X$ with $\pi_{i}\left(f^{\prime}\right)=0$ for $i \leqslant n$. There exists a normal bordism of $(f, b)$ to a homotopy equivalence if and only if it is also possible kill $\pi_{n+1}\left(f^{\prime}\right)$. In general there is an obstruction to killing $\pi_{n+1}\left(f^{\prime}\right)$, which for $m \geqslant 5$ is essentially algebraic in nature:

Wall Surgery Obstruction Theorem 1.16 ([92], 1970)

For any group $\pi$ there are defined algebraic L-groups $L_{m}(\mathbb{Z}[\pi])$ depending only on $m(\bmod 4)$, as groups of stable isomorphism classes of $(-1)^{n}$-quadratic forms over $\mathbb{Z}[\pi]$ for $m=2 n$, and as groups of stable automorphisms of such forms for $m=2 n+1$. An m-dimensional degree 1 normal map $(f, b): M^{m} \rightarrow X$ has a surgery obstruction

$$
\sigma_{*}(f, b) \in L_{m}\left(\mathbb{Z}\left[\pi_{1}(X)\right]\right)
$$

such that $\sigma_{*}(f, b)=0$ if (and for $m \geqslant 5$ only if) $(f, b)$ is bordant to a homotopy equivalence.

If $m=2 n \geqslant 6$ and $(f, b): M^{2 n} \rightarrow X$ is a degree 1 normal map such that $\pi_{i}(f)=0$ for $i \leqslant n$ the surgery obstruction is largely determined by the $(-1)^{n}$ symmetric pairing

$$
\lambda: K \times K \rightarrow \mathbb{Z}\left[\pi_{1}(X)\right]
$$

defined on the kernel $\mathbb{Z}\left[\pi_{1}(X)\right]$-module $K=\pi_{n+1}(f)$ by the intersection of immersions $S^{n} \rightarrow M^{2 n}$ which are null-homotopic in $X$. In order to kill $K$ it is necessary that there be a sufficient number of elements $x \in K$ with $\lambda(x, x)=0$ which are represented by embeddings $x: S^{n} \times D^{n} \hookrightarrow M^{2 n}$. The even-dimensional surgery obstruction will be obtained in Chapter 11, and involves a $(-1)^{n}$-quadratic refinement $\mu$ of the $(-1)^{n}$-symmetric form $(K, \lambda)$. The odd-dimensional surgery obstruction for $m=2 n+1 \geqslant 5$ will be obtained in Chapter 12 .

Example 1.17 The simply-connected surgery obstruction groups are given by:

\begin{tabular}{|c|c|c|c|c|}
\hline$m(\bmod 4)$ & 0 & 1 & 2 & 3 \\
\hline$L_{m}(\mathbb{Z})$ & $\mathbb{Z}$ & 0 & $\mathbb{Z}_{2}$ & 0 \\
\hline
\end{tabular}


The surgery obstruction of a $4 k$-dimensional normal map $(f, b): M^{4 k} \rightarrow X$ with $\pi_{1}(X)=\{1\}$ is

$$
\sigma_{*}(f, b)=\frac{1}{8} \text { signature }\left(K_{2 k}(M), \lambda\right) \in L_{4 k}(\mathbb{Z})=\mathbb{Z}
$$

with $\lambda$ the nonsingular symmetric form on the middle-dimensional homology kernel $\mathbb{Z}$-module

$$
K_{2 k}(M)=\operatorname{ker}\left(f_{*}: H_{2 k}(M) \rightarrow H_{2 k}(X)\right) .
$$

The surgery obstruction of a $(4 k+2)$-dimensional normal map $(f, b): M^{4 k+2} \rightarrow$ $X$ with $\pi_{1}(X)=\{1\}$ is

$$
\sigma_{*}(f, b)=\operatorname{Arf} \text { invariant }\left(K_{2 k+1}\left(M ; \mathbb{Z}_{2}\right), \lambda, \mu\right) \in L_{4 k+2}(\mathbb{Z})=\mathbb{Z}_{2}
$$

with $\lambda, \mu$ the nonsingular quadratic form on the middle-dimensional $\mathbb{Z}_{2}$-coefficient homology kernel $\mathbb{Z}_{2}$-module

$$
K_{2 k+1}\left(M ; \mathbb{Z}_{2}\right)=\operatorname{ker}\left(f_{*}: H_{2 k+1}\left(M ; \mathbb{Z}_{2}\right) \rightarrow H_{2 k+1}\left(X ; \mathbb{Z}_{2}\right)\right) .
$$

Surgery Exact Sequence 1.18 (Browder, Novikov, Sullivan, Wall 1962-1970) Let $m \geqslant 5$.

(i) The manifold structure set $\mathscr{S}(X)$ of an m-dimensional geometric Poincaré complex $X$ is non-empty if and only if there exists a normal map $(f, b): M^{m} \rightarrow$ $X$ with surgery obstruction

$$
\sigma_{*}(f, b)=0 \in L_{m}\left(\mathbb{Z}\left[\pi_{1}(X)\right]\right) .
$$

(ii) The structure set $\mathscr{S}(M)$ of an m-dimensional manifold $M^{m}$ fits into the surgery exact sequence of pointed sets

$$
\ldots \rightarrow L_{m+1}\left(\mathbb{Z}\left[\pi_{1}(M)\right]\right) \rightarrow \mathscr{S}(M) \rightarrow[M, G / O] \rightarrow L_{m}\left(\mathbb{Z}\left[\pi_{1}(M)\right]\right) .
$$

The surgery exact sequence will be obtained in Chapter 13. The restriction $m \geqslant 5$ is due to the use of the Whitney Embedding Theorem (1.7) in the proof, exactly as in the $h$ - and $s$-Cobordism Theorems.

The geometric surgery construction works just as well in the low dimensions $m \leqslant 4$. However, the possible geometric surgeries and their effect (e.g. on the fundamental group) are much harder to relate to algebra than in the higher dimensions. The type of algebraic surgery considered in the book thus only deals with the 'high-dimensional' part of 3- and 4-dimensional topology. 


\section{MANIFOLDS}

This chapter gives the basic constructions of geometric surgery.

Sections 2.1 and 2.2 give the official definitions of manifolds and surgery. Section 2.3 is a rapid introduction to Morse theory, including the key fact that every manifold $M$ admits a Morse function $f: M \rightarrow \mathbb{R}$. Section 2.4 introduces handles, which are the building blocks of manifolds and cobordisms. The main result of this chapter is the Handle Decomposition Theorem 2.22 : a Morse function $f: M \rightarrow \mathbb{R}$ on a manifold $M$ determines a handle decomposition

$$
M=\bigcup_{i=0}^{m}\left(h^{i} \cup h^{i} \cup \ldots \cup h^{i}\right)
$$

with one $i$-handle $h^{i}=D^{i} \times D^{m-i}$ for each critical point of $f$ of index $i$. More precisely, if $a<b \in \mathbb{R}$ are regular values of $f$ then $f^{-1}(a), f^{-1}(b) \subset M$ are codimension 1 submanifolds such that $f^{-1}(b)$ is obtained from $f^{-1}(a)$ by a sequence of surgeries, one for each critical value in $[a, b] \subset \mathbb{R}$, and $f^{-1}([a, b])$ is the union of the traces of the surgeries.

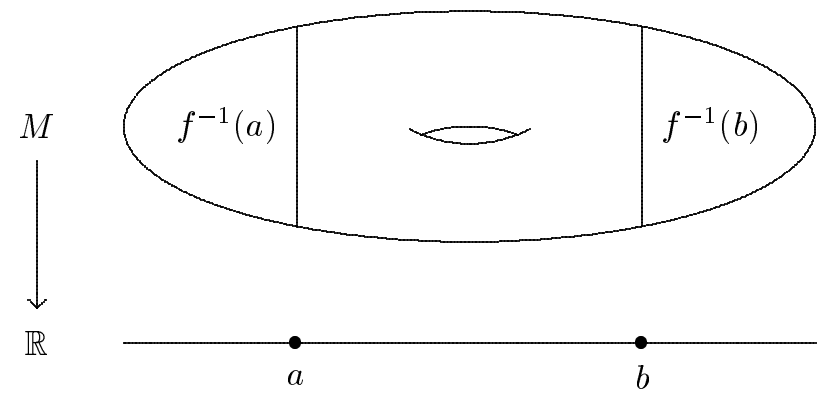

Morse functions and handle decompositions are highly non-unique. Indeed, surgery theory is essentially the study of the possible handle structures on manifolds and cobordisms.

\subsection{Differentiable manifolds}

It is assumed that the reader has already had a first course on differentiable manifolds, such as Bredon [10] or Hirsch [33]. 
Definition 2.1 An $m$-dimensional differentiable manifold $M^{m}$ is a paracompact Hausdorff topological space with a maximal atlas of charts

$$
\mathscr{U}=\left\{\left(U \subseteq M, \phi: \mathbb{R}^{m} \rightarrow U\right)\right\}
$$

of open neighbourhoods $U \subseteq M$ with a homeomorphism $\phi: \mathbb{R}^{m} \rightarrow U$, such that for every $(U, \phi),\left(U^{\prime}, \phi^{\prime}\right) \in \mathscr{U}$ the transition function

$$
\phi^{\prime-1} \phi \mid: \phi^{-1}\left(U \cap U^{\prime}\right) \rightarrow U \cap U^{\prime} \rightarrow \phi^{\prime-1}\left(U \cap U^{\prime}\right)
$$

is a diffeomorphism of open subsets of $\mathbb{R}^{m}$.

There is a corresponding notion of a differentiable manifold with boundary $(M, \partial M)$.

Definition 2.2 (i) A differentiable map of manifolds $f: N^{n} \rightarrow M^{m}$ is a map such that for any charts $\left(U \subseteq M, \phi: \mathbb{R}^{m} \rightarrow U\right) \in \mathscr{U}_{M},\left(V \subseteq N, \psi: \mathbb{R}^{n} \rightarrow V\right) \in$ $\mathscr{U}_{N}$ with $f(V) \subseteq U$ the function $\phi^{-1}(f \mid) \psi: \mathbb{R}^{n} \rightarrow \mathbb{R}^{m}$ is differentiable.

(ii) A diffeomorphism $f: N \rightarrow M$ is a differentiable map which is a homeomorphism with a differentiable inverse $f^{-1}: M \rightarrow N$.

(iii) An embedding of manifolds $f: N^{n} \hookrightarrow M^{m}$ is a differentiable map which is injective, i.e. the inclusion of a submanifold.

(iv) An isotopy between embeddings of manifolds $f_{0}, f_{1}: N^{n} \hookrightarrow M^{m}$ is a homotopy

$$
f: N \times I \rightarrow M ;(x, t) \mapsto f_{t}(x)
$$

which is an embedding $f_{t}: N \hookrightarrow M$ at each level $t \in I$.

(v) An immersion of manifolds $f: N^{n} \uparrow M^{m}$ is a differentiable map which is locally an embedding, i.e. such that for every $x \in N$ there exists a chart $(V, \psi) \in \mathscr{U}_{N}$ such that $x \in V$ and $(f \mid) \psi: \mathbb{R}^{n} \rightarrow M$ is an embedding.

(vi) A regular homotopy of immersions $f_{0}, f_{1}: N^{n} \uparrow \rightarrow M^{m}$ is a homotopy

$$
f: N \times I \rightarrow M ;(x, t) \mapsto f_{t}(x)
$$

which is an immersion $f_{t}: N \leftrightarrow M$ at each level $t \in I$.

In particular, an embedding is an immersion, and isotopic embeddings are regular homotopic.

In Chapter 5 we shall describe the neighbourhoods of submanifolds in terms of vector bundles. The following is an important special case :

Definition 2.3 An embedding of a submanifold $N^{n} \hookrightarrow M^{m}$ is framed if it extends to an embedding $N \times D^{m-n} \hookrightarrow M$. 


\subsection{Surgery}

The input of a surgery on a manifold is a framed embedding of a sphere:

Definition 2.4 (i) An $n$-embedding in an $m$-dimensional manifold $M^{m}$ is an embedding

$$
g: S^{n} \hookrightarrow M .
$$

(ii) A framed $n$-embedding in $M$ is an embedding

$$
\bar{g}: S^{n} \times D^{m-n} \hookrightarrow M,
$$

with core $n$-embedding

$$
g=\bar{g} \mid: S^{n}=S^{n} \times\{0\} \hookrightarrow M .
$$

The effect of a surgery is another manifold:

Definition 2.5 An $n$-surgery on an $m$-dimensional manifold $M^{m}$ is the surgery removing a framed $n$-embedding $\bar{g}: S^{n} \times D^{m-n} \hookrightarrow M$, and replacing it with $D^{n+1} \times S^{m-n-1}$, with effect the $m$-dimensional manifold

$$
M^{\prime m}=\operatorname{cl} .\left(M^{m} \backslash \bar{g}\left(S^{n} \times D^{m-n}\right)\right) \cup_{S^{n} \times S^{m-n-1}} D^{n+1} \times S^{m-n-1} .
$$

The applications of surgery to the classification of manifolds require a plentiful supply of framed $n$-embeddings $S^{n} \times D^{m-n} \hookrightarrow M^{m}$. The Whitney Embedding Theorem (7.2) shows that for $2 n<m$ every map $S^{n} \rightarrow M^{m}$ can be approximated by an $n$-embedding. However, in general an $n$-embedding $S^{n} \hookrightarrow M$ cannot be extended to a framed $n$-embedding $S^{n} \times D^{m-n} \hookrightarrow M$ - see 5.66 below for a specific example.

The notion of a cobordism $\left(W ; M, M^{\prime}\right)$ was defined in 1.1. The trace of an $n$-surgery removing $S^{n} \times D^{m-n} \hookrightarrow M^{m}$ was defined in 1.4 to be the cobordism $\left(W ; M, M^{\prime}\right)$ with

$$
W^{m+1}=M^{m} \times I \cup_{S^{n} \times D^{m-n} \times\{1\}} D^{n+1} \times D^{m-n} .
$$

Definition 2.6 The dual $(m-n-1)$-surgery on $M^{\prime}$ removes the dual framed $(m-n-1)$-embedding $D^{n+1} \times S^{m-n-1} \hookrightarrow M^{\prime}$ with effect $M$ and trace $\left(W ; M^{\prime}, M\right)$.

If $M$ is closed $(\partial M=\emptyset)$ then so is $M^{\prime}$. If $M$ has non-empty boundary $\partial M$ the embedding $S^{n} \times D^{m-n} \hookrightarrow M$ is required to avoid $\partial M$, so that

$$
\partial M^{\prime}=\partial M .
$$


Example 2.7 A (-1)-surgery on an $m$-dimensional manifold $M$ has effect the disjoint union

$$
M^{\prime}=M \cup S^{m} .
$$

The dual $m$-surgery on $M^{\prime}$ has effect $M$.

Example 2.8 (i) The effect on $S^{m}$ of the $n$-surgery removing the framed $n$ embedding defined in 1.2

$g: S^{n} \times D^{m-n} \hookrightarrow S^{n} \times D^{m-n} \cup D^{n+1} \times S^{m-n-1}=\partial\left(D^{n+1} \times D^{m-n}\right)=S^{m}$

is the $m$-dimensional manifold

$$
D^{n+1} \times S^{m-n-1} \cup D^{n+1} \times S^{m-n-1}=S^{n+1} \times S^{m-n-1} .
$$

The trace of the $n$-surgery $\left(W^{m+1} ; S^{m}, S^{n+1} \times S^{m-n-1}\right)$ can be viewed as

$$
W=\operatorname{cl} .\left(S^{n+1} \times D^{m-n} \backslash D^{m+1}\right),
$$

using any embedding $D^{m+1} \hookrightarrow S^{n+1} \times D^{m-n}$ in the interior.

(ii) The special case $m=1, n=0$ of (i) gives a framed 0 -embedding in $S^{1}$

$$
g: S^{0} \times D^{1}=D^{1} \cup D^{1} \hookrightarrow S^{1} .
$$

The 0-surgery on $S^{1}$ removing $g\left(S^{0} \times D^{1}\right)$ has effect the disjoint union of two circles

$$
S^{1} \times S^{0}=S^{1} \cup S^{1} .
$$

The trace is the cobordism $\left(W^{2} ; S^{1}, S^{1} \cup S^{1}\right)$ obtained from $S^{2}$ by punching out the interior of an embedding $D^{2} \cup D^{2} \cup D^{2} \hookrightarrow S^{2}$.

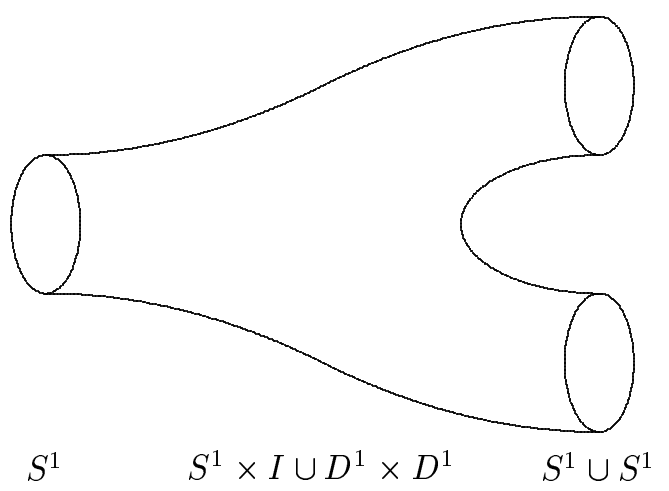

(iii) Modify the framed 0-embedding $g: S^{0} \times D^{1} \hookrightarrow S^{1}$ in (ii) by twisting one of the two embeddings of $D^{1}$ by the diffeomorphism

$$
\omega: D^{1} \rightarrow D^{1} ; t \mapsto-t,
$$


defining a different 0-embedding

$$
g_{\omega}: S^{0} \times D^{1} \stackrel{1 \cup \omega}{\longrightarrow} S^{0} \times D^{1} \stackrel{g}{\longrightarrow} S^{1}
$$

with the same core as $g$. The 0 -surgery on $S^{1}$ removing $g_{\omega}\left(S^{0} \times D^{1}\right)$ has effect a single circle $S^{1}$. The trace is the cobordism $\left(N^{2} ; S^{1}, S^{1}\right)$ obtained from the Möbius band $M^{2}$ by punching out the interior of an embedding $D^{2} \hookrightarrow M \backslash \partial M$.

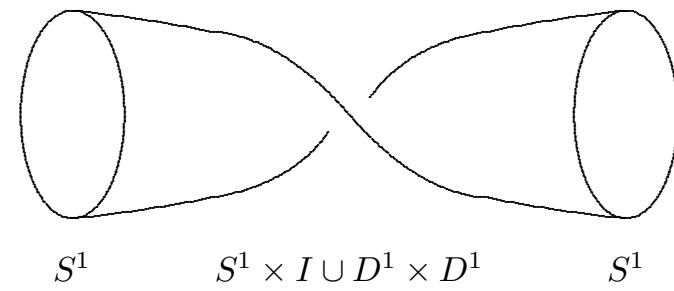

Definition 2.9 The connected sum of connected $m$-dimensional manifolds $M^{m}, M^{\prime m}$ is the connected $m$-dimensional manifold

$$
\left(M \# M^{\prime}\right)^{m}=\operatorname{cl} .\left(M \backslash D^{m}\right) \cup\left(S^{m-1} \times I\right) \cup \operatorname{cl} .\left(M^{\prime} \backslash D^{m}\right)
$$

obtained by excising the interiors of embedded discs $D^{m} \hookrightarrow M, D^{m} \hookrightarrow M^{\prime}$ and joining the boundary components $S^{m-1} \hookrightarrow \operatorname{cl} .\left(M \backslash D^{m}\right), S^{m-1} \hookrightarrow \operatorname{cl} .\left(M^{\prime} \backslash D^{m}\right)$ by $S^{m-1} \times I$.

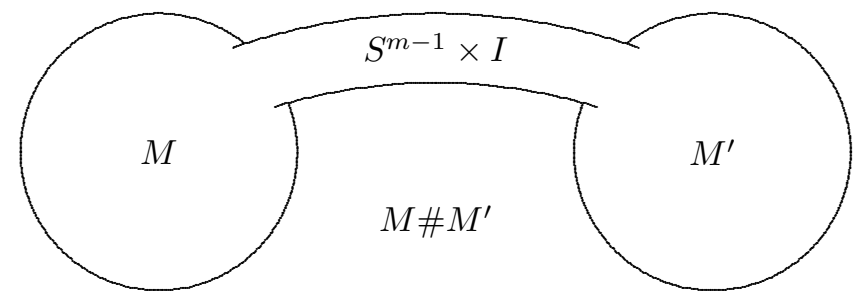

Example 2.10 The connected sum $M \# M^{\prime}$ is the effect of the 0-surgery on the disjoint union $M \cup M^{\prime}$ which removes the framed 0-embedding $S^{0} \times D^{m} \hookrightarrow$ $M \cup M^{\prime}$ defined by the disjoint union of embeddings $D^{m} \hookrightarrow M, D^{m} \hookrightarrow M^{\prime}$.

\subsection{Morse theory}

Morse theory studies differentiable manifolds $M$ by considering the critical points of differentiable functions $f: M \rightarrow \mathbb{R}$ for which the second differential is nontrivial. This section is only a very rudimentary account - see Milnor [55] for the classic exposition of Morse theory. 
It is assumed that the reader is already acquainted with $C W$ complexes, which are spaces obtained from $\emptyset$ by successively attaching cells

$$
X=\bigcup_{i=0}^{\infty}\left(D^{i} \cup D^{i} \cup \ldots \cup D^{i}\right)
$$

of increasing dimension $i$. Morse theory is used to prove that an $m$-dimensional manifold $M$ can be obtained from $\emptyset$ by successively attaching handles

$$
M=\bigcup_{i=0}^{m}\left(D^{i} \times D^{m-i} \cup D^{i} \times D^{m-i} \cup \ldots \cup D^{i} \times D^{m-i}\right)
$$

of increasing index $i$, giving $M$ the structure of a $C W$ complex. However, there is an essential difference: the cell structure of a $C W$ complex is part of the definition, whereas a handle decomposition of a manifold has to be proved to exist.

Here is the basic connection between Morse theory, handles and surgery. If $a<b \in \mathbb{R}$ are regular values of a Morse function $f: M^{m} \rightarrow \mathbb{R}$ then

$$
\left(M[a, b] ; N_{a}, N_{b}\right)=f^{-1}([a, b] ;\{a\},\{b\})
$$

is a cobordism of $(m-1)$-dimensional manifolds. If every $t \in[a, b]$ is a regular value then each $N_{t}=f^{-1}(t)$ is diffeomorphic to $N_{a}$, with a diffeomorphism

$$
\left(M[a, b] ; N_{a}, N_{b}\right) \cong N_{a} \times(I ;\{0\},\{1\}) .
$$

If $[a, b]$ consists of regular values except for one critical value of index $i$ then $\left(M[a, b] ; N_{a}, N_{b}\right)$ is the trace of an $(i-1)$-surgery on $N_{a}$, with

$$
M[a, b] \cong N_{a} \times I \cup D^{i} \times D^{m-i}
$$

obtained from $N_{a} \times I$ by attaching an $i$-handle. Thus $M$ is obtained from $\emptyset$ by attaching an $i$-handle for each critical value of $f$ with index $i$, and $M$ has the structure of a finite $C W$ complex with one $i$-cell for each $i$-handle.

The differential of a differentiable function $f: N \rightarrow M$ at $x \in N$ is defined using any charts $\left(U \subseteq M, \phi: \mathbb{R}^{m} \rightarrow U\right) \in \mathscr{U}_{M},\left(V \subseteq N, \psi: \mathbb{R}^{n} \rightarrow V\right) \in \mathscr{U}_{N}$ such that $x \in V, f(x) \in U$. The function

$$
(\phi)^{-1} f \psi: \mathbb{R}^{n} \rightarrow \mathbb{R}^{m} ; x=\left(x_{1}, x_{2}, \ldots, x_{n}\right) \mapsto\left(f_{1}(x), f_{2}(x), \ldots, f_{m}(x)\right)
$$

is differentiable, and the differential of $f$ at $x$ is the linear map given by the Jacobian $m \times n$ matrix

$$
\begin{aligned}
& d f(x)=\left(\frac{\partial f_{i}}{\partial x_{j}}\right): \mathbb{R}^{n} \rightarrow \mathbb{R}^{m} ; \\
& h=\left(h_{1}, h_{2}, \ldots, h_{n}\right) \mapsto d f(x)(h)=\left(\sum_{j=1}^{n} \frac{\partial f_{1}}{\partial x_{j}} h_{j}, \sum_{j=1}^{n} \frac{\partial f_{2}}{\partial x_{j}} h_{j}, \ldots, \sum_{j=1}^{n} \frac{\partial f_{m}}{\partial x_{j}} h_{j}\right) .
\end{aligned}
$$


Definition 2.11 Let $f: N^{n} \rightarrow M^{m}$ be a differentiable map.

(i) A regular point of $f$ is a point $x \in N$ where the differential $d f(x): \mathbb{R}^{n} \rightarrow \mathbb{R}^{m}$ is a linear map of maximal rank, i.e.

$$
\operatorname{rank}(d f(x))=\min (m, n) .
$$

(ii) A critical point of $f$ is a point $x \in N$ which is not regular.

(iii) A regular value of $f$ is a point $y \in M$ such that every $x \in f^{-1}(y) \subseteq N$ is regular (including the empty case $f^{-1}(y)=\emptyset$ ).

(iv) A critical value of $f$ is a point $y \in M$ which is not regular.

Implicit Function Theorem 2.12 The inverse image of a regular value $y \in$ $M$ of a differentiable map $f: N^{n} \rightarrow M^{m}$ is a submanifold

$$
P=f^{-1}(y) \subseteq N
$$

with

$$
\operatorname{dim}(P)=n-\min (m, n)=\left\{\begin{array}{ll}
n-m & \text { if } m \leqslant n \\
0 & \text { if } m>n
\end{array} .\right.
$$

Proof See Chapter II.1 of Bredon [10].

The Taylor expansion of a differentiable function $f: M^{m} \rightarrow \mathbb{R}$ at $x \in M$ is given in local coordinates by

$$
\begin{gathered}
f\left(x_{1}+h_{1}, x_{2}+h_{2}, \ldots, x_{m}+h_{m}\right) \\
=f\left(x_{1}, x_{2}, \ldots, x_{m}\right)+\sum_{k=1}^{\infty} \frac{1}{k !} \sum_{1 \leqslant i_{1}, i_{2}, \ldots, i_{k} \leqslant m} \frac{\partial^{k} f}{\partial x_{i_{1}} \partial x_{i_{2}} \ldots \partial x_{i_{k}}} h_{i_{1}} h_{i_{2}} \ldots h_{i_{k}} \in \mathbb{R}, \\
\left(\left(h_{1}, h_{2}, \ldots, h_{m}\right) \in \mathbb{R}^{m}\right) .
\end{gathered}
$$

The linear term in the Taylor series

$$
L\left(h_{1}, h_{2}, \ldots, h_{m}\right)=\sum_{i=1}^{m} \frac{\partial f}{\partial x_{i}} h_{i}
$$

is determined by the differential of $f$ at $x\left(=\right.$ the gradient vector $\left.\nabla f(x) \in \mathbb{R}^{m}\right)$

$$
d f(x)=\left(\frac{\partial f}{\partial x_{1}} \quad \frac{\partial f}{\partial x_{2}} \ldots \frac{\partial f}{\partial x_{m}}\right): \mathbb{R}^{m} \rightarrow \mathbb{R} ;\left(h_{1}, h_{2}, \ldots, h_{m}\right) \mapsto \sum_{i=1}^{m} \frac{\partial f}{\partial x_{i}} h_{i},
$$

which is either 0 or has the maximal rank 1 . Thus $x \in M$ is a regular point of $f$ if $d f(x) \neq 0$, and $x \in M$ is a critical point of $f$ if $d f(x)=0$. The quadratic term in the Taylor series

$$
Q\left(h_{1}, h_{2}, \ldots, h_{m}\right)=\sum_{i=1}^{m} \sum_{j=1}^{m}\left(\frac{\partial^{2} f}{\partial x_{i} \partial x_{j}}\right) h_{i} h_{j} / 2
$$


is determined by the Hessian $m \times m$ matrix of second partial derivatives

$$
H(x)=\left(\frac{\partial^{2} f}{\partial x_{i} \partial x_{j}}\right) .
$$

Definition 2.13 Let $f: M^{m} \rightarrow \mathbb{R}$ be a differentiable function on an $m$-dimensional manifold.

(i) A critical point $x \in M$ of $f$ is nondegenerate if the Hessian matrix $H(x)$ is invertible.

(ii) The index $\operatorname{Ind}(x)$ of a nondegenerate critical point $x \in M$ is the number of negative eigenvalues in $H(x)$, so that with respect to appropriate local coordinates the quadratic term in the Taylor series of $f$ near $x$ is given by

$$
Q\left(h_{1}, h_{2}, \ldots, h_{m}\right)=-\sum_{i=1}^{\operatorname{Ind}(x)}\left(h_{i}\right)^{2}+\sum_{i=\operatorname{Ind}(x)+1}^{m}\left(h_{i}\right)^{2} \in \mathbb{R} .
$$

(iii) The function $f$ is Morse if it has only nondegenerate critical points.

In dealing with Morse functions $f: M \rightarrow \mathbb{R}$ it will be assumed that the critical points $x_{1}, x_{2}, \ldots \in M$ have distinct images $f\left(x_{1}\right), f\left(x_{2}\right), \ldots \in \mathbb{R}$. The index of a critical value $f\left(x_{j}\right) \in \mathbb{R}$ is then defined by

$$
\operatorname{Ind}\left(f\left(x_{j}\right)\right)=\operatorname{Ind}\left(x_{j}\right) \geqslant 0 .
$$

Theorem 2.14 (Morse)

Every $m$-dimensional manifold $M^{m}$ admits a Morse function $f: M \rightarrow \mathbb{R}$.

Proof There exists an embedding $M^{m} \hookrightarrow S^{m+k}$ for $k$ large, by the Whitney Embedding Theorem (1.7). The function defined for any $a \in S^{m+k} \backslash M$ by

$$
f_{a}: M \rightarrow \mathbb{R} ; x \mapsto\|x-a\|
$$

is Morse for all $a$ except for a set of measure 0. See Milnor [55] or Chapter 6 of Hirsch [33] for more detailed accounts!

In fact, the set of Morse functions is dense in the function space of all differentiable functions $f: M \rightarrow \mathbb{R}$.

Example 2.15 The height function on the $m$-sphere

$$
S^{m}=\left\{\left(x_{0}, x_{1}, \ldots, x_{m}\right) \in \mathbb{R}^{m+1} \mid \sum_{k=0}^{m} x_{k}^{2}=1\right\}
$$

is a Morse function 


$$
f: S^{m} \rightarrow \mathbb{R} ;\left(x_{0}, x_{1}, \ldots, x_{m}\right) \mapsto x_{m}
$$

with a critical point $(0, \ldots, 0,-1)$ of index 0 and a critical point $(0, \ldots, 0,1)$ of index $m$.

Example 2.16 The $m$-dimensional real projective space $\mathbb{R P}^{m}$ is the quotient of $S^{m}$ by the antipodal map

$$
\mathbb{R P}^{m}=S^{m} /\left\{\left(x_{1}, x_{2}, \ldots, x_{m+1}\right) \sim\left(-x_{1},-x_{2}, \ldots,-x_{m+1}\right)\right\} .
$$

Equivalently, $\mathbb{R P}^{m}$ is the space with one point for each 1-dimensional subspace of the $(m+1)$-dimensional real vector space $\mathbb{R}^{m+1}$

$$
\left[x_{0}, x_{1}, \ldots, x_{m}\right]=\left\{\lambda\left(x_{0}, x_{1}, \ldots, x_{m}\right) \mid \lambda \in \mathbb{R}\right\} \subset \mathbb{R}^{m+1}\left(x_{k} \in \mathbb{R} \text {, not all } 0\right) .
$$

For any real numbers $\lambda_{0}<\lambda_{1}<\ldots<\lambda_{m}$ there is defined a Morse function

$$
f: \mathbb{R}^{m} \rightarrow \mathbb{R} ;\left[x_{0}, x_{1}, \ldots, x_{m}\right] \mapsto \frac{\sum_{k=0}^{m} \lambda_{k}\left(x_{k}\right)^{2}}{\sum_{k=0}^{m}\left(x_{k}\right)^{2}}
$$

with $(m+1)$ critical points $[0, \ldots, 0,1,0, \ldots, 0]$ of index $0,1, \ldots, m$.

Example 2.17 The $m$-dimensional complex projective space $\mathbb{C} \mathbb{P}^{m}$ is the $2 m$ dimensional manifold with one point for each 1-dimensional subspace of the $(m+1)$-dimensional complex vector space $\mathbb{C}^{m+1}$

$$
\left[z_{0}, z_{1}, \ldots, z_{m}\right]=\left\{\lambda\left(z_{0}, z_{1}, \ldots, z_{m}\right) \mid \lambda \in \mathbb{C}\right\} \hookrightarrow \mathbb{C}^{m+1} \quad\left(z_{k} \in \mathbb{C}, \text { not all } 0\right) .
$$

For any real numbers $\lambda_{0}<\lambda_{1}<\ldots<\lambda_{m}$ there is defined a Morse function

$$
f: \mathbb{C} \mathbb{P}^{m} \rightarrow \mathbb{R} ;\left[z_{0}, z_{1}, \ldots, z_{m}\right] \mapsto \frac{\sum_{k=0}^{m} \lambda_{k}\left|z_{k}\right|^{2}}{\sum_{k=0}^{m}\left|z_{k}\right|^{2}}
$$

with $(m+1)$ critical points $[0, \ldots, 0,1,0, \ldots, 0]$ of index $0,2, \ldots, 2 m$.

\subsection{Handles}

Definition 2.18 (i) Given an $(m+1)$-dimensional manifold with boundary $(W, \partial W)$ and an embedding

$$
S^{i-1} \times D^{m-i+1} \hookrightarrow \partial W \quad(0 \leqslant i \leqslant m+1)
$$

define the $(m+1)$-dimensional manifold with boundary $\left(W^{\prime}, \partial W^{\prime}\right)$ obtained from $W$ by attaching an $i$-handle to be 


$$
W^{\prime}=W \cup_{S^{i-1} \times D^{m-i+1}} D^{i} \times D^{m-i+1} .
$$

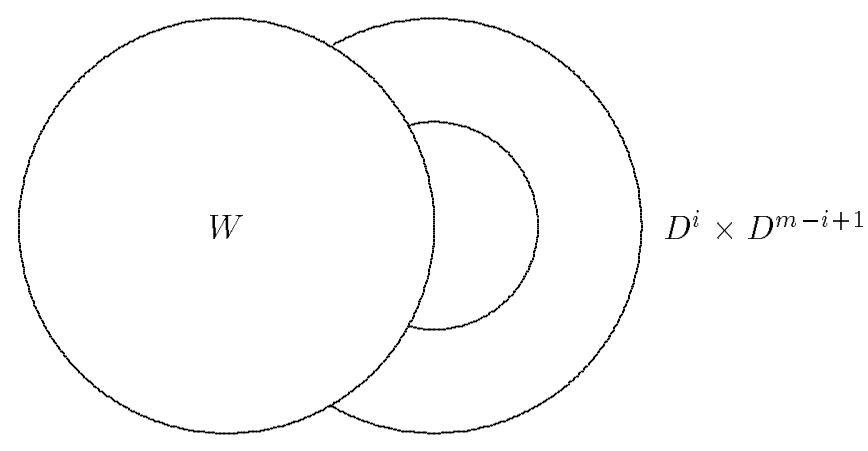

$W^{\prime}$

(ii) An elementary $(m+1)$-dimensional cobordism of index $i$ is the cobor$\operatorname{dism}\left(W ; M, M^{\prime}\right)$ obtained from $M \times I$ by attaching an $i$-handle at

$$
S^{i-1} \times D^{m-i+1} \hookrightarrow M \times\{1\}
$$

with

$$
W=M \times I \cup D^{i} \times D^{m-i+1} .
$$

(iii) The dual of an elementary $(m+1)$-dimensional cobordism $\left(W ; M, M^{\prime}\right)$ of index $i$ is the elementary $(m+1)$-dimensional cobordism $\left(W ; M^{\prime}, M\right)$ of index $(m-i+1)$ obtained by reversing the ends, and regarding the $i$-handle attached to $M \times I$ as an $(m-i+1)$-handle attached to $M^{\prime} \times I$.

Lemma 2.19 For any $0 \leqslant i \leqslant m+1$ the Morse function

$$
f: D^{m+1} \rightarrow \mathbb{R} ;\left(x_{1}, x_{2}, \ldots, x_{m+1}\right) \mapsto-\sum_{j=1}^{i} x_{j}^{2}+\sum_{j=i+1}^{m+1} x_{j}^{2}
$$

has a unique interior critical point $0 \in D^{m+1}$, which is of index $i$. The $(m+1)$ dimensional manifolds with boundary defined for $0<\epsilon<1$ by

$$
W_{-\epsilon}=f^{-1}(-\infty,-\epsilon], W_{\epsilon}=f^{-1}(-\infty, \epsilon]
$$

are such that $W_{\epsilon}$ is obtained from $W_{-\epsilon}$ by attaching an $i$-handle

$$
W_{\epsilon}=W_{-\epsilon} \cup D^{i} \times D^{m-i+1} .
$$

Here is an illustration in the case $m=i=1$ : 


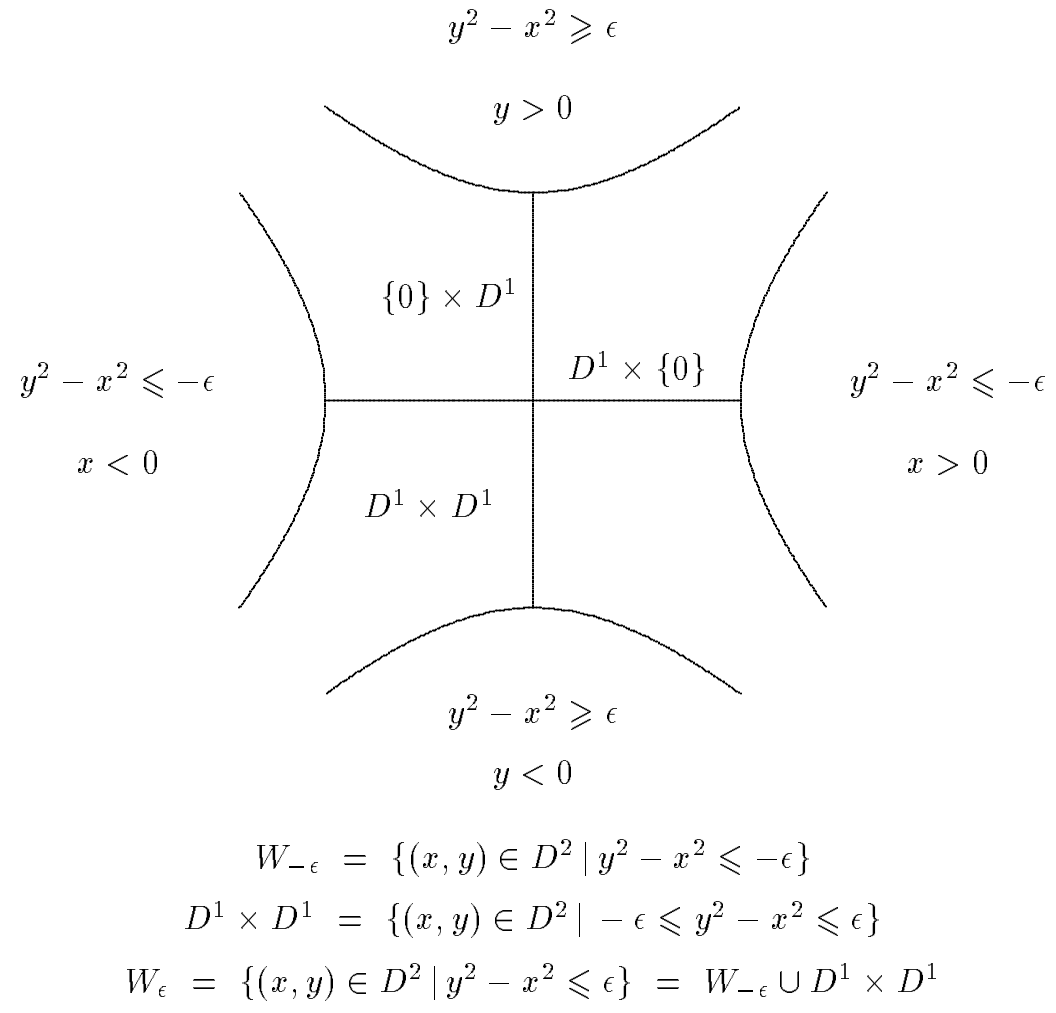

Proposition 2.20 Let $f: W^{m+1} \rightarrow I$ be a Morse function on an $(m+1)$ dimensional manifold cobordism $\left(W ; M, M^{\prime}\right)$ with

$$
f^{-1}(0)=M, f^{-1}(1)=M^{\prime},
$$

and such that all the critical points of $f$ are in the interior of $W$.

(i) If $f$ has no critical points then $\left(W ; M, M^{\prime}\right)$ is a trivial h-cobordism, with a diffeomorphism

$$
\left(W ; M, M^{\prime}\right) \cong M \times(I ;\{0\},\{1\})
$$

which is the identity on $M$.

(ii) If $f$ has a single critical point of index $i$ then $W$ is obtained from $M \times I$ by attaching an i-handle using an embedding $S^{i-1} \times D^{m-i+1} \hookrightarrow M \times\{1\}$, and $\left(W ; M, M^{\prime}\right)$ is an elementary cobordism of index $i$ with a diffeomorphism

$$
\left(W ; M, M^{\prime}\right) \cong\left(M \times I \cup D^{i} \times D^{m-i+1} ; M \times\{0\}, M^{\prime}\right) .
$$

Proof (i) See Milnor [55].

(ii) In a neighbourhood of the unique critical point $p \in W$

$$
f\left(p+\left(x_{1}, x_{2}, \ldots, x_{m+1}\right)\right)=f(p)-\sum_{j=1}^{i}\left(x_{j}\right)^{2}+\sum_{j=i+1}^{m+1}\left(x_{j}\right)^{2}
$$


with respect to a coordinate chart $\mathbb{R}^{m+1} \hookrightarrow W$ such that $0 \in \mathbb{R}^{m+1}$ corresponds to $p \in W$, with $f(p) \in \mathbb{R}$ the critical value. For any $\epsilon>0$ there are defined diffeomorphisms

$$
f^{-1}(-\infty, c-\epsilon] \cong M \times I, \quad f^{-1}[c+\epsilon, \infty) \cong M^{\prime} \times I
$$

by (i), and by 2.19 there is defined a diffeomorphism

$$
f^{-1}[c-\epsilon, c+\epsilon] \cong M \times I \cup D^{i} \times D^{m-i+1} .
$$

Attaching a handle (2.18) to a manifold with boundary is a surgery on the boundary:

Proposition 2.21 If an $(m+1)$-dimensional manifold with boundary $\left(W^{\prime}, \partial W^{\prime}\right)$ is obtained from $(W, \partial W)$ by attaching an $i$-handle

$$
W^{\prime}=W \cup_{S^{i-1} \times D^{m-i+1}} D^{i} \times D^{m-i+1}
$$

then $\partial W^{\prime}$ is obtained from $\partial W$ by an $(i-1)$-surgery

$$
\partial W^{\prime}=\operatorname{cl} .\left(\partial W \backslash\left(S^{i-1} \times D^{m-i+1}\right)\right) \cup_{S^{i-1} \times S^{m-i}} D^{i} \times S^{m-i} .
$$

Proof By construction.

Somewhat by analogy with the result that every finite-dimensional vector space has a finite basis:

Handle Decomposition Theorem 2.22 (Thom [87], Milnor [53])

(i) Every cobordism $\left(W^{m+1} ; M^{m}, M^{\prime m}\right)$ has a handle decomposition as the union of a finite sequence

$$
\left(W ; M, M^{\prime}\right)=\left(W_{1} ; M_{0}, M_{1}\right) \cup\left(W_{2} ; M_{1}, M_{2}\right) \cup \ldots \cup\left(W_{k} ; M_{k-1}, M_{k}\right)
$$

of adjoining elementary cobordisms $\left(W_{j} ; M_{j-1}, M_{j}\right)$ with index $i_{j}$, such that

$$
0 \leqslant i_{1} \leqslant i_{2} \leqslant \ldots \leqslant i_{k} \leqslant m+1, M_{0}=M, M_{k}=M^{\prime} .
$$
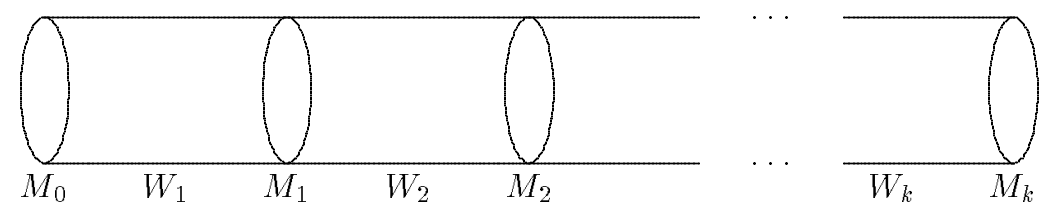

(ii) Closed m-dimensional manifolds $M, M^{\prime}$ are cobordant if and only if $M^{\prime}$ can be obtained from $M$ by a sequence of surgeries. 
Proof (i) By the relative version of (2.14) any cobordism admits a Morse function

$$
f:\left(W ; M, M^{\prime}\right) \rightarrow I
$$

with $M=f^{-1}(0), M^{\prime}=f^{-1}(1)$, and such that all the critical values are in the interior of $I$. Since $W$ is compact there is only a finite number of critical points : label them $p_{j} \in W(1 \leqslant j \leqslant k)$. Write the critical values as $c_{j}=f\left(p_{j}\right) \in \mathbb{R}$, and let $i_{j}$ be the index of $p_{j}$. It is possible to choose $f$ such that

$$
0<c_{1}<c_{2} \ldots<c_{k}<1,0 \leqslant i_{1} \leqslant i_{2} \leqslant \ldots \leqslant i_{k} \leqslant m+1 .
$$

Let $r_{j} \in I(0 \leqslant j \leqslant k)$ be regular values such that

$$
0=r_{0}<c_{1}<r_{1}<c_{2}<\ldots<r_{k-1}<c_{k}<r_{k}=1 .
$$

By 2.20 (i) each

$$
\left(W_{j} ; M_{j-1}, M_{j}\right)=f^{-1}\left(\left[r_{j-1}, r_{j}\right] ;\left\{r_{j-1}\right\},\left\{r_{j}\right\}\right)(1 \leqslant j \leqslant k)
$$

is an elementary cobordism of index $i_{j}$.

(ii) The trace of a surgery is an elementary cobordism (2.18). Thus surgeryequivalent manifolds are cobordant. Conversely, note that every elementary cobordism is the trace of a surgery, and that by (i) every cobordism $\left(W ; M, M^{\prime}\right)$ is a union of elementary cobordisms.

If $\left(W ; M, M^{\prime}\right)$ has a Morse function $f: W \rightarrow I$ with critical points of index $0 \leqslant i_{0} \leqslant i_{1} \leqslant \ldots \leqslant i_{k} \leqslant m+1$ then $W$ has a handle decomposition

$$
W=M \times I \cup h^{i_{0}} \cup h^{i_{1}} \cup \ldots \cup h^{i_{k}}
$$

with $h^{i}=D^{i} \times D^{m-i+1}$ a handle of index $i$.

Corollary 2.23 Every closed $m$-dimensional manifold $M^{m}$ can be obtained from $\emptyset$ by attaching handles. A Morse function $f: M \rightarrow \mathbb{R}$ with critical points of index $0 \leqslant i_{0} \leqslant i_{1} \leqslant \ldots \leqslant i_{k} \leqslant m$ determines a handle decomposition

$$
M=h^{i_{0}} \cup h^{i_{1}} \cup \ldots \cup h^{i_{k}},
$$

so that $M$ is a finite $m$-dimensional $C W$ complex with one $i$-cell for each critical point of index $i$.

Proof Apply 2.22 to the cobordism $(M ; \emptyset, \emptyset)$.

Strictly speaking, the above result shows that $M$ contains a subspace (not a submanifold)

$$
L=D^{i_{0}} \cup D^{i_{1}} \cup \ldots \cup D^{i_{k}}
$$

which is a finite $C W$ complex, and such that the inclusion $L \rightarrow M$ is a homotopy equivalence. 
Example 2.24 (i) The $m$-sphere $S^{m}$ has a handle decomposition consisting of a 0 -handle and an $m$-handle

$$
S^{m}=h^{0} \cup h^{m},
$$

given by the upper and lower hemispheres.

(ii) The cobordism $\left(D^{m+1} ; \emptyset, S^{m}\right)$ has a handle decomposition with one 0 -handle

$$
D^{m+1}=h^{0}
$$

The dual cobordism $\left(D^{m+1} ; S^{m}, \emptyset\right)$ has a handle decomposition with one $(m+1)$ handle

$$
D^{m+1}=S^{m} \times I \cup h^{m+1} .
$$

(iii) The torus $M=S^{1} \times S^{1}$ has a Morse function $f: M \rightarrow \mathbb{R}$ with 4 critical values. Here is a picture of the corresponding handle decomposition with the corresponding 4 handles :

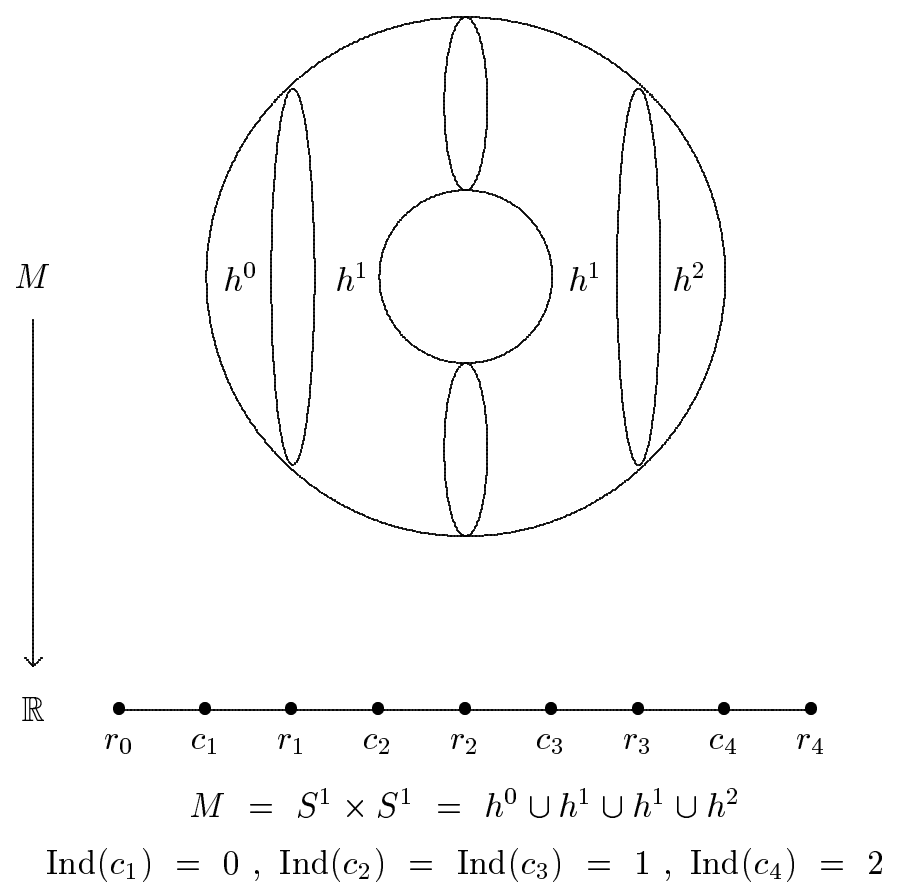

Example 2.25 The Morse function $f: \mathbb{R} \mathbb{P}^{m} \rightarrow \mathbb{R}$ of Example 2.16 has one critical point of index $i$ for $i=0,1, \ldots, m$, so that $\mathbb{R}^{m}$ has a handle decomposition of the type

$$
\mathbb{R} \mathbb{P}^{m}=h^{0} \cup h^{1} \cup \ldots \cup h^{m}
$$


Example 2.26 The Morse function $f: \mathbb{C} \mathbb{P}^{m} \rightarrow \mathbb{R}$ of Example 2.17 has one critical point of index $i$ for $i=0,2, \ldots, 2 m$, so that $\mathbb{C} \mathbb{P}^{m}$ has a handle decomposition of the type

$$
\mathbb{C} \mathbb{P}^{m}=h^{0} \cup h^{2} \cup \ldots \cup h^{2 m}
$$




\section{HOMOTOPY AND HOMOLOGY}

In order to understand how surgery theory deals with the homotopy types of manifolds it is necessary to understand how algebraic topology deals with the homotopy types of more general spaces such as $C W$ complexes. This chapter provides some of the necessary background, assuming that the reader already has some familiarity with the homotopy theory of $C W$ complexes. See Bredon [10], Hatcher [31], Whitehead [96], ... for considerably more detailed accounts of algebraic topology.

Section 3.1 reviews the homotopy groups $\pi_{*}(X)$ and the stable homotopy groups $\pi_{*}^{S}(X)$, and the Freudenthal Suspension Theorem. Section 3.2 deals with the homology and cohomology groups $H_{*}(X), H^{*}(X)$, the Steenrod squares, as well as the Universal Coefficient Theorem, the Theorems of J.H.C. Whitehead and Hurewicz, and the method of killing homotopy classes of $C W$ complexes by attaching cells, the cellular chain complex of a $C W$ complex and the handle chain complex of a manifold.

\subsection{Homotopy}

Definition 3.1 (i) A pointed space $X$ is a space together with a base point $x_{0} \in X$. A pointed map $f: X \rightarrow Y$ is a map of pointed spaces $f: X \rightarrow Y$ such that

$$
f\left(x_{0}\right)=y_{0} \in Y .
$$

A pointed homotopy between pointed maps $f, g: X \rightarrow Y$

$$
h: f \simeq g: X \rightarrow Y
$$

is a map $h: X \times I \rightarrow Y$ such that

$$
h\left(x_{0}, t\right)=y_{0} \in Y \quad(t \in I) .
$$

(ii) The homotopy set $[X, Y]$ of pointed spaces $X, Y$ is the set of pointed homotopy classes of pointed maps $f: X \rightarrow Y$.

(iii) The homotopy groups of a pointed space $X$ are

$$
\pi_{n}(X)=\left[S^{n}, X\right] \quad(n \geqslant 0)
$$

with $\pi_{0}(X)$ the set of path components, the fundamental group $\pi_{1}(X)$ nonabelian (in general), and the higher homotopy groups $\pi_{n}(X)(n \geqslant 2)$ abelian. 
Example 3.2 Here are some homotopy groups of spheres:

$$
\begin{aligned}
& \pi_{m}\left(S^{1}\right)= \begin{cases}\mathbb{Z} & \text { if } m=1 \\
0 & \text { if } m \geqslant 2,\end{cases} \\
& \pi_{m}\left(S^{n}\right)= \begin{cases}0 & \text { if } m<n \\
\mathbb{Z} & \text { if } m=n,\end{cases} \\
& \pi_{n+1}\left(S^{n}\right)= \begin{cases}\mathbb{Z} & \text { if } n=2 \\
\mathbb{Z}_{2} & \text { if } n \geqslant 3,\end{cases} \\
& \pi_{n+2}\left(S^{n}\right)= \begin{cases}0 & \text { if } n=1 \\
\mathbb{Z}_{2} & \text { if } n \geqslant 2 .\end{cases}
\end{aligned}
$$

See Section 6.1 for an account of the degree invariant detecting $\pi_{n}\left(S^{n}\right)$. See Section 5.5 for an account of the Hopf invariant $H$ used to detect $\pi_{n+1}\left(S^{n}\right)$. See Section 11.4 for an account of the Arf invariant detecting $\pi_{n+2}\left(S^{n}\right)$.

Remark 3.3 As already noted in Chapter 1 the applications of the homotopy groups to surgery on non-simply-connected manifolds will make use of the action of the fundamental group $\pi_{1}(X)$ on the higher homotopy groups $\pi_{n}(X)$

$$
\pi_{1}(X) \times \pi_{n}(X) \rightarrow \pi_{n}(X) \quad(n \geqslant 2)
$$

This action can be defined by considering elements of $\pi_{n}(X)$ as homotopy classes of pairs $(\alpha, \beta)$ consisting of an unpointed map $\alpha: S^{n} \rightarrow X$ and a path $\beta: I \rightarrow X$ from $\beta(0)=x$ to $\beta(1)=\alpha\left(1_{S^{n}}\right)$, with $1_{S^{n}} \in S^{n}$ a base point, and letting $\pi_{1}(X)$ act on $\beta$. Alternatively, use the universal cover $\widetilde{X}$ of $X$ (which may be assumed connected), identify $\pi_{n}(X)=\pi_{n}(\widetilde{X})$ with the set of unbased homotopy classes of maps $S^{n} \rightarrow \widetilde{X}$, and let $\pi_{1}(X)$ act on $\widetilde{X}$ as the group of covering translations.

Definition 3.4 The relative homotopy groups $\pi_{n}(f)(n \geqslant 1)$ of a pointed map $f: X \rightarrow Y$ consist of the pointed homotopy classes of pairs

( pointed map $\alpha: S^{n-1} \rightarrow X$, pointed null-homotopy $\beta: f \alpha \simeq *: S^{n-1} \rightarrow Y$ ), designed to fit into a long exact sequence

$$
\ldots \longrightarrow \pi_{n}(X) \stackrel{f_{*}}{\longrightarrow} \pi_{n}(Y) \longrightarrow \pi_{n}(f) \longrightarrow \pi_{n-1}(X) \longrightarrow \ldots \longrightarrow \pi_{1}(Y)
$$

For a pair of pointed spaces $(Y, X \subseteq Y)$ the relative homotopy groups $\pi_{*}(f)$ of the inclusion $f: X \rightarrow Y$ are denoted $\pi_{*}(Y, X)$. 
For any pointed map $f: X \rightarrow Y$ an element $(\alpha, \beta) \in \pi_{n}(f)$ can be represented by a commutative diagram

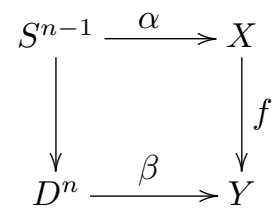

with $\alpha: S^{n-1} \rightarrow X, \beta: D^{n} \rightarrow Y$ pointed maps.

Definition 3.5 Given a space $X$ and a map $\alpha: S^{n-1} \rightarrow X$ let

$$
Y=X \cup_{\alpha} D^{n}
$$

be the space obtained from $X$ by attaching an $n$-cell at $\alpha$.

A $C W$ complex is a space obtained from $\emptyset$ by successively attaching cells of non-decreasing dimension

$$
X=\left(\bigcup D^{0}\right) \cup\left(\bigcup D^{1}\right) \cup\left(\bigcup D^{2}\right) \cup \ldots .
$$

The images of the maps $D^{n} \rightarrow X$ are called the $n$-cells of $X$.

Theorem of J.H.C.Whitehead 3.6 The following conditions on a map $f$ : $X \rightarrow Y$ of connected $C W$ complexes are equivalent:

(i) $f$ is a homotopy equivalence,

(ii) $f$ induces isomorphisms $f_{*}: \pi_{*}(X) \rightarrow \pi_{*}(Y)$,

(iii) $\pi_{*}(f)=0$.

Proof See Theorem VII.11.2 of Bredon [10].

Definition 3.7 Let $n \geqslant 1$.

(i) A space $X$ is $n$-connected if it is connected and

$$
\pi_{i}(X)=0 \quad(i \leqslant n) .
$$

(ii) A map $f: X \rightarrow Y$ of connected spaces is $n$-connected if $f_{*}: \pi_{i}(X) \rightarrow \pi_{i}(Y)$ is an isomorphism for $i<n$ and $f_{*}: \pi_{n}(X) \rightarrow \pi_{n}(Y)$ is onto, or equivalently if

$$
\pi_{i}(f)=0 \quad(i \leqslant n) .
$$

(iii) A pair of connected spaces $(Y, X \subseteq Y)$ is $n$-connected if the inclusion $f: X \rightarrow Y$ is $n$-connected, or equivalently if

$$
\pi_{i}(Y, X)=0 \quad(i \leqslant n) .
$$


Example 3.8 (i) $S^{n}$ is $(n-1)$-connected.

(ii) $\left(D^{n}, S^{n-1}\right)$ is $(n-1)$-connected.

Definition 3.9 (i) The suspension of a pointed space $X$ is the pointed space

$$
\Sigma X=S^{1} \times X /\left(S^{1} \times\{*\} \cup\{1\} \times X\right),
$$

with $* \in X, 1 \in S^{1}$ base points.

(ii) The suspension map in the homotopy groups is defined by

$$
E: \pi_{m}(X) \rightarrow \pi_{m+1}(\Sigma X) ;\left(f: S^{m} \rightarrow X\right) \mapsto\left(\Sigma f: \Sigma\left(S^{m}\right)=S^{m+1} \rightarrow \Sigma X\right) .
$$

Freudenthal Suspension Theorem 3.10 If $X$ is an $(n-1)$-connected space for some $n \geqslant 2$ then the suspension map $E: \pi_{m}(X) \rightarrow \pi_{m+1}(\Sigma X)$ is an isomorphism $m<2 n-1$ and a surjection for $m=2 n-1$.

Proof See Whitehead [96, VII.7.13].

Definition 3.11 The stable homotopy groups of spheres are

$$
\pi_{n}^{S}=\underset{k}{\varliminf_{k}} \pi_{n+k}\left(S^{k}\right) \quad(n \geqslant 0)
$$

with

$$
\pi_{2 n+2}\left(S^{n+2}\right)=\pi_{2 n+3}\left(S^{n+3}\right)=\ldots=\pi_{n}^{S}
$$

by 3.10 .

Remark 3.12 (i) The stable homotopy groups $\pi_{n}^{S}$ are finite for $n>0$ (Serre). (ii) The low-dimensional stable homotopy groups of spheres are given by:

\begin{tabular}{|c|c|c|c|c|c|c|c|c|c|c|}
\hline$n$ & 0 & 1 & 2 & 3 & 4 & 5 & 6 & 7 & 8 & 9 \\
\hline$\pi_{n}^{S}$ & $\mathbb{Z}^{\prime}$ & $\mathbb{Z}_{2}$ & $\mathbb{Z}_{2}$ & $\mathbb{Z}_{24}$ & 0 & 0 & $\mathbb{Z}_{2}$ & $\mathbb{Z}_{240}$ & $\left(\mathbb{Z}_{2}\right)^{2}$ & $\left(\mathbb{Z}_{2}\right)^{3}$ \\
\hline
\end{tabular}

\subsection{Homology}

This section summarises the aspects of (co)homology which are particularly important in keeping track of the effects of surgeries: the Universal Coefficient Theorem, relative groups, cup and cap product pairings 


$$
\begin{gathered}
\cup: H^{m}(X) \otimes_{\mathbb{Z}} H^{n}(X) \rightarrow H^{m+n}(X), \\
\cap: H_{m}(X) \otimes_{\mathbb{Z}} H^{n}(X) \rightarrow H_{m-n}(X),
\end{gathered}
$$

as well as the Steenrod squares

$$
S q^{i}: H^{r}\left(X ; \mathbb{Z}_{2}\right) \rightarrow H^{r+i}\left(X ; \mathbb{Z}_{2}\right) \quad(i \geqslant 0)
$$

See Chapters 3,4 of Hatcher [31] and/or Chapter VI of Bredon [10] for more detailed accounts.

Here are the basic definitions and properties of chain complexes, chain maps, chain contractions etc.

Let $A$ be an associative ring with 1 . An $A$-module $K$ is understood to have a left $A$-action

$$
A \times K \rightarrow K ;(a, x) \mapsto a x
$$

unless a right $A$-action is specified. If $A$ is a commutative ring there is no difference between left and right $A$-modules.

A morphism of direct sums of $A$-modules

$$
f: K=K_{1} \oplus K_{2} \oplus \ldots \oplus K_{n} \rightarrow L=L_{1} \oplus L_{2} \oplus \ldots \oplus L_{m}
$$

is given by an $m \times n$ matrix $f=\left(f_{i j}\right)$ with entries $f_{i j} \in \operatorname{Hom}_{A}\left(K_{j}, L_{i}\right)$, such that

$$
f: K \rightarrow L ;\left(x_{1}, x_{2}, \ldots, x_{n}\right) \mapsto\left(\sum_{j=1}^{n} f_{1 j}\left(x_{j}\right), \sum_{j=1}^{n} f_{2 j}\left(x_{j}\right), \ldots, \sum_{j=1}^{n} f_{m j}\left(x_{j}\right)\right) .
$$

Definition 3.13 (i) An $A$-module chain complex is a sequence of $A$-module morphisms

$$
C: \ldots \longrightarrow C_{i+1} \stackrel{d_{C}}{\longrightarrow} C_{i} \stackrel{d_{C}}{\longrightarrow} C_{i-1} \longrightarrow \ldots
$$

such that $\left(d_{C}\right)^{2}=0$. The chain complex is finite if $\left\{i \in \mathbb{Z} \mid C_{i} \neq 0\right\}$ is finite.

(ii) The homology of an $A$-module chain complex $C$ is the collection of $A$ modules

$$
H_{i}(C)=\frac{\operatorname{ker}\left(d_{C}: C_{i} \rightarrow C_{i-1}\right)}{\operatorname{im}\left(d_{C}: C_{i+1} \rightarrow C_{i}\right)} \quad(i \in \mathbb{Z}) .
$$

(iii) A chain map $f: C \rightarrow D$ is a sequence of $A$-module morphisms $f: C_{i} \rightarrow D_{i}$ such that

$$
d_{D} f=f d_{C}: C_{i} \rightarrow D_{i-1} .
$$

(iv) A chain homotopy $g: f \simeq f^{\prime}$ between chain maps $f, f^{\prime}: C \rightarrow D$ is a sequence of $A$-module morphisms $g: C_{i} \rightarrow D_{i+1}$ such that

$$
f-f^{\prime}=d_{D} g+g d_{C}: C_{i} \rightarrow D_{i} .
$$


(v) A chain equivalence is a chain map $f: C \rightarrow D$ with a chain homotopy inverse, i.e. a chain map $f^{\prime}: D \rightarrow C$ with chain homotopies

$$
g: f^{\prime} f \simeq 1: C \rightarrow C, g^{\prime}: f f^{\prime} \simeq 1: C^{\prime} \rightarrow C^{\prime} .
$$

(vi) A chain contraction of an $A$-module chain complex $C$ is a chain homotopy

$$
\Gamma: 0 \simeq 1: C \rightarrow C .
$$

(vii) The algebraic mapping cone of an $A$-module chain map $f: C \rightarrow D$ is the chain complex $\mathscr{C}(f)$ with

$d_{\mathscr{C}(f)}=\left(\begin{array}{cc}d_{D} & (-1)^{i-1} f \\ 0 & d_{C}\end{array}\right): \mathscr{C}(f)_{i}=D_{i} \oplus C_{i-1} \rightarrow \mathscr{C}(f)_{i-1}=D_{i-1} \oplus C_{i-2}$.

Proposition 3.14 (i) A chain map $f: C \rightarrow D$ induces morphisms in homology $f_{*}: H_{*}(C) \rightarrow H_{*}(D)$ which depend only on the chain homotopy class of $f$.

(ii) For any chain map $f: C \rightarrow D$ the short exact sequence of chain complexes

$$
0 \longrightarrow D \longrightarrow \mathscr{C}(f) \longrightarrow C_{*-1} \longrightarrow 0
$$

induces a long exact sequence of homology A-modules

$$
\ldots \rightarrow H_{i}(C) \stackrel{f_{*}}{\longrightarrow} H_{i}(D) \rightarrow H_{i}(f) \rightarrow H_{i-1}(C) \rightarrow \ldots
$$

with

$$
H_{i}(f)=H_{i}(\mathscr{C}(f)) .
$$

(iii) A chain map $f: C \rightarrow D$ is a chain equivalence if and only if $\mathscr{C}(f)$ is chain contractible.

(iv) A finite chain complex $C$ of projective $A$-modules is chain contractible if and only if $H_{*}(C)=0$.

(v) A chain map $f: C \rightarrow D$ of finite chain complexes of projective A-modules is a chain equivalence if and only if the morphisms $f_{*}: H_{*}(C) \rightarrow H_{*}(D)$ are isomorphisms.

Proof (i) By construction.

(ii) Every short exact sequence of $A$-module chain complexes

$$
0 \longrightarrow D \longrightarrow D^{\prime} \longrightarrow D^{\prime \prime} \longrightarrow 0
$$

induces a long exact sequence of homology $A$-modules

$$
\ldots \longrightarrow H_{i}(D) \longrightarrow H_{i}\left(D^{\prime}\right) \longrightarrow H_{i}\left(D^{\prime \prime}\right) \longrightarrow H_{i-1}(D) \longrightarrow \ldots
$$

(iii) Given a chain contraction $\Gamma: 0 \simeq 1: \mathscr{C}(f) \rightarrow \mathscr{C}(f)$ let $g, h, k$ be the morphisms defined by 


$$
\Gamma=\left(\begin{array}{cc}
k & ? \\
(-1)^{i} g & h
\end{array}\right): \mathscr{C}(f)_{i}=D_{i} \oplus C_{i-1} \rightarrow \mathscr{C}(f)_{i+1}=D_{i+1} \oplus C_{i} .
$$

Then $g: D \rightarrow C$ is a chain homotopy inverse for $f: C \rightarrow D$, with chain homotopies

$$
h: g f \simeq 1: C \rightarrow C, k: f g \simeq 1: D \rightarrow D .
$$

Conversely, if $f: C \rightarrow D$ is a chain equivalence with chain homotopy inverse $g: D \rightarrow C$ and chain homotopies $h: g f \simeq 1, k: f g \simeq 1$ then the $A$-module morphisms

$$
\begin{aligned}
\Gamma=\left(\begin{array}{cc}
1 & (-1)^{i+1}(f h-k f) \\
0 & 1
\end{array}\right)\left(\begin{array}{cc}
k & 0 \\
(-1)^{i} g & h
\end{array}\right): \\
\mathscr{C}(f)_{i}=D_{i} \oplus C_{i-1} \rightarrow \mathscr{C}(f)_{i+1}=D_{i+1} \oplus C_{i}
\end{aligned}
$$

define a chain contraction

$$
\Gamma: 0 \simeq 1: \mathscr{C}(f) \rightarrow \mathscr{C}(f) .
$$

(iv) If $C$ is any contractible chain complex then $H_{*}(C)=0$.

Conversely, suppose that $C$ is a finite projective $A$-module chain complex with $H_{*}(C)=0$. Assume inductively that there exist $A$-module morphisms $\Gamma$ : $C_{i} \rightarrow C_{i+1}$ for $i<k$ such that

$$
d_{C} \Gamma+\Gamma d_{C}=1: C_{i} \rightarrow C_{i} .
$$

The $A$-module morphism $1-\Gamma d_{C}: C_{k} \rightarrow C_{k}$ is such that

$$
d_{C}\left(1-\Gamma d_{C}\right)=\left(1-d_{C} \Gamma-\Gamma d_{C}\right) d_{C}=0: C_{k} \rightarrow C_{k-1}
$$

so that

$$
\operatorname{im}\left(1-\Gamma d_{C}: C_{k} \rightarrow C_{k}\right) \subseteq \operatorname{ker}\left(d_{C}: C_{k} \rightarrow C_{k-1}\right)=\operatorname{im}\left(d_{C}: C_{k+1} \rightarrow C_{k}\right) .
$$

Since $C_{k}$ is projective there exists an $A$-morphism $\Gamma: C_{k} \rightarrow C_{k+1}$ such that

$$
d_{C} \Gamma=1-\Gamma d_{C}: C_{k} \rightarrow C_{k}
$$

giving the inductive step in the construction of a chain contraction $\Gamma: 0 \simeq 1$ : $C \rightarrow C$.

(v) A chain equivalence $f: C \rightarrow D$ induces isomorphisms $f_{*}: H_{*}(C) \rightarrow H_{*}(D)$. For the converse apply (iv) to the algebraic mapping cone $\mathscr{C}(f)$.

Definition 3.15 (i) The dual of an $A$-module $K$ is the right $A$-module

$$
K^{*}=\operatorname{Hom}_{A}(K, A)
$$

with $A$ acting on the right by 


$$
K^{*} \times A \rightarrow K^{*} ;(f, a) \mapsto(x \mapsto f(x) a) .
$$

(ii) The dual of an $A$-module morphism $f: K \rightarrow L$ is the right $A$-module morphism

$$
f^{*}: L^{*} \rightarrow K^{*} ; g \mapsto(x \mapsto g(f(x))) .
$$

(iii) The cohomology of an $A$-module chain complex $C$ is the collection of right A-modules

$$
H^{i}(C)=\frac{\operatorname{ker}\left(d_{C}^{*}: C^{i} \rightarrow C^{i+1}\right)}{\operatorname{im}\left(d_{C}^{*}: C^{i-1} \rightarrow C^{i}\right)} \quad(i \in \mathbb{Z})
$$

where $C^{i}=\left(C_{i}\right)^{*}$.

The homology and cohomology groups of a space $X$ are defined using the singular chain complex $S(X)$, with

$$
\begin{aligned}
& S(X)_{n}=\text { free abelian group generated by maps } \sigma: \Delta^{n} \rightarrow X, \\
& d: S(X)_{n} \rightarrow S(X)_{n-1} ; \sigma \mapsto \sum_{i=0}^{n}(-1)^{i} \sigma \partial_{i}
\end{aligned}
$$

using the standard $n$-simplices

$$
\Delta^{n}=\left\{\left(x_{0}, x_{1}, \ldots, x_{n}\right) \in \mathbb{R}^{n+1} \mid 0 \leqslant x_{i} \leqslant 1, \sum_{i=0}^{n} x_{i}=1\right\}
$$

and the inclusion maps

$$
\partial_{i}: \Delta^{n-1} \rightarrow \Delta^{n} ;\left(x_{0}, x_{1}, \ldots, x_{n-1}\right) \mapsto\left(x_{0}, x_{1}, \ldots, x_{i-1}, 0, x_{i}, \ldots, x_{n-1}\right) .
$$

The (singular) homology and cohomology groups of $X$ are defined by

$$
\begin{aligned}
H_{n}(X) & =H_{n}(S(X)) \\
& =\operatorname{ker}\left(d: S_{n}(X) \rightarrow S_{n-1}(X)\right) / \operatorname{im}\left(d: S_{n+1}(X) \rightarrow S_{n}(X)\right), \\
H^{n}(X) & =H^{n}(S(X)) \\
& =\operatorname{ker}\left(d^{*}: S^{n}(X) \rightarrow S^{n+1}(X)\right) / \operatorname{im}\left(d^{*}: S^{n-1}(X) \rightarrow S^{n}(X)\right)
\end{aligned}
$$

with $S^{n}(X)=\operatorname{Hom}_{\mathbb{Z}}\left(S_{n}(X), \mathbb{Z}\right)$. For any abelian group $G$ the $G$-coefficient singular homology groups $H_{*}(X ; G)$ are defined using the $G$-coefficient singular chain complex

$$
S(X ; G)=G \otimes_{\mathbb{Z}} S(X)
$$

and the $G$-coefficient singular cohomology is defined using

$$
S^{n}(X ; G)=\operatorname{Hom}_{\mathbb{Z}}\left(S_{n}(X), G\right) .
$$

For $G=\mathbb{Z}$ these are just $H_{*}(X ; \mathbb{Z})=H_{*}(X), H^{*}(X ; \mathbb{Z})=H^{*}(X)$. 
Example 3.16 The $\mathbb{Z}$ - and $\mathbb{Z}_{2}$-coefficient homology and cohomology groups are related by exact sequences

$$
\begin{aligned}
& \ldots \rightarrow H_{n}(X) \stackrel{2}{\rightarrow} H_{n}(X) \rightarrow H_{n}\left(X ; \mathbb{Z}_{2}\right) \rightarrow H_{n-1}(X) \rightarrow \ldots \\
& \ldots \rightarrow H^{n}(X) \stackrel{2}{\rightarrow} H^{n}(X) \rightarrow H^{n}\left(X ; \mathbb{Z}_{2}\right) \rightarrow H^{n+1}(X) \rightarrow \ldots
\end{aligned}
$$

For a commutative ring $R$

$$
S^{n}(X ; R)=\operatorname{Hom}_{R}\left(S_{n}(X ; R), R\right)
$$

so that $S^{n}(X ; R)$ is the $R$-module dual of $S_{n}(X ; R)$ as in 3.15. The $R$-coefficient homology and cohomology groups $H_{*}(X ; R), H^{*}(X ; R)$ are $R$-modules which are related by evaluation morphisms

$$
H^{n}(X ; R) \rightarrow \operatorname{Hom}_{R}\left(H_{n}(X ; R), R\right) ; f \mapsto(x \mapsto f(x))
$$

Given an $R$-module $A$ let $T A \subseteq A$ be the torsion submodule

$$
T A=\{x \in A \mid s x=0 \in A \text { for some } s \neq 0 \in R\}
$$

\section{Universal Coefficient Theorem 3.17}

(i) (F-coefficient) For any field $F$ and any $n \geqslant 0$ the evaluation morphism

$$
e: H^{n}(X ; F) \rightarrow \operatorname{Hom}_{F}\left(H_{n}(X ; F), F\right) ; f \mapsto(x \mapsto f(x))
$$

is an isomorphism.

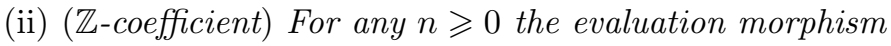

$$
e: H^{n}(X) \rightarrow \operatorname{Hom}_{\mathbb{Z}}\left(H_{n}(X), \mathbb{Z}\right) ; f \mapsto(x \mapsto f(x))
$$

is onto, and the morphism

$$
\begin{gathered}
\operatorname{ker}(e)=T H^{n}(X) \rightarrow \operatorname{Hom}_{\mathbb{Z}}\left(T H_{n-1}(X), \mathbb{Q} / \mathbb{Z}\right) ; f \mapsto\left(x \mapsto \frac{f(y)}{s}\right) \\
\left(f \in S(X)^{n}, x \in S(X)_{n-1}, y \in S(X)_{n}, s \neq 0 \in \mathbb{Z}, s x=d y\right)
\end{gathered}
$$

is an isomorphism, so that there is defined a short exact sequence

$0 \longrightarrow \operatorname{Hom}_{\mathbb{Z}}\left(T H_{n-1}(X), \mathbb{Q} / \mathbb{Z}\right) \longrightarrow H^{n}(X) \stackrel{e}{\longrightarrow} \operatorname{Hom}_{\mathbb{Z}}\left(H_{n}(X), \mathbb{Z}\right) \longrightarrow 0$ 
Proof These results follow from the structure theorem for f.g. $R$-modules with $R$ a principal ideal domain. Every f.g. $R$-module $S$ has a presentation of the type

$$
0 \longrightarrow R^{k} \stackrel{d}{\longrightarrow} R^{\ell} \longrightarrow S \longrightarrow 0
$$

with

$$
d(0, \ldots, 0,1,0, \ldots, 0)=\left(0, \ldots, 0, s_{i}, 0, \ldots, 0\right) \in R^{\ell}
$$

for some $s_{i} \neq 0 \in R(1 \leqslant i \leqslant k)$, and $S$ is a direct sum of cyclic $R$-modules

$$
S=R^{\ell-k} \oplus \bigoplus_{i=1}^{k} R / s_{i} .
$$

More generally, every finite chain complex $C$ of f.g. free $R$-modules is isomorphic to a direct sum of chain complexes of the type

$$
E[n]: \ldots \rightarrow 0 \rightarrow E_{n+1} \rightarrow E_{n}=R \rightarrow 0 \rightarrow \ldots \quad(n \in \mathbb{Z})
$$

with $E_{n+1}=R$ or 0 . For any $n \in \mathbb{Z}$ the evaluation map

$$
e: H^{n}(C) \rightarrow \operatorname{Hom}_{R}\left(H_{n}(C), R\right) ; f \mapsto(x \mapsto f(x))
$$

is onto, with a natural $R$-module isomorphism

$$
\begin{gathered}
\operatorname{ker}(e)=T H^{n}(C) \rightarrow \operatorname{Hom}_{R}\left(T H_{n-1}(C), K / R\right) ; f \mapsto\left(x \mapsto \frac{f(y)}{s}\right) \\
\left(f \in C^{n}, x \in C_{n-1}, y \in C_{n}, s \neq 0 \in R, s x=d y\right)
\end{gathered}
$$

where $K$ is the quotient field of $R$.

(i) If $R=F$ is a field then $T H^{n}(C)=0$.

(ii) The ring of integers $\mathbb{Z}$ is a principal ideal domain, with quotient field $\mathbb{Q}$ the rationals.

The homological properties of intersections of subspaces of a space $X$ are derived from the homological properties of the diagonal map

$$
\Delta: X \rightarrow X \times X ; x \mapsto(x, x),
$$

using diagonal chain approximations :

Diagonal Chain Approximation Theorem 3.18 The singular chain complex $S(X)$ of any topological space $X$ is equipped with a natural chain map

$$
\Delta_{0}: S(X) \rightarrow S(X) \otimes_{\mathbb{Z}} S(X)
$$

and natural higher chain homotopies

$$
\Delta_{i}: S(X)_{r} \rightarrow\left(S(X) \otimes_{\mathbb{Z}} S(X)\right)_{r+i} \quad(i \geqslant 1)
$$


such that

$$
\begin{gathered}
\Delta_{i}+(-1)^{i+1} T \Delta_{i}=d_{S(X) \otimes_{\mathbb{Z}} S(X)} \Delta_{i+1}+(-1)^{i} \Delta_{i+1} d_{S(X)}: \\
S(X)_{r} \rightarrow\left(S(X) \otimes_{\mathbb{Z}} S(X)\right)_{r+i} \quad(i \geqslant 0)
\end{gathered}
$$

with $T$ the transposition automorphism

$$
T: S(X)_{p} \otimes_{\mathbb{Z}} S(X)_{q} \rightarrow S(X)_{q} \otimes_{\mathbb{Z}} S(X)_{p} ; x \otimes y \mapsto(-1)^{p q} y \otimes x
$$

such that $T^{2}=1$. Naturality means that for every map $f: X \rightarrow Y$ there is defined a commutative square

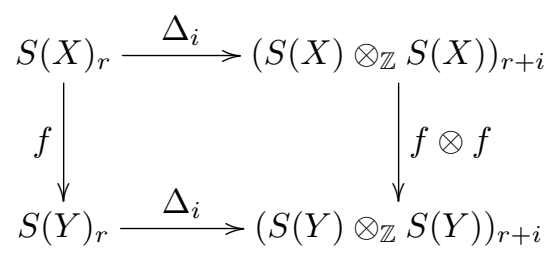

Proof See Chapter VI.16 of Bredon [10].

Remark 3.19 Diagonal chain approximations were first constructed by Alexander, Whitney and Steenrod in the 1930's using explicit formulae in simplicial homology. The singular complex diagonal chain approximations $\left\{\Delta_{i}\right\}$ are constructed by acyclic model theory. For any spaces $X, Y$ there is a natural Eilenberg-Zilber chain equivalence

$$
E_{0}: S(X \times Y) \simeq S(X) \otimes_{\mathbb{Z}} S(Y)
$$

with natural higher chain homotopies

$$
E_{i}: S(X \times Y)_{r} \rightarrow\left(S(X) \otimes_{\mathbb{Z}} S(Y)\right)_{r+i}(i \geqslant 1)
$$

such that

$$
\begin{aligned}
E_{i} T+(-1)^{i+1} T E_{i}=d_{S(X) \otimes_{\mathbb{Z} S(Y)}} E_{i+1}+(-1)^{i} E_{i+1} d_{S(X \times Y)}: & \\
S(X \times Y)_{r} & \rightarrow\left(S(X) \otimes_{\mathbb{Z}} S(Y)\right)_{r+i}(i \geqslant 0)
\end{aligned}
$$

with

$$
\begin{aligned}
& T: X \times Y \rightarrow Y \times X ;(x, y) \mapsto(y, x), \\
& T: S(X)_{p} \otimes_{\mathbb{Z}} S(Y)_{q} \rightarrow S(Y)_{q} \otimes_{\mathbb{Z}} S(X)_{p} ; a \otimes b \mapsto(-1)^{p q} b \otimes a
\end{aligned}
$$

the transposition maps. The diagonal chain approximation is obtained by taking $X=Y$ and setting

$$
\Delta_{i}=E_{i} \Delta: S(X)_{r} \stackrel{\Delta}{\longrightarrow} S(X \times X)_{r} \stackrel{E_{i}}{\longrightarrow}\left(S(X) \otimes_{\mathbb{Z}} S(X)\right)_{r+i} .
$$


Let $W$ be the standard free $\mathbb{Z}\left[\mathbb{Z}_{2}\right]$-module resolution of $\mathbb{Z}$

$$
W: \ldots \longrightarrow \mathbb{Z}\left[\mathbb{Z}_{2}\right] \stackrel{1-T}{\longrightarrow} \mathbb{Z}\left[\mathbb{Z}_{2}\right] \stackrel{1+T}{\longrightarrow} \mathbb{Z}\left[\mathbb{Z}_{2}\right] \stackrel{1-T}{\longrightarrow} \mathbb{Z}\left[\mathbb{Z}_{2}\right]
$$

The collection $\left\{E_{i}\right\}$ defines a natural chain map

$$
E: S(X \times X) \rightarrow \operatorname{Hom}_{\mathbb{Z}\left[\mathbb{Z}_{2}\right]}\left(W, S(X) \otimes_{\mathbb{Z}} S(X)\right)
$$

and $\left\{\Delta_{i}\right\}$ defines a natural chain map

$$
\Delta: S(X) \rightarrow \operatorname{Hom}_{\mathbb{Z}\left[\mathbb{Z}_{2}\right]}\left(W, S(X) \otimes_{\mathbb{Z}} S(X)\right)
$$

Definition 3.20 (i) The cup product pairing is

$\cup: H^{m}(X) \otimes_{\mathbb{Z}} H^{n}(X) \rightarrow H^{m+n}(X) ; a \otimes b \mapsto\left(a \cup b: x \mapsto \sum a\left(x^{\prime}\right) \otimes b\left(x^{\prime \prime}\right)\right)$

with $\Delta_{0}(x)=\sum x^{\prime} \otimes x^{\prime \prime}$

(ii) The cap product pairing is

$$
\cap: H_{m}(X) \otimes_{\mathbb{Z}} H^{n}(X) \rightarrow H_{m-n}(X) ; x \otimes y \mapsto x \cap y=\sum y\left(x^{\prime}\right) x^{\prime \prime} .
$$

(iii) The Steenrod squares are the cohomology operations

$S q^{i}: H^{r}\left(X ; \mathbb{Z}_{2}\right) \rightarrow H^{r+i}\left(X ; \mathbb{Z}_{2}\right) ; x \mapsto\left(y \mapsto\left\langle x \otimes x, \Delta_{r-i}(y)\right\rangle\right)\left(y \in H_{r+i}\left(X ; \mathbb{Z}_{2}\right)\right)$,

identifying $H^{*}\left(X ; \mathbb{Z}_{2}\right)=\operatorname{Hom}_{\mathbb{Z}_{2}}\left(H_{*}\left(X ; \mathbb{Z}_{2}\right), \mathbb{Z}_{2}\right)$ by the Universal Coefficient Theorem 3.17.

The cup and cap product pairings are also defined for $R$-coefficient (co)homology

$$
\begin{aligned}
& \cup: H^{m}(X ; R) \otimes_{R} H^{n}(X ; R) \rightarrow H^{m+n}(X ; R), \\
& \cap: H_{m}(X ; R) \otimes_{R} H^{n}(X ; R) \rightarrow H_{m-n}(X ; R)
\end{aligned}
$$

for any commutative ring $R$.

Definition 3.21 (i) The relative homology groups of a map $f: X \rightarrow Y$ are the relative homology groups of the induced chain map $f: S(X) \rightarrow S(Y)$

$$
H_{*}(f)=H_{*}(f: S(X) \rightarrow S(Y)),
$$

designed to fit into a long exact sequence

$$
\ldots \rightarrow H_{n}(X) \stackrel{f_{*}}{\rightarrow} H_{n}(Y) \rightarrow H_{n}(f) \rightarrow H_{n-1}(X) \rightarrow \ldots
$$


If $f: X \rightarrow Y$ is the inclusion of a subspace $X \subseteq Y$ the relative homology groups are written

$$
H_{*}(f)=H_{*}(Y, X) .
$$

(ii) The reduced homology groups of a pointed space $(X, x \in X)$ are the relative homology groups of the inclusion $i:\{x\} \hookrightarrow X$

$$
\dot{H}_{*}(X)=H_{*}(i)=H_{*}(X,\{x\})
$$

with

$$
H_{*}(X)=\dot{H}_{*}(X) \oplus H_{*}(\{x\}),
$$

so that $\dot{H}_{r}(X)=H_{r}(X)$ for $r \neq 0$, and $H_{0}(X)=\dot{H}_{0}(X) \oplus \mathbb{Z}$.

For a triple of spaces $(X, Y \subseteq X, Z \subseteq Y)$ the relative homology groups of the associated pairs fit into a long exact sequence

$$
\ldots \rightarrow H_{r}(Y, Z) \rightarrow H_{r}(X, Z) \rightarrow H_{r}(X, Y) \stackrel{\partial}{\rightarrow} H_{r-1}(Y, Z) \rightarrow \ldots .
$$

The relative cohomology groups $H^{*}(f)$ of a map $f: X \rightarrow Y$ are defined to fit into a long exact sequence

$$
\ldots \longrightarrow H^{n-1}(X) \stackrel{\delta}{\longrightarrow} H^{n}(f) \longrightarrow H^{n}(Y) \stackrel{f^{*}}{\longrightarrow} H^{n}(X) \longrightarrow \ldots .
$$

The relative cohomology groups $H^{*}(Y, X)(X \subseteq Y)$ and the reduced cohomology groups $\dot{H}^{*}(X)$ are defined by analogy with $H_{*}(Y, X)$ and $\dot{H}_{*}(X)$.

Definition 3.22 (i) The mapping cylinder of a map $f: X \rightarrow Y$ is the identification space

$$
\mathscr{M}(f)=(X \times I \cup Y) /\{(x, 1) \sim f(x) \mid x \in X\},
$$

which contains $Y$ as a deformation retract.

(ii) The mapping cone of a map $f: X \rightarrow Y$ is the pointed space

$$
\mathscr{C}(f)=\mathscr{M}(f) /\left\{(x, 0) \sim\left(x^{\prime}, 0\right) \mid x, x^{\prime} \in X\right\}
$$

with base point $[X \times\{0\}] \in \mathscr{C}(f)$.

Example 3.23 The space obtained from $X$ by attaching an $n$-cell at $\alpha: S^{n-1} \rightarrow$ $X(3.5)$ is a mapping cone

$$
X \cup_{\alpha} D^{n}=\mathscr{C}\left(\alpha: S^{n-1} \rightarrow X\right),
$$

and fits into a cofibration sequence

$$
S^{n-1} \stackrel{\alpha}{\longrightarrow} X \longrightarrow X \cup_{\alpha} D^{n} \longrightarrow S^{n} \longrightarrow \ldots .
$$


Proposition 3.24 The relative homology groups of a map $f: X \rightarrow Y$ are the reduced homology groups of the mapping cone

$$
H_{*}(f)=\dot{H}_{*}(\mathscr{C}(f: X \rightarrow Y)) .
$$

Similarly for relative cohomology.

Proof The cofibration sequence of spaces

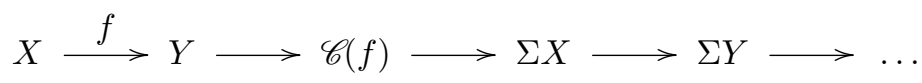

induces a long exact sequence of homology groups

$$
\ldots \rightarrow H_{n}(X) \stackrel{f_{*}}{\longrightarrow} H_{n}(Y) \rightarrow \dot{H}_{n}(\mathscr{C}(f)) \rightarrow H_{n-1}(X) \rightarrow \ldots
$$

Homology is homotopy invariant: a homotopy $h: f \simeq g: X \rightarrow Y$ induces a chain homotopy $h: f \simeq g: S(X) \rightarrow S(Y)$, so that $f_{*}=g_{*}: H_{*}(X) \rightarrow H_{*}(Y)$. If $f: X \rightarrow Y$ is a homotopy equivalence then $f: S(X) \rightarrow S(Y)$ is a chain equivalence, and $f_{*}: H_{*}(X) \rightarrow H_{*}(Y)$ is an isomorphism.

Definition 3.25 The Hurewicz map from the homotopy to the homology groups is

$$
\pi_{n}(X) \rightarrow H_{n}(X) ;\left(f: S^{n} \rightarrow X\right) \mapsto f_{*}\left[S^{n}\right]
$$

with $\left[S^{n}\right]=1 \in H_{n}\left(S^{n}\right)=\mathbb{Z}$.

For $n \geqslant 2$ there is also a $\mathbb{Z}\left[\pi_{1}(X)\right]$-module Hurewicz map

$$
\pi_{n}(X)=\pi_{n}(\tilde{X}) \rightarrow H_{n}(\tilde{X}) ;\left(f: S^{n} \rightarrow X\right) \mapsto \widetilde{f}_{*}\left[S^{n}\right]
$$

with $\tilde{X}$ the universal cover of $X$, and $\tilde{f}: S^{n} \rightarrow \widetilde{X}$ the lift of $f$ which sends the base point of $S^{n}$ to the base point of $\widetilde{X}$.

For a map $f: X \rightarrow Y$ there are also Hurewicz maps $\pi_{*}(f) \rightarrow H_{*}(\tilde{f})$ from the relative homotopy groups to the relative homology groups.

Hurewicz Theorem 3.26 (i) For a connected space $X$ the map $\pi_{1}(X) \rightarrow$ $H_{1}(X)$ is onto, with kernel the commutator subgroup $\left[\pi_{1}(X), \pi_{1}(X)\right] \triangleleft \pi_{1}(X)$ generated by the commutators $[g, h]=g h g^{-1} h^{-1}\left(g, h \in \pi_{1}(X)\right)$.

(ii) If $n \geqslant 2$ and $X$ is an $(n-1)$-connected space then $\pi_{n}(X) \rightarrow H_{n}(X)$ is an isomorphism of abelian groups.

(iii) If $n \geqslant 2$ and $f: X \rightarrow Y$ is an $(n-1)$-connected map then $\pi_{n}(f) \rightarrow H_{n}(\tilde{f})$ is an isomorphism of $\mathbb{Z}\left[\pi_{1}(X)\right]$-modules, with $\tilde{f}: \widetilde{X} \rightarrow \widetilde{Y}$ a $\pi_{1}(X)$-equivariant lift of $f$ to the universal covers $\widetilde{X}, \widetilde{Y}$ of $X, Y$. 
Proof See Theorem VII.10.7 of Bredon [10].

Corollary 3.27 (i) A connected space $X$ is n-connected if and only if $\pi_{1}(X)=$ $\{1\}$ and $H_{i}(X)=0$ for $1 \leqslant i<n$.

(ii) A map of connected spaces $f: X \rightarrow Y$ is $n$-connected if and only if $f_{*}$ : $\pi_{1}(X) \rightarrow \pi_{1}(Y)$ is an isomorphism and $H_{i}(\widetilde{f})=0$ for $i \leqslant n$.

(iii) If $Y$ is obtained from $X$ by attaching $m$-cells for $m \geqslant n$ then the pair $(Y, X)$ is $(n-1)$-connected.

(iv) If $f: X \rightarrow Y$ is an n-connected map then for any m-dimensional $C W$ complex $M$ with $m<n$ the induced function

$$
f_{*}:[M, X] \rightarrow[M, Y] ; g \mapsto f g
$$

is a bijection.

(v) If $M, N$ are $C W$ complexes such that $M$ is $m$-dimensional, $N$ is $n$-connected and $m<n$ then

$$
[M, N]=0 .
$$

Proof (i)+(ii) Immediate from the Hurewicz Theorem 3.26.

(iii) For any $\alpha: S^{m-1} \rightarrow X$ and $n \leqslant m$

$$
\pi_{n-1}\left(X \cup_{\alpha} D^{m}, X\right)=H_{n-1}\left(X \cup_{\alpha} D^{m}, X\right)=\dot{H}_{n-1}\left(S^{m}\right)=0 .
$$

(iv) It may be assumed that $Y$ is obtained from $X$ by attaching cells of dimension $j \geqslant n+1$. For an $m$-dimensional $C W$ complex $M$ attaching a cell of dimension $j \geqslant n+1$ to $X$ has no effect on $[M, X]$, since $\pi_{i}\left(S^{j}\right)=0$ for $i \leqslant m \leqslant n<j$.

(v) Any map $D^{0} \rightarrow N$ is $n$-connected, so that by (iii)

$$
[M, N]=\left[M, D^{0}\right]=0 .
$$

Example 3.28 For $n \geqslant 2$ let $X=S^{1} \vee S^{n}$ be the one-point union of a circle and an $n$-sphere.

(i) The fundamental group is $\pi_{1}(X)=\mathbb{Z}$, and the universal cover

$$
\widetilde{X}=\left(\mathbb{R} \cup\left(\mathbb{Z} \times S^{n}\right)\right) /\{k \sim(k, 1,0, \ldots, 0) \mid k \in \mathbb{Z}\} .
$$

is $(n-1)$-connected, with the Hurewicz map

$$
\pi_{n}(X)=\pi_{n}(\widetilde{X}) \rightarrow H_{n}(\widetilde{X})=\mathbb{Z}[\mathbb{Z}]
$$

a $\mathbb{Z}[\mathbb{Z}]$-module isomorphism.

(ii) The inclusion $S^{1} \hookrightarrow X$ is $(n-1)$-connected.

(iii) The projection $X \rightarrow S^{1}$ is $n$-connected. 
Definition 3.29 Given a space $X$ and an element $x \in \pi_{n}(X)(n \geqslant 1)$ let

$$
Y=X \cup_{\alpha} D^{n+1}
$$

be the space obtained from $X$ by attaching an $(n+1)$-cell $(3.5)$ at any map $\alpha: S^{n} \rightarrow X$ with $x=[\alpha] \in \pi_{n}(X)$. The operation of attaching the $(n+1)$-cell is said to kill $x$.

The method of killing of homotopy classes by attaching cells is a homotopytheoretic precursor of surgery. It does not apply directly to manifolds, since attaching a cell to a manifold results in a non-manifold space. The effect on the homotopy and homology groups of attaching an $(n+1)$-cell is given in dimensions $\leqslant n$ by :

Proposition 3.30 Let $Y=X \cup_{\alpha} D^{n+1}$ be the space obtained from a $C W$ complex $X$ by attaching an $(n+1)$-cell at $\alpha: S^{n} \rightarrow X$ to kill $x=[\alpha] \in \pi_{n}(X)$.

(i) Let $\widetilde{X}$ be a regular cover $X$ with group of covering translations $\pi$. Assume that the induced cover $\alpha^{*} \widetilde{X}$ of $S^{n}$ is trivial (which is automatically the case if $n \geqslant 2$ ), so that $\alpha: S^{n} \rightarrow X$ has a $\pi$-equivariant lift $\widetilde{\alpha}: \alpha^{*} \widetilde{X}=\pi \times S^{n} \rightarrow \widetilde{X}$ and $Y$ has a regular cover

$$
\widetilde{Y}=\tilde{X} \cup_{\widetilde{\alpha}}\left(\pi \times D^{n+1}\right) .
$$

The relative homology $\mathbb{Z}[\pi]$-modules of $(\tilde{Y}, \tilde{X} \subset \tilde{Y})$ are given by

$$
H_{i}(\tilde{Y}, \widetilde{X})=H_{i}\left(\pi \times D^{n+1}, \pi \times S^{n}\right)=\left\{\begin{array}{ll}
\mathbb{Z}[\pi] & \text { if } i=n+1 \\
0 & \text { if } i \neq n+1
\end{array} .\right.
$$

Thus

$$
H_{i}(\tilde{Y})=H_{i}(\widetilde{X}) \text { for } i \neq n, n+1
$$

and there is defined an exact sequence

$0 \longrightarrow H_{n+1}(\tilde{X}) \longrightarrow H_{n+1}(\tilde{Y}) \longrightarrow \mathbb{Z}[\pi] \stackrel{x}{\longrightarrow} H_{n}(\tilde{X}) \longrightarrow H_{n}(\tilde{Y}) \longrightarrow 0$.

In particular

$$
H_{n}(\tilde{Y})=H_{n}(\widetilde{X}) /\langle x\rangle
$$

with $\langle x\rangle \subseteq H_{n}(\tilde{X})$ the $\mathbb{Z}[\pi]$-submodule generated by the Hurewicz image $x \in$ $H_{n}(\widetilde{X})$.

(ii) The pair $(Y, X)$ is $n$-connected. For $n \geqslant 2$ the relative homotopy groups of $(Y, X)$ in dimensions $\leqslant n+1$ are given by

$$
\pi_{i}(Y, X)=\left\{\begin{array} { l } 
{ 0 } \\
{ \mathbb { Z } [ \pi _ { 1 } ( X ) ] }
\end{array} \text { if } \left\{\begin{array}{l}
i \leqslant n \\
i=n+1 .
\end{array}\right.\right.
$$

(iii) The homotopy groups of $Y$ in dimensions $\leqslant n$ are given by

$$
\pi_{i}(Y)=\left\{\begin{array} { l } 
{ \pi _ { i } ( X ) } \\
{ \pi _ { n } ( X ) / \langle x \rangle }
\end{array} \text { if } \left\{\begin{array}{l}
i<n \\
i=n
\end{array}\right.\right.
$$


where $\langle x\rangle \subseteq \pi_{n}(X)$ is the normal subgroup generated by $x$ for $n=1$, and the $\mathbb{Z}\left[\pi_{1}(X)\right]$-submodule generated by $x$ for $n \geqslant 2$.

Proof (i) Immediate from

$$
H_{*}(\tilde{Y}, \tilde{X})=\dot{H}_{*}(\tilde{Y} / \widetilde{X})=\dot{H}_{*}\left(\bigvee_{\pi} S^{n}\right) .
$$

(ii) For $n \geqslant 2 \pi_{1}(Y)=\pi_{1}(X)$, so that the universal covers $\widetilde{X}, \tilde{Y}$ of $X, Y$ define a pair $(\widetilde{Y}, \widetilde{X} \subset \widetilde{Y})$. Apply (i) with this pair and the Hurewicz Theorem (3.26) to obtain

$$
\pi_{i}(Y, X)=H_{i}(\tilde{Y}, \tilde{X})= \begin{cases}0 & \text { if } i \leqslant n \\ \mathbb{Z}\left[\pi_{1}(X)\right] & \text { if } i=n+1\end{cases}
$$

(iii) The case $n=1$ is a direct application of the Seifert-Van Kampen Theorem (Chapter III.9 of Bredon [10])

$$
\pi_{1}(Y)=\pi_{1}(X) *_{\pi_{1}\left(S^{1}\right)} \pi_{1}\left(D^{2}\right)=\pi_{1}(X) /\langle x\rangle .
$$

For $n \geqslant 2$ apply (ii) and the homotopy exact sequence

$$
\pi_{n+1}(Y, X)=\mathbb{Z}\left[\pi_{1}(X)\right] \stackrel{x}{\longrightarrow} \pi_{n}(X) \longrightarrow \pi_{n}(Y) \longrightarrow \pi_{n}(Y, X)=0 .
$$

Next, we describe the construction of the cellular chain complex $C(X)$ of a $C W$ complex $X$. We shall be particularly concerned with the cellular chain complex $C(M)$ of a manifold $M$ with the $C W$ structure determined by a handle decomposition (2.23).

Let then $X$ be a $C W$ complex

$$
X=\bigcup_{i=0}^{\infty} \bigcup_{c_{i}} D^{i}
$$

with $n$-skeleta

$$
X^{(n)}=\bigcup_{i=0}^{n} \bigcup_{c_{i}} D^{i} \subseteq X \quad(n \geqslant 0) .
$$

Definition 3.31 The cellular chain complex $C(X)$ is the chain complex with

$$
C(X)_{n}=H_{n}\left(X^{(n)}, X^{(n-1)}\right)=\mathbb{Z}^{c_{n}}
$$

the free abelian group generated by the $n$-cells $D^{n} \rightarrow X$ and

$d=\partial: C(X)_{n}=H_{n}\left(X^{(n)}, X^{(n-1)}\right) \rightarrow C(X)_{n-1}=H_{n-1}\left(X^{(n-1)}, X^{(n-2)}\right)$

the boundary in the homology of the triple $\left(X^{(n)}, X^{(n-1)}, X^{(n-2)}\right)$. 
Proposition 3.32 The homology groups of a $C W$ complex $X$ are just the homology groups of the cellular chain complex $C(X)$

$$
\begin{aligned}
H_{n}(X) & =H_{n}(C(X)) \\
& =\operatorname{ker}\left(d: C(X)_{n} \rightarrow C(X)_{n-1}\right) / \operatorname{im}\left(d: C(X)_{n+1} \rightarrow C(X)_{n}\right)
\end{aligned}
$$

and similarly for the cohomology groups

$$
\begin{aligned}
H^{n}(X) & =H^{n}(C(X)) \\
& =\operatorname{ker}\left(d^{*}: C(X)^{n} \rightarrow C(X)^{n+1}\right) / \operatorname{im}\left(d^{*}: C(X)^{n-1} \rightarrow C(X)^{n}\right)
\end{aligned}
$$

with

$$
C(X)^{n}=\operatorname{Hom}_{\mathbb{Z}}\left(C(X)_{n}, \mathbb{Z}\right)=H^{n}\left(X^{(n)}, X^{(n-1)}\right)
$$

Proof The inclusion $C(X) \hookrightarrow S(X)$ is a chain equivalence.

Let $X=(A, B \subseteq A)$ be a $C W$ pair with $A$ obtained from $B$ by attaching cells of non-decreasing dimensions $i_{1} \leqslant i_{2} \leqslant \ldots$

$$
A=B \cup D^{i_{1}} \cup D^{i_{2}} \cup \ldots .
$$

The relative skeleta of $X$ are defined by

$$
X^{(n)}=B \cup \bigcup_{i \leqslant n} D^{i}(n \geqslant 0)
$$

and are such that $B \subseteq X^{(0)} \subseteq X^{(1)} \subseteq \ldots \subseteq A$. The cellular chain complex $C(X)=C(A, B)$ is defined as in the absolute case, with $C(X)_{n}=H_{n}\left(X^{(n)}, X^{(n-1)}\right)$ the free abelian group generated by the $n$-cells, and homology

$$
H_{*}(C(X))=H_{*}(A, B) .
$$

Returning to manifolds, let $\left(W ; M, M^{\prime}\right)$ be an $(m+1)$-dimensional cobordism with a handle decomposition

$$
W=M \times I \cup h^{i_{0}} \cup h^{i_{1}} \cup \ldots \cup h^{i_{k}}
$$

where $h^{i}=D^{i} \times D^{m-i+1}$ is a handle of index $i$ and $0 \leqslant i_{0} \leqslant i_{1} \leqslant \ldots \leqslant i_{k} \leqslant m+1$, as given by 2.22 from a Morse function $f$ on $\left(W ; M, M^{\prime}\right)$. As in 2.23 the handle decomposition can be viewed as a $C W$ structure on $X=(W, M)$, with one $i$-dimensional cell for each $i$-handle, and $n$-skeleta

$$
X^{(n)}=M \times I \cup \bigcup_{i \leqslant n} h^{i} .
$$


Definition 3.33 The handle chain complex $C(W, M)$ of a cobordism $\left(W ; M, M^{\prime}\right)$ with a handle decomposition is the cellular chain complex of the corresponding relative $C W$ structure on $X=(W, M)$, with

$$
C(W, M)_{n}=H_{n}\left(X^{(n)}, X^{(n-1)}\right)
$$

the free abelian group generated by the $n$-handles, and homology $H_{*}(C(W, M))=$ $H_{*}(W, M)$.

The differentials $d: C(W, M)_{n} \rightarrow C(W, M)_{n-1}$ will be interpreted in Section 8.2 in terms of the intersections of $(n-1)$ - and $n$-handles.

Example 3.34 A closed $m$-dimensional manifold $M$ can be regarded as an $m$ dimensional cobordism $(M ; \emptyset, \emptyset)$. A handle decomposition

$$
M=h^{i_{0}} \cup h^{i_{1}} \cup \ldots \cup h^{i_{k}}
$$

determines a handle chain complex $C(M)$, with $H_{*}(C(M))=H_{*}(M)$. 


\section{POINCARÉ DUALITY}

The algebraic effect of a geometric surgery on an $m$-dimensional manifold $M$ is determined by the Poincaré duality isomorphisms $H_{*}(M) \cong H^{m-*}(M)$ between the homology and cohomology groups. The homotopy-theoretic effect of a surgery is a combination of attaching a cell and detaching a cell of complementary dimension to restore Poincaré duality.

Section 4.1 establishes Poincaré duality. The main result of this chapter is the description in Section 4.2 of the effect of a surgery on the homotopy and homology groups of a manifold. Section 4.3 recalls the classification of surfaces, and gives a complete description of the effects of surgery on surfaces. Section 4.4 describes the algebraic properties of rings with involution and sesquilinear forms needed for the intersection form of a non-simply-connected manifold. Finally, Section 4.5 gives Poincaré duality for the universal cover of a manifold.

\subsection{Poincaré duality}

The Poincaré duality isomorphisms $H_{*}(M) \cong H^{m-*}(M)$ of an orientable $m$ dimensional manifold $M$ are the global expression of the local property that every $x \in M$ has a neighbourhood $U \subseteq M$ which is diffeomorphic to $\mathbb{R}^{m}$, with

$$
H_{*}(M, M \backslash\{x\}) \cong H_{*}\left(\mathbb{R}^{m}, \mathbb{R}^{m} \backslash\{0\}\right) \cong H^{m-*}(\{0\}) \cong H^{m-*}(\{x\}) .
$$

Orientability is necessary in order to piece together these local isomorphisms to obtain global isomorphisms $H_{*}(M) \cong H^{m-*}(M)$.

Definition 4.1 Let $R$ be a commutative ring.

(i) An $m$-dimensional manifold $M$ is $R$-orientable if there exists an $R$-coefficient fundamental class, a homology class $[M] \in H_{m}(M ; R)$ such that for every $x \in M$ the $R$-module morphism

$$
H_{m}(M ; R) \rightarrow H_{m}(M, M \backslash\{x\} ; R)=H_{m}\left(\mathbb{R}^{m}, \mathbb{R}^{m} \backslash\{0\} ; R\right)=R
$$

sends $[M] \in H_{m}(M ; R)$ to a unit in $R$.

(ii) A manifold $M$ is orientable if it is $\mathbb{Z}$-orientable, and nonorientable if it is not $\mathbb{Z}$-orientable. An orientation for an orientable manifold $M$ is a choice of $\mathbb{Z}$-coefficient fundamental class $[M]$. 
(iii) The orientation character of $M$ is the cohomology class $w(M) \in H^{1}\left(M ; \mathbb{Z}_{2}\right)$ such that

$$
S q^{1}=w(M) \cup-: H^{m-1}\left(M ; \mathbb{Z}_{2}\right) \rightarrow H^{m}\left(M ; \mathbb{Z}_{2}\right)
$$

Remark 4.2 The orientation character is the first Stiefel-Whitney class of the tangent bundle $\tau_{M}, w(M)=w_{1}\left(\tau_{M}\right) \in H^{1}\left(M ; \mathbb{Z}_{2}\right)$. See Chapter 5 for bundles and characteristic classes.

Proposition 4.3 (i) Every manifold $M$ is $\mathbb{Z}_{2}$-orientable, and has a unique $\mathbb{Z}_{2}$ orientation.

(ii) $A$ manifold $M$ is orientable if and only if $w(M)=0 \in H^{1}\left(M ; \mathbb{Z}_{2}\right) . A$ connected orientable $m$-dimensional manifold $M$ has two orientations $\pm[M] \in$ $H_{m}(M)=\mathbb{Z}$.

(iii) An orientable manifold $M$ is $R$-orientable, for any commutative ring $R$.

Proof See Chapter VI.7 of Bredon [10] and/or Section 3.3 of Hatcher [31].

Poincaré Duality Theorem 4.4 For any $R$-orientable $m$-dimensional manifold $M$ cap product with $[M] \in H_{m}(M ; R)$ defines $R$-module isomorphisms

$$
[M] \cap-: H^{*}(M ; R) \cong H_{m-*}(M ; R)
$$

Proof As in Example 3.34 regard $M$ as a cobordism $(M ; \emptyset, \emptyset)$, and choose a Morse function $f: M \rightarrow \mathbb{R}$ to obtain a handle decomposition of $M$ with $R$ coefficient handle chain complex $C(M ; R)$. The opposite Morse function $-f$ : $M \rightarrow \mathbb{R}$ determines another handle decomposition on $M$, with chain complex the $m$-dual $C(M ; R)^{m-*}$. Applying the cellular approximation theorem to the identity map it is possible to approximate $1: M \rightarrow M$ by a cellular homotopy equivalence from a subdivision of the dual handlebody $C W$ structure to the handlebody $C W$ structure. The corresponding $R$-module chain equivalence $C(M ; R)^{m-*} \rightarrow C(M ; R)$ is given by the cap product $[M] \cap-$, up to chain homotopy.

Example 4.5 The complex projective space $\mathbb{C} \mathbb{P}^{m}$ is an orientable $2 m$-dimensional manifold with homology and cohomology

$$
H_{n}\left(\mathbb{C} \mathbb{P}^{m}\right)=H^{2 m-n}\left(\mathbb{C} \mathbb{P}^{m}\right)= \begin{cases}\mathbb{Z} & \text { if } 0 \leqslant n \leqslant 2 m \text { with } n \text { even, } \\ 0 & \text { otherwise. }\end{cases}
$$


Example 4.6 The $\mathbb{Z}_{2}$-homology and cohomology of the $m$-dimensional real projective space

$$
\mathbb{R P}^{m}=S^{m} /\{x \sim-x\}
$$

are given by

$$
H_{i}\left(\mathbb{R P}^{m} ; \mathbb{Z}_{2}\right)=H^{m-i}\left(\mathbb{R} \mathbb{P}^{m} ; \mathbb{Z}_{2}\right)=\mathbb{Z}_{2}(0 \leqslant i \leqslant m)
$$

and the orientation character is

$$
w\left(\mathbb{R P}^{m}\right)=(-1)^{m+1} \in H^{1}\left(\mathbb{R P}^{m} ; \mathbb{Z}_{2}\right)=\mathbb{Z}_{2} .
$$

Thus $\mathbb{R P}^{m}$ is orientable for odd $m$, and nonorientable for even $m$.

There are also versions of Poincaré duality for manifolds with boundary, and cobordisms.

Definition 4.7 Let $R$ be a commutative ring.

(i) An $(m+1)$-dimensional cobordism $\left(W ; M, M^{\prime}\right)$ is $R$-orientable if there exists an $R$-coefficient fundamental class $[W] \in H_{m+1}\left(W, M \cup M^{\prime} ; R\right)$, such that for every $x \in W \backslash\left(M \cup M^{\prime}\right)$ the $R$-module morphism

$H_{m+1}\left(W, M \cup M^{\prime} ; R\right) \rightarrow H_{m+1}(W, W \backslash\{x\} ; R)=H_{m+1}\left(\mathbb{R}^{m+1}, \mathbb{R}^{m+1} \backslash\{0\} ; R\right)=R$ sends $[W]$ to a unit of $R$, and such that

$$
\partial([W])=\left([M],-\left[M^{\prime}\right]\right) \in H_{m}\left(M \cup M^{\prime} ; R\right)=H_{m}(M ; R) \oplus H_{m}\left(M^{\prime} ; R\right)
$$

with $[M] \in H_{m}(M ; R),\left[M^{\prime}\right] \in H_{m}\left(M^{\prime} ; R\right) R$-coefficient fundamental classes. (ii) The orientation character of a cobordism $\left(W ; M, M^{\prime}\right)$ is the cohomology class $w(W) \in H^{1}\left(W ; \mathbb{Z}_{2}\right)$ such that

$$
S q^{1}=w(W) \cup-: H^{m}\left(W ; \mathbb{Z}_{2}\right) \rightarrow H^{m+1}\left(W ; \mathbb{Z}_{2}\right) .
$$

The morphism $H^{1}\left(W ; \mathbb{Z}_{2}\right) \rightarrow H^{1}\left(M ; \mathbb{Z}_{2}\right) \oplus H^{1}\left(M^{\prime} ; \mathbb{Z}_{2}\right)$ induced by the inclusion $M \cup M^{\prime} \hookrightarrow W$ sends $w(W)$ to $\left(w(M), w\left(M^{\prime}\right)\right)$. The cobordism $\left(W ; M, M^{\prime}\right)$ is orientable if $w(W)=0 \in H^{1}\left(W ; \mathbb{Z}_{2}\right)$.

Poincaré-Lefschetz Duality Theorem 4.8 For any R-orientable $(m+1)$ dimensional cobordism $\left(W ; M, M^{\prime}\right)$ cap product with $[W] \in H_{m+1}\left(W, M \cup M^{\prime} ; R\right)$ defines $R$-module isomorphisms

$$
[W] \cap-: H^{*}(W, M ; R) \cong H_{m+1-*}\left(W, M^{\prime} ; R\right) .
$$

In particular, if $(W, \partial W)$ is an $R$-oriented $(m+1)$-dimensional manifold with boundary there are defined Poincaré duality isomorphisms

$$
\begin{aligned}
& {[W] \cap-: H^{*}(W, \partial W ; R) \cong H_{m+1-*}(W ; R),} \\
& {[W] \cap-: H^{*}(W ; R) \cong H_{m+1-*}(W, \partial W ; R) .}
\end{aligned}
$$


Example $4.9\left(D^{m+1}, S^{m}\right)$ is an orientable $(m+1)$-dimensional manifold with boundary, such that

$$
H^{r}\left(D^{m+1}\right)=H_{m+1-r}\left(D^{m+1}, S^{m}\right)= \begin{cases}\mathbb{Z} & \text { if } r=0 \\ 0 & \text { otherwise }\end{cases}
$$

Poincaré duality relates the cup product structure on the cohomology of a manifold $M$ with the intersection properties of submanifolds $N \subseteq M$. If $f$ : $N^{n} \hookrightarrow M^{m}$ is an embedding of an oriented submanifold then $f_{*}[N] \in H_{n}(M)$ is a homology class (which we shall usually write as $[N] \in H_{n}(M)$ ). The Poincaré dual to a cohomology class $[N]^{*} \in H^{m-n}(M)$ determines the homology classes of (transverse) intersections of submanifolds of $M$ with $N$. The connection between Poincaré duality and intersections is particularly straightforward for deRham cohomology :

Example 4.10 The $\mathbb{R}$-coefficient cohomology group $H^{p}(M ; \mathbb{R})$ of an $m$-dimensional manifold $M^{m}$ can be expressed as the deRham cohomology groups of differential $p$-forms $\omega$ on $M$ (cf. Bott and Tu [8]). Identify

$$
H_{*}(M ; \mathbb{R})=\operatorname{Hom}_{\mathbb{R}}\left(H^{*}(M ; \mathbb{R}), \mathbb{R}\right)
$$

by the $\mathbb{R}$-coefficient Universal Coefficient Theorem (3.17). An oriented $M$ has an $\mathbb{R}$-coefficient fundamental class $[M] \in H_{m}(M ; \mathbb{R})$, such that cap product with $[M]$ defines $\mathbb{R}$-coefficient Poincaré duality isomorphism

$$
[M] \cap-: H^{m-n}(M ; \mathbb{R}) \rightarrow H_{n}(M ; \mathbb{R})
$$

An embedding of an oriented $n$-dimensional submanifold $f: N^{n} \hookrightarrow M$ represents the $\mathbb{R}$-coefficient homology class $f_{*}[N] \in H_{n}(M ; \mathbb{R})$ corresponding to integration over $N$

$$
f_{*}[N]: H^{n}(M ; \mathbb{R}) \rightarrow \mathbb{R} ; \omega \mapsto \int_{N} f^{*} \omega
$$

In fact, every $\mathbb{R}$-coefficient homology class $x \in H_{n}(M ; \mathbb{R})$ is of the form $r\left(f_{*}[N]\right)$ for some $f: N^{n} \hookrightarrow M$ and $r \in \mathbb{R}$. Write the Poincaré dual of $f_{*}[N] \in H_{n}(M ; \mathbb{R})$ as $\eta_{N} \in H^{m-n}(M ; \mathbb{R})$. If $\left(N_{1}\right)^{n_{1}},\left(N_{2}\right)^{n_{2}} \subseteq M^{n_{1}+n_{2}}$ are oriented submanifolds which intersect transversely then

$$
\eta_{N_{1}} \wedge \eta_{N_{2}}=\eta_{N_{1} \cap N_{2}} \in H^{n_{1}+n_{2}}(M ; \mathbb{R})=H_{0}(M ; \mathbb{R})=\mathbb{R}
$$

is the algebraic number of points in $N_{1} \cap N_{2}$. (See Section 7.2 below for the precise definition of transverse intersection.) 
Definition 4.11 The homology intersection pairing of an oriented $m$-dimensional manifold $M$

$$
\lambda: H_{n}(M) \times H_{m-n}(M) \rightarrow \mathbb{Z} ;(x, y) \mapsto \lambda(x, y)
$$

is defined by

$$
\lambda(x, y)=\left\langle x^{*} \cup y^{*},[M]\right\rangle \in \mathbb{Z}
$$

with $x^{*} \in H^{m-n}(M), y^{*} \in H^{n}(M)$ the Poincaré duals of $x, y$.

Proposition 4.12 The homology intersection pairing satisfies

$$
\begin{aligned}
& \lambda\left(x+x^{\prime}, y\right)=\lambda(x, y)+\lambda\left(x^{\prime}, y\right), \\
& \lambda\left(x, y+y^{\prime}\right)=\lambda(x, y)+\lambda\left(x, y^{\prime}\right), \\
& \lambda(a x, b y)=a b \lambda(x, y), \\
& \lambda(y, x)=(-1)^{n(m-n)} \lambda(x, y) \in \mathbb{Z}
\end{aligned}
$$

for every $a, b \in \mathbb{Z}, x, x^{\prime} \in H_{n}(M), y, y^{\prime} \in H_{m-n}(M)$.

Proof The cup product pairing (for any space $M$ )

$$
\cup: H^{p}(M) \times H^{q}(M) \rightarrow H^{p+q}(M) ;(x, y) \mapsto x \cup y
$$

is bilinear, and such that

$$
y \cup x=(-1)^{p q} x \cup y \in H^{p+q}(M) .
$$

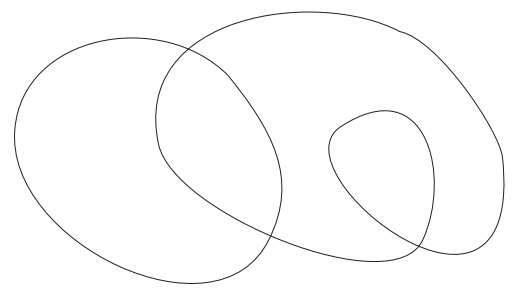

Definition 4.13 The algebraic intersection number of immersions of oriented manifolds $N_{1}^{n_{1}} \rightarrow M^{n_{1}+n_{2}}, N_{2}^{n_{2}} \rightarrow M^{n_{1}+n_{2}}$ in a connected oriented manifold

$$
\lambda\left(\left[N_{1}\right],\left[N_{2}\right]\right) \in \mathbb{Z}
$$

is the homology intersection of the homology classes $\left[N_{1}\right] \in H_{n_{1}}(M),\left[N_{2}\right] \in$ $H_{n_{2}}(M)$. 
In Chapter 7 the algebraic intersection of transversely intersecting immersions $N_{1} \rightarrow M, N_{2} \rightarrow M$ will be identified with the algebraic number of double points.

Any map of spaces $f: N \rightarrow M$ induces morphisms $f_{*}: H_{*}(N) \rightarrow H_{*}(M)$ in homology, and also morphisms $f^{*}: H^{*}(M) \rightarrow H^{*}(N)$ in cohomology.

Definition 4.14 The homology and cohomology Umkehr (= 'reverse') morphisms for a map $f: N^{n} \rightarrow M^{m}$ of oriented manifolds are the composites

$$
\begin{aligned}
& f^{!}: H_{*}(M) \cong H^{m-*}(M) \stackrel{f^{*}}{\longrightarrow} H^{m-*}(N) \cong H_{n-m+*}(N) \\
& f_{!}: H^{*}(N) \cong H_{n-*}(N) \stackrel{f_{*}}{\longrightarrow} H_{n-*}(M) \cong H^{m-n+*}(M)
\end{aligned}
$$

Proposition 4.15 Let $f: N^{n} \rightarrow M^{m}$ be a map of oriented connected manifolds. (i) The homology Umkehr $f^{!}: H_{m}(M) \rightarrow H_{n}(N)$ is such that

$$
f^{!}[M]=[N] \in H_{n}(N)
$$

(ii) The cohomology Umkehr $f$ ! : $H^{0}(N) \rightarrow H^{m-n}(M)$ sends $1 \in H^{0}(N)=\mathbb{Z}$ to the cohomology class $f_{!}(1) \in H^{m-n}(M)$ Poincaré dual to the homology class $f_{*}[N] \in H_{n}(M)$, with

$$
[M] \cap f_{!}(1)=f_{*}[N] \in H_{n}(M) .
$$

Proof (i) The generator $1 \in H^{0}(M)=\mathbb{Z}$ is Poincaré dual to the fundamental class $[M] \in H_{m}(M)$. The induced map $f^{*}: H^{0}(M)=\mathbb{Z} \rightarrow H^{0}(N)=\mathbb{Z}$ sends $1 \in H^{0}(M)$ to $f^{*}(1)=1 \in H^{0}(N)$.

(ii) By construction.

The Umkehr map will be used in the next section to describe the homology effect of surgery.

\subsection{The homotopy and homology effects of surgery}

The homotopy theoretic effect of an $n$-surgery on an $m$-dimensional manifold is a combination of attaching an $(n+1)$-cell and detaching the dual $(m-n-1)$-cell. This section will describe the effects of the cell attachments and detachments on the homotopy and homology groups. For $2 n-1 \leqslant m$ it is possible to separate the algebraic effects of attaching and detaching the cells. The algebraic effects of surgery are much more complicated in the middle-dimensional cases $m=2 n$ or $2 n+1$ (on account of self-intersections), and will be considered further in 
Chapters 11,12 . In order to deal with surgery on non-simply-connected manifolds it is also necessary to consider the effect of surgery on the homology of the universal cover, and this will be done in Chapter 10 .

Definition 4.16 An $n$-surgery on $M^{m}$ removing the framed $n$-embedding $g$ : $S^{n} \times D^{m-n} \hookrightarrow M$ kills the element $x \in \pi_{n}(M)$ represented by the core

$$
x=g \mid: S^{n} \times 0 \hookrightarrow M .
$$

The dual $(m-n-1)$-surgery on the effect $M^{\prime m}$ removing the dual framed $(m-n-1)$-embedding $g^{\prime}: D^{n+1} \times S^{m-n-1} \hookrightarrow M^{\prime}$ kills the element $x^{\prime} \in$ $\pi_{m-n-1}\left(M^{\prime}\right)$ represented by

$$
x^{\prime}=g^{\prime} \mid: 0 \times S^{m-n-1} \hookrightarrow M^{\prime} .
$$

Example 4.17 The zero element $0 \in \pi_{n}(M)$ can be killed by an $n$-surgery on $M^{m}$

$$
S^{n} \times D^{m-n} \hookrightarrow S^{n} \times D^{m-n} \cup D^{n+1} \times S^{m-n-1}=S^{m} \hookrightarrow M \# S^{m}=M
$$

with effect the connected sum

$$
M^{\prime m}=M^{m} \#\left(S^{n+1} \times S^{m-n-1}\right) .
$$

Remark 4.18 The following Proposition contains a commutative braid of the type

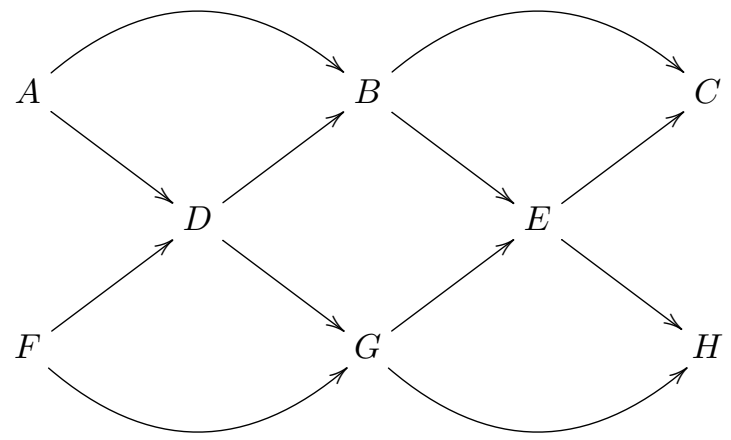

in which the sequences

$$
\begin{aligned}
& A \longrightarrow B \longrightarrow E \longrightarrow H \text {, } \\
& A \longrightarrow D \longrightarrow G \longrightarrow H \text {, } \\
& \mathrm{F} \longrightarrow \mathrm{D} \longrightarrow \mathrm{B} \longrightarrow \mathrm{C} \text {, } \\
& F \longrightarrow G \longrightarrow E \longrightarrow C
\end{aligned}
$$


are exact. It follows that there is a rudimentary Mayer-Vietoris exact sequence

$$
D \longrightarrow B \oplus G \longrightarrow E
$$

and that there is defined an isomorphism

$$
\frac{\operatorname{ker}(B \rightarrow C)}{\operatorname{im}(A \rightarrow B)} \cong \frac{\operatorname{ker}(G \rightarrow H)}{\operatorname{im}(F \rightarrow G)}
$$

Proposition 4.19 Let $\left(W ; M, M^{\prime}\right)$ be the trace of an $n$-surgery on an m-dimensional manifold $M$ removing the framed n-embedding $g: S^{n} \times D^{m-n} \hookrightarrow M$, let $g^{\prime}: D^{n+1} \times S^{m-n-1} \hookrightarrow M^{\prime}$ be the dual framed $(m-n-1)$-embedding, and let

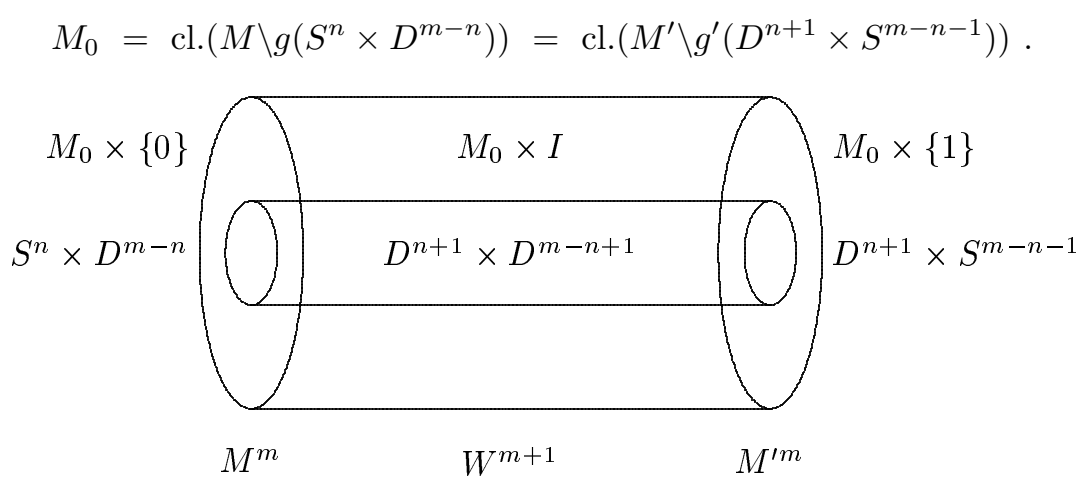

(i) The trace $W$ is homotopy equivalent to a space obtained from $M$ by attaching an $(n+1)$-cell and a space obtained from $M^{\prime}$ by attaching an $(m-n)$-cell

$$
W \simeq M \cup_{x} D^{n+1} \simeq M^{\prime} \cup_{x^{\prime}} D^{m-n},
$$

with $x=g\left|: S^{n} \hookrightarrow M, x^{\prime}=g^{\prime}\right|: S^{m-n-1} \hookrightarrow M^{\prime}$ the cores of $g, g^{\prime}$. The projection

$$
W \rightarrow W /\left(D^{n+1} \times D^{m-n}\right) \simeq M_{0} /\left(S^{n} \times S^{m-n-1}\right)
$$

is a homotopy equivalence.

(ii) The homotopy groups are such that

$$
\pi_{i}(W)= \begin{cases}\pi_{i}(M) & \text { if } i<n \\ \pi_{n}(M) /\langle x\rangle & \text { if } i=n\end{cases}
$$

with $\langle x\rangle \subseteq \pi_{n}(M)$ the normal subgroup (resp. the $\mathbb{Z}\left[\pi_{1}(M)\right]$-module) generated by $x$ for $n=1$ (resp. $n \geqslant 2$ ). Also, since $\left(W ; M, M^{\prime}\right)$ is the trace of the dual $(m-n-1)$-surgery on $M^{\prime}$ killing an element $x^{\prime} \in \pi_{m-n-1}\left(M^{\prime}\right)$

$$
\pi_{i}(W)= \begin{cases}\pi_{i}\left(M^{\prime}\right) & \text { if } i<m-n-1 \\ \pi_{m-n-1}\left(M^{\prime}\right) /\left\langle x^{\prime}\right\rangle & \text { if } i=m-n-1 .\end{cases}
$$

In particular, if $2 n+1 \leqslant m$ then 


$$
\pi_{i}\left(M^{\prime}\right)=\pi_{i}(W)=\pi_{i}(M) \text { if } i<n,
$$

and if $2 n+2 \leqslant m$

$$
\pi_{n}\left(M^{\prime}\right)=\pi_{n}(W)=\pi_{n}(M) /\langle x\rangle
$$

(iii) The homology groups of $\left(W ; M, M^{\prime}\right)$ fit into a commutative braid of exact sequences

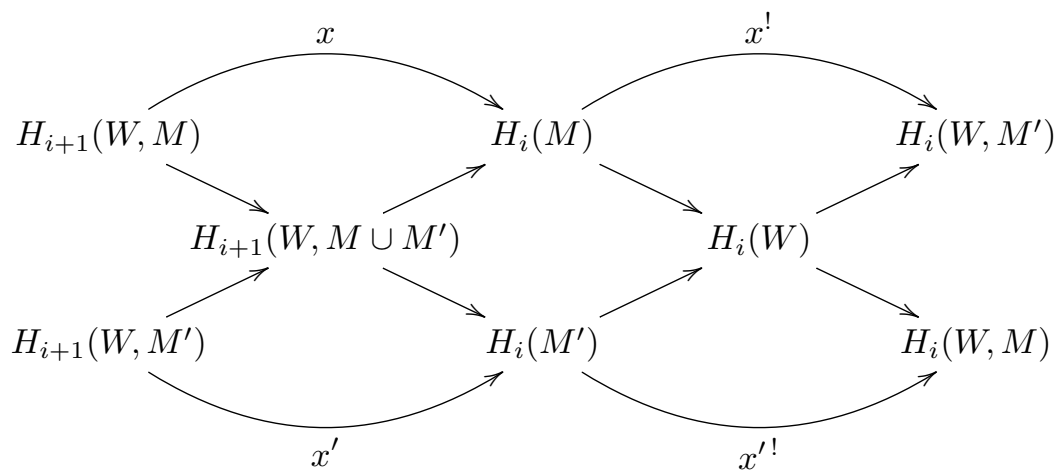

with

$$
\begin{aligned}
& H_{i}(W, M)=H_{i}\left(D^{n+1}, S^{n}\right)= \begin{cases}\mathbb{Z} & \text { if } i=n+1 \\
0 & \text { if } i \neq n+1,\end{cases} \\
& H_{i}\left(W, M^{\prime}\right)=H_{i}\left(D^{m-n}, S^{m-n-1}\right)= \begin{cases}\mathbb{Z} & \text { if } i=m-n \\
0 & \text { if } i \neq m-n\end{cases} \\
& H_{i+1}\left(W, M \cup M^{\prime}\right)=H_{i}\left(M_{0}\right) .
\end{aligned}
$$

If $M$ is orientable the Umkehr map $x^{!}$is the evaluation of the homology intersection pairing $\lambda: H_{n}(M) \times H_{m-n}(M) \rightarrow \mathbb{Z}$ on $x \in H_{n}(M)$

$$
x^{!}=\lambda(x,-): H_{m-n}(M) \rightarrow \mathbb{Z} ; y \mapsto \lambda(x, y)
$$

Similarly, if $M^{\prime}$ is orientable the Umkehr map $x^{\prime !}$ is the evaluation of the homology intersection pairing $\lambda^{\prime}: H_{m-n-1}\left(M^{\prime}\right) \times H_{n+1}\left(M^{\prime}\right) \rightarrow \mathbb{Z}$ on $x^{\prime} \in H_{m-n-1}\left(M^{\prime}\right)$

$$
x^{\prime !}=\lambda^{\prime}\left(x^{\prime},-\right): H_{n+1}\left(M^{\prime}\right) \rightarrow \mathbb{Z} ; y^{\prime} \mapsto \lambda^{\prime}\left(x^{\prime}, y^{\prime}\right) .
$$

Proof By Proposition 3.30 and the homology exact sequence of a cofibration (Proposition 3.24).

Example 4.20 The homology intersection pairing on the $n$th homology group $H_{n}(M)$ of an oriented $2 n$-dimensional manifold $M^{2 n}$ is $(-1)^{n}$-symmetric

$$
\lambda: H_{n}(M) \times H_{n}(M) \rightarrow \mathbb{Z} ;(x, y) \mapsto \lambda(x, y)=(-1)^{n} \lambda(y, x) .
$$


In order to kill an element $x \in \pi_{n}(M)$ by surgery on $M$ it is necessary (but not in general sufficient) for the Hurewicz image $x \in H_{n}(M)$ to be such that

$$
\lambda(x, x)=0 \in \mathbb{Z}
$$

- this is automatic if $n$ is odd. Moreover, if surgery on $x \in \pi_{n}(M)$ is possible and the effect $M^{2 n}$ is oriented then

$$
H_{n}\left(M^{\prime}\right)=\frac{\left\{y \in H_{n}(M) \mid \lambda(x, y)=0 \in \mathbb{Z}\right\}}{\{k x \mid k \in \mathbb{Z}\}},
$$

the quotient of the subgroup of the homology classes orthogonal to $x \in H_{n}(M)$ by the subgroup of the classes parallel to $x$. The $(-1)^{n}$-symmetric form on $H_{n}\left(M^{\prime}\right)$ is the one inherited from the form on $H_{n}(M)$

$$
\lambda^{\prime}: H_{n}\left(M^{\prime}\right) \times H_{n}\left(M^{\prime}\right) \rightarrow \mathbb{Z} ;([y],[z]) \mapsto \lambda(y, z) .
$$

As before, let $M^{\prime}$ be the $m$-dimensional manifold obtained from an $m$-dimensional manifold $M$ by an $n$-surgery killing a homotopy class $x \in \pi_{n}(M)$. If $2 n \leqslant m$ then

$$
\pi_{i}(M)=\pi_{i}(W)=\pi_{i}\left(M^{\prime}\right) \text { for } i \leqslant n-2 .
$$

If $2 n+1 \leqslant m$ then

$$
\pi_{n-1}(M)=\pi_{n-1}(W)=\pi_{n-1}\left(M^{\prime}\right)
$$

and

$$
\pi_{n}\left(M^{\prime}\right)=\pi_{n}(M) /\langle x\rangle
$$

is smaller than $\pi_{n}(M)$, in general. If $m=2 n+1$

$$
\pi_{n}(M) /\langle x\rangle=\pi_{n}(W)=\pi_{n}\left(M^{\prime}\right) /\left\langle x^{\prime}\right\rangle
$$

with $x^{\prime} \in \pi_{n}\left(M^{\prime}\right)$ the homotopy class killed by the dual $n$-surgery, so that $\pi_{n}\left(M^{\prime}\right)$ is in general neither smaller nor larger than $\pi_{n}(M)$. If $m=2 n$ it is possible for an $n$-surgery to create elements in $\pi_{n-1}\left(M^{\prime}\right)$, as in:

Example 4.21 For a $2 n$-dimensional manifold $M$ the effect of the trivial $n$ surgery killing $0 \in \pi_{n}(M)$ is the $2 n$-dimensional manifold

$$
M^{\prime 2 n}=M^{2 n} \#\left(S^{n+1} \times S^{n-1}\right)
$$

with

$$
\pi_{n-1}\left(M^{\prime}\right)=\pi_{n-1}(M) \oplus \mathbb{Z} .
$$

It is also possible for an $n$-surgery on a $2 n$-dimensional manifold to make $\pi_{n}(M)$ smaller without affecting $\pi_{n-1}(M)$, as in: 
Example 4.22 The effect of the $n$-surgery removing the framed $n$-embedding $S^{n} \times D^{n} \hookrightarrow S^{n} \times D^{n} \cup S^{n} \times D^{n}=S^{n} \times S^{n}$

is

$$
D^{n+1} \times S^{n-1} \cup S^{n} \times D^{n}=\partial\left(D^{n+1} \times D^{n}\right)=S^{2 n},
$$

with

$$
\pi_{n-1}\left(S^{2 n}\right)=\pi_{n-1}\left(S^{n} \times S^{n}\right)=0
$$

What is the effect of surgery on orientability? 0- and 1-surgeries can change orientation type:

Example 4.23 The effect of a 0-surgery on $S^{2}$ depends on the choice of framing in $S^{0} \times D^{2} \hookrightarrow S^{2}$, and is either a torus $T^{2}=S^{1} \times S^{1}$ :

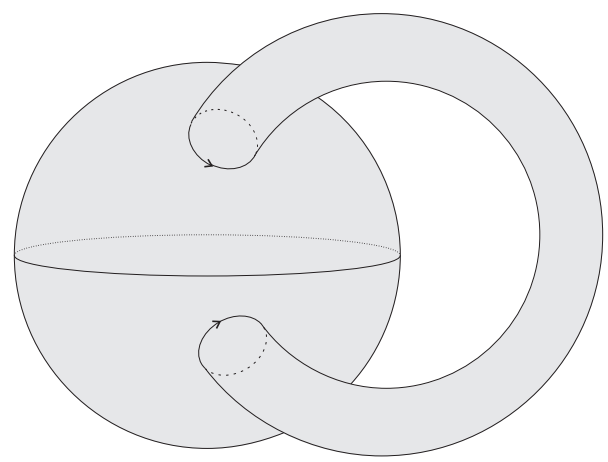

or a Klein bottle $K$ (which is nonorientable) :

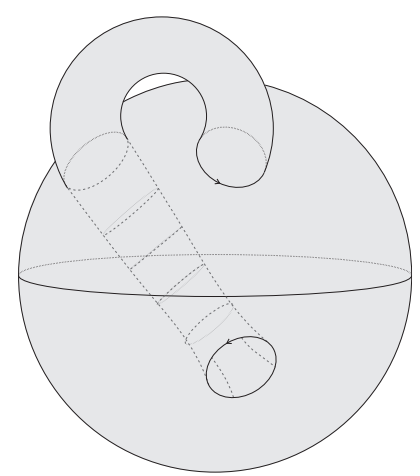

The dual 1-surgery on $K$ has effect $S^{2}$, and so changes the orientation type. 
In general, we have:

Proposition 4.24 Let $\left(W ; M, M^{\prime}\right)$ be the trace of an n-surgery on an m-dimensional manifold $M$ killing $x \in H_{n}(M)$.

(i) If $1 \leqslant n \leqslant m-2$ then $W$ and $M^{\prime}$ have the same orientation type as $M$.

(ii) If $n=m-1$ and $M$ is orientable then so are $W$ and $M^{\prime}$.

(iii) If $n=m-1$ and $M$ is nonorientable then $M^{\prime}$ is orientable if and only if

$$
x=w(M) \in H_{m-1}\left(M ; \mathbb{Z}_{2}\right)=H^{1}\left(M ; \mathbb{Z}_{2}\right) .
$$

(iv) If $n=0$ and $M$ is nonorientable then so are $W$ and $M^{\prime}$.

Proof The orientation character $w(W) \in H^{1}\left(W ; \mathbb{Z}_{2}\right)$ has images the orientation characters $w(M) \in H^{1}\left(M ; \mathbb{Z}_{2}\right), w\left(M^{\prime}\right) \in H^{1}\left(M^{\prime} ; \mathbb{Z}_{2}\right)$. The homotopy equivalences

$$
W \simeq M \cup D^{n+1} \simeq M^{\prime} \cup D^{m-n}
$$

give the relative $\mathbb{Z}_{2}$-cohomology groups in the commutative braid of exact sequences (as in 4.19)

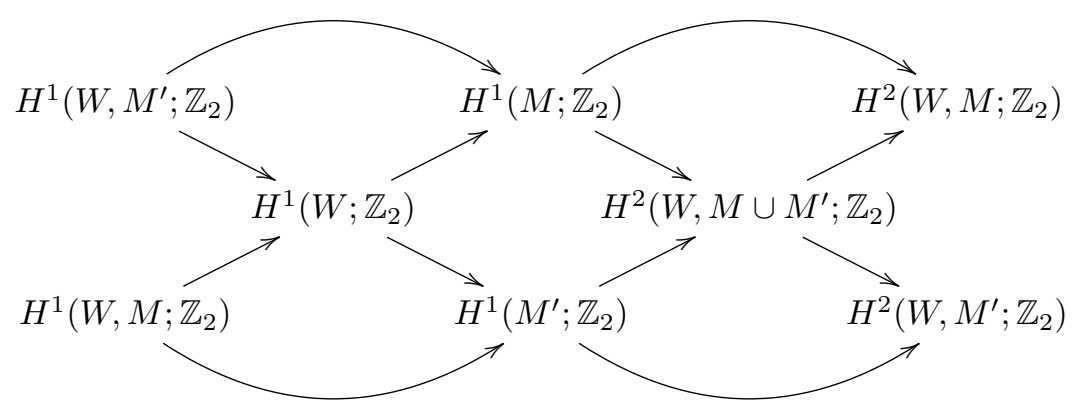

to be

$$
H^{i}\left(W, M ; \mathbb{Z}_{2}\right)=\left\{\begin{array}{ll}
\mathbb{Z}_{2} & \text { if } i=n+1 \\
0 & \text { otherwise }
\end{array}, H^{i}\left(W, M^{\prime} ; \mathbb{Z}_{2}\right)= \begin{cases}\mathbb{Z}_{2} & \text { if } i=m-n \\
0 & \text { otherwise. }\end{cases}\right.
$$

(i) If $1 \leqslant n \leqslant m-2$ then the morphisms

$$
H^{1}\left(W ; \mathbb{Z}_{2}\right) \rightarrow H^{1}\left(M ; \mathbb{Z}_{2}\right), H^{1}\left(W ; \mathbb{Z}_{2}\right) \rightarrow H^{1}\left(M^{\prime} ; \mathbb{Z}_{2}\right)
$$

are injective, so that $w(M)=0$ if and only if $w(W)=0$, and $w(W)=0$ if and only if $w\left(M^{\prime}\right)=0$.

(ii) + (iii) For $n=m-1$ the braid is given by 


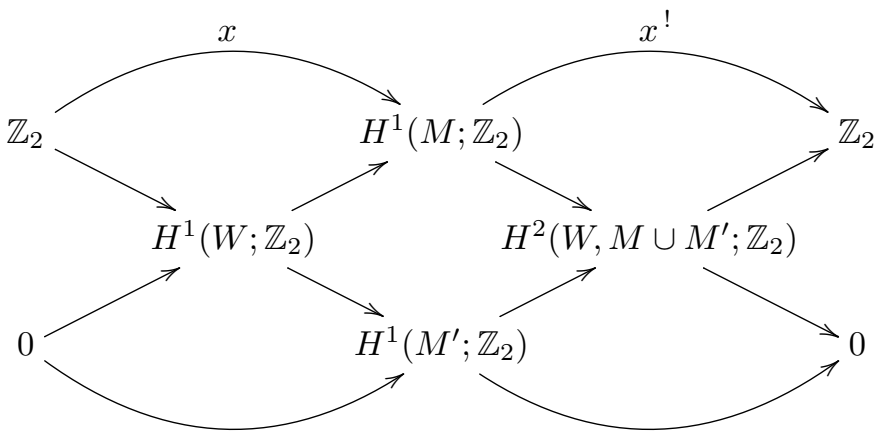

If $w(M)=0$ it follows from the injectivity of $H^{1}\left(W ; \mathbb{Z}_{2}\right) \rightarrow H^{1}\left(M ; \mathbb{Z}_{2}\right)$ that $w(W)=0$, and hence that $w\left(M^{\prime}\right)=0$.

If $w(M) \neq 0$ it follows from the injectivity of $H^{1}\left(M^{\prime} ; \mathbb{Z}_{2}\right) \rightarrow H^{2}\left(W, M \cup M^{\prime} ; \mathbb{Z}_{2}\right)$ that $w\left(M^{\prime}\right)=0$ if and only if $w(M)=x \in H^{1}\left(M ; \mathbb{Z}_{2}\right)$.

(iv) Apply (ii) to the cobordism $\left(W ; M^{\prime}, M\right)$, which is the trace of the dual $(m-1)$-surgery on $M^{\prime}$.

It is easy to describe the effect of surgery on the Euler characteristic :

Definition 4.25 The Euler characteristic of a finite $C W$ complex $X$ is

$$
\chi(X)=\sum_{i=0}^{\infty}(-1)^{i} \operatorname{dim}_{\mathbb{R}} H_{i}(X ; \mathbb{R}) \in \mathbb{Z} .
$$

Proposition 4.26 If the $m$-dimensional manifold $M^{\prime m}$ is obtained from $M^{m}$ by an n-surgery then

$$
\chi\left(M^{\prime}\right)=\chi(M)+(-1)^{n+1}\left(1+(-1)^{m}\right) \in \mathbb{Z} .
$$

Proof The effect on the Euler characteristic of attaching an $r$-cell to a space $X$ is

$$
\chi\left(X \cup D^{r}\right)=\chi(X)+(-1)^{r} .
$$

Use the homotopy equivalences given for the trace $\left(W ; M, M^{\prime}\right)$ by 4.19

$$
W \simeq M \cup D^{n+1} \simeq M^{\prime} \cup D^{m-n}
$$

to identify

$$
\chi(W)=\chi(M)+(-1)^{n+1}=\chi\left(M^{\prime}\right)+(-1)^{m-n} .
$$

Example 4.27 The $m$-sphere $S^{m}$ is obtained from $\emptyset$ by a (-1)-surgery, with Euler characteristic

$$
\chi\left(S^{m}\right)=1+(-1)^{m} .
$$


Example 4.28 The connected sum of connected $m$-dimensional manifolds $M^{m}$, $M^{\prime m}$ is the connected $m$-dimensional manifold $M \# M^{\prime}$ obtained from $M \cup M^{\prime}$ by 0 -surgery (2.10). The Euler characteristic is given by 4.26 to be

$$
\chi\left(M \# M^{\prime}\right)=\chi(M)+\chi\left(M^{\prime}\right)-\left(1+(-1)^{m}\right) .
$$

\subsection{Surfaces}

Definition 4.29 A surface is a 2-dimensional manifold.

Surfaces are excellent illustrations of the surgery construction. Every orientable surface and the nonorientable surfaces of even genus can be obtained from the empty surface by a sequence of surgeries.

Definition 4.30 The orientable surface of genus $g$ is defined for $g \geqslant 0$ to be

$$
\begin{aligned}
M(g) & =\partial\left(0 \text {-handle } D^{0} \times D^{3} \cup g \text {-handles } D^{1} \times D^{2}\right) \\
& =\text { the effect of } g \text { orientable } 0 \text {-surgeries } S^{0} \times D^{2} \hookrightarrow S^{2} \\
& =0 \text {-handle } D^{0} \times D^{2} \cup\left\{2 g \text {-handles } D^{1} \times D^{1}\right\} \cup 2 \text {-handle } D^{2} \times D^{0} \\
& =\underset{g}{\#} T^{2}
\end{aligned}
$$

the $g$-fold connected sum of the 2-torus $T^{2}=S^{1} \times S^{1}$.

In particular

$$
\begin{aligned}
& M(0)=\text { the sphere } S^{2}, \\
& M(1)=\text { the torus } T^{2}, \\
& M(2)=\text { the anchor } \operatorname{ring} T^{2} \# T^{2}
\end{aligned}
$$

Here is a picture of $M(2)$ :

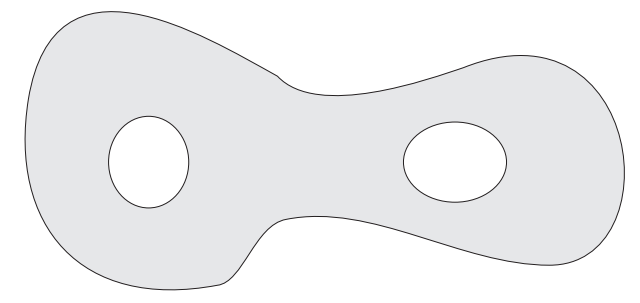

Definition 4.31 The nonorientable surface of genus $g$ is defined for $g \geqslant 1$ to be 


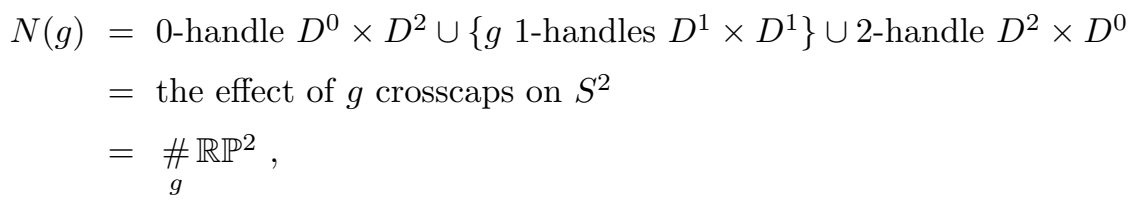

where each crosscap replaces $D^{2} \hookrightarrow S^{2}$ by an embedded Möbius band.

In particular

$$
\begin{aligned}
& N(1)=\text { the real projective space } \mathbb{R P}^{2}, \\
& N(2)=\text { the Klein bottle } K^{2} .
\end{aligned}
$$

If $g$ is even

$$
\begin{aligned}
N(g) & =\partial\left(0 \text {-handle } D^{0} \times D^{3} \cup g / 2 \text {-handles } D^{1} \times D^{2}\right) \\
& =\text { the effect of } g / 2 \text { nonorientable 0-surgeries } S^{0} \times D^{2} \hookrightarrow S^{2} \\
& =\#_{g / 2} K .
\end{aligned}
$$

If $g$ is odd

$$
\begin{aligned}
N(g) & =\text { the effect of }(g-1) / 20 \text {-surgeries } S^{0} \times D^{2} \hookrightarrow \mathbb{R P}^{2} \\
& =\mathbb{R P}^{2} \# \#{ }_{(g-1) / 2} T^{2} .
\end{aligned}
$$

Classification of Surfaces 4.32 (i) Every connected closed surface is diffeomorphic to exactly one of

$$
M(g) \quad(g \geqslant 0), N(g) \quad(g \geqslant 1) .
$$

Surfaces are classified by orientation type and Euler characteristic, with

$$
\chi(M(g))=2-2 g, \chi(N(g))=2-g .
$$

(ii) Every homotopy equivalence of surfaces $M \rightarrow M^{\prime}$ is homotopic to a diffeomorphism.

Proof See Chapter 9 of Hirsch [33] for the detailed classification of surfaces using surgery and Morse theory. The essential steps are easy to describe from the surgery-theoretic point of view, as follows. If $M$ is a connected orientable surface then $H_{1}(M)$ is a f.g. free abelian group, the homology intersection pairing (4.11) is $(-1)$-symmetric

$$
\lambda: H_{1}(M) \times H_{1}(M) \rightarrow \mathbb{Z} ;(x, y) \mapsto \lambda(x, y)=-\lambda(y, x),
$$

and there exists a basis $\left\{a_{1}, b_{1}, a_{2}, b_{2}, \ldots, a_{g}, b_{g}\right\}$ for $H_{1}(M)$ such that

$$
\lambda\left(a_{i}, b_{j}\right)=\left\{\begin{array}{ll}
1 & \text { if } i=j \\
0 & \text { if } i \neq j
\end{array}, \lambda\left(a_{i}, a_{j}\right)=\lambda\left(b_{i}, b_{j}\right)=0 .\right.
$$

The effect of an orientable 0-surgery on $M$ is to form the connected sum with a torus $T^{2}$; dually, the effect of a 1-surgery is to remove a torus. (Proposition 4.33 
below gives a detailed account of the effects of 0- and 1-surgeries on surfaces). The half-basis $\left\{a_{1}, a_{2}, \ldots, a_{g}\right\}$ for $H_{1}(M)$ can be realized by disjoint framed 1-embeddings $a_{i}: S^{1} \times D^{1} \hookrightarrow M$. The combined effect of the corresponding 1-surgeries on $M$ is a connected orientable surface $M^{\prime}$ such that

$$
M^{\prime} \# \underset{g}{\#} T^{2}=M, \pi_{1}\left(M^{\prime}\right)=H_{1}\left(M^{\prime}\right)=0,
$$

and the 2-dimensional $h$-Cobordism Theorem shows that up to diffeomorphism

$$
M^{\prime}=S^{2}, M=\underset{g}{\#} T^{2}=M(g) .
$$

The effects of 0 - and 1-surgeries on surfaces are given by:

Proposition 4.33 (i) The effect of a 0-surgery on a surface $M$ is a surface $M^{\prime}$ with

$$
\chi\left(M^{\prime}\right)=\chi(M)-2 .
$$

If $M=M(g)$ then $M^{\prime}$ is connected with $\chi\left(M^{\prime}\right)=-2 g$, so that

$$
M^{\prime}= \begin{cases}M(g+1) & \text { if orientable } \\ N(2 g+2) & \text { if nonorientable. }\end{cases}
$$

If $M=N(g)$ then $M^{\prime}$ is connected with $\chi\left(M^{\prime}\right)=-g$, so that

$$
M^{\prime}= \begin{cases}M((g+2) / 2) & \text { if orientable, with } g \text { even } \\ N(g+2) & \text { if nonorientable. }\end{cases}
$$

(ii) The effect of a 1-surgery on a surface $M$ is a surface $M^{\prime}$ with

$$
\chi\left(M^{\prime}\right)=\chi(M)+2 .
$$

If $M=M(g)$ then $M^{\prime}$ is orientable with $\chi\left(M^{\prime}\right)=4-2 g$, so that

$$
M^{\prime}= \begin{cases}M(g-1) & \text { if connected } \\ M\left(g_{1}\right) \cup M\left(g_{2}\right) & \text { if disconnected, with } g_{1}+g_{2}=g .\end{cases}
$$

If $M=N(g)$ then $\chi\left(M^{\prime}\right)=4-g$, so that

$$
M^{\prime}= \begin{cases}M((g-2) / 2) & \text { if connected and orientable } \\ N(g-2) & \text { if connected and nonorientable } \\ N\left(g_{1}\right) \cup N\left(g_{2}\right) & \text { if disconnected, both components nonorientable, } \\ & \text { with } g_{1}+g_{2}=g \\ M\left(g_{1}\right) \cup N\left(g_{2}\right) & \text { if disconnected, one component orientable, } \\ \text { with } 2 g_{1}+g_{2}=g .\end{cases}
$$


Example 4.34 Let $M=M(g)$ be the orientable surface of genus $g \geqslant 0$, and let

$$
t_{i}: T^{2}=S^{1} \times S^{1} \rightarrow M=\#_{g} T^{2}(1 \leqslant i \leqslant g)
$$

be embeddings of the $g$ tori. The homology group $H_{1}(M)=\mathbb{Z}^{2 g}$ has a basis $\left\{a_{1}, b_{1}, a_{2}, b_{2}, \ldots, a_{g}, b_{g}\right\}$ given geometrically by

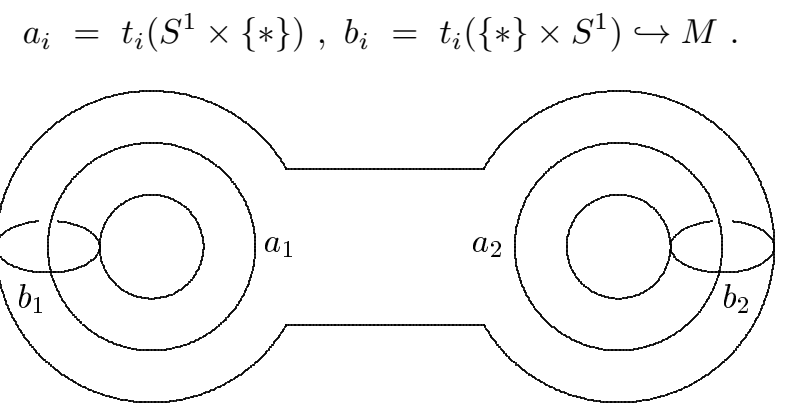

The (-1)-symmetric intersection form

$$
\lambda: H_{1}(M) \times H_{1}(M) \rightarrow \mathbb{Z}
$$

is such that

$$
\lambda\left(a_{i}, b_{j}\right)=\left\{\begin{array}{ll}
1 & \text { if } i=j \\
0 & \text { if } i \neq j
\end{array}, \lambda\left(a_{i}, a_{j}\right)=\lambda\left(b_{i}, b_{j}\right)=0\right.
$$

as in the proof of 4.3 . The function

$$
\left\{\text { isotopy classes of embeddings } S^{1} \hookrightarrow M\right\} \rightarrow H_{1}(M) ;\left(f: S^{1} \hookrightarrow M\right) \mapsto f_{*}\left[S^{1}\right]
$$

has image the subset of the elements $x \in H_{1}(M)$ which generate direct summands. Every embedding $f: S^{1} \hookrightarrow M$ has an essentially unique extension to a framed embedding $\bar{f}: S^{1} \times D^{1} \hookrightarrow M$. (A surface is orientable if and only if it is not possible to embed a Möbius band in it). By 4.33 (ii) the effect of the 1-surgery on $M$ removing $\bar{f}$ is an orientable surface $M^{\prime}$ with $\chi\left(M^{\prime}\right)=4-2 g$, which may be disconnected. There is defined an exact sequence

$$
0 \rightarrow H_{1}\left(M \backslash f\left(S^{1}\right)\right) \rightarrow H_{1}(M) \rightarrow \mathbb{Z} \rightarrow H_{0}\left(M \backslash f\left(S^{1}\right)\right) \rightarrow H_{0}(M)=\mathbb{Z} \rightarrow 0
$$

where

$$
H_{1}(M) \rightarrow \mathbb{Z} ; y \mapsto \lambda(x, y)
$$

with $x=f_{*}\left[S^{1}\right] \in H_{1}(M)$. It follows that $M^{\prime}$ is disconnected if and only if $x=0$. If $x \neq 0$ then $M^{\prime}$ is connected, and is diffeomorphic to $M(g-1)$. If $x=0$ then $M^{\prime}$ is disconnected, and

$$
M^{\prime}=M\left(g_{1}\right) \cup M\left(g_{2}\right)
$$

for some $g_{1}, g_{2} \geqslant 0$ such that $g_{1}+g_{2}=g$, with $g_{1}, g_{2}$ depending on the isotopy class of $f: S^{1} \hookrightarrow M$. 
More generally, let $M^{\prime}$ be the combined effect of $\ell$ 1-surgeries on $M$ removing disjoint framed embeddings

$$
\bar{f}_{k}: S^{1} \times D^{1} \hookrightarrow M \quad(1 \leqslant k \leqslant \ell)
$$

and killing $x_{k}=\left(f_{k}\right)_{*}\left[S^{1}\right] \in H_{1}(M)$. The trace $\left(W ; M, M^{\prime}\right)$ is orientable by 4.24. As in Proposition 4.19 (for $\ell=1$ ) there is a commutative braid of exact sequences

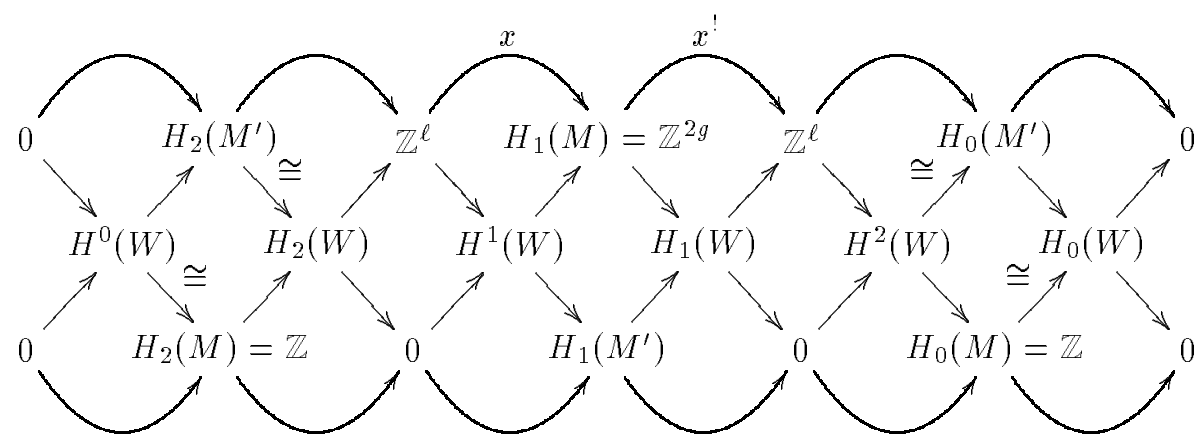

with

$$
\begin{aligned}
& W^{3}=M \times I \cup \bigcup_{\ell} D^{2} \times D^{1} \simeq M \cup \bigcup_{\ell} D^{2}, \\
& x=\left(x_{1}, x_{2}, \ldots, x_{\ell}\right): \mathbb{Z}^{\ell} \rightarrow H_{1}(M)=\mathbb{Z}^{2 g} ;\left(c_{1}, c_{2}, \ldots, c_{\ell}\right) \mapsto \sum_{k=1}^{\ell} c_{k} x_{k}, \\
& x^{!}: H_{1}(M)=\mathbb{Z}^{2 g} \rightarrow \mathbb{Z}^{\ell} ; y \mapsto\left(\lambda\left(x_{1}, y\right), \lambda\left(x_{2}, y\right), \ldots, \lambda\left(x_{\ell}, y\right)\right), \\
& H_{0}\left(M^{\prime}\right)=\mathbb{Z} \oplus \operatorname{coker}\left(x^{!}: \mathbb{Z}^{2 g} \rightarrow \mathbb{Z}^{\ell}\right), \\
& H_{1}\left(M^{\prime}\right)=\frac{\operatorname{ker}\left(x^{!}: \mathbb{Z}^{2 g} \rightarrow \mathbb{Z}^{\ell}\right)}{\operatorname{im}\left(x: \mathbb{Z}^{\ell} \rightarrow \mathbb{Z}^{2 g}\right)} \\
& H_{2}\left(M^{\prime}\right)=\mathbb{Z} \oplus \operatorname{ker}\left(x: \mathbb{Z}^{\ell} \rightarrow \mathbb{Z}^{2 g}\right) .
\end{aligned}
$$

Here are some special cases:

(i) If the homology classes $x_{1}, x_{2}, \ldots, x_{\ell} \in H_{1}(M)$ are the basis of a direct summand then

$$
M^{\prime}=M(g-\ell) .
$$

In particular, this is the case for $\ell=g, x_{i}=a_{i}(1 \leqslant i \leqslant g)$ with

$$
M^{\prime}=M(0)=S^{2} \text {. }
$$

(ii) If $x_{1}=x_{2}=\ldots=x_{\ell}=0 \in H_{1}(M)$ then

$$
M^{\prime}=M\left(g_{1}\right) \cup M\left(g_{2}\right) \cup \ldots \cup M\left(g_{\ell+1}\right)
$$

with $g_{1}+g_{2}+\ldots+g_{\ell+1}=g$. 
(iii) The picture shows $M(3)$ with three embedded circles:

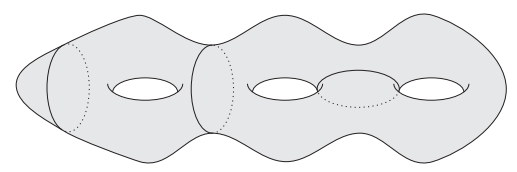

The right hand circle represents a non-zero homology class, and the effect of the corresponding 1-surgery on $M(3)$ is $M(2)$ :

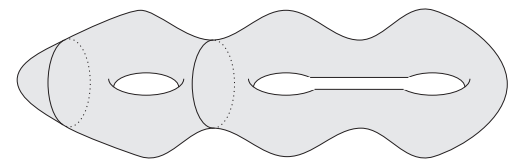

The middle circle is null-homologous, and the effect of the corresponding 1-surgery on $M(3)$ is $M(1) \cup M(2)$ :

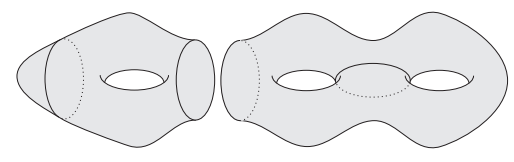

The left hand circle is null-homologous (in fact null-homotopic), and the effect of the corresponding 1-surgery on $M(3)$ is $M(0) \cup M(3)$ :

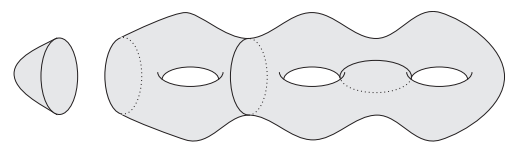

\subsection{Rings with involution}

This section is purely algebraic, developing the theory of sesquilinear forms on modules over a ring with involution $A$. The algebra will be applied in the next section to obtain the Poincaré duality and sesquilinear form on the homology of the universal cover $\widetilde{M}$ of a manifold $M$, with $A=\mathbb{Z}\left[\pi_{1}(M)\right]$.

Definition 4.35 (i) An involution on a $\operatorname{ring} A$ is a function

$$
A \rightarrow A ; a \mapsto \bar{a}
$$

satisfying

$$
\overline{(a+b)}=\bar{a}+\bar{b}, \overline{(a b)}=\bar{b} \cdot \bar{a}, \overline{\bar{a}}=a, \overline{1}=1 \in A(a, b \in A) .
$$

(ii) For a ring with involution $A$ define the transposition isomorphism 


$$
t:\{\text { left } A \text {-modules }\} \rightarrow\{\text { right } A \text {-modules }\} ; K \mapsto K^{t}
$$

with

$$
K^{t} \times A \rightarrow K^{t} ;(x, a) \mapsto \bar{a} x .
$$

(iii) For a ring with involution $A$ define the duality contravariant functor

$$
\text { * : }\{\text { left } A \text {-modules }\} \rightarrow\{\text { left } A \text {-modules }\} ; K \mapsto K^{*}
$$

sending a left $A$-module $K$ to the dual left $A$-module

$$
K^{*}=\operatorname{Hom}_{A}(K, A) \text {, }
$$

with $A$ acting by

$$
A \times K^{*} \rightarrow K^{*} ;(a, f) \mapsto(x \mapsto f(x) \cdot \bar{a}) .
$$

(This is just $\left(K^{*}\right)^{t}$ with $K^{*}$ the dual right $A$-module in the sense of 3.15). The dual of an $A$-module morphism $f: K \rightarrow L$ is the $A$-module morphism

$$
f^{*}: L^{*} \rightarrow K^{*} ; g \mapsto(x \mapsto g(f(x))) .
$$

Example 4.36 (i) A commutative ring $A$ admits the identity involution

$$
A \rightarrow A ; a \mapsto \bar{a}=a .
$$

(ii) Complex conjugation defines an involution on the ring of complex numbers $\mathbb{C}$

$$
\mathbb{C} \rightarrow \mathbb{C} ; z=a+i b \mapsto \bar{z}=a-i b .
$$

In the first instance recall the various ways of regarding bilinear pairings on vector spaces over $\mathbb{R}$. The dual of a vector space $V$ is the vector space

$$
V^{*}=\operatorname{Hom}_{\mathbb{R}}(V, \mathbb{R}) .
$$

For vector spaces $V, W$ the bilinear pairings

$$
V \times W \rightarrow \mathbb{R} ;(v, w) \mapsto \lambda(v, w)
$$

are in one-one correspondence with the linear maps

$$
V \rightarrow W^{*} ; v \mapsto(w \mapsto \lambda(v, w)) .
$$

For finite-dimensional $V$ and any $W$ there is defined a natural isomorphism

$$
V \otimes_{\mathbb{R}} W \rightarrow \operatorname{Hom}_{\mathbb{R}}\left(V^{*}, W\right) ; v \otimes w \mapsto(f \mapsto f(v) w) .
$$

In particular, for $W=\mathbb{R}$ this gives a natural isomorphism

$$
V \rightarrow V^{* *} ; v \mapsto(f \mapsto f(v)) .
$$

Thus for finite-dimensional $V, W$ there are natural identifications

$$
\{\text { bilinear pairings } V \times W \rightarrow \mathbb{R}\}=\operatorname{Hom}_{\mathbb{R}}\left(V, W^{*}\right)=V^{*} \otimes_{\mathbb{R}} W^{*} .
$$

For the applications to topology it is necessary to establish analogous properties for modules over a ring $A$ with an involution $A \rightarrow A ; a \mapsto \bar{a}$. 
Definition 4.37 A sesquilinear pairing $(K, L, \lambda)$ on $A$-modules $K, L$ is a function

$$
\lambda: K \times L \rightarrow A ;(x, y) \mapsto \lambda(x, y)
$$

such that for all $x, x^{\prime} \in K, y, y^{\prime} \in L, a, b \in A$

$$
\begin{aligned}
& \text { (i) } \lambda\left(x+x^{\prime}, y\right)=\lambda(x, y)+\lambda\left(x^{\prime}, y\right) \in A \\
& \text { (ii) } \lambda\left(x, y+y^{\prime}\right)=\lambda(x, y)+\lambda\left(x, y^{\prime}\right) \in A, \\
& \text { (iii) } \lambda(a x, b y)=b \lambda(x, y) \bar{a} \in A .
\end{aligned}
$$

Let $S(K, L)$ be the additive group of sesquilinear pairings $\lambda: K \times L \rightarrow A$.

There is an evident natural isomorphism of additive groups

$$
\begin{aligned}
& S(K, L) \rightarrow \operatorname{Hom}_{A}\left(K, L^{*}\right) ; \\
& (\lambda: K \times L \rightarrow A) \mapsto\left(\lambda: K \rightarrow L^{*} ; x \mapsto(y \mapsto \lambda(x)(y)=\lambda(x, y))\right)
\end{aligned}
$$

which is used to identify

$$
S(K, L)=\operatorname{Hom}_{A}\left(K, L^{*}\right) .
$$

Definition 4.38 (i) An $A$-module $K$ is f.g. projective if it is a direct summand of a f.g. free $A$-module $A^{n}$, that is if there exists an isomorphism

$$
K \oplus L \cong A^{n}
$$

for some $A$-module $L$ and $n \geqslant 0$.

(ii) An $A$-module $K$ is stably f.g. free if there exists an isomorphism

$$
K \oplus A^{m} \cong A^{n}
$$

for some $m, n \geqslant 0$. In particular, $K$ is f.g. projective.

Proposition 4.39 The dual of a f.g. projective A-module $K$ is a f.g. projective A-module $K^{*}$. The natural A-module morphism

$$
e_{K}: K \rightarrow K^{* *} ; x \mapsto(f \mapsto \overline{f(x)})
$$

is an isomorphism for f.g. projective $K$. The dual of a stably f.g. free A-module is stably f.g. free.

Proof For any $A$-modules $K, L$ there are evident identifications

$$
\begin{aligned}
& (K \oplus L)^{*}=K^{*} \oplus L^{*} \\
& e_{K \oplus L}=e_{K} \oplus e_{L}: K \oplus L \rightarrow(K \oplus L)^{* *}=K^{* *} \oplus L^{* *} .
\end{aligned}
$$

Thus it suffices to consider the case $K=A$, for which the result is clear. 
Use 4.39 to identify $K^{* *}=K$ for any f.g. projective $A$-module $K$.

Definition 4.40 Let $(K, L, \lambda)$ be a sesquilinear pairing. The transpose sesquilinear pairing $(L, K, T \lambda)$ is )

$$
T \lambda: L \times K \rightarrow A ;(y, x) \mapsto T \lambda(y, x)=\overline{\lambda(x, y)} .
$$

Transposition defines an isomorphism

$$
T: S(K, L) \rightarrow S(L, K) ; \lambda \mapsto T \lambda
$$

Definition 4.41 (i) The transposition isomorphism is defined for $A$-modules $K, L$ by

$$
T: K^{t} \otimes_{A} L \rightarrow L^{t} \otimes_{A} K ; x \otimes y \mapsto y \otimes x .
$$

(ii) The slant map is the morphism defined for $A$-modules $K, L$ by

$$
e_{K, L}: K^{t} \otimes_{A} L \rightarrow \operatorname{Hom}_{A}\left(K^{*}, L\right) ; x \otimes y \mapsto(f \mapsto \overline{f(x)} y)
$$

Proposition 4.42 Let $K, L$ be f.g. projective A-modules.

(i) The transposition isomorphism $T: S(K, L) \rightarrow S(L, K)$ corresponds to the duality isomorphism

$$
\begin{aligned}
*: \operatorname{Hom}_{A}\left(K, L^{*}\right) & \rightarrow \operatorname{Hom}_{A}\left(L, K^{*}\right) ; \\
\left(\lambda: K \rightarrow L^{*}\right) & \mapsto\left(\lambda^{*}: L \rightarrow K^{*} ; x \mapsto(y \mapsto \overline{\lambda(y)(x)})\right),
\end{aligned}
$$

with a commutative square of isomorphisms

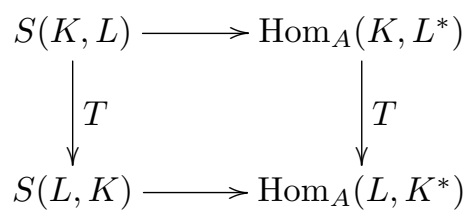

(ii) The slant map is an isomorphism

$$
e_{K, L}: K^{t} \otimes_{A} L \rightarrow S\left(K^{*}, L^{*}\right)=\operatorname{Hom}_{A}\left(K^{*}, L\right) .
$$

Duality corresponds to transposition, with a commutative square of isomorphisms

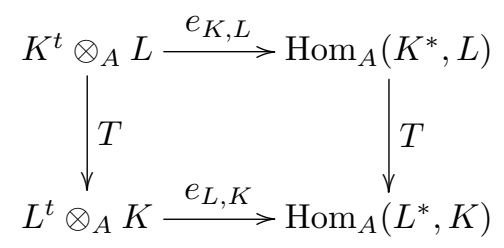


Proof It suffices to verify that $e_{K, L}$ is an isomorphism for $K=L=A$, in which case $e_{A, A}$ can be expressed as the composite of the natural isomorphisms

$$
\begin{aligned}
& A^{t} \otimes_{A} A \rightarrow A ; a \otimes b \mapsto \bar{b} a, \\
& A \rightarrow \operatorname{Hom}_{A}\left(A^{*}, A\right) ; a \mapsto(f \mapsto \overline{f(a)}) .
\end{aligned}
$$

In view of 4.42 it is possible to identify

$$
S(K, L)=\operatorname{Hom}_{A}\left(K, L^{*}\right)=\left(K^{*}\right)^{t} \otimes_{A} L^{*}
$$

for any f.g. projective $A$-modules $K, L$, with duality corresponding to transposition.

The additive group $\operatorname{Hom}_{A}\left(A^{n}, A^{m}\right)$ of the morphisms $A^{n} \rightarrow A^{m}$ between f.g. free $A$-modules is identified with the additive group $M_{m, n}(A)$ of $m \times n$ matrices $\left(a_{i j}\right)_{1 \leqslant i \leqslant m, 1 \leqslant j \leqslant n}$ with entries $a_{i j} \in A$, using the isomorphism

$$
\begin{aligned}
& M_{m, n}(A) \rightarrow \operatorname{Hom}_{A}\left(A^{n}, A^{m}\right) \\
& \left(a_{i j}\right) \mapsto\left(\left(x_{1}, x_{2}, \ldots, x_{n}\right) \mapsto\left(\sum_{j=1}^{n} x_{j} a_{1 j}, \sum_{j=1}^{n} x_{j} a_{2 j}, \ldots, \sum_{j=1}^{n} x_{j} a_{m j}\right)\right) .
\end{aligned}
$$

The composition of morphisms

$$
\begin{aligned}
\operatorname{Hom}_{A}\left(A^{p}, A^{n}\right) \times \operatorname{Hom}_{A}\left(A^{n}, A^{m}\right) & \rightarrow \operatorname{Hom}_{A}\left(A^{p}, A^{m}\right) ; \\
(f, g) & \mapsto(g f: x \mapsto(g f)(x)=g(f(x)))
\end{aligned}
$$

corresponds to the multiplication of matrices

$$
\begin{gathered}
M_{m, n}(A) \times M_{n, p}(A) \rightarrow M_{m, p}(A) ;\left(\left(a_{i j}\right),\left(b_{j k}\right)\right) \mapsto\left(c_{i k}\right) \\
c_{i k}=\sum_{j=1}^{n} a_{i j} b_{j k}(1 \leqslant i \leqslant m, 1 \leqslant k \leqslant p) .
\end{gathered}
$$

Use the isomorphism of f.g. free $A$-modules

$$
A^{m} \rightarrow\left(A^{m}\right)^{*} ;\left(x_{1}, x_{2}, \ldots, x_{m}\right) \mapsto\left(\left(y_{1}, y_{2}, \ldots, y_{m}\right) \mapsto \sum_{i=1}^{m} y_{i} \bar{x}_{i}\right)
$$

to identify

$$
\left(A^{m}\right)^{*}=A^{m}
$$

The duality isomorphism

$$
\text { * }: \operatorname{Hom}_{A}\left(A^{n}, A^{m}\right) \rightarrow \operatorname{Hom}_{A}\left(\left(A^{m}\right)^{*},\left(A^{n}\right)^{*}\right)=\operatorname{Hom}_{A}\left(A^{m}, A^{n}\right) ; f \mapsto f^{*}
$$

corresponds to the isomorphism defined by conjugate transposition of matrices 


$$
M_{m, n}(A) \rightarrow M_{n, m}(A) ; \alpha=\left(a_{i j}\right) \mapsto \alpha^{*}=\left(b_{j i}\right), b_{j i}=\bar{a}_{i j} .
$$

It is thus possible to identify

$$
S\left(A^{n}, A^{m}\right)=\operatorname{Hom}_{A}\left(A^{n}, A^{m}\right)=M_{m, n}(A),
$$

with an $m \times n$ matrix $\left(a_{i j}\right)$ corresponding to the sesquilinear form

$$
A^{n} \times A^{m} \rightarrow A ;\left(\left(y_{1}, y_{2}, \ldots, y_{n}\right),\left(x_{1}, x_{2}, \ldots, x_{m}\right)\right) \mapsto \sum_{i=1}^{m} \sum_{j=1}^{n} x_{i} a_{i j} \bar{y}_{j},
$$

and the transposition of sesquilinear pairings corresponding to the conjugate transposition of matrices.

Example 4.43 A $2 \times 2$ matrix

$$
\lambda=\left(\begin{array}{ll}
a & b \\
c & d
\end{array}\right) \in M_{2,2}(A)
$$

corresponds to the $A$-module morphism

$$
\left(\begin{array}{ll}
a & b \\
c & d
\end{array}\right): A \oplus A \rightarrow A \oplus A ;(x, y) \mapsto(x a+y b, x c+y d) .
$$

The conjugate transpose matrix

$$
\lambda^{*}=\left(\begin{array}{cc}
\bar{a} & \bar{c} \\
\bar{b} & \bar{d}
\end{array}\right) \in M_{2,2}(A)
$$

corresponds to the dual $A$-module morphism

$$
\begin{aligned}
& \left(\begin{array}{ll}
\bar{a} & \bar{c} \\
\bar{b} & \bar{d}
\end{array}\right):(A \oplus A)^{*}=A \oplus A \rightarrow(A \oplus A)^{*}=A \oplus A ; \\
& (x, y) \mapsto(x \bar{a}+y \bar{c}, x \bar{b}+y \bar{d}) .
\end{aligned}
$$

Regarded as a sesquilinear pairing $\lambda$ is

$$
\begin{aligned}
\lambda: & A \oplus A \times A \oplus A \rightarrow A ; \\
& \left(\left(x_{1}, x_{2}\right),\left(y_{1}, y_{2}\right)\right) \mapsto y_{1} a \bar{x}_{1}+y_{2} b \bar{x}_{1}+y_{1} c \bar{x}_{2}+y_{2} d \bar{x}_{2} .
\end{aligned}
$$

\subsection{Universal Poincaré duality}

In order to describe the homology effect of surgery on a non-simply-connected manifold $M$ it is necessary to deal with the Poincare duality properties of the universal cover $\widetilde{M}$. The $\mathbb{Z}_{2^{-}}$and $\mathbb{Z}$-coefficient Poincaré duality isomorphisms of a manifold $M$ obtained in Section 4.1 will now be generalised to the nonorientable case, and also to $\mathbb{Z}\left[\pi_{1}(M)\right]$-coefficient Poincaré duality isomorphisms. 
The connected regular covers of a connected space $X$ with universal cover $\widetilde{X}$ are given by

$$
\bar{X}=\tilde{X} / \rho
$$

with $\rho \triangleleft \pi_{1}(X)$ a normal subgroup. The group of covering translations $\bar{X} \rightarrow \bar{X}$ is the quotient $\pi_{1}(X) / \rho$. In particular, if $\rho \triangleleft \pi_{1}(X)$ is a subgroup of index 2 (as in the nonorientable case below) then $\bar{X}$ is a double cover of $X$.

The double cover of the infinite-dimensional projective space by the infinitedimensional sphere

$$
p: S^{\infty}=\bigcup_{n=0}^{\infty} S^{n} \rightarrow \mathbb{R P} \mathbb{P}^{\infty}=\bigcup_{n=0}^{\infty} \mathbb{R P}^{n}
$$

has the following universal property : a map $w: X \rightarrow \mathbb{R} \mathbb{P}^{\infty}$ determines a double cover of $X$

$$
X^{w}=\left\{(x, y) \in X \times S^{\infty} \mid w(x)=p(y) \in \mathbb{R P}^{\infty}\right\},
$$

and every double cover of $X$ arises in this way. The double covers of $X$ are in one-one correspondence with the elements $w \in\left[X, \mathbb{R P}^{\infty}\right]=H^{1}\left(X ; \mathbb{Z}_{2}\right)$. For a connected $X$ there is defined a bijection

$$
\begin{aligned}
H^{1}\left(X ; \mathbb{Z}_{2}\right)= & {\left[X, \mathbb{R P}^{\infty}\right] \rightarrow \operatorname{Hom}\left(\pi_{1}(X), \mathbb{Z}_{2}\right) ; } \\
& \left(w: X \rightarrow \mathbb{R P} \mathbb{P}^{\infty}\right) \mapsto\left(w_{*}: \pi_{1}(X) \rightarrow \pi_{1}\left(\mathbb{R P} \mathbb{P}^{\infty}\right)=\mathbb{Z}_{2}\right) .
\end{aligned}
$$

Definition 4.44 The orientation double cover of a connected $m$-dimensional manifold $M$ is the double cover $\bar{M}=M^{w}$ classified by the orientation character $w=w(M) \in H^{1}\left(M ; \mathbb{Z}_{2}\right)$

$$
\bar{M}=\left\{\begin{array} { l } 
{ M \cup M } \\
{ \widetilde { M } / \operatorname { k e r } ( w : \pi _ { 1 } ( M ) \rightarrow \mathbb { Z } _ { 2 } ) }
\end{array} \text { if } M \text { is } \left\{\begin{array}{l}
\text { orientable } \\
\text { nonorientable }
\end{array}\right.\right.
$$

with $\widetilde{M}$ the universal cover of $M$. The orientation double cover $\bar{M}$ is an orientable $m$-dimensional manifold.

Example 4.45 The real projective space $\mathbb{R P}^{m}$ is an $m$-dimensional manifold which is orientable for $m$ odd, and nonorientable for $m$ even. The orientation double cover is

$$
\overline{\mathbb{R P}}^{m}=\left\{\begin{array} { l } 
{ S ^ { m } } \\
{ \mathbb { R P } ^ { m } \cup \mathbb { R P } ^ { m } }
\end{array} \text { if } m \text { is } \left\{\begin{array}{l}
\text { even } \\
\text { odd. }
\end{array}\right.\right.
$$

Definition 4.46 (i) The $w$-twisted homology groups of a space $X$ with an orientation character $w \in H^{1}\left(X ; \mathbb{Z}_{2}\right)$ are

$$
H_{*}\left(X ; \mathbb{Z}^{w}\right)=H_{*}\left(S\left(X ; \mathbb{Z}^{w}\right)\right)
$$


where

$$
S\left(X ; \mathbb{Z}^{w}\right)=\mathbb{Z}^{-} \otimes_{\mathbb{Z}\left[\mathbb{Z}_{2}\right]} S\left(X^{w}\right)
$$

is the $w$-twisted singular chain complex of $X$, with $X^{w}$ the double cover of $X$ classified by $w$, and $\mathbb{Z}^{-}$the $\mathbb{Z}\left[\mathbb{Z}_{2}\right]$-module defined by $\mathbb{Z}$ with the generator $T \in \mathbb{Z}_{2}$ acting by $T(1)=-1$.

(ii) The $w$-twisted cohomology groups of $X$ are

$$
H^{*}\left(X ; \mathbb{Z}^{w}\right)=H_{*}\left(\operatorname{Hom}_{\mathbb{Z}\left[\mathbb{Z}_{2}\right]}\left(S\left(X^{w}\right), \mathbb{Z}^{-}\right)\right) .
$$

Example 4.47 For the trivial orientation character $w=0 \in H^{1}\left(X ; \mathbb{Z}_{2}\right)$ the orientation double cover is $X^{w}=X \cup X$ with $T: X^{w} \rightarrow X^{w}$ interchanging the two copies of $X$, and

$$
H_{*}\left(X ; \mathbb{Z}^{w}\right)=H_{*}(X), \quad H^{*}\left(X ; \mathbb{Z}^{w}\right)=H^{*}(X) .
$$

Proposition 4.48 (i) The w-twisted homology and cohomology groups fit into exact sequences

$$
\begin{aligned}
& \ldots \rightarrow H_{n+1}(X) \rightarrow H_{n}\left(X ; \mathbb{Z}^{w}\right) \rightarrow H_{n}\left(X^{w}\right) \stackrel{p_{*}}{\rightarrow} H_{n}(X) \rightarrow H_{n-1}\left(X ; \mathbb{Z}^{w}\right) \rightarrow \ldots, \\
& \ldots \rightarrow H^{n-1}(X) \rightarrow H^{n}\left(X ; \mathbb{Z}^{w}\right) \rightarrow H^{n}\left(X^{w}\right) \stackrel{p^{!}}{\rightarrow} H^{n}(X) \rightarrow H^{n+1}\left(X ; \mathbb{Z}^{w}\right) \rightarrow \ldots
\end{aligned}
$$

with $p: X^{w} \rightarrow X$ the covering projection, $p_{*}$ the induced morphisms in homology, and $p^{!}$the transfer map in cohomology

$$
p^{!}: H^{n}\left(X^{w}\right) \rightarrow H^{n}(X) ; f \mapsto\left(f^{!}: x \mapsto f\left(x^{w}+T\left(x^{w}\right)\right)\right)
$$

with $f: S\left(X^{w}\right) \rightarrow \mathbb{Z}$ a cocycle, using any lift of a cycle $x \in S(X)$ to a chain $x^{w} \in S\left(X^{w}\right)$.

(ii) The w-twisted (co)homology groups have cup and cap products

$$
\begin{gathered}
\cup: H^{m}\left(X ; \mathbb{Z}^{w}\right) \otimes_{\mathbb{Z}} H^{n}(X) \rightarrow H^{m+n}\left(X ; \mathbb{Z}^{w}\right), \\
\cup: H^{m}\left(X ; \mathbb{Z}^{w}\right) \otimes_{\mathbb{Z}} H^{n}\left(X ; \mathbb{Z}^{w}\right) \rightarrow H^{m+n}(X), \\
\cap: H_{m}\left(X ; \mathbb{Z}^{w}\right) \otimes_{\mathbb{Z}} H^{n}(X) \rightarrow H_{m-n}\left(X ; \mathbb{Z}^{w}\right), \\
\cap: H_{m}(X) \otimes_{\mathbb{Z}} H^{n}\left(X ; \mathbb{Z}^{w}\right) \rightarrow H_{m-n}\left(X ; \mathbb{Z}^{w}\right) .
\end{gathered}
$$

Proof (i) The short exact sequence of $\mathbb{Z}\left[\mathbb{Z}_{2}\right]$-modules

$$
0 \rightarrow \mathbb{Z}^{-} \rightarrow \mathbb{Z}\left[\mathbb{Z}_{2}\right] \rightarrow \mathbb{Z} \rightarrow 0
$$

with

$$
\begin{aligned}
& \mathbb{Z}^{-} \rightarrow \mathbb{Z}\left[\mathbb{Z}_{2}\right] ; 1 \mapsto 1-T, \\
& \mathbb{Z}\left[\mathbb{Z}_{2}\right] \rightarrow \mathbb{Z} ; a+b T \mapsto a+b
\end{aligned}
$$


determines a short exact sequence of $\mathbb{Z}$-module chain complexes

$$
0 \rightarrow \mathbb{Z}^{-} \otimes_{\mathbb{Z}\left[\mathbb{Z}_{2}\right]} S\left(X^{w}\right) \rightarrow \mathbb{Z}\left[\mathbb{Z}_{2}\right] \otimes_{\mathbb{Z}\left[\mathbb{Z}_{2}\right]} S\left(X^{w}\right) \rightarrow \mathbb{Z} \otimes_{\mathbb{Z}\left[\mathbb{Z}_{2}\right]} S\left(X^{w}\right) \rightarrow 0
$$

which can be written as

$$
0 \rightarrow S\left(X ; \mathbb{Z}^{w}\right) \rightarrow S\left(X^{w}\right) \stackrel{p_{*}}{\longrightarrow} S(X) \rightarrow 0
$$

The exact sequence for $w$-twisted homology is the corresponding long exact sequence of homology groups. Similarly for $w$-twisted cohomology. The short exact sequence of $\mathbb{Z}$-module chain complexes

$$
\begin{aligned}
0 \rightarrow \operatorname{Hom}_{\mathbb{Z}\left[\mathbb{Z}_{2}\right]}\left(S\left(X^{w}\right), \mathbb{Z}^{-}\right) \rightarrow \operatorname{Hom}_{\mathbb{Z}\left[\mathbb{Z}_{2}\right]}\left(S\left(X^{w}\right), \mathbb{Z}\left[\mathbb{Z}_{2}\right]\right) & \\
& \rightarrow \operatorname{Hom}_{\mathbb{Z}\left[\mathbb{Z}_{2}\right]}\left(S\left(X^{w}\right), \mathbb{Z}\right) \rightarrow 0
\end{aligned}
$$

can be written as

$$
0 \rightarrow \operatorname{Hom}_{\mathbb{Z}\left[\mathbb{Z}_{2}\right]}\left(S\left(X^{w}\right), \mathbb{Z}^{-}\right) \rightarrow \operatorname{Hom}_{\mathbb{Z}}\left(S\left(X^{w}\right), \mathbb{Z}\right) \rightarrow \operatorname{Hom}_{\mathbb{Z}}(S(X), \mathbb{Z}) \rightarrow 0 .
$$

The exact sequence for $w$-twisted cohomology is the corresponding long exact sequence of homology groups.

(ii) As for the ordinary cup and cap products, using a diagonal chain approximation $\Delta_{0}: S\left(X^{w}\right) \rightarrow S\left(X^{w}\right) \otimes_{\mathbb{Z}} S\left(X^{w}\right)$.

Orientation Convention 4.49 (i) Orientable manifolds $M$ are equipped with a choice of orientation, and the two copies of $M$ in the orientation double cover $\bar{M}=M \cup M$ are given opposite orientations.

(ii) Nonorientable manifolds $M$ are equipped with a choice of orientation for the orientation double cover $\bar{M}$.

Similarly for cobordisms $\left(W ; M, M^{\prime}\right)$, with $\partial W=M \cup-M^{\prime}$.

Definition 4.50 The $w$-twisted fundamental class of an $m$-dimensional manifold $M$ with orientation character $w=w(M) \in H^{1}\left(M ; \mathbb{Z}_{2}\right)$ is the $w$-twisted homology class

$$
[M]=[\bar{M}] \in H_{m}\left(M ; \mathbb{Z}^{w}\right)=\operatorname{ker}\left(p_{*}: H_{m}(\bar{M}) \rightarrow H_{m}(M)\right)
$$

given by the fundamental class $[\bar{M}] \in H_{m}(\bar{M})$ of the orientation double cover $\bar{M}=M^{w}$.

Twisted Poincaré Duality Theorem 4.51 For any m-dimensional manifold $M$ cap product with the $w$-twisted fundamental class $[M] \in H_{m}\left(M ; \mathbb{Z}^{w}\right)$ defines the w-twisted Poincaré duality isomorphisms

$$
\begin{aligned}
& {[M] \cap-: H^{*}(M) \rightarrow H_{m-*}\left(M ; \mathbb{Z}^{w}\right),} \\
& {[M] \cap-: H^{*}\left(M ; \mathbb{Z}^{w}\right) \rightarrow H_{m-*}(M) .}
\end{aligned}
$$


Proof As for the $R$-coefficient case (4.4), but using $\mathbb{Z}^{w}$-coefficients.

Example 4.52 The real projective space $\mathbb{R P}^{m}$ has a non-trivial double cover $\overline{\mathbb{R P}}^{m}=S^{m}$, so that $S^{m}$ has $\mathbb{Z}_{2}$-equivariant $C W$ structure

$$
S^{m}=\bigcup_{n=0}^{m}\left(D^{n} \cup T D^{n}\right)
$$

with quotient

$$
\mathbb{R P}^{m}=S^{m} / T=\bigcup_{n=0}^{m} D^{n} .
$$

The $\mathbb{Z}\left[\mathbb{Z}_{2}\right]$-module cellular chain complex of $S^{m}$ is

$$
C\left(S^{m}\right): \ldots \rightarrow 0 \rightarrow \mathbb{Z}\left[\mathbb{Z}_{2}\right] \rightarrow \mathbb{Z}\left[\mathbb{Z}_{2}\right] \rightarrow \ldots \rightarrow \mathbb{Z}\left[\mathbb{Z}_{2}\right]
$$

with

$d=1+(-1)^{n+1} T: C\left(S^{m}\right)_{n+1}=\mathbb{Z}\left[\mathbb{Z}_{2}\right] \rightarrow C\left(S^{m}\right)_{n}=\mathbb{Z}\left[\mathbb{Z}_{2}\right] \quad(0 \leqslant n<m)$.

(The chain complex in Remark 3.19 is $W=C\left(S^{\infty}\right)$.) The $\mathbb{Z}$-module cellular chain complexes of $\mathbb{R P}^{m}$ are given by

$$
\begin{aligned}
& C\left(\mathbb{R P}^{m}\right): \quad \ldots \rightarrow 0 \rightarrow \mathbb{Z} \stackrel{1+(-1)^{m}}{\longrightarrow} \mathbb{Z} \rightarrow \ldots \rightarrow \mathbb{Z} \stackrel{2}{\longrightarrow} \mathbb{Z} \stackrel{0}{\longrightarrow} \mathbb{Z}, \\
& C\left(\mathbb{R P}^{m} ; \mathbb{Z}^{w}\right): \quad \ldots \rightarrow 0 \rightarrow \mathbb{Z} \stackrel{1+(-1)^{m+1}}{\longrightarrow} \mathbb{Z} \rightarrow \ldots \rightarrow \mathbb{Z} \stackrel{0}{\longrightarrow} \mathbb{Z} \stackrel{2}{\longrightarrow} \mathbb{Z}
\end{aligned}
$$

$\left(\right.$ with $\left.w=w\left(\mathbb{R P}^{m}\right)=(-1)^{m+1}\right)$ and

$$
\begin{aligned}
& H_{n}\left(\mathbb{R P}^{m}\right)=H^{m-n}\left(\mathbb{R P}^{m} ; \mathbb{Z}^{w}\right)= \begin{cases}\mathbb{Z} & \text { if } n=0, \text { or if } m=n \text { is odd } \\
\mathbb{Z}_{2} & \text { if } 0<n<m \text { with } n \text { odd } \\
0 & \text { otherwise, }\end{cases} \\
& H_{n}\left(\mathbb{R P}^{m} ; \mathbb{Z}^{w}\right)=H^{m-n}\left(\mathbb{R P}^{m}\right)= \begin{cases}\mathbb{Z}^{2} & \text { if } m=n, \text { or if }(n=0 \text { and } m \text { is odd }) \\
\mathbb{Z}_{2} & \text { if } 0<n<m \text { with } n \text { even, } \\
0 & \text { or if }(n=0 \text { and } m \text { is even })\end{cases}
\end{aligned}
$$

Definition 4.53 Let $X$ be a pointed space, and let $T: X^{w} \rightarrow X^{w}$ be an involution of a pointed space which fixes the base point and is free away from the base point, such that

$$
X^{w} / \mathbb{Z}_{2}=X
$$

(i) The reduced $w$-twisted homology groups of $X$ are

$$
\dot{H}_{*}\left(X ; \mathbb{Z}^{w}\right)=H_{*}\left(\mathbb{Z}^{-} \otimes_{\mathbb{Z}\left[\mathbb{Z}_{2}\right]} \dot{S}\left(X^{w}\right)\right),
$$


with $\dot{S}\left(X^{w}\right)=S\left(X^{w},\{\right.$ pt. $\left.\}\right)$ the reduced chain complex regarded as a (free) $\mathbb{Z}\left[\mathbb{Z}_{2}\right]$-module chain complex by $T: X^{w} \rightarrow X^{w}$.

(ii) The reduced $w$-twisted cohomology groups of $X$ are

$$
\dot{H}^{*}\left(X ; \mathbb{Z}^{w}\right)=H_{*}\left(\operatorname{Hom}_{\mathbb{Z}\left[\mathbb{Z}_{2}\right]}\left(\dot{S}\left(X^{w}\right), \mathbb{Z}^{-}\right)\right)
$$

Proposition 4.54 The reduced w-twisted homology and cohomology groups fit into exact sequences

$$
\begin{aligned}
& \ldots \rightarrow \dot{H}_{n+1}(X) \rightarrow \dot{H}_{n}\left(X ; \mathbb{Z}^{w}\right) \rightarrow \dot{H}_{n}\left(X^{w}\right) \stackrel{p_{*}}{\rightarrow} \dot{H}_{n}(X) \rightarrow \dot{H}_{n-1}\left(X ; \mathbb{Z}^{w}\right) \rightarrow \ldots \\
& \ldots \rightarrow \dot{H}^{n-1}(X) \rightarrow \dot{H}^{n}\left(X ; \mathbb{Z}^{w}\right) \rightarrow \dot{H}^{n}\left(X^{w}\right) \stackrel{p^{!}}{\rightarrow} \dot{H}^{n}(X) \rightarrow \dot{H}^{n+1}\left(X ; \mathbb{Z}^{w}\right) \rightarrow \ldots
\end{aligned}
$$

Proof As for 4.48, using the short exact sequences of $\mathbb{Z}$-module chain complexes

$$
\begin{array}{r}
0 \rightarrow \mathbb{Z}^{-} \otimes_{\mathbb{Z}\left[\mathbb{Z}_{2}\right]} \dot{S}\left(X^{w}\right) \rightarrow \mathbb{Z}\left[\mathbb{Z}_{2}\right] \otimes_{\mathbb{Z}\left[\mathbb{Z}_{2}\right]} \dot{S}\left(X^{w}\right) \rightarrow \mathbb{Z} \otimes_{\mathbb{Z}\left[\mathbb{Z}_{2}\right]} \dot{S}\left(X^{w}\right) \rightarrow 0 \\
0 \rightarrow \operatorname{Hom}_{\mathbb{Z}\left[\mathbb{Z}_{2}\right]}\left(\dot{S}\left(X^{w}\right), \mathbb{Z}\right) \rightarrow \operatorname{Hom}_{\mathbb{Z}\left[\mathbb{Z}_{2}\right]}\left(\dot{S}\left(X^{w}\right), \mathbb{Z}\left[\mathbb{Z}_{2}\right]\right) \\
\rightarrow \operatorname{Hom}_{\mathbb{Z}\left[\mathbb{Z}_{2}\right]}\left(\dot{S}\left(X^{w}\right), \mathbb{Z}^{-}\right) \rightarrow 0
\end{array}
$$

We now move on to universal Poincaré duality.

Let $X$ be a space, and let $\widetilde{X}$ be a regular cover of $X$ with group of covering translations $\pi$. The action of $\pi$ on $\widetilde{X}$ by covering translations

$$
\pi \times \tilde{X} \rightarrow \widetilde{X} ;(g, x) \mapsto g x
$$

determines an action of $\mathbb{Z}[\pi]$ on $H_{*}(\tilde{X})$

$$
\mathbb{Z}[\pi] \times H_{*}(\widetilde{X}) \rightarrow H_{*}(\widetilde{X}) ;(g, x) \mapsto g_{*} x
$$

so that the homology groups $H_{*}(\widetilde{X})$ are (left) $\mathbb{Z}[\pi]$-modules. The ordinary cohomology groups $H^{*}(\widetilde{X})$ are $\mathbb{Z}[\pi]$-modules via

$$
\mathbb{Z}[\pi] \times H^{*}(\widetilde{X}) \rightarrow H^{*}(\widetilde{X}) ;(g, x) \mapsto\left(g^{-1}\right)^{*} x
$$

For finite $\pi$ the ordinary cohomology $H^{*}(\widetilde{X})$ is adequate for studying the Poincaré duality of $\widetilde{X}$ (if $X$ is a manifold, say), since then $\widetilde{X}$ is compact. However, for infinite $\pi$ and a compact $X$ the cover $\widetilde{X}$ is non-compact and it is the compactly supported cohomology $H_{\mathrm{cpt}}^{*}(\widetilde{X})$ which is relevant. Using an involution on $\mathbb{Z}[\pi]$ it is in fact possible to give a uniform treatment of the ordinary cohomology for finite $\pi$ and the compactly supported cohomology for infinite $\pi$, as follows. 
Definition 4.55 Let $\pi$ be a group together with an orientation character $w$ : $\pi \rightarrow \mathbb{Z}_{2}$, i.e. a group morphism.

(i) The group ring $\mathbb{Z}[\pi]$ is the ring with elements the linear combinations

$$
\sum_{g \in \pi} n_{g} g\left(n_{g} \in \mathbb{Z}\right)
$$

such that $\left\{g \in \pi \mid n_{g} \neq 0\right\}$ is finite. Addition and multiplication are by

$$
\begin{aligned}
& \sum_{g \in \pi} m_{g} g+\sum_{g \in \pi} n_{g} g=\sum_{g \in \pi}\left(m_{g}+n_{g}\right) g, \\
& \left(\sum_{g \in \pi} m_{g} g\right)\left(\sum_{g \in \pi} n_{g} g\right)=\sum_{g, h \in \pi}\left(m_{g} n_{h}\right) g h .
\end{aligned}
$$

(ii) The $w$-twisted involution on $\mathbb{Z}[\pi]$ is given by

$$
\text { - : } \mathbb{Z}[\pi] \rightarrow \mathbb{Z}[\pi] ; a=\sum_{g \in \pi} n_{g} g \mapsto \bar{a}=\sum_{g \in \pi} n_{g} w(g) g^{-1}\left(n_{g} \in \mathbb{Z}\right) \text {. }
$$

In the untwisted case $w(g)=+1(g \in \pi)$ this is the oriented involution on $\mathbb{Z}[\pi]$.

(iii) Let $\mathbb{Z}^{w}$ denote the right $\mathbb{Z}[\pi]$-module defined by $\mathbb{Z}$ with $\mathbb{Z}[\pi]$ acting by

$$
\mathbb{Z}^{w} \times \mathbb{Z}[\pi] \rightarrow \mathbb{Z}^{w} ;\left(m, \sum_{g \in \pi} n_{g} g\right) \mapsto \sum_{g \in \pi} w(g) m n_{g} .
$$

Definition 4.56 An oriented cover $(\widetilde{X}, \pi, w)$ of a (connected) space $X$ with an orientation character $w(X) \in H^{1}\left(X ; \mathbb{Z}_{2}\right)=\operatorname{Hom}\left(\pi_{1}(X), \mathbb{Z}_{2}\right)$ is a regular covering of $X$ with group of covering translations $\pi$, together with an orientation character $w: \pi \rightarrow \mathbb{Z}_{2}$ such that

$$
w(X): \pi_{1}(X) \longrightarrow \pi \stackrel{w}{\longrightarrow} \mathbb{Z}_{2},
$$

so that

$$
S\left(X ; \mathbb{Z}^{w}\right)=\mathbb{Z}^{w} \otimes_{\mathbb{Z}[\pi]} S(\widetilde{X})
$$

In an oriented cover $(\widetilde{X}, \pi, w)$ the double cover $X^{w}$ of $X$ pulls back to a trivial double cover $\widetilde{X}^{w}=\widetilde{X} \cup \widetilde{X}$ of $\widetilde{X}$.

Given an oriented cover $(\tilde{X}, \pi, w)$ of a space $X$ it is clear that the homology groups $H_{*}(\widetilde{X})$ are $\mathbb{Z}[\pi]$-modules (irrespective of $w$ ). The action of $\mathbb{Z}[\pi]$ is by

$$
\mathbb{Z}[\pi] \times H_{*}(\widetilde{X}) \rightarrow H_{*}(\widetilde{X}) ;\left(\sum_{g \in \pi} n_{g} g, x\right) \mapsto \sum_{g \in \pi} n_{g} g_{*}(x),
$$

with $g \in \pi$ acting by the $\mathbb{Z}$-module automorphism $g_{*}: H_{*}(\widetilde{X}) \rightarrow H_{*}(\widetilde{X})$ induced by the covering translation $g: \widetilde{X} \rightarrow \widetilde{X}$. 
Definition 4.57 The cohomology $\mathbb{Z}[\pi]$-modules of an oriented cover $(\widetilde{X}, \pi, w)$ a space $X$ are defined for $n \geqslant 0$ by

$$
\begin{aligned}
H^{n}(\tilde{X}) & =H^{n}(S(\widetilde{X})) \\
& =\operatorname{ker}\left(d^{*}: S(\widetilde{X})^{n} \rightarrow S(\widetilde{X})^{n+1}\right) / \operatorname{im}\left(d^{*}: S(\widetilde{X})^{n-1} \rightarrow S(\widetilde{X})^{n}\right)
\end{aligned}
$$

using the $w$-twisted involution on $\mathbb{Z}[\pi]$ to define

$$
S(\tilde{X})^{n}=\operatorname{Hom}_{\mathbb{Z}[\pi]}\left(S(\tilde{X})_{n}, \mathbb{Z}[\pi]\right) .
$$

Warning : the $\mathbb{Z}[\pi]$-module cohomology groups $H^{*}(\tilde{X})$ are not in general the same as the singular cohomology groups of $\widetilde{X}$, although this is the case if $X$ is a finite $C W$ complex, $\pi$ is finite and $w=+1$.

Evaluation defines $\mathbb{Z}[\pi]$-module morphisms

$$
H^{n}(\widetilde{X}) \rightarrow H_{n}(\widetilde{X})^{*} ; f \mapsto(x \mapsto f(x)) .
$$

Proposition 4.58 Let $X$ be a space with an oriented cover $(\widetilde{X}, \pi, w)$.

(i) The homology and cohomology groups of $\widetilde{X}$ are related by cap products

$$
\cap: H_{m}\left(X ; \mathbb{Z}^{w}\right) \otimes_{\mathbb{Z}} H^{n}(\tilde{X}) \rightarrow H_{m-n}(\tilde{X}) ;(x, y) \mapsto x \cap y
$$

such that for every $a \in \mathbb{Z}[\pi]$

$$
x \cap a y=a(x \cap y) \in H_{m-n}(\tilde{X}) .
$$

(ii) For any homology class $[X] \in H_{m}\left(X ; \mathbb{Z}^{w}\right)$ the cohomology intersection pairing

$$
\lambda: H^{m-n}(\tilde{X}) \times H^{n}(\tilde{X}) \rightarrow \mathbb{Z}[\pi] ;(a, b) \mapsto a([X] \cap b)
$$

is sesquilinear and such that

$$
\lambda(b, a)=(-1)^{n(m-n)} \overline{\lambda(a, b)} \in \mathbb{Z}[\pi] .
$$

The cohomology intersection pairing is the adjoint of the composite

$$
H^{m-n}(\tilde{X}) \stackrel{[X] \cap-}{\cong} H_{n}(\tilde{X}) \longrightarrow H^{n}(\tilde{X})^{*}
$$

Proof (i) + (ii) The diagonal chain approximation (3.18) is a $\mathbb{Z}[\pi]$-module chain map

$$
\widetilde{\Delta}_{0}: S(\tilde{X}) \rightarrow S(\tilde{X}) \otimes_{\mathbb{Z}} S(\widetilde{X})
$$

with $\mathbb{Z}[\pi]$ acting by

$$
\begin{aligned}
\mathbb{Z}[\pi] \times S(\tilde{X}) \otimes_{\mathbb{Z}} S(\tilde{X}) & \rightarrow S(\tilde{X}) \otimes_{\mathbb{Z}} S(\tilde{X}) ; \\
& \left(\sum_{g \in \pi} n_{g} g, y \otimes z\right) \mapsto \sum_{g \in \pi} n_{g}(g y \otimes g z) .
\end{aligned}
$$


Apply $\mathbb{Z}^{w} \otimes_{\mathbb{Z}[\pi]}$ - to obtain a $\mathbb{Z}$-module chain map

$$
\begin{aligned}
\Delta_{0}=1 \otimes \widetilde{\Delta}_{0}: & \mathbb{Z}^{w} \otimes_{\mathbb{Z}[\pi]} S(\widetilde{X})=S\left(X ; \mathbb{Z}^{w}\right) \rightarrow \\
& \mathbb{Z}^{w} \otimes_{\mathbb{Z}[\pi]}\left(S(\widetilde{X}) \otimes_{\mathbb{Z}} S(\widetilde{X})\right)=S(\widetilde{X})^{t} \otimes_{\mathbb{Z}[\pi]} S(\widetilde{X})
\end{aligned}
$$

where $S(\widetilde{X})^{t}$ denotes the right $\mathbb{Z}[\pi]$-module cellular chain complex $S(\widetilde{X})$ with the same additive structure and

$$
S(\widetilde{X})^{t} \times \mathbb{Z}[\pi] \rightarrow S(\widetilde{X})^{t} ;(x, a) \mapsto \bar{a} x .
$$

Given an $m$-chain $x \in S\left(X ; \mathbb{Z}^{w}\right)_{m}$ let

$\Delta_{0}(x)=\sum_{i} x_{i}^{\prime} \otimes x_{i}^{\prime \prime} \in\left(S(\widetilde{X})^{t} \otimes_{\mathbb{Z}[\pi]} S(\widetilde{X})\right)_{m}=\sum_{p+q=m}\left(S(\widetilde{X})_{p}^{t} \otimes_{\mathbb{Z}[\pi]} S(\widetilde{X})_{q}\right)$.

The cap product of $x$ and an $n$-cochain $y \in S(\widetilde{X})^{n}$ is the $(m-n)$-chain

$$
x \cap y=\sum_{i} \overline{y\left(x_{i}^{\prime}\right)} x_{i}^{\prime \prime} \in S(\widetilde{X})_{m-n},
$$

with $y\left(x_{i}^{\prime}\right)=0 \in \mathbb{Z}[\pi]$ if the dimension of $x_{i}^{\prime}$ is $\neq n$. The composite

$$
H_{m}\left(X ; \mathbb{Z}^{w}\right) \stackrel{\Delta_{0}}{\longrightarrow} H_{m}\left(S(\widetilde{X})^{t} \otimes_{\mathbb{Z}[\pi]} S(\widetilde{X})\right) \rightarrow H_{m}\left(\operatorname{Hom}_{\mathbb{Z}[\pi]}\left(S(\widetilde{X})^{-*}, S(\widetilde{X})\right)\right)
$$

sends a homology class $x \in H_{m}\left(X ; \mathbb{Z}^{w}\right)$ to a chain homotopy class of $\mathbb{Z}[\pi]$-module chain maps

$$
x \cap-: S(\widetilde{X})^{m-*} \rightarrow S(\tilde{X})
$$

inducing the cap products

$$
x \cap-: H^{n}(\widetilde{X}) \rightarrow H_{m-n}(\widetilde{X}) ; y \mapsto x \cap y .
$$

The symmetry property of the cohomology intersection pairing $\lambda$ follows from the existence of a $\mathbb{Z}[\pi]$-module chain homotopy

$$
\widetilde{\Delta}_{1}: \widetilde{\Delta}_{0} \simeq T \widetilde{\Delta}_{0}: S(\widetilde{X}) \rightarrow S(\widetilde{X}) \otimes_{\mathbb{Z}} S(\widetilde{X}) .
$$

Remark 4.59 For any $[X] \in H_{2 n}\left(X ; \mathbb{Z}^{w}\right), x \in H^{n}(\tilde{X})$

$$
\lambda(x, x)=(-1)^{n} \overline{\lambda(x, x)} \in \mathbb{Z}[\pi] .
$$

It can be shown (using the work of Weiss [95]) that in fact

$$
\lambda(x, x)=a+b+(-1)^{n} \bar{b} \in \mathbb{Z}[\pi]
$$

for some $a=(-1)^{n} a \in \mathbb{Z}, b \in \mathbb{Z}[\pi \backslash\{1\}]$. 
Proposition 4.60 Let $X$ be a connected space with an orientation character $w \in H^{1}\left(X ; \mathbb{Z}_{2}\right)=\operatorname{Hom}\left(\pi_{1}(X), \mathbb{Z}_{2}\right)$ and universal cover $\tilde{X}$. The $w$-twisted homology and cohomology groups of $X$ are given by

$$
\begin{aligned}
& H_{*}\left(X ; \mathbb{Z}^{w}\right)=H_{*}\left(\mathbb{Z}^{w} \otimes_{\mathbb{Z}\left[\pi_{1}(X)\right]} S(\tilde{X})\right) \\
& H^{*}\left(X ; \mathbb{Z}^{w}\right)=H_{*}\left(\operatorname{Hom}_{\mathbb{Z}\left[\pi_{1}(X)\right]}\left(S(\tilde{X})^{t}, \mathbb{Z}^{w}\right)\right) .
\end{aligned}
$$

Proof Let $X^{w}$ be the double cover classified by $w$ and identify

$$
\begin{aligned}
& \mathbb{Z}^{w} \otimes_{\mathbb{Z}\left[\pi_{1}(X)\right]} S(\tilde{X})=\mathbb{Z}^{w} \otimes_{\mathbb{Z}\left[\mathbb{Z}_{2}\right]} S\left(X^{w}\right) \\
& \left.\left.\operatorname{Hom}_{\mathbb{Z}\left[\pi_{1}(X)\right]}\left(S(\widetilde{X})^{t}, \mathbb{Z}^{w}\right)\right)=\operatorname{Hom}_{\mathbb{Z}\left[\mathbb{Z}_{2}\right]}\left(S\left(X^{w}\right), \mathbb{Z}^{w}\right)\right) .
\end{aligned}
$$

For a $C W$ complex $X$ with oriented cover $(\tilde{X}, \pi, w)$ let $C(\widetilde{X})$ be the cellular $\mathbb{Z}[\pi]$-module chain complex of $\tilde{X}$, with

$$
\begin{aligned}
C(\tilde{X})_{n} & =H_{n}\left(\tilde{X}^{(n)}, \widetilde{X}^{(n-1)}\right) \\
& =\text { free left } \mathbb{Z}[\pi] \text {-module generated by the } n \text {-cells of } X
\end{aligned}
$$

and $d: C(\widetilde{X})_{n} \rightarrow C(\widetilde{X})_{n-1}$ the boundary map of the triple $\left(\tilde{X}^{(n)}, \tilde{X}^{(n-1)}\right.$, $\left.\widetilde{X}^{(n-2)}\right)$. The homology $\mathbb{Z}[\pi]$-modules of $C(\widetilde{X})$

$$
H_{n}(C(\tilde{X}))=\operatorname{ker}\left(d: C(\tilde{X})_{n} \rightarrow C(\widetilde{X})_{n-1}\right) / \operatorname{im}\left(d: C(\tilde{X})_{n+1} \rightarrow C(\tilde{X})_{n}\right)
$$

are just the ordinary integral homology groups of $\tilde{X}$

$$
H_{*}(C(\widetilde{X}))=H_{*}(\widetilde{X}) .
$$

Use the $w$-twisted involution on $\mathbb{Z}[\pi]$ to define the dual $\mathbb{Z}[\pi]$-modules

$$
C(\tilde{X})^{n}=\left(C(\tilde{X})_{n}\right)^{*}(n \geqslant 0) .
$$

If $X$ is a finite $C W$ complex then $C(\widetilde{X})$ is a finite f.g. free $\mathbb{Z}[\pi]$-module chain complex.

Example 4.61 Let $X$ be a finite $C W$ complex with an oriented cover $(\tilde{X}, \pi, w)$ such that $w=+1$.

(i) If $\pi$ is finite the cohomology $\mathbb{Z}[\pi]$-modules $H^{*}(\widetilde{X})$ are just the ordinary cohomology groups $H^{*}(\widetilde{X})$ with the $\mathbb{Z}[\pi]$ action

$$
\mathbb{Z}[\pi] \times H^{*}(\tilde{X}) \rightarrow H^{*}(\tilde{X}) ;\left(\sum_{g} n_{g} g, x\right) \mapsto \sum_{g} n_{g}\left(g^{-1}\right)^{*}(x) .
$$

(ii) For arbitrary $\pi$

$$
H^{*}(\widetilde{X})=H_{\mathrm{cpt}}^{*}(\widetilde{X})
$$

with $H_{\text {cpt }}^{*}(\widetilde{X})$ the cohomology groups defined by integral cochains with compact support (i.e. taking non-zero values on only a finite number of cells) with the induced $\mathbb{Z}[\pi]$-module structure. 
Example 4.62 The homology and cohomology $\mathbb{Z}[\mathbb{Z}]$-modules of the oriented $\operatorname{cover}(\mathbb{R}, \mathbb{Z},+1)$ of $\mathbb{R} / \mathbb{Z}=S^{1}$ are given by

$$
H_{n}(\mathbb{R})=\left\{\begin{array}{ll}
\mathbb{Z} & n=0 \\
0 & n \neq 0
\end{array}, H^{n}(\mathbb{R})=\left\{\begin{array}{ll}
\mathbb{Z} & n=1 \\
0 & n \neq 1
\end{array} .\right.\right.
$$

Remark 4.63 For any space $Y$ there are defined cap product pairings

$$
\cap: H_{m}^{\mathrm{lf}}(Y) \otimes_{\mathbb{Z}} H_{\mathrm{cpt}}^{n}(Y) \rightarrow H_{m-n}(Y),
$$

with $H_{*}^{\text {lf }}(Y)$ the homology groups defined using the locally finite infinite chains in $Y$. Thus for any (noncompact) cover $\widetilde{X}$ of a finite $C W$ complex $X$ there are defined (infinite) transfer maps

$$
p^{!}: H_{*}(X) \rightarrow H_{*}^{\mathrm{lf}}(\widetilde{X}) ; x \mapsto \widetilde{x} .
$$

The cap product of 4.58 can be expressed as the composite

$$
\cap: H_{m}(X) \otimes_{\mathbb{Z}} H^{n}(\tilde{X}) \stackrel{p^{!} \otimes 1}{\longrightarrow} H_{m}^{\mathrm{lf}}(\widetilde{X}) \otimes_{\mathbb{Z}} H_{\mathrm{cpt}}^{n}(\widetilde{X}) \stackrel{\cap}{\longrightarrow} H_{m-n}(\tilde{X}),
$$

using 4.61 to identify $H^{*}(\widetilde{X})=H_{\mathrm{cpt}}^{*}(\widetilde{X})$.

Example 4.64 For $\widetilde{X}=X, \pi=\{1\}$ the cohomology intersection pairing is just the evaluation of the cup product

$$
\cup: H^{m-n}(X) \times H^{n}(X) \rightarrow H^{m}(X) ;(a, b) \mapsto a \cup b
$$

on $[X] \in H_{m}(X)$

$$
\lambda(a, b)=\langle a \cup b,[X]\rangle \in \mathbb{Z} .
$$

Universal Poincaré Duality Theorem 4.65 For any m-dimensional manifold $M$ and oriented cover $(\widetilde{M}, \pi, w)$ cap product with the $w$-twisted fundamental class $[M] \in H_{m}\left(M ; \mathbb{Z}^{w}\right)$ is a $\mathbb{Z}[\pi]$-module chain equivalence

$$
[M] \cap-: C(\widetilde{M})^{m-*} \rightarrow C(\widetilde{M})
$$

inducing $\mathbb{Z}[\pi]$-module isomorphisms

$$
[M] \cap-: H^{m-*}(\widetilde{M}) \rightarrow H_{*}(\widetilde{M}) .
$$

Proof As for the $R$-coefficient case (4.4), but using $\mathbb{Z}[\pi]$-coefficients.

In particular, the Universal Poincaré Duality Theorem for the orientation double cover $\left(\bar{M}, \mathbb{Z}_{2}\right.$, id. $)$ classified by $w(M) \in H^{1}\left(M ; \mathbb{Z}_{2}\right)$ is just the Twisted Poincaré Duality Theorem (4.51). The homology intersection pairing (4.11) has the following non-simply-connected generalisation: 
Definition 4.66 The homology intersection pairing of an $m$-dimensional manifold $M$ with respect to an oriented cover $(\widetilde{M}, \pi, w)$

$$
\lambda: H_{n}(\widetilde{M}) \times H_{m-n}(\widetilde{M}) \rightarrow \mathbb{Z}[\pi] ;(a, b) \mapsto \lambda(a, b)
$$

is the sesquilinear pairing defined by

$$
\lambda(a, b)=a^{*}(b) \in \mathbb{Z}[\pi]
$$

with $a^{*} \in H^{m-n}(\widetilde{M})$ the Poincaré dual of $a$, such that

$$
\lambda(b, a)=(-1)^{n(m-n)} \overline{\lambda(a, b)} \in \mathbb{Z}[\pi] .
$$

The homology intersection pairing (4.66) coincides with the cohomology intersection pairing (4.58) via the Poincaré duality isomorphisms

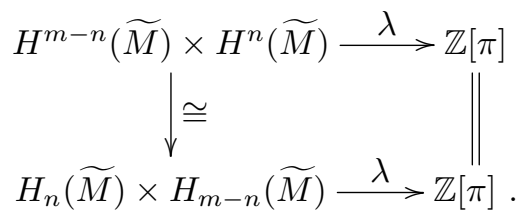

The homology intersection pairing will now be used to extend the results of Section 4.2 on the homology effect of a geometric surgery to the homology of an oriented cover, using the following equivariant version of the Umkehr map $(4.14)$.

Definition 4.67 Let $f: N^{n} \rightarrow M^{m}$ be a map of manifolds, and let $(\widetilde{M}, \pi, w)$ be an oriented cover of $M$ such that the pullback $\left(\widetilde{N}=f^{*} \widetilde{M}, \pi, w\right)$ is an oriented cover of $N$. The Umkehr $\mathbb{Z}[\pi]$-module chain map

$$
f^{!}: C(\widetilde{M}) \longrightarrow C(\widetilde{N})_{*-m+n}
$$

is defined up to chain homotopy to be the composite

$f^{!}: C(\widetilde{M}) \stackrel{([M] \cap-)^{-1}}{\simeq} C(\widetilde{M})^{m-*} \stackrel{f^{*}}{\longrightarrow} C(\widetilde{N})^{m-*} \stackrel{([N] \cap-)}{\simeq} C(\widetilde{N})_{*-m+n}$

The effect of surgery on the homology of an oriented cover is given by :

Proposition 4.68 Let $\left(W^{m+1} ; M^{m}, M^{\prime m}\right)$ be the trace of an n-surgery on an $m$-dimensional manifold $M^{m}$, with

$$
\begin{aligned}
& M^{\prime m}=\operatorname{cl} .\left(M^{m} \backslash S^{n} \times D^{m-n}\right) \cup D^{n+1} \times S^{m-n-1}, \\
& W^{m+1}=M^{m} \times I \cup D^{n+1} \times D^{m-n} .
\end{aligned}
$$


Let $\left(\left(\widetilde{W} ; \widetilde{M}, \widetilde{M^{\prime}}\right), \pi, w\right)$ be an oriented cover of $\left(W ; M, M^{\prime}\right)$, and let $x \in H_{n}(\widetilde{M})$ be the Hurewicz image of the element in $\pi_{n}(M)$ killed by the surgery. The homology $\mathbb{Z}[\pi]$-modules are such that

$$
\begin{aligned}
& H_{i}(\widetilde{W}, \widetilde{M})= \begin{cases}\mathbb{Z}[\pi] & \text { if } i=n+1 \\
0 & \text { if } i \neq n+1\end{cases} \\
& H_{i}\left(\widetilde{W}, \widetilde{M^{\prime}}\right)= \begin{cases}\mathbb{Z}[\pi] & \text { if } i=m-n \\
0 & \text { if } i \neq m-n\end{cases}
\end{aligned}
$$

with a commutative braid of exact sequences of $\mathbb{Z}[\pi]$-modules

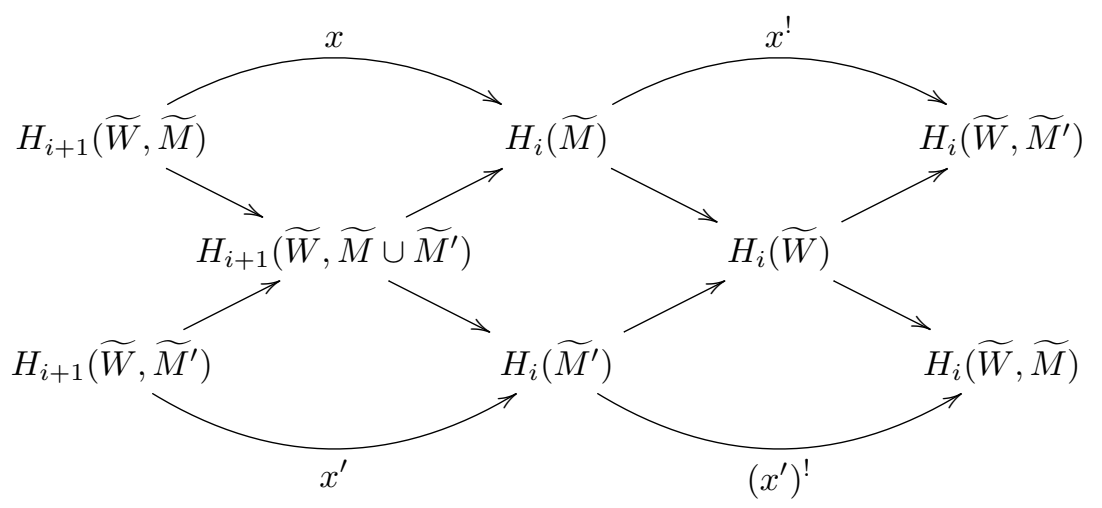

and

$$
\begin{aligned}
& H_{n+1}(\widetilde{W}, \widetilde{M})=\mathbb{Z}[\pi] \rightarrow H_{n}(\widetilde{M}) ; 1 \mapsto x \\
& H_{m-n}(\widetilde{M}) \rightarrow H_{m-n}(\widetilde{W}) \rightarrow H_{m-n}\left(\widetilde{W}, \widetilde{M^{\prime}}\right)=\mathbb{Z}[\pi] ; y \mapsto \lambda(x, y) .
\end{aligned}
$$

Proof Proposition 4.19 gives homotopy equivalences

$$
W \simeq M \cup D^{n+1} \simeq M^{\prime} \cup D^{m-n} .
$$

Thus $M^{\prime}$ is obtained from $M$ by first attaching an $(n+1)$-cell, and then "detaching" the Poincaré dual $(m-n)$-cell from $M \cup D^{n+1}$. Attaching an $(n+1)$-cell to $M$ has the algebraic effect on the cellular chain complex $C(\widetilde{M})$ of forming the algebraic mapping cone of the $\mathbb{Z}[\pi]$-module chain map $x: S^{n} \mathbb{Z}[\pi] \rightarrow C(\widetilde{M})$ representing $x \in H_{n}(\widetilde{M})$, so that the cellular chain complex of $\widetilde{W}$ is given by

$$
C(\widetilde{W})_{i}= \begin{cases}C(\widetilde{M})_{n+1} \oplus \mathbb{Z}[\pi] & \text { if } i=n+1 \\ C(\widetilde{M})_{i} & \text { otherwise } .\end{cases}
$$

Detaching an $(m-n)$-cell from $W$ has the algebraic effect on the cellular chain complex of forming the algebraic mapping cone of the $\mathbb{Z}[\pi]$-module chain map $x^{*}: C(\widetilde{W}) \rightarrow S^{m-n} \mathbb{Z}[\pi]$ representing the Poincaré dual $x^{*} \in H^{m-n}(\widetilde{M})$ of $x$, and shifting the dimension by 1 , so that

$$
C\left(\widetilde{M^{\prime}}\right)_{i}= \begin{cases}C(\widetilde{W})_{m-n-1} \oplus \mathbb{Z}[\pi] & \text { if } i=m-n-1 \\ C(\widetilde{W})_{i} & \text { otherwise }\end{cases}
$$


Eliminating $W$ from these two expressions gives

$$
C\left(\widetilde{M^{\prime}}\right)_{i}= \begin{cases}C(\widetilde{M})_{n+1} \oplus \mathbb{Z}[\pi] & \text { if } i=n+1 \neq m-n-1, \\ C(\widetilde{M})_{m-n-1} \oplus \mathbb{Z}[\pi] & \text { if } i=m-n-1 \neq n+1 \\ C(\widetilde{M})_{n+1} \oplus \mathbb{Z}[\pi] \oplus \mathbb{Z}[\pi] & \text { if } i=m-n-1=n+1 \\ C(\widetilde{M})_{i} & \text { otherwise }\end{cases}
$$

Example 4.69 For an oriented cover $(\widetilde{M}, \pi, w)$ of a $2 n$-dimensional manifold $M^{2 n}$ there is defined a $(-1)^{n}$-symmetric pairing

$$
\lambda: H_{n}(\widetilde{M}) \times H_{n}(\widetilde{M}) \rightarrow \mathbb{Z}[\pi] ;(x, y) \mapsto \lambda(x, y)
$$

with

$$
\lambda(y, x)=(-1)^{n} \overline{\lambda(x, y)} \in \mathbb{Z}[\pi]\left(x, y \in H_{n}(\widetilde{M})\right) .
$$

If $\left(W^{2 n+1} ; M^{2 n}, M^{2 n}\right)$ is the trace of an $n$-surgery on a $2 n$-dimensional manifold $M$ killing $x \in \pi_{n}(M)$ then the Hurewicz image $x \in H_{n}(\widetilde{M})$ is such that $\lambda(x, x)=$ $0 \in \mathbb{Z}[\pi]$ and

$$
H_{n}\left(\widetilde{M^{\prime}}\right)=\langle x\rangle^{\perp} /\langle x\rangle
$$

with

$$
\langle x\rangle^{\perp}=\left\{y \in H_{n}(\widetilde{M}) \mid \lambda(x, y)=0 \in \mathbb{Z}[\pi]\right\}
$$

The reader is referred to Ranicki [69] for a comprehensive theory of surgery on chain complexes - the homology effect of a geometric surgery is determined by the chain level effect of a corresponding algebraic surgery. 


\section{BUNDLES}

The algebraic and topological properties of bundles are an essential tool in understanding manifolds in general, and surgery theory in particular. This chapter brings together the basic properties of fibre bundles, fibrations and vector bundles required. Milnor and Stasheff [61] is the standard reference for vector bundles and their characteristic classes.

Section 5.1 deals with fibrations and fibre bundles with arbitrary fibres $F$. Section 5.2 considers vector bundles, which are fibre bundles with $F=\mathbb{R}^{k}$. Section 5.3 describes the tangent bundle of a manifold, and the normal bundle of a submanifold. Section 5.4 gives a necessary and sufficient bundle-theoretic condition for killing a homotopy class of a manifold by surgery, and describes the effect of surgery on the tangent bundle. Section 5.5 recalls the Hopf invariant and the $J$-homomorphism.

\subsection{Fibre bundles and fibrations}

The basic definitions and properties of fibre bundles and fibrations are reviewed.

Definition 5.1 A fibre bundle is a sequence of spaces and maps

$$
F \longrightarrow E \stackrel{p}{\longrightarrow} B
$$

with base space $B$, total space $E$ and fibre $F=p^{-1}(b)$ (for some base point $b \in B)$, together with a maximal collections of pairs

( open neighbourhood $U \subseteq B$, homeomorphism $\phi: U \times F \rightarrow p^{-1}(U)$ )

such that

$$
p \phi(y, z)=y \in X \quad(y \in B) .
$$

Example 5.2 A fibre bundle over $S^{m}$

$$
F \longrightarrow E \stackrel{p}{\longrightarrow} S^{m}
$$

is determined by a clutching map

$$
\omega: S^{m-1} \rightarrow \operatorname{Homeo}(F)
$$


to the space of homeomorphisms $F \rightarrow F$, with

$$
E=\left(D^{m} \times F\right) \cup_{(x, y) \sim(x, \omega(x)(y))}\left(D^{m} \times F\right) \quad\left(x \in S^{m-1}, y \in F\right) .
$$

More generally :

Definition 5.3 A fibration is a sequence of spaces and maps

$$
F \longrightarrow E \stackrel{p}{\longrightarrow} B
$$

with base space $B$, total space $E$ and fibre $F=p^{-1}(b)$ (for some base point $b \in B)$, such that $p$ has the homotopy lifting property: for each map $f: X \rightarrow E$ and homotopy $h: X \times I \rightarrow B$ with

$$
h(x, 0)=p f(x) \in B \quad(x \in X)
$$

there exists a homotopy $g: X \times I \rightarrow E$ such that

$$
\begin{aligned}
& g(x, 0)=f(x) \in E, \quad p g(x, t)=h(x, t) \in B \quad(x \in X, t \in I) .
\end{aligned}
$$

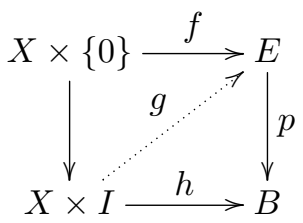

Example 5.4 A fibre bundle $F \rightarrow E \rightarrow B$ with the base space $B$ paracompact is a fibration (Whitehead [96, I.7.13]).

For every map $p: E \rightarrow B$ there exists a fibration $\mathscr{F}(p) \rightarrow \mathscr{E}(p) \rightarrow B$ such that $p: E \simeq \mathscr{E}(p) \rightarrow B$, defined as follows:

Definition 5.5 The path space fibration of a map $p: E \rightarrow B$

$$
\mathscr{F}(p) \longrightarrow \mathscr{E}(p) \stackrel{q}{\longrightarrow} B
$$

is given by

$$
\begin{aligned}
& \mathscr{E}(p)=\left\{(\omega, x) \in B^{I} \times E \mid \omega(1)=p(x) \in B\right\}, \\
& q: \mathscr{E}(p) \rightarrow B ;(\omega, x) \mapsto \omega(0), \\
& \mathscr{F}(p)=q^{-1}(b)=\left\{(\omega, x) \in B^{I} \times E \mid \omega(0)=b, \omega(1)=p(x) \in B\right\}
\end{aligned}
$$


with $B^{I}$ the topological space of maps $I \rightarrow B$ (with the compact-open topology). The maps

$$
\begin{aligned}
& \mathscr{E}(p) \rightarrow E ;(\omega, x) \mapsto x, \\
& H: E \rightarrow \mathscr{E}(p) ; x \mapsto(\omega: t \mapsto p(x), x)
\end{aligned}
$$

are inverse homotopy equivalences, with $q H \simeq p$. The fibre $\mathscr{F}(p)$ is called the mapping fibre of $p: E \rightarrow B$.

Proposition 5.6 (i) Let $p: E \rightarrow B$ be a pointed map. A pointed map $X \rightarrow \mathscr{F}(p)$ is a pair

$$
\text { ( pointed map } f: X \rightarrow E \text {, pointed null-homotopy } g: p f \simeq\{*\}: X \rightarrow B) \text {. }
$$

The pointed homotopy set $[X, \mathscr{F}(p)]$ is the set of pointed homotopy classes of pairs $(f, g)$, and if $B$ is connected there is defined an exact sequence of pointed sets

$$
\ldots \rightarrow[X, \Omega B] \rightarrow[X, \mathscr{F}(p)] \rightarrow[X, E] \rightarrow[X, B],
$$

with $\Omega B=\mathscr{F}(b:\{*\} \rightarrow B)$ the loop space of $B$ at the base point $b \in B$.

(ii) The relative homotopy groups of a pointed map of connected spaces $p: E \rightarrow B$ with connected mapping fibre $\mathscr{F}(p)$ are such that

$$
\pi_{n}(p)=\pi_{n-1}(\mathscr{F}(p)) \quad(n \geqslant 1),
$$

with an exact sequence

$$
\ldots \rightarrow \pi_{n}(\mathscr{F}(p)) \rightarrow \pi_{n}(E) \stackrel{p_{*}}{\rightarrow} \pi_{n}(B) \rightarrow \pi_{n-1}(\mathscr{F}(p)) \rightarrow \ldots \rightarrow \pi_{1}(B) .
$$

(iii) For a fibration of pointed spaces

$$
F \longrightarrow E \stackrel{p}{\longrightarrow} B
$$

with $B$ connected there is defined a homotopy equivalence $F \simeq \mathscr{F}(p)$, and for any pointed space $X$ there is defined an exact sequence of pointed sets

$$
\ldots \rightarrow[X, \Omega B] \rightarrow[X, F] \rightarrow[X, E] \rightarrow[X, B]
$$

If $F, E, B$ are all connected there is defined a long exact sequence of homotopy groups

$$
\ldots \rightarrow \pi_{n}(F) \rightarrow \pi_{n}(E) \rightarrow \pi_{n}(B) \rightarrow \pi_{n-1}(F) \rightarrow \ldots \rightarrow \pi_{1}(B) .
$$

Proof See Chapter VII.6 of Bredon [10]. 
Example 5.7 The Hopf bundles

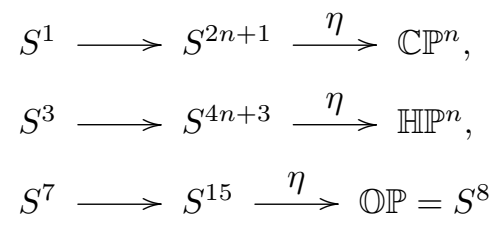

are defined over the projective spaces $\mathbb{C P}^{n}, \mathbb{H} \mathbb{P}^{n}(n \geqslant 1), \mathbb{O P}$ of the complex numbers $\mathbb{C}$, the Hamilton quaternions $\mathbb{H}$ and the Cayley octonions $\mathbb{O}$ respectively, with

$$
\begin{aligned}
\mathbb{C}= & \{x+i y \mid x, y \in \mathbb{R}\}, i^{2}=-1, \bar{i}=-i, \\
\mathbb{H}= & \{a+b i+c j+d k \mid a, b, c, d \in \mathbb{R}\}, \\
& i^{2}=j^{2}=k^{2}=-1, \bar{i}=-i, \bar{j}=-j, \bar{k}=-k, \\
& j k=-k j=i, k i=-i k=j, i j=-j i=k, \\
\mathbb{O}= & \mathbb{H} \oplus \mathbb{H}, \overline{(x, y)}=(\bar{x}, \bar{y}),\left(x_{1}, x_{2}\right)\left(y_{1}, y_{2}\right)=\left(x_{1} y_{1}-\bar{y}_{2} x_{2}, x_{2} \bar{y}_{1}+y_{2} x_{1}\right) .
\end{aligned}
$$

The complex and quaternion projective spaces

$$
\begin{aligned}
& \mathbb{C P}^{n}=\left\{\left(z_{0}, z_{1}, \ldots, z_{n}\right) \neq(0,0, \ldots, 0) \in \mathbb{C}^{n+1}\right\} /(z \sim \lambda z \text { for } \lambda \neq 0 \in \mathbb{C}), \\
& \mathbb{H}^{n}=\left\{\left(z_{0}, z_{1}, \ldots, z_{n}\right) \neq(0,0, \ldots, 0) \in \mathbb{H}^{n+1}\right\} /(z \sim \lambda z \text { for } \lambda \neq 0 \in \mathbb{H})
\end{aligned}
$$

are manifolds of dimension $2 n, 4 n$, respectively. The complex and quaternion Hopf bundle projections are given by

$$
\begin{aligned}
\eta: S^{2 n+1} \cong\left\{\left(z_{0}, z_{1}, \ldots, z_{n}\right)\right. & \left.\in \mathbb{C}^{n+1} \mid \sum_{k=0}^{n} z_{k} \bar{z}_{k}=1\right\} \\
\rightarrow \mathbb{C P}^{n} ;\left(z_{0}, z_{1}, \ldots, z_{n}\right) \mapsto\left[z_{0}, z_{1}, \ldots, z_{n}\right], & \\
\eta: S^{4 n+3} \cong\left\{\left(z_{0}, z_{1}, \ldots, z_{n}\right)\right. & \left.\in \mathbb{H}^{n+1} \mid \sum_{k=0}^{n} z_{k} \bar{z}_{k}=1\right\} \\
\rightarrow & \mathbb{H}^{n} ;\left(z_{0}, z_{1}, \ldots, z_{n}\right) \mapsto\left[z_{0}, z_{1}, \ldots, z_{n}\right]
\end{aligned}
$$

and for $n=1$ are fibre bundles of spheres

$$
\begin{aligned}
& S^{1} \longrightarrow S^{3} \stackrel{\eta}{\longrightarrow} \mathbb{C P}^{1}=S^{2}, \\
& S^{3} \longrightarrow S^{7} \stackrel{\eta}{\longrightarrow} \mathbb{H} \mathbb{P}^{1}=S^{4} .
\end{aligned}
$$

See Chapter VII.8 of Bredon [10] and Chapter 4.2 of Hatcher [31] for more detailed expositions of the complex and quaternion Hopf bundles. See $\S 3.1$ of Baez (The Octonions, Bull. A.M.S. 39, 145-205 (2002)) for the construction of the octonion projective line $\mathbb{O P}$ and the Hopf bundle $S^{7} \rightarrow S^{15} \rightarrow \mathbb{O P}=S^{8}$. 


\subsection{Vector bundles}

Definition 5.8 (i) A $k$-plane bundle (or vector bundle) $(X, \eta)$ is a fibre bundle

$$
\mathbb{R}^{k} \longrightarrow E(\eta) \stackrel{p}{\longrightarrow} X
$$

such that

(a) each fibre

$$
\eta(x)=p^{-1}(x) \quad(x \in X)
$$

is a $k$-dimensional real vector space,

(b) every $x \in X$ has a neighbourhood $U \subseteq X$ with a homeomorphism

$$
\phi: U \times \mathbb{R}^{k} \rightarrow p^{-1}(U)
$$

such that for each $u \in U$ the function

$$
\mathbb{R}^{k} \rightarrow \eta(u) ; v \mapsto \phi(u, v)
$$

is an isomorphism of vector spaces.

(ii) A bundle map

$$
(f, b):\left(X^{\prime}, \eta^{\prime}\right) \rightarrow(X, \eta)
$$

is a commutative square of maps

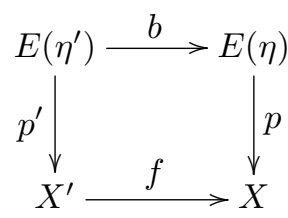

such that the restriction of $b$

$$
b\left(x^{\prime}\right): \eta^{\prime}\left(x^{\prime}\right) \rightarrow \eta\left(f\left(x^{\prime}\right)\right) ; v \mapsto b(v)
$$

is a linear map of vector spaces for each $x^{\prime} \in X^{\prime}$.

Definition 5.9 (i) The pullback of a $k$-plane bundle $\eta$ over $X$ along a map $f: X^{\prime} \rightarrow X$ is the $k$-plane bundle $f^{*} \eta$ over $X^{\prime}$ defined by

$$
E\left(f^{*} \eta\right)=\left\{\left(x^{\prime}, y\right) \in X^{\prime} \times E(\eta) \mid f\left(x^{\prime}\right)=p(y) \in X\right\}
$$

with projection

$$
p^{\prime}: E\left(f^{*} \eta\right) \rightarrow X^{\prime} ;\left(x^{\prime}, y\right) \mapsto x^{\prime}
$$

and fibres 


$$
f^{*} \eta\left(x^{\prime}\right)=\eta\left(f\left(x^{\prime}\right)\right) \quad\left(x^{\prime} \in X^{\prime}\right) .
$$

(ii) A pullback bundle map is a bundle map $(f, b):\left(X^{\prime}, \eta^{\prime}\right) \rightarrow(X, \eta)$ such that each of the linear maps

$$
b\left(x^{\prime}\right)=b \mid: \eta^{\prime}\left(x^{\prime}\right) \rightarrow \eta\left(f\left(x^{\prime}\right)\right) \quad\left(x^{\prime} \in X^{\prime}\right)
$$

is an isomorphism of vector spaces, i.e. such that the function

$$
E\left(\eta^{\prime}\right) \rightarrow E\left(f^{*} \eta\right) ; y \mapsto\left(p^{\prime}(y), b(y)\right)
$$

is a homeomorphism.

(iii) An isomorphism $b: \eta^{\prime} \rightarrow \eta$ of bundles over the same space $X$ is a pullback bundle map of the type $(1, b):\left(X, \eta^{\prime}\right) \rightarrow(X, \eta)$.

Proposition 5.10 (i) For any k-plane bundle $\eta$ over $X$ and any map $f: X^{\prime} \rightarrow$ $X$ there is defined a pullback bundle map

$$
(f, b):\left(X^{\prime}, f^{*} \eta\right) \rightarrow(X, \eta) .
$$

(ii) A pullback bundle map $(f, b):\left(X^{\prime}, \eta^{\prime}\right) \rightarrow(X, \eta)$ is a bundle map such that $(1, b):\left(X^{\prime}, \eta^{\prime}\right) \rightarrow\left(X^{\prime}, f^{*} \eta\right)$ is an isomorphism of bundles.

Proof (i) The map

$$
b: E\left(f^{*} \eta\right) \rightarrow E(\eta) ;\left(x^{\prime}, y\right) \mapsto y
$$

determines a bundle map

$$
(f, b):\left(X^{\prime}, f^{*} \eta\right) \rightarrow(X, \eta)
$$

with

$$
b\left(x^{\prime}\right): f^{*} \eta\left(x^{\prime}\right)=p^{-1}\left(x^{\prime}\right) \rightarrow \eta\left(f\left(x^{\prime}\right)\right)=p^{-1}\left(f\left(x^{\prime}\right)\right) ;\left(x^{\prime}, y\right) \mapsto y
$$

a vector space isomorphism for each $x^{\prime} \in X^{\prime}$.

(ii) Trivial.

The topology of vector bundles is closely related to the topology of the general linear and orthogonal groups:

Definition 5.11 (i) The $k$-dimensional general linear group

$$
G L(k)=\operatorname{Aut}_{\mathbb{R}}\left(\mathbb{R}^{k}\right)
$$

is the automorphism group of the standard $k$-dimensional real vector space $\mathbb{R}^{k}$, with elements the invertible $k \times k$ real matrices.

(ii) The $k$-dimensional orthogonal group

$$
O(k)=\operatorname{Aut}_{\mathbb{R}}\left(\mathbb{R}^{k}, 1\right)
$$

is the automorphism group of the standard $k$-dimensional symmetric bilinear form $\left(\mathbb{R}^{k}, 1\right)$, with elements the orthogonal $k \times k$ real matrices. 
Identify the set of all $k \times k$ matrices with the $k^{2}$-dimensional Euclidean space

$$
M_{k, k}(\mathbb{R})=\mathbb{R}^{k^{2}}
$$

and give $O(k) \subset G L(k) \subset M_{k, k}(\mathbb{R})$ the subspace topologies.

Proposition 5.12 (i) $G L(k)$ is an open manifold of dimension

$$
\operatorname{dim} G L(k)=k^{2} .
$$

(ii) $O(k)$ is a compact manifold of dimension

$$
\operatorname{dim} O(k)=\frac{1}{2} k(k-1) .
$$

(iii) The inclusion $O(k) \hookrightarrow G L(k)$ is a homotopy equivalence.

Proof (i) The determinant of $k \times k$ matrices defines a differentiable function

$$
\operatorname{det}: M_{k, k}(\mathbb{R})=\mathbb{R}^{k^{2}} \rightarrow \mathbb{R} ; a \mapsto \operatorname{det}(a)
$$

such that

$$
G L(k)=M_{k, k}(\mathbb{R}) \backslash \operatorname{det}^{-1}(0) \subset \mathbb{R}^{k^{2}}
$$

is an open subset.

(ii) A $k \times k$ matrix $a=\left(a_{i j}\right)$ is orthogonal if and only if

$$
a^{T} a=I
$$

where $a^{T}=\left(a_{j i}\right)$ is the transpose $k \times k$ matrix. The function

$$
f: \mathbb{R}^{k^{2}} \rightarrow \mathbb{R}^{k(k+1) / 2} ; a \mapsto\left\{\left(a^{T} a-I\right)_{i j} \mid 1 \leqslant i \leqslant j \leqslant k\right\}
$$

has regular value 0 , with

$$
O(k)=f^{-1}(0) \subset M_{k, k}(\mathbb{R})=\mathbb{R}^{k^{2}} .
$$

The Implicit Function Theorem (2.12) gives that $O(k)$ is a manifold with

$$
\operatorname{dim} O(k)=k^{2}-k(k+1) / 2=k(k-1) / 2 .
$$

The subspace $O(k) \subset \mathbb{R}^{k^{2}}$ is closed and bounded, and hence compact.

(iii) The Gram-Schmidt orthonormalisation process defines a homotopy inverse $G L(k) \rightarrow O(k)$ to the inclusion $O(k) \hookrightarrow G L(k)$. 
Example 5.13 An orthogonal map $\mathbb{R}^{2} \rightarrow \mathbb{R}^{2}$ is a rotation or a reflection, so that the orthogonal group $O(2)$ is diffeomorphic to the disjoint union of two circles, with a diffeomorphism

$$
\begin{aligned}
& S^{1} \cup S^{1}=S^{1} \times\{ \pm 1\} \rightarrow O(2) ; \\
& \begin{cases}((\cos \theta, \sin \theta),+1) \mapsto\left(\begin{array}{cc}
\cos \theta & -\sin \theta \\
\sin \theta & \cos \theta
\end{array}\right) & (\text { rotation through } \theta) \\
((\cos \theta, \sin \theta),-1) \mapsto\left(\begin{array}{cc}
\cos \theta & \sin \theta \\
\sin \theta & -\cos \theta
\end{array}\right) & (\text { reflection in } \theta / 2) .\end{cases}
\end{aligned}
$$

Definition 5.14 The base space $X$ of a $k$-plane bundle $\eta$ is covered by open neighbourhoods $U \subseteq X$ with homeomorphisms $\phi: U \times \mathbb{R}^{k} \rightarrow p^{-1}(U)$ such that

(i) $p \phi(x, v)=x \in X$ for all $x \in U, v \in \mathbb{R}^{k}$,

(ii) for any $(U, \phi),\left(U^{\prime}, \phi^{\prime}\right)$ with non-empty intersection $U \cap U^{\prime}$ the transition functions

$$
\phi^{\prime-1} \phi \mid:\left(U \cap U^{\prime}\right) \times \mathbb{R}^{k} \rightarrow p^{-1}\left(U \cap U^{\prime}\right) \rightarrow\left(U \cap U^{\prime}\right) \times \mathbb{R}^{k}
$$

are of the form $(x, v) \mapsto(x, h(x)(v))$ for some continuous function

$$
h=h_{U}^{U^{\prime}}: U \cap U^{\prime} \rightarrow G L(k)
$$

satisfying the usual compatibility conditions

$$
h_{U}^{U^{\prime \prime}}(x)=h_{U^{\prime}}^{U^{\prime \prime}}(x) h_{U}^{U^{\prime}}(x): \mathbb{R}^{k} \rightarrow \mathbb{R}^{k}\left(x \in U \cap U^{\prime} \cap U^{\prime \prime}\right) .
$$

It follows from Proposition 5.12 (iii) that the transition functions of a vector bundle can always be deformed to be of the form

$$
\left(U \cap U^{\prime}\right) \times \mathbb{R}^{k} \rightarrow\left(U \cap U^{\prime}\right) \times \mathbb{R}^{k} ;(x, v) \mapsto(x, h(x)(v))
$$

with $h: U \cap U^{\prime} \rightarrow O(k)$. From now on, we shall only consider bundles of this type. The transition functions preserve the standard inner products on the fibres, so that each vector $v \in E(\eta)$ has a length $\|v\| \geqslant 0$.

Definition 5.15 (i) A $k$-plane bundle $\eta$ over a space $X$ is trivial if it is isomorphic to the bundle $\epsilon^{k}$ with projection

$$
p: E\left(\epsilon^{k}\right)=X \times \mathbb{R}^{k} \rightarrow X ;(x, y) \mapsto x .
$$

(ii) A framing (or trivialisation) of a $k$-plane bundle $\eta$ is an isomorphism to the trivial $k$-plane bundle 


$$
b: \eta \cong \epsilon^{k} .
$$

(iii) Two framings $b_{0}, b_{1}$ of a $k$-plane bundle $\eta$ over $X$ are isomorphic if there exists a continuous family of framings

$$
b_{t}: \eta \cong \epsilon^{k} \quad(0 \leqslant t \leqslant 1),
$$

or equivalently if $b_{0}, b_{1}$ extend to a framing of the $k$-plane bundle $\eta \times I$ over $X \times I$ with total space $E(\eta \times I)=E(\eta) \times I$.

(iv) A section of a $k$-plane bundle $\eta$ over a space $X$ with projection $p: E(\eta) \rightarrow X$ is a map $s: X \rightarrow E(\eta)$ such that

$$
p s=1: X \stackrel{s}{\longrightarrow} E(\eta) \stackrel{p}{\longrightarrow} X .
$$

Thus for each $x \in X$ there is given a continuous choice of element $s(x) \in \eta(x)$.

(v) A section $s$ of $\eta$ is non-zero if $s(x) \neq 0(x) \in \eta(x)$ for every $x \in X$.

(vi) The zero section of $\eta$ is the section

$$
z: X \rightarrow E(\eta) ; x \mapsto 0(x) .
$$

(vii) The Whitney sum of a $j$-plane bundle $\alpha$ over $X$ and a $k$-plane bundle $\beta$ over $X$ is the $(j+k)$-plane bundle $\alpha \oplus \beta$ over $X$ defined by

$$
E(\alpha \oplus \beta)=\left\{(u, v) \in E(\alpha) \times E(\beta) \mid p_{\alpha}(u)=p_{\beta}(v) \in X\right\}
$$

with fibres

$$
(\alpha \oplus \beta)(x)=\alpha(x) \oplus \beta(x) \quad(x \in X) .
$$

Example 5.16 The trivial $k$-plane bundle $\epsilon^{k}$ over a space $X$ is the $k$-fold Whitney sum of the trivial 1-plane (= line) bundle

$$
\epsilon^{k}=\epsilon \oplus \epsilon \oplus \ldots \oplus \epsilon,
$$

with $k$ linearly independent sections. For any map $f: Y \rightarrow X$ the pullback $f^{*} \epsilon^{k}$ is (isomorphic to) the trivial $k$-plane bundle $\epsilon^{k}$ over $Y$.

Proposition 5.17 A k-plane bundle $\eta$ admits a non-zero section (5.15) if and only if it is isomorphic to $\eta^{\prime} \oplus \epsilon$ for a $(k-1)$-plane bundle $\eta^{\prime}$.

Proof Given a non-zero section $s: X \rightarrow E(\eta)$ define $\eta^{\prime}$ by

$$
E\left(\eta^{\prime}\right)=\bigcup_{x \in X} \eta(x) /\langle s(x)\rangle
$$

with $\langle s(x)\rangle \subseteq \eta(x)$ the 1-dimensional subspace spanned by $s(x) \neq 0 \in \eta(x)$. 
Definition 5.18 Let $\eta$ be a $k$-plane bundle over a space $X$.

(i) The disk bundle of $\eta$ is

$$
D(\eta)=\{v \in E(\eta) \mid\|v\| \leqslant 1\} .
$$

(ii) The sphere bundle of $\eta$ is

$$
S(\eta)=\{v \in E(\eta) \mid\|v\|=1\} .
$$

(iii) The Thom space of $\eta$ is the pointed space

$$
T(\eta)=D(\eta) / S(\eta)
$$

which is the one-point compactification of $E(\eta)$.

The disk and sphere bundles are the total spaces of a fibre bundle

$$
\left(D^{k}, S^{k-1}\right) \rightarrow(D(\eta), S(\eta)) \rightarrow X .
$$

Example 5.19 (i) For any vector bundle $\eta$ over a space $X$ the Thom space of $\eta \oplus \epsilon$ is the suspension (3.9) of the Thom space of $\eta$

$$
T(\eta \oplus \epsilon)=\Sigma T(\eta) .
$$

(ii) For the trivial $k$-plane bundle $\epsilon^{k}$ over a space $X$

$$
\left(D\left(\epsilon^{k}\right), S\left(\epsilon^{k}\right)\right)=X \times\left(D^{k}, S^{k-1}\right), T\left(\epsilon^{k}\right)=\Sigma^{k}\left(X_{+}\right)
$$

where $X_{+}=X \cup\{$ pt. $\}$.

Proposition 5.20 The Thom space $T(\eta)$ of a $k$-plane bundle $\eta$ over a $C W$ complex $X$ has the structure of a $C W$ complex with one 0-cell (at the base point) and one $(n+k)$-cell for each $n$-cell of $X$.

Proof By definition, $X$ is obtained from $\emptyset$ by successively attaching $n$-cells. It therefore suffices to consider the effect on the Thom space $T(\eta)$ of attaching an $n$-cell to $X$ along a map $\alpha: S^{n-1} \rightarrow X$ with a trivialisation $\beta: \alpha^{*} \eta \cong \epsilon^{k}$ over $S^{n-1}$, giving a $k$-plane bundle $\omega$ over $X \cup_{\alpha} D^{n}$ with $\left.\omega\right|_{X}=\eta$. The Thom space $T(\omega)$ is obtained from $T(\eta)$ by attaching an $(n+k)$-cell

$$
T(\omega)=T(\eta) \cup_{\gamma} D^{n+k}
$$

with

$$
\gamma: S^{n+k-1} \hookrightarrow T\left(\alpha^{*} \eta\right) \cong T\left(\epsilon^{k}\right)=S^{k} \vee S^{n+k-1} \longrightarrow T(\eta) .
$$


$\mathbb{Z}_{2}$-coefficient Thom Isomorphism Theorem 5.21 Every $k$-plane bundle $\eta$ over a $C W$ complex $X$ has a mod2 Thom class $U_{\eta} \in \dot{H}^{k}\left(T(\eta) ; \mathbb{Z}_{2}\right)$ such that for each $x \in X$ the morphism

$$
\left(i_{x}\right)^{*}: \dot{H}^{k}\left(T(\eta) ; \mathbb{Z}_{2}\right) \rightarrow \dot{H}^{k}\left(T\left(\left(i_{x}\right)^{*} \eta\right) ; \mathbb{Z}_{2}\right)=\dot{H}^{k}\left(S^{k} ; \mathbb{Z}_{2}\right)=\mathbb{Z}_{2}
$$

induced by the inclusion $i_{x}:\{x\} \hookrightarrow X$ sends $U_{\eta}$ to the non-zero element in $\mathbb{Z}_{2}$. The cap and cup products define isomorphisms

$$
\begin{aligned}
& U_{\eta} \cap-: \dot{H}_{*}\left(T(\eta) ; \mathbb{Z}_{2}\right) \rightarrow H_{*-k}\left(X ; \mathbb{Z}_{2}\right), \\
& U_{\eta} \cup-: H^{*}\left(X ; \mathbb{Z}_{2}\right) \rightarrow \dot{H}^{*+k}\left(T(\eta) ; \mathbb{Z}_{2}\right) .
\end{aligned}
$$

Proof The reduced $\mathbb{Z}_{2}$-coefficient cellular chain complex of $T(\eta)$ may be identified with the cellular chain complex of $X$ with a $k$-fold dimension shift

$$
\dot{C}\left(T(\eta) ; \mathbb{Z}_{2}\right)=C\left(X ; \mathbb{Z}_{2}\right)_{*-k} .
$$

(See Theorem 4D.10 of Hatcher [31], Theorem VI.11.3 of Bredon [10], Lemma 18.2 of Milnor and Stasheff [61] for more detailed accounts.)

Definition 5.22 Let $V$ be a real vector space, and let $k \geqslant 1$ be finite and such that $k \leqslant \operatorname{dim}(V) \leqslant \infty$.

(i) The Grassmann manifold $G_{k}(V)$ is the space of $k$-dimensional subspaces $W \subseteq V$.

(ii) The canonical $k$-plane bundle over $G_{k}(V)$

$$
\gamma_{k}(V)=\left\{(W, x) \mid W \in G_{k}(V), x \in W\right\}
$$

has projection

$$
\gamma_{k}(V) \rightarrow G_{k}(V) ;(W, x) \mapsto W
$$

Bundle Classification Theorem 5.23 (Steenrod [86])

Let $X$ be a finite $C W$ complex.

(i) Every $k$-plane bundle $\eta$ over $X$ is (isomorphic to) the pullback $f^{*} \gamma_{k}\left(\mathbb{R}^{\infty}\right)$ of the canonical $k$-plane bundle $1_{k}=\gamma_{k}\left(\mathbb{R}^{\infty}\right)$ over the classifying space

$$
B O(k)=G_{k}\left(\mathbb{R}^{\infty}\right)
$$

along a map $f: X \rightarrow B O(k)$.

(ii) The isomorphism classes of $k$-plane bundles over $X$ are in one-one correspondence with the homotopy classes of maps $X \rightarrow B O(k)$.

(iii) The trivial $k$-plane bundle $\epsilon^{k}$ is classified by the trivial map $\{*\}: X \rightarrow$ $B O(k)$.

(iv) The pullback $f^{*} \eta$ of a $k$-plane bundle $\eta: X \rightarrow B O(k)$ along a map $f: X^{\prime} \rightarrow$ $X$ is classified by the composite

$$
f^{*} \eta: X^{\prime} \stackrel{f}{\rightarrow} X \stackrel{\eta}{\rightarrow} B O(k) .
$$


Proof See Chapter 5 of Milnor and Stasheff [61].

In particular, the identity map $1_{k}: B O(k) \rightarrow B O(k)$ classifies $\gamma_{k}\left(\mathbb{R}^{\infty}\right)$. On the level of classifying spaces, the Whitney sum is given by a map

$$
\oplus: B O(j) \times B O(k) \rightarrow B O(j+k) .
$$

Definition 5.24 (i) An orthonormal $n$-frame $b=\left(b_{1}, b_{2}, \ldots, b_{n}\right)$ in an inner product space $W$ is an ordered set of $n$ orthonormal vectors, with

$$
b_{i} \bullet b_{j}=\delta_{i j}=\left\{\begin{array}{ll}
1 & \text { if } i=j \\
0 & \text { if } i \neq j
\end{array} .\right.
$$

(ii) The Stiefel $n$-frame manifold $V_{n}(W)$ of an inner product space $W$ is the space of orthonormal $n$-frames in $W$. In particular, the Stiefel $n$-frame manifold of $\mathbb{R}^{m}$ is denoted ${ }^{1}$

$$
V_{m, n}=V_{n}\left(\mathbb{R}^{m}\right) \text {. }
$$

(iii) The Stiefel $n$-frame bundle $V_{n}(\eta)$ of an $m$-plane bundle $\eta$ over $X$ with $n \leqslant m$ is the fibre bundle

$$
V_{m, n} \rightarrow V_{n}(\eta) \rightarrow X
$$

with total space

$$
V_{n}(\eta)=\bigcup_{x \in X} V_{n}(\eta(x))
$$

Proposition 5.25 (i) The Stiefel manifold $V_{m, n}$ of orthonormal $n$-frames in $\mathbb{R}^{m}$ is a compact manifold of dimension

$$
\operatorname{dim} V_{m, n}=(2 m-n-1) n / 2 .
$$

(ii) The Grassmann manifold $G_{n}\left(\mathbb{R}^{m}\right)$ of $n$-dimensional subspaces in $\mathbb{R}^{m}$ is a compact manifold of dimension

$$
\operatorname{dim} G_{n}\left(\mathbb{R}^{m}\right)=n(m-n) .
$$

(iii) The Stiefel manifolds $V_{m, n}, V_{m, m-n}$ and the Grassmann manifold $G_{n}\left(\mathbb{R}^{m}\right)$ fit into a commutative braid of fibrations

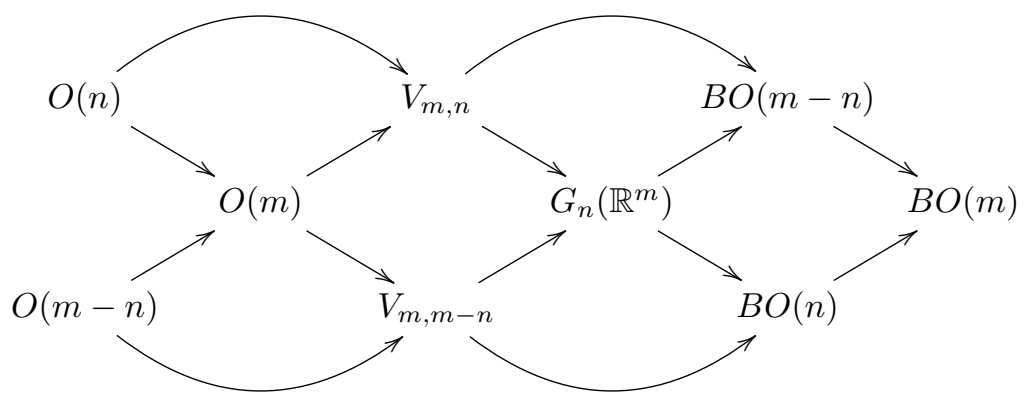

\footnotetext{
${ }^{1}$ Some authors (e.g. Browder [11] and Wall [92]) use a different terminology, with $V_{m, n}$
} denoting the Stiefel manifold of orthonormal $m$-frames in $\mathbb{R}^{m+n}$. 
with

$$
\begin{aligned}
& V_{m, n}=O(m) / O(m-n), \quad V_{m, m-n}=O(m) / O(n), \\
& G_{n}\left(\mathbb{R}^{m}\right)=G_{m-n}\left(\mathbb{R}^{m}\right)=O(m) /(O(n) \times O(m-n)), \\
& B O(n)=G_{n}\left(\mathbb{R}^{\infty}\right) .
\end{aligned}
$$

Proof (i) The Stiefel manifold

$$
V_{m, n}=\left\{\left(b_{1}, b_{2}, \ldots, b_{n}\right) \in \prod_{n} S^{m-1} \mid b_{i} \bullet b_{j}=\delta_{i j}\right\}
$$

is a closed subspace of the compact Hausdorff space $\prod_{n} S^{m-1}$, so that it is also a compact Hausdorff space. Use the standard unit vectors

$$
e_{i}=(0, \ldots, 0,1,0, \ldots, 0) \in \mathbb{R}^{m} \quad(1 \leqslant i \leqslant m)
$$

to define the projection

$$
O(m) \rightarrow V_{m, n} ; \omega \mapsto\left(\omega\left(e_{m-n+1}\right), \omega\left(e_{m-n+2}\right), \ldots, \omega\left(e_{m}\right)\right)
$$

of a fibre bundle

$$
O(m-n) \rightarrow O(m) \rightarrow V_{m, n} .
$$

Applying the dimension formula given by Proposition 5.12 (ii) we have

$$
\begin{aligned}
\operatorname{dim} V_{m, n} & =\operatorname{dim} O(m)-\operatorname{dim} O(m-n) \\
& =(m(m-1)-(m-n)(m-n-1)) / 2 \\
& =(2 m-n-1) n / 2 .
\end{aligned}
$$

(ii) The map

$$
V_{m, n} \rightarrow G_{n}\left(\mathbb{R}^{m}\right) ;\left(b_{1}, b_{2}, \ldots, b_{n}\right) \mapsto \operatorname{span}\left(b_{1}, b_{2}, \ldots, b_{n}\right)
$$

is the projection of a fibre bundle with fibre $O(n)$, so

$$
\begin{aligned}
\operatorname{dim} G_{n}\left(\mathbb{R}^{m}\right) & =\operatorname{dim} V_{m, n}-\operatorname{dim} O(n) \\
& =(2 m-n-1) n / 2-n(n-1) / 2 \\
& =n(m-n) .
\end{aligned}
$$

(Alternatively, note that for any $n$-dimensional subspace $U \subseteq \mathbb{R}^{m}$ the orthogonal complement $U^{\perp} \subseteq \mathbb{R}^{m}$ is $(m-n)$-dimensional, and the graphs of linear maps $f: U \rightarrow U^{\perp}$

$$
\Gamma_{f}=\{(x, f(x)) \mid x \in U\} \subseteq U \oplus U^{\perp}=\mathbb{R}^{m}
$$

define an open neighbourhood $\left\{\Gamma_{f}\right\} \subseteq G_{n}\left(\mathbb{R}^{m}\right)$ of $U \in G_{n}\left(\mathbb{R}^{m}\right)$ with

$$
\left\{\Gamma_{f}\right\}=\operatorname{Hom}_{\mathbb{R}}\left(U, U^{\perp}\right)=\mathbb{R}^{n(m-n)}
$$


up to homeomorphism.)

(iii) The Stiefel $n$-frame bundle of the universal $m$-plane bundle $1_{m}$

$$
V_{m, n} \rightarrow V_{n}\left(1_{m}\right) \rightarrow G_{m}\left(\mathbb{R}^{\infty}\right)
$$

has total space

$$
V_{n}\left(1_{m}\right)=\left\{(W, b) \mid W \subset \mathbb{R}^{\infty}, \operatorname{dim}_{\mathbb{R}}(W)=m, b \in V_{n}(W)\right\}
$$

and projection

$$
V_{n}\left(1_{m}\right) \rightarrow G_{m}\left(\mathbb{R}^{\infty}\right) ;(W, b) \mapsto W
$$

The map

$$
V_{n}\left(1_{m}\right) \rightarrow G_{m-n}\left(\mathbb{R}^{\infty}\right) ;(W, b) \mapsto \operatorname{span}(b)^{\perp}
$$

is a homotopy equivalence, so there is defined a fibration

$V_{m, n}=O(m) / O(m-n) \rightarrow G_{m-n}\left(\mathbb{R}^{\infty}\right)=B O(m-n) \rightarrow G_{m}\left(\mathbb{R}^{\infty}\right)=B O(m)$.

Example 5.26 An element $x \in V_{m, 1}$ is a unit vector $x \in \mathbb{R}^{m}$, so

$$
V_{m, 1}=S^{m-1} \text {. }
$$

Vector bundles over $S^{n}$ are constructed as follows:

Definition 5.27 Given a map $\omega: S^{n-1} \rightarrow O(k)$ define a $k$-plane bundle $\eta_{\omega}$ : $S^{n} \rightarrow B O(k)$ over $S^{n}=D^{n} \cup D^{n}$ with total space

$$
E\left(\eta_{\omega}\right)=D^{n} \times \mathbb{R}^{k} \cup_{(x, y) \sim(x, \omega(x)(y))} D^{n} \times \mathbb{R}^{k} \quad\left(x \in S^{n-1}, y \in \mathbb{R}^{k}\right)
$$

and clutching map $\omega(5.2)$.

Proposition 5.28 (Steenrod [86])

(i) The function

$$
\pi_{n-1}(O(k)) \rightarrow \pi_{n}(B O(k)) ; \omega \mapsto \eta_{\omega}
$$

is an isomorphism.

(ii) The loop space of the classifying space $B O(k)$ is such that there are defined homotopy equivalences

$$
\Omega B O(k) \simeq O(k) \simeq G L(k),
$$

and

$$
\pi_{*}(B O(k))=\pi_{*-1}(O(k))=\pi_{*-1}(G L(k)) .
$$


Proof Every $k$-plane bundle over $D^{n}$ is trivial. For every $k$-plane bundle $\eta$ over $S^{n}=D^{n} \cup D^{n}$ the restrictions to the two $D^{n}$ 's are trivial, which are glued together using an automorphism $\omega: \epsilon^{k} \cong \epsilon^{k}$ of the trivial $k$-plane bundle $\epsilon^{k}$ over $S^{n-1}$. Thus $\eta$ is isomorphic to $\eta_{\omega}$ for some $\omega: S^{n-1} \rightarrow O(k)$.

Proposition 5.29 (i) Every vector bundle $\eta: X \rightarrow B O(k)$ has a stable inverse, a vector bundle $-\eta: X \rightarrow B O(j)$ ( $j$ large) such that

$$
\eta \oplus-\eta=\epsilon^{j+k}: X \rightarrow B O(j+k) .
$$

(ii) A $k$-plane bundle $\eta$ can be framed (= is trivial) if and only if the classifying map $\eta: X \rightarrow B O(k)$ is null-homotopic, with the isomorphism classes of framings $b: \eta \cong \epsilon^{k}$ in (unnatural) one-one correspondence with $[X, O(k)]$.

Proof This is a direct consequence of the Bundle Classification Theorem (5.23).

Definition 5.30 (i) A stable isomorphism between a $k$-plane bundle $\eta$ and a $k^{\prime}$-plane bundle $\eta^{\prime}$ over the same space $X$ is a bundle isomorphism

$$
b: \eta \oplus \epsilon^{j} \cong \eta^{\prime} \oplus \epsilon^{j^{\prime}}
$$

for some $j, j^{\prime} \geqslant 0$ with $j+k=j^{\prime}+k^{\prime}$.

(ii) A stable bundle over $X$ is an equivalence class of bundles $\eta$ over $X$, subject to the equivalence relation

$$
\begin{gathered}
\eta \sim \eta^{\prime} \text { if there exists a stable isomorphism } \eta \oplus \epsilon^{j} \cong \eta^{\prime} \oplus \epsilon^{j^{\prime}} \\
\text { for some } j, j^{\prime} \geqslant 0 .
\end{gathered}
$$

(iii) A $k$-plane bundle $\eta$ is stably trivial if $\eta \oplus \epsilon^{j}$ is trivial for some $j \geqslant 0$.

Proposition 5.31 The isomorphism classes of stable bundles over a finite $C W$ complex $X$ are in one-one correspondence with the homotopy classes of maps $X \rightarrow B O$ to the classifying space

$$
B O=\varliminf_{k} B O(k),
$$

the direct limit with respect to the inclusions $B O(k) \rightarrow B O(k+1)$ passing from $\eta$ to $\eta \oplus \epsilon$.

Proof Immediate from the Bundle Classification Theorem 5.23.

Remark 5.32 The homotopy groups $\pi_{*}(B O)$ are 8-periodic by the Bott periodicity theorem, and are given by : 


\begin{tabular}{|c|c|c|c|c|c|c|c|c|}
\hline$n(\bmod 8)$ & 0 & 1 & 2 & 3 & 4 & 5 & 6 & 7 \\
\hline$\pi_{n}(B O)$ & $\mathbb{Z}$ & $\mathbb{Z}_{2}$ & $\mathbb{Z}_{2}$ & 0 & $\mathbb{Z}$ & 0 & 0 & 0 \\
\hline
\end{tabular}

Proposition 5.33 (Steenrod [86])

(i) The pair $(B O(k+1), B O(k))$ is $k$-connected.

(ii) If $k>m$ then two $k$-plane vector bundles $\eta$, $\eta^{\prime}$ over an $m$-dimensional finite $C W$ complex $X$ are isomorphic if and only if they are stably isomorphic.

(iii) The Stiefel manifold $V_{m, n}$ is $(m-n-1)$-connected.

Proof (i) The inclusion $B O(k) \rightarrow B O(k+1)$ fits into a fibration sequence

$$
V_{k+1,1}=S^{k} \rightarrow B O(k) \rightarrow B O(k+1),
$$

the $k$-sphere bundle of the universal $(k+1)$-plane bundle. (See Proposition 5.83 (ii) for the special case of the tangent bundle $\left.\tau_{S^{k+1}}: S^{k+1} \rightarrow B O(k+1)\right)$. The pair $(B O(k+1), B O(k))$ is $k$-connected, with

$$
\pi_{j}(B O(k+1), B O(k))=\pi_{j-1}\left(S^{k}\right)=0 \text { for } j \leqslant k .
$$

(ii) It follows from (i) that for $k>m$ each map

$$
[X, B O(k)] \rightarrow[X, B O(k+1)] \rightarrow \ldots \rightarrow[X, B O]
$$

is a bijection.

(iii) It follows from (i) that the pair $(B O(m), B O(m-n))$ is $(m-n)$-connected, so that

$$
\pi_{k}\left(V_{m, n}\right)=\pi_{k+1}(B O(m), B O(m-n))=0 \text { for } k \leqslant m-n-1 .
$$

Every cohomology class $c \in H^{j}(B O(k))$ determines a characteristic class, associating to each $k$-plane bundle $\eta: X \rightarrow B O(k)$ a cohomology class

$$
c(\eta)=\eta^{*}(c) \in H^{j}(X) .
$$

Characteristic classes are the basic algebraic topology invariants of vector bundles.

Remark 5.34 (i) See Chapters 7,8 of Milnor and Stasheff [61] for the definition of the characteristic Stiefel-Whitney classes of a $k$-plane bundle $\eta: X \rightarrow$ $B O(k)$

$$
w_{i}(\eta) \in H^{i}\left(X ; \mathbb{Z}_{2}\right) \quad(i \geqslant 0)
$$

which Thom characterised by 


$$
S q^{i}\left(U_{\eta}\right)=w_{i}(\eta) \cup U_{\eta} \in \dot{H}^{k+i}\left(T(\eta) ; \mathbb{Z}_{2}\right),
$$

with $U_{\eta} \in \dot{H}^{k}\left(T(\eta) ; \mathbb{Z}_{2}\right)$ the $\bmod 2$ Thom class. The universal Stiefel-Whitney classes $w_{i}=w_{i}\left(1_{k}\right) \in H^{i}\left(B O(k) ; \mathbb{Z}_{2}\right)$ are the generators of the mod 2 cohomology of $B O(k)$

$$
H^{*}\left(B O(k) ; \mathbb{Z}_{2}\right)=\mathbb{Z}_{2}\left[w_{1}, w_{2}, \ldots, w_{k}\right] .
$$

(ii) See Chapter 15 of [61] for the definition of the characteristic Pontrjagin classes of a $k$-plane bundle $\eta: X \rightarrow B O(k)$

$$
p_{j}(\eta) \in H^{4 j}(X) \quad(j=0,1,2, \ldots)
$$

and the computation

$$
H^{*}(B O(k) ; \mathbb{Q})=\mathbb{Q}\left[p_{1}, p_{2}, \ldots, p_{[k / 2]}\right] .
$$

The $k$-dimensional general linear group has two components

$$
G L(k)=G L^{+}(k) \cup G L^{-}(k)
$$

with

$$
\begin{aligned}
& G L^{+}(k)=\{a \in G L(k) \mid \operatorname{det}(a)>0\}, \\
& G L^{-}(k)=\{a \in G L(k) \mid \operatorname{det}(a)<0\} .
\end{aligned}
$$

The determinant defines a surjective group morphism

$$
\operatorname{det}: G L(k) \rightarrow \mathbb{R} \backslash\{0\} ; a \mapsto \operatorname{det}(a)
$$

and

$$
G L^{+}(k)=\operatorname{ker}\left(\operatorname{sign}(\operatorname{det}): G L(k) \rightarrow \mathbb{Z}_{2}\right) \triangleleft G L(k)
$$

is a normal subgroup of index 2 .

Definition 5.35 (i) An orientation for a $k$-dimensional real vector space $V$ is an equivalence class of (ordered) bases, with two bases equivalent if they differ by an element of $G L^{+}(k)$. There are exactly $\left[G L(k): G L^{+}(k)\right]=2$ orientations for $V$.

(ii) A $k$-plane bundle $\eta: X \rightarrow B O(k)$ is orientable if the transition functions $h: U \cap U^{\prime} \rightarrow G L(k)$ (5.8) are orientation-preserving, that is $h\left(U \cap U^{\prime}\right) \subseteq G L^{+}(k)$.

(iii) A $k$-plane bundle is nonorientable if it is not orientable.

(iv) An orientation for an orientable $k$-plane bundle $\eta: X \rightarrow B O(k)$ is a compatible choice of orientation for each of the $k$-dimensional vector spaces $\eta(x)$ $(x \in X)$. An oriented bundle is an orientable bundle with a choice of orientation.

(v) The $k$-dimensional special orthogonal group

$$
S O(k)=\{a \in O(k) \mid \operatorname{det}(a)=1\}
$$

is the index 2 subgroup of $O(k)$ consisting of the orientation-preserving elements. The inclusion $S O(k) \hookrightarrow G L^{+}(k)$ is a homotopy equivalence, by the GramSchmidt orthonormalisation process. 
The orientability of a vector bundle $\eta: X \rightarrow B O(k)$ is determined by the first Stiefel-Whitney class $w_{1}(\eta) \in H^{1}\left(X ; \mathbb{Z}_{2}\right)$.

Proposition 5.36 (i) A k-plane bundle $\eta: X \rightarrow B O(k)$ is orientable if and only if $w_{1}(\eta)=0 \in H^{1}\left(X ; \mathbb{Z}_{2}\right)$.

(ii) For any $\eta: X \rightarrow B O(k)$ over a connected $C W$ complex $X$ the double cover $\bar{X}$ of $X$ classified by $w_{1}(\eta) \in H^{1}\left(X ; \mathbb{Z}_{2}\right)$ is such that there are exactly two lifts of $\eta$ to an oriented bundle $\bar{\eta}: \bar{X} \rightarrow B S O(k)$. In particular, $B S O(k)$ is the double cover of $B O(k)$ classified by the universal first Stiefel-Whitney class $w_{1} \in$ $H^{1}\left(B O(k) ; \mathbb{Z}_{2}\right)=\mathbb{Z}_{2}$.

(iii) An orientable $k$-plane bundle $\eta: X \rightarrow B O(k)$ over a connected space $X$ has two orientations.

Proof See Bredon [10, VI.17.2].

Example 5.37 Vector bundles over $S^{1}$ are determined by their orientation type, as follows. The first Stiefel-Whitney class defines an isomorphism

$$
w_{1}: \pi_{1}(B O(k)) \rightarrow H^{1}\left(S^{1} ; \mathbb{Z}_{2}\right)=\mathbb{Z}_{2} ; \omega \mapsto w_{1}(\omega)
$$

An orientable $k$-plane bundle $\omega: S^{1} \rightarrow B O(k)$ is isomorphic to the trivial bundle $\epsilon^{k}$. A nonorientable $k$-plane bundle $\omega: S^{1} \rightarrow B O(k)$ is isomorphic to the Whitney sum $\mu \oplus \epsilon^{k-1}$, with $\mu: S^{1} \rightarrow B O(1)=\mathbb{R} \mathbb{P}^{\infty}$ the nonorientable 1-plane bundle with total space the open Möbius band

$$
E(\mu)=\mathbb{R} \times I /\{(x, 0) \sim(-x, 1)\}
$$

The 1-disk bundle $D(\mu)$ is the closed Möbius band :

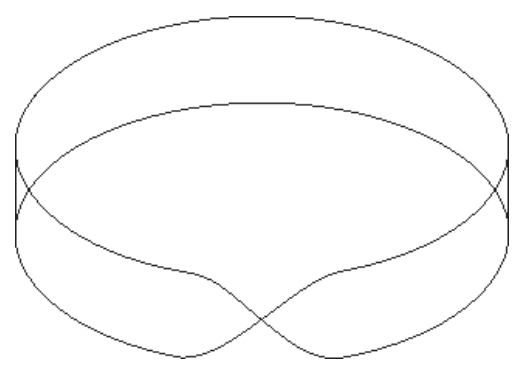

Given a finite-dimensional real vector space $W$ let $\widetilde{W}$ denote $W$ with a choice of orientation. 
Definition 5.38 For any real vector space $V$ let $\widetilde{G}_{k}(V)$ be the Grassmann manifold of $k$-dimensional subspaces $W \subseteq V$ with a choice of orientation $\widetilde{W}$, and let

$$
\widetilde{\gamma}_{k}(V)=\left\{(\widetilde{W}, x) \mid \widetilde{W} \in \widetilde{G}_{k}(V), x \in W\right\}
$$

The projection

$$
\widetilde{\gamma}_{k}(V) \rightarrow \widetilde{G}_{k}(V) ;(\widetilde{W}, x) \mapsto \widetilde{W}
$$

is an oriented $k$-plane bundle. Also, the projection

$$
\widetilde{G}_{k}(V) \rightarrow G_{k}(V) ; \widetilde{W} \mapsto W
$$

is a double covering.

Oriented Bundle Classification Theorem 5.39 The isomorphism classes of oriented $k$-plane bundles over a finite $C W$ complex $X$ are in one-one correspondence with the homotopy classes of maps $X \rightarrow B S O(k)$ to the classifying space

$$
B S O(k)=\widetilde{G}_{k}\left(\mathbb{R}^{\infty}\right) .
$$

The identity map $\widetilde{1}_{k}: B S O(k) \rightarrow B S O(k)$ classifies the universal oriented $k$ plane bundle

$$
\mathbb{R}^{k} \rightarrow \widetilde{\gamma}_{k}\left(\mathbb{R}^{\infty}\right) \rightarrow B S O(k) .
$$

In fact, $B S O(k)=B O(k)^{w_{1}}$ is the orientation double cover of $B O(k)$ classified by the first Stiefel-Whitney class of the universal unoriented $k$-plane bundle $1_{k}$ : $B O(k) \rightarrow B O(k)$

$$
w_{1}\left(1_{k}\right)=1 \in H^{1}\left(B O(k) ; \mathbb{Z}_{2}\right)=\mathbb{Z}_{2} .
$$

Proof As for the unoriented case (5.23)

Example 5.40 The low-dimensional special orthogonal groups are given by

$$
S O(1)=\{1\}, S O(2)=S^{1}, S O(3)=\mathbb{R P}^{3} .
$$

(See 5.13 for $S O(2)$, and Bredon [10,III.10] for $S O(3)$ ).

Example 5.41 The Hopf bundle $\eta: S^{2} \rightarrow B S O(2)$ (5.7) is obtained by the construction of 5.27 with clutching map the diffeomorphism

$$
\omega: S^{1} \rightarrow S O(2) ;(\cos \theta, \sin \theta) \mapsto\left(\begin{array}{cc}
\cos \theta & -\sin \theta \\
\sin \theta & \cos \theta
\end{array}\right),
$$

such that

$$
(D(\eta), S(\eta))=\left(\operatorname{cl} .\left(\mathbb{C} \mathbb{P}^{2} \backslash D^{4}\right), S^{3}\right), T(\eta)=D(\eta) / S(\eta)=\mathbb{C} \mathbb{P}^{2} .
$$


Thom Isomorphism Theorem 5.42 An oriented k-plane bundle $\eta: X \rightarrow$ $B S O(k)$ has a Thom class $U_{\eta} \in \dot{H}^{k}(T(\eta))$ such that for each $x \in X$ the morphism

$$
\left(i_{x}\right)^{*}: \dot{H}^{k}(T(\eta)) \rightarrow \dot{H}^{k}\left(T\left(\left(i_{x}\right)^{*} \eta\right)\right)=\dot{H}^{k}\left(S^{k}\right)=\mathbb{Z}
$$

induced by the inclusion $i_{x}:\{x\} \hookrightarrow X$ sends $U_{\eta}$ to $1 \in \mathbb{Z}$. Cap and cup products define isomorphisms

$$
\begin{aligned}
& U_{\eta} \cap-: \dot{H}_{*}(T(\eta)) \rightarrow H_{*-k}(X), \\
& U_{\eta} \cup-: H^{*}(X) \rightarrow \dot{H}^{*+k}(T(\eta)) .
\end{aligned}
$$

Proof As in the unoriented $\mathbb{Z}_{2}$-coefficient case (5.21), with

$$
\dot{C}(T(\eta))=C(X)_{*-k}
$$

Corollary 5.43 A choice of orientation for a vector bundle $\eta: X \rightarrow B O(k)$ corresponds to a choice of Thom class $U_{\eta} \in \dot{H}^{k}(T(\eta))$, which is unique up to sign on each component.

Definition 5.44 (i) The Euler class $e(\eta) \in H^{n}(N)$ of an oriented $n$-plane bundle $\eta: N \rightarrow B S O(n)$ over a finite $C W$ complex $N$ is characterised by

$$
U_{\eta} \cup U_{\eta}=e(\eta) \cup U_{\eta} \in \dot{H}^{2 n}(T(\eta))
$$

with $U_{\eta} \in \dot{H}^{n}(T(\eta))$ the Thom class, and $-\cup U_{\eta}: H^{n}(N) \cong \dot{H}^{2 n}(T(\eta))$ the Thom isomorphism. The Euler class is such that

$$
e(\eta)=(-1)^{n} e(\eta) \in H^{n}(N)
$$

(ii) The Euler number of an oriented $n$-plane bundle $\eta: N \rightarrow B S O(n)$ over a connected oriented $n$-dimensional manifold $N$ is

$$
\chi(\eta)=e(\eta) \in H^{n}(N)=H_{0}(N)=\mathbb{Z} .
$$

Note that $\chi(\eta)=0$ for odd $n$, on account of $e(\eta)=(-1)^{n} e(\eta)$

Proposition 5.45 The Euler class $e(\eta) \in H^{n}(N)$ of an n-plane bundle $\eta: N \rightarrow$ $B S O(n)$ is the primary obstruction to the existence of a non-zero section of $\eta$.

Proof See Milnor and Stasheff [61] (pp. 98, 130, 147). 
Remark 5.46 See Chapter 15 of Milnor and Stasheff [61] for the computation

$$
H^{*}(B S O(k) ; \mathbb{Q})= \begin{cases}\mathbb{Q}\left[p_{1}, p_{2}, \ldots, p_{j}\right][e] /\left(e^{2}=p_{j}\right) & \text { if } k=2 j \\ \mathbb{Q}\left[p_{1}, p_{2}, \ldots, p_{j}\right] & \text { if } k=2 j+1\end{cases}
$$

with $p_{i} \in H^{4 i}(B S O(k) ; \mathbb{Q})$ the rational Pontrjagin classes, and $e \in H^{2 j}(B S O(2 j) ; \mathbb{Q})$ the universal Euler class.

\subsection{The tangent and normal bundles}

Definition 5.47 (i) The tangent bundle of an $m$-dimensional manifold $M^{m}$ with atlas $\mathscr{U}$ is the $m$-plane bundle $\tau_{M}: M \rightarrow B O(m)$ with total space the open $2 m$-dimensional manifold

$$
E\left(\tau_{M}\right)=\left(\coprod_{(U, \phi) \in \mathscr{U}} U \times \mathbb{R}^{m}\right) / \sim
$$

with $\sim$ the equivalence relation defined by

$$
\left(x \in U, h \in \mathbb{R}^{m}\right) \sim\left(x^{\prime} \in U^{\prime}, h^{\prime} \in \mathbb{R}^{m}\right)
$$

if

$$
x=x^{\prime} \in U \cap U^{\prime} \subseteq M \quad, \quad d\left(\phi^{\prime-1} \phi \mid\right)\left(\phi^{-1}(x)\right)(h)=h^{\prime} \in \mathbb{R}^{m} .
$$

and projection

$$
p: E\left(\tau_{M}\right) \rightarrow M ;(x, h) \mapsto x
$$

The tangent space to $x \in M$ is the $m$-dimensional vector space

$$
\tau_{M}(x)=\left(\coprod_{(U, \phi) \in \mathscr{U}, x \in U}\{x\} \times \mathbb{R}^{m}\right) / \sim
$$

such that

$$
E\left(\tau_{M}\right)=\bigcup_{x \in M} \tau_{M}(x)
$$

(ii) The differential of a differentiable map $f: N^{n} \rightarrow M^{m}$ is the bundle map $d f: \tau_{N} \rightarrow \tau_{M} ;\left(x \in V, h \in \mathbb{R}^{n}\right) \mapsto\left(f(x) \in U, d\left(\phi^{-1}(f \mid) \psi\right)\left(\psi^{-1}(x)\right)(h) \in \mathbb{R}^{m}\right)$.

A differentiable map $f: N^{n} \rightarrow M^{m}$ is given in local coordinates by

$$
f: \mathbb{R}^{n} \rightarrow \mathbb{R}^{m} ; x=\left(x_{1}, x_{2}, \ldots, x_{n}\right) \mapsto\left(f_{1}(x), f_{2}(x), \ldots, f_{m}(x)\right) .
$$

The differential of $f$ is the bundle map

$$
d f: \tau_{N} \rightarrow \tau_{M}
$$


given in local coordinates by the Jacobian matrix

$$
\begin{aligned}
& d f(x)=\left(\frac{\partial f_{i}}{\partial x_{j}}\right): \tau_{N}(x)=\mathbb{R}^{n} \rightarrow \tau_{M}(f(x))=\mathbb{R}^{m} ; \\
& \left(h_{1}, h_{2}, \ldots, h_{n}\right) \mapsto\left(\sum_{j=1}^{n} \frac{\partial f_{1}}{\partial x_{j}} h_{j}, \sum_{j=1}^{n} \frac{\partial f_{2}}{\partial x_{j}} h_{j}, \ldots, \sum_{j=1}^{n} \frac{\partial f_{m}}{\partial x_{j}} h_{j}\right) .
\end{aligned}
$$

Proposition 5.48 (i) A differentiable map of manifolds $f: N^{n} \rightarrow M^{m}$ with $n \leqslant m$ is an immersion if and only if the differential at each $x \in N$ is an injective linear map

$$
d f(x): \tau_{N}(x) \rightarrow \tau_{M}(f(x)),
$$

or equivalently such that the Jacobian $m \times n$ matrix $\left(\frac{\partial f_{i}}{\partial x_{j}}\right)$ at each $x \in N$ has rank $n$.

(ii) The orientation character of $M$ is the first Stiefel-Whitney class of the tangent bundle $\tau_{M}$

$$
w(M)=w_{1}\left(\tau_{M}\right) \in H^{1}\left(M ; \mathbb{Z}_{2}\right) .
$$

An m-dimensional manifold $M$ is orientable (4.1) if and only if the tangent bundle $\tau_{M}: M \rightarrow B O(m)$ is orientable.

An immersion $f: N^{n} \rightarrow M^{m}$ induces injections of tangent spaces

$$
d f(x): \tau_{N}(x) \rightarrow \tau_{M}(f(x)) \quad(x \in N),
$$

which we shall use to identify $\tau_{N}(x)$ with a subspace of $\tau_{M}(f(x))$. Choosing a metric on $M$ there is defined an inner product

$$
\langle,\rangle: \tau_{M}(f(x)) \times \tau_{M}(f(x)) \rightarrow \mathbb{R} ;(v, w) \mapsto\langle v, w\rangle
$$

and the orthogonal complement of $\tau_{N}(x)$

$$
\tau_{N}(x)^{\perp}=\left\{v \in \tau_{M}(f(x)) \mid\left\langle v, \tau_{N}(x)\right\rangle=0\right\}
$$

is a subspace such that

$$
\tau_{N}(x) \oplus \tau_{N}(x)^{\perp}=\tau_{M}(f(x)) .
$$

Definition 5.49 The normal bundle $\nu_{f}: N \rightarrow B O(m-n)$ of an immersion $f: N^{n} \uparrow M^{m}$ is the $(m-n)$-plane bundle over $N$ with

$$
E\left(\nu_{f}\right)=\bigcup_{x \in N} \nu_{f}(x)
$$

the union of the orthogonal complements

$$
\nu_{f}(x)=\tau_{N}(x)^{\perp} \subseteq \tau_{M}(f(x)),
$$

and is such that

$$
\tau_{N} \oplus \nu_{f}=f^{*} \tau_{M}: N \rightarrow B O(m) .
$$


The normal bundle $\nu_{f}: N \rightarrow B O(m-n)$ of an immersion $f: N^{n} \uparrow M^{m}$ of oriented manifolds has a unique orientation such that $\tau_{N} \oplus \nu_{f}=f^{*} \tau_{M}$ as oriented bundles.

Tubular Neighbourhood Theorem 5.50 An embedding $f: N^{n} \hookrightarrow M^{m}$ extends to a codimension 0 embedding $E\left(\nu_{f}\right) \hookrightarrow M^{m}$ of the total space of the normal $(m-n)$-plane bundle $\nu_{f}: N \rightarrow B O(m-n)$. Similarly for an immersion $f: N \leftrightarrow M$, with $E\left(\nu_{f}\right) \leftrightarrow M$.

Proof See Theorem 11.1 of Milnor and Stasheff [61], or Theorem II.11.14 of Bredon [10].

Example 5.51 For any $m \geqslant 1$ the normal 1-plane bundle of the embedding of real projective spaces

$$
\mathbb{R} \mathbb{P}^{m}=G_{1}\left(\mathbb{R}^{m+1}\right) \hookrightarrow \mathbb{R} \mathbb{P}^{m+1}=G_{1}\left(\mathbb{R}^{m+2}\right)
$$

is classified by the inclusion

$$
\nu_{\mathbb{R}^{P} \hookrightarrow} \hookrightarrow \mathbb{R}^{m+1}: \quad: \mathbb{R} \mathbb{P}^{m}=G_{1}\left(\mathbb{R}^{m+1}\right) \rightarrow \mathbb{R P}^{\infty}=G_{1}\left(\mathbb{R}^{\infty}\right)=B O(1) .
$$

In particular, for $m=1$ the normal bundle of the embedding $\mathbb{R P}^{1}=S^{1} \hookrightarrow \mathbb{R P}^{2}$ is the non-trivial 1-plane bundle

$$
\mu=\nu_{S^{1} \hookrightarrow \mathbb{R}^{2}}: S^{1} \rightarrow B O(1)
$$

with $D^{1}$-bundle $D(\mu)$ the Möbius band and $S^{0}$-bundle

$$
S(\mu)=S^{1} \rightarrow S^{1} ; z \mapsto z^{2}
$$

the non-trivial double cover of $S^{1}$.

Proposition 5.52 (i) If $f: N^{n} \rightarrow M^{m}, g: M^{m} \rightarrow L^{\ell}$ are immersions with normal bundles $\nu_{f}: N \rightarrow B O(m-n), \nu_{g}: M \rightarrow B O(\ell-m)$ then the composite immersion $g f: N^{n} \rightarrow L^{\ell}$ has normal bundle

$$
\nu_{g f}=\nu_{f} \oplus f^{*} \nu_{g}: N \rightarrow B O(\ell-n) .
$$

(ii) The Umkehr map of an embedding $f: N^{n} \hookrightarrow M^{m}$ of an oriented $n$ dimensional manifold $N$ as submanifold of an oriented $m$-dimensional $M$ is given by

$$
f^{!}: H_{*}(M) \rightarrow H_{*}(M, M \backslash f(N)) \cong \dot{H}_{*}\left(T\left(\nu_{f}\right)\right) \cong H_{*-m+n}(N)
$$

The evaluation of the Thom class

$$
U_{\nu_{f}} \in \dot{H}^{m-n}\left(T\left(\nu_{f}\right)\right) \cong H^{m-n}(M, M \backslash f(N))
$$


on the homology class $f_{*}[N] \in H_{n}(M)$ is

$$
\left\langle U_{\nu_{f}}, f_{*}[N]\right\rangle=1 \in \mathbb{Z}
$$

(iii) The fundamental class $[M] \in H_{m}(M)$ of an oriented m-dimensional manifold $M$ is related to the Thom class of $\tau_{M}$

$$
U_{\tau_{M}} \in \dot{H}^{m}\left(T\left(\tau_{M}\right)\right) \cong H^{m}(M \times M, M \times M \backslash \Delta(M))
$$

by

$$
\left\langle U_{\tau_{M}}, \Delta_{*}[M]\right\rangle=1 \in \mathbb{Z},
$$

with $\Delta_{*}[M] \in H_{m}(M \times M, M \times M \backslash \Delta(M))$. The Euler number of $\tau_{M}$ is just the Euler characteristic of $M$

$$
\chi\left(\tau_{M}\right)=\chi(M) \in \mathbb{Z} .
$$

Proof (i) + (ii) By construction.

(iii) Use the identification of the tangent bundle of $M$ with the normal bundle of the diagonal embedding

$$
\Delta: M \hookrightarrow M \times M ; x \mapsto(x, x)
$$

that is

$$
\tau_{M}=\nu_{\Delta}: M \rightarrow B O(m)
$$

(Milnor and Stasheff $[61,11.5])$.

Example 5.53 The Euler number of $\tau_{S^{n}}: S^{n} \rightarrow B S O(n)$ is

$$
\chi\left(\tau_{S^{n}}\right)=\chi\left(S^{n}\right)=1+(-1)^{n} \in \mathbb{Z} .
$$

Definition 5.54 The $n$-dimensional regular homotopy group $I_{n}(M)$ of an $m$-dimensional manifold $M$ is the $\mathbb{Z}\left[\pi_{1}(M)\right]$-module of regular homotopy classes of immersions $f: S^{n} \uparrow M^{m}$ with a choice of lift $\widetilde{f}: S^{n} \uparrow \widetilde{M}$ to the universal cover $\widetilde{M}$ of $M$, with addition by connected sum.

Proposition 5.55 (i) The isomorphism class $\nu_{f} \in[N, B O(m-n)]$ of the normal bundle of an immersion $f: N^{n} \rightarrow M^{m}$ is a regular homotopy invariant.

(ii) The normal bundle defines a $\mathbb{Z}\left[\pi_{1}(M)\right]$-module morphism

$$
\nu: I_{n}(M) \rightarrow \pi_{n}(B O(m-n)) ;\left(f: S^{n} \leftrightarrow M\right) \mapsto \nu_{f}
$$

with the trivial $\mathbb{Z}\left[\pi_{1}(M)\right]$-action on $\pi_{n}(B O(m-n))$. 
Proof (i) The track of a regular homotopy $\left\{f_{t}: N \leftrightarrow M \mid t \in I\right\}$ of immersions $f_{0}, f_{1}: N^{n} \leftrightarrow M^{m}$ is a level-preserving immersion

$$
f: N \times I \leftrightarrow M \times I ;(x, t) \mapsto\left(f_{t}(x), t\right) .
$$

The normal bundle of $f$ defines a homotopy of classifying maps

$$
\nu_{f}: \nu_{f_{0}} \simeq \nu_{f_{1}}: N \rightarrow B O(m-n)
$$

corresponding to a bundle isomorphism $\nu_{f_{0}} \cong \nu_{f_{1}}$.

(ii) The normal bundle of an immersion $f: S^{n} \uparrow M^{m}$ is an $(m-n)$-plane bundle $\nu_{f}: S^{n} \rightarrow B O(m-n)$, such that

$$
E\left(\nu_{f}\right)=D^{n} \times \mathbb{R}^{m-n} \cup_{(x, y) \sim\left(x, c_{f}(x)(y)\right)} D^{n} \times \mathbb{R}^{m-n} \quad\left(x \in S^{n-1}, y \in \mathbb{R}^{m-n}\right)
$$

for a clutching map $c_{f}: S^{n-1} \rightarrow O(m-n)$. The isomorphism class of $\nu_{f}$ is the homotopy class of $c_{f}$

$$
\nu_{f}=c_{f} \in \pi_{n}(B O(m-n))=\pi_{n-1}(O(m-n)) .
$$

The connected sum of immersions $f, g: S^{n} \uparrow M^{m}$ is an immersion

$$
f \# g: S^{n} \# S^{n}=S^{n} \rightarrow M^{m}
$$

with clutching map

$$
c_{f \# g}=c_{f} c_{g}: S^{n-1} \rightarrow O(m-n) ; x \mapsto c_{f}(x) c_{g}(x)
$$

so that

$$
\nu_{f \# g}=\nu_{f}+\nu_{g} \in \pi_{n}(B O(m-n))
$$

Definition 5.56 A framing of an immersion $g: N^{n} \rightarrow M^{m}$ is a framing of the normal bundle $\nu_{g}: N \rightarrow B O(m-n)$

$$
b: \nu_{g} \cong \epsilon^{m-n} .
$$

Example 5.57 For any $n<m$ the embedding

$$
S^{n} \times D^{m-n} \hookrightarrow S^{m}=\partial\left(D^{n+1} \times D^{m-n}\right)=S^{n} \times D^{m-n} \cup D^{n+1} \times S^{m-n-1}
$$

gives a standard framed embedding

$$
\left(g: S^{n} \hookrightarrow S^{m}, b: \nu_{g} \cong \epsilon^{m-n}\right) .
$$

The framing can be altered by any element $\omega \in \pi_{n}(O(m-n))$, using the automorphism $\omega: \epsilon^{m-n} \cong \epsilon^{m-n}$ of the trivial $(m-n)$-plane bundle over $S^{n}$ it classifies. 
Proposition 5.58 The framings $b$ (if any) of an embedding $g: N^{n} \hookrightarrow M^{m}$ are in one-one correspondence with extensions of $g$ to an embedding $\bar{g}: N \times D^{m-n} \hookrightarrow$ $M^{m}$. Similarly for an immersion $g: N \uparrow M$, with extensions $\bar{g}: N \times D^{m-n} \uparrow \rightarrow$ $M$.

Proof By the Tubular Neighbourhood Theorem (5.50).

Proposition 5.59 For any $m$-dimensional manifold $M^{m}$ and $k>m$ there exists an embedding $M \hookrightarrow S^{m+k}$, and for $k>m+1$ any two embeddings $M \hookrightarrow S^{m+k}$ are isotopic.

Proof This is a special case of the Whitney Embedding Theorem $(1.7,7.2)$ applied to a constant map $\{*\}: M \rightarrow S^{m+k}$, noting that $M$ is an $m$-dimensional $C W$ complex $(2.23)$ and $\left[M, S^{m+k}\right]=0$ for any $k \geqslant 1$ by 3.27 .

Definition 5.60 (i) A normal bundle of an $m$-dimensional manifold is the normal bundle (5.49)

$$
\nu_{M}=\nu_{g}: M \rightarrow B O(k)
$$

of any embedding $g: M \hookrightarrow S^{m+k}(k \geqslant 1)$, such that

$$
\tau_{M} \oplus \nu_{M}=\epsilon^{m+k}: M \rightarrow B O(m+k)
$$

(ii) The stable normal bundle of an $m$-dimensional manifold $M$ is the stable bundle

$$
\nu_{M}: M \rightarrow B O
$$

represented by the normal $k$-plane bundle $\nu: M \rightarrow B O(k)$ of any embedding $M \hookrightarrow S^{m+k}$ ( $k$ large), such that

$$
\tau_{M} \oplus \nu_{M}=\epsilon^{\infty}: M \rightarrow B O
$$

More precisely, any embedding $g: M^{m} \hookrightarrow S^{m+k}$ with $k \geqslant 1$ is null-homotopic (by 3.27) and the pullback $g^{*} \tau_{S^{m+k}}: M \rightarrow B O(m+k)$ is trivial, allowing an identification of the tangent space $\tau_{M}(x)$ at each $x \in M$ with an $m$-dimensional subspace of

$$
\tau_{S^{m+k}}(g(x))=g^{*} \tau_{S^{m+k}}(x)=\mathbb{R}^{m+k} .
$$

The map

$$
\tau_{M}: M \rightarrow G_{m}\left(\mathbb{R}^{m+k}\right) ; x \mapsto \tau_{M}(x)
$$

represents the classifying map

$$
\tau_{M}: M \rightarrow \underset{k}{\lim } G_{m}\left(\mathbb{R}^{m+k}\right)=B O(m) .
$$


The orthogonal complements with respect to the standard inner product on $\mathbb{R}^{m+k}$ define a map

$$
\nu_{M}: M \rightarrow G_{k}\left(\mathbb{R}^{m+k}\right) ; x \mapsto \nu_{M}(x)=\tau_{M}(x)^{\perp}
$$

representing the classifying map

$$
\nu_{M}: M \rightarrow \varliminf_{m} G_{k}\left(\mathbb{R}^{m+k}\right)=B O(k),
$$

such that

$$
\tau_{M} \oplus \nu_{M}=\epsilon^{m+k}: M \rightarrow B O(m+k) .
$$

Example 5.61 The normal bundle of $S^{m}$ is trivial

$$
\nu_{S^{m}}=\epsilon^{k}: S^{m} \rightarrow B O(k)
$$

since already the standard embedding $S^{m} \hookrightarrow S^{m+1}$ has trivial normal bundle

$$
\nu_{S^{m} \hookrightarrow S^{m+1}}=\epsilon: S^{m} \rightarrow B O(1) .
$$

The stable normal bundle is also trivial

$$
\nu_{S^{m}}=\epsilon^{\infty}: S^{m} \rightarrow B O .
$$

Proposition 5.62 The normal bundle $\nu_{g}: S^{n} \rightarrow B O(m-n)$ of an immersion $g: S^{n} \rightarrow M^{m}$ is such that:

$$
\begin{aligned}
& \nu_{g} \oplus \tau_{S^{n}}=g^{*} \tau_{M} \in \pi_{n}(B O(m)), \\
& \nu_{g} \oplus \epsilon^{n+1}=g^{*}\left(\tau_{M} \oplus \epsilon\right) \in \pi_{n}(B O(m+1)), \\
& \nu_{g}=-g^{*}\left(\nu_{M}\right) \in \pi_{n}(B O) .
\end{aligned}
$$

Proof Stabilise the identity given by 5.49

$$
\nu_{g} \oplus \tau_{S^{n}}=g^{*} \tau_{M}: S^{n} \rightarrow B O(m)
$$

by adding $\epsilon$ and using the isomorphism $\tau_{S^{n}} \oplus \epsilon \cong \epsilon^{n+1}$ given by 5.61 to obtain the isomorphism

$$
\nu_{g} \oplus \epsilon^{n+1} \cong g^{*}\left(\tau_{M} \oplus \epsilon\right) .
$$

Stabilise further by adding $g^{*} \nu_{M}$ to obtain a stable isomorphism

$$
\nu_{g} \oplus g^{*} \nu_{M} \cong \epsilon^{\infty} .
$$




\subsection{Surgery and bundles}

Recall from 2.5 that an $n$-surgery on an $m$-dimensional manifold $M^{m}$ removes a framed $n$-embedding $\bar{g}: S^{n} \times D^{m-n} \hookrightarrow M$ to obtain the $m$-dimensional manifold

$$
M^{\prime}=\operatorname{cl} .\left(M^{m} \backslash \bar{g}\left(S^{n} \times D^{m-n}\right)\right) \cup_{S^{n} \times S^{m-n-1}} D^{n+1} \times S^{m-n-1} .
$$

The $n$-surgery kills the homotopy class $[g] \in \pi_{n}(M)$ of the core $n$-embedding $g=\bar{g} \mid: S^{n} \times\{0\} \hookrightarrow M$.

Theorem 5.63 Let $M^{m}$ be an m-dimensional manifold. The following conditions on an element $x \in \pi_{n}(M)$ are equivalent:

(i) $x$ can be killed by an $n$-surgery on $M$,

(ii) $x$ can be represented by a framed n-embedding $\bar{g}: S^{n} \times D^{m-n} \hookrightarrow M$,

(iii) $x$ can be represented by an n-embedding $g: S^{n} \hookrightarrow M^{m}$ with trivial normal bundle $\nu_{g}: S^{n} \rightarrow B O(m-n)$.

Proof (i) $\Longleftrightarrow$ (ii) By definition.

(ii) $\Longleftrightarrow$ (iii) By 5.58 the framings $b$ (if any) of an embedding $g: S^{n} \hookrightarrow M^{m}$ are in one-one correspondence with extensions to an embedding $\bar{g}: S^{n} \times D^{m-n} \hookrightarrow M^{m}$.

It follows that below the middle dimension the possibility of killing an element of $\pi_{n}\left(M^{m}\right)$ is entirely determined by the stable normal bundle $\nu_{M}: M \rightarrow B O$.

Corollary 5.64 If $2 n<m$ an element $x \in \pi_{n}(M)$ can be killed by surgery if and only if $\left(\nu_{M}\right)_{*}(x)=0 \in \pi_{n}(B O)$.

Proof The Whitney Embedding Theorem $(1.7,7.2)$ shows that $x$ can be represented by an $n$-embedding $g: S^{n} \hookrightarrow M$. The result now follows from the identity $\nu_{g}=-g^{*}\left(\nu_{M}\right) \in \pi_{n}(B O)$ given by 5.62 and the stability result $\pi_{n}(B O(m-n))=$ $\pi_{n}(B O)$ of 5.33 .

Here are some examples of homotopy classes which cannot be killed by surgery :

Example 5.65 For $m \geqslant 2$ the generator $1 \in \pi_{1}\left(\mathbb{R} \mathbb{P}^{m}\right)=\mathbb{Z}_{2}$ is represented by the standard 1-embedding

$$
g: S^{1}=\mathbb{R} \mathbb{P}^{1} \hookrightarrow \mathbb{R P}^{m}
$$

with normal bundle 


$$
\nu_{g}=(m-1) \mu=\left\{\begin{array} { l } 
{ \mu \oplus \epsilon ^ { m - 2 } } \\
{ \epsilon ^ { m - 1 } }
\end{array} \text { if } m \text { is } \left\{\begin{array}{l}
\text { even } \\
\text { odd },
\end{array}\right.\right.
$$

with $\mu: S^{1} \rightarrow B O(1)$ the nontrivial 1-plane bundle. The first Stiefel-Whitney class is

$$
w_{1}\left(\nu_{g}\right)=(-1)^{m-1} \in H^{1}\left(S^{1} ; \mathbb{Z}_{2}\right)=\mathbb{Z}_{2}=\{ \pm 1\} .
$$

Thus if $m$ is even the generator $1 \in \pi_{1}\left(\mathbb{R P}^{m}\right)$ cannot be represented by a framed 1 -embedding, and so cannot be killed by a 1 -surgery on $\mathbb{R P}^{m}$.

The 1-embedding $S^{1} \hookrightarrow \mathbb{R}^{m}$ in 5.65 cannot be framed since it is not even orientable. There exist $n$-embeddings $S^{n} \hookrightarrow M^{m}$ with orientable non-trivial normal bundles, as in:

Example 5.66 For $m \geqslant 2$ the generator $1 \in \pi_{2}\left(\mathbb{C} \mathbb{P}^{m}\right)=\mathbb{Z}$ is represented by the standard 1-embedding

$$
g: S^{2}=\mathbb{C} \mathbb{P}^{1} \hookrightarrow \mathbb{C} \mathbb{P}^{m}
$$

with oriented normal bundle

$$
\nu_{g}=(m-1) \eta=\left\{\begin{array} { l } 
{ \eta \oplus \epsilon ^ { 2 m - 4 } } \\
{ \epsilon ^ { 2 m - 2 } }
\end{array} : S ^ { 2 } \rightarrow B S O ( 2 m - 2 ) \text { if } m \text { is } \left\{\begin{array}{l}
\text { even } \\
\text { odd }
\end{array}\right.\right.
$$

with $\eta: S^{2} \rightarrow B S O(2)$ the Hopf bundle (5.41). The second Stiefel-Whitney class is

$$
w_{2}\left(\nu_{g}\right)=(-1)^{m-1} \in H^{2}\left(S^{2} ; \mathbb{Z}_{2}\right)=\mathbb{Z}_{2}=\{ \pm 1\} .
$$

If $m$ is even $1 \in \pi_{2}\left(\mathbb{C} \mathbb{P}^{m}\right)$ cannot be represented by a framed 2-embedding, and so cannot be killed by a 2 -surgery on $\mathbb{C} \mathbb{P}^{m}$. For odd $m=2 n+1$ it is possible to kill the generator $1 \in \pi_{2}\left(\mathbb{C} \mathbb{P}^{2 n+1}\right)=\mathbb{Z}$ by surgery; the map

$$
\mathbb{C} \mathbb{P}^{2 n+1} \rightarrow \mathbb{H} \mathbb{P}^{n} ;\left[z_{1}, z_{2}, \ldots, z_{2 n+2}\right] \mapsto\left(z_{1}+z_{2} j, z_{3}+z_{4} j, \ldots, z_{2 n+1}+z_{2 n+2} j\right)
$$

is the projection of a fibre bundle with fibre $S^{2}$, and the total pair of the corresponding fibre bundle

$$
\left(D^{3}, S^{2}\right) \rightarrow\left(E, \mathbb{C} \mathbb{P}^{2 n+1}\right) \rightarrow \mathbb{H} \mathbb{P}^{n}
$$

defines a null-cobordism of $\mathbb{C} \mathbb{P}^{2 n+1}$.

The following result describes the number of ways of killing an element $x \in$ $\pi_{n}(M)$ by surgery on $M$, and also describes the behaviour of $\tau_{M}$ and $\nu_{M}$ under surgery.

Theorem 5.67 Let $g: S^{n} \hookrightarrow M^{m}$ be an n-embedding in an m-dimensional manifold. 
(i) The extensions of $g$ to a framed $n$-embedding $\bar{g}: S^{n} \times D^{m-n} \hookrightarrow M$ are in oneone correspondence with the framings $\bar{g}: \nu_{g} \cong \epsilon^{m-n}$ of $\nu_{g}: S^{n} \rightarrow B O(m-n)$.

(ii) An extension of $g$ to a framed $n$-embedding $\bar{g}$ determines a stable framing $a_{\bar{g}}: g^{*} \tau_{M} \oplus \epsilon \cong \epsilon^{m+1}$ of $g^{*} \tau_{M}: S^{n} \rightarrow B O(m)$.

(iii) Let $\left(W ; M, M^{\prime}\right)$ be the trace of the $n$-surgery on $M$ determined by an extension of $g$ to $\bar{g}$. The tangent bundle of $W$ is classified by

$$
\tau_{W} \simeq \tau_{M} \oplus \epsilon \cup a_{\bar{g}}: W \simeq M \cup_{g} D^{n+1} \rightarrow B O(m+1) .
$$

(iv) Let $\nu_{M}: M \rightarrow B O(k)$ be the normal bundle of an embedding $M^{m} \hookrightarrow S^{m+k}$ ( $k$ large). An extension of $g$ to $\bar{g}$ determines a framing $c_{\bar{g}}: g^{*} \nu_{M} \cong \epsilon^{k}$ of $g^{*} \nu_{M}$ : $S^{n} \rightarrow B O(k)$. The embedding $M \hookrightarrow S^{m+k}$ extends to an embedding

$$
\left(W^{m+1}, M^{m}\right) \hookrightarrow\left(D^{m+k+1}, S^{m+k}\right)
$$

with normal bundle

$$
\nu_{W} \simeq \nu_{M} \cup c_{\bar{g}}: W \simeq M \cup_{g} D^{n+1} \rightarrow B O(k)
$$

Proof (i) By the Tubular Neighbourhood Theorem (5.50).

(ii) Stabilise the identity of $m$-plane bundles over $S^{n}$

$$
\tau_{S^{n}} \oplus \nu_{g}=g^{*} \tau_{M}: S^{n} \rightarrow B O(m)
$$

by adding $\epsilon$

$$
\left(\tau_{S^{n}} \oplus \epsilon\right) \oplus \nu_{g}=g^{*}\left(\tau_{M} \oplus \epsilon\right): S^{n} \rightarrow B O(m+1)
$$

and combine the framing $\bar{g}: \nu_{g} \cong \epsilon^{m-n}$ with the framing given by $5.57,5.61$

$$
\tau_{S^{n}} \oplus \epsilon=\left.\tau_{S^{n+1}}\right|_{S^{n}} \cong \epsilon^{n+1}
$$

to obtain the stable framing $a_{\bar{g}}: g^{*}\left(\tau_{M} \oplus \epsilon\right) \cong \epsilon^{m+1}$.

(iii) The tangent bundle of

$$
W=M \times I \cup_{\bar{g}} D^{n+1} \times D^{m-n}
$$

is obtained by glueing together

$$
\tau_{M \times I}=\left(\tau_{M} \oplus \epsilon\right) \times I: M \times I \rightarrow B O(m+1)
$$

and

$$
\tau_{D^{n+1} \times D^{m-n}}=\epsilon^{m+1}: D^{n+1} \times D^{m-n} \rightarrow B O(m+1)
$$

using the framing

$$
a_{\bar{g}}:\left.\tau_{M \times I}\right|_{\bar{g}\left(S^{n} \times D^{m-n}\right)} \cong \epsilon^{m+1} .
$$

(iv) The normal bundle of $W \hookrightarrow D^{m+k+1}$ is obtained by glueing together 


$$
\nu_{M \times I \hookrightarrow D^{m+k+1}}=\left(\nu_{M} \oplus \epsilon\right) \times I: M \times I \rightarrow B O(k)
$$

and

$$
\nu_{D^{n+1} \times D^{m-n} \hookrightarrow D^{m+k+1}}=\epsilon^{k}: D^{n+1} \times D^{m-n} \rightarrow B O(k)
$$

using the framing

$$
c_{\bar{g}}:\left.\nu_{M \times I \hookrightarrow D^{m+k+1}}\right|_{\bar{g}\left(S^{n} \times D^{m-n}\right)} \cong \epsilon^{k} .
$$

Here are the effects of the $n$-surgeries on $S^{m}$ removing all the extensions of the standard $n$-embedding $S^{n} \hookrightarrow S^{m}(5.57)$ to a framed $n$-embedding $S^{n} \times D^{m-n} \hookrightarrow$ $S^{m}$.

Proposition 5.68 An element

$$
\omega \in \pi_{n}(O(m-n))=\pi_{n+1}(B O(m-n))
$$

classifies an $(m-n)$-plane bundle over $S^{n+1}$, with an associated $\left(D^{m-n}, S^{m-n-1}\right)$ bundle

$$
\left(D^{m-n}, S^{m-n-1}\right) \rightarrow(D(\omega), S(\omega)) \rightarrow S^{n+1} .
$$

The effect of the n-surgery on $S^{m}$ removing the framed $n$-embedding

$g_{\omega}: S^{n} \times D^{m-n} \hookrightarrow S^{m}=S^{n} \times D^{m-n} \cup D^{n+1} \times S^{m-n-1} ;(x, y) \mapsto(x, \omega(x)(y))$

is the $(m-n-1)$-sphere bundle over $S^{n+1}$

$$
S(\omega)=D^{n+1} \times S^{m-n-1} \cup_{\omega} D^{n+1} \times S^{m-n-1} .
$$

The trace $\left(W(\omega)^{m+1} ; S^{m}, S(\omega)\right)$ is given by

$$
W(\omega)^{m+1}=\operatorname{cl} .\left(D(\omega) \backslash D^{m+1}\right),
$$

with tangent and stable normal bundle

$$
\begin{aligned}
& \tau_{W(\omega)} \simeq 0 \vee\left(\tau_{S^{n+1}} \oplus \omega\right): W(\omega) \simeq S^{m} \vee S^{n+1} \rightarrow B O(m+1), \\
& \nu_{W(\omega)} \simeq 0 \vee-\omega: W(\omega) \simeq S^{m} \vee S^{n+1} \rightarrow B O
\end{aligned}
$$

for any stable inverse $-\omega: S^{n+1} \rightarrow B O$ of $\omega: S^{n+1} \rightarrow B O(m-n)$.

Proof The zero section is an embedding $z: S^{n+1} \hookrightarrow D(\omega)$ with normal bundle $\omega: S^{n+1} \rightarrow B O(m-n)$, such that

$$
\tau_{S^{n+1}} \oplus \omega=z^{*} \tau_{D(\omega)}: S^{n+1} \rightarrow B O(m+1) .
$$


The sphere bundle $S(\omega)$ of $\omega: S^{n+1} \rightarrow B O(m-n)$ is an $m$-dimensional manifold which is orientable if and only if $\omega$ is orientable; this is the case if $n \neq 0$, or if $n=0$ and $w_{1}(\omega)=0 \in H^{1}\left(S^{1} ; \mathbb{Z}_{2}\right)=\mathbb{Z}_{2}$.

Example 5.69 Let $m=1, n=0$ in 5.68. The two elements in $\pi_{1}(B O(1))=\mathbb{Z}_{2}$ correspond to the two 1-plane bundles over $S^{1}(5.37)$

$$
\omega_{0}, \omega_{1}: S^{1} \rightarrow B O(1)
$$

with $\omega_{0}$ trivial and $\omega_{1}$ non-trivial. The corresponding 0-embeddings

$$
g_{\omega_{0}}, g_{\omega_{1}}: S^{0} \times D^{1} \hookrightarrow S^{1}
$$

have the same core embeddings

$$
g_{\omega_{0}}\left|=g_{\omega_{1}}\right|: S^{0} \hookrightarrow S^{1}
$$

but different framings, exactly as in 2.8 (ii). The effects of the 0-surgeries are

$$
\begin{aligned}
& S\left(\omega_{0}\right)=\text { the trivial double cover of } S^{1}=S^{1} \times S^{0}=S^{1} \cup S^{1} \\
& S\left(\omega_{1}\right)=\text { the non-trivial double cover of } S^{1}=S^{1} .
\end{aligned}
$$

Example 5.70 Let $m=2, n=0$ in 5.68. The two elements in $\pi_{1}(B O(2))=\mathbb{Z}_{2}$ correspond to the two 2-plane bundles over $S^{1}$ (5.37), say

$$
\omega_{0}, \omega_{1}: S^{1} \rightarrow B O(2)
$$

with $\omega_{0}=\epsilon^{2}$ the trivial bundle and $\omega_{1}=\mu \oplus \epsilon$ the nonorientable 2-plane bundle, with $\mu: S^{1} \rightarrow B O(1)$ the non-trivial 1-plane bundle (as in 5.51). The $\left(D^{1}, S^{0}\right)$ bundle

$$
\left(D^{1}, S^{0}\right) \rightarrow(D(\mu), S(\mu))=\left(M^{2}, S^{1}\right) \rightarrow S^{1}
$$

has $M^{2}$ the Möbius band. The total spaces of the $S^{1}$-bundles over $S^{1}$ associated to $\omega_{0}, \omega_{1}$

$$
S^{1} \rightarrow S\left(\omega_{i}\right) \rightarrow S^{1}(i=0,1)
$$

are given by

$$
\begin{aligned}
S\left(\omega_{0}\right) & =S^{1} \times S^{1}=\text { the torus } T^{2} \\
S\left(\omega_{1}\right) & =\left\{(x, y) \in D(\mu) \times S(\epsilon) \cup S(\mu) \times D(\epsilon) \mid[x]=[y] \in S^{1}\right\} \\
& =M^{2} \times S^{0} \cup S^{1} \times D^{1}=\text { the Klein bottle } K^{2}
\end{aligned}
$$

(cf. Example 4.22). The torus $T^{2}$ is obtained from $S^{2}$ by the 0 -surgery removing the 0 -embedding

$$
g_{\omega_{0}}: S^{0} \times D^{2} \hookrightarrow S^{0} \times D^{2} \cup D^{1} \times S^{1}=\partial\left(D^{1} \times D^{2}\right)=S^{2},
$$


and the Klein bottle $K^{2}$ is obtained from $S^{2}$ by the 0 -surgery removing the 0-embedding

$$
g_{\omega_{1}}: S^{0} \times D^{2} \hookrightarrow S^{0} \times D^{2} \cup D^{1} \times S^{1}=\partial\left(D^{1} \times D^{2}\right)=S^{2} .
$$

Example 5.71 Let $m=2 n+1$ in 5.68 , and let

$$
\omega=\tau_{S^{n+1}} \in \pi_{n}(S O(n+1))=\pi_{n+1}(B S O(n+1)) .
$$

The total space of the tangent $S^{n}$-bundle over $S^{n+1}$

$$
S O(n+1) / S O(n)=S^{n} \rightarrow V_{n+2,2} \rightarrow S O(n+2) / S O(n+1)=S^{n+1}
$$

is the Stiefel manifold of orthonormal 2-frames in $\mathbb{R}^{n+2}(5.24)$

$$
S(\omega)^{2 n+1}=V_{n+2,2}=S O(n+2) / S O(n) .
$$

Proposition 5.72 An element

$$
(\delta \omega, \omega) \in \pi_{n+1}(O, O(m-n))=\pi_{n+2}(B O, B O(m-n))
$$

classifies an $(m-n)$-plane bundle $\omega: S^{n+1} \rightarrow B O(m-n)$ together with a stable framing $\delta \omega: \omega \simeq\{*\}: S^{n+1} \rightarrow B O$. As in 5.68 the effect of the $n$-surgery on $S^{m}$ removing the framed $n$-embedding

$g_{\omega}: S^{n} \times D^{m-n} \hookrightarrow S^{m}=S^{n} \times D^{m-n} \cup D^{n+1} \times S^{m-n-1} ;(x, y) \mapsto(x, \omega(x)(y))$

is the $(m-n-1)$-sphere bundle over $S^{n+1}$

$$
S(\omega)=D^{n+1} \times S^{m-n-1} \cup_{\omega} D^{n+1} \times S^{m-n-1}
$$

with $(m-n)$-disk bundle

$$
D(\omega)=D^{n+1} \times D^{m-n} \cup_{\omega} D^{n+1} \times D^{m-n} .
$$

The trace $\left(W(\omega)^{m+1} ; S^{m}, S(\omega)\right)$ is given by

$$
W(\omega)^{m+1}=\operatorname{cl} .\left(D(\omega) \backslash D^{m+1}\right),
$$

with stable normal bundle

$$
\nu_{W(\omega)} \simeq 0 \vee \omega: W(\omega) \simeq S^{m} \vee S^{n+1} \rightarrow B O .
$$

The stable framing $\delta \omega$ of $\omega$ determines an extension of the standard framing of $\tau_{S^{m}}$ to a framing $b_{\delta \omega}$ of $\tau_{W(\omega)}$.

Proof By construction. 
Definition 5.73 An $m$-dimensional manifold $M$ is parallelisable if the tangent bundle $\tau_{M}: M \rightarrow B O(m)$ is trivial.

Bott-Milnor Sphere Parallelisability Theorem 5.74 ([9], [51])

The sphere $S^{n}$ is parallelisable if and only if $n=1,3,7$.

Example 5.75 The element $(1,1) \in H_{n}\left(S^{n} \times S^{n}\right)=\mathbb{Z} \oplus \mathbb{Z}$ is represented by the diagonal embedding $\Delta: S^{n} \hookrightarrow S^{n} \times S^{n}$ with $\nu_{\Delta}=\tau_{S^{n}}: S^{n} \rightarrow B O(n)$. For $n \neq 1,3,7$ it is not possible to kill $(1,1)$ by surgery.

\subsection{The Hopf invariant and the $J$-homomorphism}

Definition 5.76 (i) The Hopf invariant $H(f) \in \mathbb{Z}$ of a map $f: S^{2 n+1} \rightarrow S^{n+1}$ with $n \geqslant 1$ is determined by the cup product structure of the mapping cone

$$
X=S^{n+1} \cup_{f} D^{2 n+2}
$$

with

$$
a \cup a=H(f) b \in H^{2 n+2}(X),
$$

where $a=1 \in H^{n+1}(X)=\mathbb{Z}, b=1 \in H^{2 n+2}(X)=\mathbb{Z}$.

(ii) The mod2 Hopf invariant $H_{2}(g) \in \mathbb{Z}_{2}$ of a map $g: S^{\ell} \rightarrow S^{m}$ with $\ell>m \geqslant 1$ is determined by the Steenrod squares on the mod2 cohomology of the mapping cone

$$
Y=S^{m} \cup_{g} D^{\ell+1}
$$

with

$$
S q^{\ell-m+1}=H_{2}(g): H^{m}\left(Y ; \mathbb{Z}_{2}\right)=\mathbb{Z}_{2} \rightarrow H^{\ell+1}\left(Y ; \mathbb{Z}_{2}\right)=\mathbb{Z}_{2}
$$

If $\ell=2 n+1, m=n+1$ then $H_{2}(g) \in \mathbb{Z}_{2}$ is the $\bmod 2$ reduction of the Hopf invariant $H(g) \in \mathbb{Z}$ in (i).

Example 5.77 The Hopf maps $\eta: S^{3} \rightarrow S^{2}, \eta: S^{7} \rightarrow S^{4}, \eta: S^{15} \rightarrow S^{8}(5.7)$ each have Hopf invariant $H(\eta)=1$.

Proposition 5.78 (i) The Hopf invariant defines a morphism of groups

$$
H: \pi_{2 n+1}\left(S^{n+1}\right) \rightarrow \mathbb{Z} ; f \mapsto H(f)
$$

which is an isomorphism for $n=1$.

(ii) The mod2 Hopf invariant defines an isomorphism

$$
H_{2}: \pi_{m+1}\left(S^{m}\right) \rightarrow \mathbb{Z}_{2} ; g \mapsto H_{2}(g) \quad(m \geqslant 3) .
$$

(iii) The Hopf invariant is such that $H(f)=(-1)^{n+1} H(f)$, so that 


$$
H=0: \pi_{2 n+1}\left(S^{n+1}\right) \rightarrow \mathbb{Z} \text { for even } n
$$

(iv) The suspension map $E$ and the Hopf invariant map $H$ fit into an exact sequence

$$
\pi_{2 n}\left(S^{n}\right) \stackrel{E}{\longrightarrow} \pi_{2 n+1}\left(S^{n+1}\right) \stackrel{H}{\longrightarrow} \mathbb{Z} \stackrel{P}{\longrightarrow} \pi_{2 n-1}\left(S^{n}\right)
$$

Proof (i) See Proposition 4B.1 of Hatcher [31]. The homotopy exact sequence of the Hopf fibration $S^{1} \rightarrow S^{3} \rightarrow S^{2}$ is

$$
\pi_{3}\left(S^{1}\right)=0 \longrightarrow \pi_{3}\left(S^{3}\right)=\mathbb{Z} \longrightarrow \pi_{3}\left(S^{2}\right) \longrightarrow \pi_{2}\left(S^{1}\right)=0 .
$$

(ii) See Theorem VII.8.3 of Bredon [10].

(iii) The cup product pairing for any space $X$

$$
\cup: H^{p}(X) \times H^{q}(X) \rightarrow H^{p+q}(X) ;(x, y) \mapsto x \cup y
$$

is such that

$$
x \cup y=(-1)^{p q}(y \cup x) .
$$

Now take $X$ as in 5.76, with $p=q=n+1$.

(iv) This is part of the EHP exact sequence of homotopy theory

$$
\ldots \rightarrow \pi_{m}(X) \stackrel{E}{\rightarrow} \pi_{m+1}(\Sigma X) \stackrel{H}{\rightarrow} \pi_{m}(X \wedge X) \stackrel{P}{\rightarrow} \pi_{m-1}(X) \rightarrow \ldots
$$

which holds for any $(n-1)$-connected space $X$ with $m \leqslant 3 n-2$ (Whitehead [96], p.548). Here $X=S^{n}, m=2 n$.

\section{Hopf Invariant 1 Theorem of Adams 5.79 ([2])}

There exists a map $f: S^{2 n+1} \rightarrow S^{n+1}$ with Hopf invariant $H(f)=1$ if and only if $n=1,3,7$.

It follows that for odd $n$ the image of the Hopf invariant map is

$$
\operatorname{im}\left(H: \pi_{2 n+1}\left(S^{n+1}\right) \rightarrow \mathbb{Z}\right)= \begin{cases}\mathbb{Z} & \text { if } n=1,3,7 \\ 2 \mathbb{Z} & \text { if } n \neq 1,3,7\end{cases}
$$

\section{Definition 5.80 The $J$-homomorphism}

$$
J: \pi_{m}(S O(k)) \rightarrow \pi_{m+k}\left(S^{k}\right) ; \omega \mapsto J(\omega)
$$

sends the homotopy class of a map $\omega: S^{m} \rightarrow S O(k)$ to the homotopy class of the composite

$$
\begin{aligned}
J(\omega): S^{m+k}= & S^{m} \times D^{k} \cup D^{m+1} \times S^{k-1} \stackrel{\text { proj. }}{\longrightarrow}\left(S^{m} \times D^{k}\right) /\left(S^{m} \times S^{k-1}\right) \\
& \stackrel{b_{\omega}}{\longrightarrow}\left(S^{m} \times D^{k}\right) /\left(S^{m} \times S^{k-1}\right) \stackrel{\text { proj. }}{\longrightarrow} D^{k} / S^{k-1}=S^{k}
\end{aligned}
$$

with $b_{\omega}: \epsilon^{k} \cong \epsilon^{k}$ the automorphism of the trivial $k$-plane bundle over $S^{m}$ adjoint to $\omega$

$$
b_{\omega}: E\left(\epsilon^{k}\right)=S^{m} \times \mathbb{R}^{k} \rightarrow S^{m} \times \mathbb{R}^{k} ;(x, y) \mapsto(x, \omega(x)(y))
$$


In other words, the $J$-homomorphism sends $\omega: S^{m} \rightarrow S O(k)$ to the component $J(\omega): S^{m+k} \rightarrow S^{k}$ of the self-homeomorphism of the Thom space of $\epsilon^{k}: S^{m} \rightarrow B S O(k)$ induced by $b_{\omega}$

$$
\begin{aligned}
\left(b_{\omega}\right)_{*} & =\left(\begin{array}{cc}
1 & 0 \\
J(\omega) & 1
\end{array}\right): \\
T\left(\epsilon^{k}\right) & =\left(S^{m} \times D^{k}\right) /\left(S^{m} \times S^{k-1}\right)=S^{m+k} \vee S^{k} \rightarrow S^{m+k} \vee S^{k} .
\end{aligned}
$$

Example 5.81 The Hopf bundle $\eta$ over $S^{2}(5.7)$ is the normal bundle of the embedding

$$
\mathbb{C} \mathbb{P}^{1}=G_{1}\left(\mathbb{C}^{2}\right)=S^{2} \hookrightarrow \mathbb{C} \mathbb{P}^{2}=G_{1}\left(\mathbb{C}^{3}\right)
$$

(extending the terminology of 5.22 to complex vector spaces), that is

$$
\eta=\nu_{S^{2} \hookrightarrow \mathbb{C} \mathbb{P}^{2}}: S^{2} \rightarrow B S O(2) .
$$

In this case, the $J$-homomorphism $J: \pi_{1}(S O(2)) \rightarrow \pi_{3}\left(S^{2}\right)$ is an isomorphism sending the generator

$$
\omega=1 \in \pi_{1}(S O(2))=\pi_{1}\left(S^{1}\right)=\mathbb{Z}
$$

to the element $\eta \in \pi_{3}\left(S^{2}\right)$ of Hopf invariant 1, with inverse

$$
H: \pi_{3}\left(S^{2}\right) \rightarrow \pi_{1}(S O(2))=\mathbb{Z} ; \eta \mapsto H(\eta) .
$$

The Hopf bundle $\eta$ has Euler number

$$
\chi(\eta)=e(\eta)=1 \in \mathbb{Z}
$$

and Thom space

$$
T(\eta)=S^{2} \cup_{\eta} D^{4} \simeq \mathbb{C} \mathbb{P}^{2}
$$

Proposition 5.82 (i) The Euler number map

$$
\chi: \pi_{n+1}(B S O(n+1)) \rightarrow \mathbb{Z} ; \omega \mapsto \chi(\omega)
$$

is the composite

$$
\chi=H J: \pi_{n+1}(B S O(n+1)) \stackrel{J}{\rightarrow} \pi_{2 n+1}\left(S^{n+1}\right) \stackrel{H}{\rightarrow} \mathbb{Z} .
$$

(ii) The inclusion $S O(k) \rightarrow S O(k+1)$ corresponds under the J-homomorphism to the suspension $E$ in the homotopy groups of spheres, with a commutative square

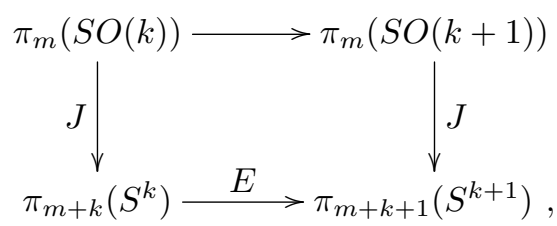


and there is also defined a stable J-homomorphism

$$
J: \pi_{m}(S O)=\underset{k}{\varliminf_{m}} \pi_{m}(S O(k)) \rightarrow \pi_{m}^{S}=\underset{k}{\lim _{m+k}} \pi_{m}\left(S^{k}\right) .
$$

Moreover,

$$
\begin{aligned}
& \pi_{m}(S O(m+2))=\pi_{m}(S O(m+3))=\ldots=\pi_{m}(S O) \\
& \pi_{2 m+2}\left(S^{m+2}\right)=\pi_{2 m+3}\left(S^{m+3}\right)=\ldots=\pi_{m}^{S}
\end{aligned}
$$

(iii) The Thom space of the bundle $\omega: S^{m+1} \rightarrow B S O(k)$ with clutching map $\omega: S^{m} \rightarrow S O(k)$ is given up to homotopy equivalence by

$$
T(\omega)=S^{k} \cup_{J(\omega)} D^{m+k+1} .
$$

Proof (i) For any $(n+1)$-plane bundle $\omega: S^{n+1} \rightarrow B S O(n+1)$ the Euler class $\chi(\omega) \in H^{n+1}\left(S^{n+1}\right)=\mathbb{Z}$ is the obstruction to the existence of a non-zero section of $\omega$, and the Hopf invariant $H J(\omega) \in \mathbb{Z}$ is the obstruction to desuspending $J(\omega): S^{2 n+1} \rightarrow S^{n+1}$.

(ii) By construction and the Freudenthal Suspension Theorem (3.10).

(iii) See Milnor [50].

Here is some basic information concerning the Stiefel manifold of orthonormal 2 -frames in $\mathbb{R}^{n+2}$

$$
V_{n+2,2}=O(n+2) / O(n)=S O(n+2) / S O(n)
$$

which will play an important role in the bundle theoretic aspects of surgery on $n$-spheres in $2 n$-dimensional manifolds (Chapters 10,11).

Proposition 5.83 (i) The Stiefel manifold $V_{n+2,2}$ fits into a fibration

$$
V_{n+2,2} \rightarrow B S O(n) \rightarrow B S O(n+2)
$$

with a long exact sequence of homotopy groups

$\ldots \rightarrow \pi_{m}\left(V_{n+2,2}\right) \rightarrow \pi_{m}(B S O(n)) \rightarrow \pi_{m}(B S O(n+2)) \rightarrow \pi_{m-1}\left(V_{n+2,2}\right) \rightarrow \ldots$

An element $x \in \pi_{m}\left(V_{n+2,2}\right)$ is an equivalence class of pairs $x=(\delta \omega, \omega)$ with $\omega: S^{m} \rightarrow B S O(n)$ an oriented $n$-plane bundle over $S^{m}$ and $\delta \omega: \omega \oplus \epsilon^{2} \cong \epsilon^{n+2}$ a 2-stable trivialisation.

(ii) The Stiefel manifold $V_{n+2,2}$ is the tangent $n$-sphere bundle of $S^{n+1}$

$$
S^{n} \rightarrow V_{n+2,2}=S\left(\tau_{S^{n+1}}\right) \rightarrow S^{n+1}
$$

which fits into a commutative braid of fibrations 


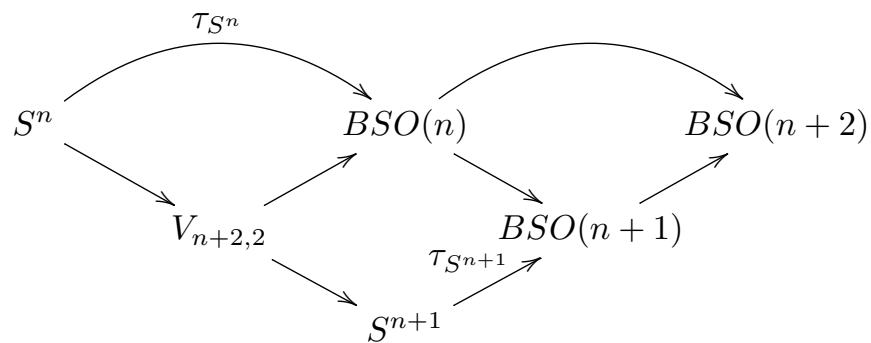

An element of $V_{n+2,2}$ can be regarded as a pair $\left(x \in S^{n+1}, y \in \tau_{S^{n+1}}(x)\right)$ with $\|y\|=1$, so that

$$
V_{n+2,2} \rightarrow S^{n+1} ;(x, y) \mapsto x
$$

$V_{n+2,2}$ is an orientable $(n-1)$-connected $(2 n+1)$-dimensional manifold with

$$
H_{i}\left(V_{n+2,2}\right)= \begin{cases}\mathbb{Z} & \text { if } i=0,2 n+1 \\ Q_{(-1)^{n}}(\mathbb{Z}) & \text { if } i=n \\ 0 & \text { otherwise }\end{cases}
$$

where

$$
Q_{(-1)^{n}}(\mathbb{Z})=\mathbb{Z} /\left\{1+(-1)^{n+1}\right\}= \begin{cases}\mathbb{Z} & \text { if } n \text { is even } \\ \mathbb{Z}_{2} & \text { if } n \text { is odd. }\end{cases}
$$

(iii) The projection

$$
p: S O(n+1) \rightarrow S O(n+1) / S O(n)=S^{n}
$$

induces the map

$$
p_{*}: \pi_{n}(S O(n+1))=\pi_{n+1}(B S O(n+1)) \rightarrow \pi_{n}\left(S^{n}\right)=\mathbb{Z}
$$

which sends an $(n+1)$-plane bundle $\omega: S^{n+1} \rightarrow B S O(n+1)$ to the obstruction

$$
p_{*}(\omega)=H J(\omega)=\chi(\omega) \in H^{n+1}\left(S^{n+1}\right)=\mathbb{Z}
$$

to the existence of a non-zero section of $\omega: S^{n+1} \rightarrow B S O(n+1)$.

(iv) The homotopy groups of $V_{n+2,2}, B S O(n), B S O(n+1), B S O(n+2)$ fit into a commutative braid of exact sequences:

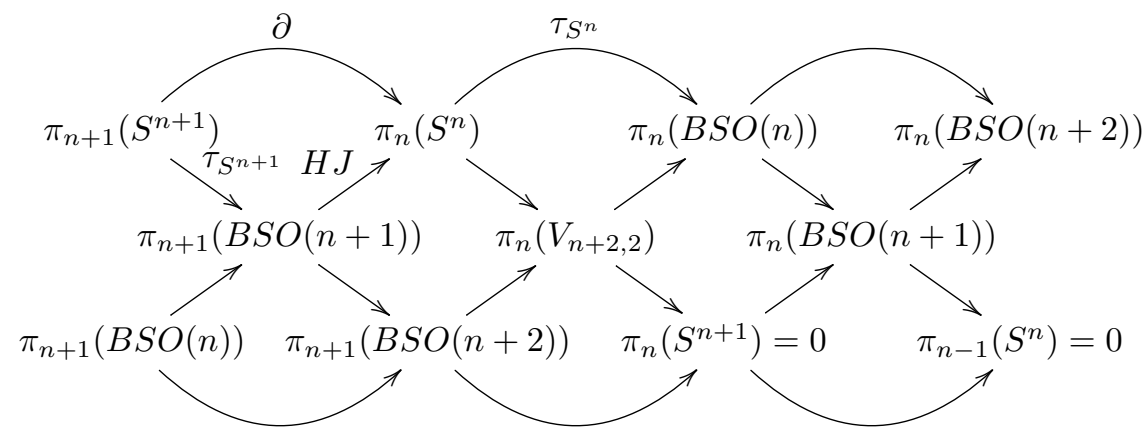

with 


$$
\begin{aligned}
& \partial=\chi\left(S^{n+1}\right)=1+(-1)^{n+1}: \pi_{n+1}\left(S^{n+1}\right)=\mathbb{Z} \rightarrow \pi_{n}\left(S^{n}\right)=\mathbb{Z}, \\
& \pi_{n}\left(S^{n}\right)=\mathbb{Z} \rightarrow \pi_{n}(B S O(n)) ; 1 \mapsto \tau_{S^{n}}, \\
& \pi_{n}(B S O(n+1))=\pi_{n}(B S O(n+2))=\ldots=\pi_{n}(B S O) \\
& \pi_{n+1}(B S O(n+2))=\pi_{n+1}(B S O(n+3))=\ldots=\pi_{n+1}(B S O), \\
& \pi_{n}\left(V_{n+2,2}\right)=\pi_{n+1}(B S O(n+2), B S O(n)) \\
& \quad=\pi_{n+1}(B S O(n+3), B S O(n)) \\
& \quad=\ldots=\pi_{n+1}(B S O, B S O(n))=Q_{(-1)^{n}}(\mathbb{Z}) .
\end{aligned}
$$

(v) The morphism

$$
\pi_{n}\left(V_{n+2,2}\right)=Q_{(-1)^{n}}(\mathbb{Z}) \rightarrow \pi_{n}(B S O(n)) ; 1 \mapsto \tau_{S^{n}}
$$

is $\left\{\begin{array}{l}\text { injective } \\ 0\end{array}\right.$ for $\left\{\begin{array}{l}n \neq 1,3,7 \\ n=1,3,7 .\end{array}\right.$

Proof (i)+(ii)+(iii)+(iv) See Chapter 25.6 of Steenrod [86].

(v) Immediate from the Bott-Milnor Sphere Parallelisability Theorem (5.74). 


\section{COBORDISM THEORY}

We have already seen in Chapter 2 that every cobordism is a union of the traces of a finite sequence of consecutive surgeries. However, this result is not useful in the cobordism classification of manifolds, since there are so many possibilities of performing surgeries. This chapter describes the Sard-Thom Transversality Theorem, the Pontrjagin-Thom construction of a homotopy class of maps from a bordism class of manifolds, and the Thom Cobordism Theorem establishing the isomorphism between the cobordism groups and the homotopy groups of Thom spaces. In Chapter 13 the isomorphism between the framed cobordism groups and the stable homotopy groups of spheres will be used in the surgery classification of exotic spheres. More generally, the surgery exact sequence will make use of the expression of the bordism set of normal maps as a homotopy group.

\subsection{Cobordism and transversality}

The basic definition of cobordism (1.1) is now repeated.

Definition 6.1 A cobordism of closed $m$-dimensional manifolds $M^{m}, M^{\prime m}$ is an $(m+1)$-dimensional manifold $W^{m+1}$ with boundary

$$
\partial W=M \cup-M^{\prime}
$$

where $-M^{\prime}$ denotes $M^{\prime}$ with the opposite orientation.

Remark 6.2 The definition of homology due to Poincaré was motivated by the invariance of integration on cobordant submanifolds. If $\omega$ is a closed differential $n$-form on an $m$-dimensional manifold $M^{m}$ then for any closed oriented $n$-dimensional submanifold $N^{n} \hookrightarrow M^{m}$ it is possible to define the integral

$$
\int_{N} \omega \in \mathbb{R} .
$$

For disjoint submanifolds $N^{n}, N^{\prime n} \hookrightarrow M^{m}$ related by an oriented cobordism $\left(W ; N, N^{\prime}\right)$ which is also a submanifold $W^{n+1} \hookrightarrow M^{m}$

$$
\int_{N} \omega-\int_{N^{\prime}} \omega=\int_{W} d \omega=0 \in \mathbb{R}
$$


by Stokes' theorem. In modern terminology integration along submanifolds gives the isomorphism of the $\mathbb{R}$-coefficient Universal Coefficient Theorem

$$
H^{n}(M ; \mathbb{R}) \rightarrow \operatorname{Hom}_{\mathbb{R}}\left(H_{n}(M ; \mathbb{R}), \mathbb{R}\right) ;[\omega] \mapsto\left([N] \mapsto \int_{N} \omega\right)
$$

with $[\omega] \in H^{n}(M ; \mathbb{R})$ the deRham cohomology class of the form $\omega$, and $[N] \in$ $H_{n}(M ; \mathbb{R})$ the homology class of a submanifold $N^{n} \hookrightarrow M^{m}$ (cf. Remark 4.10).

Proposition 6.3 Cobordism is an equivalence relation on manifolds.

Proof Every manifold $M$ is cobordant to itself by the product cobordism

$$
M \times(I ;\{0\},\{1\})=(M \times I ; M \times\{0\}, M \times\{1\}),
$$

with $I=[0,1]$ the unit interval. The union of adjoining cobordisms

$$
\left(W ; M, M^{\prime}\right),\left(W^{\prime} ; M^{\prime}, M^{\prime \prime}\right)
$$

is a cobordism

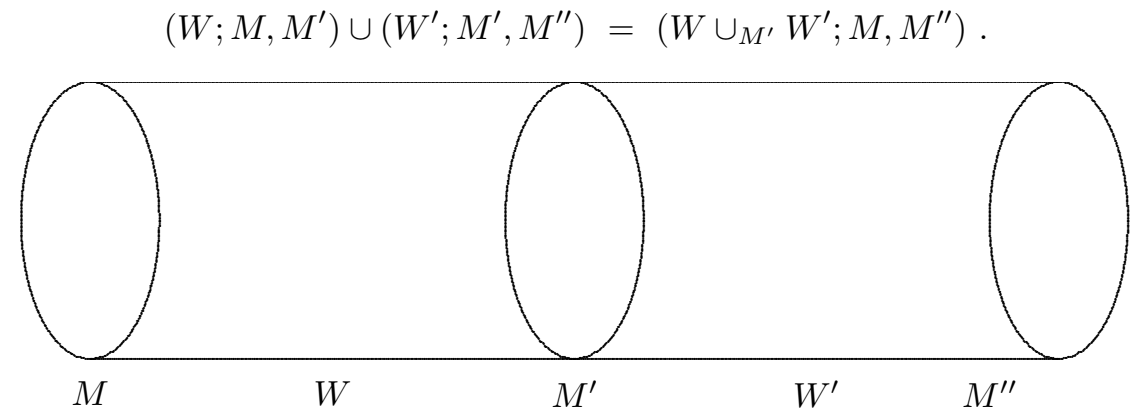

Diffeomorphic manifolds are cobordant.

Definition 6.4 The $m$-dimensional bordism set $\mathfrak{B}_{m}(N, X, \eta)$ is defined for an $n$-manifold $N^{n}$ and a $k$-plane bundle $\eta: X \rightarrow B O(k)$ to be the set of equivalence classes of pullback bundle maps from $m$-dimensional submanifolds $M^{m} \hookrightarrow N^{n}(m=n-k)$

$$
(f, b):\left(M, \nu_{M \hookrightarrow N}\right) \rightarrow(X, \eta)
$$

with respect to the bordism relation :

$$
(M,(f, b)) \sim\left(M^{\prime},\left(f^{\prime}, b^{\prime}\right)\right)
$$


if there exists a submanifold $W^{m+1} \hookrightarrow N \times I$ with a pullback bundle map

$$
(F, B):(W, \nu) \rightarrow(X, \eta) \quad\left(\nu=\nu_{W \hookrightarrow N \times I}\right)
$$

such that

$$
\begin{aligned}
& \left.(F, B)\right|_{M}=(f, b):(W, \nu) \cap(N \times\{0\})=\left(M, \nu_{M \hookrightarrow N}\right) \rightarrow(X, \eta), \\
& \left.(F, B)\right|_{M^{\prime}}=\left(f^{\prime}, b^{\prime}\right):(W, \nu) \cap(N \times\{1\})=\left(M^{\prime}, \nu_{M^{\prime} \hookrightarrow N}\right) \rightarrow(X, \eta) .
\end{aligned}
$$

In particular, there is defined a cobordism $\left(W^{m+1} ; M^{m}, M^{\prime m}\right)$ such that the normal 1-plane bundles $\nu_{M \hookrightarrow W}, \nu_{M^{\prime} \hookrightarrow W}$ are trivial. Only cobordisms with this property will be considered.

The computation of the bordism sets only became feasible with the development of transversality.

Definition 6.5 A map $g: N^{n} \rightarrow T(\eta)$ from an $n$-manifold $N$ to the Thom space (5.18) of a $k$-plane bundle $\eta: X \rightarrow B O(k)$ is transverse at the zero section $X \hookrightarrow T(\eta)$ if the inverse image is a closed $(n-k)$-dimensional submanifold

$$
M^{n-k}=g^{-1}(X) \subseteq N
$$

with normal $k$-plane bundle

$$
\nu_{M \hookrightarrow N}=f^{*} \eta: M \stackrel{f}{\rightarrow} X \stackrel{\eta}{\rightarrow} B O(k)
$$

the pullback of $\eta$ to $M$ along the restriction $f=g \mid: M \rightarrow X$, so that there is defined a pullback bundle map

$$
(f, b):\left(M, \nu_{M \hookrightarrow N}\right) \rightarrow(X, \eta)
$$

Sard-Thom Transversality Theorem 6.6 ([88])

Every continuous map $N^{n} \rightarrow T(\eta)$ from an $n$-dimensional manifold to the Thom space $T(\eta)$ of a $k$-plane bundle $\eta: X \rightarrow B O(k)$ is homotopic to a map $g: N \rightarrow$ $T(\eta)$ which is transverse at the zero section $X \hookrightarrow T(\eta)$, with a pullback bundle map

$$
(f, b):\left(M, \nu_{M \hookrightarrow N}\right) \rightarrow(X, \eta)
$$

with $f=g \mid: M=g^{-1}(X) \rightarrow X$.

Proof See Bredon [10, II.6] or Hirsch [33, Chapter 3]. 
Example 6.7 Let $f: N^{n} \rightarrow K^{k}$ be a differentiable function from an $n$-dimensional manifold $N$ to a $k$-dimensional manifold $K$, with $m=n-k \geqslant 0$, and let $x \in K$ be a regular value. The normal bundle of $X=\{x\} \hookrightarrow K$ is the (trivial!) $k$-plane bundle $\epsilon^{k}: X \rightarrow B O(k)$ with Thom space

$$
T\left(\epsilon^{k}\right)=S^{k} .
$$

If $U \cong \mathbb{R}^{k} \subseteq K$ is a neighbourhood of $x \in K$ then the composite

$$
g: N \stackrel{f}{\rightarrow} K \rightarrow K /(K \backslash U)=T\left(\epsilon^{k}\right)=S^{k}
$$

is transverse at the zero section $X \hookrightarrow T\left(\epsilon^{k}\right)$. The inverse image is the $m$ dimensional submanifold

$$
M=g^{-1}(x)=f^{-1}(x) \subseteq N
$$

with normal bundle

$$
\nu_{M \hookrightarrow N}=\epsilon^{k}: N \rightarrow B O(k),
$$

as already considered in the Implicit Function Theorem (2.12).

Pontrjagin-Thom Construction 6.8 Given an embedding $f: M^{m} \hookrightarrow N^{n}$ with normal bundle $\nu_{f}: M \rightarrow B O(n-m)$ and tubular neighbourhood $E\left(\nu_{f}\right) \hookrightarrow N$ the projection defines a map

$$
g: N \rightarrow N /\left(N \backslash E\left(\nu_{f}\right)\right)=D\left(\nu_{f}\right) / S\left(\nu_{f}\right)=T\left(\nu_{f}\right)
$$

which is transverse at the zero section $M \hookrightarrow T\left(\nu_{f}\right)$, with

$$
g \mid=\text { id. }: g^{-1}(M)=M \rightarrow M .
$$

Example 6.9 Apply the Pontrjagin-Thom construction to an embedding $f$ : $S^{0} \hookrightarrow S^{n}(n \geqslant 1)$, to obtain a map

$$
g: S^{n} \rightarrow S^{n} /\left(S^{n} \backslash\left(S^{0} \times D^{n}\right)\right)=S^{n} \vee S^{n}
$$

representing

$$
(1,1) \in \pi_{n}\left(S^{n} \vee S^{n}\right)= \begin{cases}\mathbb{Z} * \mathbb{Z} & \text { if } n=1 \\ \mathbb{Z} \oplus \mathbb{Z} & \text { if } n \geqslant 2 .\end{cases}
$$

Here is a picture for $n=1$ :

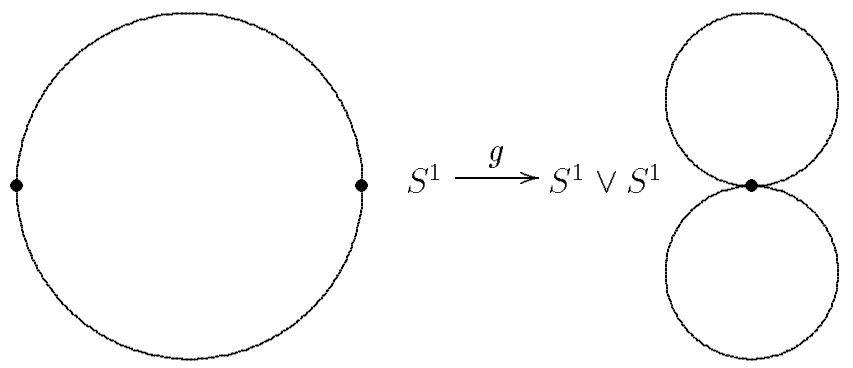


Thom Cobordism Theorem 6.10 ([88])

For any $n$-dimensional manifold $N^{n}$ and $k$-plane bundle $\eta: X \rightarrow B O(k)$ the bordism set $\mathfrak{B}_{m}(N, X, \eta)(m=n-k)$ is in natural one-one correspondence with the homotopy classes of maps $N \rightarrow T(\eta)$

$$
\mathfrak{B}_{m}(N, X, \eta)=[N, T(\eta)] .
$$

Proof The function in one direction is given by the Sard-Thom Transversality Theorem (6.6)

$$
[N, T(\eta)] \rightarrow \mathfrak{B}_{m}(N, X, \eta) ; g \mapsto(M,(f, b))
$$

with $g$ transverse at the zero section $X \hookrightarrow T(\nu)$, and $f$ the restriction

$$
f=g \mid: M^{m}=g^{-1}(X) \rightarrow X .
$$

The inverse function is defined by the Pontrjagin-Thom construction (6.8)

$$
\mathfrak{B}_{m}(N, X, \eta) \rightarrow[N, T(\eta)] ;(M, f, b) \mapsto h
$$

with $h$ the composite

$$
h: N \rightarrow N /\left(N \backslash E\left(\nu_{M \hookrightarrow N}\right)\right)=T\left(\nu_{M \hookrightarrow N}\right) \stackrel{T(b)}{\rightarrow} T(\eta) .
$$

The identification $\mathfrak{B}_{m}(N, X, \eta)=[N, T(\eta)]$ of 6.10 is already interesting in the case $m=0, X=\{*\}, \eta=\epsilon^{n}$ when $T(\eta)=S^{n}$, when the bordism set $\mathfrak{B}_{0}(N, X, \eta)=\left[N, S^{n}\right]$ is an abelian group isomorphic to $\mathbb{Z}$ by the degree, as follows.

Definition 6.11 The degree of a map of oriented connected $n$-dimensional manifolds $f: N^{n} \rightarrow M^{n}$ is the integer $\operatorname{deg}(f) \in \mathbb{Z}$ characterised by

$$
f_{*}[N]=\operatorname{deg}(f)[M] \in H_{n}(M)=\mathbb{Z}
$$

Example 6.12 Given a differentiable map $f: N^{n} \rightarrow M^{n}$ of oriented connected $n$-dimensional manifolds and a regular value $x \in M$ let

$$
f^{-1}(x)=\left\{y_{1}, y_{2}, \ldots, y_{k}\right\} \subseteq N .
$$

For each $i=1,2, \ldots, k$ there is defined an isomorphism of oriented $n$-dimensional vector spaces

$$
d f\left(y_{i}\right): \tau_{N}\left(y_{i}\right) \rightarrow \tau_{M}(x)
$$

Set 


$$
\epsilon_{i}=\left\{\begin{array} { l } 
{ + 1 } \\
{ - 1 }
\end{array} \text { if } d f ( y _ { i } ) \text { is } \left\{\begin{array}{l}
\text { orientation-preserving } \\
\text { orientation-reversing. }
\end{array}\right.\right.
$$

It follows from the commutative square

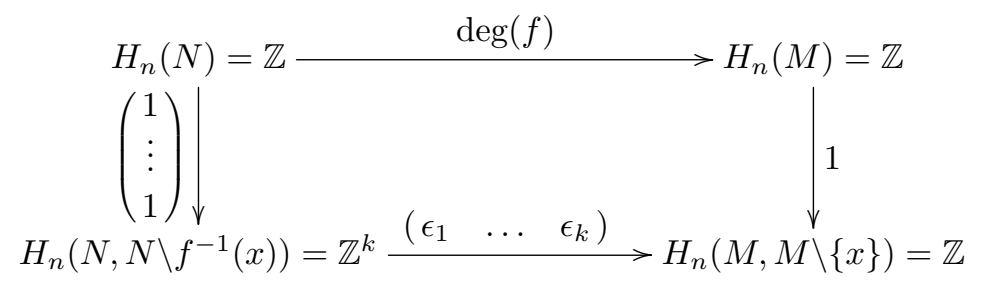

that the degree is given by

$$
\operatorname{deg}(f)=\sum_{i=1}^{k} \epsilon_{i} \in \mathbb{Z},
$$

as in the original definition of Brouwer.

Proposition 6.13 (Hopf)

For any connected oriented $n$-dimensional manifold $N$ the degree (6.11) of maps $f: N \rightarrow S^{n}$ defines an isomorphism

$$
d: \mathfrak{B}_{0}\left(N,\{*\}, \epsilon^{n}\right)=\left[N, S^{n}\right] \rightarrow \mathbb{Z} ; f \mapsto \operatorname{deg}(f) .
$$

Proof The inverse isomorphism $d^{-1}: \mathbb{Z} \rightarrow\left[N, S^{n}\right]$ sends $1 \in \mathbb{Z}$ to the homotopy class of the degree 1 map $f: N \rightarrow S^{n}$ constructed using any embedding $D^{n} \hookrightarrow N$ and the Pontrjagin-Thom construction (6.8)

$$
f: N \stackrel{\text { proj. }}{\longrightarrow} N /\left(N \backslash D^{n}\right)=D^{n} / S^{n-1}=S^{n} .
$$

See Chapter 6 of Milnor [56] for a more detailed exposition.

\subsection{Framed cobordism}

The identification of 6.13 for $N=S^{n}$

$$
\mathfrak{B}_{0}\left(S^{n},\{*\}, \epsilon^{n}\right)=\pi_{n}\left(S^{n}\right)=\mathbb{Z}
$$

can be viewed as the computation of the 0-dimensional framed cobordism group. Framed cobordism was an important early special case of bordism :

Definition 6.14 A framing of an $m$-dimensional manifold $M^{m}$ is an embed$\operatorname{ding} M \hookrightarrow S^{m+k}$ together with a framing of the normal bundle

$$
b: \nu_{M} \cong \epsilon^{k} \text {. }
$$

The pair $(M, b)$ is a framed manifold. 
A manifold $M^{m}$ can be framed if and only if the tangent bundle $\tau_{M}: M \rightarrow$ $B O(m)$ is stably trivial.

Example 6.15 Let $f: \mathbb{R}^{m+k} \rightarrow \mathbb{R}^{k}$ be a differentiable function for which $0 \in \mathbb{R}^{k}$ is a regular value. The inverse image

$$
M=f^{-1}(0) \subseteq \mathbb{R}^{m+k}=S^{m+k} \backslash\{\mathrm{pt} .\}
$$

is a framed $m$-dimensional manifold, by Example 6.7 .

\section{Definition 6.16 The framed cobordism ring}

$$
\Omega_{*}^{f r}=\sum_{m=0}^{\infty} \Omega_{m}^{f r}
$$

is the graded ring of cobordism classes $[M, b]$ of framed $m$-dimensional manifolds $(M, b)$. The addition is by disjoint union

$$
[M, b]+\left[M^{\prime}, b^{\prime}\right]=\left[M \cup M^{\prime}, b \cup b^{\prime}\right] \in \Omega_{m}^{f r},
$$

and the multiplication is by cartesian product

$$
\Omega_{m}^{f r} \times \Omega_{n}^{f r} \rightarrow \Omega_{m+n}^{f r} ;([M, b],[N, c]) \mapsto[M \times N, b \times c] .
$$

Pontrjagin Framed Cobordism Theorem 6.17 ([66])

The framed cobordism ring is isomorphic to the ring of the stable homotopy groups of spheres

$$
\Omega_{m}^{f r}=\pi_{m}^{S}(m \geqslant 0)
$$

Proof For each $k \geqslant 06.10$ gives identifications

$$
\mathfrak{B}_{m}\left(S^{m+k},\{*\}, \epsilon^{k}\right)=\pi_{m+k}\left(S^{k}\right)
$$

and

$$
\Omega_{m}^{f r}=\varliminf_{k} \mathfrak{B}_{m}\left(S^{m+k},\{*\}, \epsilon^{k}\right)=\varliminf_{k} \pi_{m+k}\left(S^{k}\right)=\pi_{m}^{S}
$$

Proposition 6.18 (i) In terms of framed cobordism the J-homomorphism (5.80) is the map

$$
J: \pi_{m}(S O(k)) \rightarrow \pi_{m+k}\left(S^{k}\right)=\mathfrak{B}_{m}\left(S^{m+k}, *, \epsilon^{k}\right) ; \omega \mapsto\left(S^{m}, b_{\omega}\right)
$$

which sends the homotopy class of $\omega: S^{m} \rightarrow S O(k)$ to the framed cobordism class of the submanifold $S^{m} \hookrightarrow S^{m+k}$ with the framing 


$$
b_{\omega}: \nu_{S^{m} \hookrightarrow S^{m+k}}=\epsilon^{k} \cong \epsilon^{k}
$$

where $\nu_{S^{m} \hookrightarrow S^{m+k}}=\epsilon^{k}$ refers to the standard framing given by the standard embedding

$$
S^{m} \hookrightarrow S^{m+k}=S^{m} \times D^{k} \cup D^{m+1} \times S^{k-1}
$$

(ii) The image of $J: \pi_{m}(S O) \rightarrow \pi_{m}^{S}=\Omega_{m}^{f r}$ consists of the framed cobordism classes of spheres $\left(S^{m}, c\right)$ with a choice of framing.

Proof (i) The inverse image $J(\omega)^{-1}(*)=S^{m} \subset S^{m+k}$ has the framing $b_{\omega}$.

(ii) Immediate from (i).

Remark 6.19 Surgery theory grew out of the classification of homotopy spheres, i.e. manifolds $\Sigma^{m}$ homotopy equivalent to $S^{m}$. Given a framed $m$-dimensional manifold $(M, b)$ one can ask two questions :

(i) Is $(M, b)$ framed cobordant to a sphere $\left(S^{m}, c\right)$ ?

(ii) Is $(M, b)$ framed cobordant to a homotopy sphere $\left(\Sigma^{m}, c\right)$ ?

By (6.18) question (i) is equivalent to asking if $(M, b) \in \Omega_{m}^{f r}$ is in the image of $J$, and is thus answered by homotopy theory. (The image of $J$ was much studied in the 1960's, notably by Adams.) Question (ii) is answered by surgery theory: the simply-connected surgery obstruction morphism

$$
\sigma_{*}: \Omega_{m}^{f r}=\pi_{m}^{S} \rightarrow L_{m}(\mathbb{Z}) \quad(m \geqslant 0)
$$

is such that $\sigma_{*}(M, b)=0$ if (and for $m \geqslant 5$ only if) $(M, b)$ is framed cobordant to a homotopy sphere - see Section 13.3 for a further discussion.

Example 6.20 The $J$-homomorphism

$$
J: \pi_{1}(S O(n)) \rightarrow \pi_{n+1}\left(S^{n}\right)=\mathfrak{B}_{1}\left(S^{n+1}, *, \epsilon^{n}\right) \quad(n \geqslant 1)
$$

is an isomorphism.

(i) The inverse of the isomorphism for $n=2$

$$
J: \pi_{1}(S O(2))=\pi_{1}\left(S^{1}\right)=\mathbb{Z} \rightarrow \pi_{3}\left(S^{2}\right)=\mathfrak{B}_{1}\left(S^{3}, *, \epsilon^{2}\right)
$$

is given by the Hopf invariant (5.76), as follows. Given an embedding $k: S^{1} \hookrightarrow S^{3}$ (which in general may be knotted) extend $k$ to an embedding $E\left(\nu_{k}\right) \hookrightarrow S^{3}$ as a tubular neighbourhood. Let $X=S^{3} \backslash k\left(S^{1}\right)$ be the knot complement, and let $p: X \rightarrow S^{1}$ be a map representing the generator $1 \in H^{1}(X)=\mathbb{Z}$. (The 
computation $H^{1}(X)=\mathbb{Z}$ is an easy application of the Mayer-Vietoris exact sequence to

$$
S^{3}=E\left(\nu_{k}\right) \cup_{S\left(\nu_{k}\right)} \text { cl. }\left(S^{3} \backslash E\left(\nu_{k}\right)\right),
$$

noting that the inclusion cl. $\left(S^{3} \backslash E\left(\nu_{k}\right)\right) \rightarrow X$ is a homotopy equivalence). Given a framing $b: \nu_{k} \cong \epsilon^{2}$ use the diffeomorphism

$$
b: E\left(\nu_{k}\right) \cong S^{1} \times \mathbb{R}^{2}
$$

to define a disjoint embedding

$$
k_{b}: S^{1} \hookrightarrow S^{3} ; x \mapsto b^{-1}(x,(1,0)) .
$$

The degree of the composite map

$$
p \circ k_{b}: S^{1} \stackrel{k_{b}}{\longrightarrow} X \stackrel{p}{\longrightarrow} S^{1}
$$

is the linking number of $i\left(S^{1}\right), k_{b}\left(S^{1}\right) \hookrightarrow S^{3}$, defining an invariant

$$
H\left(S^{1}, k, b\right)=d\left(p \circ k_{b}\right) \in \mathbb{Z}
$$

of the framed cobordism class $\left(S^{1}, k, b\right) \in \mathfrak{B}_{1}\left(S^{3}, *, \epsilon^{2}\right)$. The Hopf invariant isomorphism

$$
H: \pi_{3}\left(S^{2}\right) \rightarrow \mathbb{Z} ;\left(f: S^{3} \rightarrow S^{2}\right) \mapsto \sum_{f^{-1}(*)} H\left(S^{1}, k, b\right)
$$

sends a map $f: S^{3} \rightarrow S^{2}$ to the sum of the invariants for the components of the framed submanifold

$$
f^{-1}(*)=\bigcup S^{1} \hookrightarrow S^{3} .
$$

The isomorphism

$$
J: \pi_{1}(S O(2))=\pi_{1}\left(S^{1}\right)=\mathbb{Z} \rightarrow \pi_{3}\left(S^{2}\right)=\mathfrak{B}_{1}\left(S^{3}, *, \epsilon^{2}\right)
$$

sends a map $\omega: S^{1} \rightarrow S O(2)=S^{1}(5.13)$ with degree $d$ to a map $J(\omega): S^{3} \rightarrow S^{2}$ with Hopf invariant $d$. In particular, the map $1: S^{1} \rightarrow S^{1}$ of degree 1 has image the map $\eta=J(1): S^{3} \rightarrow S^{2}$ of Hopf invariant 1 , with $J(1)^{-1}(*)=S^{1} \hookrightarrow S^{3}$ the standard embedding with a nonstandard framing $b_{\eta}: \nu_{S^{1} \hookrightarrow S^{3}} \cong \epsilon^{2}$.

(ii) The inverse of the isomorphism for $n \geqslant 3$

$$
J: \pi_{1}(S O(n))=\mathbb{Z}_{2} \rightarrow \pi_{n+1}\left(S^{n}\right)=\mathfrak{B}_{1}\left(S^{n+1}, *, \epsilon^{n}\right)
$$

is given by the stable (i.e. mod 2) Hopf invariant. The stable $J$-homomorphism is an isomorphism

$$
J: \pi_{1}(S O)=\mathbb{Z}_{2} \rightarrow \Omega_{1}^{f r}=\pi_{1}^{S}
$$

and $\Omega_{1}^{f r}$ is generated by $\left(S^{1}, b_{\eta}\right)$. 
Example 6.21 The $J$-homomorphism

$$
J: \pi_{2}(S O(n))=0 \rightarrow \pi_{n+2}\left(S^{n}\right)=\mathfrak{B}_{2}\left(S^{n+2}, *, \epsilon^{n}\right) \quad(n \geqslant 1)
$$

is an isomorphism for $n=1$, but not an isomorphism for $n \geqslant 2$. Pontrjagin [66] showed that (in modern terminology)

$$
\Omega_{2}^{f r}=\pi_{n+2}\left(S^{n}\right)=\pi_{2}^{S}=L_{2}(\mathbb{Z})=\mathbb{Z}_{2}(n \geqslant 2),
$$

detected by the Arf invariant, with the generator represented by $T^{2}$ with an exotic framing $b$ (cf. Example 11.77 below).

\subsection{Unoriented and oriented cobordism}

Definition 6.22 The unoriented cobordism ring

$$
\mathfrak{N}_{*}=\sum_{m=0}^{\infty} \mathfrak{N}_{m}
$$

is the graded ring with $\mathfrak{N}_{m}$ the abelian group of cobordism classes $[M]$ of closed $m$-dimensional manifolds $M^{m}$, with addition by disjoint union and multiplication by cartesian product.

The Thom space of the universal $k$-plane bundle $1_{k}: B O(k) \rightarrow B O(k)$ is denoted by $M O(k)$. The effect on the Thom space of passing from a $k$-plane bundle $\eta: X \rightarrow B O(k)$ to the stabilisation $\eta \oplus \epsilon: X \rightarrow B O(k+1)$ is such a suspension

$$
T(\eta \oplus \epsilon)=\Sigma T(\eta)
$$

In particular, the classifying map $B O(k) \rightarrow B O(k+1)$ for $1_{k} \oplus \epsilon$ induces a map of Thom spaces

$$
T\left(1_{k} \oplus \epsilon\right)=\Sigma M O(k) \rightarrow T\left(1_{k+1}\right)=M O(k+1),
$$

defining a spectrum

$$
M O=\{\Sigma M O(k) \rightarrow M O(k+1) \mid k \geqslant 0\} .
$$

Unoriented Cobordism Theorem 6.23 (Thom [88])

The unoriented cobordism groups are isomorphic to the homotopy groups of the spectrum $M O$

$$
\mathfrak{N}_{m}=\underset{k}{\lim _{m+k}} \pi_{m O(k)) \quad(m \geqslant 0)} .
$$

Proof For each $k \geqslant 06.10$ gives

$$
\mathfrak{B}_{m}\left(S^{m+k}, B O(k), 1_{k}\right)=\pi_{m+k}(M O(k)) .
$$


For every $m$-dimensional manifold $M$ the disjoint union $M \cup M$ is the boundary of $M \times I$, so that every element of $\mathfrak{N}_{m}$ is of exponent 2 .

By reducing the geometry to homotopy theory and algebra Thom computed $\mathfrak{N}_{*}$ to be the polynomial algebra over $\mathbb{Z}_{2}$

$$
\mathfrak{N}_{*}=\mathbb{Z}_{2}\left[x_{i} \mid i \geqslant 1, i \neq 2^{j}-1\right]
$$

with one generator $x_{i}$ in each dimension $i \neq 2^{j}-1$, with $x_{i}=\left[\mathbb{R P}^{i}\right]$ if $i$ is even. The unoriented cobordism class of an $m$-dimensional manifold $M^{m}$ is determined by the Stiefel-Whitney numbers

$$
w_{I}(M)=\left\langle w_{1}(M)^{i_{1}} w_{2}(M)^{i_{2}} \ldots w_{n}(M)^{i_{n}},[M]\right\rangle \in \mathbb{Z}_{2}
$$

defined for any sequence $I=\left(i_{1}, i_{2}, \ldots, i_{n}\right)$ of integers $i_{j} \geqslant 0$ such that

$$
i_{1}+2 i_{2}+\ldots+n i_{n}=m
$$

with $w_{i}(M)=w_{i}\left(\tau_{M}\right) \in H^{i}\left(M ; \mathbb{Z}_{2}\right)$ the $i$ th Stiefel-Whitney class of the tangent bundle $\tau_{M}: M \rightarrow B O(m)$.

\section{Definition 6.24 The oriented cobordism ring}

$$
\Omega_{*}=\sum_{m=0}^{\infty} \Omega_{m}
$$

is the graded ring with $\Omega_{m}$ the abelian group of cobordism classes $[M]$ of closed oriented $m$-dimensional manifolds $M^{m}$.

The Thom space of the universal $k$-plane bundle $1_{k}: B S O(k) \rightarrow B S O(k)$ is denoted by $M S O(k)$. As in the unoriented case there is defined a spectrum

$$
M S O=\{\Sigma M S O(k) \rightarrow M S O(k+1) \mid k \geqslant 0\} .
$$

Oriented Cobordism Theorem 6.25 (Thom [88])

The oriented cobordism groups are isomorphic to the homotopy groups of the spectrum $M S O$

$$
\Omega_{m}=\varliminf_{k} \pi_{m+k}(M S O(k)) \quad(m \geqslant 0) .
$$

Proof For each $k \geqslant 06.10$ gives

$$
\mathfrak{B}_{m}\left(S^{m+k}, B S O(k), 1_{k}\right)=\pi_{m+k}(M S O(k)) .
$$


Thom showed that

$$
\Omega_{*} \otimes \mathbb{Q}=\mathbb{Q}\left[y_{4 i} \mid i \geqslant 1\right]
$$

with one generator $y_{4 i}=\left[\mathbb{C} P^{2 i}\right]$ in dimension $4 i$ for each $i \geqslant 1$. Milnor and Wall then sharpened the result, proving that $\Omega_{*}$ only has 2 -torsion, and that the oriented cobordism class of an oriented $m$-dimensional manifold $M^{m}$ is determined by the Stiefel-Whitney numbers $w_{I}(M) \in \mathbb{Z}_{2}$ and the Pontrjagin numbers

$$
p_{J}(M)=\left\langle p_{1}(M)^{j_{1}} p_{2}(M)^{j_{2}} \ldots p_{n}(M)^{j_{n}},[M]\right\rangle \in \mathbb{Z}
$$

defined for any sequence $J=\left(j_{1}, j_{2}, \ldots, j_{n}\right)$ of integers $j_{k} \geqslant 0$ such that

$$
4 j_{1}+8 j_{2}+\ldots+4 n j_{n}=m,
$$

with $p_{j}(M)=p_{j}\left(\tau_{M}\right) \in H^{4 j}(M)$ the $j$ th Pontrjagin class of the tangent bundle $\tau_{M}: M \rightarrow B O(m)$. The forgetful maps from framed to oriented cobordism

$$
\Omega_{m}^{f r} \rightarrow \Omega_{m} \quad(m \geqslant 1)
$$

are 0, since the Stiefel-Whitney and Pontrjagin numbers of a framed manifold are 0 .

Remark 6.26 The low-dimensional cobordism groups are given by:

\begin{tabular}{|c|c|c|c|c|c|c|c|c|c|}
\hline$m$ & 0 & 1 & 2 & 3 & 4 & 5 & 6 & 7 & 8 \\
\hline $\mathfrak{N}_{m}$ & $\mathbb{Z}_{2}$ & 0 & $\mathbb{Z}_{2}$ & 0 & $\left(\mathbb{Z}_{2}\right)^{2}$ & $\mathbb{Z}_{2}$ & $\left(\mathbb{Z}_{2}\right)^{3}$ & $\mathbb{Z}_{2}$ & $\left(\mathbb{Z}_{2}\right)^{5}$ \\
\hline$\Omega_{m}$ & $\mathbb{Z}$ & 0 & 0 & 0 & $\mathbb{Z}$ & $\mathbb{Z}_{2}$ & 0 & 0 & $\mathbb{Z}^{2}$ \\
\hline
\end{tabular}

\subsection{Signature}

Definition 6.27 (i) A symmetric form $(K, \lambda)$ over $\mathbb{R}$ is a finite-dimensional real vector space $K$ together with a bilinear pairing

$$
\lambda: K \times K \rightarrow \mathbb{R} ;(x, y) \mapsto \lambda(x, y)
$$

such that

$$
\lambda(x, y)=\lambda(y, x) \in \mathbb{R} .
$$

The form is nonsingular if the adjoint linear map

$$
\operatorname{adjoint}(\lambda): K \rightarrow K^{*}=\operatorname{Hom}_{\mathbb{R}}(K, \mathbb{R}) ; x \mapsto(y \mapsto \lambda(x, y))
$$

is an isomorphism.

(ii) A morphism of symmetric forms over $\mathbb{R}$ 


$$
f:(K, \lambda) \rightarrow\left(K^{\prime}, \lambda^{\prime}\right)
$$

is a linear map $f: K \rightarrow K^{\prime}$ such that

$$
\lambda^{\prime}(f(x), f(y))=\lambda(x, y) \in \mathbb{R} \quad(x, y \in K) .
$$

The morphism is an isomorphism if $f: K \rightarrow K^{\prime}$ is an isomorphism.

Diagonal forms are particularly important:

Definition 6.28 Given $\lambda_{i} \in \mathbb{R}(1 \leqslant i \leqslant n)$ define the diagonal symmetric form over $\mathbb{R}$

$$
\operatorname{diag}\left(\lambda_{1}, \lambda_{2}, \ldots, \lambda_{n}\right)=\left(\mathbb{R}^{n}, \lambda\right)
$$

with

$$
\lambda\left(\left(x_{1}, x_{2}, \ldots, x_{n}\right),\left(y_{1}, y_{2}, \ldots, y_{n}\right)\right)=\sum_{i=1}^{n} \lambda_{i} x_{i} y_{i} \in \mathbb{R} .
$$

Spectral Theorem 6.29 For every symmetric form $(K, \lambda)$ over $\mathbb{R}$ there exists a basis $\left\{v_{1}, v_{2}, \ldots, v_{n}\right\}$ of $K$ with

$$
\lambda\left(v_{i}, v_{j}\right)= \begin{cases}\lambda_{i} & \text { if } i=j \\ 0 & \text { if } i \neq j\end{cases}
$$

for some $\lambda_{i} \in \mathbb{R}$, corresponding to an isomorphism

$$
f: \operatorname{diag}\left(\lambda_{1}, \lambda_{2}, \ldots, \lambda_{n}\right) \rightarrow(K, \lambda)
$$

with

$$
f: \mathbb{R}^{n} \rightarrow K ;\left(x_{1}, x_{2}, \ldots, x_{n}\right) \mapsto \sum_{i=1}^{n} x_{i} v_{i}
$$

Proof For an arbitrary basis $\left\{u_{1}, u_{2}, \ldots, u_{n}\right\}$ of $K$ choose a basis for each eigenspace of the symmetric $n \times n$ matrix $\left(\lambda\left(u_{i}, u_{j}\right)\right)$. Apply the Gram-Schmidt process to each of these bases, to obtain an orthonormal basis for $K$ consisting of eigenvectors $\left\{v_{1}, v_{2}, \ldots, v_{n}\right\}$.

Definition 6.30 (i) A symmetric form $(K, \lambda)$ over $\mathbb{R}$ is positive definite if

$$
\lambda(x, x)>0 \quad(x \in K \backslash\{0\}),
$$

in which case it is nonsingular. Similarly for negative definite with $\lambda(x, x)<0$.

(ii) The positive index of a symmetric form $(K, \lambda)$ over $\mathbb{R}$ is

$$
\operatorname{Ind}_{+}(K, \lambda)=\max \operatorname{dim}(U) \geqslant 0,
$$

the maximum dimension of a subspace $U \subseteq K$ such that the restricted form $(U, \lambda \mid)$ is positive definite. Similarly for the negative index $\operatorname{Ind}_{-}(K, \lambda)$.

(iii) The signature of a symmetric form $(K, \lambda)$ over $\mathbb{R}$ is

$$
\sigma(K, \lambda)=\operatorname{Ind}_{+}(K, \lambda)-\operatorname{Ind}_{-}(K, \lambda) \in \mathbb{Z} .
$$


Remark 6.31 (i) Nonsingular forms are sometimes called nondegenerate forms. The index of a symmetric form is usually defined to be the negative index. Signature used to be called the inertia of a symmetric form, and (confusingly) also the index.

(ii) An inner product space $(K, \lambda)$ is a vector space $K$ together with a positive definite symmetric form $\lambda$. The signature of an inner product space is

$$
\sigma(K, \lambda)=\operatorname{Ind}_{+}(K, \lambda)=\operatorname{dim}(K)
$$

Example 6.32 The diagonal form $\operatorname{diag}\left(\lambda_{1}, \lambda_{2}, \ldots, \lambda_{n}\right)$ is such that

$$
\operatorname{Ind}_{ \pm}\left(\operatorname{diag}\left(\lambda_{1}, \lambda_{2}, \ldots, \lambda_{n}\right)\right)=I_{ \pm}
$$

with $I_{+}$(resp. $I_{-}$) the number of strictly positive (resp. negative) $\lambda_{i}$ 's.

The indices and signature are isomorphism invariants, so that for any symmetric form $(K, \lambda)$ and any diagonal form $\operatorname{diag}\left(\lambda_{1}, \lambda_{2}, \ldots, \lambda_{n}\right)$ isomorphic to $(K, \lambda)\left(\right.$ with $\left.n=\operatorname{dim}_{\mathbb{R}}(K)\right)$

$$
\begin{aligned}
& \operatorname{Ind}_{ \pm}(K, \lambda)=\operatorname{Ind}_{ \pm}\left(\operatorname{diag}\left(\lambda_{1}, \lambda_{2}, \ldots, \lambda_{n}\right)\right)=I_{ \pm} \\
& \sigma(K, \lambda)=\sigma\left(\operatorname{diag}\left(\lambda_{1}, \lambda_{2}, \ldots, \lambda_{n}\right)\right)=I_{+}-I_{-} .
\end{aligned}
$$

Furthermore

$$
\operatorname{Ind}_{+}(K, \lambda)+\operatorname{Ind}_{-}(K, \lambda)=I_{+}+I_{-}=n-r
$$

where

$$
\begin{aligned}
r & =\operatorname{dim}_{\mathbb{R}}\{x \in K \mid \lambda(x, y)=0 \in \mathbb{R} \text { for all } y \in K\} \\
& =\left|\left\{i \mid \lambda_{i}=0\right\}\right|
\end{aligned}
$$

with $r=0$ if and only if $(K, \lambda)$ is nonsingular.

Symmetric forms over $\mathbb{R}$ are classified by rank and index, according to the following classic 19th century result.

Sylvester's Law of Inertia 6.33 Two symmetric forms $(K, \lambda),\left(K^{\prime}, \lambda^{\prime}\right)$ over $\mathbb{R}$ are isomorphic if and only if

$$
\operatorname{dim}_{\mathbb{R}}(K)=\operatorname{dim}_{\mathbb{R}}\left(K^{\prime}\right), \operatorname{Ind}_{ \pm}(K, \lambda)=\operatorname{Ind}_{ \pm}\left(K^{\prime}, \lambda^{\prime}\right)
$$

Proof By the Spectral Theorem 6.29 every symmetric form $(K, \lambda)$ is isomorphic to $\operatorname{diag}(1, \ldots, 1,-1, \ldots,-1,0, \ldots, 0)$, with $\operatorname{Ind}_{+}(K) 1$ 's, Ind- $(K)(-1)$ 's and $\operatorname{dim}_{\mathbb{R}}(K)-\operatorname{Ind}_{+}(K)-\operatorname{Ind}_{-}(K) 0$ 's. 
Definition 6.34 The nonsingular hyperbolic symmetric form over $\mathbb{R}$

$$
H(L)=\left(L \oplus L^{*}, \lambda\right)
$$

is defined for any finite-dimensional real vector space $L$ by

$$
\lambda: L \oplus L^{*} \times L \oplus L^{*} \rightarrow \mathbb{R} ;((x, f),(y, g)) \mapsto f(y)+g(x),
$$

with

$$
\operatorname{Ind}_{+} H(L)=\operatorname{Ind}_{-} H(L)=\operatorname{dim}_{\mathbb{R}}(L) \in \mathbb{N}, \sigma(H(L))=0 \in \mathbb{Z} .
$$

Proposition 6.35 Two nonsingular symmetric forms $(K, \lambda),\left(K^{\prime}, \lambda^{\prime}\right)$ over $\mathbb{R}$ are related by an isomorphism

$$
f:(K, \lambda) \oplus H(L) \rightarrow\left(K^{\prime}, \lambda^{\prime}\right) \oplus H\left(L^{\prime}\right)
$$

for some finite-dimensional real vector spaces $L, L^{\prime}$ if and only if

$$
\sigma(K, \lambda)=\sigma\left(K^{\prime}, \lambda^{\prime}\right) \in \mathbb{Z} .
$$

Proof Immediate from the Spectral Theorem 6.29 and Sylvester's Law of Inertia 6.33 , on noting that for any real numbers $\lambda, \mu$ with $\mu<0<\lambda$ there is defined an isomorphism of symmetric forms

$$
\left(\begin{array}{cc}
\frac{1}{\sqrt{\lambda}} & \frac{1}{2 \sqrt{\lambda}} \\
\frac{1}{\sqrt{-\mu}} & \frac{-1}{2 \sqrt{-\mu}}
\end{array}\right): H(\mathbb{R}) \rightarrow \operatorname{diag}(\lambda, \mu) .
$$

Definition 6.36 (i) Given a symmetric form $(K, \lambda)$ over $\mathbb{R}$ and a subspace $L \subseteq$ $K$ let

$$
L^{\perp}=\{x \in K \mid \lambda(x, y)=0 \in \mathbb{R} \text { for all } y \in L\} .
$$

(ii) A lagrangian of a nonsingular symmetric form $(K, \lambda)$ over $\mathbb{R}$ is a subspace $L \subseteq K$ such that

$$
L=L^{\perp}
$$

Proposition 6.37 The following conditions on a nonsingular symmetric form $(K, \lambda)$ over $\mathbb{R}$ are equivalent:

(i) $(K, \lambda)$ is isomorphic to a hyperbolic form,

(ii) $\sigma(K, \lambda)=0 \in \mathbb{Z}$,

(iii) $(K, \lambda)$ admits a lagrangian $L$. 
Proof (i) $\Longleftrightarrow$ (ii) Immediate from 6.35 .

(i) $\Longrightarrow$ (iii) The hyperbolic form $H(L)$ has lagrangian $L$.

(iii) $\Longrightarrow$ (i) Choose a direct summand $M$ to $L$ in $K$. The linear maps

$$
\begin{aligned}
& f: M \rightarrow L^{*} ; x \mapsto(y \mapsto \lambda(x, y)), \\
& g: M \stackrel{\lambda \mid}{\rightarrow} M^{*} \stackrel{\left(f^{-1}\right)^{*}}{\longrightarrow} L^{* *}=L, \\
& h=\left(\begin{array}{cc}
1 & g / 2 \\
0 & f
\end{array}\right): K=L \oplus M \rightarrow L \oplus L^{*}
\end{aligned}
$$

are such that $h:(K, \lambda) \cong H(L)$ is an isomorphism of symmetric forms.

Definition 6.38 The intersection form of a closed oriented $4 k$-dimensional manifold $M^{4 k}$ is the nonsingular symmetric form over $\mathbb{R}\left(H^{2 k}(M ; \mathbb{R}), \lambda\right)$ with

$$
\lambda: H^{2 k}(M ; \mathbb{R}) \times H^{2 k}(M ; \mathbb{R}) \rightarrow \mathbb{R} ;(x, y) \mapsto\langle x \cup y,[M]\rangle
$$

The signature of $M$ is the signature of the intersection form

$$
\sigma(M)=\sigma\left(H^{2 k}(M ; \mathbb{R}), \lambda\right) \in \mathbb{Z}
$$

The intersection form $\left(H^{2 k}(M ; \mathbb{R}), \lambda\right)$ is just the $\mathbb{R}$-coefficient version of the homology intersection form of Definition 4.11, identifying $H^{2 k}(M ; \mathbb{R})=H_{2 k}(M ; \mathbb{R})$ by Poincaré duality.

Example 6.39 The intersection form of $S^{2 k} \times S^{2 k}$ is hyperbolic (6.34)

$$
\left(H^{2 k}\left(S^{2 k} \times S^{2 k} ; \mathbb{R}\right), \lambda\right)=H(\mathbb{R})=\left(\mathbb{R} \oplus \mathbb{R},\left(\begin{array}{ll}
0 & 1 \\
1 & 0
\end{array}\right)\right),
$$

so that the signature is

$$
\sigma\left(S^{2 k} \times S^{2 k}\right)=\sigma(H(\mathbb{R}))=0
$$

Example 6.40 The intersection form of the complex projective space $\mathbb{C} \mathbb{P}^{2 k}$ is the symmetric form

$$
\left(H^{2 k}\left(\mathbb{C} \mathbb{P}^{2 k} ; \mathbb{R}\right), \lambda\right)=(\mathbb{R}, 1)
$$

and the signature is

$$
\sigma\left(\mathbb{C} \mathbb{P}^{2 k}\right)=\sigma(\mathbb{R}, 1)=1
$$

We refer to Hirzebruch [34] and Chapters 15,19 of Milnor and Stasheff [61] for the definition of the $\mathscr{L}$-genus of an oriented vector bundle $\eta: M \rightarrow B S O$

$$
\mathscr{L}(\eta)=\mathscr{L}\left(p_{1}(\eta), p_{2}(\eta), \ldots\right) \in H^{4 *}(M ; \mathbb{Q})
$$


The $4 k$-dimensional component

$$
\mathscr{L}_{k}(M)=\mathscr{L}_{k}\left(p_{1}(M), p_{2}(M), \ldots, p_{k}(M)\right) \in H^{4 k}(M ; \mathbb{Q})
$$

is a polynomial in the Pontrjagin classes of the form

$$
\mathscr{L}_{k}\left(p_{1}, p_{2}, \ldots, p_{k}\right)=s_{k} p_{k}+\text { terms with } p_{j} \text { for } j<k
$$

with

$$
s_{k}=\frac{2^{2 k}\left(2^{2 k-1}-1\right)}{(2 k) !} B_{k}
$$

involving the $k$ th Bernoulli number $B_{k}$. The $\mathscr{L}$-genus of an oriented manifold $M$ is defined by

$$
\mathscr{L}(M)=\mathscr{L}\left(\tau_{M}\right)=\mathscr{L}\left(p_{1}(M), p_{2}(M), \ldots\right) \in H^{4 *}(M ; \mathbb{Q})
$$

with $p_{k}(M)=p_{k}\left(\tau_{M}\right) \in H^{4 k}(M)$ the $k$ th Pontrjagin class.

Hirzebruch Signature Theorem 6.41 (1952)

The signature of a closed oriented $4 k$-dimensional manifold $M^{4 k}$ is given by

$$
\sigma(M)=\left\langle\mathscr{L}_{k}(M),[M]\right\rangle \in \mathbb{Z}
$$

Proof The first proof was a direct consequence of Thom's computation

$$
\Omega_{*} \otimes \mathbb{Q}=\mathbb{Q}\left[y_{4 k} \mid k \geqslant 1\right]
$$

with $y_{4 k}=\left[\mathbb{C P}^{2 k}\right]$. For any $n \geqslant 1$ the Pontrjagin classes of the $n$-dimensional complex projective space $\mathbb{C} \mathbb{P}^{m}$ are given by

$$
p_{j}\left(\mathbb{C} \mathbb{P}^{m}\right)=p_{j}\left(\tau_{\mathbb{C P}^{m}}\right)=\left(\begin{array}{c}
m+1 \\
j
\end{array}\right) \in H^{4 j}\left(\mathbb{C} \mathbb{P}^{m}\right)=\mathbb{Z} \quad(0 \leqslant j \leqslant m / 2)
$$

(Milnor and Stasheff [61,p.177]). For $m=2 k$ the evaluation

$$
\left\langle\mathscr{L}_{k}\left(\mathbb{C} \mathbb{P}^{2 k}\right),\left[\mathbb{C} \mathbb{P}^{2 k}\right]\right\rangle=1 \in \mathbb{Z}
$$

coincides with the signature of $\mathbb{C} \mathbb{P}^{2 k}$

$$
\sigma\left(\mathbb{C} \mathbb{P}^{2 k}\right)=\sigma\left(H^{2 k}\left(\mathbb{C} \mathbb{P}^{2 k}\right), \lambda\right)=\sigma(\mathbb{Z}, 1)=1 \in \mathbb{Z}
$$

See Hirzebruch [34] and Chapter 19 of [61] for further details. 
Example 6.42 The first three $\mathscr{L}$-polynomials are given by

$$
\begin{aligned}
& \mathscr{L}_{1}\left(p_{1}\right)=\frac{1}{3} p_{1} \\
& \mathscr{L}_{2}\left(p_{1}, p_{2}\right)=\frac{1}{45}\left(7 p_{2}-p_{1}^{2}\right) \\
& \mathscr{L}_{3}\left(p_{1}, p_{2}, p_{3}\right)=\frac{1}{945}\left(62 p_{3}-13 p_{2} p_{1}+2 p_{1}^{3}\right) .
\end{aligned}
$$

and

$$
\begin{aligned}
p_{1}\left(\mathbb{C} \mathbb{P}^{2}\right)=3, \sigma\left(\mathbb{C} \mathbb{P}^{2}\right)=\left\langle\mathscr{L}_{1}\left(\mathbb{C} \mathbb{P}^{2}\right),\left[\mathbb{C} \mathbb{P}^{2}\right]\right\rangle=1, \\
p_{1}\left(\mathbb{C} \mathbb{P}^{4}\right)=5, p_{2}\left(\mathbb{C} \mathbb{P}^{4}\right)=10, \sigma\left(\mathbb{C} \mathbb{P}^{4}\right)=\left\langle\mathscr{L}_{2}\left(\mathbb{C} \mathbb{P}^{4}\right),\left[\mathbb{C} \mathbb{P}^{4}\right]\right\rangle=1, \\
p_{1}\left(\mathbb{C} \mathbb{P}^{6}\right)=7, p_{2}\left(\mathbb{C} \mathbb{P}^{6}\right)=21, p_{3}\left(\mathbb{C} \mathbb{P}^{6}\right)=35, \\
\sigma\left(\mathbb{C} \mathbb{P}^{6}\right)=\left\langle\mathscr{L}_{3}\left(\mathbb{C} \mathbb{P}^{6}\right),\left[\mathbb{C} \mathbb{P}^{6}\right]\right\rangle=1 .
\end{aligned}
$$

The signature is also defined for an oriented $4 k$-dimensional manifold with boundary $(M, \partial M)$, but there is no corresponding expression in terms of the $\mathscr{L}$-genus $\mathscr{L}(M) \in H^{4 *}(M ; \mathbb{Q})$. (For one thing, it is not possible to evaluate $\mathscr{L}_{k}(M) \in H^{4 k}(M ; \mathbb{Q})$ on the fundamental class $[M] \in H_{4 k}(M, \partial M ; \mathbb{Q})$, and in any case $H^{4 k}(M ; \mathbb{Q})=H_{0}(M, \partial M ; \mathbb{Q})=0$ if $\partial M$ and $M$ have the same number of path components).

Proposition 6.43 The signature of closed oriented $4 k$-dimensional manifolds is an oriented cobordism invariant, with

$$
\sigma(M)=\sigma(N) \in \mathbb{Z}
$$

for any oriented $(4 k+1)$-dimensional cobordism $(W ; M, N)$.

Proof It suffices to prove that $\sigma(\partial W)=0$ for an oriented $(4 k+1)$-dimensional manifold with boundary $(W, \partial W)$. Let

$$
(K, \lambda)=\left(H^{2 k}(\partial W ; \mathbb{R}),[\partial W] \cap-\right)
$$

be the intersection form of $\partial W$, with $[\partial W] \in H_{4 k}(\partial W ; \mathbb{R})$. The Poincaré duality isomorphisms define an isomorphism of exact sequences

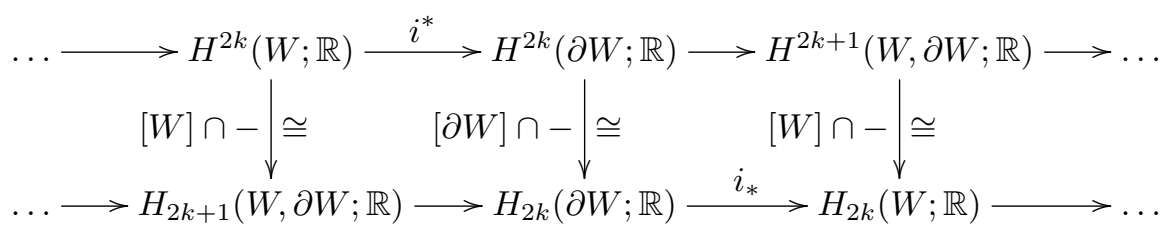


with $i: \partial W \rightarrow W$ the inclusion. It follows that the inclusion $j: L \rightarrow K$ of the subspace

$$
L=\operatorname{im}\left(i^{*}: H^{2 k}(W ; \mathbb{R}) \rightarrow H^{2 k}(\partial W ; \mathbb{R})\right) \subseteq K=H^{2 k}(\partial W ; \mathbb{R})
$$

is such that there is defined an exact sequence

$$
0 \longrightarrow L \stackrel{j}{\longrightarrow} K \stackrel{j^{*} \lambda}{\longrightarrow} L^{*} \longrightarrow 0
$$

and $L$ is a lagrangian $(6.36)$ in $(K, \lambda)$. By $6.37 j$ extends to an isomorphism of symmetric forms

$$
H(L)=\left(L \oplus L^{*},\left(\begin{array}{ll}
0 & 1 \\
1 & 0
\end{array}\right)\right) \rightarrow(K, \lambda),
$$

and the signature of $\partial W$ is

$$
\sigma(\partial W)=\sigma(K, \lambda)=\sigma(H(L))=0 \in \mathbb{Z} .
$$

In particular, the signature defines morphisms on the $4 k$-dimensional oriented cobordism group

$$
\sigma: \Omega_{4 k} \rightarrow \mathbb{Z} ;[M] \mapsto \sigma(M) .
$$

Example 6.44 The signature map is an isomorphism

$$
\sigma: \Omega_{4} \rightarrow \mathbb{Z} ;[M] \mapsto \sigma(M),
$$

with $\sigma\left(\mathbb{C} \mathbb{P}^{2}\right)=1$. 


\section{EMBEDDINGS, IMMERSIONS AND SINGULARITIES}

The one-one correspondence between algebraic and geometric surgeries below and in the middle dimension is central to surgery theory. This chapter describes the algebraic and geometric intersection properties of submanifolds used to establish this one-one correspondence in subsequent chapters.

Section 7.1 states the Whitney Embedding and Immersion Theorems : every map of manifolds $f: N^{n} \rightarrow M^{m}$ is arbitrarily close to an embedding if $2 n<$ $m$, and arbitrarily close to an immersion if $2 n=m$. Section 7.2 treats the algebraic and geometric intersections of immersions, including self-intersections. Section 7.3 describes the Whitney trick for removing pairs of double points in manifolds of dimension $m \geqslant 5$. Section 7.4 is a brief account of the Smale-Hirsch classification theory of immersions. Finally, Section 7.5 describes the unique type of singularities arising for generic maps $f: N^{n} \rightarrow M^{2 n-1}$.

\subsection{The Whitney Immersion and Embedding Theorems}

Here are the statements:

Whitney Immersion Theorem 7.1 ([97], [99])

For $2 n \leqslant m$ every map $f: N^{n} \rightarrow M^{m}$ is homotopic to an immersion $N \rightarrow M$, and for $2 n+1 \leqslant m$ any two homotopic immersions are regular homotopic.

Whitney Embedding Theorems 7.2 ([97], [99])

(i) For $2 n+1 \leqslant m$ every map $f: N^{n} \rightarrow M^{m}$ is homotopic to an embedding $N \hookrightarrow M$, and for $2 n+2 \leqslant m$ any two homotopic embeddings are isotopic.

(ii) For $n \geqslant 3$ and $\pi_{1}(M)=\{1\}$ every map $f: N^{n} \rightarrow M^{2 n}$ is homotopic to an embedding $N \hookrightarrow M$.

A map $f: N^{n} \rightarrow M^{m}$ with $2 n \geqslant m$ cannot in general be approximated by embeddings. Example 11.33 gives an explicit map which cannot be approximated, with $m=2 n$.

A map $f: N^{n} \rightarrow M^{m}$ with $2 n-1 \geqslant m$ cannot in general be approximated by immersions. See Section 7.5 below for the unique type of singularity arising in the case $m=2 n-1$. 
The essential difference between immersions and embeddings are the double points :

Definition 7.3 (i) The ordered double point set of a map $f: N \rightarrow M$ is the set

$$
S_{2}(f)=\left\{\left(x_{1}, x_{2}\right) \in N \times N \mid x_{1} \neq x_{2} \in N, f\left(x_{1}\right)=f\left(x_{2}\right) \in M\right\} .
$$

(ii) The unordered double point set of a map $f: N \rightarrow M$ is the set

$$
S_{2}[f]=S_{2}(f) / \mathbb{Z}_{2}
$$

with $\mathbb{Z}_{2}$ acting by $\left(x_{1}, x_{2}\right) \mapsto\left(x_{2}, x_{1}\right)$. The projection

$$
S_{2}(f) \rightarrow S_{2}[f] ;\left(x_{1}, x_{2}\right) \mapsto\left[x_{1}, x_{2}\right]
$$

is a double covering map.

Proposition 7.4 An embedding $N \hookrightarrow M$ is an immersion $N \hookrightarrow M$ with no double points.

If $f: N^{n} \leftrightarrow M^{m}$ is a generic 'self-transverse' immersion of an $n$-dimensional manifold $N$ in an $m$-dimensional manifold $M$ then $S_{2}(f)$ is a $(2 n-m)$-dimensional manifold. Thus for $2 n<m$ a generic immersion is an embedding.

The approximation theorems $(7.1,7.2)$ for maps of manifolds are global versions of the following results for linear maps of vector spaces.

Let $M_{m, n}(\mathbb{R})$ be the vector space of $m \times n$ matrices $\left(a_{i j}\right)_{1 \leqslant i \leqslant m, 1 \leqslant j \leqslant n}$ with

coefficients $a_{i j} \in \mathbb{R}$, with $m$ rows and $n$ columns. The function

$$
\begin{aligned}
& M_{m, n}(\mathbb{R}) \rightarrow \operatorname{Hom}_{\mathbb{R}}\left(\mathbb{R}^{n}, \mathbb{R}^{m}\right) ; \\
& A=\left(a_{i j}\right) \mapsto\left(f_{A}:\left(x_{1}, x_{2}, \ldots, x_{n}\right) \mapsto\left(\sum_{j=1}^{n} a_{1 j} x_{j}, \sum_{j=1}^{n} a_{2 j} x_{j}, \ldots, \sum_{j=1}^{n} a_{m j} x_{j}\right)\right)
\end{aligned}
$$

is an isomorphism of vector spaces, such that

$$
f_{A B}=f_{A} f_{B}: \mathbb{R}^{p} \rightarrow \mathbb{R}^{n} \rightarrow \mathbb{R}^{m}
$$

for $A \in M_{m, n}(\mathbb{R}), B \in M_{n, p}(\mathbb{R})$. Regarding $M_{m, n}(\mathbb{R})$ as a differentiable manifold there is an evident identification

$$
M_{m, n}(\mathbb{R})=\mathbb{R}^{m n} .
$$

The invertible elements of $M_{m, m}(\mathbb{R})$ define the general linear group under multiplication

$$
G L_{m}(\mathbb{R})=M_{m, m}(\mathbb{R}) \backslash \operatorname{det}^{-1}(0) \subset M_{m, m}(\mathbb{R})
$$

an open $m^{2}$-dimensional submanifold. 
Proposition 7.5 For $k \leqslant n \leqslant m$ the subset $R_{k} \subset M_{m, n}(\mathbb{R})$ consisting of the $m \times n$ matrices having rank exactly $k$ is an open submanifold of $M_{m, n}(\mathbb{R})$ of dimension

$$
\operatorname{dim}\left(R_{k}\right)=k(m+n-k) .
$$

Proof The $m \times n$ matrix

$$
J_{k}=\left(\begin{array}{cc}
I_{k} & 0 \\
0 & 0
\end{array}\right)
$$

has rank $k$. An $m \times n$ matrix $X$ has rank $k$ if and only if

$$
X=A J_{k} B^{-1}
$$

for some $A \in G L_{m}(\mathbb{R}), B \in G L_{n}(\mathbb{R})$. Now $A J_{k} B^{-1}=A^{\prime} J_{k} B^{\prime-1}$ if and only if

$$
\left(A^{\prime-1} A\right) J_{k}\left(B^{-1} B\right)^{-1}=J_{k},
$$

so that $R_{k}$ is the coset space

$$
R_{k}=G / H
$$

with

$$
\begin{aligned}
G & =G L_{m}(\mathbb{R}) \times G L_{n}(\mathbb{R}), \\
H & =\left\{(A, B) \in G \mid A J_{k} B^{-1}=J_{k}\right\} .
\end{aligned}
$$

Invertible matrices

$$
A=\left(\begin{array}{cc}
A_{1} & A_{2} \\
A_{3} & A_{4}
\end{array}\right) \in G L_{m}(\mathbb{R}), B=\left(\begin{array}{cc}
B_{1} & B_{2} \\
B_{3} & B_{4}
\end{array}\right) \in G L_{n}(\mathbb{R})
$$

with $A_{1}, B_{1} \in M_{k, k}(\mathbb{R})$ are such that $(A, B) \in H$ if and only if

$$
\begin{aligned}
& A_{1}=B_{1} \in G L_{k}(\mathbb{R}), A_{3}=0 \in M_{m-k, k}(\mathbb{R}), A_{4} \in G L_{m-k}(\mathbb{R}), \\
& B_{2}=0 \in M_{k, n-k}(\mathbb{R}), B_{4} \in G L_{m-k}(\mathbb{R}),
\end{aligned}
$$

so that $H$ is the subgroup of $G$ consisting of the pairs of the form

$$
(A, B)=\left(\left(\begin{array}{cc}
L & M \\
0 & N
\end{array}\right),\left(\begin{array}{cc}
L & 0 \\
P & Q
\end{array}\right)\right)
$$

with

$$
\begin{aligned}
& L \in G L_{k}(\mathbb{R}), M \in M_{k, m-k}(\mathbb{R}), \\
& N \in G L_{m-k}(\mathbb{R}), P \in M_{n-k, k}(\mathbb{R}), Q \in G L_{n-k}(\mathbb{R}) .
\end{aligned}
$$

Thus

$$
\begin{aligned}
\operatorname{dim}(G) & =m^{2}+n^{2}, \\
\operatorname{dim}(H) & =k^{2}+k(m-k)+(m-k)^{2}+(n-k) k+(n-k)^{2} \\
& =m^{2}+n^{2}-k(m+n-k),
\end{aligned}
$$

and

$$
\operatorname{dim}\left(R_{k}\right)=\operatorname{dim}(G)-\operatorname{dim}(H)=k(m+n-k) .
$$


Note that $k(m+n-k)$ is an increasing function of $k$ for $k \leqslant n \leqslant m$, so that

$$
\begin{aligned}
\operatorname{dim}\left(R_{0}\right)=0 & \leqslant \operatorname{dim}\left(R_{1}\right)=m+n-1 \leqslant \ldots \\
& \leqslant \operatorname{dim}\left(R_{n-1}\right)=(m+1)(n-1) \leqslant \operatorname{dim}\left(R_{n}\right)=m n .
\end{aligned}
$$

Example 7.6 (i) If $m=2 n$ then

$$
\operatorname{dim}\left(R_{n-2}\right)=2 n^{2}-2 n-4, \operatorname{dim}\left(R_{n-1}\right)=(2 n+1)(n-1) .
$$

(ii) If $m=2 n-1$ then

$$
\operatorname{dim}\left(R_{n-2}\right)=2 n^{2}-3 n-2, \operatorname{dim}\left(R_{n-1}\right)=2 n(n-1) .
$$

Here is a sketch proof of the Whitney Immersion Theorem (7.1):

Proposition 7.7 If $2 n \leqslant m$ every map $f: N^{n} \rightarrow M^{m}$ is arbitrarily close to an immersion $f^{\prime}: N \uparrow M$.

Sketch proof Arbitrarily close means that given a metric $d$ on $M$ and $\epsilon>0$ there is an immersion $f^{\prime}: N \leftrightarrow M$ such that

$$
d(f(x), g(x))<\epsilon \text { for all } x \in N .
$$

First, it is possible to approximate $f$ arbitrarily closely by a $C^{\infty}$ map, which will also be called $f$. Near each point of $N$ it can be assumed that local coordinates chosen, so that $f$ can be regarded as a differentiable map $f: \mathbb{R}^{n} \rightarrow \mathbb{R}^{m}$. The differential of $f$ is a differentiable map

$$
\text { df }: \mathbb{R}^{n} \rightarrow M_{m, n}(\mathbb{R})=R_{0} \cup R_{1} \cup \ldots \cup R_{n} ; x \mapsto(y \mapsto d f(x)(y))
$$

which is arbitrarily close to a map $F: \mathbb{R}^{n} \rightarrow M_{m, n}(\mathbb{R})$ which is transverse at each of the submanifolds $R_{k} \subset M_{m, n}(\mathbb{R})(0 \leqslant k \leqslant n-1)$ with

$$
\begin{aligned}
\operatorname{dim} F^{-1}\left(R_{k}\right) & =n-\left(m n-\operatorname{dim}\left(R_{k}\right)\right) \\
& \leqslant n-\left(m n-\operatorname{dim}\left(R_{n-1}\right)\right) \\
& =n-m n+(n-1)(m+1)=2 n-m-1<0 .
\end{aligned}
$$

Thus $d f$ is arbitrarily close to a map $F: \mathbb{R}^{n} \rightarrow M_{m, n}(\mathbb{R})$ with

$$
F\left(\mathbb{R}^{n}\right) \subseteq M_{m, n}(\mathbb{R}) \backslash\left(R_{0} \cup R_{1} \cup \ldots \cup R_{n-1}\right)=R_{n}
$$

(i.e. each $F(x): \mathbb{R}^{n} \rightarrow \mathbb{R}^{m}$ is injective). Any map $F$ with $F\left(\mathbb{R}^{n}\right) \subseteq R_{n}$ is the differential of an immersion $\mathbb{R}^{n} \rightarrow \mathbb{R}^{m}$. Also, the set of immersions is open in the $C^{r}$-topology (for any $r \geqslant 1$ ) on the set of differentiable functions $\mathbb{R}^{n} \rightarrow \mathbb{R}^{m}$. Hence, using standard pasting arguments the result follows.

Here is a sketch proof of the Whitney Embedding Theorem (7.2) (i) below the middle dimension : 
Proposition 7.8 If $2 n+1 \leqslant m$ every immersion $f: N^{n} \rightarrow M^{m}$ is arbitrarily close to an embedding $f^{\prime}: N \hookrightarrow M$.

Sketch proof The diagonal $\Delta(M) \subset M \times M$ is an $m$-dimensional submanifold of the $2 m$-dimensional manifold $M \times M$, and

$$
\operatorname{im}(f \times f: N \times N \rightarrow M \times M) \subset M \times M
$$

is a union of submanifolds of dimension $\leqslant 2 n$. As $m+2 n<2 m$ an arbitrarily small modification of $f$ will avoid $\Delta(M)$.

Definition 7.9 A double point $x=\left(x_{1}, x_{2}\right) \in S_{2}(f)$ of an immersion $f: N^{n}$ ৭ $M^{2 n}$ is transverse if the linear map of tangent spaces

$$
\left(d f\left(x_{1}\right) d f\left(x_{2}\right)\right): \tau_{N}\left(x_{1}\right) \oplus \tau_{N}\left(x_{2}\right) \rightarrow \tau_{M}(f(x))
$$

is an isomorphism.

The triple point set of a map $f: N \rightarrow M$ is defined by

$S_{3}(f)=\left\{\left(x_{1}, x_{2}, x_{3}\right) \in N \times N \times N \mid x_{i} \neq x_{j}\right.$ for $\left.i \neq j, f\left(x_{1}\right)=f\left(x_{2}\right)=f\left(x_{3}\right) \in M\right\}$.

Definition 7.10 An immersion $f: N^{n} \rightarrow M^{2 n}$ is self-transverse if it has no triple points, and only a finite number of transverse double points.

Proposition 7.11 Every immersion $f: N^{n} \rightarrow M^{2 n}$ is arbitrarily close to a self-transverse immersion.

Proof The triple point set $S_{3}(f)$ of an immersion $f: N^{n} \rightarrow M^{m}$ is generically $(3 n-2 m)$-dimensional, and $3 n-2 m<0$ in our case $m=2 n$.

By general position arguments it can be assumed that

$$
\operatorname{im}(f \times f: N \times N \uparrow M \times M) \cap \Delta(M)
$$

is a set of dimension 0 , so that $S_{2}(f)$ must be discrete. If there exists a double point which is not transverse then an arbitrarily small modification of $f$ will either remove the double point or change the map to one for which this rank condition holds.

See Theorems 2.12, 2.13 of Hirsch [33] for more detailed proofs of the Whitney Immersion and Embedding Theorems. 


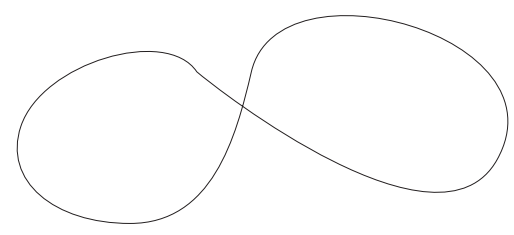

The figure 8 immersion $S^{1} \leftrightarrow S^{2}$ has the following high-dimensional generalisation to immersions with a single double point:

Proposition 7.12 For each $n \geqslant 1$ and $\epsilon= \pm 1$ there exists a null-homotopic self-transverse immersion $f_{n}^{\epsilon}: S^{n} \leftrightarrow S^{2 n}$ with an ordered double point $x^{\epsilon} \in$ $S_{2}\left(f_{n}^{\epsilon}\right)$ such that

$$
I\left(x^{\epsilon}\right)=\epsilon, S_{2}\left[f_{n}^{\epsilon}\right]=\left\{\left[x^{\epsilon}\right]\right\},
$$

and oriented normal bundle

$$
\nu_{f_{n}^{\epsilon}}=-\epsilon \tau_{S^{n}}: S^{n} \rightarrow B S O(n)
$$

Proof For $n=1$, take $f_{1}^{+}: S^{1} \rightarrow S^{2}$ to be the figure 8 immersion, and let $f_{1}^{-}=f_{1}^{+} r: S^{1} \leftrightarrow S^{2}$ with

$$
r: S^{1} \rightarrow S^{1} ;(\cos \theta, \sin \theta) \mapsto(\cos \theta,-\sin \theta)
$$

For $n \geqslant 2$ use the following construction of immersions $S^{n} \rightarrow \mathbb{R}^{2 n}$, which may be composed with the standard embedding $\mathbb{R}^{2 n} \hookrightarrow S^{2 n}$. Start with

$$
\begin{aligned}
& D_{1}^{n}=\left\{\left(x_{1}, \ldots, x_{n}, 0, \ldots, 0\right) \in \mathbb{R}^{2 n} \mid \sum_{i=1}^{n} x_{i}^{2} \leqslant 1\right\} \\
& D_{2}^{n}=\left\{\left(0, \ldots, 0, y_{1}, \ldots, y_{n}\right) \in \mathbb{R}^{2 n} \mid \sum_{i=1}^{n} y_{i}^{2} \leqslant 1\right\} .
\end{aligned}
$$

The intersections

$$
S^{2 n-1} \cap D_{1}^{n}=S_{1}^{n-1}, S^{2 n-1} \cap D_{2}^{n}=S_{2}^{n-1}
$$

are linked spheres in $S^{2 n-1}$. For $0 \leqslant t \leqslant 1$ let

$$
\phi_{t}=\left(\begin{array}{cc}
\cos (\pi t / 2) I_{n} & \sin (\pi t / 2) I_{n} \\
-\sin (\pi t / 2) I_{n} & \cos (\pi t / 2) I_{n}
\end{array}\right): \mathbb{R}^{2 n} \rightarrow \mathbb{R}^{2 n} .
$$

The embeddings

$$
\bar{\phi}_{t}: S^{n-1} \hookrightarrow \mathbb{R}^{2 n} ; x \mapsto \sqrt{1+\sin ^{2}(\pi t)} \cdot \phi_{t}(x) \quad(0 \leqslant t \leqslant 1)
$$

define an isotopy between $\bar{\phi}_{0}\left(S^{2 n-1}\right)=S_{1}^{2 n-1}$ and $\bar{\phi}_{1}\left(S^{2 n-1}\right)=S_{2}^{2 n-1}$ in the complement of the unit ball in $\mathbb{R}^{2 n}$. The map 
$f_{n}^{+}: S^{n}=D^{n} \times\{0,1\} \cup S^{n-1} \times I \rightarrow \mathbb{R}^{2 n} ;\left\{\begin{array}{l}\left(x_{1}, \ldots, x_{n}, 0\right) \mapsto\left(x_{1}, \ldots, x_{n}, 0, \ldots, 0\right) \\ \left(y_{1}, \ldots, y_{n}, 1\right) \mapsto\left(0, \ldots, 0, y_{1}, \ldots, y_{n}\right) \\ (z, t) \mapsto \bar{\phi}_{t}(z)\end{array}\right.$

is the desired immersion of $S^{n}$ in $\mathbb{R}^{2 n}$. To obtain the immersion $f_{n}^{-}$with a double point of complementary index modify $\phi_{t}$ to

$$
\phi_{t}^{\prime}=\mu_{t} \phi_{t} \text { where } \mu_{t}=\left(\begin{array}{cccc}
I_{n-1} & 0 & 0 & 0 \\
0 & \cos (\pi t) & \sin (\pi t) & 0 \\
0 & -\sin (\pi t) & \cos (\pi t) & 0 \\
0 & 0 & 0 & I_{n-1}
\end{array}\right) .
$$

\subsection{Algebraic and geometric intersections}

The homology intersection pairing (4.66) of an oriented cover $(\widetilde{M}, \pi, w)$ of an $m$-dimensional manifold $M$

$$
\lambda: H_{n}(\widetilde{M}) \times H_{m-n}(\widetilde{M}) \rightarrow \mathbb{Z}[\pi]
$$

will now be related to the geometric intersections of transverse submanifolds and immersed submanifolds. The two most important cases are:

(i) the identity cover, with $\widetilde{M}=M, \pi=\{1\}$,

(ii) the universal cover, with $\pi=\pi_{1}(M), w=w(M)$.

Definition 7.13 A $\pi$-trivial map $f: N^{n} \rightarrow M^{m}$ is a map from an oriented manifold $N$ such that the composite

$$
\pi_{1}(N) \stackrel{f_{*}}{\longrightarrow} \pi_{1}(M) \longrightarrow \pi
$$

is trivial, so that the pullback cover $\widetilde{N}=f^{*} \widetilde{M}$ of $N$ is trivial, together with a choice of $\pi$-equivariant lift $\widetilde{f}: \widetilde{N}=\pi \times N \rightarrow \widetilde{M}$ of $f$.

A $\pi$-trivial map $f: N^{n} \rightarrow M^{m}$ represents a homology class

$$
\widetilde{f}_{*}[N] \in H_{n}(\widetilde{M})
$$

which will usually be denoted $[N] \in H_{n}(\widetilde{M})$. The normal bundle $\nu_{f}: N \rightarrow$ $B O(m-n)$ of a $\pi$-trivial immersion $f: N^{n} \rightarrow M^{m}$ is oriented, since $\tau_{N}$ is oriented (by hypothesis),

$$
\tau_{N} \oplus \nu_{f}=f^{*} \tau_{M}: M \rightarrow B O(m)
$$

and 


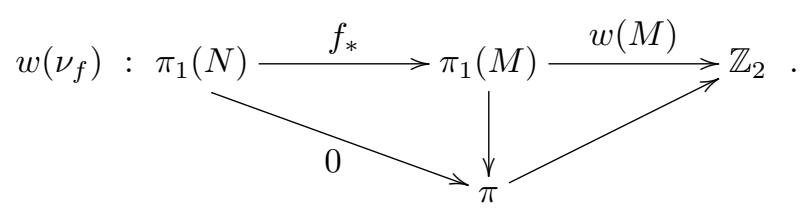

Definition 7.14 The algebraic intersection of $\pi$-trivial maps $f_{1}: N_{1}^{n_{1}} \rightarrow$ $M^{n_{1}+n_{2}}, f_{2}: N_{2}^{n_{2}} \rightarrow M^{n_{1}+n_{2}}$ is

$$
\lambda^{\text {alg }}\left(N_{1}, N_{2}\right)=\lambda\left(\left[N_{1}\right],\left[N_{2}\right]\right) \in \mathbb{Z}[\pi] .
$$

In terms of the $\mathbb{Z}[\pi]$-module Umkehr chain maps (4.67)

$$
\lambda^{a l g}\left(N_{1}, N_{2}\right)=\left(f_{1}^{!} \otimes f_{2}^{!}\right) \Delta[M] \in H_{0}\left(C\left(\tilde{N}_{1}\right)^{t} \otimes_{\mathbb{Z}[\pi]} C\left(\tilde{N}_{2}\right)\right)=\mathbb{Z}[\pi],
$$

with $C\left(\widetilde{N}_{1}\right)^{t}$ as defined in the proof of 4.58 (except that we are now working with the cellular chain complexes $C$ rather than the singular chain complexes $S$ ) and

$$
\left(f_{i}\right)^{!}: C(\widetilde{M}) \rightarrow C\left(\widetilde{N}_{i}\right)_{*-m+n_{i}} \quad(i=1,2) .
$$

In 7.22 below the algebraic intersection $\lambda^{\text {alg }}\left(N_{1}, N_{2}\right)$ of transverse immersions will be identified with the geometric intersection.

Example 7.15 Let $\eta: N \rightarrow B S O(n)$ be an oriented $n$-plane bundle over an oriented connected $n$-dimensional manifold $N$. The total pair of the fibre bundle

$$
\left(D^{n}, S^{n-1}\right) \rightarrow(D(\eta), S(\eta)) \rightarrow N
$$

is an oriented $2 n$-dimensional manifold with boundary

$$
(M, \partial M)=(D(\eta), S(\eta))
$$

The zero section of $\eta$ is an embedding

$$
f: N \hookrightarrow M=D(\eta) ; x \mapsto(x, 0)
$$

which is a homotopy equivalence with an Umkehr chain equivalence

$$
f^{!}: C(M, \partial M) \simeq C(M)^{2 n-*} \stackrel{f^{*}}{\longrightarrow} C(N)^{2 n-*} \simeq C(N)_{*-n}
$$

and

$$
\lambda^{a l g}(N, N)=\left(f^{!} \otimes f^{!}\right) \Delta_{(M, \partial M)}[M] \in H_{0}(N \times N)=\mathbb{Z}
$$

with $[M] \in H_{2 n}(M, \partial M)$ the fundamental class. The Umkehr chain equivalence is just the Thom chain equivalence

$$
f^{!}=U_{\eta} \cap-: \dot{C}(T(\eta))=C(M, \partial M) \stackrel{\simeq}{\longrightarrow} C(N)_{*-n}
$$


with a commutative diagram

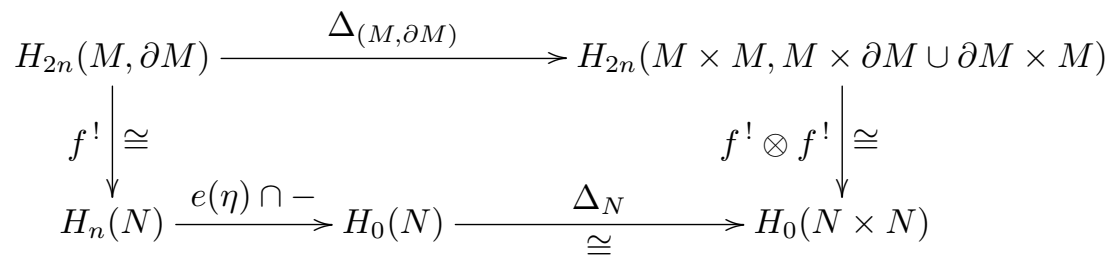

The algebraic intersection of $f: N \rightarrow M$ with itself is therefore the Euler number of $\eta$

$$
\lambda^{\text {alg }}(N, N)=\Delta_{N}(e(\eta) \cap[N])=\chi(\eta) \in H_{0}(N \times N)=\mathbb{Z},
$$

the image of the Euler class $e(\eta) \in H^{n}(N)$ under the Poincaré duality isomorphism

$$
[N] \cap-: H^{n}(N) \stackrel{\cong}{\longrightarrow} H_{0}(N)=\mathbb{Z}
$$

Example 7.16 For any $k \geqslant 1$ define the embedding of complex projective spaces

$$
N^{2 k}=\mathbb{C} \mathbb{P}^{k} \hookrightarrow M^{4 k}=\mathbb{C P}^{2 k} ;\left[z_{0}, z_{1}, \ldots, z_{k}\right] \mapsto\left[z_{0}, z_{1}, \ldots, z_{k}, 0, \ldots, 0\right]
$$

with $\left[z_{0}, z_{1}, \ldots, z_{k}\right] \subset \mathbb{C}^{k+1}$ the 1 -dimensional subspace spanned by $\left(z_{0}, z_{1}, \ldots, z_{k}\right) \in$ $\mathbb{C}^{k+1}$. The normal bundle

$$
\nu_{\mathbb{C} \mathbb{P}^{k} \hookrightarrow \mathbb{C} \mathbb{P}^{2 k}}=k \eta: \mathbb{C} \mathbb{P}^{k} \rightarrow B S O(2 k)
$$

is the Whitney sum of $k$ copies of the canonical (complex) line bundle

$$
\nu_{\mathbb{C} \mathbb{P}^{k} \hookrightarrow \mathbb{C} \mathbb{P}^{k+1}}=\eta: \mathbb{C P}^{k} \rightarrow B S O(2)=\mathbb{C} \mathbb{P}^{\infty}
$$

with $S(\eta)=S^{2 k+1}$, and

$$
[N]=1 \in H_{2 k}(M)=\mathbb{Z}, \lambda(N, N)=\chi(k \eta)=1 \in \mathbb{Z} .
$$

Definition 7.17 (i) The double point set of maps $f_{i}: N_{i} \rightarrow M(i=1,2)$ is defined by

$$
\begin{aligned}
S_{2}\left(f_{1}, f_{2}\right) & =\left\{\left(x_{1}, x_{2}\right) \in N_{1} \times N_{2} \mid f_{1}\left(x_{1}\right)=f_{2}\left(x_{2}\right) \in M\right\} \\
& =\left(f_{1} \times f_{2}\right)^{-1}(\Delta(M))
\end{aligned}
$$

with

$$
\Delta(M)=\{(x, x) \mid x \in M\} \subset M \times M
$$

the diagonal subspace.

(ii) A double point $x=\left(x_{1}, x_{2}\right) \in S_{2}\left(f_{1}, f_{2}\right)$ of immersions $f_{1}: N_{1}^{n_{1}} \rightarrow M^{n_{1}+n_{2}}$, $f_{2}: N_{1}^{n_{2}} \rightarrow M^{n_{1}+n_{2}}$ is transverse if the linear map

$$
d f(x)=\left(d f_{1}\left(x_{1}\right) d f_{2}\left(x_{2}\right)\right): \tau_{N_{1}}\left(x_{1}\right) \oplus \tau_{N_{2}}\left(x_{2}\right) \rightarrow \tau_{M}(f(x))
$$

is an isomorphism.

(iii) Immersions $f_{i}: N_{i}^{n_{i}} \rightarrow M^{n_{1}+n_{2}}(i=1,2)$ have transverse intersection (or are transverse) if each double point is transverse and $S_{2}\left(f_{1}, f_{2}\right)$ is finite. 
At a transverse double point $x=\left(x_{1}, x_{2}\right) \in S_{2}\left(f_{1}, f_{2}\right)$ of $\pi$-trivial immersions $f_{1}: N_{1}^{n_{1}} \rightarrow M^{n_{1}+n_{2}}, f_{2}: N_{2}^{n_{2}} \leftrightarrow M^{n_{1}+n_{2}}$ let $g(x) \in \pi$ be the unique covering translation $\widetilde{M} \rightarrow \widetilde{M}$ such that

$$
\widetilde{f}_{2}\left(x_{2}\right)=g(x) \tilde{f}_{1}\left(x_{1}\right) \in \widetilde{M} .
$$

The lifted immersions $g(x) \tilde{f}_{1}: N_{1} \rightarrow \widetilde{M}, \widetilde{f}_{2}: N_{2} \rightarrow \widetilde{M}$ have a transverse double point

$$
\widetilde{x}=\left(x_{1}, x_{2}\right) \in S_{2}\left(g(x) \tilde{f}_{1}, \tilde{f}_{2}\right)
$$

and there is defined an isomorphism of oriented $m$-dimensional vector spaces

$$
d \widetilde{f}(x)=\left(d\left(g(x) \tilde{f}_{1}\right) d \widetilde{f}_{2}\right): \tau_{N_{1}}\left(x_{1}\right) \oplus \tau_{N_{2}}\left(x_{2}\right) \rightarrow \tau_{\widetilde{M}}\left(\widetilde{f}_{1}\left(x_{1}\right)\right) .
$$

Definition 7.18 The equivariant index $I(x) \in \mathbb{Z}[\pi]$ of a transverse double point $x=\left(x_{1}, x_{2}\right) \in S_{2}\left(f_{1}, f_{2}\right)$ is

$$
I(x)=w(x) g(x) \in\{ \pm \pi\} \subset \mathbb{Z}[\pi]
$$

with

$$
w(x)= \begin{cases}+1 & \text { if } d \widetilde{f}(x) \text { preserves orientations } \\ -1 & \text { otherwise }\end{cases}
$$

Remark 7.19 (i) The definition of the double point set in 7.17 also applies in the case $f_{1}=f_{2}=f: N \rightarrow M$. In this case

$$
S_{2}(f, f)=\Delta(N) \cup S_{2}(f) \text { (disjoint union) }
$$

with $S_{2}(f)$ the ordered double point set of 7.3. The definition of the equivariant index $I(x) \in \mathbb{Z}[\pi]$ in 7.18 applies just as well to a transverse ordered double point $x=\left(x_{1}, x_{2}\right) \in S_{2}(f)$ of a $\pi$-trivial immersion $f: N^{n} \rightarrow M^{2 n}$.

(ii) The effect on the equivariant index of a change of order in the double point is given by

$$
I\left(x_{2}, x_{1}\right)=(-1)^{n_{1} n_{2}} \overline{I\left(x_{1}, x_{2}\right)} \in \mathbb{Z}[\pi]
$$

with $\mathbb{Z}[\pi] \rightarrow \mathbb{Z}[\pi] ; a \mapsto \bar{a}$ the $w$-twisted involution (4.55), since there is defined a commutative diagram of isomorphisms of oriented $m$-dimensional vector spaces

$$
\begin{aligned}
& \tau_{N_{1}}\left(x_{1}\right) \oplus \tau_{N_{2}}\left(x_{2}\right) \stackrel{\left(d \tilde{f}_{1} d\left(g(x)^{-1} \tilde{f}_{2}\right)\right)}{\longrightarrow} \tau_{\widetilde{M}}\left(\tilde{f}_{1}\left(x_{1}\right)\right) \\
& \left.\left(\begin{array}{ll}
0 & 1 \\
1 & 0
\end{array}\right)\right|_{\downarrow} \mid d g(x) \\
& \tau_{N_{2}}\left(x_{2}\right) \oplus \tau_{N_{1}}\left(x_{1}\right) \stackrel{\left(d \tilde{f}_{2} d\left(g(x) \tilde{f}_{1}\right)\right)}{\longrightarrow} \tau_{\widetilde{M}}\left(\tilde{f}_{2}\left(x_{2}\right)\right) .
\end{aligned}
$$

(iii) If $\widetilde{f}_{i}^{\prime}: N_{i} \leftrightarrow \widetilde{M}(i=1,2)$ are other lifts of $f_{i}$ let $h_{i} \in \pi$ be the unique elements such that 


$$
\widetilde{f}_{i}^{\prime}(y)=h_{i} \widetilde{f}_{i}(y) \in \widetilde{M} \text { for all } y \in N .
$$

The effect of changing the lifts is given by

$$
I^{\prime}(x)=h_{2} I(x) w\left(h_{1}\right) h_{1}^{-1} \in \mathbb{Z}[\pi] .
$$

Definition 7.20 The geometric intersection of transverse $\pi$-trivial immersions $f_{1}: N^{n_{1}} \leftrightarrow M^{n_{1}+n_{2}}, f_{2}: N_{2}^{n_{2}} \uparrow M^{n_{1}+n_{2}}$ is

$$
\lambda^{g e o}\left(N_{1}, N_{2}\right)=\sum_{x \in S_{2}\left(f_{1}, f_{2}\right)} I(x) \in \mathbb{Z}[\pi] .
$$

Likewise for a self-transverse $\pi$-trivial immersion $f: N^{n} \hookrightarrow M^{2 n}$

$$
\lambda^{g e o}(N, N)=\sum_{x \in S_{2}(f)} I(x) \in \mathbb{Z}[\pi] .
$$

For any immersion $f: N^{n} \hookrightarrow M^{m}$ there exists a differentiable function

$$
e: M \rightarrow \mathbb{R}^{p}=D^{p} \backslash S^{p-1}(p \text { large })
$$

such that the immersion

$$
f^{\prime}: N \leftrightarrow M \times D^{p} ; x \mapsto(f(x), e(x))
$$

is an embedding approximating $f$, with a regular homotopy to the immersion

$$
f \times 0: N \rightarrow M \times D^{p} ; x \mapsto(f(x), 0)
$$

and normal bundle

$$
\nu_{f^{\prime}}=\nu_{f \times 0}=\nu_{f} \oplus \epsilon^{p}: N \rightarrow B O(m-n+p) .
$$

Definition 7.21 (i) The stable $\pi$-equivariant geometric Umkehr map of an immersion $f: N^{n} \leftrightarrow M^{m}$

$$
F: \Sigma^{p} \widetilde{M}_{+} \rightarrow \Sigma^{p} T\left(\nu_{\widetilde{f}}\right)
$$

is defined by collapsing the complement of $E\left(\nu_{\widetilde{f}^{\prime}}\right) \subset \widetilde{M} \times D^{p}$

$$
\begin{aligned}
F: & \left(\widetilde{M} \times D^{p}\right) /\left(\widetilde{M} \times S^{p-1}\right)=\Sigma^{p} \widetilde{M}_{+} \\
& \rightarrow\left(\widetilde{M} \times D^{p}\right) /\left(\left(\widetilde{M} \times D^{p}\right) \backslash E\left(\nu_{\widetilde{f}^{\prime}}\right)\right)=T\left(\nu_{\widetilde{f}^{\prime}}\right)=\Sigma^{p} T\left(\nu_{\widetilde{f}}\right)
\end{aligned}
$$

with $\widetilde{M}_{+}=\widetilde{M} \cup\{$ pt. $\}$. The geometric Umkehr map $F$ induces the $\pi$-equivariant homology Umkehr map

$$
F_{*}=f^{\prime !}=f^{!}: \dot{H}_{*+p}\left(\Sigma^{p} \widetilde{M}_{+}\right)=H_{*}(\widetilde{M}) \rightarrow \dot{H}_{*+p}\left(T\left(\nu_{\widetilde{f}^{\prime}}\right)\right)=H_{*-m+n}(\widetilde{N}) \text {. }
$$


(ii) For an embedding $f: N \hookrightarrow M$ take $p=0$ in (i) to obtain the unstable $\pi$-equivariant geometric Umkehr map

$$
F=\text { projection }: \widetilde{M}_{+} \rightarrow \widetilde{M} /\left(\widetilde{M} \backslash E\left(\nu_{\widetilde{f}}\right)\right)=T\left(\nu_{\widetilde{f}}\right)
$$

which is just the $\pi$-equivariant map given by the Pontrjagin-Thom construction (6.8) for the embedding $\tilde{f}: \widetilde{N} \hookrightarrow \widetilde{M}$.

Proposition 7.22 The algebraic and geometric intersections of transverse $\pi$ trivial immersions $f_{i}: N_{1}^{n_{1}} \rightarrow M^{n_{1}+n_{2}}, f_{2}: N_{2}^{n_{2}} \rightarrow M^{n_{1}+n_{2}}$ coincide

$$
\lambda^{\text {alg }}\left(N_{1}, N_{2}\right)=\lambda^{g e o}\left(N_{1}, N_{2}\right) \in \mathbb{Z}[\pi] .
$$

Proof Approximate the immersions $f_{i}$ by embeddings

$$
f_{i}^{\prime}: N_{i} \hookrightarrow M \times D^{p_{i}} ; x_{i} \mapsto\left(f_{i}\left(x_{i}\right), e_{i}\left(x_{i}\right)\right) \quad\left(p_{i} \text { large }\right),
$$

which determine geometric Umkehr maps for $f_{i}$

$$
F_{i}: \Sigma^{p_{i}} \widetilde{M}_{+} \rightarrow \Sigma^{p_{i}} T\left(\nu_{\widetilde{f}_{i}}\right)(i=1,2) .
$$

Let $p=p_{1}+p_{2}$. The embedding

$$
\begin{aligned}
f^{\prime}: N=S_{2}\left(f_{1}, f_{2}\right) \hookrightarrow & M \times D^{p}=M \times D^{p_{1}} \times D^{p_{2}} ; \\
x=\left(x_{1}, x_{2}\right) & \mapsto\left(f(x), e_{1}\left(x_{1}\right), e_{2}\left(x_{2}\right)\right)
\end{aligned}
$$

approximates $f$, and determines a $\pi$-equivariant geometric Umkehr map $F$ : $\Sigma^{p} \widetilde{M}_{+} \rightarrow \Sigma^{p} T\left(\nu_{\widetilde{f}}\right)$ inducing the Umkehr $\mathbb{Z}[\pi]$-module chain map $f^{!}: C(\widetilde{M}) \rightarrow$ $C(\tilde{N})_{*-m+n}$. The Umkehr $\mathbb{Z}[\pi]$-module chain maps

$$
f_{i}^{!}: C(\widetilde{M}) \rightarrow C\left(\widetilde{N}_{i}\right)_{*+n_{i}-m} \quad(i=1,2)
$$

are induced by stable $\pi$-equivariant geometric Umkehr maps

$$
\begin{aligned}
F_{i}:\left(\widetilde{M} \times D^{p_{i}}\right) / & \left(\widetilde{M} \times S^{p_{i}-1}\right)=\Sigma^{p_{i}} \widetilde{M}_{+} \rightarrow \\
& \left(\widetilde{M} \times D^{p_{i}} \backslash E\left(\nu_{\widetilde{f}_{i}^{\prime}}\right)\right) /\left(\widetilde{M} \times S^{p_{i}-1}\right)=\Sigma^{p_{i}} T\left(\nu_{\widetilde{f}_{i}}\right) .
\end{aligned}
$$

The commutative square of geometric Umkehr and diagonal maps

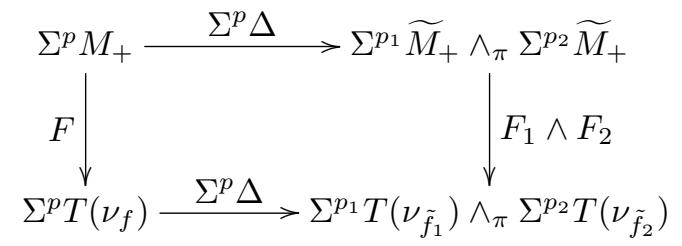

induces a commutative square of morphisms in homology 


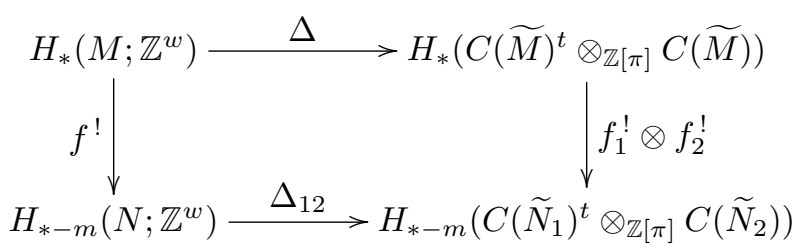

with

$$
\Delta_{12}: N=\tilde{N} / \pi=S_{2}\left(f_{1}, f_{2}\right) \hookrightarrow \widetilde{N}_{1} \times_{\pi} \tilde{N}_{2}
$$

the inclusion. (Terminology: If a group $\pi$ acts on spaces $X, Y$ then

$$
X \times_{\pi} Y=(X \times Y) /\{(x, y) \sim(g x, g y) \mid g \in \pi, x \in X, y \in Y\} .
$$

If a group $\pi$ acts on pointed spaces $X, Y$ by base point preserving maps then

$$
X \wedge_{\pi} Y=(X \wedge Y) /\{(x, y) \sim(g x, g y) \mid g \in \pi, x \in X, y \in Y\} .
$$

The smash product of pointed spaces $X, Y$ is the pointed space

$$
X \wedge Y=(X \times Y) /\left(X \times\left\{y_{0}\right\} \cup\left\{x_{0}\right\} \times Y\right),
$$

with $x_{0} \in X, y_{0} \in Y$ the base points.) The evaluation of the fundamental class $[M] \in H_{m}\left(M ; \mathbb{Z}^{w}\right)$ both ways round the square gives

$$
\begin{aligned}
\lambda^{\operatorname{alg}\left(N_{1}, N_{2}\right)} & =\left(f_{1}^{!} \otimes f_{2}^{!}\right) \Delta[M]=\Delta_{12} f^{!}[M]=\Delta_{12}[N] \\
& =\lambda^{g e o}\left(N_{1}, N_{2}\right) \in \mathbb{Z}[\pi] .
\end{aligned}
$$

Example 7.23 For any oriented manifolds $N_{1}^{n_{1}}, N_{2}^{n_{2}}$ and points $x_{1} \in N_{1}, x_{2} \in$ $N_{2}$ the submanifolds

$$
N_{1}^{n_{1}}=N_{1} \times\left\{x_{2}\right\}, N_{2}^{n_{2}}=\left\{x_{1}\right\} \times N_{2} \subseteq M^{n_{1}+n_{2}}=N_{1} \times N_{2}
$$

are transverse with a single point of intersection

$$
\left(N_{1} \times\left\{x_{2}\right\}\right) \cap\left(\left\{x_{1}\right\} \times N_{2}\right)=\left\{\left(x_{1}, x_{2}\right)\right\} \subseteq N_{1} \times N_{2},
$$

and 7.22 applies with

$$
\lambda^{\text {alg }}\left(N_{1}, N_{2}\right)=\lambda^{g e o}\left(N_{1}, N_{2}\right)=1 \in \mathbb{Z} .
$$

Proposition 7.24 For any $\pi$-trivial immersions of manifolds $f_{1}: N_{1}^{n_{1}} \leftrightarrow M^{n_{1}+n_{2}}$, $f_{2}: N_{2}^{n_{2}} \rightarrow M^{n_{1}+n_{2}}$ there exists a regular homotopy $f_{2} \simeq f_{2}^{\prime}: N_{2}=N_{2}^{\prime} \rightarrow M$ such that $f_{1}, f_{2}^{\prime}$ are transverse, with

$$
\lambda^{\text {alg }}\left(N_{1}, N_{2}\right)=\lambda^{\text {alg }}\left(N_{1}, N_{2}^{\prime}\right)=\lambda^{g e o}\left(N_{1}, N_{2}^{\prime}\right) \in \mathbb{Z}[\pi] .
$$

If $f_{1}, f_{2}$ are embeddings the regular homotopy can be chosen to be an isotopy. 
Proof Adapt Corollary II.15.6 of Bredon [10] (which deals with embeddings) to immersions.

Proposition 7.25 (i) The algebraic self-intersection of a $\pi$-trivial embedding $f: N^{n} \hookrightarrow M^{2 n}$ is the Euler number (5.44) of the normal n-plane bundle $\nu_{N \hookrightarrow M}$ : $N \rightarrow B S O(n)$

$$
\lambda^{a l g}(N, N)=\chi\left(\nu_{N \hookrightarrow M}\right) \in \mathbb{Z} \hookrightarrow \mathbb{Z}[\pi] .
$$

(ii) For any oriented n-plane bundle $\eta: N^{n} \rightarrow B S O(n)$ over an $n$-dimensional manifold $N$ the zero section in the $D^{n}$-bundle

$$
N \hookrightarrow D(\eta) ; x \mapsto(x, 0)
$$

is isotopic to a submanifold $N^{\prime} \subset D(\eta)$ intersecting $N$ transversely in $\chi(\eta)$ points (counted algebraically), so that the algebraic self-intersection of $N$ in $D(\eta)$ is given by

$$
\lambda^{\operatorname{alg}}(N, N)=\lambda^{g e o}\left(N, N^{\prime}\right)=\chi(\eta) \in \mathbb{Z} .
$$

Proof (i) Use the Tubular Neighbourhood Theorem (5.50) to embed the total space of the normal bundle $\nu_{N \hookrightarrow M}: N \rightarrow B S O(n)$ as a codimension 0 submanifold $E\left(\nu_{N \hookrightarrow M}\right) \subseteq M$, and apply 7.15.

(ii) Apply 7.22 (with $\pi=\{1\}$ ), 7.24 and (i), noting that isotopic embeddings have isomorphic normal bundles.

Example 7.26 For any $\pi$-trivial immersion $f: N^{n} \uparrow M^{2 n}$ take

$$
f_{1}=f_{2}=f: N_{1}=N_{2}=N \rightarrow M
$$

in 7.24, to obtain a regular homotopic immersion $f^{\prime}: N \hookrightarrow \rightarrow M$ such that $f$ and $f^{\prime}$ are transverse, with

$$
\lambda^{a l g}(N, N)=\lambda^{a l g}\left(N, N^{\prime}\right)=\lambda^{g e o}\left(N, N^{\prime}\right) \in \mathbb{Z}[\pi]
$$

\subsection{The Whitney trick}

Whitney trick for removing double points 7.27 ([99])

(i) Let $f_{1}: N_{1}^{n_{1}} \hookrightarrow M^{m}, f_{2}: N_{2}^{n_{2}} \hookrightarrow M^{m}$ be $\pi_{1}(M)$-trivial embeddings with $n_{1}+n_{2}=m$ and $N_{1}, N_{2}$ connected, such that

$$
\begin{array}{ll}
\text { either } & n_{1}, n_{2} \geqslant 3, \\
\text { or } & n_{1}=2, n_{2} \geqslant 3 \text { with } \pi_{1}(M) \cong \pi_{1}\left(M \backslash N_{1}\right) .
\end{array}
$$


Given two transverse double points

$$
x=\left(x_{1}, x_{2}\right), y=\left(y_{1}, y_{2}\right) \in S_{2}\left(f_{1}, f_{2}\right)
$$

such that

$$
I(x)=-I(y) \in \mathbb{Z}\left[\pi_{1}(M)\right] .
$$

There exists an isotopy of $f_{1}$ to an embedding $f_{1}^{\prime}: N_{1} \hookrightarrow M$ with double point set

$$
S_{2}\left(f_{1}^{\prime}, f_{2}\right)=S_{2}\left(f_{1}, f_{2}\right) \backslash\{[x],[y]\} .
$$

(ii) Let $f: N^{n} \leftrightarrow M^{2 n}$ be a $\pi_{1}(M)$-trivial immersion with $n \geqslant 3$, with $N$ connected. Given two transverse ordered double points

$$
x=\left(x_{1}, x_{2}\right), y=\left(y_{1}, y_{2}\right) \in S_{2}(f)
$$

such that

$$
I(x)=-I(y) \in \mathbb{Z}\left[\pi_{1}(M)\right], \quad[x] \neq[y] \in S_{2}[f]
$$

there exists a regular homotopy of $f$ to an immersion $f^{\prime}: N^{n} \rightarrow M^{2 n}$ with unordered double point set

$$
S_{2}\left[f^{\prime}\right]=S_{2}[f] \backslash\{[x],[y]\}
$$

Proof See Theorem 6.6 of Milnor [57] for case (i), generalising the removal of transverse double points of curves in the plane by sliding over the closed area bounded by the portions of the curves between the double points.

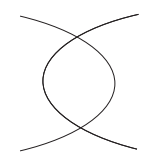

The condition $I(x)=-I(y)$ means that

$$
w(x)=-w(y) \in\{ \pm 1\}, g(x)=g(y) \in \pi_{1}(M)
$$

and the dimension conditions ensure the existence of a 'Whitney disc' $D^{2} \subseteq M$. The proof of case (ii) is essentially the same.

The following proof (of (ii)) is due to Jim Milgram, and is a bundle-theoretic interpretation of the original construction ([99]).

Choose differentiable maps

$$
\gamma_{i}: I=[0,1] \rightarrow N \quad(i=1,2)
$$

with endpoints

$$
\gamma_{i}(0)=x_{i}, \gamma_{i}(1)=y_{i},
$$


such that both curves avoid the double points of $f$ except at their endpoints. Consequently, the map

$$
\omega: S^{1}=I /\{0 \sim 1\} \rightarrow M^{2 n} ; t \mapsto \begin{cases}f \gamma_{1}(2 t) & \text { if } 0 \leqslant t \leqslant 1 / 2 \\ f \gamma_{2}(2(1-t)) & \text { if } 1 / 2 \leqslant t \leqslant 1\end{cases}
$$

is an embedding which is differentiable except at two points. Consider the pullback $\omega^{*} \tau_{M}: S^{1} \rightarrow B O(m)$ of the tangent bundle $\tau_{M}: M \rightarrow B O(m)$. Let

$$
g=g(x)=g(y) \in \pi_{1}(M)
$$

and lift $\omega$ to an embedding in the universal cover $\widetilde{M}$ of $M$

$$
\tilde{\omega}: S^{1}=I /\{0 \sim 1\} \rightarrow \widetilde{M} ; t \mapsto \begin{cases}g \widetilde{f} \gamma_{1}(2 t) & \text { if } 0 \leqslant t \leqslant 1 / 2 \\ \widetilde{f} \gamma_{2}(2(1-t)) & \text { if } 1 / 2 \leqslant t \leqslant 1\end{cases}
$$

such that

$$
\omega: S^{1} \stackrel{\tilde{\omega}}{\rightarrow} \widetilde{M} \rightarrow M .
$$

Now $\widetilde{M}$ is simply connected, so that both $\tilde{\omega}$ and $\omega$ are null-homotopic, and the pullback bundle

$$
\tilde{\omega}^{*} \tau_{\widetilde{M}}=\omega^{*} \tau_{M}: S^{1} \rightarrow B O(2 n)
$$

is trivial. Choose a metric on $M^{m}$ so that at the double point $x=\left(x_{1}, x_{2}\right) \in S_{2}(f)$ the subspaces

$$
d f\left(x_{1}\right) \tau_{N}\left(x_{1}\right)^{n}, d f\left(x_{2}\right) \tau_{N}\left(x_{2}\right)^{n} \subseteq \tau_{M}(f(x))^{2 n}
$$

are orthogonal, and similarly at the double point $y \in S_{2}(f)$. This gives a splitting of the orientable $2 n$-plane bundle

$$
\omega^{*} \tau_{M}=\alpha_{1} \oplus \alpha_{2}: S^{1} \rightarrow B O(2 n)
$$

as a Whitney sum of nonorientable bundles

$$
\alpha_{1}=\left.\left.\tau_{N}\right|_{\gamma_{1}} \cup \nu_{f}\right|_{\gamma_{2}}, \alpha_{2}=\left.\left.\nu_{f}\right|_{\gamma_{1}} \cup \tau_{N}\right|_{\gamma_{2}}: S^{1} \rightarrow B O(n) .
$$

The condition $I(x)=-I(y)$ is exactly what is needed to ensure that

$$
\alpha_{i} \cong \mu \oplus \epsilon^{n-1}
$$

with $\mu$ the nonorientable line bundle over $S^{1}$.

Next, extend the 1-dimensional tangent section along $\gamma_{1}$ to a Möbius band section $\beta_{1} \hookrightarrow \alpha_{1}$ and the 1-dimensional tangent section on $\gamma_{2}$ to a 1-dimensional Möbius band section $\beta_{2} \hookrightarrow \alpha_{2}$, defining non-trivial line bundles $\beta_{1}, \beta_{2}: S^{1} \rightarrow$ $B O(1)\left(\right.$ with $\left.\beta_{1} \cong \beta_{2} \cong \mu\right)$ such that

$$
\alpha_{1}=\beta_{1} \oplus \epsilon^{n-1}, \alpha_{2}=\beta_{2} \oplus \epsilon^{n-1}: S^{1} \rightarrow B O(n) .
$$

Moreover

$$
\beta_{1} \oplus \beta_{2}=\epsilon^{2}: S^{1} \rightarrow B O(2),
$$

since $\beta_{1}, \beta_{2}$ are perpendicular at every point. This defines, using the Tubular Neighbourhood Theorem (5.50), an embedding $D^{2} \times S^{1} \hookrightarrow M^{2 n}$ extending $\omega\left(S^{1}\right)$ 
which we can use to construct a differentiable embedding $D^{1} \times S^{1} \hookrightarrow M^{2 n}$ containing $\omega\left(S^{1}\right)$ in its interior and contained in the image of $D^{2} \times S^{1}$. We regard this $D^{1} \times S^{1}$ as a closed collar neighborhood of the boundary in the unit ball $D^{2} \subset \mathbb{R}^{2}$, and we now want to extend this embedding to an embedding $D^{2} \subset M^{2 n}$.

Lemma 7.28 Let $f: N^{n} \rightarrow M^{m}$ be an immersion with image $V=f(N) \subseteq M$. If $m \geqslant 5$ and $m-n \geqslant 3$ then

$$
\pi_{1}(M \backslash V)=\pi_{1}(M) .
$$

Proof The morphism $\pi_{1}(M \backslash V) \rightarrow \pi_{1}(M)$ induced by inclusion is surjective, since every map $S^{1} \rightarrow M$ can be moved away from $V$ by general position. In order to prove that the morphism is injective consider an element $x \in \operatorname{ker}\left(\pi_{1}(M \backslash V) \rightarrow\right.$ $\pi_{1}(M)$ ), which may be represented by a commutative square

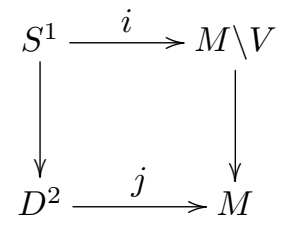

with $i$ an embedding. Since $m \geqslant 5 j$ is homotopic to an embedding (leaving the embedding $i$ fixed). Now ensure that $V \cap j\left(D^{2}\right)=\emptyset$. By general position move $j\left(D^{2}\right)$ away from $V$ by an arbitrarily small perturbation leaving $j$ an embedding, and leaving $i$ alone on $S^{1}$. The result is an embedded $j\left(D^{2}\right) \subset M \backslash V$ with $\partial\left(j\left(D^{2}\right)\right)=i\left(S^{1}\right)$, so that $x=1 \in \pi_{1}(M \backslash V)$.

Returning to the proof of 7.27 , apply 7.28 to obtain a differentiably embedded disk $D^{2} \subseteq M \backslash f(N)$ with $\partial\left(D^{2}\right)$ one of the two boundary components of the embedded $D^{1} \times S^{1}$ above. It can even be arranged that this disk together with the $S^{1} \times D^{1}$ gives an embedding $D^{2} \hookrightarrow M$ including $\gamma$ in its interior and intersecting $f(N)$ only in a small extension of $\gamma$ beyond the corner points.

The obstruction to extending the splitting $\left.\nu_{D^{2} \hookrightarrow M}\right|_{\gamma}=\epsilon^{n-1} \oplus \epsilon^{n-1}$ to the entire normal bundle of $D^{2}$ is an element in $\pi_{1}(O(2(n-1)))=\mathbb{Z}_{2}$. Moreover, $\pi_{1}(O(n-1))$ maps onto this group since $n \geqslant 3$. Thus the framing can be changed on one of the two $\epsilon^{n-1}$ 's over $S^{1}$ to extend the framing across $D^{2}$.

Finally, leave the immersion alone near $\gamma_{1}$ in $N$ and change it near $\gamma_{2}$ using the isotopy given on p. 74 of Milnor [57]. This defines a regular homotopy of $f: N \leftrightarrow M$ to an immersion without this pair of unordered double points, completing the proof of 7.27 .

The Whitney trick in the embedding case (i) is the key ingredient in the handle cancellation of the $(m+1)$-dimensional $h$ - and $s$-Cobordism Theorems $(m \geqslant 5)-$ see Chapter 8 . 
The Whitney trick in the immersion case (ii) is the essential geometric ingredient of the surgery classification theory of manifolds of dimension $m \geqslant 5$.

Remark 7.29 In certain cases it is also possible to perform the Whitney trick for $m=4$, and so extend the theory to 4-dimensional manifolds. But this is much harder - see Freedman [25], Freedman and Quinn [26].

In order to kill an element $x \in \pi_{n}(M)$ by surgery on a $2 n$-dimensional manifold $M^{2 n}$ it is necessary to represent $x$ by an embedding $S^{n} \hookrightarrow M^{2 n}$ with trivial normal bundle. By Proposition 7.11 it is possible to represent $x$ by a selftransverse immersion $S^{n} \uparrow M^{2 n}$. In order to further deform such an immersion to an embedding it is necessary to remove the double points.

The Whitney trick gives the geometric realizability of algebraic intersections :

Corollary 7.30 Let $f_{1}: N_{1}^{n_{1}} \hookrightarrow M^{n_{1}+n_{2}}, f_{2}: N_{2}^{n_{2}} \hookrightarrow M^{n_{1}+n_{2}}$ be $\pi_{1}(M)$-trivial embeddings with algebraic intersection

$$
\lambda^{a l g}\left(N_{1}, N_{2}\right)=\sum_{g \in \pi_{1}(M)} a_{g} g \in \mathbb{Z}\left[\pi_{1}(M)\right] \quad\left(a_{g} \in \mathbb{Z}\right)
$$

If the dimension hypotheses of Theorem 7.27 are satisfied (either $n_{1} \geqslant 3, n_{2} \geqslant 3$ or $\left.n_{1}=2, n_{2} \geqslant 3, \pi_{1}\left(M \backslash f_{1}\left(N_{1}\right)\right)=\pi_{1}(M)\right)$ there is an isotopy of $f_{2}$ to an embedding $f_{2}^{\prime}: N_{2}=N_{2}^{\prime} \hookrightarrow M$ such that $f_{1}, f_{2}^{\prime}$ are transverse and $S_{2}\left(f_{1}, f_{2}^{\prime}\right)=$ $N_{1} \cap N_{2}^{\prime}$ has exactly $\sum_{g \in \pi_{1}(M)}\left|a_{g}\right|$ points.

Proof By 7.24 it may be assumed that $f_{1}, f_{2}$ intersect transversely, so that by 7.22

$$
\lambda^{\text {alg }}\left(N_{1}, N_{2}\right)=\lambda^{g e o}\left(N_{1}, N_{2}\right) \in \mathbb{Z}\left[\pi_{1}(M)\right]
$$

and

$$
\sum_{x \in S_{2}\left(f_{1}, f_{2}\right)} I(x)=\sum_{g \in \pi_{1}(M)} a_{g} g \in \mathbb{Z}\left[\pi_{1}(M)\right] .
$$

For each $g \in \pi_{1}(M)$

$$
\left|\left\{x \in S_{2}\left(f_{1}, f_{2}\right) \mid g(x)=g\right\}\right|=\left|a_{g}\right|+2 b_{g} \geqslant 0
$$

for some $b_{g} \geqslant 0$. There are $b_{g}$ pairs of algebraically cancelling double points, which can be geometrically cancelled by 7.27 (i).

The Whitney trick is necessary for the proof of the middle-dimensional Embedding Theorem 7.2 (ii) : 
Corollary 7.31 If $n \geqslant 3$ and $\pi_{1}(M)=\{1\}$ every map $f: N^{n} \rightarrow M^{2 n}$ is homotopic to an embedding.

Proof By 7.11 it may be assumed that $f$ is a self-transverse immersion. Define the self-intersection number of $f$ by algebraically counting the unordered double points

$$
\mu(f)=\sum_{x \in S_{2}[f]} I(x) \in Q_{(-1)^{n}}(\mathbb{Z})=\mathbb{Z} /\left\{1-(-1)^{n}\right\}= \begin{cases}\mathbb{Z} & \text { if } n \text { is even } \\ \mathbb{Z}_{2} & \text { if } n \text { is odd }\end{cases}
$$

(See Chapter 11 for a more extensive account of the self-intersections of an immersion $\left.N^{n} \rightarrow M^{2 n}\right)$. Let $\epsilon \in\{ \pm 1\}$ be the sign of $-\mu(f)$ for $n$ even, and $\epsilon=-1$ for $n$ odd. The connected sum of $f$ and $\mu(f)$ copies of the standard single double point immersion $f_{n}^{\epsilon}: S^{n} \uparrow S^{2 n}(7.12)$ is an immersion

$$
f^{\prime}=f \# \mu(f) f_{n}^{\epsilon}: N=N \# S^{n} \rightarrow M=M \# S^{2 n}
$$

which is homotopic to $f$ with

$$
\mu\left(f^{\prime}\right)=0 \in Q_{(-1)^{n}}(\mathbb{Z})
$$

The unordered double points of $f^{\prime}$ can be paired off in algebraically cancelling pairs. These can be cancelled by 7.27 (ii), so that $f^{\prime}$ is regular homotopic to an immersion $f^{\prime \prime}: N \leftrightarrow M$ with no double points, i.e. an embedding.

The self-intersections of immersions $f: N^{n} \rightarrow M^{2 n}$ in the non-simplyconnected case $\pi_{1}(M) \neq\{1\}$ will be considered in Chapter 11 .

\subsection{The Smale-Hirsch classification of immersions}

The Theorem identifies the regular homotopy classes of immersions $N^{n} \rightarrow M^{m}$ for $n<m$ with the homotopy classes of injective bundle maps $\tau_{N} \rightarrow \tau_{M}$. See Smale [81] and Hirsch [32] for the original sources, and Adachi [1] for a more recent account of immersion theory.

Definition 7.32 (i) For any $m$-dimensional manifold $M$ and $n \leqslant m$ the $n$ frame bundle $F_{n}(M)$ is the Stiefel $n$-frame bundle (5.24) of the tangent bundle $\tau_{M}$

$$
V_{m, n} \rightarrow F_{n}(M)=V_{n}\left(\tau_{M}\right) \rightarrow M
$$

with total space

$$
V_{n}\left(\tau_{M}\right)=\bigcup_{x \in M} V_{n}\left(\tau_{M}(x)\right)
$$

and fibre the Stiefel manifold of orthonormal $n$-frames in $\mathbb{R}^{m}$ 


$$
V_{m, n}=V_{n}\left(\mathbb{R}^{m}\right)=O(m) / O(m-n) .
$$

In particular, for $m=n$ this is the principal $O(m)$-bundle of $m$-frames in $\tau_{M}$

$$
V_{m, m}=O(m) \rightarrow F_{m}(M) \rightarrow M
$$

(ii) For any $m$-dimensional manifold $M$ and $n$-dimensional manifold $N$ with $n \leqslant m$ the frame bundle $F(N, M)$ is the fibre bundle

$$
F_{n}(M) \rightarrow F(N, M)=F_{n}(N) \times_{O(n)} F_{n}(M) \rightarrow F_{n}(N) / O(n)=N .
$$

Example 7.33 For any $n \geqslant 1$ there is a natural identification

$$
F_{n}\left(S^{n}\right)=O(n+1) .
$$

An element $(x, a) \in F_{n}\left(S^{n}\right)$ is given by $x \in S^{n}$ together with an orthonormal $n$-frame $a=\left(a_{1}, a_{2}, \ldots, a_{n}\right)$ of

$$
\tau_{S^{n}}(x)=\operatorname{span}(x)^{\perp} \subseteq \mathbb{R}^{n+1},
$$

which can be identified with an orthonormal $(n+1)$-frame $\left(a_{1}, a_{2}, \ldots, a_{n}, x\right)$ of

$$
\tau_{S^{n}}(x) \oplus \operatorname{span}(x)=\mathbb{R}^{n+1},
$$

and hence with an element of $O(n+1)$.

Proposition 7.34 Let $n \leqslant m$.

(i) For any $m$-dimensional manifold $M$ there is defined a homotopy commutative braid of fibration sequences

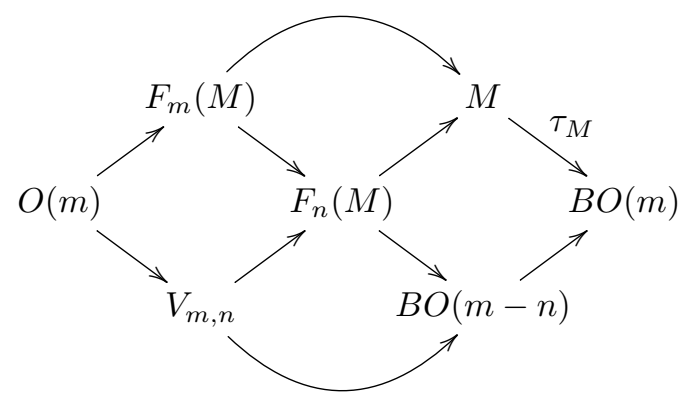

(ii) For any $n$-dimensional manifold $N$ there is defined a fibre bundle

$$
V_{m, n} \rightarrow V_{m, n} \times_{O(n)} F_{n}(N) \rightarrow N
$$

which fits into a homotopy commutative braid of fibration sequences 


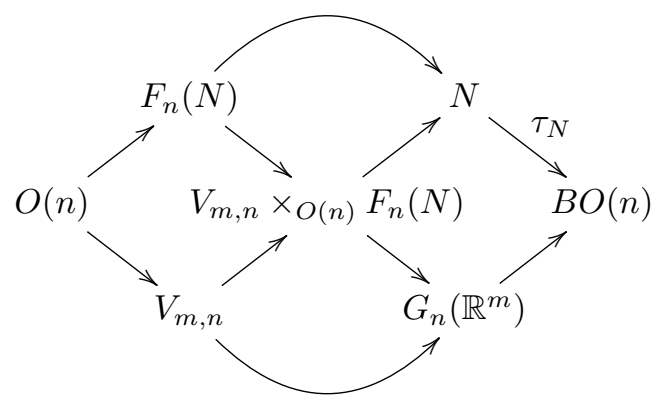

with $G_{n}\left(\mathbb{R}^{m}\right)=V_{m, n} / O(n)$ the Grassmann manifold (5.22) of $n$-dimensional subspaces of $\mathbb{R}^{m}$.

(iii) For any $m$-dimensional manifold $M$ and $n$-dimensional manifold $N$ there is defined a homotopy commutative braid of fibration sequences

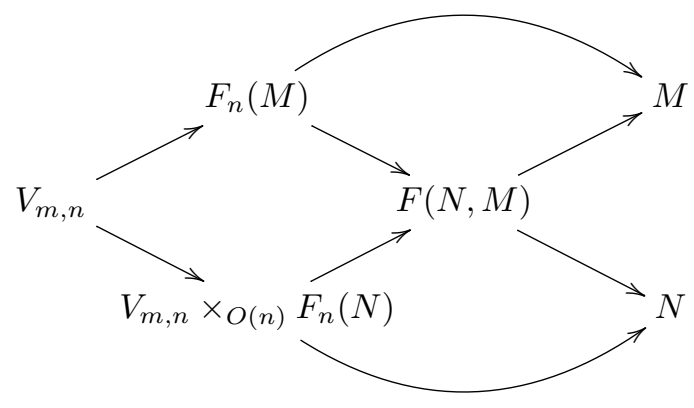

Smale-Hirsch Immersion Classification Theorem 7.35 ([81], [32])

Let $M^{m}, N^{n}$ be manifolds, with $1 \leqslant n<m$. The following sets of equivalence classes are in one-one correspondence:

(i) the regular homotopy classes of immersions $f: N \leftrightarrow M$,

(ii) the homotopy classes of injective bundle maps $\tau_{N} \rightarrow \tau_{M}$,

(iii) the homotopy classes of sections of the frame bundle $F_{n}(M) \rightarrow F(N, M) \stackrel{p}{\rightarrow} N$, i.e. maps $s: N \rightarrow F(N, M)$ such that $p s=1: N \rightarrow N$.

Sketch proof. In fact, only the correspondences will be stated.

An element $[x, y, a, b] \in F(N, M)$ is an $O(n)$-equivalence of quadruples

$$
x \in M, y \in N, a \in V_{n}\left(\tau_{M}(x)\right), b \in V_{n}\left(\tau_{N}(y)\right)
$$

with $\omega \in O(n)$ acting by

$$
\omega(x, y, a, b)=(x, y, \omega(a), \omega(b)) .
$$

Define vector bundles over $F(N, M)$ 


$$
\begin{aligned}
& \alpha: F(N, M) \rightarrow N \stackrel{\tau_{N}}{\rightarrow} B O(n), \\
& \beta: F(N, M) \rightarrow B O(m-n), \\
& \gamma: F(N, M) \rightarrow B O(m)
\end{aligned}
$$

with total spaces

$$
\begin{aligned}
& E(\alpha)=\bigcup_{[x, y, a, b] \in F(N, M)} \operatorname{span}(a), \\
& E(\beta)=\bigcup_{[x, y, a, b] \in F(N, M)} \operatorname{span}(a)^{\perp}, \\
& E(\gamma)=\bigcup_{[x, y, a, b] \in F(N, M)} \tau_{M}(x)
\end{aligned}
$$

and such that

$$
\begin{aligned}
& \alpha \oplus \beta=\gamma: F(N, M) \rightarrow B O(m), \\
& \alpha: F(N, M) \rightarrow N \stackrel{\tau_{N}}{\rightarrow} B O(n) .
\end{aligned}
$$

(i) $\Longleftrightarrow$ (ii) The differential of an immersion $f: N \rightarrow M$ is an injective bundle map $d f: \tau_{N} \rightarrow \tau_{M}$.

(i) $\Longrightarrow$ (iii) An immersion $f: N \hookrightarrow M$ determines the section

$$
s: N \rightarrow F(N, M) ; y \mapsto[f(y), y, d f(y)(b), b]
$$

of the frame bundle, for an arbitrary $b \in V_{n}\left(\tau_{N}(y)\right)$. The pullbacks of $\alpha, \beta, \gamma$ along $s$ are then such that

$$
\begin{aligned}
& s^{*} \alpha=\tau_{N}: N \rightarrow B O(n), \\
& s^{*} \beta=\nu_{f}: N \rightarrow B O(m-n), \\
& s^{*} \gamma=f^{*} \tau_{M}: N \rightarrow B O(m) .
\end{aligned}
$$

(iii) $\Longrightarrow$ (i) A section $s: N \rightarrow F(N, M)$ determines an immersion $f: N \hookrightarrow M$ given up to regular homotopy by

$$
f: N \stackrel{s}{\longrightarrow} F(N, M) \longrightarrow M
$$

with a (homotopy) commutative diagram

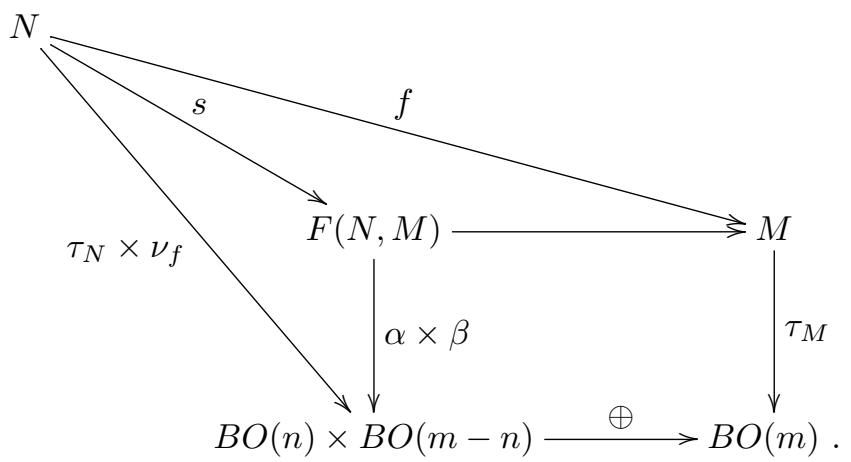


The Immersion Classification Theorem (7.35) relates the regular homotopy groups of immersions $S^{n} \rightarrow M^{m}$ for $n \leqslant m-2$ to the homotopy groups of $M$ and of the stable Stiefel space $V_{m-n}$.

We shall also need the regular homotopy groups of framed immersions:

Definition 7.36 The $n$-dimensional framed regular homotopy group $I_{n}^{f r}(M)$ is the $\mathbb{Z}\left[\pi_{1}(M)\right]$-module of regular homotopy classes of framed immersions $f$ : $S^{n} \times D^{m-n} \rightarrow M^{m}$, with addition by connected sum.

Definition 7.37 The stable Stiefel space is defined for $d \geqslant 0$ to be

$$
V_{d}=\bigcup_{k} V_{d+k, k}
$$

the space of orthonormal $k$-frames in $\mathbb{R}^{d+k}$ ( $k$ large).

Lemma 7.38 The stable Stiefel space $V_{d}$ is $(d-1)$-connected, fits into a fibration

$$
V_{d} \rightarrow B O(d) \rightarrow B O,
$$

and

$$
\pi_{j}\left(V_{d}\right)=\pi_{j}\left(V_{d+k, k}\right) \quad(j \leqslant d+k-2) .
$$

Proof By 5.25 and 5.33 the unstable Stiefel manifolds $V_{d+k, k}$ are $(d-1)$ connected and fit into commutative braids of fibrations

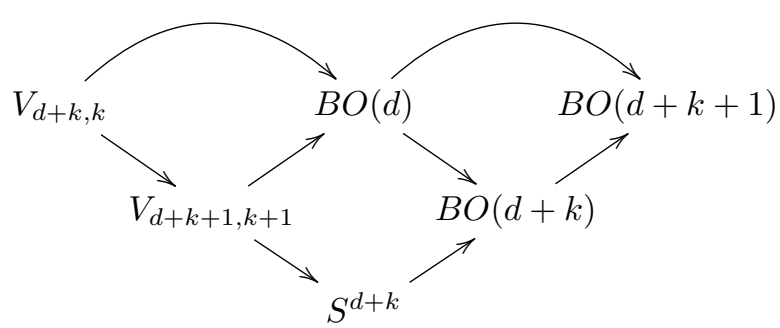

Proposition 7.39 (i) For $n \leqslant m-2$ the regular homotopy groups $I_{n}(M)$, $I_{n}^{f r}(M)$ fit into commutative braids of exact sequences 


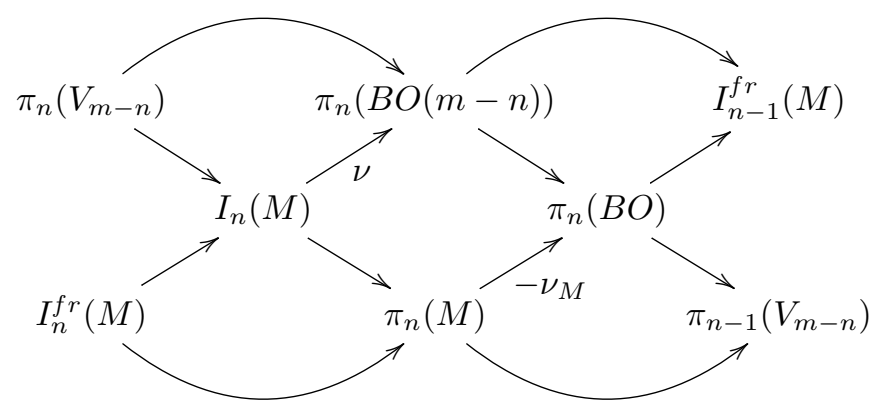

with

$\pi_{n}\left(V_{m-n}\right)=I_{n}\left(S^{m}\right) \rightarrow I_{n}(M) ;\left(g: S^{n} \uparrow S^{m}\right) \mapsto\left(g: S^{n} \uparrow M \# S^{m}=M\right)$.

(ii) For $2 n+1 \leqslant m \pi_{n}\left(V_{m-n}\right)=0$ and

$$
I_{n}(M)=\pi_{n}(M)
$$

with every element of $I_{n}(M)$ represented by an embedding $S^{n} \hookrightarrow M$. For $2 n+2 \leqslant$ $m I_{n}(M)=\pi_{n}(M)$ is the group of isotopy classes of embeddings $S^{n} \hookrightarrow M$.

Proof (i) By the Whitney Immersion Theorem (7.1) for every map $g: S^{n} \rightarrow M$ there exists a map $h: S^{n} \rightarrow \mathbb{R}^{k}$ ( $k$ large) such that $g \times h: S^{n} \rightarrow M \times \mathbb{R}^{k}$ is an embedding, with the normal bundle

$$
\nu_{g \times h}: S^{n} \rightarrow B O(m-n+k)
$$

such that

$$
\tau_{S^{n}} \oplus \nu_{g \times h}=g^{*} \tau_{M} \oplus \epsilon^{k}: S^{n} \rightarrow B O(m+k) .
$$

By the Smale-Hirsch Immersion Classification Theorem (7.35) the regular homotopy classes of immersions $g^{\prime}: S^{n} \rightarrow M$ homotopic to $g$ are in one-one correspondence with the reductions of $\nu_{g \times h}: S^{n} \rightarrow B O(m-n+k)$ to an $(m-n)$-plane bundle $\nu=\nu_{g^{\prime}}: S^{n} \rightarrow B O(m-n)$. The stabilisation map $B O(m-n) \rightarrow B O(m-n+k)$ fits into a fibration (as in 5.25)

$$
V_{m-n+k, k} \rightarrow B O(m-n) \rightarrow B O(m-n+k)
$$

so there is defined an exact sequence

$$
\ldots \rightarrow \pi_{n}\left(V_{m-n+k, k}\right) \rightarrow I_{n}(M) \rightarrow \pi_{n}(M) \rightarrow \pi_{n-1}\left(V_{m-n+k, k}\right) \rightarrow \ldots
$$

which can be written as

$$
\ldots \rightarrow \pi_{n}\left(V_{m-n}\right) \rightarrow I_{n}(M) \rightarrow \pi_{n}(M) \rightarrow \pi_{n-1}\left(V_{m-n}\right) \rightarrow \ldots .
$$

Similarly for $I_{n}^{f r}(M)$.

(ii) From $5.33 V_{m-n}$ is $(m-n-1)$-connected, and $2 n+1 \leqslant m$, so that there is an exact sequence

$$
\pi_{n}\left(V_{m-n}\right)=0 \rightarrow I_{n}(M) \rightarrow \pi_{n}(M) \rightarrow \pi_{n-1}\left(V_{m-n}\right)=0 .
$$

The connection with embeddings is given by the Whitney Embedding Theorem (7.2). 
Example 7.40 For $M=S^{m}, n \leqslant m-2$ Proposition 7.39 gives

$$
\begin{aligned}
& I_{n}\left(S^{m}\right)=\pi_{n}\left(V_{m-n}\right)=\pi_{n+1}(B O, B O(m-n)) \\
& I_{n}^{f r}\left(S^{m}\right)=\pi_{n+1}(B O(m))=\pi_{n+1}(B O) .
\end{aligned}
$$

In particular

$$
I_{n}\left(S^{m}\right)=0 \text { if } 2 n+1 \leqslant m
$$

and

$$
I_{n}\left(S^{2 n}\right)=\pi_{n+1}(B O, B O(n))=Q_{(-1)^{n}}(\mathbb{Z})= \begin{cases}\mathbb{Z} & \text { if } n \equiv 0(\bmod 2) \\ \mathbb{Z}_{2} & \text { if } n \equiv 1(\bmod 2)\end{cases}
$$

\subsection{Singularities}

In Chapter 11 we shall be considering the self-intersections of immersed $n$ manifolds in $2 n$-manifolds, in order to decide which $n$-dimensional homology classes can be killed by surgery. In order to compare the self-intersections of homotopic immersions we shall need to consider maps of the type $f: N^{n+1} \rightarrow$ $M^{2 n+1}$. In these dimensions the general position arguments used to deform $f$ into an immersion (7.1) do not apply, and $f$ has generic singularities. These were first described by Whitney [100]. (This section was written by Jim Milgram).

By the dimension count in 7.6 a map $f: N^{n+1} \rightarrow M^{2 n+1}$ can be deformed by an arbitrarily small modification to a map which intersects the singular set only in isolated points, and there intersects regularly (so it has rank $n$ and the two tangent planes together span the tangent space of $M_{2 n+1, n+1}(\mathbb{R})$ at the point). Note that changing local coordinates in $N^{n+1}$ and $M^{2 n+1}$ affects the description of the singular point via the faithful action of $G L_{2 n+1}(\mathbb{R}) \times G L_{n+1}(\mathbb{R})$ on the $2 n(n+1)$-dimensional submanifold $R_{n} \subset M_{2 n+1, n+1}(\mathbb{R})$ of the $(2 n+1) \times(n+1)$ matrices of rank $n$. Hence it can be assumed that the intersection is at the matrix $\left(\begin{array}{cc}I_{n} & 0 \\ 0 & 0\end{array}\right)$

Lemma 7.41 The tangent space $\tau_{R_{n}}(J)$ at the $(2 n+1) \times(n+1)$ matrix

$$
J=\left(\begin{array}{cc}
I_{n} & 0 \\
0 & 0
\end{array}\right)
$$

can be identified with the subspace of $M_{2 n+1, n+1}(\mathbb{R})$ consisting of all matrices of the form $\left(\begin{array}{cc}A & B \\ C & 0\end{array}\right)$ where $A$ is $n \times n, B$ is $n \times 1$, and $C$ is $(n+1) \times n$. 
Proof An infinitesimal transformation $\left(I_{2 n+1}+\Delta, I_{n+1}+\Delta^{\prime}\right) \operatorname{acts}$ on $\left(\begin{array}{cc}I_{n} & 0 \\ 0 & 0\end{array}\right)$ by sending it to $\left(\begin{array}{cc}I_{n}+\Delta_{1}-\Delta_{1}^{\prime} & -\Delta_{2}^{\prime} \\ \Delta_{3} & 0\end{array}\right)$. From this the lemma is immediate.

Taking account of the fact that the two tangent planes span $\tau_{R_{n}}(J)$ (and which can even be assumed to be orthogonal in the usual metric), there are obtained two generic forms for the map near the singular point differing only by an orientation :

Definition 7.42 The generic singularities

$$
f_{n}^{+}, f_{n}^{-}: \mathbb{R}^{n+1} \rightarrow \mathbb{R}^{2 n+1}
$$

are given by

$$
\begin{aligned}
& f_{n}^{+}\left(x_{1}, x_{2}, \ldots, x_{n}, x_{n+1}\right) \\
& \quad=\left(x_{1}, x_{2}, \ldots, x_{n}, x_{n+1}^{2}, x_{n+1} x_{n}, x_{n+1} x_{n-1}, \ldots, x_{n+1} x_{1}\right) \\
& \quad f_{n}^{-}\left(x_{1}, x_{2}, \ldots, x_{n}, x_{n+1}\right) \\
& \quad=\left(x_{1}, x_{2}, \ldots, x_{n}, x_{n+1}^{2},-x_{n+1} x_{n}, x_{n+1} x_{n-1}, \ldots, x_{n+1} x_{1}\right) .
\end{aligned}
$$

Remark 7.43 The double point locus of $f_{n}^{ \pm}$is the $x_{n+1}$-axis, where

$$
f_{n}^{ \pm}\left(0, \ldots, 0, x_{n+1}\right)=f_{n}^{ \pm}\left(0, \ldots, 0,-x_{n+1}\right)
$$

Moreover, if $N(x)=\sum_{i=1}^{n+1} x_{i}^{2}$, then

$$
N\left(f_{n}^{ \pm}(x)\right)=N(x)-x_{n+1}^{2}(1-N(x)) .
$$

It follows that the unit sphere is taken to the unit sphere under $f_{n}^{ \pm}$and there is a single double point immersion. More generally, it can be checked that for all $n$ the indices of the immersions are $(-1)^{n+1}$ for $f_{n}^{+}$, and $(-1)^{n}$ for $f_{n}^{-}$. Additionally, for each sphere $S_{r}^{n}$ of radius $r<1, f_{n}^{ \pm}\left(S_{r}^{n}\right)$ is an immersed sphere with a single double point, in a surface contained in the ball of radius $r$ in $\mathbb{R}^{2 n+1}$.

Next, consider a general differentiable map $f: N^{n+1} \rightarrow M^{2 n+1}$, with $n \geqslant 1$, and $N^{n+1}$ compact. By working as in Proposition 7.11 it may be assumed that $f$ has no triple points, and the singularities are generic, looking locally like either $f_{n}^{+}$or $f_{n}^{-}$, in appropriate local coordinate systems. Moreover, if $N^{n+1}$ has a boundary, it can be assumed that $f$ is an embedding on the boundary. The double point locus is the set of all the double points of $f$ in $M^{2 n+1}$, which can 
be assumed to consist of a series of differentiable curves, either closed or with endpoints. The singularities must all occur as endpoints of double point curves, and conversely, every endpoint must be a singularity.

At each end assign an index according to the index of the double point on restricting to spheres near the singularity, as above. Moreover, if $n \geqslant 2$, then draw non-intersecting paths disjoint from the double point locus in $\operatorname{im}(f)$ between singular points of + and - types, and modify the map in a neighbourhood of these paths to replace the pair of singularities by a path of double points joining their images.

A neighbourhood $T$ of the path consists of the unit ball near each singularity, and a tube $D^{2 n} \times I$ joining the two balls. The intersection of $\partial T$ with $\operatorname{im}(f)$ is a $D^{n}$ immersed with a single double point on the upper hemisphere of each sphere, and $S^{n-1} \times I$ along the boundary of the tube. Now, give a new extension of $f$ along the interior of $T$ by making it be the single double point immersion of $D^{n} \times t$ at each level $D^{2 n} \times t$ of the tube, and filling in appropriately on the upper and lower balls. This modification has the effect of removing the pair of singularities, and replacing them by a path of double points. In particular, this shows that all double point paths in the image which begin and end at singularities can be replaced by non-singular double point paths. But since the boundary was assumed to be embedded this shows that the original map could in fact be deformed to an immersion.

Slightly more generally :

Whitney Singularity Theorem 7.44 ([100])

If $f:\left(N^{n+1}, \partial N\right) \rightarrow\left(M^{2 n+1}, \partial M\right)$ is a map with $n \geqslant 2$ and $f \mid \partial N$ an embedding, then $f$ is homotopic to an immersion leaving the boundary fixed. Additionally, if $f \mid \partial N$ is merely an immersion with $k$ index +1 and $s$ index -1 double points, then $f$ is homotopic rel$\partial N$ to a map (also denoted by $f$ ) which is an immersion except at precisely $|k-s|$ interior points where it has a generic singularity, and each singularity has the same index. 
WHITEHEAD TORSION

Algebraic $K$-theory deals with modules and their automorphisms, while algebraic $L$-theory deals with quadratic forms and their automorphisms. Whitehead torsion is an algebraic $K$-theory invariant comparing the cell structures of homotopy equivalent finite $C W$ complexes, while the surgery obstruction is an algebraic $L$-theory invariant of the handlebody structure of the kernel of a normal map. In fact, there are many formal similarities between the applications of algebraic $K$ - and $L$-theory to manifolds, and the applications of Whitehead torsion to manifold structures are precursors to the applications of surgery theory.

This chapter outlines the proofs of the $h$ - and $s$-Cobordism Theorems stated in Chapter 1, which use Whitehead torsion to study the possible handle decompositions of an $h$-cobordism $\left(W^{m+1} ; M, M^{\prime}\right)$. There are no handles precisely when $\left(W ; M, M^{\prime}\right)$ is diffeomorphic to $M \times(I ;\{0\},\{1\})$. The Whitehead torsion $\tau=\tau(M \hookrightarrow W) \in W h\left(\pi_{1}(W)\right)$ is an algebraic $K$-theory invariant such that $\tau=0$ if (and for $m \geqslant 5$ only if) there exists a decomposition with no handles. The vanishing $\tau=0$ was the first systematic condition for deciding if a homotopy equivalence $M \rightarrow M^{\prime}$ is homotopic to a diffeomorphism.

Section 8.1 describes the algebraic properties of the Whitehead group, and constructs the Whitehead torsion of a homotopy equivalence. Section 8.2 describes the proofs of the $h$ - and $s$-Cobordism Theorems, which realize geometrically the algebraically allowed handle cancellations.

\subsection{The Whitehead group}

See Milnor [58] and Cohen [19] for more detailed accounts, and Rosenberg [78] for a general introduction to algebraic $K$-theory.

Let $A$ be an associative ring with 1. As in Section 4.4 regard an $m \times n$ matrix $\left(\phi_{i j}\right)$ with entries $\phi_{i j} \in A$ as an $A$-module morphism

$$
\phi: A^{n} \rightarrow A^{m} ;\left(x_{1}, x_{2}, \ldots, x_{n}\right) \mapsto\left(\sum_{j=1}^{n} x_{j} \phi_{1 j}, \sum_{j=1}^{n} x_{j} \phi_{2 j}, \ldots, \sum_{j=1}^{n} x_{j} \phi_{m j}\right) .
$$

The $A$-module isomorphism

$$
M_{m, n}(A) \rightarrow \operatorname{Hom}_{A}\left(A^{n}, A^{m}\right) ;\left(\phi_{i j}\right) \mapsto \phi
$$


will be used as an identification. A morphism $f: L \rightarrow K$ of based f.g. free $A$-modules is determined by the $m \times n$ matrix $\phi=\left(\phi_{i j}\right)$ of the coefficients

$$
f\left(c_{j}\right)=\sum_{i=1}^{m} \phi_{i j} b_{i} \in K \quad(1 \leqslant j \leqslant n)
$$

with $\left(b_{1}, b_{2}, \ldots, b_{m}\right)$ the basis of $K$ and $\left(c_{1}, c_{2}, \ldots, c_{n}\right)$ the basis of $L$.

Let $A^{\bullet} \hookrightarrow A$ be the multiplicative group of units in the ring $A$.

Definition 8.1 (i) The $n$th general linear group of $A$

$$
G L_{n}(A)=M_{n, n}(A)^{\bullet} \hookrightarrow M_{n, n}(A)
$$

is the multiplicative group of units in the ring of $n \times n$ matrices in $A$. The injections

$$
M_{n, n}(A) \rightarrow M_{n+1, n+1}(A) ; \phi \mapsto\left(\begin{array}{cc}
\phi & 0 \\
0 & 1
\end{array}\right)
$$

preserve matrix multiplication, and restrict to injections of groups $G L_{n}(A) \rightarrow$ $G L_{n+1}(A)$.

(ii) The infinite general linear group of $A$ is the union

$$
G L(A)=\bigcup_{n=1}^{\infty} G L_{n}(A) \text {. }
$$

Whitehead Lemma 8.2 The commutator subgroup of the infinite general linear group

$$
E(A)=[G L(A), G L(A)] \triangleleft G L(A)
$$

is the normal subgroup generated by the elementary matrices $\left(\begin{array}{ll}1 & a \\ 0 & 1\end{array}\right)$.

Proof See Proposition 2.1.4 of Rosenberg [78].

Definition 8.3 (i) The torsion group $K_{1}(A)$ is the abelian group

$$
K_{1}(A)=G L(A) / E(A) .
$$

(ii) The reduced torsion group of $A$ is the abelian group

$$
\widetilde{K}_{1}(A)=K_{1}(A) /\{\tau(-1)\} .
$$

In dealing with Whitehead torsion it is convenient to assume that the ring $A$ is such that $A^{m}$ is isomorphic to $A^{n}$ if and only if $m=n$. (This is the case if $A=\mathbb{Z}[\pi]$ is a group ring). The rank of a f.g. free $A$-module is then well-defined, and the matrix of an isomorphism $f: L \rightarrow K$ of based f.g. free $A$-modules of rank $n$ is an element $\phi \in G L_{n}(A)$. 
Example 8.4 (i) For a commutative ring $A$ the inclusion $A^{\bullet} \rightarrow K_{1}(A)$ is split by the determinant map

$$
\operatorname{det}: K_{1}(A) \rightarrow A^{\bullet} ; \tau(\phi) \mapsto \operatorname{det}(\phi)
$$

and the torsion group of $A$ splits as

$$
K_{1}(A)=A^{\bullet} \oplus S K_{1}(A)
$$

with

$$
S K_{1}(A)=\operatorname{ker}\left(\operatorname{det}: K_{1}(A) \rightarrow A^{\bullet}\right) .
$$

(ii) If $A$ is a field then det : $K_{1}(A) \rightarrow A^{\bullet}$ is an isomorphism and

$$
S K_{1}(A)=0
$$

Definition 8.5 The torsion of an isomorphism $f: L \rightarrow K$ of based f.g. free $A$-modules of rank $n$ is the torsion of the corresponding invertible matrix $\phi \in$ $G L_{n}(A)$

$$
\tau(f)=[\phi] \in K_{1}(A) .
$$

The isomorphism is simple if $\tau(f)=0 \in K_{1}(A)$.

The geometric applications of $K_{1}(A)$ make use of :

Definition 8.6 The Whitehead group of a group $\pi$ is the abelian group

$$
W h(\pi)=K_{1}(\mathbb{Z}[\pi]) /\{\tau( \pm g) \mid g \in \pi\}
$$

Example 8.7 (i) The Whitehead group is trivial

$$
W h(\pi)=0
$$

in the following cases:

(a) $\pi=\{1\}$ is trivial,

(b) $\pi=\pi_{1}(M)$ is the fundamental group of a surface $M=M(g)$ or $N(g)$ (Section $4.3)$,

(c) $\pi=\mathbb{Z}^{m}$ is a free abelian group, for any $m \geqslant 1$.

(ii) The Whitehead group version of the Novikov conjecture is that $W h(\pi)=0$ if $\pi=\pi_{1}(M)$ is the fundamental group of a compact $m$-dimensional manifold $M$ with universal cover $\widetilde{M}=\mathbb{R}^{m}$ (as in (b) above with $\widetilde{M(g)}=\widehat{N(g+1)}=\mathbb{R}^{2}$ $(g \geqslant 1)$, and (c) with $\left.M=T^{m}, \widetilde{M}^{m}=\mathbb{R}^{m}\right)$. The conjecture has been verified in many cases - see Ferry, Ranicki and Rosenberg [24] for a survey of results. 
Example 8.8 (i) The Whitehead group $W h(\pi)$ of a finite group $\pi$ is finitely generated, with rank $r(\pi)-q(\pi)$ the difference between the number $r(\pi)$ of irreducible real representations of $\pi$ and the number $q(\pi)$ of irreducible rational representations of $\pi$ (Bass [6]). More explicitly, $r(\pi)$ is the number of conjugacy classes of unordered pairs $\left\{g, g^{-1}\right\}$ in $\pi$, and $q(\pi)$ is the number of conjugacy classes of cyclic subgroups of $\pi$.

(ii) For a finite abelian group $\pi$ the determinant is a split surjection

$$
\operatorname{det}: K_{1}(\mathbb{Z}[\pi]) \rightarrow \mathbb{Z}[\pi]^{\bullet}
$$

(iii) The Whitehead group $W h\left(\mathbb{Z}_{m}\right)$ of a cyclic group $\mathbb{Z}_{m}$ is trivial for $m=$ $2,3,4,6$, and is a free abelian group of rank $\geqslant 1$ for $m \neq 2,3,4,6$. (See Proposition 8.37 below for the precise formula). In particular, $W h\left(\mathbb{Z}_{5}\right)=\mathbb{Z}$ with generator $\tau\left(1-t+t^{2}\right)$.

The following properties of contractible finite chain complexes are necessary for the definition of torsion.

Lemma 8.9 Let $C$ be a contractible finite based f.g. free A-module chain complex.

(i) For any chain contraction $\Gamma: 0 \simeq 1: C \rightarrow C$ the $A$-module morphism

$d+\Gamma=\left(\begin{array}{cccc}d & 0 & 0 & \ldots \\ \Gamma & d & 0 & \ldots \\ 0 & \Gamma & d & \ldots \\ \vdots & \vdots & \vdots & \end{array}\right): C_{\text {odd }}=C_{1} \oplus C_{3} \oplus C_{5} \ldots \rightarrow C_{\text {even }}=C_{0} \oplus C_{2} \oplus C_{4} \ldots$

is an isomorphism of based f.g. free A-modules, so that $\tau(d+\Gamma) \in K_{1}(A)$ is defined.

(ii) If $\Gamma, \Gamma^{\prime}: 0 \simeq 1: C \rightarrow C$ are two chain contractions then

$$
\tau(d+\Gamma)=\tau\left(d+\Gamma^{\prime}\right) \in K_{1}(A) .
$$

Proof (i) The inverse is given by

$$
(d+\Gamma)^{-1}=\left(\begin{array}{cccc}
1 & 0 & 0 & \ldots \\
\Gamma^{2} & 1 & 0 & \ldots \\
0 & \Gamma^{2} & 1 & \ldots \\
\vdots & \vdots & \vdots &
\end{array}\right)^{-1}\left(\begin{array}{cccc}
\Gamma & d & 0 & \ldots \\
0 & \Gamma & d & \ldots \\
0 & 0 & \Gamma & \ldots \\
\vdots & \vdots & \vdots &
\end{array}\right) \quad: C_{\text {even }} \rightarrow C_{\text {odd }}
$$

(ii) The $A$-module morphisms

$$
\Delta_{i}=\left(\Gamma^{\prime}-\Gamma\right) \Gamma: C_{i} \rightarrow C_{i+2}
$$

are such that 


$$
\left(d+\Gamma^{\prime}\right)=\left(1+\Delta_{\text {even }}\right)(d+\Gamma)\left(1+\Delta_{\text {odd }}\right)^{-1}: C_{\text {odd }} \rightarrow C_{\text {even }}
$$

with

$$
\tau\left(1+\Delta_{\text {even }}\right)=\tau\left(1+\Delta_{\text {odd }}\right)=0 \in K_{1}(A) .
$$

Definition 8.10 The torsion of a contractible finite chain complex $C$ of based f.g. free $A$-modules is

$$
\tau(C)=\tau\left(d+\Gamma: C_{\text {odd }} \rightarrow C_{\text {even }}\right) \in K_{1}(A)
$$

for any chain contraction $\Gamma: 0 \simeq 1: C \rightarrow C$.

Torsion is such that

$$
\begin{aligned}
& \tau\left(f \oplus f^{\prime}\right)=\tau(f)+\tau\left(f^{\prime}\right), \\
& \tau(g f)=\tau(f)+\tau(g) \in K_{1}(A)
\end{aligned}
$$

for any isomorphisms $f: L \rightarrow K, f^{\prime}: L^{\prime} \rightarrow K^{\prime}, g: K \rightarrow J$ of based f.g. free $A$-modules. Also, for any contractible finite chain complexes $C, D$ of based f.g. free $A$-modules

$$
\tau(C \oplus D)=\tau(C)+\tau(D) \in \widetilde{K}_{1}(A)
$$

Proposition 8.11 The reduced torsion $\tau(C) \in \widetilde{K}_{1}(A)$ of a contractible finite chain complex $C$ of based f.g. free A-modules is such that $\tau(C)=0$ if and only if $C$ is simple isomorphic to a direct sum of elementary complexes

$$
\ldots \longrightarrow 0 \longrightarrow A \stackrel{1}{\longrightarrow} A \longrightarrow 0 \longrightarrow \ldots .
$$

The torsion of a chain equivalence is defined to be the torsion of the algebraic mapping cone :

Definition 8.12 (i) The torsion of a chain equivalence $f: C \rightarrow D$ of finite chain complexes of based f.g. free $A$-modules is

$$
\tau(f)=\tau(\mathscr{C}(f)) \in K_{1}(A) .
$$

(ii) The Whitehead torsion of a homotopy equivalence $f: X \rightarrow Y$ of finite $C W$ complexes is the torsion of the induced chain equivalence $\tilde{f}: C(\tilde{X}) \rightarrow C(\widetilde{Y})$ of the cellular chain complexes

$$
\tau(f)=\tau(\mathscr{C}(\widetilde{f})) \in W h\left(\pi_{1}(X)\right) .
$$

The homotopy equivalence is simple if $\tau(f)=0 \in W h\left(\pi_{1}(X)\right)$. 
Example 8.13 (i) A diffeomorphism $f: M^{m} \rightarrow N^{m}$ is a simple homotopy equivalence. A complete proof requires the verification that any two handle decompositions are related by a sequence of elementary modifications - this uses the triangulability of differentiable manifolds and the combinatorial invariance of Whitehead torsion.

(ii) It follows from (i) that a homotopy equivalence of closed manifolds which is not simple cannot be homotopic to a diffeomorphism.

\subsection{The $h$ - and $s$-Cobordism Theorems}

This section contains a brief account of the $h$ - and $s$-Cobordism Theorems stated in Chapter 1. See Milnor [57], [58], Kervaire [37] for further details.

By Theorem 2.22 every cobordism $\left(W ; M, M^{\prime}\right)$ has a handle decomposition

$$
W=M \times I \cup h^{i_{0}} \cup h^{i_{1}} \cup \ldots \cup h^{i_{k}}
$$

which determines a $C W$ structure on $W$ relative to $M$ with one $i$-cell for each $i$-handle. The $\mathbb{Z}$-coefficient handle chain complex $C(W, M)(3.33)$ will now be generalised to a $\mathbb{Z}\left[\pi_{1}(W)\right]$-coefficient handle chain complex, and the differentials will be interpreted in terms of intersections of the core spheres. The handle decomposition expresses $W$ as a union of a finite sequence

$$
\left(W ; M, M^{\prime}\right)=\left(W_{1} ; M_{0}, M_{1}\right) \cup\left(W_{2} ; M_{1}, M_{2}\right) \cup \ldots \cup\left(W_{k} ; M_{k-1}, M_{k}\right)
$$

of adjoining elementary cobordisms $\left(W_{j} ; M_{j-1}, M_{j}\right)$ with

$$
W_{j}=M_{j-1} \times I \cup h^{i_{j}}, h^{i_{j}}=D^{i_{j}} \times D^{m+1-i_{j}}
$$

such that

$$
0 \leqslant i_{1} \leqslant i_{2} \leqslant \ldots \leqslant i_{k} \leqslant m+1, M_{0}=M, M_{k}=M .
$$

We shall call $W_{j}$ an $i_{j}$-handle, although strictly speaking it is $h^{i_{j}}$ which is the $i_{j}$-handle. For each $i=0,1, \ldots, m+1$ let $(W(i) ; M, M(i))$ be the cobordism defined by the union of all the $i^{\prime}$-handles with $i^{\prime} \leqslant i$

$$
W(i)=\bigcup_{i_{j} \leqslant i} W_{j} .
$$

Regard all the $(i+1)$-handles $W_{j}$ as being attached simultaneously at an embedding

$$
\bigcup S^{i} \times D^{m-i} \hookrightarrow M(i)
$$

so that

$$
W(i+1)=W(i) \cup \bigcup D^{i+1} \times D^{m-i} .
$$


It is also possible to reverse the $i$-handles $W_{j^{\prime}}$, and regard them as dual $(m+1-i)$ handles $\bar{W}_{k-j^{\prime}}$ of $\left(W ; M^{\prime}, M\right)$ attached simultaneously at an embedding

$$
\bigcup D^{i} \times S^{m-i} \hookrightarrow M(i)
$$

such that the cores $\{0\} \times S^{m-i} \subset M(i)$ of these handles intersect the cores $S^{i} \times\{0\} \subset M(i)$ of the $(i+1)$-handles $W_{j}$ transversely.

Definition 8.14 The handle chain complex $C(\widetilde{W}, \widetilde{M})$ of an $(m+1)$-dimensional cobordism $\left(W ; M, M^{\prime}\right)$ with a handle decomposition

$$
W=M \times I \cup h^{i_{0}} \cup h^{i_{1}} \cup \ldots \cup h^{i_{k}}
$$

is the cellular $\mathbb{Z}\left[\pi_{1}(W)\right]$-module chain complex of the relative $C W$ pair $C(\widetilde{W}, \widetilde{M})$ with $\widetilde{W}$ the universal cover of $W, \widetilde{M} \hookrightarrow \widetilde{W}$ the induced cover of $M \hookrightarrow W$, with $\pi_{1}(W) i$-cells for each $i$-handle $h^{i}=D^{i} \times D^{m+1-i}$.

Example 8.15 The handle chain complex of $\mathbb{R} \mathbb{P}^{m}=h^{0} \cup h^{1} \cup \ldots \cup h^{m}(2.25)$ is

$$
\begin{aligned}
C\left(\widetilde{\mathbb{R P}}^{m}\right): C_{m} & =\mathbb{Z}\left[\mathbb{Z}_{2}\right] \stackrel{1+(-1)^{m} T}{\longrightarrow} C_{m-1}=\mathbb{Z}\left[\mathbb{Z}_{2}\right] \longrightarrow \\
\longrightarrow & C_{2}=\mathbb{Z}\left[\mathbb{Z}_{2}\right] \stackrel{1+T}{\longrightarrow} C_{1}=\mathbb{Z}\left[\mathbb{Z}_{2}\right] \stackrel{1-T}{\longrightarrow} C_{0}=\mathbb{Z}\left[\mathbb{Z}_{2}\right]
\end{aligned}
$$

with $\widetilde{\mathbb{R P}}^{m}=S^{m}$.

The differentials in the handle chain complex are determined by the algebraic intersections of the handles of successive index. The embeddings of the cores $S^{i} \hookrightarrow M(i)$ are $\pi_{1}(W)$-trivial, since they extend to maps $D^{i+1} \rightarrow W$. (For $i \neq 0,1 S^{i}$ is simply-connected, anyway).

Definition 8.16 The algebraic intersection of an $(i+1)$-handle $W_{j}$ and an $i$-handle $W_{j^{\prime}}$ in an $(m+1)$-dimensional cobordism $\left(W ; M, M^{\prime}\right)$ is the intersection of the core $S^{i} \times\{0\} \hookrightarrow M(i)^{m}$ of $W_{j}$ and the core $\{0\} \times S^{m-i} \hookrightarrow M(i)^{m}$ of the $(m+1-i)$-handle $\bar{W}_{j^{\prime}}$ of $\left(W ; M^{\prime}, M\right)$ dual to $W_{j^{\prime}}$

$$
\lambda\left(W_{j}, W_{j^{\prime}}\right)=\lambda\left(S^{i}, S^{m-i}\right) \in \mathbb{Z}\left[\pi_{1}(W)\right] .
$$

In view of 7.22 there is no need to distinguish between the algebraic and geometric intersections here.

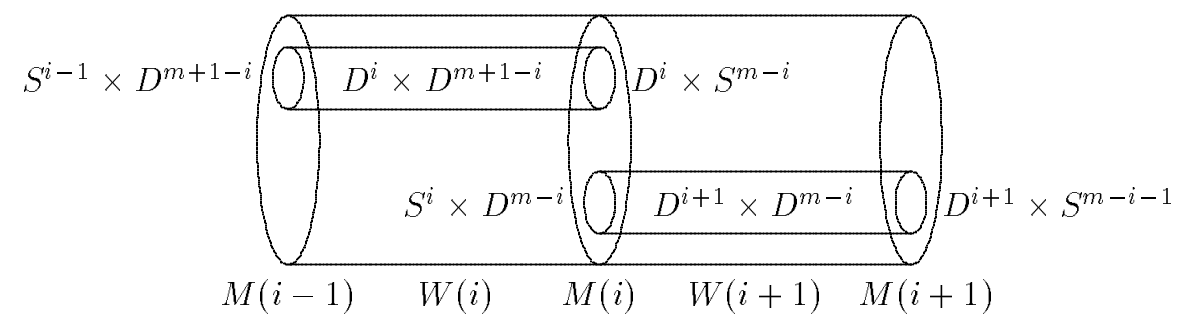


Proposition 8.17 (i) The homotopy theoretic effect of attaching an $i$-handle to an $(m+1)$-dimensional manifold with boundary $(W, \partial W)$ is that of attaching an i-cell, that is

$$
W^{\prime}=W \cup D^{i} \times D^{m-i+1} \simeq W \cup D^{i}
$$

(ii) A handle decomposition of a cobordism

$$
\left(W ; M, M^{\prime}\right)=\left(W_{1} ; M_{0}, M_{1}\right) \cup\left(W_{2} ; M_{1}, M_{2}\right) \cup \ldots \cup\left(W_{k} ; M_{k-1}, M_{k}\right)
$$

as a union of $k$ adjacent elementary cobordisms determines a relative $C W$ structure on the pair $X=(W, M)$

$$
W \simeq M \cup D^{i_{1}} \cup D^{i_{2}} \cup \ldots \cup D^{i_{k}}
$$

with $i_{j}$ the index of $\left(W_{j} ; M_{j-1}, M_{j}\right)$. The differentials in the cellular $\mathbb{Z}\left[\pi_{1}(W)\right]$ module chain complex $C=C(\widetilde{W}, \widetilde{M})$ are given by the algebraic intersections of the handles of adjoining index

$$
\begin{aligned}
& d: C_{i+1}=H_{i+1}\left(\widetilde{X}^{(i+1)}, \widetilde{X}^{(i)}\right)=\mathbb{Z}\left[\pi_{1}(W)\right]^{b_{i+1}} \\
& \rightarrow C_{i}=H_{i}\left(\widetilde{X}^{(i)}, \tilde{X}^{(i-1)}\right)=\mathbb{Z}\left[\pi_{1}(W)\right]^{b_{i}} ;\left[W_{j^{\prime}}\right] \mapsto \sum_{i_{j}=i} \lambda\left(W_{j}, W_{j^{\prime}}\right)\left[W_{j}\right] .
\end{aligned}
$$

(iii) Let $\left(W ; M, M^{\prime}\right)$ be an $(m+1)$-dimensional cobordism. Given a Morse function $f: W \rightarrow I$ and a corresponding handle decomposition

$$
\left(W ; M, M^{\prime}\right)=\left(W_{1} ; M_{0}, M_{1}\right) \cup\left(W_{2} ; M_{1}, M_{2}\right) \cup \ldots \cup\left(W_{k} ; M_{k-1}, M_{k}\right)
$$

let $f^{\prime}=1-f: W \rightarrow I$ be the opposite Morse function, corresponding to the dual handle decomposition

$$
\left(W^{\prime} ; M^{\prime}, M\right)=\left(W_{1}^{\prime} ; M_{0}^{\prime}, M_{1}^{\prime}\right) \cup\left(W_{2}^{\prime} ; M_{1}^{\prime}, M_{2}^{\prime}\right) \cup \ldots \cup\left(W_{k}^{\prime} ; M_{k-1}^{\prime}, M_{k}^{\prime}\right)
$$

with

$$
W^{\prime}=-W, W_{i}^{\prime}=-W_{k-i}, M_{i}^{\prime}=M_{k-i}(0 \leqslant i \leqslant k)
$$

where $-W$ denotes $W$ with the opposite orientation on the universal cover $\widetilde{W}$. The relative cellular $\mathbb{Z}\left[\pi_{1}(W)\right]$-module chain complexes of $(W, M),\left(W, M^{\prime}\right)$ are related by

$$
C\left(\widetilde{W}, \widetilde{M^{\prime}}\right)=C(\widetilde{W}, \widetilde{M})^{m+1-*}
$$

Proof (i) The inclusion

$$
\left(D^{i}, S^{i-1}\right) \times\{0\} \hookrightarrow\left(W^{\prime}, W\right)
$$

is a relative homotopy equivalence.

(ii) By (i) attaching an $i$-handle has the homotopy theoretic effect of attaching 
an $i$-cell.

(iii) The dual of an $i_{j}$-handle $W_{j}$ is the $\left(m+1-i_{j}\right)$-handle $\bar{W}_{k-j}$. The algebraic intersection of an $i_{j}$-handle $W_{i}$ and an $i_{j^{\prime}}$-handle $W_{j}$ with $i_{j^{\prime}}=i_{j}+1$ is related to the algebraic intersection of the dual $\left(m+1-i_{j}\right)$-handle $\bar{W}_{k-j}$ and the dual $\left(m+1-i_{j}\right)$-handle $\bar{W}_{k-j^{\prime}}$ by

$$
\lambda\left(W_{j}, W_{j^{\prime}}\right)=\lambda\left(\bar{W}_{k-j}, \bar{W}_{k-j^{\prime}}\right) \in \mathbb{Z}\left[\pi_{1}(W)\right] .
$$

Recall that an $h$-cobordism $\left(W ; M, M^{\prime}\right)$ is a cobordism such that the inclusions $M \hookrightarrow W, M^{\prime} \hookrightarrow W$ are homotopy equivalences, and that an $h$-cobordism is trivial if there exists a diffeomorphism

$$
\left(W ; M, M^{\prime}\right) \cong M \times(I ;\{0\},\{1\})
$$

which is the identity on $M$. The $s$-Cobordism Theorem asserts that for $m \geqslant 5$ an $(m+1)$-dimensional $h$-cobordism $\left(W ; M, M^{\prime}\right)$ is trivial if and only if the contractible based f.g. free $\mathbb{Z}\left[\pi_{1}(W)\right]$-module chain complex $C(\widetilde{W}, \widetilde{M})$ is such that the bases can be modified by elementary operations to be such that the differentials send basis elements to basis elements. The $h$-Cobordism Theorem (1.9) states that for $m \geqslant 5$ every $(m+1)$-dimensional $h$-cobordism $\left(W ; M, M^{\prime}\right)$ with $\pi_{1}(W)=\{1\}$ is trivial. The $s$-Cobordism Theorem (1.11) is the generalisation of the $h$-Cobordism Theorem to non-simply-connected $h$-cobordisms.

Definition 8.18 The torsion of an $h$-cobordism $\left(W ; M, M^{\prime}\right)$ is

$$
\tau\left(W ; M, M^{\prime}\right)=\tau(M \hookrightarrow W) \in W h\left(\pi_{1}(W)\right) .
$$

Proposition 8.19 The torsion of an h-cobordism $\left(W ; M, M^{\prime}\right)$ is the torsion

$$
\tau\left(W ; M, M^{\prime}\right)=\tau(C(\widetilde{W}, \widetilde{M})) \in W h\left(\pi_{1}(W)\right)
$$

of the contractible cellular $\mathbb{Z}\left[\pi_{1}(W)\right]$-module chain complex $C=C(\widetilde{W}, \widetilde{M})$ determined by any handle decomposition

$$
\left(W ; M, M^{\prime}\right)=\left(W_{1} ; M_{0}, M_{1}\right) \cup\left(W_{2} ; M_{1}, M_{2}\right) \cup \ldots \cup\left(W_{k} ; M_{k-1}, M_{k}\right),
$$

with $\widetilde{W}, \widetilde{M}$ the universal covers of $W, M$, and $C_{i}=\mathbb{Z}\left[\pi_{1}(W)\right]^{b_{i}}$ the based f.g. free $\mathbb{Z}\left[\pi_{1}(W)\right]$-module of rank $b_{i}=$ the number of $i$-handles.

Definition 8.20 An $s$-cobordism is an $h$-cobordism $\left(W ; M, M^{\prime}\right)$ such that

$$
\tau\left(W ; M, M^{\prime}\right)=0 \in W h\left(\pi_{1}(W)\right) .
$$


Proposition 8.21 (i) A trivial h-cobordism is an s-cobordism.

(ii) An h-cobordism is trivial if and only if it admits the empty handle decomposition.

Proof (i) A trivial $h$-cobordism $\left(W ; M, M^{\prime}\right)$ admits the empty handle decomposition, with cellular chain complex $C(\widetilde{W}, \widetilde{M})=0$.

(ii) Trivial.

The $s$-Cobordism Theorem is the converse of 8.21 (i) in dimensions $\geqslant 6:$ an $s$-cobordism is a trivial $h$-cobordism.

Proposition 8.22 Let $m \geqslant 5$, and let $M$ be a closed $m$-dimensional manifold with fundamental group $\pi_{1}(M)=\pi$. For every element $\tau \in W h(\pi)$ there exists an h-cobordism $\left(W ; M, M^{\prime}\right)$ with $\tau\left(W ; M, M^{\prime}\right)=\tau \in W h(\pi)$.

Proof Represent $\tau \in W h(\pi)$ by $A \in G L_{k}(\mathbb{Z}[\pi])$, and let $\left(W ; M, M^{\prime}\right)$ be the $(m+1)$-dimensional $h$-cobordism with $k 2$-handles and $k 3$-handles attached to the 2-handles using $A$

$$
W=M \times I \cup \bigcup_{k} D^{2} \times D^{m-1} \cup_{A} \bigcup_{k} D^{3} \times D^{m-2} .
$$

The condition $m \geqslant 5$ is required to realize the prescribed algebraic intersections in $A$ by geometric intersections in

$$
M(2)=\left(M \backslash \bigcup_{k} S^{1} \times D^{m-1}\right) \cup \bigcup_{k} D^{2} \times S^{m-2},
$$

using the non-simply-connected Whitney trick as in 7.30.

Definition 8.23 A cobordism $\left(W ; M, M^{\prime}\right)$ has an $(i, i+1)$-index handle decomposition if it has a handle decomposition involving handles of index $i, i+1$ only, so that

$$
\begin{aligned}
\left(W ; M, M^{\prime}\right)=\left(W_{1} ; M_{0}, M_{1}\right) & \cup\left(W_{2} ; M_{1}, M_{2}\right) \cup \ldots \cup\left(W_{j} ; M_{j-1}, M_{j}\right) \\
& \cup\left(W_{j+1} ; M_{j}, M_{j+1}\right) \cup \ldots \cup\left(W_{k} ; M_{k-1}, M_{k}\right)
\end{aligned}
$$

with $W_{1}, W_{2}, \ldots, W_{j}$ elementary cobordisms of index $i$ and $W_{j+1}, W_{j+2}, \ldots, W_{k}$ elementary cobordisms of index $i+1$, for some $j$ with $0 \leqslant j \leqslant k$.

Here is a picture of a cobordism with an $(i, i+1)$-index handle decomposition

$$
\left(W ; M, M^{\prime}\right)=\left(W_{1} ; M_{0}, M_{1}\right) \cup\left(W_{2} ; M_{1}, M_{2}\right)
$$

consisting of an elementary cobordism $W_{1}$ of index $i$ and an elementary cobor$\operatorname{dism} W_{2}$ of index $i+1$. 


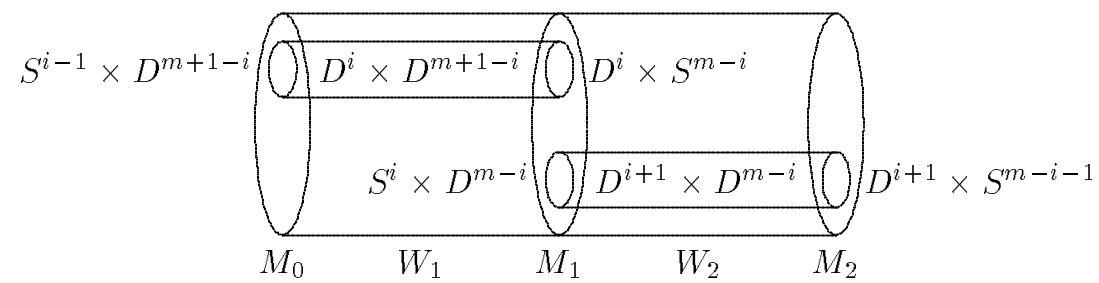

The following handle cancellation result is the geometric step in the proofs of the $h$ - and $s$-Cobordism Theorems :

Handle Cancellation Lemma 8.24 Let $\left(W ; M, M^{\prime}\right)$ be an $(m+1)$-dimensional cobordism with an $(i, i+1)$-index handle decomposition

$$
\left(W ; M, M^{\prime}\right)=\left(W_{1} ; M_{0}, M_{1}\right) \cup\left(W_{2} ; M_{1}, M_{2}\right)
$$

involving a handle $W_{1}$ of index $i$ and a handle $W_{2}$ of index $i+1$. If the core of the attaching framed $i$-embedding $S^{i} \times D^{m-i} \hookrightarrow M_{1}$ of the $(i+1)$-handle $W_{2}$ and the core of the attaching framed $(m-i)$-embedding $D^{i} \times S^{m-i} \hookrightarrow M_{1}$ of the $(m-i+1)$-handle $\bar{W}_{1}$ on $M_{1} \times I$ dual to the $i$-handle $W_{1}$ on $M_{0} \times I$ intersect transversely with a single double point

$$
\left(S^{i} \times\{0\}\right) \cap\left(\{0\} \times S^{m-i}\right)=\{\text { pt. }\} \subset M_{1}
$$

then $\left(W ; M, M^{\prime}\right)$ is a trivial $h$-cobordism with a diffeomorphism

$$
\left(W ; M, M^{\prime}\right) \cong M \times(I ;\{0\},\{1\})
$$

Proof See Milnor [57] and Kosinski [42,VI.7].

Example 8.25 The letter $S$ is a 1-dimensional cobordism, such that vertical projection is a Morse function with a critical point of index 0 and a critical point of index 1, so that $S$ has a handle decomposition with a 0 -handle and a 1-handle. The handles satisfy the hypothesis of 8.24 , so that $S$ is diffeomorphic to the unit interval $I$.

Example 8.26 (i) For any $i \leqslant m$ regard $S^{m}$ as

$$
S^{m}=\partial\left(D^{i} \times D^{m-i+1}\right)=S^{i-1} \times D^{m-i+1} \cup D^{i} \times S^{m-i}
$$

as in 1.3 (ii), and define $(m+1)$-dimensional elementary cobordisms of index $i, i+1$

$$
\begin{aligned}
& \left(W_{1} ; M_{0}, M_{1}\right)=\left(S^{m} \times I \cup D^{i} \times D^{m-i+1} ; S^{m}, S^{i} \times S^{m-i}\right) \\
& \left(W_{2} ; M_{1}, M_{2}\right)=\left(S^{i} \times S^{m-i} \times I \cup D^{i+1} \times D^{m-i} ; S^{i} \times S^{m-i}, S^{m}\right) .
\end{aligned}
$$


The cores of the attaching embeddings of the $(i+1)$-handle $W_{2}$ and the dual $(m-i+1)$-handle $\bar{W}_{1}$ intersect transversely with a single intersection point

$$
S^{i} \times\{0\} \cap\{0\} \times S^{m-i}=\{\text { pt. }\} \hookrightarrow M_{1}=S^{i} \times S^{m-i}
$$

and there is defined a diffeomorphism

$$
\begin{aligned}
& \left(W_{1} \cup W_{2} ; M_{0}, M_{2}\right) \cong S^{m} \times(I ;\{0\},\{1\}) \text {. }
\end{aligned}
$$

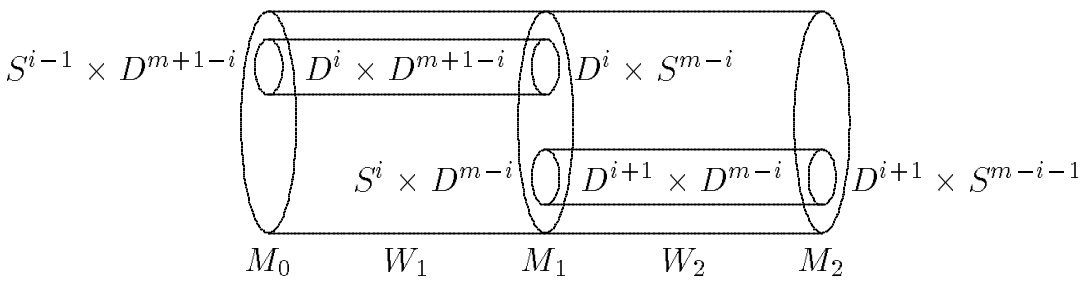

(ii) For $m=0, i=0$ the cancellation of a 0 -handle on $S^{0} \times I$ and a 1-handle is given by

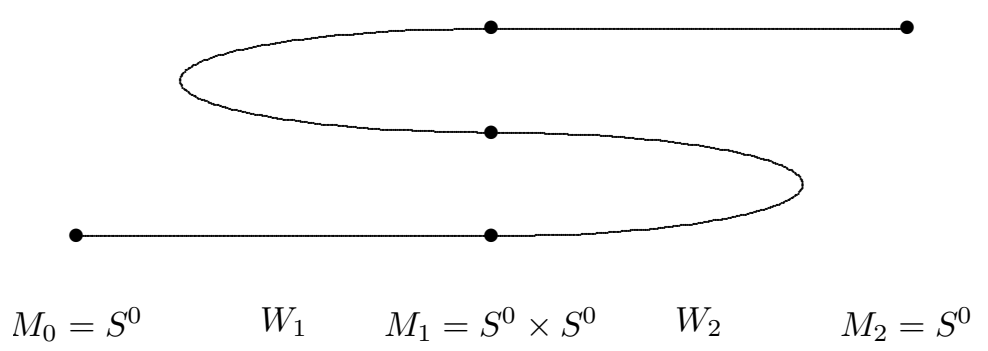

(iii) For $m=1, i=1$ the cancellation of a 1-handle on $S^{1} \times I$ and a 2-handle is given by

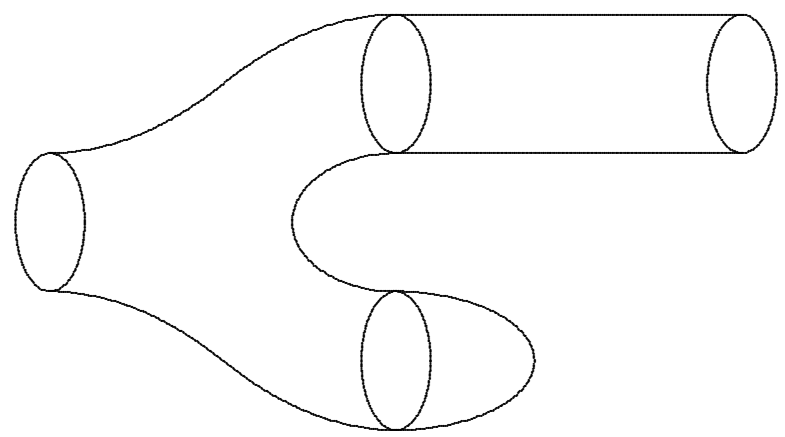

$S^{1} \quad W_{1} \quad S^{1} \times S^{0} \quad W_{2} \quad S^{1}$

(iv) For $m=2, i=1$ the cancellation of a 1-handle on $S^{2} \times I$ and a 2-handle can be viewed as follows. Embed the solid torus $S^{1} \times D^{2}$ in the interior of $D^{3}$, and embed a smaller copy of $D^{3}$ inside $S^{1} \times D^{2}$. The space between the two $D^{3}$ 's 
is a union of a 1-handle on $S^{2} \times I$ and a cancelling 2-handle, which is a trivial $h$-cobordism diffeomorphic to the product $S^{2} \times(I ;\{0\},\{1\})$.

Translating the Handle Cancellation Lemma 8.24 into the language of surgery :

Corollary 8.27 Let $\left(W_{1} ; M_{0}, M_{1}\right)$ be the trace of an $(i-1)$-surgery on an $m$ dimensional manifold $M=M_{0}$ removing the framed $(i-1)$-embedding $S^{i-1} \times$ $D^{m-i+1} \hookrightarrow M$. Let $\left(W_{2} ; M_{1}, M_{2}\right)$ be the trace of an $i$-surgery on $M_{1}$ removing a framed $i$-embedding $S^{i} \times D^{m-i} \hookrightarrow M_{1}$. If the core of $S^{i} \times D^{m-i} \hookrightarrow M_{1}$ and the core of $D^{i} \times S^{m-i} \hookrightarrow M_{1}$ intersect transversely with a single intersection point then the union

$$
\left(W ; M, M^{\prime}\right)=\left(W_{1} ; M_{0}, M_{1}\right) \cup\left(W_{2} ; M_{1}, M_{2}\right)
$$

is a trivial h-cobordism, with a diffeomorphism

$$
\left(W ; M, M^{\prime}\right) \cong M \times(I ;\{0\},\{1\}) .
$$

Definition 8.28 A $\pi_{1}$-cobordism is a cobordism $\left(W ; M, M^{\prime}\right)$ such that the inclusions $M \rightarrow W, M^{\prime} \rightarrow W$ induce isomorphisms

$$
\pi_{1}(M) \cong \pi_{1}(W) \quad, \quad \pi_{1}\left(M^{\prime}\right) \cong \pi_{1}(W) .
$$

Example 8.29 (i) An $(m+1)$-dimensional cobordism $\left(W ; M, M^{\prime}\right)$ with a handle decomposition involving only handles of index $\geqslant 3$ and $\leqslant m-2$ is a $\pi_{1}$-cobordism. (ii) An $h$-cobordism is a $\pi_{1}$-cobordism.

Proposition 8.30 An $(m+1)$-dimensional $\pi_{1}$-cobordism $\left(W^{m+1} ; M, M^{\prime}\right)$ is an $h$-cobordism if and only if the cellular $\mathbb{Z}\left[\pi_{1}(W)\right]$-module chain complex $C(\widetilde{W}, \widetilde{M})$ is contractible, with $\widetilde{W}, \widetilde{M}$ the universal covers of $W, M$.

Proof By J.H.C.Whitehead's Theorem (3.6) the inclusion $M \hookrightarrow W$ is a homotopy equivalence if and only if the chain map $C(\widetilde{M}) \rightarrow C(\widetilde{W})$ is a $\mathbb{Z}\left[\pi_{1}(W)\right]$ module chain equivalence, which is the case if and only if $C(\widetilde{W}, \widetilde{M})$ is contractible. By Poincaré duality (4.4) there is a chain equivalence

$$
C\left(\widetilde{W}, \widetilde{M}^{\prime}\right)^{m+1-*} \simeq C(\widetilde{W}, \widetilde{M}),
$$

so that $M \hookrightarrow W$ is a homotopy equivalence if and only if $M^{\prime} \hookrightarrow W$ is a homotopy equivalence.

The first step in the proofs of the $h$ - and $s$-Cobordism Theorems is to arrange an $(i, i+1)$-index handle decomposition: 
Proposition 8.31 For $m \geqslant 5$ every $(m+1)$-dimensional h-cobordism $\left(W ; M, M^{\prime}\right)$ admits an $(i, i+1)$-index handle decomposition

$$
\left(W ; M, M^{\prime}\right)=\left(W_{1} ; M_{0}, M_{1}\right) \cup\left(W_{2} ; M_{1}, M_{2}\right) \cup \ldots \cup\left(W_{k} ; M_{k-1}, M_{k}\right)
$$

for any $i$ with $2 \leqslant i \leqslant m-2$.

Proof Let $\widetilde{W}, \widetilde{M}$ be the universal covers of $W, M$, and let

$$
\pi_{1}(M)=\pi_{1}(W)=\pi
$$

The cellular chain complex of $C(\widetilde{W}, \widetilde{M})$ is a contractible $\mathbb{Z}[\pi]$-module chain complex

$$
C(\widetilde{W}, \widetilde{M})=C: C_{m+1} \rightarrow C_{m} \rightarrow \ldots \rightarrow C_{0}
$$

with $C_{i}=\mathbb{Z}[\pi]^{b_{i}}$ the based f.g. free $\mathbb{Z}[\pi]$-module generated by the $b_{i}$ handles of index $i$.

The proof that for any $i \leqslant m-2$ there exists a handle decomposition for $\left(W ; M, M^{\prime}\right)$ with handles of index $\geqslant i$ proceeds by cancelling handles of increasing index, as follows.

Assume inductively that $\left(W ; M, M^{\prime}\right)$ only has handles of index $\geqslant j$, so that $C_{i}=0$ for $i<j$ and $d: C_{j+1} \rightarrow C_{j}$ is onto. Choose a splitting $\mathbb{Z}[\pi]$-module morphism $\Gamma: C_{j} \rightarrow C_{j+1}$, so that

$$
d \Gamma=1: C_{j} \rightarrow C_{j}
$$

and let $C^{\prime}, C^{\prime \prime}$ be the contractible based f.g. free $\mathbb{Z}[\pi]$-module chain complexes defined by

$$
\begin{aligned}
& C^{\prime}: \ldots \rightarrow C_{j+4} \stackrel{d}{\rightarrow} C_{j+3} \stackrel{\left(\begin{array}{l}
d \\
0
\end{array}\right)}{\longrightarrow} C_{j+2} \oplus C_{j} \\
& \stackrel{\left(\begin{array}{cc}
d & \Gamma \\
0 & -1
\end{array}\right)}{\longrightarrow} C_{j+1} \oplus C_{j} \stackrel{\left(\begin{array}{ll}
d & 1
\end{array}\right)}{\longrightarrow} C_{j} \rightarrow 0 \rightarrow \ldots, \\
& C^{\prime \prime}: \ldots \rightarrow C_{j+4} \stackrel{d}{\rightarrow} C_{j+3} \\
& \stackrel{\left(\begin{array}{l}
d \\
0
\end{array}\right)}{\longrightarrow} C_{j+2} \oplus C_{j} \stackrel{\left(\begin{array}{ll}
d & \Gamma
\end{array}\right)}{\longrightarrow} C_{j+1} \rightarrow 0 \rightarrow \ldots
\end{aligned}
$$

with $C_{i}^{\prime}=0$ for $i<j$ and $C_{i}^{\prime \prime}=0$ for $i \leqslant j$. If $j \leqslant m-3$ then by the Whitney trick (7.27) it is possible to realize $C^{\prime}$ by a handle decomposition of $\left(W ; M, M^{\prime}\right)$ with $b_{j+1}+b_{j}$ handles of index $j+1$ and $b_{j+2}+b_{j}$ handles of index $j+2$. The $b_{j}$ handles of index $j+1$ cancel with the $b_{j}$ handles of index $j$ (by 8.24, using $m \geqslant 5)$, so that $\left(W ; M, M^{\prime}\right)$ has a handle decomposition realizing $C^{\prime \prime}$.

Thus for any $i \leqslant m-2$ there exists a decomposition for $\left(W ; M, M^{\prime}\right)$ with handles of index $\geqslant i$ only. The proof that there exists a decomposition for $\left(W ; M, M^{\prime}\right)$ 
with handles of index $\leqslant i+1$ now follows by duality: the handles of index $j$ in $\left(W ; M, M^{\prime}\right)$ are the handles of index $(m+1-j)$ in $\left(-W ; M^{\prime}, M\right)$.

In fact, for any chain contraction $\Gamma: 0 \simeq 1: C \rightarrow C$ there exists an $(i, i+1)$ index handle decomposition of $\left(W ; M, M^{\prime}\right)$ with $c i$-handles and $c(i+1)$-handles, where

$$
c=\sum_{r} b_{i+2 r}=\sum_{r} b_{i+2 r+1},
$$

and cellular chain complex

$$
\ldots \rightarrow 0 \rightarrow \mathbb{Z}[\pi]^{c}=\bigoplus_{r} C_{i+2 r+1} \stackrel{d+\Gamma}{\longrightarrow} \mathbb{Z}[\pi]^{c}=\bigoplus_{r} C_{i+2 r} \rightarrow 0 \rightarrow \ldots
$$

(cf. Definition 8.10).

Proposition 8.32 An $(m+1)$-dimensional $\pi_{1}$-cobordism $\left(W ; M, M^{\prime}\right)$ with an $(i, i+1)$-index handle decomposition

$$
\left(W ; M, M^{\prime}\right)=\left(W_{1} ; M_{0}, M_{1}\right) \cup\left(W_{2} ; M_{1}, M_{2}\right) \cup \ldots \cup\left(W_{k} ; M_{k-1}, M_{k}\right)
$$

is an $h$-cobordism if and only if the number of $i$-handles is equal to the number of $(i+1)$-handles, say $c$, and the $c \times c$ matrix of intersection numbers with entries in $\mathbb{Z}[\pi]$

$$
\lambda=\left(\lambda\left(W_{j}, W_{k+c}\right)\right) \in M_{c, c}\left(\mathbb{Z}\left[\pi_{1}(W)\right]\right)
$$

is invertible, with $W_{j}(1 \leqslant j \leqslant c)$ running over all the $i$-handles and $W_{k+c}$ $(1 \leqslant k \leqslant c)$ running over all the $(i+1)$-handles.

Proof This is a special case of 8.30 , with

$$
C(\widetilde{W}, \widetilde{M}): \ldots \longrightarrow 0 \longrightarrow \mathbb{Z}\left[\pi_{1}(W)\right]^{c} \stackrel{\lambda}{\longrightarrow} \mathbb{Z}\left[\pi_{1}(W)\right]^{c} \longrightarrow 0 \longrightarrow \ldots
$$

The $s$-Cobordism and $h$-Cobordism Theorems $(1.9,1.11)$ are now stated again, and their proofs are outlined:

$s$-Cobordism Theorem 8.33 (Barden-Mazur-Stallings)

An $(m+1)$-dimensional $h$-cobordism $\left(W^{m+1} ; M, M^{\prime}\right)$ with $m \geqslant 5$ is trivial if and only if it is an s-cobordism.

Proof A trivial $h$-cobordism is an $s$-cobordism (8.21).

Conversely, suppose that $\left(W ; M, M^{\prime}\right)$ is an $s$-cobordism, so that by 8.31 there 
exists an $(i, i+1)$-index handle decomposition for some $i$ with $2 \leqslant i \leqslant m-2$, with $2 c$ handles, and intersection matrix $\lambda \in G L_{c}\left(\mathbb{Z}\left[\pi_{1}(W)\right]\right)$ such that

$$
\tau\left(W ; M, M^{\prime}\right)=\tau(\lambda)=0 \in W h\left(\pi_{1}(W)\right) .
$$

For some $b \geqslant 0$ there exist elementary matrices

$$
E_{1}, E_{2}, \ldots, E_{k} \in G L_{b+c}\left(\mathbb{Z}\left[\pi_{1}(W)\right]\right)
$$

such that

$$
\lambda \oplus 1_{b}=E_{1} E_{2} \ldots E_{k} \in G L_{b+c}\left(\mathbb{Z}\left[\pi_{1}(W)\right]\right) .
$$

It is possible to realize this identity by adding $b$ trivially cancelling $i$ - and $(i+1)$ handles, and to rearrange the handles in such a way that the new intersection matrix $\lambda^{\prime} \in G L_{b+c}\left(\mathbb{Z}\left[\pi_{1}(W)\right]\right)$ of the $i$ - and $(i+1)$-handles is diagonal with entries $\pm g \in \pi_{1}(W)$. Apply the Whitney trick (7.27) to realize these algebraic intersections geometrically : each of the $(b+c)$ pairs of $i$ - and $(i+1)$-handles is such that the core and the core of the dual handle intersect transversely in a single point, and so may be cancelled (8.24) leaving the empty handle decomposition of a trivial $h$-cobordism.

h-Cobordism Theorem 8.34 (Smale [83])

An $(m+1)$-dimensional $h$-cobordism $\left(W^{m+1} ; M, M^{\prime}\right)$ with $m \geqslant 5$ and $\pi_{1}(M)=$ $\{1\}$ is trivial.

Proof This is the special case $\pi_{1}=\{1\}$ of the $s$-Cobordism Theorem, noting that $W h(\{1\})=0$.

\subsection{Lens spaces}

Lens spaces are 3-dimensional manifolds which provided the first examples of a divergence between the homotopy and diffeomorphism classifications of manifolds. See Milnor [58], deRham et al. [20] and Cohen [19] for a more detailed exposition.

The classification of lens spaces depends on the computation of the Whitehead group $W h\left(\mathbb{Z}_{m}\right)$ : indeed, this application was one of the original motivations for the development of Whitehead torsion. See Chapter 14E of Wall [92] for the surgery classification in the $P L$ category of the high-dimensional 'fake lens spaces', which are manifolds with cyclic fundamental group and universal cover a sphere of dimension $\geqslant 5$. This led to the classification of free actions of finite groups on high-dimensional spheres (Madsen, Thomas and Wall [46]), one of the early triumphs of non-simply-connected surgery theory. 
Write

$$
\begin{aligned}
& \mathbb{Z}_{m}=\left\langle t \mid t^{m}\right\rangle, \quad N=\sum_{i=0}^{m-1} t^{i} \in \mathbb{Z}\left[\mathbb{Z}_{m}\right], \\
& A_{m}=\mathbb{Z}\left[\mathbb{Z}_{m}\right], \quad B_{m}=\mathbb{Q}\left[\mathbb{Z}_{m}\right], \quad R_{m}=B_{m} /(N) .
\end{aligned}
$$

The determinant map det $: K_{1}(\Lambda) \rightarrow \Lambda^{\bullet}$ is an isomorphism for each of the rings $\Lambda=A_{m}, B_{m}, \mathbb{Q}, R_{m}$. Define the augmentation

$$
\epsilon: A_{m} \rightarrow \mathbb{Z} ; \sum_{i} n_{i} t^{i} \mapsto \sum_{i} n_{i}
$$

and similarly for $\epsilon: B_{m} \rightarrow \mathbb{Q}$. Use the ring isomorphism

$$
B_{m} \rightarrow \mathbb{Q} \times R_{m} ; x \mapsto(\epsilon(x), x-N \epsilon(x) / m)
$$

to identify

$$
B_{m}=\mathbb{Q} \times R_{m}
$$

Definition 8.35 The Whitehead group of $R_{m}$ is

$$
W h\left(R_{m}\right)=R_{m}^{\bullet} /\left\{q(t-N / m)^{n} \mid q \in \mathbb{Q}^{\bullet}, n \in \mathbb{Z}\right\} .
$$

The computation of $W h\left(\mathbb{Z}_{m}\right)$ uses the inclusion $A_{m} \rightarrow B_{m}=\mathbb{Q} \times R_{m}$ and the composite

$$
K_{1}\left(A_{m}\right)=A_{m}^{\bullet} \rightarrow K_{1}\left(B_{m}\right)=B_{m}^{\bullet}=\mathbb{Q}^{\bullet} \times R_{m}^{\bullet} \rightarrow K_{1}\left(R_{m}\right)=R_{m}^{\bullet} .
$$

It turns out that $W h\left(R_{m}\right)$ is a free abelian group detected by Reidemeister torsion, and that $W h\left(\mathbb{Z}_{m}\right) \rightarrow W h\left(R_{m}\right)$ is an injection with the image detected by certain congruences.

Every unit $r \in \mathbb{Z}_{m}^{\bullet}$ determines a unit

$$
u(r)=t^{r}-1+N / m \in B_{m}^{\bullet}
$$

with inverse

$$
u(r)^{-1}=\sum_{i=1}^{m} i t^{r(i-1)} / m+(1-m) N / 2 m \in B_{m}^{\bullet},
$$

and such that

$$
u(-r)=t^{-r}(2 N / m-1) u(r) \in B_{m}^{\bullet} .
$$

The unit $u(r)$ is determined by the identity

$$
u(r)=t^{r}-1 \in R_{m}^{\bullet} .
$$

Let $\phi(m)=\left|\mathbb{Z}_{m}^{\bullet}\right|$ be the Euler function counting the number of positive integers $<m$ coprime to $m$, and let

$$
1=r_{1}<r_{2}<\ldots<r_{k} \quad(k=\phi(m) / 2)
$$

be the enumeration of the integers $r$ coprime to $m$ with $1 \leq r \leq[m / 2]$. 
Proposition 8.36 Every unit $u \in B_{m}^{\bullet}$ has a unique factorisation

$$
u=t^{n}(p N / m+q(1-N / m)) u\left(r_{1}\right)^{a_{1}} u\left(r_{2}\right)^{a_{2}} \ldots u\left(r_{k}\right)^{a_{k}} \in B_{m}^{\bullet}
$$

with $n \in \mathbb{Z}, p, q \in \mathbb{Q}^{\bullet}, a_{i} \in \mathbb{Z}$. The Reidemeister torsion function

$$
\begin{aligned}
& \Delta: W h\left(R_{m}\right) \rightarrow \mathbb{Z}^{k} ; \\
& u=\left(t^{r_{1}}-1\right)^{a_{1}}\left(t^{r_{2}}-1\right)^{a_{2}} \ldots\left(t^{r_{k}}-1\right)^{a_{k}} \mapsto \Delta(u)=\left(a_{1}, a_{2}, \ldots, a_{k}\right)
\end{aligned}
$$

is an isomorphism.

Proof The essential ingredient is the Franz independence lemma.

Proposition 8.37 (i) The units of $A_{m}=\mathbb{Z}\left[\mathbb{Z}_{m}\right]$ are of the type $\pm t^{n} u(n \in \mathbb{Z})$, with $u \in A_{m}^{\bullet}$ the unique unit determined by the equations

$$
u \prod_{a_{i}<0}\left(t^{r_{i}}-1\right)^{-a_{i}}= \pm \prod_{a_{j}>0}\left(t^{r_{j}}-1\right)^{a_{j}} \in A_{m}
$$

for a $k$-tuple $\left(a_{1}, a_{2}, \ldots, a_{k}\right) \in \mathbb{Z}^{k}$ satisfying

(a) $a_{1}+a_{2}+\ldots+a_{k}=0 \in \mathbb{Z}$

(b) $\left(r_{1}\right)^{a_{1}}\left(r_{2}\right)^{a_{2}} \ldots\left(r_{k}\right)^{a_{k}} \equiv \pm 1(\bmod m)$.

The sign \pm in $(\mathrm{b})$ is such that

$$
\epsilon(u)= \pm 1 \in \mathbb{Z}^{\bullet}=\{ \pm 1\}
$$

(ii) The Whitehead group $W h\left(\mathbb{Z}_{m}\right)$ of the cyclic group $\mathbb{Z}_{m}$ is free abelian of rank $[m / 2]+1-\delta(m)$, with $\delta(m)$ the number of divisors of $m$. The Reidemeister torsion map

$$
\Delta: W h\left(\mathbb{Z}_{m}\right)=\mathbb{Z}^{[m / 2]+1-\delta(m)} \rightarrow W h\left(R_{m}\right)=\mathbb{Z}^{k}
$$

is an injection onto the subgroup of $\mathbb{Z}^{k}$ consisting of the elements $\left(a_{1}, a_{2}, \ldots, a_{k}\right) \in$ $\mathbb{Z}^{k}$ satisfying the conditions (a) and (b) of (i).

Proof See Milnor [58, $\S 12]$.

Example 8.38 (i) If $m \geqslant 3$ is prime then

$$
\delta(m)=2, \phi(m)=m-1, k=(m-1) / 2, r_{j}=j
$$

and

$$
W h\left(\mathbb{Z}_{m}\right)=\mathbb{Z}^{(m-3) / 2}, W h\left(R_{m}\right)=\mathbb{Z}^{(m-1) / 2}
$$


(ii) For $m=5$ the unit $u=1-t+t^{2} \in \mathbb{Z}\left[\mathbb{Z}_{5}\right]$ is a generator of $W h\left(\mathbb{Z}_{5}\right)=\mathbb{Z}$ with

$$
\begin{aligned}
& u=t^{2}(-1+2 N / 5)(t-1+N / 5)^{2}\left(t^{2}-1+N / 5\right)^{-2}, \\
& \Delta(u)=(2,-2) \in \operatorname{im}\left(W h\left(\mathbb{Z}_{5}\right)\right) \hookrightarrow W h\left(R_{5}\right)=\mathbb{Z}^{2} .
\end{aligned}
$$

Given an invertible $2 \times 2$ matrix with entries in $\mathbb{Z}$

$$
M=\left(\begin{array}{ll}
a & b \\
c & d
\end{array}\right) \in G L_{2}(\mathbb{Z})
$$

use complex multiplication in $S^{1}=\{z \in \mathbb{C}|| z \mid \leq 1\}$ to define a diffeomorphism

$$
f_{M}: S^{1} \times S^{1} \rightarrow S^{1} \times S^{1} ;(x, y) \mapsto\left(x^{a} y^{b}, x^{c} y^{d}\right)
$$

inducing

$$
\begin{gathered}
\left(f_{M}\right)_{*}=M: H_{1}\left(S^{1} \times S^{1}\right)=\mathbb{Z} \oplus \mathbb{Z} \rightarrow H_{1}\left(S^{1} \times S^{1}\right)=\mathbb{Z} \oplus \mathbb{Z} ; \\
(x, y) \mapsto(a x+b y, c x+d y) .
\end{gathered}
$$

The identification space

$$
L=S^{1} \times D^{2} \cup_{M} S^{1} \times D^{2}
$$

is obtained by glueing together two copies of the solid torus $S^{1} \times D^{2}$ along the boundary using the diffeomorphism $M: \partial\left(S^{1} \times D^{2}\right)=S^{1} \times S^{1} \rightarrow S^{1} \times S^{1}$.

Definition 8.39 The lens space $L(m, n)$ is the closed oriented 3-dimensional manifold defined for coprime integers $m, n \geqslant 0$ to be the identification space

$$
L(m, n)=S^{1} \times D^{2} \cup_{M} S^{1} \times D^{2}
$$

with $M=\left(\begin{array}{cc}p & m \\ q & n\end{array}\right) \in G L_{2}(\mathbb{Z})$ for any $p, q \in \mathbb{Z}$ such that $n p-m q=1$.

Proposition 8.40 (i) The oriented diffeomorphism class of $L(m, n)$ depends only on the class of $M$ under the equivalence relation

$$
M \sim A M B \text { for } A=\left(\begin{array}{ll}
1 & 0 \\
a & 1
\end{array}\right), B=\left(\begin{array}{ll}
1 & 0 \\
b & 1
\end{array}\right) \in G L_{2}(\mathbb{Z})
$$

with $a, b \in \mathbb{Z}$. In particular, there is an orientation-preserving diffeomorphism

$$
L(m, n) \cong L(m, a m+n)
$$

for any $a \in \mathbb{Z}$.

(ii) $L(m,-n)$ is $L(m, n)$ with the opposite orientation.

(iii) $L(m, n)$ is parallelisable, i.e. the tangent bundle $\tau_{L(m, n)}: L(m, n) \rightarrow B O(3)$ is trivial. 
Example 8.41 (i) $L(0,1)=S^{2} \times S^{1}$.

(ii) $L(1, n)=S^{3}$.

(iii) $L(2,1)=S O(3)=\mathbb{R P}^{3}$, the tangent $S^{1}$-bundle of $S^{2}$.

Proposition 8.42 For $m \geqslant 2$

$$
L(m, n)=S^{3} / \mathbb{Z}_{m}
$$

is the quotient of the free $\mathbb{Z}_{m}$-action on the 3 -sphere

$$
S^{3}=\left\{\left.\left(z_{1}, z_{2}\right) \in \mathbb{C}^{2}|| z_{1}\right|^{2}+\left|z_{2}\right|^{2}=1\right\}
$$

generated by

$$
t: S^{3} \rightarrow S^{3} ;\left(z_{1}, z_{2}\right) \mapsto\left(z_{1} \omega, z_{2} \omega^{n}\right)
$$

with $\omega=\exp (2 \pi i / m)$. The fundamental group is

$$
\pi_{1}(L(m, n))=\mathbb{Z}_{m}=\left\langle t \mid t^{m}\right\rangle .
$$

The cellular $A_{m}$-module chain complex of the universal cover $\widetilde{L}(m, n)=S^{3}$ is given by

$$
C(\widetilde{L}(m, n)): \ldots \longrightarrow 0 \longrightarrow A_{m} \stackrel{t^{n}-1}{\longrightarrow} A_{m} \stackrel{N}{\longrightarrow} A_{m} \stackrel{t-1}{\longrightarrow} A_{m}
$$

with $N=1+t+t^{2}+\ldots+t^{m-1} \in A_{m}$.

For the remainder of Section 8.3 it is assumed that $m \geqslant 2$.

Definition 8.43 The Reidemeister torsion of a lens space $L(m, n)$ with respect to a particular choice of generator $t \in \pi_{1}(L(m, n))=\mathbb{Z}_{m}$ is the torsion of the induced contractible based f.g. free $R_{m}$-module chain complex $R_{m} \otimes_{A_{m}}$ $C(\widetilde{L}(m, n))$

$$
\begin{aligned}
\Delta_{t}(L(m, n)) & =\tau\left(R_{m} \otimes_{A_{m}} C(\widetilde{L}(m, n))\right) \\
& =\left(t^{n}-1\right)(t-1) \in W h\left(R_{m}\right)=\mathbb{Z}^{k} \quad(k=\phi(m) / 2) .
\end{aligned}
$$

It is clear that $m=m^{\prime}$ is a necessary condition for lens spaces $L(m, n)$, $L\left(m^{\prime}, n^{\prime}\right)$ to be homotopy equivalent.

Proposition 8.44 The following conditions on two lens spaces $L(m, n), L\left(m, n^{\prime}\right)$ are equivalent:

(i) $L(m, n), L\left(m, n^{\prime}\right)$ are homotopy equivalent, 
(ii) there exist units $u \in A_{m}^{\bullet}, r \in \mathbb{Z}_{m}^{\bullet}$ such that

$$
u\left(t^{n^{\prime} r}-1\right)\left(t^{r}-1\right)=\left(t^{n}-1\right)(t-1) \in B_{m},
$$

(iii) $n \equiv \pm n^{\prime} r^{2}(\bmod m)$ for some $r \in \mathbb{Z}_{m}^{\bullet}$,

(iv) for some generators $t \in \pi_{1}(L(m, n))=\mathbb{Z}_{m}, t^{\prime} \in \pi_{1}\left(L\left(m, n^{\prime}\right)\right)=\mathbb{Z}_{m}$

$$
\Delta_{t}(L(m, n))-\Delta_{t^{\prime}}\left(L\left(m, n^{\prime}\right)\right) \in \operatorname{im}\left(W h\left(\mathbb{Z}_{m}\right) \rightarrow W h\left(R_{m}\right)\right) .
$$

If $L(m, n), L\left(m, n^{\prime}\right)$ satisfy these conditions there is a unique homotopy class of homotopy equivalences $h: L\left(m, n^{\prime}\right) \rightarrow L(m, n)$ with

$$
h_{*}\left(t^{\prime}\right)=t^{r} \in \pi_{1}(L(m, n))=\mathbb{Z}_{m}
$$

and

$$
\begin{aligned}
\tau(h) & =\Delta_{t}(L(m, n))-\Delta_{t^{\prime}}\left(L\left(m, n^{\prime}\right)\right)=\tau(u) \\
& =\tau\left(\left(t^{n}-1\right)(t-1)\left(t^{n^{\prime} r}-1\right)^{-1}\left(t^{r}-1\right)^{-1}\right) \in \operatorname{im}\left(W h\left(\mathbb{Z}_{m}\right) \rightarrow W h\left(R_{m}\right)\right) .
\end{aligned}
$$

The homotopy equivalence $h$ preserves orientations if and only if

$$
n \equiv n^{\prime} r^{2}(\bmod m)
$$

i.e. if $\epsilon(u)=+1 \in \mathbb{Z} \bullet$.

Proof (i) $\Longrightarrow$ (ii) Given a homotopy equivalence $h: L\left(m, n^{\prime}\right) \rightarrow L(m, n)$ choose generators

$$
t \in \pi_{1}(L(m, n))=\mathbb{Z}_{m}, \quad t^{\prime} \in \pi_{1}\left(L\left(m, n^{\prime}\right)\right)=\mathbb{Z}_{m},
$$

so that $h_{*}\left(t^{\prime}\right)=t^{r} \in \mathbb{Z}_{m}$ for some $r \in \mathbb{Z}_{m}^{\bullet}$. Regarding $t, t^{\prime}$ as generating covering translations

$$
\begin{aligned}
& t: \widetilde{L}(m, n)=S^{3} \rightarrow \widetilde{L}(m, n)=S^{3}, \\
& t^{\prime}: \widetilde{L}\left(m, n^{\prime}\right)=S^{3} \rightarrow \widetilde{L}\left(m, n^{\prime}\right)=S^{3}
\end{aligned}
$$

it is possible to lift $h$ to a homotopy equivalence

$$
\widetilde{h}: \widetilde{L}\left(m, n^{\prime}\right)=S^{3} \rightarrow \widetilde{L}(m, n)=S^{3}
$$

such that

$$
\widetilde{h} t^{\prime}=t^{r} \widetilde{h}: S^{3} \rightarrow S^{3} .
$$

The induced cellular $A_{m}$-module chain map is given by

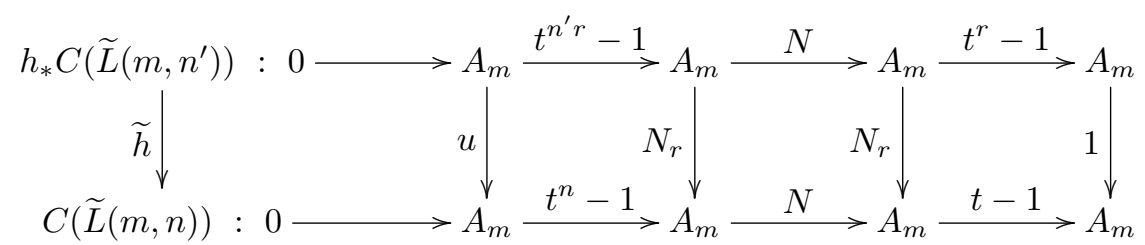


with

$$
N_{r}=1+t+\ldots+t^{r-1} \in A_{m}
$$

and $u \in A_{m}^{\bullet}$ the unique unit such that

$$
u\left(t^{n}-1\right)(t-1)=\left(t^{n^{\prime} r}-1\right)\left(t^{r}-1\right) \in B_{m} .
$$

(ii) $\Longleftrightarrow$ (iii) $\Longleftrightarrow$ (iv) Immediate from 8.37

(iii) $\Longrightarrow$ (i) See Cohen $[19, \S 29]$ for the explicit construction of a homotopy equivalence $h: L\left(m, n^{\prime}\right) \rightarrow L(m, n)$ realizing every $n \equiv \pm n^{\prime} r^{2}(\bmod m)$.

Example 8.45 The lens spaces $L(5,1), L(5,2)$ are not homotopy equivalent, even though they have isomorphic homotopy, homology and cohomology groups; $L(5,1), L(5,2)$ have different Reidemeister torsions $\Delta(8.36)$ and different homology linking pairings $(12.44)$ on $H_{1}(L(5,1))=H_{1}(L(5,2))=\mathbb{Z}_{5}$.

Proposition 8.46 The following conditions on two lens spaces $L(m, n), L\left(m, n^{\prime}\right)$ are equivalent:

(i) $L(m, n), L\left(m, n^{\prime}\right)$ are diffeomorphic,

(ii) $L(m, n), L\left(m, n^{\prime}\right)$ are simple homotopy equivalent,

(iii) $L(m, n), L\left(m, n^{\prime}\right)$ are oriented $h$-cobordant,

(iv) $n \equiv \pm n^{\prime} r^{2}(\bmod m)$ with $r \equiv 1$ or $n(\bmod m)$,

(v) for some generators $t \in \pi_{1}(L(m, n))=\mathbb{Z}_{m}, t^{\prime} \in \pi_{1}\left(L\left(m, n^{\prime}\right)\right)=\mathbb{Z}_{m}$

$$
\Delta_{t^{\prime}}\left(L\left(m, n^{\prime}\right)\right)=\Delta_{t}(L(m, n)) \in W h\left(R_{m}\right) .
$$

If $L(m, n), L\left(m, n^{\prime}\right)$ satisfy these conditions there is a diffeomorphism

$$
h: L\left(m, n^{\prime}\right) \rightarrow L(m, n)
$$

such that $h_{*}\left(t^{\prime}\right)=t^{r} \in \pi_{1}(L(m, n))$. The diffeomorphism $h$ preserves orientations if and only if $n \equiv n^{\prime} r^{2}(\bmod m)$.

Example 8.47 (i) The lens spaces $L(7,1)$ and $L(7,2)$ are homotopy equivalent but not diffeomorphic. The Reidemeister torsions are

$$
\Delta_{t}(L(7,1))=(t-1)^{2}, \quad \Delta_{t}(L(7,2))=(t-1)\left(t^{2}-1\right),
$$

so that there exists an orientation-preserving homotopy equivalence $h: L(7,1) \rightarrow$ $L(7,2)$ with $h_{*}(t)=t^{3}$. The torsion of $h$ is non-zero

$$
\tau(h)=(t-1)\left(t^{2}-1\right)\left(t^{3}-1\right)^{-2} \neq 0 \in \operatorname{im}\left(W h\left(\mathbb{Z}_{7}\right) \rightarrow W h\left(R_{7}\right)\right),
$$

so that $h$ is not homotopic to a diffeomorphism. (In general, it is not possible to deduce that manifolds $M, N$ are not diffeomorphic just because a particular 
homotopy equivalence $M \rightarrow N$ is not homotopic to a diffeomorphism).

(ii) The lens spaces $L(7,1)$ and $L(7,2)$ are homotopy equivalent but not $h$ cobordant. Milnor [54] showed that the homotopy equivalence

$$
h \times 1: L(7,1) \times S^{4} \rightarrow L(7,2) \times S^{4}
$$

is realized by a non-trivial 8 -dimensional $h$-cobordism

$$
\left(W^{8} ; L(7,1) \times S^{4}, L(7,2) \times S^{4}\right)
$$

(with torsion $\tau(h)$ ) such that the boundary components $L(7,1) \times S^{4}, L(7,2) \times S^{4}$ are not diffeomorphic. 


\section{POINCARÉ COMPLEXES AND SPHERICAL FIBRATIONS}

This chapter describes the homotopy theoretic analogues of manifolds and vector bundles, namely Poincaré complexes and spherical fibrations. An $m$ dimensional Poincaré complex $X$ is a space with the universal coefficient Poincaré duality properties of an $m$-dimensional manifold. Any space which is homotopy equivalent to a manifold is a Poincaré complex. Surgery theory investigates the extent to which a Poincaré complex is homotopy equivalent to a manifold.

A spherical fibration is a fibration of the type

$$
S^{k-1} \rightarrow E \rightarrow B
$$

such as the sphere bundle of a $k$-plane bundle over $B$. In general, spherical fibrations do not come from vector bundles, and Poincaré complexes are not homotopy equivalent to manifolds.

A Poincaré complex $X$ is homotopy equivalent to a manifold if and only if :

(i) there exists a manifold $M$ with a degree 1 normal map $(f, b): M^{m} \rightarrow X$,

(ii) there exists $(f, b)$ as in (i) with a sequence of surgeries on $M$ such that the trace $\left(W^{m+1} ; M^{m}, M^{\prime m}\right)$ is the domain of a degree 1 normal bordism

$$
\left((F, B) ;(f, b),\left(f^{\prime}, b^{\prime}\right)\right):\left(W ; M, M^{\prime}\right) \rightarrow X \times(I ;\{0\},\{1\})
$$

with $f^{\prime}: M^{\prime} \rightarrow X$ a homotopy equivalence.

The Browder-Novikov theory shows that for any dimension $m$ the bordism classes of degree 1 normal maps $(f, b)$ as in (i) are in natural one-one correspondence with the vector bundle structures (if any!) on the Spivak normal fibration $X$. The surgery obstruction theory for deciding if a degree 1 normal map $(f, b)$ is in fact normal bordant to a homotopy equivalence as in (ii) will be developed in Chapters 11,12.

Sections 9.1, 9.2 give the basic properties of Poincaré complexes and spherical fibrations. Section 9.3 describes the Spivak normal fibration $\nu_{X}$ of a Poincaré complex $X$, the homotopy theoretic analogue of the stable normal bundle of a manifold. Section 9.4 describes the Browder-Novikov theory for deciding if a Poincaré complex $X$ admits a degree 1 normal map. 


\subsection{Geometric Poincaré complexes}

Definition 9.1 An $m$-dimensional geometric Poincaré complex $X$ is a finite $C W$ complex with an orientation character $w(X): \pi_{1}(X) \rightarrow \mathbb{Z}_{2}$ and with a fundamental class homology class $[X] \in H_{m}\left(X ; \mathbb{Z}^{w(X)}\right)$ such that the cap products are isomorphisms

$$
[X] \cap-: H^{*}(\widetilde{X}) \rightarrow H_{m-*}(\widetilde{X}),
$$

with $\tilde{X}$ the universal cover of $X$.

Example 9.2 (i) An $m$-dimensional manifold $M$ is an $m$-dimensional geometric Poincaré complex, by the Poincaré Duality Theorem (4.4).

(ii) If $\left(M_{+}, \partial M_{+}\right),\left(M_{-}, \partial M_{-}\right)$are $m$-dimensional manifolds with boundary and $h: \partial M_{+} \rightarrow \partial M_{-}$is a homotopy equivalence then the identification space

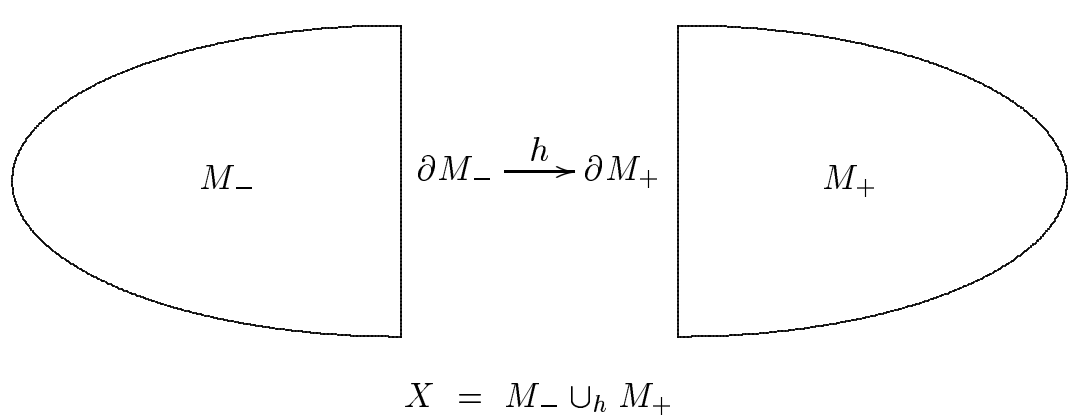

is an $m$-dimensional geometric Poincaré complex $X$.

Example 9.3 Browder [11] proved that a finite $H$-space is a geometric Poincaré complex. This was the example which motivated the development of Poincaré complexes as a tool for classifying manifold structures in homotopy theory.

Definition 9.4 The signature of a $4 k$-dimensional oriented geometric Poincaré complex $X$ is the signature of the $\mathbb{R}$-coefficient homology intersection form

$$
\sigma(X)=\sigma\left(H^{2 k}(X ; \mathbb{R}), \lambda\right) \in \mathbb{Z}
$$

exactly as for a manifold (6.38).

Remark 9.5 The following results were obtained by Browder [12], prior to the development of the general theory for deciding if a geometric Poincaré complex $X$ is homotopy equivalent to a manifold. 
An $m$-dimensional geometric Poincaré complex $X$ with $\pi_{1}(X)=\{1\}$ is homotopy equivalent to a manifold if and only if there exist a vector bundle $\eta: X \rightarrow B S O(j)$ ( $j$ large) and a map $\rho: S^{m+j} \rightarrow T(\eta)$ such that the composite

$$
\pi_{m+j}(T(\eta)) \stackrel{\text { Hurewicz }}{\longrightarrow} \dot{H}_{m+j}(T(\eta)) \stackrel{\left[U_{\eta}\right] \cap-}{\longrightarrow} H_{m}(X)
$$

sends $\rho$ to the fundamental class $[X] \in H_{m}(X)$, with $U_{\eta} \in \dot{H}^{j}(T(\eta))$ the Thom class, and for even $m$ subject to the additional condition:

(i) in the case $m=4 k$ the signature of $X$ is given by the formula in the Hirzebruch Signature Theorem (6.41)

$$
\sigma(X)=\left\langle\mathscr{L}_{k}(-\eta),[X]\right\rangle \in \mathbb{Z}
$$

with $\eta$ playing the role of the stable normal bundle,

(ii) in the case $m=4 k+2$ the $\mathbb{Z}_{2}$-valued Arf invariant (11.60 below) of the self-intersection quadratic form $\mu: K_{2 k+1}\left(M ; \mathbb{Z}_{2}\right) \rightarrow \mathbb{Z}_{2}$ on

$$
K_{2 k+1}\left(M ; \mathbb{Z}_{2}\right)=\operatorname{ker}\left(f_{*}: H_{2 k+1}\left(M ; \mathbb{Z}_{2}\right) \rightarrow H_{2 k+1}\left(X ; \mathbb{Z}_{2}\right)\right)
$$

is 0 .

The proof that such $\eta, \rho$ determine a manifold in the homotopy type of $M$ proceeded by making $\rho$ transverse regular at the zero section $X \hookrightarrow T(\eta)$ to obtain a degree 1 map

$$
f=\rho \mid: M^{4 k}=\rho^{-1}(X) \rightarrow X
$$

and a bundle map $b: \nu_{M} \rightarrow \eta$ (i.e. a 'normal map' $(f, b)$ ) which can always be made a homotopy equivalence by surgeries on $M$ for odd $m$, with a surgery obstruction as in (ii) for even $m$.

Example 9.6 It follows from the Hirzebruch Signature Theorem (6.41) that for a degree $d$ cover $\widetilde{M}$ of an oriented $4 k$-dimensional manifold $M$

$$
\sigma(\widetilde{M})=d \sigma(M) \in \mathbb{Z}
$$

Wall [91] constructed 4-dimensional geometric Poincaré complexes of the form

$$
X=D^{0} \cup D^{1} \cup \bigcup_{10} D^{2} \cup D^{3} \cup D^{4}
$$

with $\pi_{1}(X)=\mathbb{Z}_{p}(p$ prime $)$, and

$$
\sigma(\tilde{X}) \neq p \sigma(X) \in \mathbb{Z}
$$

with $\widetilde{X}$ the universal cover. Such $X$ are not homotopy equivalent to manifolds. 
Example 9.7 Eckmann and Linnell [22] proved that every 2-dimensional geometric Poincaré complex is homotopy equivalent to a surface.

See Wall [91] for the basic homotopy theoretic properties of geometric Poincaré complexes. Chapter 19 of Ranicki [71] and Klein [40] are general surveys of their properties.

Proposition 9.8 Given an m-dimensional geometric Poincaré complex $X$ and an oriented cover $(\widetilde{X}, \pi, w)$ there are defined Poincaré duality $\mathbb{Z}[\pi]$-module isomorphisms

$$
[X] \cap-: H^{*}(\widetilde{X}) \rightarrow H_{m-*}(\widetilde{X})
$$

Proof Let $\bar{X}$ be the universal cover of $X$. A chain map $C \rightarrow D$ of finite chain complexes of projective $A$-modules is a chain equivalence if and only if it induces isomorphisms $H_{*}(C) \cong H_{*}(D)$ in homology, for any ring $A$. Cap product with an $m$-cycle $[X]$ representing the fundamental class $[X] \in H_{m}\left(X ; \mathbb{Z}^{w(X)}\right)$ defines a $\mathbb{Z}\left[\pi_{1}(X)\right]$-module chain map

$$
[X] \cap-: C(\bar{X})^{m-*} \rightarrow C(\bar{X})
$$

which induces the Poincaré duality isomorphisms in homology, and is thus a chain equivalence. The morphism of rings with involution $\mathbb{Z}\left[\pi_{1}(X)\right] \rightarrow \mathbb{Z}[\pi]$ is such that

$$
\mathbb{Z}[\pi] \otimes_{\mathbb{Z}\left[\pi_{1}(X)\right]} C(\bar{X})=C(\tilde{X})
$$

and the $\mathbb{Z}\left[\pi_{1}(X)\right]$-module chain equivalence $[X] \cap-: C(\bar{X})^{m-*} \rightarrow C(\bar{X})$ induces a $\mathbb{Z}[\pi]$-module chain equivalence $[X] \cap-: C(\widetilde{X})^{m-*} \rightarrow C(\tilde{X})$.

Corollary 9.9 An m-dimensional geometric Poincaré complex $X$ has universal coefficient Poincaré duality, such that for any $\mathbb{Z}\left[\pi_{1}(X)\right]$-module $\Lambda$ there are defined Poincaré duality isomorphisms

$$
[X] \cap-: H^{m-*}(X ; \Lambda) \rightarrow H_{*}(X ; \Lambda) \text {. }
$$

Proof Let $\bar{X}$ be the universal cover of $X$, and apply $\Lambda \otimes_{\mathbb{Z}\left[\pi_{1}(X)\right]}-$ to the $\mathbb{Z}\left[\pi_{1}(X)\right]$-module chain equivalence

$$
[X] \cap-: C(\bar{X})^{m-*} \rightarrow C(\bar{X})
$$

to obtain a $\mathbb{Z}$-module chain equivalence

$$
\begin{aligned}
1 \otimes[X] \cap-: \Lambda \otimes_{\mathbb{Z}\left[\pi_{1}(X)\right]} & C(\bar{X})^{m-*}=C(\bar{X} ; \Lambda)^{m-*} \\
& \rightarrow \Lambda \otimes_{\mathbb{Z}\left[\pi_{1}(X)\right]} C(\bar{X})=C(\bar{X} ; \Lambda) .
\end{aligned}
$$


Example 9.10 Let $\left(M_{1}, \partial M_{1}\right),\left(M_{2}, \partial M_{2}\right)$ be $m$-dimensional manifolds with boundary, and let $f: \partial M_{1} \rightarrow \partial M_{2}$ be a homotopy equivalence of the boundaries. The identification space

$$
X=M_{1} \cup_{f}-M_{2}
$$

is an $m$-dimensional geometric Poincaré complex. If $f$ is homotopic to a diffeomorphism then $X$ is homotopy equivalent to an $m$-dimensional manifold.

Given an $m$-dimensional geometric Poincaré complex $X$ let $-X$ denote the $m$ dimensional geometric Poincaré complex with the same underlying $C W$ complex and orientation character, and

$$
[-X]=-[X] \in H_{m}\left(X ; \mathbb{Z}^{w(X)}\right) .
$$

Definition 9.11 (i) An $m$-dimensional geometric Poincaré pair is a finite $C W$ pair $(X, \partial X \hookrightarrow X)$ with an orientation character $w(X): \pi_{1}(X) \rightarrow \mathbb{Z}_{2}$ and with a fundamental homology class

$$
[X] \in H_{m}\left(X, \partial X ; \mathbb{Z}^{w(X)}\right)
$$

such that the cap products are $\mathbb{Z}\left[\pi_{1}(X)\right]$-module isomorphisms

$$
[X] \cap-: H^{*}(\tilde{X}) \rightarrow H_{m-*}(\tilde{X}, \partial \tilde{X})
$$

with $\tilde{X}$ the universal cover of $X$ and $\partial \widetilde{X} \hookrightarrow \widetilde{X}$ the corresponding cover of $\partial X$, and such that $\partial X$ is an $(m-1)$-dimensional geometric Poincaré complex with

$$
\begin{aligned}
& w(\partial X): \pi_{1}(\partial X) \rightarrow \pi_{1}(X) \stackrel{w(X)}{\rightarrow} \mathbb{Z}_{2}, \\
& {[\partial X]=\partial[X] \in H_{m-1}\left(\partial X ; \mathbb{Z}^{w(\partial X)}\right) .}
\end{aligned}
$$

(ii) A geometric Poincaré cobordism $\left(X ; \partial_{0} X, \partial_{1} X\right)$ is a geometric Poincaré pair $(X, \partial X)$ such that the boundary is a disjoint union

$$
\partial X=\partial_{0} X \cup-\partial_{1} X
$$

Example 9.12 An $m$-dimensional manifold with boundary $(M, \partial M)$ is an $m$ dimensional geometric Poincaré pair with the orientation character $w(M)=$ $w_{1}\left(\nu_{M}\right)$ of the stable normal bundle $\nu_{M}: M \rightarrow B O$.

Example 9.13 The mapping cylinder of a homotopy equivalence $f: X \simeq Y$ of $m$-dimensional geometric Poincaré complexes

$$
Z=(X \times I \cup Y) /\{(x, 1) \sim f(x) \mid x \in X\}
$$

defines an $(m+1)$-dimensional geometric Poincaré cobordism $(Z ; X, Y)$. If $X$ and $Y$ are $m$-dimensional manifolds and $f$ is homotopic to a diffeomorphism then $(Z ; X, Y)$ is homotopy equivalent rel $\partial$ to an $(m+1)$-dimensional manifold $h$-cobordism $\left(W^{m+1} ; X, Y\right)$. 
A connected $m$-dimensional manifold $M$ has a handle decomposition with one 0-handle and one $m$-handle

$$
M=h^{0} \cup \bigcup h^{1} \cup \ldots \cup \bigcup h^{m-1} \cup h^{m} .
$$

The following analogue for geometric Poincaré complexes will be useful in dealing with surgery obstructions in Chapters 10,11,12 below.

Proposition 9.14 Every connected $m$-dimensional geometric Poincaré complex $X$ is homotopy equivalent to one of the type $X_{0} \cup D^{m}$ for a connected $m$ dimensional geometric Poincaré pair $\left(X_{0}, S^{m-1}\right)$.

Proof This is the Poincaré Disc Theorem of Wall [91] (Thm. 2.4).

\subsection{Spherical fibrations}

Fibrations $F \rightarrow E \rightarrow B$ with the fibre $F$ a sphere are to geometric Poincaré complexes as vector bundles are to manifolds.

Definition 9.15 (i) A $(k-1)$-spherical fibration $\alpha$ over a space $X$ is a fibration sequence

$$
S^{k-1} \rightarrow S(\alpha) \rightarrow X
$$

in which case there is defined a fibration of pairs

$$
\left(D^{k}, S^{k-1}\right) \rightarrow(D(\alpha), S(\alpha)) \rightarrow X
$$

with $D(\alpha)$ the mapping cylinder $(3.22)$

$$
D(\alpha)=\mathscr{M}(S(\alpha) \rightarrow X)
$$

(ii) A fibre homotopy equivalence $\alpha \simeq \beta$ of $(k-1)$-spherical fibrations over $X$ is a homotopy equivalence $S(\alpha) \simeq S(\beta)$ which is compatible up to homotopy with the maps to $X$.

(iii) $\mathrm{A}(k-1)$-spherical fibration $\alpha$ over $X$ is trivial if it is fibre homotopy equivalent to the $(k-1)$-spherical fibration $\epsilon^{k}$ with

$$
\left(D\left(\epsilon^{k}\right), S\left(\epsilon^{k}\right)\right)=X \times\left(D^{k}, S^{k-1}\right) .
$$

(iv) A fibre homotopy trivialisation of a $(k-1)$-spherical fibration $\alpha$ is a fibre homotopy equivalence $\alpha \simeq \epsilon^{k}$. 
Example 9.16 The sphere bundle of a $k$-plane bundle $\eta$ over a space $X$ is the $(k-1)$-spherical fibration $J \eta$ over $X$ with

$$
S(J \eta)=S(\eta) \subset E(\eta)
$$

the subset of vectors of length 1 (with respect to some metric). Isomorphic vector bundles over the same space $X$ have fibre homotopy equivalent spherical fibrations.

A vector bundle over a finite $C W$ complex $X$ is obtained by glueing together trivial vector bundles $U \times \mathbb{R}^{k}$ over some neighbourhoods $U \subseteq X$ which cover $X$, using compatible vector bundle isomorphisms on overlaps. Similarly, a spherical fibration over $X$ is obtained by glueing together trivial fibrations $U \times S^{k-1}$ over some neighbourhoods $U \subseteq X$ which cover $X$, using compatible fiber-preserving homotopy equivalences on overlaps.

\section{Fibration Classification Theorem 9.17 (Stasheff [85])}

The fibre homotopy classes of $(k-1)$-spherical fibrations over a finite $C W$ complex $X$ are in one-one correspondence with the homotopy classes of maps $X \rightarrow B G(k)$ to the classifying space $B G(k)$ of the monoid $G(k)$ of homotopy equivalences $S^{k-1} \rightarrow S^{k-1}$.

See Chapter 3 of Madsen and Milgram [45] for an account of spherical fibrations and their classifying spaces.

Proposition 9.18 (i) The trivial $(k-1)$-spherical fibration $\epsilon^{k}$ is classified by the trivial map $*: X \rightarrow B G(k)$.

(ii) Every spherical fibration $\alpha: X \rightarrow B G(k)$ has a stable inverse, a spherical fibration $-\alpha: X \rightarrow B G(j)$ (j large) such that

$$
\alpha \oplus-\alpha=\epsilon^{j+k}: X \rightarrow B G(j+k)
$$

(iii) $A(k-1)$-spherical fibration $\alpha$ can be fibre homotopy trivialised if and only if the classifying map $\alpha: X \rightarrow B G(k)$ is null-homotopic, with the choices of fibre homotopy trivialisation $b: \alpha \simeq \epsilon^{k}$ classified by $[X, G(k)]$.

Proof By analogy with 5.29.

The standard vector bundle operations have analogues for spherical fibrations : 
Definition 9.19 (i) The pullback of a spherical fibration $\alpha: X \rightarrow B G(k)$ along a map $f: Y \rightarrow X$ is the spherical fibration $f^{*} \alpha: Y \rightarrow B G(k)$ with

$$
S\left(f^{*} \alpha\right)=\{(x, y) \in S(\alpha) \times Y \mid[x]=f(y) \in X\} .
$$

(ii) The product of spherical fibrations $\alpha: X \rightarrow B G(j), \beta: Y \rightarrow B G(k)$ is the spherical fibration $\alpha \times \beta: X \times Y \rightarrow B G(j+k)$ with

$$
(D(\alpha \times \beta), S(\alpha \times \beta))=(D(\alpha) \times D(\beta), D(\alpha) \times S(\beta) \cup S(\alpha) \times D(\beta)) .
$$

(iii) The Whitney sum of spherical fibrations

$$
\alpha: X \rightarrow B G(j) \quad, \quad \beta: X \rightarrow B G(k)
$$

is the spherical fibration

$$
\alpha \oplus \beta=\Delta^{*}(\alpha \times \beta): X \rightarrow B G(j+k)
$$

with

$$
\Delta: X \rightarrow X \times X ; x \mapsto(x, x)
$$

so that

$$
S(\alpha \oplus \beta)=\{(x, y) \in S(\alpha) \times D(\beta) \cup D(\alpha) \times S(\beta) \mid[x]=[y] \in X\} .
$$

(iv) A section of a $(k-1)$-spherical fibration $\alpha: X \rightarrow B G(k)$ is a fibre homotopy equivalence $\alpha \simeq \alpha^{\prime} \oplus \epsilon$ for a $(k-2)$-spherical fibration $\alpha^{\prime}: X \rightarrow B G(k-1)$.

(v) A stable fibre homotopy equivalence $\alpha \simeq_{s} \beta$ between spherical fibrations $\alpha: X \rightarrow B G(j), \beta: X \rightarrow B G(k)$ is a fibre homotopy equivalence

$$
\alpha \oplus \epsilon^{p} \simeq \beta \oplus \epsilon^{q}: X \rightarrow B G(n)
$$

with $p, q, n$ such that $j+p=k+q=n$.

(vi) A stable spherical fibration over $X$ is an equivalence class of spherical fibrations $\alpha$ over $X$, subject to the equivalence relation

$$
\alpha \sim \beta \text { if there exists a stable fibre homotopy equivalence } \alpha \simeq_{s} \beta \text {. }
$$

For any $\ell \geqslant 1$ the $\ell$-fold loop space $\Omega^{\ell} S^{\ell}$ of pointed maps $S^{\ell} \rightarrow S^{\ell}$ has one component for each integer, with degree defining a bijection

$$
\pi_{0}\left(\Omega^{\ell} S^{\ell}\right) \rightarrow \mathbb{Z} ;\left(f: S^{\ell} \rightarrow S^{\ell}\right) \mapsto \operatorname{deg}(f) .
$$

For any $d \in \mathbb{Z}$ let $\left(\Omega^{\ell} S^{\ell}\right)_{d}$ be the component of degree $d$ pointed maps $S^{\ell} \rightarrow S^{\ell}$. The components are all homotopy equivalent, and the higher homotopy groups are just the homotopy groups of spheres

$$
\pi_{n}\left(\left(\Omega^{\ell} S^{\ell}\right)_{d}\right)=\pi_{n+\ell}\left(S^{\ell}\right) \quad(n \geqslant 1) .
$$

For any $k \geqslant 1$ the monoid of pointed homotopy equivalences $S^{k-1} \rightarrow S^{k-1}$

$$
F(k)=\left(\Omega^{k-1} S^{k-1}\right)_{1} \cup\left(\Omega^{k-1} S^{k-1}\right)_{-1}
$$

is related to the monoid $G(k)$ of homotopy equivalences $S^{k-1} \rightarrow S^{k-1}$ by a fibration

$$
F(k) \rightarrow G(k) \rightarrow S^{k-1}
$$


Proposition 9.20 (i) The fibre homotopy classes of $(k-1)$-spherical fibrations with a section over a finite $C W$ complex $X$ are in one-one correspondence with the homotopy classes of maps $X \rightarrow B F(k)$ to the classifying space $B F(k)$ of $F(k)$. Moreover, $B F(k)$ is the total space of the universal $(k-1)$-spherical fibration $1_{k}: B G(k) \rightarrow B G(k)$

$$
S^{k-1} \rightarrow B F(k) \rightarrow B G(k) .
$$

The homotopy groups of $B F(k)$ are given by

$$
\pi_{n}(B F(k))=\pi_{n-1}(F(k))= \begin{cases}\pi_{n+k-2}\left(S^{k-1}\right) & \text { if } n>1 \\ \mathbb{Z}_{2} & \text { if } n=1 .\end{cases}
$$

(ii) The passage from $k$-plane bundles to $(k-1)$-spherical fibrations defines a map

$$
J: B O(k) \rightarrow B G(k) ; \eta \mapsto J \eta
$$

Let $J$ also denote the composite

$$
J: B O(k) \stackrel{J}{\longrightarrow} B G(k) \longrightarrow B F(k+1)
$$

with

$$
B G(k) \rightarrow B F(k+1) ; \alpha \mapsto \alpha \oplus \epsilon
$$

The induced morphism of homotopy groups is the J-homomorphism (5.80)

$$
J: \pi_{n}(B O(k))=\pi_{n-1}(O(k)) \rightarrow \pi_{n}(B F(k+1))=\pi_{n+k-1}\left(S^{k}\right) \quad(n>1) .
$$

(iii) The sphere bundle of the universal $k$-plane bundle over $B O(k)$ is the pullback of the universal $(k-1)$-spherical fibration over $B G(k)$ along $J: B O(k) \rightarrow B G(k)$. The mapping fibres

$$
\begin{aligned}
& G(k) / O(k)=\mathscr{F}(J: B O(k) \rightarrow B G(k)), \\
& F(k) / O(k-1)=\mathscr{F}(J: B O(k-1) \rightarrow B F(k))
\end{aligned}
$$

are homotopy equivalent, and fit into a commutative braid of fibrations

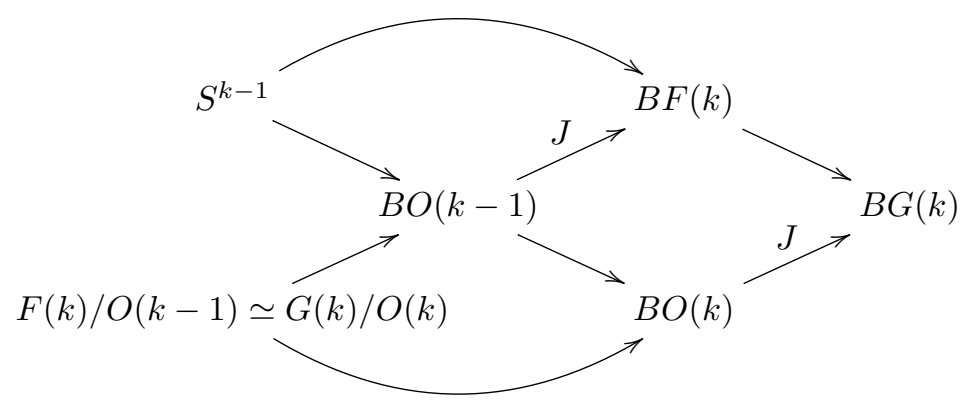

inducing a commutative braid of exact sequences 


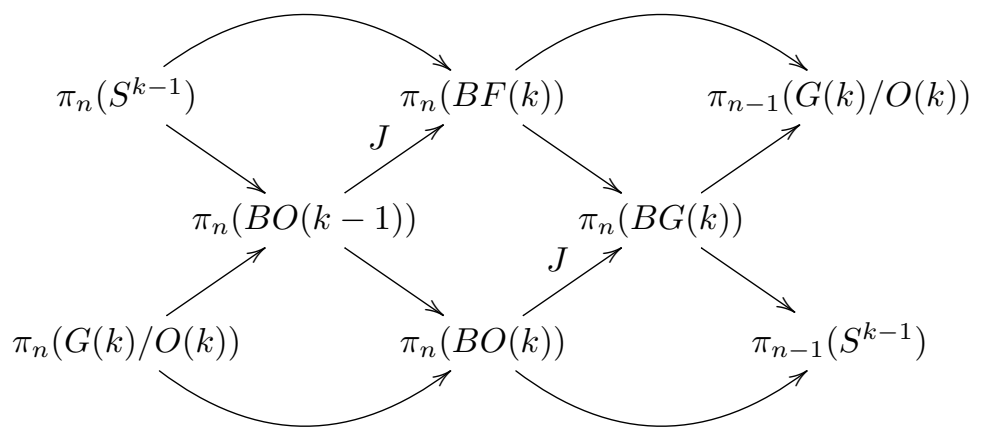

(iv) Stabilisation $\alpha \mapsto \alpha \oplus \epsilon$ defines maps

$$
\ldots \rightarrow B G(k) \rightarrow B F(k+1) \rightarrow B G(k+1) \rightarrow B F(k+2) \rightarrow \ldots
$$

such that the direct limits

$$
B F=\varliminf_{k} B F(k) \quad, \quad B G=\varliminf_{k} B G(k)
$$

are homotopy equivalent

$$
B F \simeq B G
$$

The homotopy groups of the stable classifying spaces are given by

$$
\pi_{n}(B F)=\pi_{n}(B G)= \begin{cases}\underline{\lim _{k}} \pi_{n+k-2}\left(S^{k-1}\right)=\pi_{n-1}^{S} & \text { if } n>1 \\ \mathbb{Z}_{2} & \text { if } n=1\end{cases}
$$

The space BG classifies stable spherical fibrations; the passage from stable vector bundles to stable spherical fibrations defines a map $J: B O \rightarrow B G$ inducing the stable J-homomorphism

$$
J: \pi_{*}(B O)=\pi_{*-1}(O) \rightarrow \pi_{*}(B G)=\pi_{*-1}^{S}(*>1) .
$$

The direct limit

$$
G / O=\underset{k}{\varliminf_{k}} G(k) / O(k)
$$

is the mapping fibre of $J: B O \rightarrow B G$, so that there is defined a fibration (up to homotopy)

$$
G / O \rightarrow B O \stackrel{J}{\rightarrow} B G
$$

inducing a long exact sequence

$$
\ldots \rightarrow \pi_{n}(G / O) \rightarrow \pi_{n}(B O) \stackrel{J}{\rightarrow} \pi_{n}(B G) \rightarrow \pi_{n-1}(G / O) \rightarrow \ldots
$$


Proposition 9.21 (i) For a finite $C W$ complex $X$ the homotopy classes of maps $X \rightarrow G / O$ are in one-one correspondence with the equivalence classes of pairs

( a stable vector bundle $\eta$ over $X$, a fibre homotopy trivialisation of J $\eta$ ) .

(ii) A stable spherical fibration $\alpha: X \rightarrow B G$ over a finite $C W$ complex $X$ admits a bundle reduction if and only if the classifying map $\alpha: X \rightarrow B G$ lifts (up to homotopy) to a map $\widetilde{\alpha}: X \rightarrow B O$, and the different such reductions are classified by the maps $X \rightarrow G / O$.

Remark 9.22 The homotopy groups $\pi_{*}(B O)$ are infinite for $* \equiv 0(\bmod 4)$ (namely $\mathbb{Z}$ ), whereas the homotopy groups $\pi_{*}(B G)$ are finite. The low-dimensional homotopy groups are given in the following table.

\begin{tabular}{|c|c|c|c|c|c|c|c|c|c|c|}
\hline$n$ & 1 & 2 & 3 & 4 & 5 & 6 & 7 & 8 & 9 & 10 \\
\hline$\pi_{n}(G / O)$ & 0 & $\mathbb{Z}_{2}$ & 0 & $\mathbb{Z}$ & 0 & $\mathbb{Z}_{2}$ & 0 & $\mathbb{Z} \oplus \mathbb{Z}_{2}$ & $\left(\mathbb{Z}_{2}\right)^{2}$ & $\mathbb{Z}_{2} \oplus \mathbb{Z}_{3}$ \\
$\pi_{n}(B O)$ & $\mathbb{Z}_{2}$ & $\mathbb{Z}_{2}$ & 0 & $\mathbb{Z}$ & 0 & 0 & 0 & $\mathbb{Z}$ & $\mathbb{Z}_{2}$ & $\mathbb{Z}_{2}$ \\
$\pi_{n}(B G)$ & $\mathbb{Z}_{2}$ & $\mathbb{Z}_{2}$ & $\mathbb{Z}_{2}$ & $\mathbb{Z}_{24}$ & 0 & 0 & $\mathbb{Z}_{2}$ & $\mathbb{Z}_{240}$ & $\left(\mathbb{Z}_{2}\right)^{2}$ & $\left(\mathbb{Z}_{2}\right)^{3}$ \\
\hline
\end{tabular}

It is possible to extend the fibration sequence $G / O \rightarrow B O \rightarrow B G$ to the right

$$
G / O \rightarrow B O \rightarrow B G \rightarrow B(G / O)
$$

(Boardman and Vogt [7]), so that a stable spherical fibration $\alpha: X \rightarrow B G$ admits a bundle reduction if and only if the composite

$$
t(\alpha): X \stackrel{\alpha}{\longrightarrow} B G \longrightarrow B(G / O)
$$

is null-homotopic.

Definition 9.23 The Thom space of a $(k-1)$-spherical fibration $\alpha: X \rightarrow$ $B G(k)$ is the pointed space given by the mapping cone (3.22) of the projection $S(\alpha) \rightarrow X$

$$
T(\alpha)=\mathscr{C}(S(\alpha) \rightarrow X)
$$

Proposition 9.24 The Thom space $T(\eta)$ of a $k$-plane bundle $\eta: X \rightarrow B O(k)$ is pointed homotopy equivalent to the Thom space $T(J \eta)$ of the sphere bundle J $\eta: X \rightarrow B G(k)$

$$
T(\eta) \simeq T(J \eta)
$$


Proof The zero section is a homotopy equivalence $X \rightarrow D(\eta)$, so that

$$
\begin{aligned}
T(J \eta) & =\mathscr{C}(S(J \eta) \rightarrow X) \\
& \simeq \mathscr{C}(S(\eta) \rightarrow D(\eta)) \simeq D(\eta) / S(\eta)=T(\eta)
\end{aligned}
$$

Example 9.25 (i) The Thom space of the $(k-1)$-spherical fibration over $S^{n}$ classified by

$$
\omega \in \pi_{n}(B G(k))=\pi_{n-1}(G(k))
$$

with image

$$
[\omega] \in \pi_{n}(B F(k+1))=\pi_{n-1}(F(k+1))= \begin{cases}\pi_{n+k-1}\left(S^{k}\right) & \text { if } n>1 \\ \mathbb{Z}_{2} & \text { if } n=1\end{cases}
$$

is given up to homotopy equivalence by

$$
T(\omega)=S^{k} \cup_{[\omega]} D^{n+k} .
$$

If $n=1$ then

$$
T(\omega)= \begin{cases}S^{k} \vee S^{k+1} & \text { if } \omega=\epsilon^{k} \text { is trivial } \\ S^{k} \cup_{2} D^{k+1}=\Sigma^{k-1}\left(\mathbb{R P}^{2}\right) & \text { if } \omega=\mu \oplus \epsilon^{k-1} \text { is nontrivial. }\end{cases}
$$

(ii) The Thom space of the $k$-plane bundle over $S^{n}(n>1)$ classified by

$$
\omega \in \pi_{n}(B O(k))=\pi_{n-1}(O(k))
$$

is given up to homotopy equivalence by

$$
T(\omega)=S^{k} \cup_{[J \omega]} D^{n+k},
$$

with $J \omega \in \pi_{n}(B G(k))$ classifying the sphere bundle and

$$
[J \omega] \in \operatorname{im}(J) \subseteq \pi_{n}(B F(k+1))=\pi_{n+k-1}\left(S^{k}\right)
$$

the image of $\omega$ under the $J$-homomorphism $J: \pi_{n-1}(O(k)) \rightarrow \pi_{n+k-1}\left(S^{k}\right)$ $(5.80)$.

Definition 9.26 The orientation character of a $(k-1)$-spherical fibration $\alpha: X \rightarrow B G(k)$ is the first Stiefel-Whitney class $w_{1}(\alpha) \in H^{1}\left(X ; \mathbb{Z}_{2}\right)$ regarded as a group morphism

$$
w_{1}(\alpha): \pi_{1}(X) \rightarrow \mathbb{Z}_{2}=\{ \pm 1\}
$$

Proposition 9.27 (i) The orientation character of $\alpha: X \rightarrow B G(k)$ is such that $w_{1}(\alpha)=0$ if and only if $\alpha$ can be oriented, i.e. if the homotopy equivalences $S^{k-1} \rightarrow S^{k-1}$ on overlapping product neighbourhoods preserve orientations. 
(ii) The classifying space $B S G(k)$ for oriented $(k-1)$-spherical fibrations is the double cover of $B G(k)$ classified by the first Stiefel-Whitney class

$$
w_{1}\left(1_{k}\right)=1 \in H^{1}\left(B G(k) ; \mathbb{Z}_{2}\right)=\mathbb{Z}_{2}
$$

of the universal $(k-1)$-spherical fibration $1_{k}: B G(k) \rightarrow B G(k)$.

By analogy with the Thom Isomorphism Theorem (5.42) for oriented vector bundles :

Fibration Thom Isomorphism 9.28 An oriented $(k-1)$-spherical fibration $\alpha: X \rightarrow B S G(k)$ has a Thom class $U_{\alpha} \in \dot{H}^{k}(T(\alpha))$ such that the cap and cup products define isomorphisms

$$
\begin{aligned}
& U_{\alpha} \cap-: \dot{H}_{*}(T(\alpha)) \rightarrow H_{*-k}(X), \\
& U_{\alpha} \cup-: H^{*}(X) \rightarrow \dot{H}^{*+k}(T(\alpha)) .
\end{aligned}
$$

Proof The Thom space $T(\alpha)$ has the structure of a $C W$ complex with one 0 -cell (at the base point) and one $(n+k)$-cell for each $n$-cell of $X$, and

$$
U_{\alpha}= \pm 1 \in \dot{H}^{k}(T(\alpha))=\mathbb{Z} .
$$

Again, there is a twisted version in the nonorientable case:

Twisted Fibration Thom Isomorphism 9.29 $A(k-1)$-spherical fibration $\alpha: X \rightarrow B G(k)$ with orientation character $w=w_{1}(\alpha) \in H^{1}\left(X ; \mathbb{Z}_{2}\right)$ has a $w$ twisted Thom class $U_{\alpha} \in \dot{H}^{k}\left(T(\alpha) ; \mathbb{Z}^{w}\right)$ which is unique up to sign, such that the cap and cup products define isomorphisms

$$
\begin{aligned}
& U_{\alpha} \cap-: \dot{H}_{*}(T(\alpha)) \rightarrow H_{*-k}\left(X ; \mathbb{Z}^{w}\right), \\
& U_{\alpha} \cup-: H^{*}(X) \rightarrow \dot{H}^{*+k}\left(T(\alpha) ; \mathbb{Z}^{w}\right) .
\end{aligned}
$$

\subsection{The Spivak normal fibration}

The Spivak normal fibration of an $m$-dimensional geometric Poincaré complex $X$ is a stable spherical fibration $\nu_{X}: X \rightarrow B G$, which is the homotopy theoretic analogue of the stable normal bundle $\nu_{M}: M \rightarrow B O$ of a manifold $M$. The first stage of the surgery programme for deciding if $X$ is homotopy equivalent 
to a manifold is to decide if there exists a degree 1 normal map $(f, b): M \rightarrow X$ from an $m$-dimensional manifold $M^{m}$. Such a normal map is called a 'normal invariant' for $X$, and exists if and only if $\nu_{X}: X \rightarrow B G$ admits a vector bundle reduction $\tilde{\nu}_{X}: X \rightarrow B O$.

A finite $m$-dimensional simplicial complex $K$ admits an embedding $K \hookrightarrow$ $S^{m+k}$ for $k \geqslant m+1$, by the simplicial complex version of the Whitney Embedding Theorem (1.7). Every finite $C W$ complex $X$ is homotopy equivalent to a finite simplicial complex $K$, by the simplicial approximation theorem. It follows that $X$ can be embedded in $S^{N}$, for a sufficiently large $N$, with a closed regular neighbourhood $Y \subseteq S^{N}$ containing $X$ as a deformation retract and such that $Y$ is an $N$-dimensional manifold with boundary $\partial Y$. (Here, closed is in the sense of topology, not as a manifold). The image of the fundamental class $\left[S^{N}\right]=1 \in$ $H_{N}\left(S^{N}\right)=\mathbb{Z}$ under the projection

$$
\rho: S^{N} \rightarrow S^{N} /\left(S^{N} \backslash Y\right)=Y / \partial Y
$$

is the fundamental class of $(Y, \partial Y)$

$$
\rho_{*}\left[S^{N}\right]=[Y] \in \dot{H}_{N}(Y / \partial Y)=H_{N}(Y, \partial Y) .
$$

If $X$ is an $m$-dimensional manifold then $(Y, \partial Y)$ is the $\left(D^{k}, S^{k-1}\right)$-bundle over $X$ determined by the normal $k$-plane bundle $\nu_{X \hookrightarrow S^{N}}: X \rightarrow B O(k)$ with $k=N-m$.

Definition 9.30 A Spivak normal structure $\left(\nu_{X}, \rho_{X}\right)$ for an $m$-dimensional geometric Poincaré complex $X$ is a $(k-1)$-spherical fibration $\nu_{X}: X \rightarrow B G(k)$ together with a map $\rho_{X}: S^{m+k} \rightarrow T\left(\nu_{X}\right)$ such that the orientation character of $X$ is the orientation character of $\nu_{X}$

$$
w(X)=w_{1}\left(\nu_{X}\right): \pi_{1}(X) \rightarrow \mathbb{Z}_{2},
$$

and the fundamental class of $X$ is given by

$$
[X]=U_{\nu_{X}} \cap h_{*}\left(\rho_{X}\right) \in H_{m}\left(X ; \mathbb{Z}^{w(X)}\right)
$$

with

$$
\begin{aligned}
& h_{*}=\text { Hurewicz map : } \pi_{m+k}^{S}\left(T\left(\nu_{X}\right)\right) \rightarrow \dot{H}_{m+k}\left(T\left(\nu_{X}\right)\right), \\
& U_{\nu_{X}} \cap-=\text { Thom iso. : } \dot{H}_{m+k}\left(T\left(\nu_{X}\right)\right) \rightarrow H_{m}\left(X ; \mathbb{Z}^{w}\right) .
\end{aligned}
$$

The stable spherical fibration $\nu_{X}: X \rightarrow B G$ is the Spivak normal fibration of $X$.

Theorem 9.31 (i) A finite $C W$ complex $X$ is an m-dimensional geometric Poincaré complex if and only if for any closed regular neighbourhood $(Y, \partial Y)$ of an embedding $X \hookrightarrow S^{m+k}$ ( $k$ large) the mapping fibre of the inclusion $\partial Y \rightarrow Y$ is a homotopy $(k-1)$-sphere. 
(ii) For an m-dimensional geometric Poincaré complex $X$ an embedding $X \hookrightarrow$ $S^{m+k}$ with closed regular neighbourhood $(Y, \partial Y)$ determines a Spivak normal structure $\left(\nu_{X}, \rho_{X}\right)$ with

$$
\begin{aligned}
& S^{k-1} \rightarrow S\left(\nu_{X}\right)=\partial Y \rightarrow Y \simeq X \\
& \rho_{X}=\text { projection }: S^{m+k} \rightarrow Y / \partial Y=T\left(\nu_{X}\right) .
\end{aligned}
$$

(iii) Any two Spivak normal structures $(\nu, \rho),\left(\nu^{\prime}, \rho^{\prime}\right)$ on an m-dimensional geometric Poincaré complex $X$ are related by a stable fibre homotopy equivalence $c: \nu \simeq \nu^{\prime}$ such that

$$
T(c)_{*}(\rho)=\rho^{\prime} \in \pi_{m+k^{\prime}}^{S}\left(T\left(\nu^{\prime}\right)\right) .
$$

Proof Let $(Y, \partial Y)$ be a closed regular neighbourhood of an embedding $X \hookrightarrow$ $S^{N}$ (N large). Write

$$
\pi=\pi_{1}(X)=\pi_{1}(Y)=\pi_{1}(\partial Y),
$$

and let $\widetilde{X}, \widetilde{Y}, \partial \widetilde{Y}$ be the universal covers of $X, Y, \partial Y$. The reduced cohomology of the quotient space $\tilde{Y} / \partial \tilde{Y}$ is isomorphic to the homology of $\widetilde{X}$, with

$$
\dot{H}^{*}(\tilde{Y} / \partial \tilde{Y})=H^{*}(\tilde{Y}, \partial \tilde{Y})=H_{N-*}(\tilde{Y})=H_{N-*}(\tilde{X}) .
$$

Let $w: \pi \rightarrow \mathbb{Z}_{2}$ be an orientation character. The following conditions are equivalent :

(i) the mapping fibre

$$
F=\mathscr{F}(\partial Y \rightarrow Y)
$$

is homotopy equivalent to $S^{k-1}$, defining a spherical fibration

$$
\nu_{X}: X \simeq Y \rightarrow B G(k)
$$

with orientation character $w_{1}\left(\nu_{X}\right)=w$,

(ii) there exists a Thom class $U \in \dot{H}^{k}\left(Y / \partial Y ; \mathbb{Z}^{w}\right)$ such that the cup products

$$
U \cup-: H^{*}(\widetilde{Y}) \rightarrow \dot{H}^{*+k}(\widetilde{Y} / \partial \widetilde{Y})
$$

are isomorphisms (using involutions on $\mathbb{Z}[\pi]$ which differ by $w$ to define $H^{*}(\widetilde{Y})$ and $\left.\dot{H}^{*+k}(\widetilde{Y} / \partial \widetilde{Y})\right)$,

(iii) there exists a fundamental class $[X] \in H_{m}\left(X ; \mathbb{Z}^{w}\right)$ such that the cap products

$$
[X] \cap-: H^{m-*}(\widetilde{X}) \rightarrow H_{*}(\widetilde{X})
$$

are isomorphisms,

(iv) there exists $\nu_{X}: X \rightarrow B G(k)$ such that

$$
(Y, \partial Y) \simeq\left(D\left(\nu_{X}\right), S\left(\nu_{X}\right)\right)
$$

with $w=w_{1}\left(\nu_{X}\right)$. 
If these conditions are satisfied the Thom class $U_{\nu_{X}} \in \dot{H}^{k}\left(T\left(\nu_{X}\right) ; \mathbb{Z}^{w}\right)(w=$ $\left.w_{1}\left(\nu_{X}\right)\right)$ corresponds to the fundamental class $[X] \in H_{m}\left(X ; \mathbb{Z}^{w}\right)$, the Thom isomorphisms for $\nu_{X}$ correspond to the Poincaré duality isomorphisms for $X$, with a commutative diagram of isomorphisms

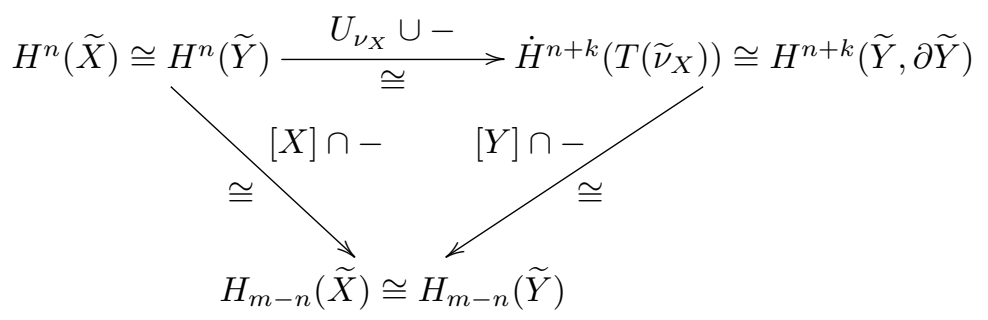

The Spivak normal structure $\left(\nu_{X}, \rho_{X}\right)$ is given by $\nu_{X}$ with

$$
\rho_{X}: S^{N} \rightarrow S^{N} / \operatorname{cl} .\left(S^{N} \backslash Y\right)=Y / \partial Y=T\left(\nu_{X}\right) .
$$

See Atiyah [5], Spivak [84], Wall [91], Browder [15], Ranicki [69] for further details.

There is also a relative version:

Proposition 9.32 An m-dimensional geometric Poincaré pair $(X, \partial X)$ carries an equivalence class of relative Spivak normal structures

$$
\left(\nu_{X}: X \rightarrow B G(k),\left(\rho_{X}, \rho_{\partial X}\right):\left(D^{m+k}, S^{m+k-1}\right) \rightarrow\left(T\left(\nu_{X}\right), T\left(\nu_{\partial X}\right)\right)\right)
$$

with

$$
\begin{aligned}
& \nu_{\partial X}: \partial X \rightarrow X \stackrel{\nu_{X}}{\rightarrow} B G(k), \\
& {[X]=U_{\nu_{X}} \cap h_{*}\left(\rho_{X}, \rho_{\partial X}\right) \in H_{m}\left(X, \partial X ; \mathbb{Z}^{w}\right) .}
\end{aligned}
$$

Although a homotopy equivalence of manifolds need not preserve the normal bundles it does preserve the Spivak normal fibrations. This is a special case of:

Corollary 9.33 A homotopy equivalence of geometric Poincaré complexes preserves the Spivak normal fibrations.

Proof The mapping cylinder of a homotopy equivalence $X \simeq Y$ of $m$-dimensional geometric Poincaré complexes defines an $(m+1)$-dimensional geometric Poincaré cobordism $(Z ; X, Y)$ (9.11) with Spivak normal fibration

$$
\nu_{Z} \simeq \nu_{X}: Z=\mathscr{M}(X \rightarrow Y) \simeq X \rightarrow B G .
$$




\subsection{Browder-Novikov theory}

The isomorphism class of the stable normal bundle $\nu_{M}: M \rightarrow B O$ of an $m$ dimensional manifold $M^{m}$ is a diffeomorphism invariant but not a homotopy invariant. If $g: M^{\prime m} \rightarrow M^{m}$ is a homotopy equivalence of $m$-dimensional manifolds then the stable bundles $\nu_{M^{\prime}}, g^{*} \nu_{M}: M^{\prime} \rightarrow B O$ are fibre homotopy equivalent, but not in general isomorphic. (See Example 13.26 for the construction of a homotopy equivalence $g$ with $\nu_{M^{\prime}}$ not isomorphic to $g^{*} \nu_{M}$ ). Thus the Stiefel-Whitney classes are the same, but the Pontrjagin classes may be different. Similarly for the characteristic numbers, so that homotopy equivalent oriented manifolds $M$, $M^{\prime}$ are unoriented cobordant but not necessarily oriented cobordant.

In order for a homotopy equivalence of manifolds $M^{m} \simeq M^{\prime m}$ to be homotopic to a diffeomorphism it is therefore necessary that it preserve the normal bundles. This condition is necessary but not sufficient : the homotopy equivalence $M \simeq W \simeq M^{\prime}$ determined by an $h$-cobordism $\left(W^{m+1} ; M^{m}, M^{\prime m}\right)$ preserves normal bundles, but if the $h$-cobordism is not diffeomorphic to $M \times(I ;\{0\},\{1\})$ there may not exist a homotopy to diffeomorphism.

In the applications of cobordism theory to the problem of deciding if a space $X$ is homotopy equivalent to a manifold it is convenient to consider maps $f$ : $M \rightarrow X$ such that the stable normal bundle $\nu_{M}: M \rightarrow B O$ is the pullback along $f$ of a stable bundle $\eta: X \rightarrow B O$.

Definition 9.34 (i) An m-dimensional normal map

$$
(f, b):\left(M, \nu_{M}\right) \rightarrow(X, \eta)
$$

is a map $f: M \rightarrow X$ from an $m$-dimensional manifold $M$ to a $C W$ complex $X$ together with a stable pullback bundle map $b: \nu_{M} \rightarrow \eta$ over $f$, with $\nu_{M}: M \rightarrow$ $B O$ the stable normal bundle of $M$ and $\eta: X \rightarrow B O$ a stable bundle over $X$.

(ii) A normal bordism is a normal map

$$
\left((F, B) ;(f, b),\left(f^{\prime}, b^{\prime}\right)\right):\left(W ; M, M^{\prime}\right) \rightarrow X \times(I ;\{0\},\{1\})
$$

from a cobordism $\left(W^{m+1} ; M^{m}, M^{\prime m}\right)$.

Proposition 9.35 Let $X$ be a space with a $k$-plane bundle $\eta: X \rightarrow B O(k)$. The normal bordism classes of $m$-dimensional normal maps $(f, b):\left(M, \nu_{M}\right) \rightarrow(X, \eta)$ are in bijective correspondence with the elements of the stable homotopy group

$$
\pi_{m+k}^{S}(T(\eta))=\varliminf_{j} \pi_{m+j+k}\left(\Sigma^{j} T(\eta)\right)
$$


Proof Immediate from the identifications

$$
\mathfrak{B}_{m}\left(S^{m+j+k}, X, \eta \oplus \epsilon^{j}\right)=\pi_{m+j+k}\left(T\left(\eta \oplus \epsilon^{j}\right)\right)(j \geqslant 0)
$$

given by 6.10 , and $T\left(\eta \oplus \epsilon^{j}\right)=\Sigma^{j} T(\eta)$.

We shall be mainly concerned with normal maps $(f, b): M \rightarrow X$ from manifolds $M$ to Poincaré complexes which are of degree 1 . Degree is defined by :

Definition 9.36 The degree of a map $f: M \rightarrow X$ of connected $m$-dimensional geometric Poincaré complexes such that $f^{*} w(X)=w(M) \in H^{1}\left(M ; \mathbb{Z}_{2}\right)$ is the integer $\operatorname{deg}(f) \in \mathbb{Z}$ such that

$$
f_{*}[M]=\operatorname{deg}(f)[X] \in H_{m}\left(X ; \mathbb{Z}^{w}\right)=\mathbb{Z} .
$$

Definition 9.37 A normal homotopy equivalence is a normal map $(f, b)$ : $\left(M, \nu_{M}\right) \rightarrow(X, \eta)$ such that $f: M \rightarrow X$ is a homotopy equivalence.

A normal homotopy equivalence has degree 1 .

Surgery obstruction theory investigates the extent to which a normal map is bordant to a normal homotopy equivalence. Browder and Novikov applied the Spivak normal structure and the transversality construction of normal maps to the existence and uniqueness of manifold structures in the homotopy type of a geometric Poincaré complex, dealing with the following questions :

When is an m-dimensional geometric Poincaré complex homotopy equivalent to an m-dimensional manifold?

When is a homotopy equivalence of $m$-dimensional manifolds $h$-cobordant to a diffeomorphism?

In both cases there is a two-stage obstruction theory, with a homotopy-theoretic primary obstruction and a surgery-theoretic secondary obstruction.

A geometric Poincaré complex $X$ has a Whitehead torsion

$$
\tau(X)=\tau\left([X] \cap-: C(\widetilde{X})^{m-*} \rightarrow C(\tilde{X})\right) \in W h\left(\pi_{1}(X)\right) .
$$

A manifold $M$ has $\tau(M)=0$, so that there is also a version of surgery theory for answering the questions :

When is an $m$-dimensional geometric Poincaré complex $X$ with $\tau(X)=0$ simple homotopy equivalent to an m-dimensional manifold? 
When is a simple homotopy equivalence of $m$-dimensional manifolds s-cobordant to a diffeomorphism?

By the $s$-Cobordism Theorem (8.33) for $m \geqslant 5$ the last question is equivalent to asking if the simple homotopy equivalence is homotopic to a diffeomorphism. The version of surgery theory taking into account Whitehead torsion will be considered (briefly) in Chapter 13.

By the results quoted in Section 9.2, the obstruction to a bundle reduction of the Spivak normal fibration $\nu_{X}: X \rightarrow B G$ of a geometric Poincaré complex $X$ is the homotopy class of a map $t\left(\nu_{X}\right): X \rightarrow B(G / O)$.

Proposition 9.38 An m-dimensional geometric Poincaré complex $X$ is homotopy equivalent to an $m$-dimensional manifold if and only if there exists a nullhomotopy $t\left(\nu_{X}\right) \simeq\{*\}: X \rightarrow B(G / O)$ such that the corresponding bordism class of degree 1 normal maps $(f, b): M^{m} \rightarrow X$ contains a homotopy equivalence.

For $m \geqslant 5$ a degree 1 normal map $(f, b): M^{m} \rightarrow X$ is bordant to a homotopy equivalence if and only if the surgery obstruction $\sigma_{*}(f, b) \in L_{m}\left(\mathbb{Z}\left[\pi_{1}(X)\right]\right)$ to be defined in Chapter 11 ( $m$ even) and Chapter $12(m$ odd $)$ is $\sigma_{*}(f, b)=0$.

The criterion of 9.38 for the existence of a manifold structure in a homotopy type also applies in the relative case:

Corollary 9.39 Let $(X, \partial X)$ be an m-dimensional geometric Poincaré pair.

(i) $(X, \partial X)$ is homotopy equivalent to an $m$-dimensional manifold with boundary if and only if there exists a null-homotopy $t\left(\nu_{X}\right) \simeq\{*\}: X \rightarrow B(G / O)$ such that the corresponding bordism class of degree 1 normal maps $(f, b):\left(M^{m}, \partial M\right) \rightarrow$ $(X, \partial X)$ contains a homotopy equivalence.

(ii) If $\partial X$ is already an $(m-1)$-dimensional manifold, then $(X, \partial X)$ is rel$\partial$ homotopy equivalent to an $m$-dimensional manifold with boundary if and only if there exists a null-homotopy $t\left(\nu_{X}\right) \simeq\{*\}: X / \partial X \rightarrow B(G / O)$ such that the corresponding relว bordism class of degree 1 normal maps $(f, b):\left(M^{m}, \partial M\right) \rightarrow$ $(X, \partial X)$ contains a homotopy equivalence.

A normal invariant is a realization of the Spivak normal structure by a vector bundle:

Definition 9.40 (i) A normal invariant $(\eta, \rho)$ for an $m$-dimensional geometric Poincaré complex $X$ is a vector bundle $\eta: X \rightarrow B O(k)$ ( $k$ large) with orientation character $w_{1}(\eta)=w(X) \in H^{1}\left(X ; \mathbb{Z}_{2}\right)$, together with a map $\rho: S^{m+k} \rightarrow T(\eta)$ to the Thom space of $\eta$ such that 


$$
U_{\eta} \cap h_{*}(\rho)=[X] \in H_{m}\left(X ; \mathbb{Z}^{w(X)}\right) .
$$

(ii) An equivalence of normal invariants $(\eta, \rho),\left(\eta^{\prime}, \rho^{\prime}\right)$ on an $m$-dimensional geometric Poincaré complex $X$ is a stable bundle isomorphism $c: \eta \simeq \eta^{\prime}$ such that

$$
T(c)_{*}(\rho)=\rho^{\prime} \in \pi_{m+k^{\prime}}^{S}\left(T\left(\eta^{\prime}\right)\right) .
$$

(iii) The normal structure set $\mathscr{T}(X)$ of an $m$-dimensional geometric Poincaré complex $X$ is the set of equivalence classes of normal invariants.

Example 9.41 Any embedding $M \hookrightarrow S^{m+k}$ of an $m$-dimensional manifold $M^{m}$ with tubular neighbourhood $(Y, \partial Y)$ determines a normal invariant $(\eta, \rho)$ with

$$
\begin{aligned}
& \eta=\nu_{M \hookrightarrow S^{m+k}}: M \rightarrow B O(k), \\
& \left(D^{k}, S^{k-1}\right) \rightarrow(D(\eta), S(\eta))=(Y, \partial Y) \rightarrow M, \\
& \rho=\text { projection }: S^{m+k} \rightarrow Y / \partial Y=T(\eta) .
\end{aligned}
$$

Any two such embeddings determine equivalent normal invariants. The Spivak normal fibration of $M$ is the stable sphere bundle $J \nu_{M}: M \rightarrow B G$ of the stable normal bundle $\nu_{M}: M \rightarrow B O$.

When does a geometric Poincaré complex admit a normal invariant?

Browder-Novikov Normal Invariant Theorem 9.42 ([14], [63])

The following conditions on an $m$-dimensional geometric Poincaré complex $X$ are equivalent:

(i) The normal structure set $\mathscr{T}(X)$ is non-empty, i.e. $X$ admits a normal invariant.

(ii) There exists a degree 1 normal map $(f, b): M^{m} \rightarrow X$.

(iii) The Spivak normal fibration $\nu_{X}: X \rightarrow B G$ admits a bundle reduction $\eta: X \rightarrow B O$.

(iv) The composite

$$
t\left(\nu_{X}\right): X \stackrel{\nu_{X}}{\longrightarrow} B G \longrightarrow B(G / O)
$$

is null-homotopic.

Proof (i) $\Longrightarrow$ (ii), (iii) $\Longrightarrow$ (ii) Given a normal invariant $(\eta, \rho)$ on $X$ make $\rho: S^{m+k} \rightarrow T(\eta)$ transverse at the zero section $X \hookrightarrow T(\eta)$, obtaining a degree 1 normal map

$$
(f, b): M^{m}=\rho^{-1}(X) \rightarrow X .
$$

(ii) $\Longrightarrow$ (i) Given a degree 1 normal map $(f, b): M \rightarrow X$ with $b: \nu_{M} \rightarrow \eta$ use an embedding $M^{m} \hookrightarrow S^{m+k}$ of an $m$-dimensional manifold $M$ to define a normal invariant $\left(\nu_{M}, \rho_{M}\right)$, with 


$$
\begin{aligned}
& \nu_{M}=\nu_{M \hookrightarrow S^{m+k}}: M \rightarrow B O(k), \\
& \rho_{M}=\text { proj. }: S^{m+k} \rightarrow S^{m+k} /\left(S^{m+k} \backslash D\left(\nu_{M}\right)\right)=T\left(\nu_{M}\right) .
\end{aligned}
$$

Define a normal invariant $(\eta, \rho)$ on $X$ by

$$
\rho=T(b) \rho_{M}: S^{m+k} \stackrel{\rho_{M}}{\longrightarrow} T\left(\nu_{M}\right) \stackrel{T(b)}{\longrightarrow} T(\eta) .
$$

(ii) $\Longrightarrow$ (iii) For any normal invariant $(\eta, \rho)$ of $X$ the sphere bundle $J \eta: X \rightarrow B G$ is the Spivak normal fibration $\nu_{X}$, by 9.42 .

(iii) $\Longleftrightarrow$ (iv) The fibration sequence $B O \rightarrow B G \rightarrow B(G / O)$ induces an exact sequence of pointed sets

$$
[X, B O] \rightarrow[X, B G] \rightarrow[X, B(G / O)]
$$

Thus the first obstruction to an $m$-dimensional geometric Poincaré complex $X$ being homotopy equivalent to a manifold is the homotopy class of the composite

$$
t\left(\nu_{X}\right): X \stackrel{\nu_{X}}{\longrightarrow} B G \longrightarrow B(G / O)
$$

Proposition 9.43 Let $X$ be an $m$-dimensional geometric Poincaré complex which admits a normal invariant.

(i) The normal structure set $\mathscr{T}(X)$ is in natural bijective correspondence with the set of normal bordism classes of degree 1 normal maps $(f, b): M \rightarrow X$ for varying reductions $\eta: X \rightarrow B O$ of the Spivak normal fibration $\nu_{X}: X \rightarrow B G$.

(ii) The normal structure set $\mathscr{T}(X)$ is in unnatural bijective correspondence with the set $[X, G / O]$ of fibre homotopy trivialised stable vector bundles over $X$.

Proof (i) Immediate from the transversality construction of a normal map $(f, b): M \rightarrow X$ from a normal invariant $(\eta, \rho)$, with $M=\rho^{-1}(X)$.

(ii) An element $(\alpha, \beta) \in[X, G / O]$ is a vector bundle $\alpha: X \rightarrow B O(j)$ (j large) together with a fibre homotopy trivialisation $\beta: J \alpha \simeq\{*\}: X \rightarrow B G(j)$. Given a normal invariant $\left(\eta: X \rightarrow B O(k), \rho: S^{m+k} \rightarrow T(\eta)\right)$ define a normal invariant $\left(\eta^{\prime}, \rho^{\prime}\right)$ by

$$
\begin{aligned}
& \eta^{\prime}=\eta \oplus \alpha: X \rightarrow B O(j+k) \\
& \rho^{\prime}: S^{m+j+k} \stackrel{\Sigma^{j} \rho}{\longrightarrow} \Sigma^{j} T(\eta)=T\left(\eta \oplus \epsilon^{j}\right) \stackrel{1 \oplus T(\beta)^{-1}}{\longrightarrow} T\left(\eta^{\prime}\right) .
\end{aligned}
$$

The construction defines a bijection

$$
\iota_{\eta, \rho}:[X, G / O] \rightarrow \mathscr{T}(X) ;(\alpha, \beta) \mapsto\left(\eta^{\prime}, \rho^{\prime}\right) .
$$

The bijection is unnatural in that it depends on the choice of normal invariant $(\eta, \rho)$. 
Example 9.44 See Milnor [50] for the original identification

$$
\mathscr{T}\left(S^{m}\right)=\pi_{m}(G / O) .
$$

Example 9.45 The Spivak normal fibration $\nu_{M}: M \rightarrow B G$ of a manifold $M$ has a canonical vector bundle reduction, namely the stable normal bundle $\widetilde{\nu}_{M}: M \rightarrow B O$, and the normal structure set $\mathscr{T}(M)$ is in natural bijective correspondence with $[M, G / O]$. The bijective correspondence

$$
\mathscr{T}(M) \rightarrow[M, G / O] ;((f, b): N \rightarrow M) \mapsto(\alpha, \beta)
$$

is defined by sending a normal map $(f, b): N \rightarrow M$ to the fibre homotopy trivialised stable vector bundle $(\alpha, \beta)$ over $M$ with $b: \widetilde{\nu}_{N} \rightarrow \widetilde{\nu}_{M} \oplus \alpha$.

Example 9.46 The following construction exhibits a geometric Poincaré complex $X$ without a normal invariant, i.e. such that the Spivak normal fibration $\nu_{X}: X \rightarrow B G$ is not reducible to a vector bundle $\widetilde{\nu}_{X}: X \rightarrow B O$. A fortiori $X$ is not homotopy equivalent to a manifold. The construction uses the fact that the total space of a fibration

$$
F \rightarrow E \rightarrow B
$$

with the base $B$ an $m$-dimensional geometric Poincaré complex and the fibre $F$ an $n$-dimensional geometric Poincaré complex is an $(m+n)$-dimensional geometric Poincaré complex $E$ (Gottlieb [28]). In particular, the total space of an $n$-spherical fibration $\omega: S^{m} \rightarrow B G(n+1)$ over $S^{m}$ is an $(m+n)$-dimensional geometric Poincaré complex $S(\omega)$

$$
S^{n} \rightarrow S(\omega) \rightarrow S^{m}
$$

If $\omega$ admits a section, say

$$
\omega=\omega_{1} \oplus \epsilon: S^{m} \rightarrow B F(n+1)
$$

for some $\omega_{1}: S^{m} \rightarrow B G(n)$, the total space $S(\omega)$ has a cell structure

$$
S(\omega)=\left(S^{m} \vee S^{n}\right) \cup_{\left[\iota_{m}, \iota_{n}\right]+\theta(\omega)} D^{m+n},
$$

with $\left[\iota_{m}, \iota_{n}\right] \in \pi_{m+n-1}\left(S^{m} \vee S^{n}\right)$ the Whitehead product of $\iota_{m} \in \pi_{m}\left(S^{m}\right)$ and $\iota_{n} \in \pi_{n}\left(S^{n}\right)$ (the attaching map of the top cell in $S\left(\epsilon^{n+1}\right)=S^{m} \times S^{n}$ ) and

$$
\theta(\omega)=\text { adjoint of } \omega: S^{m+n-1} \rightarrow S^{n} .
$$

The Thom space of $\omega$ has a cell structure

$$
T(\omega)=S^{n+1} \cup_{\Sigma \theta(\omega)} D^{m+n+1} .
$$

The Spivak normal fibration of $S(\omega)$ is classified by 


$$
\nu_{S(\omega)}: S(\omega) \longrightarrow S^{m} \stackrel{-\omega}{\longrightarrow} B F(k)(k \text { large })
$$

and the Thom space of $\nu_{S(\omega)}$ has the cell structure

$$
T\left(\nu_{S(\omega)}\right)=\left(S^{k} \cup_{\Sigma \theta(-\omega)} D^{m+k}\right) \vee S^{n+k} \vee S^{m+n+k}
$$

In the special case

$$
m=3, n=2, \omega=1 \in \pi_{3}(B F(3))=\pi_{4}\left(S^{2}\right)=\mathbb{Z}_{2}
$$

there is obtained a 5-dimensional geometric Poincaré complex $X=S(\omega)$ such that the Spivak normal fibration $\nu_{X}: X \rightarrow B G$ does not have a vector bundle reduction - see Gitler and Stasheff [27] and Madsen and Milgram [45, p.33]. In this case the composite

$$
t\left(\nu_{X}\right): X \stackrel{\nu_{X}}{\longrightarrow} B G \longrightarrow B(G / O)
$$

does not admit a null-homotopy.

Next, consider the uniqueness of manifold structures in a homotopy type.

Proposition 9.47 A homotopy equivalence $f: M^{\prime} \rightarrow M$ of $m$-dimensional manifolds determines a fibre homotopy trivialisation

$$
t(f): J\left(\nu_{M}-\left(f^{-1}\right)^{*}\left(\nu_{M^{\prime}}\right)\right) \simeq\{*\}: M \rightarrow B G
$$

of the stable bundle

$$
\nu_{M}-\left(f^{-1}\right)^{*}\left(\nu_{M^{\prime}}\right)=\nu_{M} \oplus\left(-\left(f^{-1}\right)^{*}\left(\nu_{M^{\prime}}\right)\right): M \rightarrow B O .
$$

The classifying map $t(f): M \rightarrow G / O$ is null-homotopic if and only if there exists an extension of $f$ to a degree 1 normal bordism

$$
((F, B) ; 1,(f, b)):\left(W^{m+1} ; M^{m}, M^{\prime m}\right) \rightarrow M \times(I ;\{0\},\{1\}) .
$$

Proof Let

$$
\begin{aligned}
& \left(\nu_{M}: M \rightarrow B O(k), \rho_{M}: S^{m+k} \rightarrow T\left(\nu_{M}\right)\right), \\
& \left(\nu_{M^{\prime}}: M^{\prime} \rightarrow B O(k), \rho_{M^{\prime}}: S^{m+k} \rightarrow T\left(\nu_{M^{\prime}}\right)\right)
\end{aligned}
$$

be the normal invariants determined by embeddings $M, M^{\prime} \hookrightarrow S^{m+k}$. The fibre homotopy trivialisation $t(f)$ of $J\left(\nu_{M}-\left(f^{-1}\right)^{*}\left(\nu_{M^{\prime}}\right)\right): M \rightarrow B G$ is defined by the unique map of stable spherical fibrations $a: J \nu_{M^{\prime}} \rightarrow J \nu_{M}$ over $f: M^{\prime} \simeq M$ such that

$$
T(a)_{*}\left(\rho_{M^{\prime}}\right)=\rho_{M} \in \pi_{m+k}^{S}\left(T\left(\nu_{M}\right)\right)
$$

given by 9.31 (iii). There exists an extension of $f$ to a degree 1 normal map $(f, b): M^{\prime} \rightarrow M$ if and only if there exists a null-homotopy 


$$
\nu_{M}-\left(f^{-1}\right)^{*}\left(\nu_{M^{\prime}}\right) \simeq\{*\}: M \rightarrow B O
$$

with the bordism class given by 9.43 to be

$(f, b)=T(b)_{*}\left(\rho_{M^{\prime}}\right) \in \operatorname{im}\left(\pi_{m+k}^{S}\left(T\left(\nu_{M}\right)\right) \rightarrow \mathscr{T}(M)\right)=\operatorname{im}([M, G] \rightarrow[M, G / O])$.

The following conditions on $(f, b)$ are equivalent:

(i) $(f, b)=(1,1) \in \mathscr{T}(M)$,

(ii) $T(b)_{*}\left(\rho_{M^{\prime}}\right)=\rho_{M} \in \pi_{m+k}^{S}\left(T\left(\nu_{M}\right)\right)$,

(iii) $J b \simeq a: J \nu_{M^{\prime}} \rightarrow J \nu_{M}$.

There exists an extension $(f, b)$ satisfying these conditions if and only if there exists a null-homotopy $t(f) \simeq\{*\}: M \rightarrow G / O$.

The necessary and sufficient condition $t(f) \simeq\{*\}: M \rightarrow G / O$ for a homotopy equivalence $f: M^{\prime m} \simeq M^{m}$ of $m$-dimensional manifolds to be bordant to $1: M \simeq$ $M$ is non-trivial - see the classification of exotic spheres in Section 13.3.

Assuming $t(f) \simeq\{*\}: M \rightarrow G / O$ the next stage of the surgery programme to decide if a homotopy equivalence $f: M^{\prime m} \simeq M^{m}$ of $m$-dimensional manifolds is homotopic to a diffeomorphism is to investigate the extent to which a degree 1 normal bordism

$$
(F, B):\left(W ; M, M^{\prime}\right) \rightarrow M \times(I ;\{0\},\{1\})
$$

determined by a choice of null-homotopy $t(f) \simeq\{*\}$ is bordant rel $\partial$ to a homotopy equivalence $\left(F^{\prime}, B^{\prime}\right):\left(W^{\prime} ; M, M^{\prime}\right) \simeq M \times(I ;\{0\},\{1\})$.

Proposition 9.48 A homotopy equivalence $f: M^{\prime m} \simeq M^{m}$ of $m$-dimensional manifolds is h-cobordant to a diffeomorphism if and and only if there exists a null-homotopy $t(f) \simeq\{*\}: M \rightarrow G / O$ such that the corresponding relo bordism class of degree 1 normal maps

$$
((F, B) ; 1,(f, b)):\left(W^{m+1} ; M^{m}, M^{\prime m}\right) \rightarrow M \times(I ;\{0\},\{1\})
$$

contains a homotopy equivalence.

For $m \geqslant 5$ the degree 1 normal map $(F, B):\left(W ; M, M^{\prime}\right) \rightarrow M \times(I ;\{0\},\{1\})$ in 9.48 is bordant to a homotopy equivalence rel $\partial$ if and only if the rel $\partial$ surgery obstruction $\sigma_{*}(F, B) \in L_{m+1}\left(\mathbb{Z}\left[\pi_{1}(M)\right]\right)$ defined in Chapters 11 and 12 is $\sigma_{*}(F, B)=$ 0 .

The formal similarity between the criteria of 9.38 and 9.48 for the existence and uniqueness of manifold structures in homotopy types has a straightforward 
explanation. The mapping cylinder of a homotopy equivalence $f: M^{\prime m} \simeq M^{m}$ of $m$-dimensional manifolds defines an $(m+1)$-dimensional geometric Poincaré bordism $\left(W ; M, M^{\prime}\right)$ with Spivak normal fibration

$$
\nu_{W} \simeq J \nu_{M}: W \simeq M \rightarrow B G .
$$

Now $f$ is $h$-cobordant to a diffeomorphism if and only if $\left(W ; M, M^{\prime}\right)$ is rel $\partial$ homotopy equivalent to an $(m+1)$-dimensional manifold cobordism $\left(W^{m+1} ; M, M^{\prime}\right)$, which is necessarily an $h$-cobordism. The primary obstruction $t(f): M \rightarrow G / O$ for $f$ to be $h$-cobordant to a diffeomorphism is identified with the primary obstruction

$$
t\left(\nu_{W}\right): W / \partial W \simeq \Sigma M \rightarrow B(G / O)
$$

for $(W, \partial W)$ to be rel $\partial$ homotopy equivalent to an $(m+1)$-dimensional manifold with boundary

$$
t(f)=t\left(\nu_{W}\right) \in[\Sigma M, B(G / O)]=[M, G / O] .
$$

If this primary obstruction vanishes there exists a degree 1 normal map

$$
(F, B):\left(W^{\prime} ; M, M^{\prime}\right) \rightarrow M \times(I ;\{0\},\{1\})
$$

with $f \mid=$ id. $: M \rightarrow M, F \mid=f: M^{\prime} \rightarrow M$. The secondary obstruction is the rel $\partial$ surgery obstruction $\sigma_{*}(F, B) \in L_{m+1}\left(\mathbb{Z}\left[\pi_{1}(M)\right]\right)$ to the existence of a rel $\partial$ normal bordism of $(F, B)$ to a homotopy equivalence.

The main result of surgery obstruction theory is that for $m \geqslant 5$ an $m$ dimensional degree 1 normal map $(f, b): M \rightarrow X$ is bordant to a homotopy equivalence if and only if an algebraic $L$-theory obstruction

$$
\sigma_{*}(f, b) \in L_{m}\left(\mathbb{Z}\left[\pi_{1}(X)\right]\right)
$$

is 0 . There are two distinct ways of obtaining the surgery obstruction :

(i) The original method of Wall [90],[92], using geometric surgery below the middle dimension. In Chapter 10 it will be shown that for $m=2 n($ resp. $2 n+1)$ $(f, b)$ is bordant to an $n$-connected degree 1 normal map $\left(f^{\prime}, b^{\prime}\right): M^{\prime} \rightarrow X$, with $K_{i}\left(M^{\prime}\right)=0$ for $i \neq n$ (resp. $\left.n, n+1\right)$. The $L$-group $L_{2 n}(A)$ (resp. $\left.L_{2 n+1}(A)\right)$ of a ring with involution $A$ is the Witt group of nonsingular $(-1)^{n}$-quadratic forms (resp. formations), which will be described in Chapter 11 (resp. 12). The surgery obstruction

$$
\sigma_{*}(f, b)=\sigma_{*}\left(f^{\prime}, b^{\prime}\right) \in L_{m}\left(\mathbb{Z}\left[\pi_{1}(X)\right]\right)
$$

is the class of the form (resp. formation) associated to the kernel Poincaré duality $K^{*}\left(M^{\prime}\right) \cong K_{m-*}\left(M^{\prime}\right)$.

(ii) The chain complex method of Ranicki [69],[76] in which the surgery obstruction $\sigma_{*}(f, b) \in L_{m}\left(\mathbb{Z}\left[\pi_{1}(X)\right]\right)$ is obtained directly from the chain level version of the kernel Poincaré duality $K^{*}(M) \cong K_{m-*}(M)$, without preliminary geometric surgeries below the middle dimension. 


\section{SURGERY ON MAPS}

An $m$-dimensional normal map $(f, b): M \rightarrow X$ is an $m$-dimensional manifold $M^{m}$ together with a map $f: M \rightarrow X$ and a bundle map $b: \nu_{M} \rightarrow \eta$ from a stable normal bundle of $M$ to a vector bundle $\eta$ over $X$.

Section 10.1 extends the notion of surgery on a manifold to surgery on a normal map. An $n$-surgery on a normal map $(f, b): M \rightarrow X$ killing $x \in \pi_{n+1}(f)$ starts with an embedding $S^{n} \times D^{m-n} \hookrightarrow M$ such that $(f, b)$ extends to a normal map

$$
\left((F, B) ;(f, b),\left(f^{\prime}, b^{\prime}\right)\right):\left(W ; M, M^{\prime}\right) \rightarrow X \times(I ;\{0\},\{1\}),
$$

with $\left(W ; M, M^{\prime}\right)$ the trace of the $n$-surgery on $M$ removing $S^{n} \times D^{m-n} \hookrightarrow M$ and killing $\partial x \in \pi_{n}(M)$. The main result of this chapter is Theorem 10.30: if $m=2 n$ or $2 n+1$ it is possible to kill the homotopy groups $\pi_{*}(f)$ for $* \leqslant n$, and $(f, b)$ is normal bordant to an $n$-connected normal map.

Which elements $x \in \pi_{n+1}(f)$ can be killed by an $n$-surgery on an $m$-dimensional normal map $(f, b): M^{m} \rightarrow X$ ? By definition, $x$ is a homotopy class of commutative squares

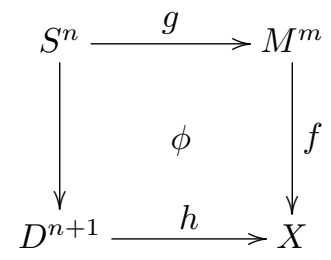

and $x$ can be killed by surgery on $(f, b)$ precisely when there exists a representative with $g: S^{n} \hookrightarrow M$ an embedding such that the normal bundle $\nu_{g}: S^{n} \rightarrow B O(m-n)$ can be framed compatibly with the bundle map $b: \nu_{M} \rightarrow \eta$. There are two distinct ways of proceeding:

(i) First embeddings then framings.

The "b-framing obstruction" of any representative $\phi$ with $g: S^{n} \uparrow M$ an immersion is an element

$$
\nu_{b}(\phi) \in \pi_{n+1}(B O, B O(m-n))
$$

measuring the extent to which the normal bundle $\nu_{g}: S^{n} \rightarrow B O(m-n)$ can be framed in a way compatible with the stable framing of $\nu_{g}$ determined 
by $b: \nu_{M} \rightarrow \eta$ and $h: D^{n+1} \rightarrow X$. The element $x \in \pi_{n+1}(f)$ can be killed by an $n$-surgery on $(f, b)$ if and only if it has a representative $\phi$ with $g$ an embedding and $b$-framing obstruction $\nu_{b}(\phi)=0$. This is the method favoured by Kervaire and Milnor [38] and Browder [14]. It is particularly effective in the simply-connected case $\pi_{1}(M)=\pi_{1}(X)=\{1\}$ for $2 n \leqslant m$ with $m \geqslant 5$, since by the Whitney Embedding Theorem (7.2) every element $x \in \pi_{n+1}(f)$ has a representative $\phi$ with $g$ an embedding.

(ii) First framings then embeddings.

By the Smale-Hirsch Immersion Classification Theorem (7.35) it is possible for $n \leqslant m-2$ to represent each $x \in \pi_{n+1}(f)$ by a diagram $\phi$ with $g: S^{n} \leftrightarrow$ $M^{m}$ an immersion such that $\nu_{g}: S^{n} \rightarrow B O(m-n)$ is framed in a manner compatible with $b$ (so that $\nu_{b}(\phi)=0$ ). Any two such representatives are regular homotopic. Section 10.2 gives the basic properties of the regular homotopy groups of immersions and their applications to surgery theory. An element $x \in \pi_{n+1}(f)$ can be killed by an $n$-surgery on $(f, b)$ if and only if there exists such a representative $\phi$ with $g: S^{n} \hookrightarrow M^{m}$ an embedding. This is the method favoured by Wall [92]. It is more elaborate than (i), but it is better suited to the classification of the effects of the surgeries.

The kernel $\mathbb{Z}\left[\pi_{1}(X)\right]$-modules of a map $f: M \rightarrow X$

$$
K_{*}(M)=H_{*+1}(\widetilde{f}: \widetilde{M} \rightarrow \widetilde{X})
$$

are defined in Section 10.3, with $\widetilde{X}$ the universal cover of $X$ and $\widetilde{M}=f^{*} \widetilde{X}$ the pullback cover of $M$. A map $f$ of $C W$ complexes is a homotopy equivalence if and only if $f_{*}: \pi_{1}(M) \rightarrow \pi_{1}(X)$ is an isomorphism and $K_{*}(M)=0$.

Given an $m$-dimensional degree 1 normal map $(f, b): M^{m} \rightarrow X$ we should like (if possible) to construct a bordant degree 1 normal map $\left(f_{\infty}, b_{\infty}\right): M_{\infty} \rightarrow X$ which is a homotopy equivalence, with $K_{*}\left(M_{\infty}\right)=0$. The standard operating procedure of surgery theory is to try and kill the successive kernel homology modules $K_{i}(M)$ for $i=0,1,2, \ldots$ by surgeries on $(f, b)$, to obtain a sequence of bordant degree 1 normal maps $\left(f_{i}, b_{i}\right): M_{i} \rightarrow X$ which are $i$-connected, with $K_{j}\left(M_{i}\right)=0$ for $j<i$. Start with

$$
\left(f_{0}, b_{0}\right)=(f, b): M_{0}=M \rightarrow X .
$$

Assume inductively that $\left(f_{0}, b_{0}\right),\left(f_{1}, b_{1}\right), \ldots,\left(f_{i}, b_{i}\right)$ have already been constructed. At this stage we have the question:

Is it possible to kill $K_{i}\left(M_{i}\right)$ by surgery, to obtain a bordant $(i+1)$-connected degree 1 normal map $\left(f_{i+1}, b_{i+1}\right): M_{i+1} \rightarrow X$ ?

In Section 10.4 the Whitney Embedding Theorem (7.1) will be used to prove that for $2 i+1 \leqslant m$ every element $x \in \pi_{i+1}\left(f_{i}\right)$ can be killed by an $n$-surgery. In 
Section 10.5 it will be proved that $\pi_{i+1}\left(f_{i}\right)$ is a f.g. $\mathbb{Z}\left[\pi_{1}(X)\right]$-module, and that for $2 i+2 \leqslant m\left(f_{i}, b_{i}\right)$ is bordant to an $(i+1)$-connected degree 1 normal map $\left(f_{i+1}, b_{i+1}\right): M_{i+1} \rightarrow X$.

Thus for an $m$-dimensional degree 1 normal map $(f, b): M \rightarrow X$ with $m=2 n$ or $2 n+1$ there is a bordant $n$-connected degree 1 normal map $\left(f_{n}, b_{n}\right): M_{n} \rightarrow$ $X$. However, there is a surgery obstruction (to be defined in Chapters 11 and 12 ) to killing $K_{n}\left(M_{n}\right)$ to obtain a bordant $(n+1)$-connected degree 1 normal map $\left(f_{n+1}, b_{n+1}\right): M_{n+1} \rightarrow X$, which takes value in the surgery obstruction group $L_{m}\left(\mathbb{Z}\left[\pi_{1}(X)\right]\right)$. If the surgery obstruction is 0 then $\left(f_{n+1}, b_{n+1}\right)$ can be constructed. Now $\left(f_{n+1}, b_{n+1}\right)$ is a homotopy equivalence by the Theorem of J.H.C. Whitehead (3.6) and Poincaré duality, so that we can set

$$
\left(f_{n+1}, b_{n+1}\right)=\left(f_{n+2}, b_{n+2}\right)=\ldots=\left(f_{\infty}, b_{\infty}\right) .
$$

For a $2 n$-dimensional normal map $(f, b): M^{2 n} \rightarrow X$ it is possible to represent every element $x \in \pi_{n+1}(f)$ by a framed $n$-immersion $\phi$ in $(f, b)$, by the Whitney Immersion Theorem (7.1). However, in general $x$ is not represented by a framed $n$-embedding, so that $x$ cannot be killed by an $n$-surgery on $(f, b)$. For $n \geqslant 3$ it is possible to represent $x$ by a framed $n$-embedding if and only if a certain self-intersection obstruction $\mu(x)$ in a quotient of $\mathbb{Z}\left[\pi_{1}(M)\right]$ is 0 . The surgery obstruction of an $n$-connected $2 n$-dimensional normal map $(f, b)$ is due to the fact that $\phi$ may not be represented by a framed $n$-embedding. See Chapter 11 for the self-intersection form $\mu$ and the $2 n$-dimensional surgery obstruction.

For a $(2 n+1)$-dimensional normal map $(f, b): M^{2 n+1} \rightarrow X$ with $n \geqslant 2$ it is possible to represent every element $x \in \pi_{n+1}(f)$ by a framed $n$-embedding $\phi$ in $(f, b)$. The Wall surgery obstruction of an $n$-connected $(2 n+1)$-dimensional normal map $(f, b)$ is due to the fact that even though it is possible to kill every element $x \in \pi_{n+1}(f)=K_{n}(M)$ by an $n$-surgery on $(f, b)$ there are many ways of doing so, none of which need reduce the size of $K_{n}(M)$. See Chapter 12 for the $(2 n+1)$-dimensional surgery obstruction.

\subsection{Surgery on normal maps}

Recall from Definition 2.4 that an $n$-embedding in an $m$-dimensional manifold $M$ is an embedding $g: S^{n} \hookrightarrow M$.

Definition 10.1 Let $M^{m}$ be an $m$-dimensional manifold with a map $f: M \rightarrow X$ to a space $X$.

(i) An $n$-immersion in $M$ is an immersion

$$
g: S^{n} \rightarrow M .
$$

(ii) A framed $n$-immersion in $M$ is an immersion 


$$
\bar{g}: S^{n} \times D^{m-n} \uparrow M \text {. }
$$

(iii) An $n$-immersion $\phi$ in $f$ is a commutative square

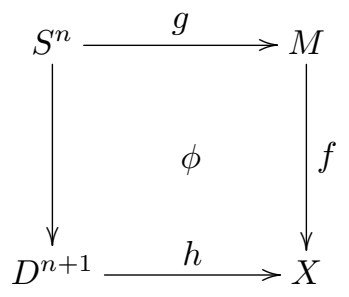

with $g: S^{n} \rightarrow M$ an $n$-immersion in $M$. An $n$-embedding in $f$ is an $n$ immersion $\phi$ with $g$ an $n$-embedding in $M$.

(iv) A framed $n$-immersion $\Phi$ in $f$ is a commutative square

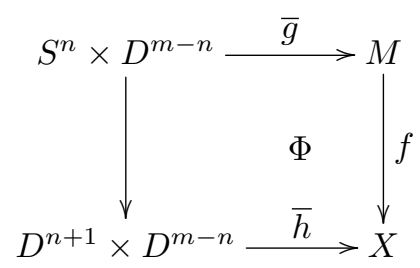

with $\bar{g}: S^{n} \times D^{m-n} \rightarrow M$ a framed $n$-immersion in $M$. A framed $n$-embedding in $f$ is a framed $n$-immersion $\phi$ with $\bar{g}$ an embedding.

(v) The core of a framed $n$-immersion $\Phi$ is the $n$-immersion $\phi$ defined by the restrictions $g, h$ of $\bar{g}, \bar{h}$. Similarly for $n$-embeddings.

(vi) The $n$-surgery on $f$ removing a framed $n$-embedding $\Phi$ is the operation of $n$-surgery on $M$ removing $\bar{g}: S^{n} \times D^{m-n} \hookrightarrow M$ with effect

$$
M^{\prime}=\operatorname{cl} .\left(M \backslash \bar{g}\left(S^{n} \times D^{m-n}\right)\right) \cup D^{n+1} \times S^{m-n-1}
$$

together with the extension of $f$ to a trace bordism

$$
\left(F ; f, f^{\prime}\right):\left(W ; M, M^{\prime}\right) \rightarrow X \times(I ;\{0\},\{1\})
$$

given by

$$
F=f \times 1 \cup \bar{h}: W=M \times I \cup_{\bar{g}} D^{n+1} \times D^{m-n} \rightarrow X \times I
$$

The $n$-surgery kills the homotopy class of the core

$$
x=[\phi]=(h, g) \in \pi_{n+1}(f) .
$$

The homotopy theoretic effect in dimensions $\leqslant n+1$ of an $n$-surgery on a map is given by: 
Proposition 10.2 The relative homotopy groups in the trace of an $n$-surgery on an $m$-dimensional map $f: M \rightarrow X$

$$
\left(F ; f, f^{\prime}\right):\left(W^{m+1} ; M^{m}, M^{\prime m}\right) \rightarrow X \times(I ;\{0\},\{1\})
$$

killing $x \in \pi_{n+1}(f)$ are such that

$$
\pi_{i}(F)= \begin{cases}\pi_{i}(f) & \text { if } i \leqslant n \\ \pi_{n+1}(f) /\langle x\rangle & \text { if } i=n+1,\end{cases}
$$

with $\langle x\rangle \subseteq \pi_{n+1}(f)$ the $\mathbb{Z}\left[\pi_{1}(X)\right]$-module generated by $x$. Also, $F$ is the trace of the dual $(m-n-1)$-surgery on $f^{\prime}: M^{\prime} \rightarrow X$ killing an element $x^{\prime} \in \pi_{m-n}\left(f^{\prime}\right)$, so that

$$
\pi_{j}(F)= \begin{cases}\pi_{j}\left(f^{\prime}\right) & \text { if } j \leqslant m-n-1 \\ \pi_{m-n}\left(f^{\prime}\right) /\left\langle x^{\prime}\right\rangle & \text { if } j=m-n\end{cases}
$$

Proof The elements

$$
x=[\phi]=(h, g) \in \pi_{n+1}(f), x^{\prime}=\left[\phi^{\prime}\right]=\left(h^{\prime}, g^{\prime}\right) \in \pi_{m-n}\left(f^{\prime}\right)
$$

are represented by the core $n$-embedding and the dual core $(m-n-1)$-embedding
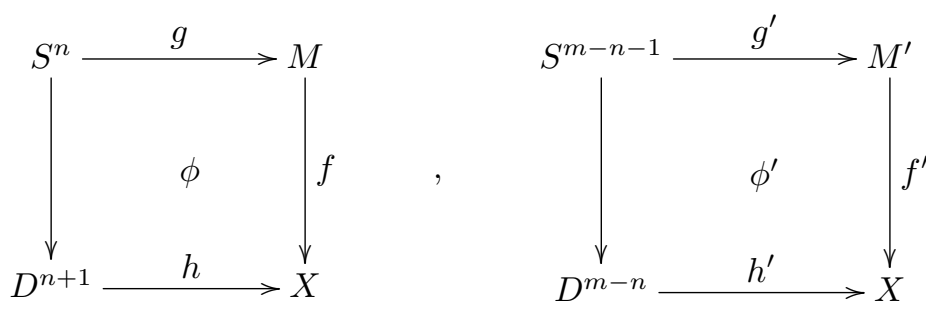

with

$$
F \simeq f \cup h \simeq f^{\prime} \cup h^{\prime}: W \simeq M \cup_{g} D^{n+1} \simeq M^{\prime} \cup_{g^{\prime}} D^{m-n} \rightarrow X .
$$

Corollary 10.3 For $2 n+2 \leqslant m$ an n-connected map $f: M^{m} \rightarrow X$ can be made $(n+1)$-connected by $n$-surgeries if and only if there exists a finite set of $\mathbb{Z}\left[\pi_{1}(X)\right]$-module generators $x_{1}, x_{2}, \ldots, x_{k} \in \pi_{n+1}(f)=K_{n}(M)$ which can be killed by $n$-surgeries on $f$.

Proof The condition $2 n+2 \leqslant m$ ensures that the effect of an $n$-surgery killing an element $x \in \pi_{n+1}(f)$ is a map $f^{\prime}: M^{\prime m} \rightarrow X$ such that

$$
\pi_{i}\left(f^{\prime}\right)=\pi_{i}(F)= \begin{cases}\pi_{i}(f) & \text { if } i \leqslant n \\ \pi_{n+1}(f) /\langle x\rangle & \text { if } i=n+1 .\end{cases}
$$


Definition 10.4 The framing obstruction of an $n$-immersion in a map $f$ : $M^{m} \rightarrow X$

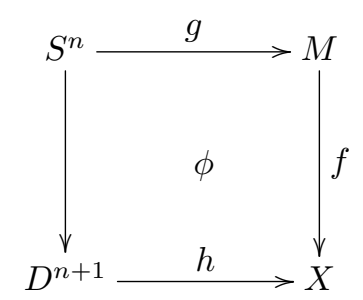

is the isomorphism class of the normal bundle of $g$

$$
\nu(\phi)=\nu_{g} \in \pi_{n}(B O(m-n)) .
$$

Proposition 10.5 Let $f: M \rightarrow X$ be a map from an m-dimensional manifold $M^{m}$. For any element $x \in \pi_{n+1}(f)$ the following conditions are equivalent:

(i) $x$ can be killed by an n-surgery on $f$,

(ii) $x$ can be represented by a framed $n$-embedding $\Phi$ in $f$,

(iii) $x$ can be represented by an $n$-embedding $\phi$ in $f$ with framing obstruction

$$
\nu(\phi)=0 \in \pi_{n}(B O(m-n)) .
$$

Proof (i) $\Longleftrightarrow$ (ii) Immediate from the definitions (10.1).

(ii) $\Longleftrightarrow$ (iii) An $n$-embedding $\phi$ in $f$ extends to a framed $n$-embedding $\Phi$ precisely when the normal bundle $\nu_{g}$ can be framed, by the Tubular Neighbourhood Theorem (5.50).

We now move on to surgery on a normal map (1.15)

$$
(f, b): M \rightarrow X
$$

with $f: M^{m} \rightarrow X$ a map from an $m$-dimensional manifold and $b: \nu_{M} \rightarrow \eta$ a pullback bundle map over $f$.

Definition 10.6 A framed $n$-embedding $(\Phi, B)$ in $(f, b)$ (or a $b$-framed $n$ embedding $(\Phi, B)$ in $f)$ is a framed $n$-embedding in $f$

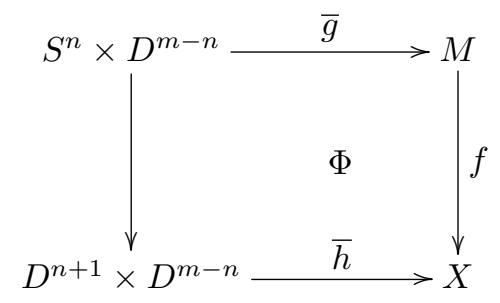

together with an extension of $(f, b)$ to a normal map on the trace of the $n$-surgery on $f$ removing $\Phi$ 


$$
(F, B):\left(W ; M, M^{\prime}\right) \rightarrow X \times(I ;\{0\},\{1\})
$$

with

$$
F: W=M \times I \cup_{\bar{g}} D^{n+1} \times D^{m-n} \rightarrow X \times I
$$

such that

$$
F \simeq f \cup h: W \simeq M \cup_{g} D^{n+1} \rightarrow X
$$

The normal map

$$
\left(f^{\prime}, b^{\prime}\right)=(F, B) \mid: M^{\prime}=\operatorname{cl} .\left(M \backslash \bar{g}\left(S^{n} \times D^{m-n}\right)\right) \cup D^{n+1} \times S^{m-n-1} \rightarrow X
$$

bordant to $(f, b)$ by $(F, B)$ is the effect of the $n$-surgery on $(f, b)$ removing $(\Phi, B)$ and killing the homotopy class $x=[\phi] \in \pi_{n+1}(f)$.

Example 10.7 Every manifold $M$ admits a normal map $\left(\nu_{M}, 1\right): M \rightarrow X=$ $B O$. A framed $n$-embedding $(\Phi, B)$ in $\left(\nu_{M}, 1\right)$ is just a framed $n$-embedding in $M$, and surgery on $(f, b)$ is just surgery on $M$.

Definition 10.8 Let $(f, b): M \rightarrow X$ be a normal map, and let $\phi$ be an $n$ immersion in $f$

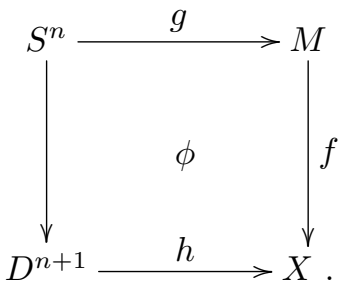

The normal bundle $\nu_{g}: S^{n} \rightarrow B O(m-n)$ has a stable framing

$$
\delta \nu_{h, b}: \nu_{g} \oplus \epsilon^{k} \cong \epsilon^{m-n+k}
$$

determined by an embedding $e: M \hookrightarrow S^{m+k}$ ( $k$ large), the identities

$$
\begin{aligned}
& \nu_{g} \oplus g^{*} \nu_{e}=\nu_{e g}, \\
& \nu_{e}=\nu_{M} \cong f^{*} \eta, \\
& g^{*} \nu_{e} \cong g^{*} f^{*} \eta \cong_{h} \epsilon^{k}
\end{aligned}
$$

and the canonical framing $\nu_{e g} \cong \epsilon^{m-n+k}$ of the normal bundle of the immersion $e g: S^{n} \uparrow S^{m+k}$ The $b$-framing obstruction of $\phi$ is

$$
\nu_{b}(\phi)=\left(\delta \nu_{h, b}, \nu_{g}\right) \in \pi_{n+1}(B O, B O(m-n)) .
$$

Lemma 10.9 The normal bundle $\nu_{i}: S^{n} \rightarrow B O(k)$ of an immersion $i: S^{n} \uparrow$ $S^{n+k}$ ( $k$ large) is equipped with a canonical class of isomorphisms $\nu_{j}: \nu_{i} \cong \epsilon^{k}$, which corresponds to a canonical class of null-homotopies of a classifying map

$$
\nu_{j}: \nu_{i} \simeq\{*\}: S^{n} \rightarrow B O(k),
$$

with $\nu_{j} \sim \nu_{j}^{\prime}$ if $\left(\nu_{j}^{\prime}\right)^{-1} \nu_{j}=0 \in \pi_{n}(O(k))$. 
Proof Relate $i$ to the standard embedding $i_{0}: S^{n} \hookrightarrow S^{n+k}$ by a regular homotopy $i_{t}: S^{n} \rightarrow S^{n+k}(0 \leqslant t \leqslant 1)$, with $i_{1}=i$. Use this to translate the standard bundle isomorphisms

$$
\nu_{j_{0}}: \nu_{i_{0}} \oplus \epsilon^{n+1} \cong \nu_{i_{0}} \oplus \tau_{S^{n}} \oplus \epsilon \cong i_{0}^{*} \tau_{S^{n+k}} \oplus \epsilon \cong \epsilon^{n+k+1}
$$

from $i_{0}$ to $i$. Any two choices of regular homotopies $\left\{i_{t}\right\},\left\{i_{t}^{\prime}\right\}$ are related by a regular homotopy of the track immersions $S^{n} \times I \uparrow S^{n+k} \times I$.

Next, consider the manifold $M$. For definiteness, let $\nu_{M}=\nu_{e}: M \rightarrow B O(k)$ be the normal bundle of an embedding $e: M \hookrightarrow S^{m+k}$ ( $k$ large), and let $\eta$ : $X \rightarrow B O(k)$ be likewise a $k$-plane bundle, so that $b: \nu_{M} \rightarrow \eta$ is a map of $k$-plane bundles. The normal bundle of the composite immersion

$$
g^{\prime}=e g: S^{n} \hookrightarrow M^{m} \hookrightarrow S^{m+k}
$$

is trivial, with a trivialisation

$$
\nu_{g^{\prime}}=\nu_{g} \oplus g^{*} \nu_{M} \simeq\{*\}: S^{n} \rightarrow B O(m-n+k) .
$$

The normal bundle $\nu_{g}: S^{n} \rightarrow B O(m-n)$ is a stable inverse of the pullback $g^{*} \nu_{M}: S^{n} \rightarrow B O$ of the stable normal bundle $\nu_{M}: M \rightarrow B O$, that is

$$
\nu_{g} \oplus g^{*} \nu_{M}=\epsilon^{\infty}: S^{n} \rightarrow B O .
$$

Similarly for a normal map $(f, b): M \rightarrow X$ : the map of $k$-plane bundles $b$ : $\nu_{M} \rightarrow \eta$ and the null-homotopy $h: g f \simeq *: S^{n} \rightarrow X$ determine a null-homotopy

$$
g^{*} \nu_{M}=g^{*} f^{*} \eta \simeq *: S^{n} \rightarrow B O(k)
$$

which in general is not compatible with the canonical null-homotopy of $\nu_{g^{\prime}}$. Combining the two null-homotopies into a single null-homotopy and passing to the limit as $k \rightarrow \infty$ there is obtained a commutative square

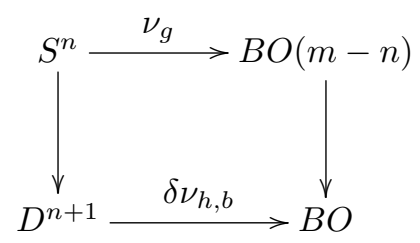

representing the $b$-framing obstruction

$$
\nu_{b}(\phi)=\left(\delta \nu_{h, b}, \nu_{g}\right) \in \pi_{n+1}(B O, B O(m-n)) .
$$

Proposition 10.10 Let $(f, b): M \rightarrow X$ be a normal map from an $m$-dimensional manifold $M^{m}$. For any element $x \in \pi_{n+1}(f)$ the following conditions are equivalent: 
(i) $x$ can be killed by an n-surgery on $(f, b)$,

(ii) $x$ can be represented by a framed $n$-embedding $(\Phi, B)$ in $(f, b)$,

(iii) $x$ can be represented by an $n$-embedding $\phi$ in $f$ with b-framing obstruction

$$
\nu_{b}(\phi)=0 \in \pi_{n+1}(B O, B O(m-n)) .
$$

Proof (i) $\Longleftrightarrow$ (ii) Immediate from the definitions (10.6).

(ii) $\Longleftrightarrow$ (iii) An $n$-embedding $\phi$ in $f$ extends to a framed $n$-embedding $\Phi$ precisely when the normal bundle $\nu_{g}$ can be framed, by the Tubular Neighbourhood Theorem (5.50).

By analogy with 10.3 :

Corollary 10.11 For $2 n+2 \leqslant m$ an n-connected normal map $(f, b): M^{m} \rightarrow X$ can be made $(n+1)$-connected by $n$-surgeries if and only if there exists a finite set of $\mathbb{Z}\left[\pi_{1}(X)\right]$-module generators $x_{1}, x_{2}, \ldots, x_{k} \in \pi_{n+1}(f)=K_{n}(M)$ which can be killed by $n$-surgeries on $(f, b)$.

\subsection{The regular homotopy groups}

The regular homotopy groups $I_{n}(M)$ of immersions $g: S^{n} \rightarrow M^{m}(5.54)$ will now be generalised to the relative regular homotopy homotopy groups $I_{n+1}(f)$ for a map $f: M^{m} \rightarrow X$, with an exact sequence

$$
\ldots \rightarrow \pi_{n+1}(X) \rightarrow I_{n+1}(f) \rightarrow I_{n}(M) \rightarrow \pi_{n}(X) \rightarrow \ldots .
$$

The groups $I_{n+1}(f)$ will be used to formulate Wall's immersion-theoretic approach to surgery on normal maps $(f, b): M \rightarrow X$. The case $m=2 n$ will be used in the $2 n$-dimensional surgery obstruction theory of Chapter 11.

Definition 10.12 (i) The relative regular homotopy group $I_{n+1}(f)$ of a map $f: M^{m} \rightarrow X$ from an $m$-dimensional manifold $M$ to any space $X$ is the $\mathbb{Z}\left[\pi_{1}(X)\right]$-module of regular homotopy classes of $n$-immersions in $f$, with addition by connected sum.

(ii) The relative framed regular homotopy group $I_{n+1}^{f r}(f)$ of a map $f$ : $M^{m} \rightarrow X$ from an $m$-dimensional manifold $M$ to any space $X$ is the $\mathbb{Z}\left[\pi_{1}(X)\right]$ module of regular homotopy classes of framed $n$-immersions in $f$, with addition by connected sum.

Proposition 10.13 Let $f: M^{m} \rightarrow X$ be a map from an m-dimensional manifold $M$, and let $n \leqslant m-2$.

(i) The relative regular homotopy groups $I_{n+1}(f), I_{n+1}^{f r}(f)$ fit into a commutative braid of exact sequences of $\mathbb{Z}\left[\pi_{1}(M)\right]$-modules 


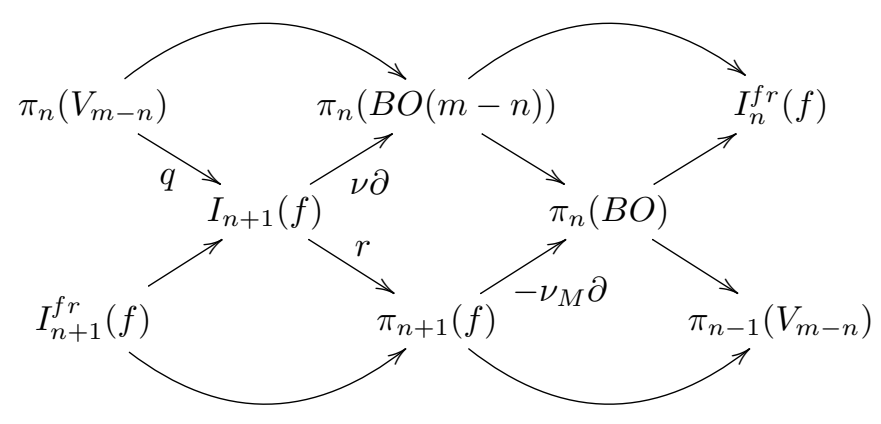

with

$$
I_{n+1}^{f r}(f)=\pi_{n+1}\left(f \times \nu_{M}: M \rightarrow X \times B O\right) .
$$

(ii) The absolute and relative regular homotopy groups are related by commutative braids of exact sequences

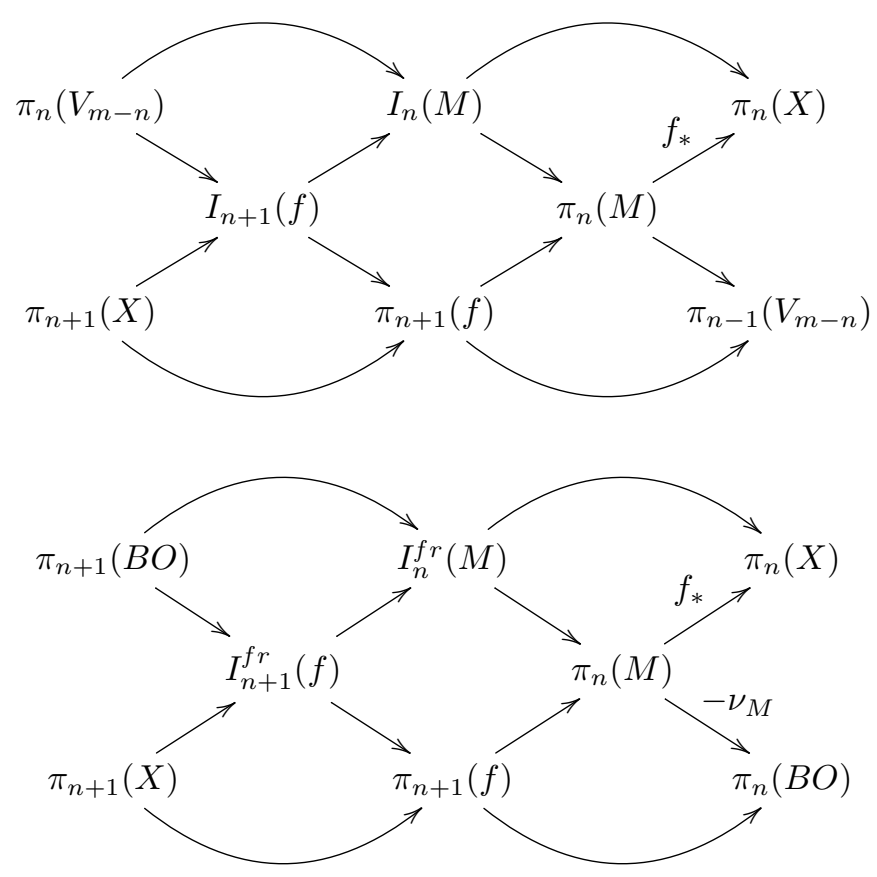

Proof These are just the relative cases of 7.39 , with

$$
V_{m-n}=\bigcup_{k} V_{m-n+k, k}
$$

the stable Stiefel space (7.37). 
Definition 10.14 Let $(f, b): M \rightarrow X$ be an $m$-dimensional normal map, and let $n \leqslant m-2$.

(i) The $b$-framing section of the forgetful map $I_{n+1}(f) \rightarrow \pi_{n+1}(f)$ is the $\mathbb{Z}\left[\pi_{1}(X)\right]$-module morphism

$$
s_{b}: \pi_{n+1}(f) \rightarrow I_{n+1}(f) ; \phi \mapsto s_{b}(\phi)
$$

sends a commutative square

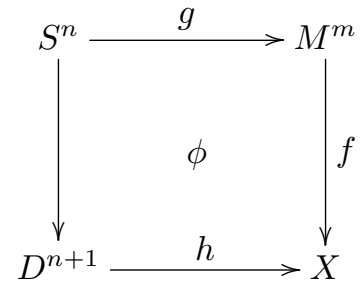

to the regular homotopy class of the framed $n$-immersion

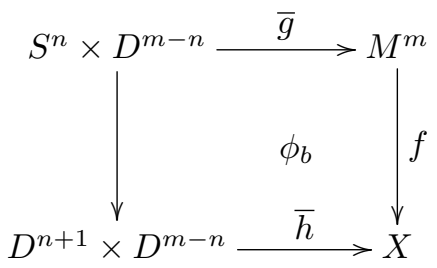

determined by the stable bundle isomorphism

$$
\delta \nu_{h, b}: \tau_{S^{n}} \oplus \nu_{g} \oplus \epsilon^{\infty} \cong g^{*} \tau_{M} \oplus \epsilon^{\infty}
$$

and the Hirsch-Smale Immersion Classification Theorem (7.35).

(ii) The $b$-framing section

$$
s_{b}^{f r}: \pi_{n+1}(f) \rightarrow I_{n+1}^{f r}(f)
$$

of the forgetful map $I_{n+1}^{f r}(f) \rightarrow \pi_{n+1}(f)$ is defined in the same way as $s_{b}$.

Theorem 10.15 (Wall [92, Theorem 1.1])

Let $(f, b): M^{m} \rightarrow X$ be an m-dimensional normal map, and let $n \leqslant m-2$. An element $x \in \pi_{n+1}(f)$ can be killed by an $n$-surgery on $(f, b)$ if and only if the regular homotopy class $s_{b}^{f r}(x) \in I_{n+1}^{f r}(f)$ of framed $n$-immersions in $(f, b)$ contains a representative $(g, h)$ with $g: S^{n} \rightarrow M^{m}$ an embedding.

Proof Such a representative is a $b$-framed $n$-embedding, i.e. an $n$-surgery. 
Proposition 10.16 For $n \leqslant m-2$ a stable bundle map $b: \nu_{M} \rightarrow \eta$ over $f: M^{m} \rightarrow X$ determines a decomposition of the relative regular homotopy group of immersions $I_{n+1}(f)$ as a direct sum system of $\mathbb{Z}\left[\pi_{1}(X)\right]$-modules

$$
\pi_{n+1}(B O, B O(m-n))=\pi_{n}\left(V_{m-n}\right) \stackrel{q}{\longleftarrow} I_{n+1}(f) \stackrel{r}{\rightleftarrows} \underset{s_{b}}{\rightleftarrows} \pi_{n+1}(f)
$$

such that:

(i) for every $(h, g) \in I_{n+1}(f)$

$$
t_{b}(h, g)=\left(\nu_{h}, \nu_{g}\right) \in \pi_{n+1}(B O, B O(m-n))
$$

is the b-framing obstruction (10.8),

(ii) for every $x \in \pi_{n+1}(f)$

$$
s_{b}(x)=(h, g) \in I_{n+1}(f)
$$

is the unique element with image $r(h, g)=x \in \pi_{n+1}(f)$ which admits a b-framing.

Similarly for the relative regular homotopy group of framed $n$-immersions $I_{n+1}^{f r}(f)$, with a direct sum system of $\mathbb{Z}\left[\pi_{1}(X)\right]$-modules

$$
\pi_{n+1}(B O)=\pi_{n+1}(B O(m)) \stackrel{q^{f r}}{\longleftarrow} I_{t_{b}^{f r}}^{f r}(f) \stackrel{r^{f r}}{\longleftarrow} \pi_{n+1}^{f r}(f)
$$

such that

$$
s_{b}: \pi_{n+1}(f) \stackrel{s_{b}^{f r}}{\longrightarrow} I_{n+1}^{f r}(f) \longrightarrow I_{n+1}(f) .
$$

Proof Suppose given a map $g: S^{n} \rightarrow M^{m}$ and a stable isomorphism of bundles over $S^{n}$

$$
c: \tau_{S^{n}} \oplus \epsilon^{m-n+\infty} \cong g^{*} \tau_{M} \oplus \epsilon^{\infty} .
$$

Since $n \leqslant m-2$

$$
\pi_{n}(B O(m))=\pi_{n}(B O)
$$

and it is possible to destabilise $c$ and regard it as a bundle isomorphism

$$
c: \tau_{S^{n}} \oplus \epsilon^{m-n} \cong g^{*} \tau_{M} .
$$

By 7.35 such a pair $(g, c)$ determines a regular homotopy class of framed $n$ immersions $g^{\prime}: S^{n} \times D^{m-n} \leftrightarrow M$ homotopic to $g$. An element $x \in \pi_{n+1}(f)$ is represented by a commutative square 


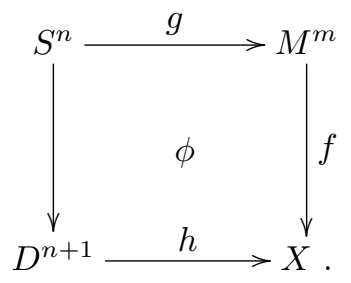

Now $b$ and $h$ determine a stable isomorphism of bundles over $S^{n}$

$$
c: g^{*} \nu_{M} \cong(f g)^{*}(\eta) \cong \epsilon^{\infty}
$$

which may be regarded as a stable isomorphism

$$
c: \tau_{S^{n}} \oplus \epsilon^{m-n+\infty} \cong g^{*} \tau_{M} \oplus \epsilon^{\infty}
$$

(using a standard isomorphism $\tau_{S^{n}} \oplus \epsilon \cong \epsilon^{n+1}$ ). As above, $c$ determines a regular homotopy class of framed $n$-immersions $g^{\prime}: S^{n} \rightarrow M$ in the homotopy class of $g$, such that $f g^{\prime}: S^{n} \rightarrow X$ is equipped with a null-homotopy $h^{\prime}: D^{n+1} \rightarrow X$. Set

$$
s_{b}: \pi_{n+1}(f) \rightarrow I_{n+1}(f) ; \phi \mapsto \phi^{\prime}
$$

with

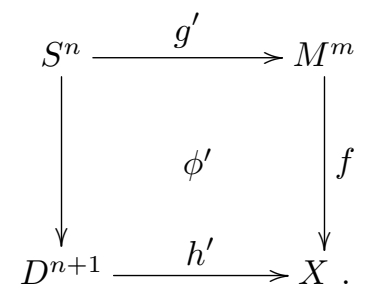

\subsection{Kernels}

The kernel homology and cohomology $\mathbb{Z}[\pi]$-modules of a map $f: M \rightarrow X$ with respect to an oriented cover $(\widetilde{X}, \pi, w)$ of $X$ are defined by

$$
K_{*}(M)=H_{*+1}(\tilde{f}: \widetilde{M} \rightarrow \widetilde{X}), K^{*}(M)=H^{*+1}(\tilde{f}: \widetilde{M} \rightarrow \widetilde{X})
$$

with $\widetilde{M}=f^{*} \widetilde{X}$ the pullback cover of $M$. The kernel homology and cohomology modules of an $m$-dimensional degree 1 normal map $(f, b): M \rightarrow X$ are such that

$$
H_{*}(\widetilde{M})=K_{*}(M) \oplus H_{*}(\widetilde{X}), H^{*}(\widetilde{M})=K^{*}(M) \oplus H^{*}(\widetilde{X})
$$

with Poincaré duality isomorphisms

$$
K^{*}(M) \cong K_{m-*}(M)
$$


The extent to which a map $f: M \rightarrow X$ of connected $C W$ complexes fails to be a homotopy equivalence is measured by the relative homotopy groups $\pi_{*}(f)$ (3.4), with an exact sequence

$$
\ldots \longrightarrow \pi_{n+1}(M) \stackrel{f_{*}}{\longrightarrow} \pi_{n+1}(X) \longrightarrow \pi_{n+1}(f) \longrightarrow \pi_{n}(M) \longrightarrow \ldots .
$$

If $f_{*}: \pi_{1}(M) \rightarrow \pi_{1}(X)$ is an isomorphism then $\pi_{n+1}(f)$ is the set of homotopy classes of commutative squares

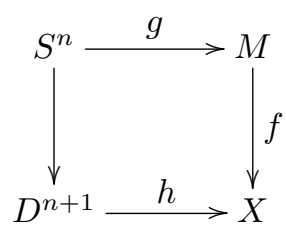

together with a reference path in $M$ from $g(1)$ to the base point $* \in M$. The fundamental group $\pi_{1}(X)$ acts on $\pi_{n+1}(f)$ by changing the reference path, giving $\pi_{n+1}(f)$ the structure of a $\mathbb{Z}\left[\pi_{1}(X)\right]$-module. The Hurewicz map

$$
\pi_{n+1}(f)=\pi_{n+1}(\tilde{f}) \rightarrow H_{n+1}(\tilde{f})
$$

is a $\mathbb{Z}\left[\pi_{1}(X)\right]$-module morphism, with $\tilde{f}: \widetilde{M} \rightarrow \widetilde{X}$ a $\pi_{1}(X)$-equivariant lift of $f$ to the universal cover $\widetilde{X}$ of $X$ and the pullback cover $\widetilde{M}=f^{*} \widetilde{X}$ of $X$.

Definition 10.17 Let $X$ be a space with an oriented cover $(\tilde{X}, \pi, w)$, and let $f: M \rightarrow X$ be a map with a lift to a $\pi$-equivariant map $\tilde{f}: \widetilde{M} \rightarrow \widetilde{X}$ from the pullback cover $\widetilde{M}=f^{*} \widetilde{X}$.

(i) The homology kernel $\mathbb{Z}[\pi]$-modules of $f$ are

$$
K_{n}(M)=H_{n+1}(\tilde{f}: \widetilde{M} \rightarrow \widetilde{X})
$$

with an exact sequence

$$
\ldots \rightarrow H_{n+1}(\tilde{X}) \rightarrow K_{n}(M) \rightarrow H_{n}(\widetilde{M}) \stackrel{\widetilde{f}_{*}}{\longrightarrow} H_{n}(\tilde{X}) \rightarrow K_{n-1}(M) \rightarrow \ldots
$$

(ii) The cohomology kernel $\mathbb{Z}[\pi]$-modules of $f$ are defined by

$$
K^{n}(M)=H^{n+1}(\widetilde{f}: \widetilde{M} \rightarrow \widetilde{X})
$$

with an exact sequence

$$
\ldots \rightarrow K^{n-1}(M) \rightarrow H^{n}(\widetilde{X}) \stackrel{\tilde{f}^{*}}{\longrightarrow} H^{n}(\widetilde{M}) \rightarrow K^{n}(M) \rightarrow H^{n+1}(\widetilde{X}) \rightarrow \ldots
$$

where

$$
H^{*}(\tilde{X})=H_{*}\left(\operatorname{Hom}_{\mathbb{Z}[\pi]}(C(\widetilde{X}), \mathbb{Z}[\pi])\right)
$$


Proposition 10.18 The following conditions on a map $f: M \rightarrow X$ of connected $C W$ complexes are equivalent:

(i) $f$ is a homotopy equivalence,

(ii) the relative homotopy groups are $\pi_{*}(f)=0$,

(iii) $f_{*}: \pi_{1}(M) \rightarrow \pi_{1}(X)$ is an isomorphism of groups, and the kernel $\mathbb{Z}\left[\pi_{1}(X)\right]$ modules are $K_{*}(M)=0$.

Proof (i) $\Longleftrightarrow$ (ii) by the Theorem of J.H.C.Whitehead (3.6).

(ii) $\Longleftrightarrow$ (iii) by the Hurewicz Theorem (3.26).

Proposition 10.19 Let $n \geqslant 2$, and let $f: M \rightarrow X$ be a map of connected $C W$ complexes.

(i) The map $f$ is n-connected if and only if $f_{*}: \pi_{1}(M) \rightarrow \pi_{1}(X)$ is an isomorphism of groups and the kernel $\mathbb{Z}\left[\pi_{1}(X)\right]$-modules are such that

$$
K_{i}(M)=0 \text { for } i<n
$$

(ii) If $f$ is n-connected

$$
\pi_{i+1}(f)=K_{i}(M)=0 \text { for } i<n
$$

and the Hurewicz map is an isomorphism of $\mathbb{Z}\left[\pi_{1}(X)\right]$-modules

$$
\pi_{n+1}(f) \cong K_{n}(M) \text {. }
$$

Moreover, the kernel homology and cohomology $\mathbb{Z}[\pi]$-modules with respect to any oriented cover $(\widetilde{X}, \pi, w)$ of $X$ are such that

$$
K_{i}(M)=K^{i}(M)=0 \text { for } i<n,
$$

and the evaluation map is an isomorphism of $\mathbb{Z}[\pi]$-modules

$$
K^{n}(M) \rightarrow K_{n}(M)^{*} ; y \mapsto(x \mapsto y(x))
$$

Proof By the Universal Coefficient Theorem (3.17) and the Hurewicz Theorem (3.26).

The kernel homology and cohomology of a degree 1 map $f: M \rightarrow X$ of $m$-dimensional geometric Poincaré complexes are shown in 10.21 to be such that there are natural direct sum decompositions

$$
H_{*}(\widetilde{M})=K_{*}(M) \oplus H_{*}(\widetilde{X}), H^{*}(\widetilde{M})=K^{*}(M) \oplus H^{*}(\widetilde{X})
$$

with Poincaré duality isomorphisms 


$$
K^{m-*}(M) \cong K_{*}(M) .
$$

Although there does not exist an $m$-dimensional geometric Poincaré complex with homology $K_{*}(M)$ and cohomology $K^{*}(M)$ there does exist a $\mathbb{Z}\left[\pi_{1}(X)\right]$ module chain complex $C$ (defined in the proof of 10.21 below) such that

$$
H_{*}(C)=K_{*}(M), H^{*}(C)=K^{*}(M), C^{m-*} \simeq C .
$$

For a map of pairs $(M, \partial M) \rightarrow(X, \partial X)$ there are defined relative homology and cohomology kernel $\mathbb{Z}[\pi]$-modules $K_{*}(M, \partial M), K^{*}(M, \partial M)$, to fit into exact sequences of $\mathbb{Z}[\pi]$-modules

$$
\begin{aligned}
& \ldots \rightarrow K_{n}(\partial M) \rightarrow K_{n}(M) \rightarrow K_{n}(M, \partial M) \rightarrow K_{n-1}(\partial M) \rightarrow \ldots, \\
& \ldots \rightarrow K^{n-1}(\partial M) \rightarrow K^{n}(M, \partial M) \rightarrow K^{n}(M) \rightarrow K^{n}(\partial M) \rightarrow \ldots .
\end{aligned}
$$

A symmetric bilinear form on a vector space $V$

$$
\alpha: V \times V \rightarrow \mathbb{R} ;(x, y) \mapsto \alpha(x, y)=\alpha(y, x)
$$

is nonsingular if the adjoint linear map

$$
\alpha=\alpha^{*}: V \rightarrow V^{*}=\operatorname{Hom}_{\mathbb{R}}(V, \mathbb{R}) ; x \mapsto(y \mapsto \alpha(x, y))
$$

is an isomorphism. A morphism of vector spaces with symmetric bilinear forms

$$
f:(V, \alpha) \rightarrow(W, \beta)
$$

is a linear map $f: V \rightarrow W$ such that

$$
\beta(f(x), f(y))=\alpha(x, y) \in \mathbb{R}(x, y \in V),
$$

or equivalently if

$$
f^{*} \beta f=\alpha: V \rightarrow V^{*},
$$

with

$$
f^{*}: W^{*} \rightarrow V^{*} ; g \mapsto(x \mapsto g(f(x))) .
$$

Thus if $(V, \alpha)$ is nonsingular the linear map defined by

$$
f^{!}=\alpha^{-1} f^{*} \beta: W \stackrel{\beta}{\longrightarrow} W^{*} \stackrel{f^{*}}{\longrightarrow} V^{*} \stackrel{\alpha^{-1}}{\longrightarrow} V
$$

splits $f$, with

$$
f^{!} f=1: V \rightarrow V .
$$

The symmetric bilinear form $(K, \lambda)$ defined by

$$
\begin{aligned}
& K=\operatorname{ker}\left(f^{!}: W \rightarrow V\right), \\
& \lambda: K \times K \rightarrow \mathbb{R} ;(x, y) \mapsto \lambda(x, y)=\beta(x, y)
\end{aligned}
$$


is such that there are defined inverse isomorphisms of symmetric bilinear forms

$$
(V, \alpha) \oplus(K, \lambda) \underset{f^{!} \oplus h}{\stackrel{f \oplus g}{\rightleftarrows}}(W, \beta)
$$

with

$$
g=\text { inclusion }: K \rightarrow W, h=1-f f^{!}: W \rightarrow K .
$$

The fact that isometries of nonsingular symmetric bilinear forms split off in this manner has a direct generalisation to the splitting of Poincaré duality structures for a degree 1 map, making use of the $\mathbb{Z}[\pi]$-module version of the Umkehr chain map (4.14) :

Definition 10.20 Let $f: M \rightarrow X$ be a degree 1 map of $m$-dimensional geometric Poincaré complexes, and let $\widetilde{f}: \widetilde{M} \rightarrow \widetilde{X}$ be a $\pi$-equivariant lift of $f$, for an oriented cover $(\widetilde{X}, \pi, w)$ of $X$ with pullback cover $\widetilde{M}=f^{*} \widetilde{X}$. The Umkehr $\mathbb{Z}[\pi]$-module chain map is given by

$$
\begin{aligned}
f^{!}: C(\widetilde{X}) \stackrel{([X] \cap-)^{-1}}{\longrightarrow} C(\widetilde{X})^{m-*} \stackrel{\widetilde{f}^{*}}{\longrightarrow} \\
C(\widetilde{M})^{m-*} \stackrel{[M] \cap-}{\longrightarrow} C(\widetilde{M}),
\end{aligned}
$$

using a chain homotopy inverse to the Poincaré duality chain equivalence of $X$.

The Umkehr of 10.20 is an evident generalisation of the Umkehr chain map in 4.67 .

Proposition 10.21 For a degree 1 map $f: M \rightarrow X$ of m-dimensional geometric Poincaré complexes the Poincaré duality isomorphisms of $\widetilde{M}$ split as

$$
\begin{aligned}
& {[M] \cap-=([M] \cap-) \oplus([X] \cap-):} \\
& H^{n}(\widetilde{M})=K^{n}(M) \oplus H^{n}(\widetilde{X}) \rightarrow H_{m-n}(\widetilde{M})=K_{m-n}(M) \oplus H_{m-n}(\widetilde{X}) .
\end{aligned}
$$

In particular, the homology and cohomology kernel modules are related by Poincaré duality isomorphisms

$$
[M] \cap-: K^{n}(M) \rightarrow K_{m-n}(M) .
$$

Proof It will be shown that $\tilde{f}: C(\widetilde{M}) \rightarrow C(\widetilde{X})$ is a chain homotopy split surjection, with a particular splitting $f^{!}: C(\widetilde{X}) \rightarrow C(\widetilde{M})$ such that the algebraic mapping cone $\mathscr{C}\left(f^{!}\right)(3.13)$ has $m$-dimensional Poincaré duality, and such that the homology and cohomology are precisely $K_{*}(M)$ and $K^{*}(M)$. By the naturality of the cap product and $f_{*}[M]=[X]$ there is defined a chain homotopy commutative diagram 


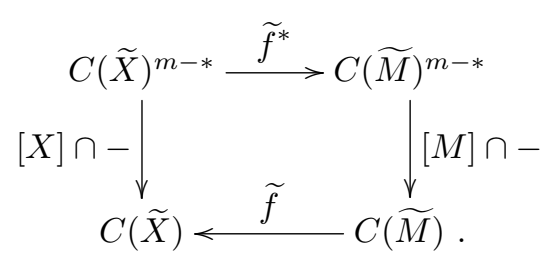

Thus there exists a chain homotopy

$$
\widetilde{f} f^{!} \simeq 1: C(\widetilde{X}) \rightarrow C(\widetilde{X})
$$

and there is defined a chain homotopy direct sum system

$$
\mathscr{C}\left(f^{!}\right) \underset{g}{\rightleftarrows} C(\widetilde{M}) \underset{f^{!}}{\rightleftarrows} C(\widetilde{X})
$$

with $e: C(\widetilde{M}) \rightarrow \mathscr{C}\left(f^{!}\right)$the inclusion (which is a chain homotopy split surjection), and $g: \mathscr{C}\left(f^{!}\right) \rightarrow C(\widetilde{M})$ the chain homotopy split injection determined up to chain homotopy by $e, \widetilde{f}, f$ ! . The system specifies identifications

$$
K_{*}(M)=H_{*}\left(\mathscr{C}\left(f^{!}\right)\right), K^{*}(M)=H^{*}\left(\mathscr{C}\left(f^{!}\right)\right) .
$$

The cap product chain equivalence

$$
[M] \cap-: C(\widetilde{M})^{m-*} \rightarrow C(\widetilde{M})
$$

defines a chain equivalence of direct sum systems

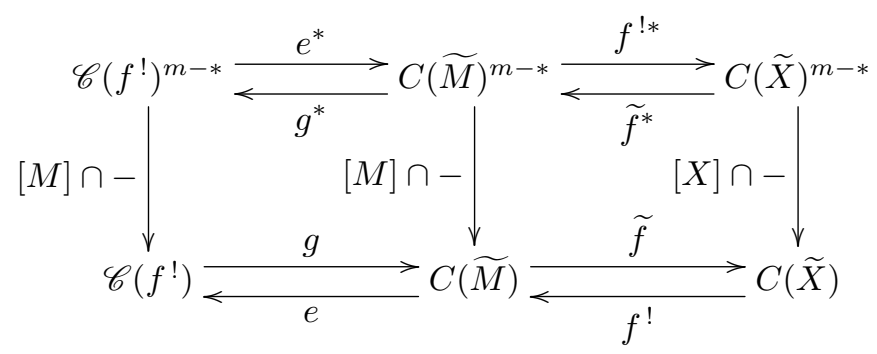

since there exist chain homotopies

$$
\begin{aligned}
& \tilde{f}([M] \cap-) e^{*} \simeq 0: \mathscr{C}\left(f^{!}\right)^{m-*} \rightarrow C(\widetilde{X}), \\
& e([M] \cap-) \tilde{f}^{*} \simeq 0: C(\widetilde{X})^{m-*} \rightarrow \mathscr{C}\left(f^{!}\right),
\end{aligned}
$$

using the existence of a chain homotopy

$$
[M] \cap-\simeq T([M] \cap-): C(\widetilde{M})^{m-*} \rightarrow C(\widetilde{M})
$$

given by the chain homotopy symmetry of the diagonal chain approximation (3.18). 
Definition 10.22 The homology intersection pairing on the kernel $\mathbb{Z}[\pi]$ modules of an $m$-dimensional oriented map $f: M \rightarrow X$ with respect to an oriented cover $(\widetilde{X}, \pi, w)$ of $X$ are the sesquilinear pairings

$$
\lambda: K_{n}(M) \times K_{m-n}(M) \rightarrow \mathbb{Z}[\pi]
$$

inherited from the intersection pairing of 4.66

$$
\lambda: H_{n}(\widetilde{M}) \times H_{m-n}(\widetilde{M}) \rightarrow \mathbb{Z}[\pi]
$$

using the natural maps $K_{*}(M) \rightarrow H_{*}(\widetilde{M})$.

The intersection pairings determine the effect of surgery on the homology kernel of a normal map :

Proposition 10.23 Let $(f, b): M^{m} \rightarrow X$ be an m-dimensional normal map, and let

$$
\left((F, B) ;(f, b),\left(f^{\prime}, b^{\prime}\right)\right):\left(W^{m+1} ; M^{m}, M^{\prime m}\right) \rightarrow X \times(I ;\{0\},\{1\})
$$

be the trace of an n-surgery on $(f, b)$ killing an element of $x \in \pi_{n+1}(f)$, and let

$$
M_{0}=\operatorname{cl} .\left(M \backslash\left(S^{n} \times D^{m-n}\right)\right) .
$$

(i) The kernel $\mathbb{Z}[\pi]$-modules are such that

$$
\begin{aligned}
& K_{i}(W, M)= \begin{cases}\mathbb{Z}[\pi] & \text { if } i=n+1 \\
0 & \text { if } i \neq n+1\end{cases} \\
& K_{i}\left(W, M^{\prime}\right)= \begin{cases}\mathbb{Z}[\pi] & \text { if } i=m-n \\
0 & \text { if } i \neq m-n\end{cases}
\end{aligned}
$$

with a commutative braid of exact sequences of $\mathbb{Z}[\pi]$-modules

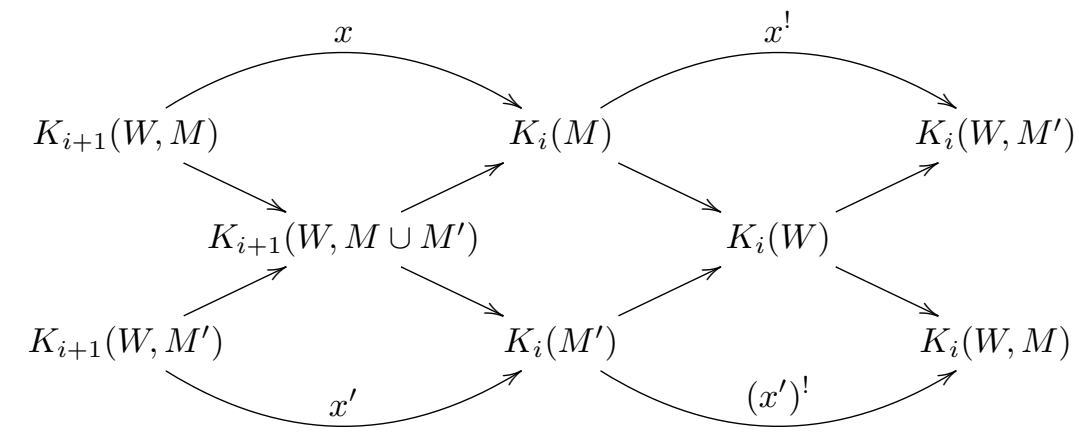

such that

$$
\begin{aligned}
& x: K_{n+1}(W, M)=\mathbb{Z}[\pi] \rightarrow K_{n}(M) ; 1 \mapsto x, \\
& x^{!}: K_{m-n}(M) \rightarrow K_{m-n}(W) \rightarrow K_{m-n}\left(W, M^{\prime}\right)=\mathbb{Z}[\pi] ; y \mapsto \lambda(x, y) .
\end{aligned}
$$

(ii) If $X$ is an $m$-dimensional geometric Poincaré complex and $(f, b)$ is a degree 1 normal map then

$$
K_{*+1}\left(W, M \cup M^{\prime}\right)=K_{*}\left(M_{0}\right)
$$


Proof (i) Working as in the proof of 4.68 it is possible to express $\mathscr{C}\left(f^{\prime !}\right)$ up to chain equivalence in terms of $\mathscr{C}\left(f^{!}\right)$as

$$
\mathscr{C}\left(f^{\prime !}\right)_{i}= \begin{cases}\mathscr{C}\left(f^{!}\right)_{n+1} \oplus \mathbb{Z}[\pi] & \text { if } i=n+1 \neq m-n-1 \\ \mathscr{C}\left(f^{!}\right)_{m-n-1} \oplus \mathbb{Z}[\pi] & \text { if } i=m-n-1 \neq n+1 \\ \mathscr{C}\left(f^{!}\right)_{n+1} \oplus \mathbb{Z}[\pi] \oplus \mathbb{Z}[\pi] & \text { if } i=m-n-1=n+1 \\ \mathscr{C}\left(f^{!}\right)_{i} & \text { otherwise }\end{cases}
$$

(ii) By the Poincaré disc theorem (9.14) it may be assumed that $X=X_{0} \cup D^{m}$ for an $m$-dimensional geometric Poincaré pair $\left(X_{0}, S^{m-1}\right)$, and that the trace degree 1 normal map can be expressed as a union of degree 1 normal maps

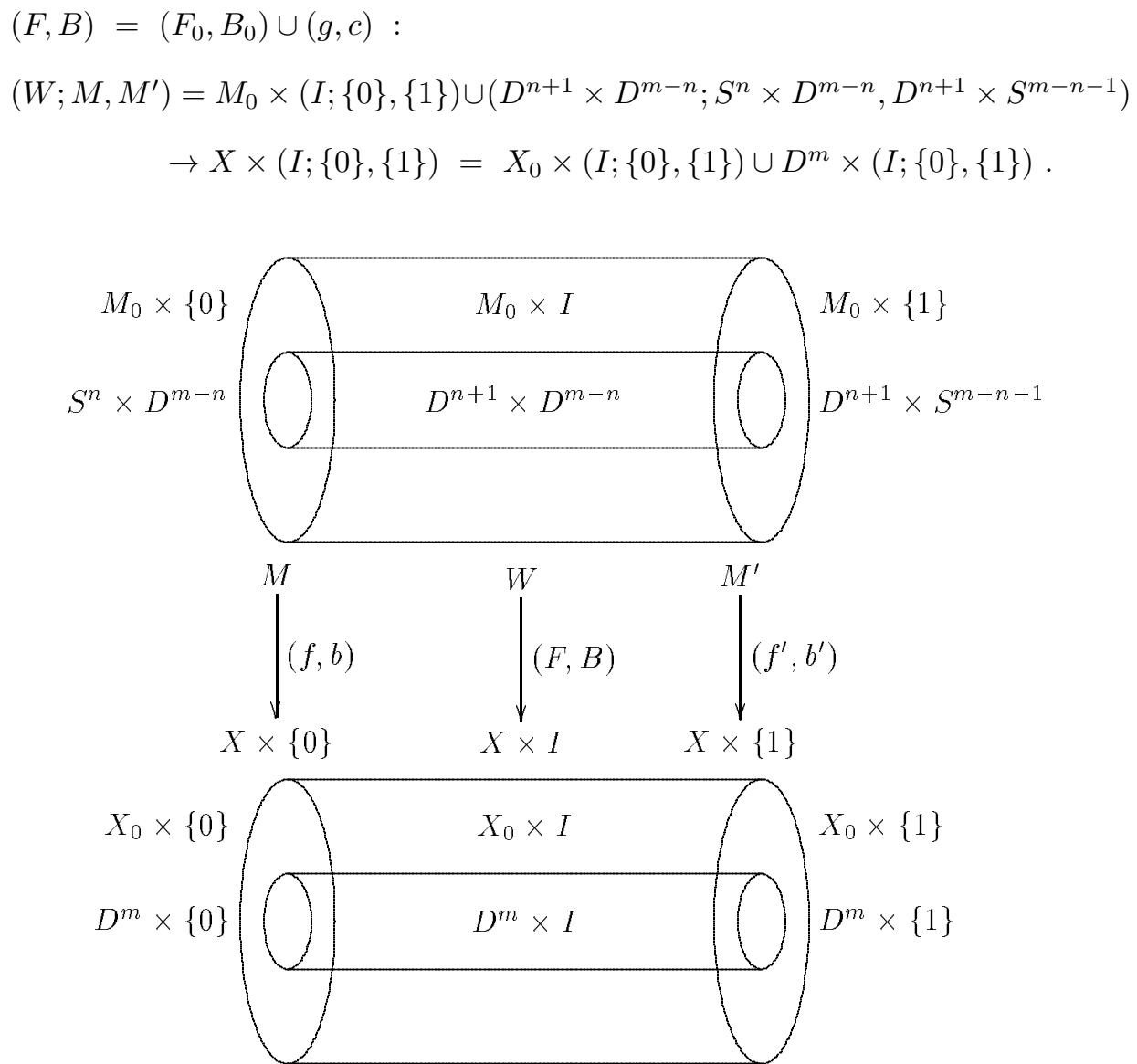

The isomorphism $K_{*}\left(M_{0}\right) \cong K_{*+1}\left(W, M \cup M^{\prime}\right)$ follows from the commutative braid of exact sequences 


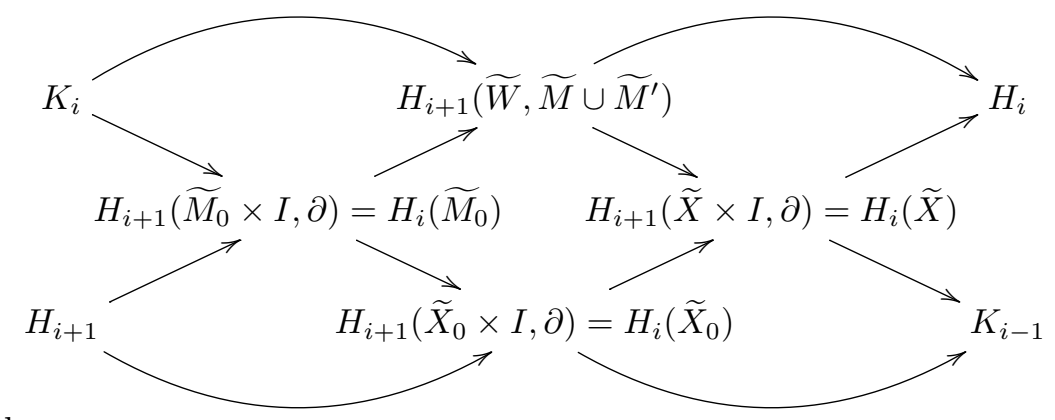

where

$$
\begin{aligned}
& H_{i}=\mathbb{Z}[\pi] \otimes_{\mathbb{Z}} H_{i}\left(D^{m}, S^{m-1}\right)=\mathbb{Z}[\pi] \otimes_{\mathbb{Z}} H_{i+1}\left(D^{m+1}, S^{m}\right) \\
&= \begin{cases}\mathbb{Z}[\pi] & \text { if } i=m \\
0 & \text { if } i \neq m,\end{cases} \\
& K_{i}=K_{i}\left(M_{0}\right)=K_{i+1}\left(W, M \cup M^{\prime}\right) .
\end{aligned}
$$

\subsection{Surgery below the middle dimension}

An element $x \in \pi_{n}(M)$ can be killed by an $n$-surgery on an $m$-dimensional manifold $M^{m}$ if and only if it can be represented by an embedding $g: S^{n} \hookrightarrow M^{m}$ with trivial normal bundle $\nu_{g}: S^{n} \rightarrow B O(m-n)$. Below the middle dimension, this condition is purely homotopy-theoretic:

Proposition 10.24 (Repetition of 5.64) If $2 n+1 \leqslant m$ an element $x \in \pi_{n}(M)$ can be killed by $n$-surgery on $M^{m}$ if and only if $\left(\nu_{M}\right)_{*}(x)=0 \in \pi_{n}(B O)$.

Proof (A more detailed version of the proof of By the Whitney Embedding Theorem (7.2) it is possible to represent $x$ by an embedding $g: S^{n} \subset M^{m}$. Let $\nu_{M}=\nu_{e}: M \rightarrow B O(k)$ be the normal bundle of an embedding $e: M \hookrightarrow S^{m+k}(k$ large), and let $\eta: X \rightarrow B O(k)$ be likewise a $k$-plane bundle, so that $b: \nu_{M} \rightarrow \eta$ is a map of $k$-plane bundles. The normal bundle of the composite embedding

$$
g^{\prime}=e g: S^{n} \hookrightarrow M^{m} \hookrightarrow S^{m+k}
$$

is trivial, with 10.9 giving a trivialisation

$$
\nu_{g^{\prime}}=\nu_{g} \oplus g^{*} \nu_{M} \simeq\{*\}: S^{n} \rightarrow B O(m-n+k) .
$$

The normal bundle $\nu_{g}: S^{n} \rightarrow B O(m-n)$ is a stable inverse of the pullback $g^{*} \nu_{M}: S^{n} \rightarrow B O$ of the stable normal bundle $\nu_{M}: M \rightarrow B O$, that is

$$
\nu_{g} \oplus g^{*} \nu_{M}=\epsilon^{\infty}: S^{n} \rightarrow B O .
$$

Thus

$$
\nu_{g}=-g^{*} \nu_{M}=-\left(\nu_{M}\right)_{*}(x) \in \pi_{n}(B O(m-n))=\pi_{n}(B O),
$$

and $\nu_{g}=0$ if and only if $\left(\nu_{M}\right)_{*}(x)=0$. 
Surgery is always possible below the middle dimension of a normal map, and the homology effect is given by :

Proposition 10.25 Let $(f, b): M^{m} \rightarrow X$ be an $m$-dimensional normal map. (i) If $2 n+1 \leqslant m$ every element $x \in \pi_{n+1}(f)$ can be killed by an $n$-surgery on $(f, b)$. Let

$$
\left((F, B) ;(f, b),\left(f^{\prime}, b^{\prime}\right)\right):\left(W ; M, M^{\prime}\right) \rightarrow X \times(I ;\{0\},\{1\})
$$

be the trace of an n-surgery on $(f, b)$ killing $x$, and let $x^{\prime} \in \pi_{m-n}\left(f^{\prime}\right)$ be the relative homotopy class killed by the dual $(m-n-1)$-surgery. The kernel homology $\mathbb{Z}\left[\pi_{1}(X)\right]$-modules are such that

$$
\begin{aligned}
& K_{i}(M)=K_{i}(W) \text { for } i \neq n, n+1, \\
& K_{i}\left(M^{\prime}\right)=K_{i}(W) \text { for } i \neq m-n-1, m-n
\end{aligned}
$$

with exact sequences

$$
\begin{aligned}
0 \longrightarrow K_{n+1}(M) \longrightarrow & K_{n+1}(W) \longrightarrow \mathbb{Z}\left[\pi_{1}(X)\right] \\
& \stackrel{x}{\longrightarrow} K_{n}(M) \longrightarrow K_{n}(W) \longrightarrow 0 \\
0 \longrightarrow K_{m-n}\left(M^{\prime}\right) \longrightarrow & \longrightarrow K_{m-n}(W) \longrightarrow \mathbb{Z}\left[\pi_{1}(X)\right] \\
& \stackrel{x^{\prime}}{\longrightarrow} K_{m-n-1}\left(M^{\prime}\right) \longrightarrow K_{m-n-1}(W) \longrightarrow 0 .
\end{aligned}
$$

(ii) For $2 n+2<m$ there are exact sequences

$$
\begin{aligned}
0 \longrightarrow K_{n+1}(M) \longrightarrow & K_{n+1}\left(M^{\prime}\right) \stackrel{x^{\prime} !}{\longrightarrow} \mathbb{Z}\left[\pi_{1}(X)\right] \\
& \stackrel{x}{\longrightarrow} K_{n}(M) \longrightarrow K_{n}\left(M^{\prime}\right) \longrightarrow 0 \\
0 \longrightarrow K_{m-n}\left(M^{\prime}\right) \stackrel{x^{!}}{\longrightarrow} \mathbb{Z}\left[\pi_{1}(X)\right] & K_{m-n}(M) \stackrel{x^{\prime}}{\longrightarrow} K_{m-n-1}\left(M^{\prime}\right) \longrightarrow K_{m-n-1}(M) \longrightarrow 0 .
\end{aligned}
$$

(iii) For $m=2 n+2$ there are exact sequences

$$
\begin{aligned}
0 \longrightarrow K_{n+1}(M) \longrightarrow \operatorname{coker}\left(x^{\prime}: \mathbb{Z}\left[\pi_{1}(X)\right] \rightarrow K_{n+1}\left(M^{\prime}\right)\right) \stackrel{x^{\prime !}}{\longrightarrow} \mathbb{Z}\left[\pi_{1}(X)\right] \\
\stackrel{x}{\longrightarrow} K_{n}(M) \longrightarrow K_{n}\left(M^{\prime}\right) \longrightarrow 0 \\
0 \longrightarrow K_{n+2}\left(M^{\prime}\right) \longrightarrow K_{n+2}(M) \stackrel{x^{!}}{\longrightarrow} \mathbb{Z}\left[\pi_{1}(X)\right] \\
\stackrel{x^{\prime}}{\longrightarrow} \operatorname{ker}\left(x^{\prime !}: K_{n+1}\left(M^{\prime}\right) \rightarrow \mathbb{Z}\left[\pi_{1}(X)\right]\right) \longrightarrow K_{n+1}(M) \longrightarrow 0 .
\end{aligned}
$$

(iv) For $m=2 n+1$ there is a commutative braid of exact sequences 


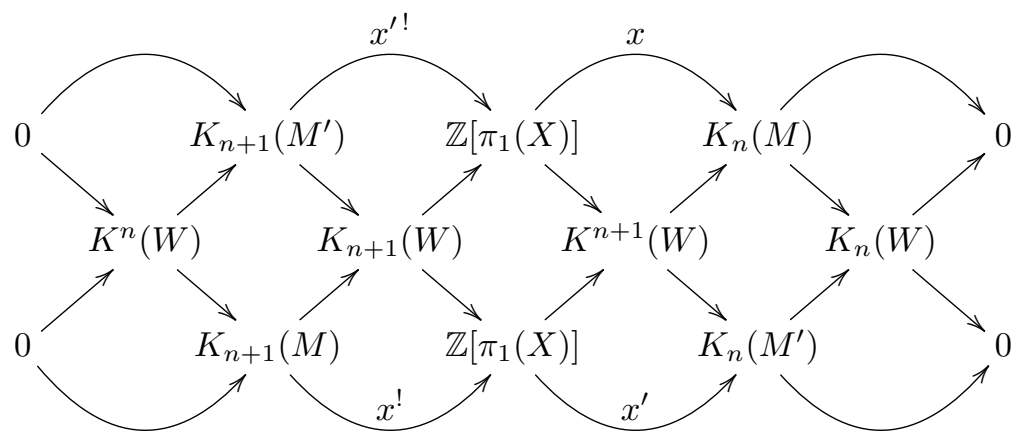

Proof For $2 n+1 \leqslant m$ the relative regular homotopy groups of Section 10.2 are such that

$$
I_{n+1}(f)=\pi_{n+1}(f), I_{n+1}^{f r}(f)=s_{b}^{f r}\left(\pi_{n+1}(f)\right) \oplus \pi_{n+1}(B O) .
$$

By the Whitney Immersion and Embedding Theorem $(7.1,7.2) x \in \pi_{n+1}(f)$ can represented by an $n$-immersion $\phi=(h, g)$ with $g: S^{n} \leftrightarrow M^{m}$ an embedding. The $b$-framing obstruction $\nu_{b}(\phi)=\left(\nu_{h}, \nu_{g}\right)$ of 10.8 takes its value in $\pi_{n+1}(B O, B O(m-n))=0$, and Theorem 10.15 applies to give that $x$ can be killed by surgery. The homology kernels $K_{*}\left(M^{\prime}\right), K_{*}(W)$ are given by Proposition 10.23 .

\subsection{Finite generation}

The main results of this section are:

(i) if $m=2 n$ or $2 n+1$ every $m$-dimensional normal map $(f, b): M \rightarrow X$ is normal bordant to an $n$-connected normal map,

(ii) for an $n$-connected map of finite $C W$ complexes $f: M \rightarrow X$ the first nonvanishing relative homotopy group $\pi_{n+1}(f)=K_{n}(M)$ is a finitely generated (f.g.) $\mathbb{Z}\left[\pi_{1}(X)\right]$-module,

(iii) for an $n$-connected degree 1 map $f: M \rightarrow X$ of $2 n$-dimensional geometric Poincaré complexes $K_{n}(M)$ is a stably f.g. free $\mathbb{Z}\left[\pi_{1}(X)\right]$-module.

These results make use of the duality properties of the homology and cohomology of finite chain complexes of f.g. projective $A$-modules for a ring with involution $A$. In the application $A=\mathbb{Z}\left[\pi_{1}(X)\right]$.

Lemma 10.26 Let $A$ be a ring with involution, and let $C$ be a finite f.g. projective $A$-module chain complex.

(i) If $H_{i}(C)=0$ for $i<n$ then $H_{n}(C)$ is a f.g. A-module, and the evaluation map is an A-module isomorphism

$$
H^{n}(C) \rightarrow H_{n}(C)^{*} ; f \mapsto(x \mapsto f(x))
$$


(ii) If $H_{i}(C)=0$ for $i<n$ and $H^{j}(C)=0$ for $j>n$ then $H_{n}(C)$ and $H^{n}(C)$ are dual f.g. projective A-modules, with isomorphisms

$$
\begin{aligned}
& H_{n}(C) \oplus \sum_{i \in \mathbb{Z}} C_{n+2 i+1} \cong \sum_{j \in \mathbb{Z}} C_{n+2 j}, \\
& H^{n}(C) \oplus \sum_{i \in \mathbb{Z}} C^{n+2 i+1} \cong \sum_{j \in \mathbb{Z}} C^{n+2 j} .
\end{aligned}
$$

In particular, if the A-modules $C_{r}(r \geqslant 0)$ are f.g. free then $H_{n}(C)$ and $H^{n}(C)$ are dual stably f.g. free A-modules.

Proof (i) By standard homological algebra there exist $A$-module morphisms $\Gamma: C_{i} \rightarrow C_{i+1}$ for $i<n$ such that

$$
d \Gamma+\Gamma d=1: C_{i} \rightarrow C_{i} \text { for } i<n
$$

The $A$-module morphisms

$$
\begin{aligned}
& g: \operatorname{ker}\left(d: C_{n} \rightarrow C_{n-1}\right) \oplus C_{n-1} \oplus C_{n-3} \oplus \ldots \rightarrow C_{n} \oplus C_{n-2} \oplus C_{n-4} \oplus \ldots, \\
& h: C_{n} \oplus C_{n-2} \oplus C_{n-4} \oplus \ldots \rightarrow \operatorname{ker}\left(d: C_{n} \rightarrow C_{n-1}\right) \oplus C_{n-1} \oplus C_{n-3} \oplus \ldots
\end{aligned}
$$

defined by

$$
\begin{aligned}
& g\left(x_{n}, x_{n-1}, x_{n-3}, \ldots\right)= \\
& \left(x_{n}+\Gamma\left(x_{n-1}\right), d\left(x_{n-1}\right)+\Gamma\left(x_{n-3}\right), d\left(x_{n-3}\right)+\Gamma\left(x_{n-5}\right), \ldots\right), \\
& h\left(y_{n}, y_{n-2}, y_{n-4}, \ldots\right)= \\
& \left((1-\Gamma d)\left(y_{n}\right), d\left(y_{n}\right)+\Gamma\left(y_{n-2}\right), d\left(y_{n-2}\right)+\Gamma\left(y_{n-4}\right), \ldots\right)
\end{aligned}
$$

are inverse isomorphisms

$$
\operatorname{ker}\left(d: C_{n} \rightarrow C_{n-1}\right) \oplus C_{n-1} \oplus C_{n-3} \oplus \ldots \cong C_{n} \oplus C_{n-2} \oplus C_{n-4} \oplus \ldots .
$$

Thus $\operatorname{ker}\left(d: C_{n} \rightarrow C_{n-1}\right)$ is a f.g. projective $A$-module, and the $A$-module

$$
H_{n}(C)=\operatorname{ker}\left(d: C_{n} \rightarrow C_{n-1}\right) / \operatorname{im}\left(d: C_{n+1} \rightarrow C_{n}\right)
$$

is finitely generated. The f.g. projective $A$-module chain complex $C^{\prime}$ defined by

$$
\begin{aligned}
& C_{r}^{\prime}=0 \text { for } r<n, \\
& d^{\prime}=\left\{\begin{array}{l}
d+\Gamma: C_{n+1}^{\prime}=\sum_{i \geqslant 0} C_{n+1-2 i} \rightarrow C_{n}^{\prime}=\sum_{j \geqslant 0} C_{n-2 j} \\
d \oplus 0: C_{n+2}^{\prime}=C_{n+2} \rightarrow C_{n+1}^{\prime}=C_{n+1} \oplus \sum_{i \geqslant 1} C_{n+1-2 i} \\
d: C_{r}^{\prime}=C_{r} \rightarrow C_{r-1}^{\prime}=C_{r-1} \text { for } r \geqslant n+2
\end{array}\right.
\end{aligned}
$$

is chain equivalent to $C$, so that

$$
\begin{aligned}
& H_{n}(C)=H_{n}\left(C^{\prime}\right)=\operatorname{coker}\left(d^{\prime}: C_{n+1}^{\prime} \rightarrow C_{n}^{\prime}\right), \\
& H^{n}(C)=H^{n}\left(C^{\prime}\right)=\operatorname{ker}\left(d^{\prime *}: C^{\prime n} \rightarrow C^{\prime n+1}\right) .
\end{aligned}
$$

The evaluation map $H^{n}\left(C^{\prime}\right) \rightarrow H_{n}\left(C^{\prime}\right)^{*}$ is an isomorphism because $C_{n-1}^{\prime}=0$. Here is the argument in detail. Since $C_{n}^{\prime}$ is projective, if an element $f \in C^{\prime n}$ is 
such that $f(x)=0 \in A$ for all $x \in C_{n}^{\prime}$ then $f=0$. This shows that the evaluation map is injective. For surjectivity, given $g \in H_{n}\left(C^{\prime}\right)^{*}$ use the projectivity of $C_{n}^{\prime}$ to lift $g: H_{n}\left(C^{\prime}\right) \rightarrow A$ to an $A$-module morphism $h: C_{n}^{\prime} \rightarrow A$ such that $h d^{\prime}(y)=0 \in A$ for all $y \in C_{n+1}^{\prime}$, so that $h \in \operatorname{ker}\left(d^{\prime *}\right)=H^{n}\left(C^{\prime}\right)$ has image $g$ under the evaluation map.

(ii) In this case there also exist $A$-module morphisms $\Gamma: C_{j} \rightarrow C_{j+1}$ for $j>n$ with

$$
d \Gamma+\Gamma d=1: C_{j} \rightarrow C_{j+1} \text { for } j>n .
$$

This uses the involution on $A$, specifically the property of the f.g. projective $A$-modules $C_{j}$ that the natural double duality $A$-module morphisms are isomorphisms

$$
e_{C_{j}}: C_{j} \rightarrow C_{j}^{* *} ; x \mapsto(f \mapsto \overline{f(x)}) .
$$

The $A$-module morphisms

$$
\begin{aligned}
& H_{n}(C) \oplus \sum_{i \in \mathbb{Z}} C_{n+2 i+1} \rightarrow \sum_{j \in \mathbb{Z}} C_{n+2 j} ; \\
& \left(\left[x_{n}\right], \sum_{i} x_{n+2 i+1}\right) \mapsto(1-d \Gamma)\left(x_{n}\right)+\sum_{i}\left(d\left(x_{n+2 i+1}\right)+\Gamma\left(x_{n+2 i+1}\right)\right), \\
& \sum_{j \in \mathbb{Z}} C_{n+2 j} \rightarrow H_{n}(C) \oplus \sum_{i \in \mathbb{Z}} C_{n+2 i+1} ; \\
& \sum_{j} y_{n+2 j} \mapsto\left(\left[(1-\Gamma d)\left(y_{n}\right)\right], \sum_{j}\left(d\left(y_{n+2 j}\right)+\Gamma(1-\Gamma d)\left(y_{n+2 j}\right)\right)\right)
\end{aligned}
$$

define inverse isomorphisms

$$
H_{n}(C) \oplus \sum_{i \in \mathbb{Z}} C_{n+2 i+1} \cong \sum_{j \in \mathbb{Z}} C_{n+2 j} .
$$

The dual $A$-module morphisms define inverse isomorphisms

$$
H^{n}(C) \oplus \sum_{i \in \mathbb{Z}} C^{n+2 i+1} \cong \sum_{j \in \mathbb{Z}} C^{n+2 j},
$$

identifying $H^{n}(C)=H_{n}(C)^{*}$ by (i).

Remark 10.27 If $C$ is a finite based f.g. free $A$-module chain complex such that $H_{*}(C)=0$ then $H^{*}(C)=0$ and the isomorphism used in the proof of 10.26 (with $n=0$ )

$$
d+\Gamma: \sum_{i} C_{2 i+1} \rightarrow \sum_{j} C_{2 j}
$$

is the one used to define the Whitehead torsion of $C$ in 8.10 .

The following result gives the finite generation of the relative homotopy group in the Hurewicz dimension:

Proposition 10.28 Let $n \geqslant 2$, and let $f: M \rightarrow X$ be an n-connected map of $C W$ complexes, so that $f_{*}: \pi_{1}(M) \rightarrow \pi_{1}(X)$ is an isomorphism and $\pi_{i+1}(f)=$ 
$K_{i}(M)=0$ for $i \leqslant n-1$.

(i) If $M$ has a finite $n$-skeleton and $X$ has a finite $(n+1)$-skeleton then $\pi_{n+1}(f)=$ $K_{n}(M)$ is a f.g. $\mathbb{Z}\left[\pi_{1}(X)\right]$-module.

(ii) If $M$ and $X$ are finite $C W$ complexes and also $K^{i}(M)=0$ for $i \geqslant n+1$ then $K_{n}(M)$ is a stably f.g. free $\mathbb{Z}\left[\pi_{1}(X)\right]$-module, with the evaluation map a $\mathbb{Z}\left[\pi_{1}(X)\right]$-module isomorphism

$$
K^{n}(M) \rightarrow K_{n}(M)^{*} ; y \mapsto(x \mapsto\langle y, x\rangle) .
$$

Proof Let $\tilde{f}: \widetilde{M} \rightarrow \widetilde{X}$ be a $\pi_{1}(X)$-equivariant lift of $f$ to the universal covers $\widetilde{M}, \widetilde{X}$ of $M, X$. By the Hurewicz Theorem (3.26) there are identifications of $\mathbb{Z}\left[\pi_{1}(X)\right]$-modules

$$
\begin{array}{rlrl}
\pi_{i}(f)=\pi_{i}(\widetilde{f})=H_{i}(\widetilde{f}) & =K_{i-1}(M) & \text { for } i \leqslant n+1 \\
& =0 & & \text { for } i \leqslant n .
\end{array}
$$

The relative homology modules $H_{*}(\widetilde{f})=K_{*-1}(M)$ of the induced $\mathbb{Z}\left[\pi_{1}(X)\right]$ module chain map $\widetilde{f}: C(\widetilde{M}) \rightarrow C(\widetilde{X})$ of the cellular chain complexes

$$
H_{*}(\widetilde{f})=H_{*}(C(\widetilde{f}))
$$

are the homology of the algebraic mapping cone $\mathscr{C}(\widetilde{f})(3.22)$. The chain complex $C=\mathscr{C}(\widetilde{f})$ is such that

(a) $C_{i}=0$ for $i<0$,

(b) $C_{i}$ is a f.g. free $\mathbb{Z}\left[\pi_{1}(X)\right]$-module for $i \leqslant n+1$,

(c) $H_{i}(C)=0$ for $i \leqslant n$,

and in the case (ii) also

(d) $C_{i}=0$ for all $i>m$, for some $m \geqslant 0$,

(e) $C_{i}$ is a f.g. free $\mathbb{Z}\left[\pi_{1}(X)\right]$-module for $i \geqslant n+2$,

(f) $H^{i}(C)=0$ for $i \geqslant n+2$.

By 10.26 the $\mathbb{Z}\left[\pi_{1}(X)\right]$-module

$$
\begin{aligned}
K_{n}(M) & =\pi_{n+1}(f)=H_{n+1}(\tilde{f}) \\
& =\operatorname{ker}\left(d: C_{n+1} \rightarrow C_{n}\right) / \operatorname{im}\left(d: C_{n+2} \rightarrow C_{n+1}\right)
\end{aligned}
$$

is f.g. in case (i) and stably f.g. free in case (ii).

Corollary 10.29 Let $f: M \rightarrow X$ be a degree 1 map of m-dimensional geometric Poincaré complexes, and let $K_{*}(M), K^{*}(M)$ be the homology and cohomology kernel $\mathbb{Z}[\pi]$-modules with respect to an oriented cover $(\tilde{X}, \pi, w)$ of $X$. If $f$ is $n$-connected then: 
(i) $K_{r}(M)=K^{r}(M)=0$ for $r<n$,

(ii) $K_{n}(M)$ is a f.g. $\mathbb{Z}[\pi]$-module, and the evaluation map is a $\mathbb{Z}[\pi]$-module isomorphism $K^{n}(M) \cong K_{n}(M)^{*}$,

(iii) if $m=2 n$ then $K_{n}(M)$ is a stably f.g. free $\mathbb{Z}[\pi]$-module,

(iv) if $m=2 n-2$ or $2 n-1$ then $K_{*}(M)=0$,

(v) if $m=2 n-2$ or $2 n-1, n \geqslant 2$, and $(\widetilde{X}, \pi, w)$ is the universal oriented cover then $f$ is a homotopy equivalence.

Proof (i) By the Universal Coefficient Theorem (3.17).

(ii) + (iii) Immediate from 10.28 (i) + (ii).

(iv) $K_{i}(M)=0$ for $i \leqslant n-1$ by the Hurewicz Theorem, so that by 10.28 (ii)

$$
K^{i}(M)=K_{i}(M)^{*}=0 \text { for } i \leqslant n-1 .
$$

The kernel Poincaré duality established in 10.21 gives

$$
K_{j}(M)=K^{m-j}(M)=0 \text { for } j \geqslant n
$$

since $m-j \leqslant n-1$, and so $K_{*}(M)=0$.

(v) For $n \geqslant 2$ and $\left(\widetilde{X}, \pi_{1}(X), w(X)\right)$ the universal oriented cover the Whitehead Theorem (3.6) and (iv) give that $f$ is a homotopy equivalence.

Theorem 10.30 (Surgery below the middle dimension.)

(i) Let $(f, b): M \rightarrow X$ be an m-dimensional normal map with $X$ a connected $C W$ complex with a finite $n$-skeleton. If $2 n \leqslant m$ then $(f, b)$ is bordant to an $n$-connected normal map $\left(f^{\prime}, b^{\prime}\right): M^{\prime} \rightarrow X$.

(ii) Let $(f, b): M \rightarrow X$ be an $m$-dimensional degree 1 normal map. If $2 n \leqslant m$ then $(f, b)$ is bordant to an $n$-connected degree 1 normal map $\left(f^{\prime}, b^{\prime}\right): M^{\prime} \rightarrow X$.

Proof (i) It suffices to prove that if $(f, b)$ is $(n-1)$-connected and $2 n \leqslant m$ then $(f, b)$ is bordant to an $n$-connected normal map $\left(f^{\prime}, b^{\prime}\right): M^{\prime} \rightarrow X$.

Consider first the case $n=0$. The connected sum (2.9) of the components of $M$ is a connected manifold $M^{\prime}$. The effect of the corresponding 0 -surgeries on $(f, b)$ is a bordant 0-connected normal map $\left(f^{\prime}, b^{\prime}\right): M^{\prime} \rightarrow X$.

Similarly in the case $n=1$. The $C W$ complex $X$ has a finite 1-skeleton, so that the fundamental group $\pi_{1}(X)$ is finitely generated, and hence so is

$$
\pi_{1}(f)=\pi_{1}(X) /\left\langle f_{*}\left(\pi_{1}(M)\right)\right\rangle
$$

with $\left\langle f_{*}\left(\pi_{1}(M)\right)\right\rangle \triangleleft \pi_{1}(X)$ the normal subgroup generated by $f_{*}\left(\pi_{1}(M)\right) \subseteq$ $\pi_{1}(X)$. Kill a finite of set of generators of $\pi_{1}(f)$ by 0 -surgeries on $(f, b)$, with effect a bordant 1-connected normal map $\left(f^{\prime}, b^{\prime}\right): M^{\prime} \rightarrow X$.

Next, consider the case $n \geqslant 2$, so that

$$
K_{i}(M)=\pi_{i+1}(f)=0 \text { for } i \leqslant n-2
$$

by the Hurewicz Theorem (3.26). The $\mathbb{Z}\left[\pi_{1}(X)\right]$-module $K_{n-1}(M)=\pi_{n}(f)$ is f.g. by Corollary 10.29, and can be killed by Corollary 10.11, thus obtaining a 
normal bordant $n$-connected normal map $\left(f^{\prime}, b^{\prime}\right): M^{\prime} \rightarrow X$.

(ii) Apply (i), noting that for a degree 1 normal map $(f, b): M \rightarrow X$ the target $X$ is a finite $C W$ complex, and that $f_{*}: H_{0}(M) \rightarrow H_{0}(X)$ is onto.

In the case $m=2 n$ it is possible to represent every element $x \in \pi_{n+1}(f)$ by an $n$-immersion $(h, g)$ with $b$-framing obstruction

$$
\left(\nu_{h}, \nu_{g}\right)=0 \in \pi_{n+1}(B O, B O(n)),
$$

but there is an obstruction in $\mathbb{Z}\left[\pi_{1}(M) \backslash\{1\}\right]$ to representing $x$ by an $n$-immersion with $g$ an embedding. The surgery obstruction of an $n$-connected $2 n$-dimensional degree 1 normal map $(f, b)$ is due to the fact that it may not be possible to kill an element $x \in \pi_{n+1}(f)=K_{n}(M)$ by an $n$-surgery on $(f, b)$ because it may not be possible to represent $\partial x \in \pi_{n}(M)$ by an embedding $S^{n} \times D^{n} \hookrightarrow M^{2 n}$. See Chapter 11 for a detailed account of the $2 n$-dimensional surgery obstruction.

The surgery obstruction of an $n$-connected $(2 n+1)$-dimensional degree 1 normal map $(f, b)$ is due to the fact that even though it is possible to kill every element $x \in \pi_{n+1}(f)=K_{n}(M)$ by an $n$-surgery on $(f, b)(10.25)$ there are many ways of doing so, none of which need reduce the size of $\pi_{n+1}(f)$. See Chapter 12 for a detailed account of the $(2 n+1)$-dimensional surgery obstruction. 


\section{THE EVEN-DIMENSIONAL SURGERY OBSTRUCTION}

This chapter defines the Wall surgery obstruction of a $2 n$-dimensional degree $1 \operatorname{map}(f, b): M \rightarrow X$

$$
\sigma_{*}(f, b) \in L_{2 n}\left(\mathbb{Z}\left[\pi_{1}(X)\right]\right) .
$$

The main result is that $\sigma_{*}(f, b)=0$ if (and for $n \geqslant 3$ only if) $(f, b)$ is normal bordant to a homotopy equivalence.

Section 11.1 develops the general theory of $\epsilon$-quadratic forms $(K, \lambda, \mu)$ over a ring with involution $A$, with $\epsilon= \pm 1$. Section 11.2 constructs the kernel $(-1)^{n}$ quadratic form $\left(K_{n}(M), \lambda, \mu\right)$ over $\mathbb{Z}\left[\pi_{1}(X)\right]$ of an $n$-connected $2 n$-dimensional degree 1 normal map $(f, b)$. Section 11.3 describes the algebraic effect on the kernel form of a geometric surgery on $(f, b)$. The $2 n$-dimensional surgery obstruction group $L_{2 n}(A)$ of equivalence classes of nonsingular $(-1)^{n}$-quadratic forms over $A$ is defined in Section 11.4. The even-dimensional surgery obstruction is defined in Section 11.5.

\subsection{Quadratic forms}

Let $A$ be a ring with involution $A \rightarrow A ; a \mapsto \bar{a}$. The general theory of sesquilinear pairings of $A$-modules was already developed in Section 4.4. As in 4.37, given $A$-modules $K, L$ let $S(K, L)$ denote the additive group of sesquilinear pairings $\lambda: K \times L \rightarrow A$.

Definition 11.1 Let $K$ be an $A$-module.

(i) The sesquilinear group of $K$ is the additive group

$$
\begin{aligned}
S(K) & =S(K, K) \\
& =\{\text { sesquilinear pairings } \lambda: K \times K \rightarrow A\} .
\end{aligned}
$$

(ii) The $\epsilon$-transposition involution is given for $\epsilon= \pm 1$ by

$$
T_{\epsilon}: S(K) \rightarrow S(K) ; \lambda \mapsto T_{\epsilon} \lambda=\epsilon(T \lambda),
$$

such that

$$
T_{\epsilon} \lambda(x, y)=\epsilon \overline{\lambda(y, x)} \in A,\left(T_{\epsilon}\right)^{2}=\mathrm{id} .: S(K) \rightarrow S(K) .
$$

(iii) The $\epsilon$-symmetric group of $K$ is the additive group 


$$
Q^{\epsilon}(K)=\operatorname{ker}\left(1-T_{\epsilon}: S(K) \rightarrow S(K)\right) .
$$

(iv) The $\epsilon$-quadratic group of $K$ is the additive group

$$
Q_{\epsilon}(K)=\operatorname{coker}\left(1-T_{\epsilon}: S(K) \rightarrow S(K)\right) .
$$

(v) The $\epsilon$-symmetrisation morphism is given by

$$
1+T_{\epsilon}: Q_{\epsilon}(K) \rightarrow Q^{\epsilon}(K) ; \psi \mapsto \psi+T_{\epsilon} \psi .
$$

(vi) Write

$$
\widehat{Q}^{\epsilon}(K)=\frac{\operatorname{ker}\left(1-T_{\epsilon}: S(K) \rightarrow S(K)\right)}{\operatorname{im}\left(1+T_{\epsilon}: S(K) \rightarrow S(K)\right)},
$$

so that there is defined an exact sequence

$$
0 \rightarrow \widehat{Q}^{-\epsilon}(K) \rightarrow Q_{\epsilon}(K) \stackrel{1+T_{\epsilon}}{\longrightarrow} Q^{\epsilon}(K) \rightarrow \widehat{Q}^{\epsilon}(K) \rightarrow 0 .
$$

For $\epsilon=+1$ it is customary to refer to $\epsilon$-symmetric and $\epsilon$-quadratic objects as symmetric and quadratic, as in the commutative case. For $\epsilon=-1$ the customary terminology is skew-symmetric (or symplectic) and skew-quadratic.

For $K=A$ there is an isomorphism of additive groups with involution

$$
A \rightarrow S(A) ; a \mapsto((x, y) \mapsto y a \bar{x})
$$

and there are natural identifications

$$
\begin{aligned}
& Q^{\epsilon}(A)=\{a \in A \mid \epsilon \bar{a}=a\}, \\
& Q_{\epsilon}(A)=A /\{b-\epsilon \bar{b} \mid b \in A\}, \\
& 1+T_{\epsilon}: Q_{\epsilon}(A) \rightarrow Q^{\epsilon}(A) ; a \mapsto a+\epsilon \bar{a}, \\
& \widehat{Q}^{\epsilon}(A)=\frac{\{a \in A \mid \epsilon \bar{a}=a\}}{\{b+\epsilon \bar{b} \mid b \in A\}} .
\end{aligned}
$$

Example 11.2 Let $A=\mathbb{Z}$. The $\epsilon$-symmetric and $\epsilon$-quadratic groups of $K=\mathbb{Z}$ are given by

$$
\begin{aligned}
& Q^{\epsilon}(\mathbb{Z})= \begin{cases}\mathbb{Z} & \text { if } \epsilon=+1 \\
0 & \text { if } \epsilon=-1,\end{cases} \\
& Q_{\epsilon}(\mathbb{Z})= \begin{cases}\mathbb{Z} & \text { if } \epsilon=+1 \\
\mathbb{Z}_{2} & \text { if } \epsilon=-1,\end{cases} \\
& \widehat{Q}^{\epsilon}(\mathbb{Z})= \begin{cases}\mathbb{Z}_{2} & \text { if } \epsilon=+1 \\
0 & \text { if } \epsilon=-1,\end{cases}
\end{aligned}
$$

with generators represented by $1 \in \mathbb{Z}$, and with 


$$
1+T_{+}=2: Q_{+}(\mathbb{Z})=\mathbb{Z} \rightarrow Q^{+}(\mathbb{Z})=\mathbb{Z} .
$$

The quadratic $Q$-groups $Q_{\epsilon}(\mathbb{Z})$ have already featured in Chapters 5 and 7, with

$$
I_{n}\left(S^{2 n}\right)=\pi_{n+1}(B O, B O(n))=Q_{(-1)^{n}}(\mathbb{Z}) .
$$

Definition 11.3 (i) An $\epsilon$-symmetric form $(K, \lambda)$ over $A$ is an $A$-module $K$ together with an element $\lambda \in Q^{\epsilon}(K)$. Thus $\lambda$ is a sesquilinear pairing

$$
\lambda: K \times K \rightarrow A ;(x, y) \mapsto \lambda(x, y)
$$

such that for all $x, y \in K$

$$
\lambda(x, y)=\epsilon \overline{\lambda(y, x)} \in A .
$$

(ii) A morphism of $\epsilon$-symmetric forms

$$
f:(K, \lambda) \rightarrow\left(K^{\prime}, \lambda^{\prime}\right)
$$

is an $A$-module morphism $f: K \rightarrow K^{\prime}$ such that

$$
\lambda^{\prime}(f(x), f(y))=\lambda(x, y) \in A .
$$

(iii) The adjoint of an $\epsilon$-symmetric form $(K, \lambda)$ is the $A$-module morphism

$$
K \rightarrow K^{*} ; x \mapsto(y \mapsto \lambda(x, y))
$$

which is also denoted by $\lambda$, and is such that

$$
T_{\epsilon} \lambda=\lambda: K \rightarrow K^{*} .
$$

(iv) An $\epsilon$-symmetric form $(K, \lambda)$ is nonsingular if $\lambda: K \rightarrow K^{*}$ is an isomorphism of $A$-modules.

Example 11.4 The symmetric form $(A, \lambda)$ defined by

$$
\lambda=1: A \rightarrow A^{*} ; a \mapsto(b \mapsto b \bar{a})
$$

is nonsingular.

Example 11.5 Let $X$ be a $2 n$-dimensional geometric Poincaré complex, and let $(\widetilde{X}, \pi, w)$ be an oriented cover. The cohomology intersection pairing (4.58)

$$
\lambda: H^{n}(\tilde{X}) \times H^{n}(\tilde{X}) \rightarrow \mathbb{Z}[\pi]
$$

is a $(-1)^{n}$-symmetric form over $\mathbb{Z}[\pi]$ with the $w$-twisted involution. Using the Poincaré duality isomorphism $H^{n}(\widetilde{X}) \cong H_{n}(\widetilde{X})$ this can also be regarded as a homology intersection $(-1)^{n}$-symmetric form

$$
\lambda: H_{n}(\tilde{X}) \times H_{n}(\tilde{X}) \rightarrow \mathbb{Z}[\pi] .
$$


The homology intersection form $\left(\operatorname{im}\left(\pi_{n}(M) \rightarrow H_{n}(\widetilde{M})\right), \lambda\right)$ of a $2 n$-dimensional manifold $M^{2 n}$ with universal cover $\widetilde{M}$ was interpreted in Section 7.2 in terms of geometric intersection numbers. In particular, it was shown that if an element $x \in \pi_{n}(M)$ can be killed by an $n$-surgery then

$$
\lambda(x, x)=0 \in \mathbb{Z}\left[\pi_{1}(M)\right] .
$$

However, the condition $\lambda(x, x)=0$ given by the symmetric structure alone is not sufficient for an element $x \in \pi_{n}(M)$ to be a potential surgery victim. It will also be necessary to consider quadratic structure.

Definition 11.6 (i) An $\epsilon$-quadratic form $(K, \lambda, \mu)$ over $A$ is an $\epsilon$-symmetric form $(K, \lambda)$ together with a function

$$
\mu: K \rightarrow Q_{\epsilon}(A) ; x \mapsto \mu(x)
$$

such that for all $x, y \in K, a \in A$

(a) $\mu(x+y)-\mu(x)-\mu(y)=\lambda(x, y) \in Q_{\epsilon}(A)$,

(b) $\mu(x)+\epsilon \overline{\mu(x)}=\lambda(x, x) \in \operatorname{im}\left(1+T_{\epsilon}: Q_{\epsilon}(A) \rightarrow Q^{\epsilon}(A)\right)$,

(c) $\mu(a x)=a \mu(x) \bar{a} \in Q_{\epsilon}(A)$.

(ii) An $\epsilon$-quadratic form $(K, \lambda, \mu)$ is nonsingular if $(K, \lambda)$ is nonsingular, i.e. if $\lambda: K \rightarrow K^{*}$ is an isomorphism of $A$-modules.

(iii) A morphism of $\epsilon$-quadratic forms

$$
f:(K, \lambda, \mu) \rightarrow\left(K^{\prime}, \lambda^{\prime}, \mu^{\prime}\right)
$$

is a morphism of the underlying $\epsilon$-symmetric forms $f:(K, \lambda) \rightarrow\left(K^{\prime}, \lambda^{\prime}\right)$ such that

$$
\mu^{\prime}(f(x))=\mu(x) \in Q_{\epsilon}(A) .
$$

(iv) For any f.g. projective $A$-module $L$ define the nonsingular hyperbolic $\epsilon$ quadratic form over $A$ by

$$
H_{\epsilon}(L)=\left(L \oplus L^{*}, \lambda, \mu\right)
$$

with

$$
\begin{gathered}
\lambda=\left(\begin{array}{ll}
0 & 1 \\
\epsilon & 0
\end{array}\right): L \oplus L^{*} \rightarrow\left(L \oplus L^{*}\right)^{*}=L^{*} \oplus L ; \\
(x, f) \mapsto((y, g) \mapsto f(y)+\epsilon \overline{g(x)}), \\
\mu: L \oplus L^{*} \rightarrow Q_{\epsilon}(A) ;(x, f) \mapsto f(x) .
\end{gathered}
$$

Proposition 11.7 If $2 \in A$ is invertible there is an identification of categories $\{\epsilon$-quadratic forms over $A\}=\{\epsilon$-symmetric forms over $A\}$. 
Proof The $\epsilon$-symmetrisation map $1+T_{\epsilon}: Q_{\epsilon}(K) \rightarrow Q^{\epsilon}(K)$ is an isomorphism for any $A$-module $K$, with inverse

$$
Q^{\epsilon}(K) \rightarrow Q_{\epsilon}(K) ; \lambda \mapsto\left((x, y) \mapsto \frac{1}{2} \lambda(x, y)\right)
$$

For any $\epsilon$-quadratic form $(K, \lambda, \mu)$ over $A$ the $\epsilon$-quadratic function $\mu$ is determined by the $\epsilon$-symmetric pairing $\lambda$, with

$$
\mu(x)=\frac{1}{2} \lambda(x, x) \in Q_{\epsilon}(A)
$$

In particular, for $A=\mathbb{R}$ the hyperbolic quadratic form $H_{+1}(L)$ defined in 11.6 (iv) is essentially the same as the hyperbolic symmetric form $H(L)$ of 6.34 .

In Section 11.2 it will be shown that an $n$-connected $2 n$-dimensional degree 1 normal map $(f, b): M^{2 n} \rightarrow X$ determines a nonsingular $(-1)^{n}$-quadratic form $\left(K_{n}(M), \lambda, \mu\right)$ over $\mathbb{Z}\left[\pi_{1}(X)\right]$. The $(-1)^{n}$-quadratic function

$$
\mu: K_{n}(M) \rightarrow Q_{(-1)^{n}}\left(\mathbb{Z}\left[\pi_{1}(X)\right]\right)
$$

will be defined using geometric self-intersection numbers, and it will be shown that $\mu(x)=0$ if (and for $n \geqslant 3$ only if) $x \in K_{n}(M)$ can be killed by surgery. The main result of even-dimensional surgery obstruction theory is that for $n \geqslant 3(f, b)$ is bordant to a homotopy equivalence if and only if the $(-1)^{n}$-quadratic form $\left(K_{n}(M), \lambda, \mu\right)$ over $\mathbb{Z}\left[\pi_{1}(X)\right]$ is stably hyperbolic, i.e. there exists an isomorphism of forms

$$
\left(K_{n}(M), \lambda, \mu\right) \oplus H_{(-1)^{n}}(F) \cong H_{(-1)^{n}}\left(F^{\prime}\right)
$$

for some f.g. free $\mathbb{Z}\left[\pi_{1}(X)\right]$-modules $F, F^{\prime}$.

The $\epsilon$-quadratic structures $(\lambda, \mu)$ on a f.g. projective $A$-module $K$ will now be shown to correspond to the elements of the $\epsilon$-quadratic group of 11.1

$$
Q_{\epsilon}(K)=\operatorname{coker}\left(1-T_{\epsilon}: S(K) \rightarrow S(K)\right) .
$$

The pair of functions $(\lambda, \mu)$ used to define an $\epsilon$-quadratic form $(K, \lambda, \mu)$ can thus be replaced by an equivalence class of $A$-module morphisms $\psi: K \rightarrow K^{*}$ such that

$$
\lambda(x, y)=\psi(x)(y)+\epsilon \overline{\psi(y)(x)} \in A, \mu(x)=\psi(x)(x) \in Q_{\epsilon}(A)
$$

Only forms on f.g. projective $A$-modules will be considered from now on.

Definition 11.8 (i) A split $\epsilon$-quadratic form $(K, \psi)$ over $A$ is a f.g. projective $A$-module $K$ together with an element $\psi \in S(K)$. 
(ii) An equivalence of split $\epsilon$-quadratic forms $(K, \psi),\left(K, \psi^{\prime}\right)$ on the same f.g. projective $A$-module $K$ is an element $\chi \in Q_{-\epsilon}(K)$ such that

$$
\psi^{\prime}-\psi=\chi-\epsilon \chi^{*}: K \rightarrow K^{*} .
$$

(iii) A morphism of split $\epsilon$-quadratic forms

$$
(f, \chi):(K, \psi) \rightarrow\left(K^{\prime}, \psi^{\prime}\right)
$$

is an $A$-module morphism $f \in \operatorname{Hom}_{A}\left(K, K^{\prime}\right)$ together with an element $\chi \in$ $Q_{-\epsilon}(K)$ such that

$$
f^{*} \psi^{\prime} f-\psi=\chi-\epsilon \chi^{*}: K \rightarrow K^{*}
$$

Proposition 11.9 (i) The $\epsilon$-quadratic structures $(\lambda, \mu)$ on a f.g. projective $A$ module $K$ are in one-one correspondence with the equivalence classes $\psi \in Q_{\epsilon}(K)$ of split $\epsilon$-quadratic forms $(K, \psi)$ over $A$.

(ii) Every morphism of $\epsilon$-quadratic forms $f:(K, \lambda, \mu) \rightarrow\left(K^{\prime}, \lambda^{\prime}, \mu^{\prime}\right)$ lifts to a morphism of split $\epsilon$-quadratic forms $(f, \chi):(K, \psi) \rightarrow\left(K^{\prime}, \psi^{\prime}\right)$.

Proof (i) Given a split $\epsilon$-quadratic form $(K, \psi)$ define an $\epsilon$-quadratic form $(K, \lambda, \mu)$ by

$$
\begin{aligned}
& \lambda=\left(1+T_{\epsilon}\right) \psi: K \rightarrow K^{*} ; x \mapsto(y \mapsto \psi(x)(y)+\epsilon \overline{\psi(y)(x)}) \\
& \mu: K \rightarrow Q_{\epsilon}(A) ; x \mapsto \psi(x)(x) .
\end{aligned}
$$

Conversely, let $(K, \lambda, \mu)$ be an $\epsilon$-quadratic form over $A$, with $K$ f.g. projective. Choose a f.g. projective $A$-module $L$ such that $K \oplus L$ is f.g. free of rank $k$, let $\left\{x_{1}, x_{2}, \ldots, x_{k}\right\}$ be a basis for $K \oplus L$, and let

$$
\lambda_{i j}=(\lambda \oplus 0)\left(x_{i}, x_{j}\right) \in A(1 \leqslant i<j \leqslant k) .
$$

Choose representatives $\mu_{i} \in A$ of $\mu\left(x_{i}\right) \in Q_{\epsilon}(A)(1 \leqslant i \leqslant k)$, and define the $A$-module morphism

$$
\begin{aligned}
\psi_{K \oplus L}: K \oplus L & \rightarrow(K \oplus L)^{*} ; \\
\sum_{i=1}^{k} a_{i} x_{i} & \mapsto\left(\sum_{j=1}^{k} b_{j} x_{j} \mapsto \sum_{i=1}^{k} b_{i} \mu_{i} \bar{a}_{i}+\sum_{1 \leqslant i<j \leqslant k} b_{j} \lambda_{i j} \bar{a}_{i}\right) .
\end{aligned}
$$

The $A$-module morphism defined by

$$
\psi: K \stackrel{\text { inclusion }}{\longrightarrow} K \oplus L \stackrel{\psi_{K \oplus L}}{\longrightarrow}(K \oplus L)^{*}=K^{*} \oplus L^{*} \stackrel{\text { projection }}{\longrightarrow} K^{*}
$$

is such that

$$
\begin{aligned}
& \lambda=\psi+\epsilon \psi^{*}: K \rightarrow K^{*}, \\
& \mu(x)=\psi(x)(x) \in Q_{\epsilon}(A)(x \in K) .
\end{aligned}
$$

(ii) Immediate from (i). 
Thus $Q_{\epsilon}(K)$ is both the group of isomorphism classes of $\epsilon$-quadratic and split $\epsilon$-quadratic structures on a f.g. projective $A$-module $K$.

For a f.g. free $A$-module $K=A^{k}$ :

Proposition 11.10 An $\epsilon$-quadratic form $\left(A^{k}, \lambda, \mu\right)$ over $A$ is determined by a $k \times k$-matrix $\lambda=\left\{\lambda_{i j} \in A \mid 1 \leqslant i, j \leqslant k\right\}$ such that

$$
\bar{\lambda}_{i j}=\epsilon \lambda_{j i} \in A
$$

and a collection of elements $\mu=\left\{\mu_{i} \in Q_{\epsilon}(A) \mid 1 \leqslant i \leqslant k\right\}$ such that

$$
\mu_{i}+\epsilon \bar{\mu}_{i}=\lambda_{i i} \in Q^{\epsilon}(A) .
$$

Choosing any representatives $\mu_{i} \in A$ of $\mu_{i} \in Q_{\epsilon}\left(A^{k}\right)$ there is defined a split $\epsilon$ quadratic form $\left(A^{k}, \psi\right)$ with $\psi=\left\{\psi_{i j} \in A \mid 1 \leqslant i, j \leqslant k\right\}$ the $k \times k$ matrix defined by

$$
\psi_{i j}= \begin{cases}\lambda_{i j} & \text { if } i<j \\ \mu_{i} & \text { if } i=j \\ 0 & \text { otherwise }\end{cases}
$$

Proof This is the essence of Proposition 11.9 for $K=A^{k}$.

Example 11.11 For a f.g. projective $A$-module $P$ the hyperbolic $\epsilon$-quadratic form $H_{\epsilon}(P)=\left(P \oplus P^{*}, \lambda, \mu\right)(11.6)$ corresponds to the element $\psi \in Q_{\epsilon}\left(P \oplus P^{*}\right)$ represented by the sesquilinear pairing

$$
\psi=\left(\begin{array}{ll}
0 & 1 \\
0 & 0
\end{array}\right): P \oplus P^{*} \rightarrow\left(P \oplus P^{*}\right)^{*}=P^{*} \oplus P .
$$

For any $\epsilon$-symmetric form $(K, \lambda)$ and $x \in K$

$$
\lambda(x, x) \in Q^{\epsilon}(A) .
$$

Definition 11.12 An $\epsilon$-symmetric form $(K, \lambda)$ is even if for all $x \in K$

$$
\lambda(x, x) \in \operatorname{im}\left(1+T_{\epsilon}: Q_{\epsilon}(A) \rightarrow Q^{\epsilon}(A)\right) .
$$

Proposition 11.13 If $1+T_{\epsilon}: Q_{\epsilon}(A) \rightarrow Q^{\epsilon}(A)$ is an injection there is an identification of categories

$\{\epsilon$-quadratic forms over $A\}=\{$ even $\epsilon$-symmetric forms over $A\}$. 
Proof Given an even $\epsilon$-symmetric form $(K, \lambda)$ over $A$ there is a unique function $\mu: K \rightarrow Q_{\epsilon}(A)$ such that for all $x \in K$

$$
\left(1+T_{\epsilon}\right)(\mu(x))=\lambda(x, x) \in Q^{\epsilon}(A),
$$

which then automatically satisfies the conditions of 11.6 for $(K, \lambda, \mu)$ to be an $\epsilon$-quadratic form.

Example 11.14 (i) The symmetrisation map

$$
1+T=2: Q_{+}(\mathbb{Z})=\mathbb{Z} \rightarrow Q^{+}(\mathbb{Z})=\mathbb{Z}
$$

is an injection, so that quadratic forms over $\mathbb{Z}$ coincide with the even symmetric forms.

(ii) The skew-symmetrisation map

$$
1-T=0: Q_{-}(\mathbb{Z})=\mathbb{Z}_{2} \rightarrow Q^{-}(\mathbb{Z})=0
$$

is not an injection, and there is an essential difference between skew-quadratic and skew-symmetric forms over $\mathbb{Z}$ (Arf invariant - see 11.60 below).

Definition 11.15 (i) A ring with involution $A$ is pure if it splits as a $\mathbb{Z}\left[\mathbb{Z}_{2}\right]$ module

$$
A=\mathbb{Z} \oplus \widetilde{A}
$$

Write the components of elements $a \in A$ as

$$
\left(a_{1}, \tilde{a}\right) \in \mathbb{Z} \oplus \widetilde{A},
$$

calling $a_{1}$ the integral component of $a$ and $\tilde{a}$ the reduced component of $a$. If $(K, \lambda, \mu)$ is an $\epsilon$-quadratic form write

$$
\lambda=\lambda_{1} \oplus \tilde{\lambda}, \mu=\mu_{1} \oplus \tilde{\mu} .
$$

(ii) The $Q$-groups of a pure $A$ split as direct sums of the $Q$-groups of $\mathbb{Z}$ and reduced $Q$-groups

$$
\begin{aligned}
& Q^{\epsilon}(A)=Q^{\epsilon}(\mathbb{Z}) \oplus \widetilde{Q}^{\epsilon}(A), \\
& Q_{\epsilon}(A)=Q_{\epsilon}(\mathbb{Z}) \oplus \widetilde{Q}_{\epsilon}(A), \\
& \widehat{Q}^{\epsilon}(A)=\widehat{Q}^{\epsilon}(\mathbb{Z}) \oplus \widetilde{\widehat{Q}}^{\epsilon}(A)
\end{aligned}
$$

with $Q^{\epsilon}(\mathbb{Z}), Q_{\epsilon}(\mathbb{Z}), \widehat{Q}^{\epsilon}(\mathbb{Z})$ as in 11.2 and

$$
\begin{aligned}
& \widetilde{Q}^{\epsilon}(A)=\{a \in \widetilde{A} \mid \epsilon \bar{a}=a\}, \\
& \widetilde{Q}_{\epsilon}(A)=\widetilde{A} /\{a-\epsilon \bar{a} \mid a \in \widetilde{A}\}, \\
& \widetilde{\widehat{Q}}^{\epsilon}(A)=\frac{\{a \in \widetilde{A} \mid \epsilon \bar{a}=a\}}{\{b+\epsilon \bar{b} \mid b \in \widetilde{A}\}} .
\end{aligned}
$$


Example 11.16 A group $\operatorname{ring} \mathbb{Z}[\pi]$ with a $w$-twisted involution is pure, with

$$
\mathbb{Z}[\pi]=\mathbb{Z} \oplus \mathbb{Z}[\pi \backslash\{1\}] .
$$

Proposition 11.17 If $A$ is a pure ring with involution such that there is a $\mathbb{Z}\left[\mathbb{Z}_{2}\right]$-module splitting

$$
A=\mathbb{Z} \oplus(B \oplus \bar{B})
$$

then the morphisms of additive groups

$$
\begin{aligned}
& B \rightarrow \widetilde{Q}_{\epsilon}(A) ; b \mapsto(b, 0)=(0, \epsilon \bar{b}), \\
& 1+T_{\epsilon}: \widetilde{Q}_{\epsilon}(A) \rightarrow \widetilde{Q}^{\epsilon}(A) ;(b, 0) \mapsto(b, \epsilon \bar{b})
\end{aligned}
$$

are isomorphisms. The reduced component $\widetilde{\mu}$ of the function $\mu$ in any $\epsilon$-quadratic form $(K, \lambda, \mu)$ over $A$ is determined by the reduced component $\tilde{\lambda}$ of $\lambda$, with

$$
\left(1+T_{\epsilon}\right) \widetilde{\mu}(x)=\tilde{\lambda}(x, x) \in \widetilde{Q}^{\epsilon}(A)(x \in K) .
$$

Proof The $\widehat{Q}$-groups of any pure ring with involution $A=\mathbb{Z} \oplus \widetilde{A}$ split as

$$
\widehat{Q}^{\epsilon}(A)=\widehat{Q}^{\epsilon}(\mathbb{Z}) \oplus \widetilde{\widehat{Q}}^{\epsilon}(A),
$$

and the exact sequence

$$
0 \longrightarrow \widehat{Q}_{-\epsilon}(A) \longrightarrow Q_{\epsilon}(A) \stackrel{1+T_{\epsilon}}{\longrightarrow} Q^{\epsilon}(A) \rightarrow \widehat{Q}^{\epsilon}(A) \rightarrow 0
$$

is the direct sum of an integral and a reduced exact sequence. If $\widetilde{A}=B \oplus \bar{B}$ then $\widetilde{\widehat{Q}}^{\epsilon}(A)=0$, so the reduced exact sequence collapses to an isomorphism

$$
1+T_{\epsilon}: \widetilde{Q}_{\epsilon}(A) \rightarrow \widetilde{Q}^{\epsilon}(A) .
$$

The integral components of an $\epsilon$-quadratic form $(K, \lambda, \mu)$ over $\mathbb{Z}[\pi]$ are invariant under the group action

$$
\lambda_{1}(g x, g y)=\lambda_{1}(x, y), \mu_{1}(g x)=\mu_{1}(x)(x, y \in K, g \in \pi) \text {, }
$$

and so may be regarded as defining an $\epsilon$-quadratic form $\left(K_{1}, \lambda_{1}, \mu_{1}\right)$ over $\mathbb{Z}$ on the induced $\mathbb{Z}$-module $K_{1}=\mathbb{Z} \otimes_{\mathbb{Z}[\pi]} K$.

Example 11.18 If $\pi$ is a group without 2-torsion, then a decomposition

$$
\pi=\{1\} \cup S \cup S^{-1}
$$

determines a $\mathbb{Z}\left[\mathbb{Z}_{2}\right]$-module splitting of the group ring with a $w$-twisted involution 


$$
\mathbb{Z}[\pi]=\mathbb{Z} \oplus\left(\mathbb{Z}[S] \oplus \mathbb{Z}\left[S^{-1}\right]\right)
$$

satisfying the hypothesis of 11.15 , so that up to isomorphism

$$
\widetilde{Q}_{\epsilon}(\mathbb{Z}[\pi])=\widetilde{Q}^{\epsilon}(\mathbb{Z}[\pi])=\mathbb{Z}[S] .
$$

A quadratic form over $\mathbb{Z}[\pi]$ is the same as an even symmetric form. A skewquadratic form $(K, \lambda, \mu)$ over $\mathbb{Z}[\pi]$ is the same as a skew-symmetric form $(K, \lambda)$ over $\mathbb{Z}[\pi]$ together with a function $\mu_{1}: K_{1} \rightarrow Q_{-}(\mathbb{Z})=\mathbb{Z}_{2}$ such that $\left(K_{1}, \lambda_{1}, \mu_{1}\right)$ is a skew-quadratic form over $\mathbb{Z}$. There exists such $\mu_{1}$ for every skew-symmetric form $(K, \lambda)$ over $\mathbb{Z}[\pi]$ with $K$ f.g. projective, but it is not unique.

Remark 11.19 If $A=\mathbb{Z} \oplus B \oplus \bar{B}$ (as in 11.17) the element $\psi \in Q_{\epsilon}\left(A^{k}\right)$ associated to an $\epsilon$-quadratic form $\left(A^{k}, \lambda, \mu\right)$ is determined by $\lambda$ and the integral components

$$
\mu_{1}=\left\{\left(\mu_{i}\right)_{1} \in Q_{\epsilon}(\mathbb{Z}) \mid 1 \leqslant i \leqslant m\right\} .
$$

\subsection{The kernel form}

The kernel form $\left(K_{n}(M), \lambda, \mu\right)$ of an $n$-connected $2 n$-dimensional degree 1 normal map $(f, b): M \rightarrow X$ is defined using the intersection and self-intersection properties of immersions $g: S^{n} \stackrel{\rightarrow}{\rightarrow} M^{2 n}$. The intersection pairing (4.66) on the homology of the universal cover $\widetilde{M}$

$$
\lambda: H_{n}(\widetilde{M}) \times H_{n}(\widetilde{M}) \rightarrow \mathbb{Z}\left[\pi_{1}(M)\right]
$$

is such that

$$
\lambda(g, g)=(-1)^{n} \overline{\lambda(g, g)} \in Q^{(-1)^{n}}\left(\mathbb{Z}\left[\pi_{1}(M)\right]\right)
$$

counts each unordered double point $[x, y] \in S_{2}[g]$ (7.3) twice, once as $(x, y)$ and once as $(y, x)$. The quadratic self-intersection defined in this section

$$
\mu(g) \in Q_{(-1)^{n}}\left(\mathbb{Z}\left[\pi_{1}(M)\right]\right)
$$

counts each unordered double point once only.

The main result of this section is the Wall Embedding Theorem (11.25) that for $n \geqslant 3$ :

(i) an immersion $g: N^{n} \rightarrow M^{2 n}$ with $\pi_{1}(N)=\{1\}$ is regular homotopic to an embedding if and only if

$$
\mu(g)=0 \in Q_{(-1)^{n}}\left(\mathbb{Z}\left[\pi_{1}(M)\right]\right)
$$


(ii) for a $2 n$-dimensional normal map $(f, b): M \rightarrow X$ the quadratic selfintersection of $b$-framed immersions $g: S^{n} \rightarrow M$ defines a function

$$
\mu: \pi_{n+1}(f) \rightarrow Q_{(-1)^{n}}\left(\mathbb{Z}\left[\pi_{1}(X)\right]\right)
$$

such that an element $x \in \pi_{n+1}(f)$ can be killed by surgery on $(f, b)$ if and only if $\mu(x)=0$.

The double point set of a map $g: N \rightarrow M$ with itself is

$$
\begin{aligned}
S_{2}(g, g) & =\{(x, y) \in N \times N \mid g(x)=g(y) \in M\} \\
& =S_{2}(g) \cup \Delta(N) \text { (disjoint union), }
\end{aligned}
$$

with

$$
S_{2}(g)=\left\{(x, y) \in S_{2}(g, g) \mid x \neq y\right\}
$$

the ordered double point set (7.3). The ordered double points $(x, y) \in S_{2}(g)$ come in pairs $(x, y),(y, x)$. As in (7.3) the unordered double point set is defined by

$$
\begin{aligned}
S_{2}[g] & =S_{2}(g) / \mathbb{Z}_{2} \\
& =\{(x, y) \in N \times N \mid x \neq y \in N, g(x)=g(y) \in M\} /\{(x, y) \sim(y, x)\} .
\end{aligned}
$$

Proposition 11.20 The homology self-intersection of an immersion $g: N^{n} \rightarrow$ $M^{2 n}$ is related to the ordered double points by

$$
\lambda(g, g)=\sum_{(x, y) \in S_{2}(g)} I(x, y)+\chi(g) \in Q^{(-1)^{n}}\left(\mathbb{Z}\left[\pi_{1}(M)\right]\right),
$$

with $I(x, y)$ the equivariant intersection index (7.18) and

$$
\chi(g)=\chi\left(\nu_{g}\right) \in Q^{(-1)^{n}}(\mathbb{Z})= \begin{cases}\mathbb{Z} & \text { if } n \equiv 0(\bmod 2) \\ 0 & \text { if } n \equiv 1(\bmod 2)\end{cases}
$$

the Euler number (5.44) of the normal bundle.

Proof The immersion $g: S^{n} \rightarrow M$ extends to an immersion $\bar{g}: E\left(\nu_{g}\right) \uparrow \rightarrow M$ of the total space of the normal bundle. Use $\bar{g}$ to move $g$ away from itself, as follows. Let $s_{0}: N \hookrightarrow E\left(\nu_{g}\right)$ be the zero section, and let $s_{t}: N \hookrightarrow E\left(\nu_{g}\right)(0 \leqslant t \leqslant 1)$ be an isotopy between $s_{0}$ and a section $s_{1}: N \hookrightarrow E\left(\nu_{g}\right)$ which is zero at only a finite number of points, such that $g_{t}=\bar{g} s_{t}: N \uparrow M$ defines a regular homotopy between $g_{0}=g$ and an immersion $g_{1}: N \leftrightarrow M$ without triple points and only transverse double points. By the Poincaré-Hopf theorem (Milnor [56, p.35])

$$
\left|S_{2}\left(s_{0}, s_{1}\right)\right|=|\chi(g)| \geqslant 0 .
$$

For each $(x, y) \in S_{2}(g)$ there is a unique $y_{1} \in N$ in the neighbourhood of $y \in N$ with 


$$
g(x)=g(y)=g_{1}\left(y_{1}\right) \in M .
$$

The double point set of $g_{0}$ and $g_{1}$ is the disjoint union

$$
S_{2}\left(g_{0}, g_{1}\right)=\left\{\left(x, y_{1}\right) \mid(x, y) \in S_{2}(g)\right\} \cup S_{2}\left(s_{0}, s_{1}\right) .
$$

Now apply 7.22 to identify

$$
\begin{aligned}
\lambda(g, g) & =\lambda^{a l g}(g, g)=\lambda^{a l g}\left(g_{0}, g_{1}\right) \\
& =\lambda^{g e o}\left(g_{0}, g_{1}\right)=\sum_{(x, y) \in S_{2}(g)} I(x, y)+\chi(g) \in Q^{(-1)^{n}}\left(\mathbb{Z}\left[\pi_{1}(M)\right]\right) .
\end{aligned}
$$

The formula of 11.20 gives a necessary homological condition for an element $x \in \pi_{n}(M)$ to be killed by surgery on a $2 n$-dimensional manifold $M^{2 n}$, namely that the Hurewicz image $x \in H_{n}(\widetilde{M})$ be such that

$$
\lambda(x, x)=0 \in Q^{(-1)^{n}}\left(\mathbb{Z}\left[\pi_{1}(M)\right]\right),
$$

with $\widetilde{M}$ the universal cover of $M$. In general, this is not a sufficient condition, since the formula counts the ordered double points of an immersion $g: S^{n} \rightarrow$ $M^{2 n}$ representing $x$, rather than the unordered double points. In order to count the unordered double points it is necessary to factor out the effect on the equivariant index of the change of ordering.

The equivariant index $I(x) \in \mathbb{Z}\left[\pi_{1}(M)\right]$ of a transverse ordered double point $x=\left(x_{1}, x_{2}\right) \in S_{2}(g)$ of an immersion $g: N^{n} \rightarrow M^{2 n}$ with a lift $\widetilde{g}: N \rightarrow \widetilde{M}$ was defined in (7.18) to be

$$
I(x)=w(x) a(x) \in\left\{ \pm \pi_{1}(M)\right\} \subset \mathbb{Z}\left[\pi_{1}(M)\right]
$$

with $a(x) \in \pi_{1}(M)$ such that

$$
\widetilde{g}\left(x_{2}\right)=a(x) \widetilde{g}\left(x_{1}\right) \in \widetilde{M}
$$

and

$$
w(x)=\left\{\begin{array} { l } 
{ + 1 } \\
{ - 1 }
\end{array} \quad \text { if } d \widetilde { g } ( x ) \left\{\begin{array}{l}
\text { preserves } \\
\text { reverses }
\end{array}\right.\right. \text { orientations }
$$

with

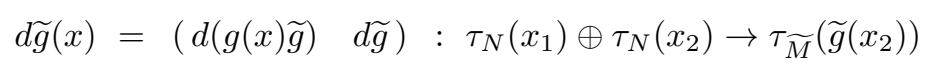

an isomorphism of oriented vector spaces. By 7.19 the effect of reversing the order on the equivariant index is given by

$$
I\left(x_{2}, x_{1}\right)=(-1)^{n} \overline{I\left(x_{1}, x_{2}\right)} \in \mathbb{Z}\left[\pi_{1}(M)\right] .
$$


Definition of the Wall $\mu$-form 11.21 ([92,5.2])

Let $g: N^{n} \rightarrow M^{2 n}$ be a self-transverse immersion with a lift $\widetilde{g}: N \leftrightarrow \widetilde{M}$ to the universal cover $\widetilde{M}$ of $M$.

(i) The equivariant index of an unordered double point $x=\left[x_{1}, x_{2}\right] \in S_{2}[g]$ is

$$
I[x]=[I(x)] \in Q_{(-1)^{n}}\left(\mathbb{Z}\left[\pi_{1}(M)\right]\right) .
$$

(ii) The geometric self-intersection of $g$ is the sum of the equivariant indices of the unordered double points

$$
\mu(g)=\sum_{[x] \in S_{2}[g]} I[x] \in Q_{(-1)^{n}}\left(\mathbb{Z}\left[\pi_{1}(M)\right]\right) .
$$

Example 11.22 An embedding $g: N^{n} \hookrightarrow M^{2 n}$ has $S_{2}[g]=\emptyset$, so that the geometric self-intersection is

$$
\mu(g)=0 \in Q_{(-1)^{n}}\left(\mathbb{Z}\left[\pi_{1}(M)\right]\right) .
$$

Example 11.23 The immersion $f_{n}^{\epsilon}: S^{n} \rightarrow S^{2 n}$ of 7.12 has a single unordered double point $\left[x^{\epsilon}, y^{\epsilon}\right] \in S_{2}\left[f_{n}^{\epsilon}\right]$ with $I\left[x^{\epsilon}, y^{\epsilon}\right]=\epsilon \in \mathbb{Z}$ and geometric self-intersection

$$
\mu\left(f_{n}^{\epsilon}\right)=I\left[x^{\epsilon}, y^{\epsilon}\right]=\epsilon \in Q_{(-1)^{n}}(\mathbb{Z}) .
$$

Proposition 11.24 (i) The geometric self-intersection $\mu(g)$ of an immersion $g: N^{n} \rightarrow M^{2 n}$ is a regular homotopy invariant.

(ii) If an immersion $g: N^{n} \rightarrow M^{2 n}$ is regular homotopic to an embedding then $\mu(g)=0$.

(iii) The homological self-intersection of an immersion $g: N^{n} \rightarrow M^{2 n}$ is related to the geometric self-intersection $\lambda(g, g)$ and the Euler number of the normal bundle $\nu_{g}$ by

$$
\lambda(g, g)=\mu(g)+(-1)^{n} \overline{\mu(g)}+\chi(g) \in Q^{(-1)^{n}}\left(\mathbb{Z}\left[\pi_{1}(M)\right]\right) .
$$

(iv) The connected sum of immersions $g: N^{n} \rightarrow M^{2 n}, g^{\prime}: N^{\prime n} \rightarrow M^{2 n}$ is an immersion

$$
g^{\prime \prime}=g \# g^{\prime}: N^{\prime \prime n}=N \# N^{\prime} \rightarrow M
$$

with geometric self-intersection

$$
\mu\left(g^{\prime \prime}\right)=\mu(g)+\mu\left(g^{\prime}\right)+\lambda\left(g, g^{\prime}\right) \in Q_{(-1)^{n}}\left(\mathbb{Z}\left[\pi_{1}(M)\right]\right)
$$

and Euler number

$$
\chi\left(g^{\prime \prime}\right)=\chi(g)+\chi\left(g^{\prime}\right) \in Q^{(-1)^{n}}(\mathbb{Z})
$$

(v) The effect on $\mu(g)$ of changing the lift $\widetilde{g}: N \rightarrow \widetilde{M}$ to a $\widetilde{g}: N \rightarrow \widetilde{M}$ for some $a \in \pi_{1}(M)$ is given by 


$$
\mu(a g)=w(a) a \mu(g) a^{-1} \in Q_{(-1)^{n}}\left(\mathbb{Z}\left[\pi_{1}(M)\right]\right) .
$$

(vi) The geometric self-intersections of immersions $g_{0}, g_{1}: N^{n} \rightarrow M^{2 n}$ related by a generic homotopy $g_{t}: N \rightarrow M(0 \leqslant t \leqslant 1)$ are such that

$$
\mu\left(g_{1}\right)=\mu\left(g_{0}\right)+\omega(g) \in Q_{(-1)^{n}}\left(\mathbb{Z}\left[\pi_{1}(M)\right]\right),
$$

with $\omega(g) \in Q_{(-1)^{n}}(\mathbb{Z})$ the number of generic singularities (7.42) in the track of $g_{t}$

$$
g: N \times I \rightarrow M \times I ;(x, t) \mapsto\left(g_{t}(x), t\right) .
$$

The normal bundles are such that

$$
\begin{aligned}
& \nu_{g_{1}}=\nu_{g_{0}} \# \omega(g) \tau_{S^{n}}: N=N \# S^{n} \rightarrow B O(n), \\
& \nu_{g_{1}} \oplus \epsilon^{\infty}=\nu_{g_{0}} \oplus \epsilon^{\infty}: N \rightarrow B O .
\end{aligned}
$$

In particular, if $g_{t}$ is a regular homotopy then $\omega(g)=0$ and

$$
\begin{aligned}
& \mu\left(g_{1}\right)=\mu\left(g_{0}\right) \in Q_{(-1)^{n}}\left(\mathbb{Z}\left[\pi_{1}(M)\right]\right), \\
& \nu_{g_{1}}=\nu_{g_{0}}: N \rightarrow B O(n) .
\end{aligned}
$$

Proof (i) The track of a regular homotopy $g_{t}: N^{n} \rightarrow M^{2 n}(0 \leqslant t \leqslant 1)$ is an immersion of an $(n+1)$-dimensional manifold in a $(2 n+1)$-dimensional manifold

$$
N \times I \leftrightarrow M \times I ;(x, t) \mapsto\left(g_{t}(x), t\right)
$$

with a 1-dimensional unordered double point set with components:

(a) $k_{1}$ paths joining unordered double points of $g_{0}$,

(b) $k_{2}$ paths joining unordered double points of $g_{1}$,

(c) $k_{3}$ paths joining unordered double points of $g_{0}$ and $g_{1}$,

(d) $k_{4}$ circles in the interior

for some $k_{1}, k_{2}, k_{3}, k_{4} \geqslant 0$.

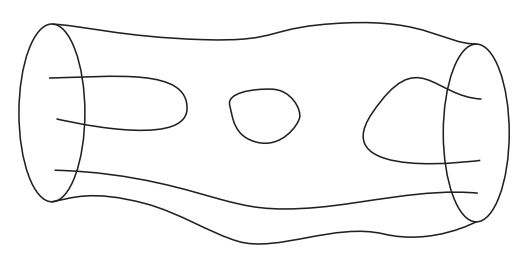

The unordered double point set $S_{2}\left(g_{1}\right)$ is obtained from $S_{2}\left(g_{0}\right)$ by removing $k_{1}$ cancelling pairs of double points and introducing $k_{2}$ cancelling pairs. Each pair contributes

$$
a-(-1)^{n} w(a) a^{-1}=0 \in Q_{(-1)^{n}}\left(\mathbb{Z}\left[\pi_{1}(M)\right]\right)
$$

to the self-intersection form $\mu$, with $a \in \pi_{1}(M)$ the equivariant index, so that $\mu\left(g_{0}\right)=\mu\left(g_{1}\right)$. 
(ii) By (i) and 11.22.

(iii) By 11.20.

(iv) The ordered double point set of $g^{\prime \prime}$ is the disjoint union

$$
S_{2}\left(g^{\prime \prime}\right)=S_{2}(g) \cup S_{2}\left(g^{\prime}\right) \cup S_{2}\left(g^{\prime}, g^{\prime \prime}\right) \cup S_{2}\left(g^{\prime \prime}, g^{\prime}\right),
$$

so that the unordered double point set is

$$
S_{2}\left[g^{\prime \prime}\right]=S_{2}[g] \cup S_{2}\left[g^{\prime}\right] \cup S_{2}\left(g^{\prime}, g^{\prime \prime}\right) .
$$

(v) By 7.19 (ii) at any ordered double point $\left(x_{1}, x_{2}\right) \in S_{2}(g)$ the effect on the equivariant index of a change of lift from $\widetilde{g}$ to $\widetilde{g}^{\prime}=a \widetilde{g}: N \rightarrow \widetilde{M}\left(a \in \pi_{1}(M)\right)$ is

$$
\widetilde{I}^{\prime}\left(x_{1}, x_{2}\right)=w(a) a \widetilde{I}\left(x_{1}, x_{2}\right) a^{-1} \in \mathbb{Z}\left[\pi_{1}(M)\right] .
$$

(vi) The singularities of $g$ consist of closed circles in the interior and paths with endpoints in the boundary (as in (i)) and at the generic singularities (7.42), which are cones on immersed $n$-spheres in the boundary. Thus in passing from $t=0$ to $t=1$ the double points in $S_{2}\left[g_{t}\right]$ are created and die in pairs away from the singularities, and are created and die individually at the singularities. At each singularity a copy of $\pm \tau_{S^{n}}$ is spliced to the normal bundle.

The geometric self-intersection $\mu$ has the following key property :

Wall Embedding Theorem 11.25 ([92, 5.2])

For $n \geqslant 3$ an immersion $g: N^{n} \rightarrow M^{2 n}$ with $\pi_{1}(N)=\{1\}$ is regular homotopic to an embedding if and only if

$$
\mu(g)=0 \in Q_{(-1)^{n}}\left(\mathbb{Z}\left[\pi_{1}(M)\right]\right) .
$$

Proof The geometric self-intersection is such that $\mu(g)=0$ if the number of unordered double points of $g$ is even and it is possible to match them up in pairs $\left[x_{1}, y_{1}\right],\left[x_{2}, y_{2}\right] \in S_{2}[g]$ with lifts to ordered double points $\left(x_{1}, y_{1}\right),\left(x_{2}, y_{2}\right) \in$ $S_{2}(g)$ such that

$$
I\left(x_{1}, y_{1}\right)=-I\left(x_{2}, y_{2}\right) \in \mathbb{Z}\left[\pi_{1}(M)\right] .
$$

Apply the Whitney trick (7.27) to each pair in turn, deforming $g$ by a sequence of regular homotopies to an immersion without double points, i.e. an embedding.

Remark 11.26 For any immersion $g: N^{n} \rightarrow M^{2 n}$ it is possible to deform the immersion

$$
N \leftrightarrow M \times \mathbb{R} ; x \mapsto(g(x), 0)
$$

by a regular homotopy to an embedding $g^{\prime}: N \hookrightarrow M \times \mathbb{R}$ (7.2) with normal bundle 


$$
\nu_{g^{\prime}}=\nu_{g} \oplus \epsilon: N \rightarrow B S O(n+1) .
$$

The Pontrjagin-Thom construction (6.8) gives a geometric Umkehr map for $g$

$$
G: \Sigma M_{+}=(M \times \mathbb{R})_{+} \rightarrow T\left(\nu_{g^{\prime}}\right)=\Sigma T\left(\nu_{g}\right)
$$

which depends on the choice of $g^{\prime}$. If $g: N^{n} \rightarrow S^{2 n}$ is a framed immersion then

$$
\nu_{g}=\epsilon^{n}, T\left(\nu_{g}\right)=\Sigma^{n} N_{+}
$$

and $g^{\prime}: N \hookrightarrow S^{2 n} \times \mathbb{R}$ is a framed embedding. The geometric self-intersection of $g$ is the image of the Hopf invariant $H(f) \in \mathbb{Z}$ (5.76) of the composite

$$
f: S^{2 n+1} \stackrel{G}{\longrightarrow} T\left(\nu_{g^{\prime}}\right)=\Sigma T\left(\nu_{g}\right)=\Sigma^{n+1} N_{+} \stackrel{\text { proj. }}{\longrightarrow} S^{n+1}
$$

that is

$$
\mu(g)=[H(f)] \in Q_{(-1)^{n}}(\mathbb{Z})=\mathbb{Z} /\left\{1+(-1)^{n+1}\right\},
$$

(Koschorke and Sanderson [41]). By the Adams Hopf Invariant 1 Theorem (5.79) there exists a map $f: S^{2 n+1} \rightarrow S^{n+1}$ with $H(f) \equiv 1(\bmod 2)$ if and only if $n=1,3,7$. Thus for a framed immersion $g: N^{n} \rightarrow S^{2 n}$

$$
\mu(g)=0 \in Q_{(-1)^{n}}(\mathbb{Z}) \text { for } n \neq 1,3,7 .
$$

The geometric definition (11.21) of the self-intersection form $\mu(g) \in Q_{(-1)^{n}}(\mathbb{Z}[\pi])$ of an immersion $g: N^{n} \rightarrow M^{2 n}$ is quite delicate, in that it is only a regular homotopy invariant and not a homotopy invariant. The decomposition of the group ring as a direct sum

$$
\mathbb{Z}\left[\pi_{1}(M)\right]=\mathbb{Z} \oplus \mathbb{Z}\left[\pi_{1}(M) \backslash\{1\}\right]
$$

and the splitting of the $Q$-groups (11.15) gives a decomposition of $\mu(g)$ into integral and reduced components

$\mu(g)=\left(\mu_{0}(g), \widetilde{\mu}(g)\right) \in Q_{(-1)^{n}}\left(\mathbb{Z}\left[\pi_{1}(M)\right]\right)=Q_{(-1)^{n}}(\mathbb{Z}) \oplus Q_{(-1)^{n}}\left(\mathbb{Z}\left[\pi_{1}(M) \backslash\{1\}\right]\right)$.

The reduced component $\widetilde{\mu}(g)$ will now be shown to depend only on the homotopy class of $g: N \leftrightarrow M$. (In fact, the visible symmetric construction of Weiss [95] can be used to prove that $\widetilde{\mu}(g)$ only depends on the homology class $\widetilde{g}[N] \in H_{n}(\widetilde{M})$.)

For any group $\pi$ with orientation character $w: \pi \rightarrow \mathbb{Z}_{2}$ write

$$
\widetilde{\mathbb{Z}}[\pi]=\mathbb{Z}[\pi \backslash\{1\}]=\left\{\sum_{g \in \pi} n_{g} g \in \mathbb{Z}[\pi] \mid n_{1}=0 \in \mathbb{Z}\right\} .
$$

The direct sum decomposition 


$$
\mathbb{Z}[\pi]=\mathbb{Z} \oplus \widetilde{\mathbb{Z}}[\pi]
$$

is respected by the $w$-twisted involution, and the $\epsilon$-quadratic $Q$-groups of $\mathbb{Z}[\pi]$ split as

$$
Q_{\epsilon}(\mathbb{Z}[\pi])=Q_{\epsilon}(\mathbb{Z}) \oplus \widetilde{Q}_{\epsilon}(\mathbb{Z}[\pi])
$$

(as in 11.15) with the reduced $\epsilon$-quadratic $Q$-groups given by

$$
\widetilde{Q}_{\epsilon}(\mathbb{Z}[\pi])=\widetilde{\mathbb{Z}}[\pi] /\{a-\epsilon \bar{a} \mid a \in \widetilde{\mathbb{Z}}[\pi]\} .
$$

Definition 11.27 (i) For any element $x=\sum_{a \in \pi} n_{a} a \in \mathbb{Z}[\pi]$ write the components as

$$
x=\left(x_{1}, \widetilde{x}\right) \in \mathbb{Z}[\pi]=\mathbb{Z} \oplus \widetilde{\mathbb{Z}}[\pi]
$$

with

$$
x_{1}=n_{1} \in \mathbb{Z}, \quad \widetilde{x}=\sum_{a \neq 1 \in \pi} n_{a} a \in \widetilde{\mathbb{Z}}[\pi] .
$$

(ii) The components of the intersection $\lambda\left(g_{1}, g_{2}\right)$ of homology classes $g_{1}, g_{2} \in$ $H_{n}\left(\widetilde{M}^{2 n}\right)$ are written as

$$
\lambda\left(g_{1}, g_{2}\right)=\left(\lambda_{1}\left(g_{1}, g_{2}\right), \widetilde{\lambda}\left(g_{1}, g_{2}\right)\right) \in \mathbb{Z}\left[\pi_{1}(M)\right]=\mathbb{Z} \oplus \widetilde{\mathbb{Z}}\left[\pi_{1}(M)\right]
$$

with $\lambda_{1}\left(g_{1}, g_{2}\right)$ the integral intersection and $\widetilde{\lambda}\left(g_{1}, g_{2}\right)$ the reduced intersection of $g_{1}, g_{2}$.

(iii) The components of the geometric self-intersection $\mu(g)$ of an immersion $g: N^{n} \rightarrow M^{2 n}$ are written as

$$
\mu(g)=\left(\mu_{1}(g), \widetilde{\mu}(g)\right) \in Q_{(-1)^{n}}\left(\mathbb{Z}\left[\pi_{1}(M)\right]\right)=Q_{(-1)^{n}}(\mathbb{Z}) \oplus \widetilde{Q}_{(-1)^{n}}\left(\mathbb{Z}\left[\pi_{1}(M)\right]\right)
$$

with $\mu_{1}(g)$ the integral self-intersection and $\widetilde{\mu}(g)$ the reduced self-intersection of $g$.

Proposition 11.28 Let $g: S^{n} \rightarrow M^{2 n}$ be a self-transverse immersion with a lift $\widetilde{g}: S^{n} \rightarrow \widetilde{M}$ to the universal cover $\widetilde{M}$ of $M$.

(i) The integral self-intersection $\mu_{1}(g) \in Q_{(-1)^{n}}(\mathbb{Z})$ is a regular homotopy invariant of $g$ such that $\mu_{1}(g)=0$ if (and for $n \geqslant 3$ only if) $\widetilde{g}$ is regular homotopic to an embedding $S^{n} \hookrightarrow \widetilde{M}$.

(ii) The reduced self-intersection $\widetilde{\mu}(g) \in \widetilde{Q}_{(-1)^{n}}\left(\mathbb{Z}\left[\pi_{1}(M)\right]\right)$ is a homotopy invariant of $g$ such that $\widetilde{\mu}(g)=0$ if (and for $n \geqslant 3$ only if) $g$ is homotopic to an embedding $g^{\prime}: S^{n} \hookrightarrow M$.

(iii) The coefficients $n_{a} \in \mathbb{Z}\left(a \in \pi_{1}(M), a^{2} \neq 1\right)$ in

$$
\mu(g)=\sum_{a \in \pi_{1}(M)} n_{a} a \in Q_{(-1)^{n}}\left(\mathbb{Z}\left[\pi_{1}(M)\right]\right)
$$

are determined by $\lambda(g, g) \in Q^{(-1)^{n}}\left(\mathbb{Z}\left[\pi_{1}(M)\right]\right)$. 
Proof (i) This is a special case of 11.24 (i).

(ii) The homotopy invariance of $\widetilde{\mu}(g)$ is given by 11.24 (vi), and it is clear that $\widetilde{\mu}(g)=0$ for an embedding $g$. Conversely, assume that $n \geqslant 3$ and that $\widetilde{\mu}(g)=0$. The connected sum of $g$ and $\mu_{1}(g)$ copies of $f_{n}^{-}: S^{n} \rightarrow S^{2 n}$ is a homotopic immersion

$$
g^{\prime}=g \# \mu_{1}(g) f_{n}^{-}: N \# S^{n}=N \leftrightarrow M \# S^{2 n}=M
$$

with

$$
\mu\left(g^{\prime}\right)=\mu(g)-\mu_{1}(g)=\widetilde{\mu}(g)=0 \in Q_{(-1)^{n}}\left(\mathbb{Z}\left[\pi_{1}(M)\right]\right) .
$$

By the Wall Embedding Theorem (11.25) $g^{\prime}$ is regular homotopic to an embedding, and so $g$ is homotopic to an embedding.

(iii) Let

$$
\alpha=\left\{a \in \pi_{1}(M) \mid a^{2} \neq 1\right\}, \beta=\left\{b \in \pi_{1}(M) \mid b^{2}=1, b \neq 1\right\},
$$

so that

$$
\pi_{1}(M)=\{1\} \cup \alpha \cup \beta
$$

Choose partitions

$$
\alpha=\alpha^{+} \cup \alpha^{-}, S_{2}(b \widetilde{g}, \widetilde{g})=S_{2}^{+}(b \widetilde{g}, \widetilde{g}) \cup S_{2}^{-}(b \widetilde{g}, \widetilde{g})(b \in \beta)
$$

such that

$$
\alpha^{-}=\left\{a^{-1} \mid a \in \alpha^{+}\right\}, S_{2}^{-}(b \widetilde{g}, \widetilde{g})=\left\{\left(x_{2}, x_{1}\right) \mid\left(x_{1}, x_{2}\right) \in S_{2}^{+}(b \widetilde{g}, \widetilde{g})\right\} .
$$

The reduced self-intersection is given by

$$
\widetilde{\mu}(g)=\sum_{a \in \alpha^{+}} m_{a} a+\sum_{b \in \beta} n_{b} b \in \widetilde{Q}_{(-1)^{n}}\left(\mathbb{Z}\left[\pi_{1}(M)\right]\right),
$$

with

$$
m_{a}=\sum_{x \in S_{2}(a \widetilde{g}, \widetilde{g})} I(x), n_{b}=\sum_{y \in S_{2}^{+}(b \widetilde{g}, \widetilde{g})} I(y) \in \mathbb{Z} .
$$

The $(-1)^{n}$-symmetrisation map

$$
\begin{aligned}
& 1+T_{(-1)^{n}}: \\
& \widetilde{Q}_{(-1)^{n}}\left(\mathbb{Z}\left[\pi_{1}(M)\right]\right)=\sum_{a \in \alpha^{+}} \mathbb{Z} \oplus \sum_{b \in \beta} Q_{(-1)^{n} w(b)}(\mathbb{Z}) \rightarrow \widetilde{Q}^{(-1)^{n}}\left(\mathbb{Z}\left[\pi_{1}(M)\right]\right) \\
& \left(\sum_{a \in \alpha^{+}} m_{g} g, \sum_{b \in \beta} n_{b} b\right) \mapsto \sum_{a \in \alpha^{+}} m_{a}\left(a+(-1)^{n} w(a) a^{-1}\right)+\sum_{b \in \beta} n_{b}\left(1+(-1)^{n} w(b)\right) b
\end{aligned}
$$

is an injection on $\sum_{a \in \alpha^{+}} \mathbb{Z}$, so that the coefficients $m_{a} \in \mathbb{Z}\left(a \in \alpha^{+}\right)$are determined by the $(-1)^{n}$-symmetrisation of $\mu(g)$

$$
\begin{aligned}
\left(1+T_{(-1)^{n}}\right) \mu(g) & =\mu(g)+(-1)^{n} \overline{\mu(g)} \\
& =\lambda(g, g)-\chi(g) \in Q^{(-1)^{n}}\left(\mathbb{Z}\left[\pi_{1}(M)\right]\right) .
\end{aligned}
$$

(If $n$ is even this is also the case for the coefficients $n_{b} \in Q_{(-1)^{n}}(\mathbb{Z})(b \in \beta)$ with $w(b)=+1$.) 
Remark 11.29 In the simply-connected case $\pi_{1}(M)=\{1\}$ the reduced geometric self-intersection $\widetilde{\mu}(g)$ takes values in $\widetilde{Q}_{(-1)^{n}}(\mathbb{Z})=0$, and 11.28 shows that for $n \geqslant 3$ every map $g: N^{n} \rightarrow M^{2 n}$ is homotopic to an embedding $g^{\prime}: N \hookrightarrow M$, as in the original embedding theorem of Whitney [98]. In the non-simply-connected case $\pi_{1}(M) \neq\{1\}$ with $n \geqslant 3$ every map $g: N^{n} \rightarrow M^{2 n}$ of a simply-connected manifold $N$ is homotopic to an immersion $g^{\prime}: N \leftrightarrow M$ with a lift to an embed$\operatorname{ding} \widetilde{g}^{\prime}: N \hookrightarrow \widetilde{M}$ in the universal cover $\widetilde{M}$, so that $\mu_{1}\left(g^{\prime}\right)=0 \in Q_{(-1)^{n}}(\mathbb{Z})$.

We now restrict attention to the case $N^{n}=S^{n}$, that is immersions $g: S^{n} \rightarrow$ $M^{2 n}$ in a $2 n$-dimensional manifold $M$.

Proposition 11.30 Let $n \geqslant 1$, and let $(1, b): S^{2 n} \rightarrow S^{2 n}$ be the identity normal map.

(i) The b-framing obstruction (10.8) of an $n$-immersion

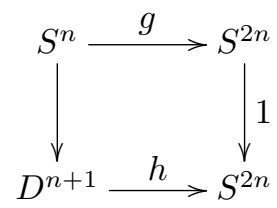

and the self-intersection of $g$ define an isomorphism

$$
\begin{aligned}
I_{n}\left(S^{2 n}\right)=I_{n+1}\left(1: S^{2 n}\right. & \left.\rightarrow S^{2 n}\right)=\pi_{n+1}(B S O, B S O(n)) \\
& \rightarrow Q_{(-1)^{n}}(\mathbb{Z}) ;(h, g)=\left(\nu_{h}, \nu_{g}\right) \mapsto-\mu(g) .
\end{aligned}
$$

The choice of sign ensures that the diagram

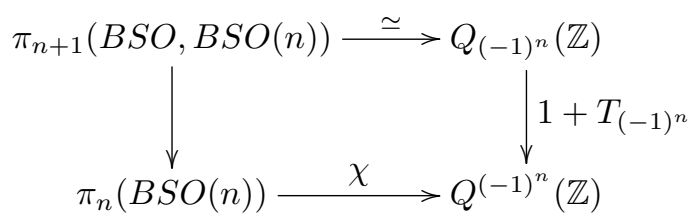

commutes. Thus for $n \equiv 0(\bmod 2)$

$$
\mu(g)=-\frac{1}{2} \chi\left(\nu_{g}\right) \in Q_{+1}(\mathbb{Z})=\mathbb{Z} .
$$

(ii) The single double point immersion $f_{n}^{+}: S^{n} \leftrightarrow S^{2 n}$ (7.12) represents the generator

$$
\mu\left(f_{n}^{+}\right)=\left(g_{n}^{+}, f_{n}^{+}\right)=1 \in Q_{(-1)^{n}}(\mathbb{Z})= \begin{cases}\mathbb{Z} & \text { if } n \equiv 0(\bmod 2) \\ \mathbb{Z}_{2} & \text { if } n \equiv 1(\bmod 2)\end{cases}
$$

with

$$
\pi_{n+1}(B S O, B S O(n))=Q_{(-1)^{n}}(\mathbb{Z}) \rightarrow \pi_{n}(B S O(n)) ; 1 \mapsto \nu_{f_{n}^{+}}=-\tau_{S^{n}} .
$$

(iii) For $n \neq 1,3,7$ the following conditions on an $n$-immersion $g: S^{n} \rightarrow S^{2 n}$ are equivalent: 
(a) $g$ is regular homotopic to the standard embedding $S^{n} \hookrightarrow S^{2 n}$,

(b) $\mu(g)=0 \in Q_{(-1)^{n}}(\mathbb{Z})$,

(c) $g$ is framed, i.e. $\nu_{g}=0 \in \pi_{n}(B S O(n))$.

Proof Every immersion $g: S^{n} \rightarrow S^{2 n}$ is null-homotopic, so by 11.24 (i)

$$
\lambda(g, g)=\left(1+(-1)^{n}\right) \mu(g)+\chi\left(\nu_{g}\right)=0 \in Q^{(-1)^{n}}(\mathbb{Z}) .
$$

From 5.83 we have

$$
\pi_{i}\left(V_{n+2,2}\right)=H_{i}\left(V_{n+2,2}\right)= \begin{cases}0 & \text { if } 1 \leqslant i \leqslant n-1 \\ Q_{(-1)^{n}}(\mathbb{Z}) & \text { if } i=n\end{cases}
$$

with the generator

$1=\left(\delta \tau_{S^{n}}, \tau_{S^{n}}\right) \in I_{n}\left(S^{2 n}\right)=\pi_{n+1}(B S O, B S O(n))=\pi_{n}\left(V_{n+2,2}\right)=Q_{(-1)^{n}}$

represented by the tangent bundle of the $n$-sphere $\tau_{S^{n}}: S^{n} \rightarrow B S O(n)$ with the stable trivialisation $\delta \tau_{S^{n}}: \tau_{S^{n}} \oplus \epsilon^{k} \cong \epsilon^{n+k}$ determined by the standard embedding $S^{n} \hookrightarrow S^{n+k}(k \geqslant 1)$. This generator is the $b$-framing obstruction $\nu_{b}\left(g_{n}^{+}, f_{n}^{+}\right) \in \pi_{n+1}(B S O, B S O(n))$ of the immersion $f_{n}^{+}: S^{n} \rightarrow S^{2 n}$ of 7.12, regarded as an $n$-immersion in the identity normal map $(1,1): S^{2 n} \rightarrow S^{2 n}$

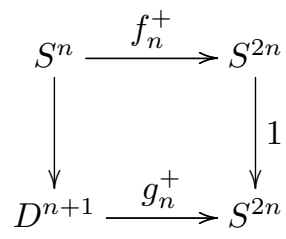

using any null-homotopy $g_{n}^{+}$, with

$$
\begin{aligned}
& \mu\left(f_{n}^{+}\right)=1 \in Q_{(-1)^{n}}(\mathbb{Z}), \\
& \lambda\left(f_{n}^{+}, f_{n}^{+}\right)=0 \in Q^{(-1)^{n}}(\mathbb{Z}), \\
& \chi\left(f_{n}^{+}\right)=\chi\left(-\tau_{S^{n}}\right)=-\left(1+(-1)^{n}\right) \\
& =\lambda\left(f_{n}^{+}, f_{n}^{+}\right)-\left(1-(-1)^{n}\right) \mu\left(f_{n}^{+}\right) \in Q^{(-1)^{n}}(\mathbb{Z}) .
\end{aligned}
$$

If $n \neq 1,3,7$ then $\tau_{S^{n}} \neq 0 \in \pi_{n}(B S O(n))$ (5.74) and there is defined a short exact sequence

$$
0 \rightarrow Q_{(-1)^{n}}(\mathbb{Z}) \rightarrow \pi_{n}(B S O(n)) \rightarrow \pi_{n}(B S O) \rightarrow 0
$$


Example 11.31 The Stiefel manifold of orthonormal 2 -frames in $\mathbb{R}^{3}$ can be expressed as

$$
V_{3,2}=S O(3)=S^{3} /\{ \pm 1\}=\mathbb{R P}^{3}
$$

using $S O(1)=\{1\}$ and regarding $S^{3}$ as the group of unit quaternions. The fundamental group is

$$
\pi_{1}\left(V_{3,2}\right)=\mathbb{Z}_{2}=Q_{-1}(\mathbb{Z})
$$

with the generator $1=\left(\delta \tau_{S^{1}}, \tau_{S^{1}}\right) \in I_{1}\left(S^{2}\right)=\mathbb{Z}_{2}$ corresponding to the figure 8 immersion $f_{1}^{+}: S^{1} \leftrightarrow S^{2}$ with geometric self-intersection $\mu\left(f_{1}^{+}\right)=1 \in \mathbb{Z}_{2}$.

Remark 11.32 (i) The $J$-homomorphism (5.80) and the Hopf invariant (5.76) define natural transformations of exact sequences

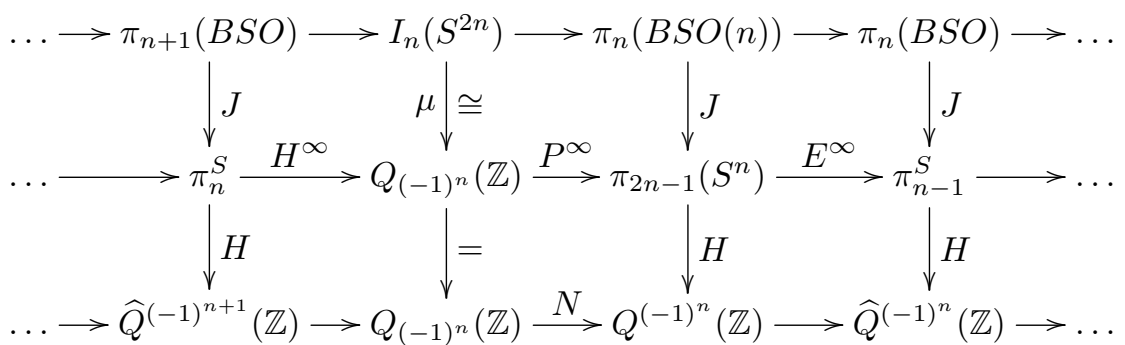

with

$$
\begin{aligned}
& \widehat{Q}^{(-1)^{n}}(\mathbb{Z})=\left\{x \in \mathbb{Z} \mid\left(1+(-1)^{n+1}\right) x=0\right\} /\left\{\left(1+(-1)^{n}\right) y \mid y \in \mathbb{Z}\right\} \\
&= \begin{cases}\mathbb{Z}_{2} & \text { if } n \equiv 0(\bmod 2) \\
0 & \text { if } n \equiv 1(\bmod 2),\end{cases} \\
& N=1+(-1)^{n}: Q_{(-1)^{n}}(\mathbb{Z}) \rightarrow Q^{(-1)^{n}}(\mathbb{Z}) .
\end{aligned}
$$

The morphism

$$
I_{n}\left(S^{2 n}\right) \rightarrow \pi_{n}(B S O(n)) ;\left(g: S^{n} \rightarrow S^{2 n}\right) \mapsto \nu_{g}
$$

injective for $n \neq 1,3,7$.

(ii) The morphism

$$
\pi_{n+1}(B S O, B S O(n))=Q_{(-1)^{n}}(\mathbb{Z}) \rightarrow \pi_{n}(B S O(n)) ; 1 \mapsto \nu_{f_{n}^{+}}=-\tau_{S^{n}}
$$

trivial if and only if $n=1,3,7$ by the Bott-Milnor theorem (5.74). For $n=1,3,7$ the geometric Umkehr map $F: S^{2 n+1} \rightarrow S^{n+1}$ of $f_{n}^{+}$has Hopf invariant 1, and $\left(f_{n}^{+}\right)^{\prime}: N=S^{n} \hookrightarrow S^{2 n+1}$ is the standard embedding with the exotic framing.

(iii) The composite of the universal (double) covering projection

$$
p: S^{n} \rightarrow \mathbb{R P}^{n} ; x=\left(x_{0}, x_{1}, \ldots, x_{n}\right) \mapsto p(x)=\left[x_{0}, x_{1}, \ldots, x_{n}\right]
$$

and any immersion $f: \mathbb{R P}^{n} \rightarrow S^{2 n}$ is an immersion

$$
g=p f: S^{n} \uparrow \mathbb{R P}^{n} \rightarrow S^{2 n} .
$$

The geometric self-intersection is such that $\mu(g) \neq 0$ if and only if $n=2^{j}-1$ for some $j \geqslant 1$ (Brown [16]). 
As in 11.27 write the $(-1)^{n}$-symmetric intersection pairing as

$$
\lambda=\left(\lambda_{1}, \widetilde{\lambda}\right): \pi_{n}(M) \times \pi_{n}(M) \rightarrow \mathbb{Z}\left[\pi_{1}(M)\right]=\mathbb{Z} \oplus \widetilde{\mathbb{Z}}\left[\pi_{1}(M)\right] .
$$

Note that $\lambda_{1}: \pi_{n}(M) \times \pi_{n}(M) \rightarrow \mathbb{Z}$ determines $\lambda$ by

$$
\lambda(x, y)=\sum_{a \in \pi_{1}(M)} \lambda_{1}(a x, y) a \in \mathbb{Z}\left[\pi_{1}(M)\right] .
$$

Example 11.33 (Rees $[77, \S 5])$ For $n \geqslant 2$ let

$$
p: S^{n} \rightarrow \mathbb{R P}^{n} ; x=\left(x_{0}, x_{1}, \ldots, x_{n}\right) \mapsto p(x)=\left[x_{0}, x_{1}, \ldots, x_{n}\right]
$$

be the universal (double) covering projection. The immersion

$$
g: S^{n} \rightarrow \mathbb{R P}^{n} \times D^{n} ; x=\left(x_{0}, x_{1}, \ldots, x_{n}\right) \mapsto\left(p(x),\left(x_{1}, \ldots, x_{n}\right)\right)
$$

has a single unordered double point, namely

$$
\begin{aligned}
g(1,0, \ldots, 0) & =g(-1,0, \ldots, 0) \\
& =([ \pm 1,0, \ldots, 0],(0, \ldots, 0)) \in \mathbb{R}^{n} \times D^{n} .
\end{aligned}
$$

The geometric self-intersection is

$$
\mu(g)=(0, T) \in Q_{(-1)^{n}}\left(\mathbb{Z}\left[\mathbb{Z}_{2}^{(-1)^{n+1}}\right]\right)=Q_{(-1)^{n}}(\mathbb{Z}) \oplus \widetilde{Q}_{(-1)^{n}}\left(\mathbb{Z}\left[\mathbb{Z}_{2}^{(-1)^{n+1}}\right]\right),
$$

where $\mathbb{Z}\left[\mathbb{Z}_{2}^{(-1)^{n+1}}\right]$ denotes the group ring $\mathbb{Z}\left[\pi_{1}\left(\mathbb{R} \mathbb{P}^{n}\right)\right]=\mathbb{Z}\left[\mathbb{Z}_{2}\right]$ with the involution determined by $w_{1}\left(\mathbb{R P}^{n}\right)=(-1)^{n+1} \in H^{1}\left(\mathbb{R P}^{n} ; \mathbb{Z}_{2}\right)=\mathbb{Z}_{2}=\{ \pm 1\}$

$$
\mathbb{Z}\left[\mathbb{Z}_{2}^{(-1)^{n+1}}\right] \rightarrow \mathbb{Z}\left[\mathbb{Z}_{2}^{(-1)^{n+1}}\right] ; a+b T \mapsto a+(-1)^{n+1} b T .
$$

The reduced self-intersection is non-zero

$$
\widetilde{\mu}(g)=T \neq 0 \in \widetilde{Q}_{(-1)^{n}}\left(\mathbb{Z}\left[\mathbb{Z}_{2}^{(-1)^{n+1}}\right]\right)=\mathbb{Z}_{2},
$$

so that $g: S^{n} \rightarrow \mathbb{R P}^{n} \times D^{n}$ is not homotopic to an embedding.

The connected sum of immersions $g, g^{\prime}: S^{n} \rightarrow M^{2 n}$ is an immersion

$$
g \# g^{\prime}: S^{n} \# S^{n}=S^{n} \leftrightarrow M^{2 n} .
$$

Proposition 11.34 Let $M^{2 n}$ be a $2 n$-dimensional manifold, with $n \geqslant 3$.

(i) The $\mathbb{Z}\left[\pi_{1}(M)\right]$-module $I_{n}(M)$ of regular homotopy classes of immersions $g$ : $S^{n} \rightarrow M^{2 n}$ is isomorphic to the $\mathbb{Z}\left[\pi_{1}(M)\right]$-module

$$
Q_{(-1)^{n}}(\mathbb{Z}) \times_{\lambda_{1}} \pi_{n}(M)=\left\{(x, y) \mid x \in Q_{(-1)^{n}}(\mathbb{Z}), y \in \pi_{n}(M)\right\}
$$

with addition and $\mathbb{Z}\left[\pi_{1}(M)\right]$-action by 


$$
\begin{aligned}
& (x, y)+\left(x^{\prime}, y^{\prime}\right)=\left(x+x^{\prime}+\lambda_{1}\left(y, y^{\prime}\right), y+y^{\prime}\right), \\
& \left(\sum_{a \in \pi_{1}(M)} n_{a} a\right)(x, y)=\left(n_{1} x, \sum_{a \in \pi_{1}(M)} n_{a} a y\right)
\end{aligned}
$$

The isomorphism is given by

$$
I_{n}(M) \rightarrow Q_{(-1)^{n}}(\mathbb{Z}) \times_{\lambda_{1}} \pi_{n}(M) ; g \mapsto\left(\mu_{1}(g),[g]\right)
$$

with $[g] \in \pi_{n}(M)$ the homotopy class of a regular homotopy class of immersions $g: S^{n} \rightarrow M^{2 n}$. The element $(1,0) \in I_{n}(M)$ is represented by the null-homotopic immersion

$$
f_{n}^{+}: S^{n} \rightarrow M \# S^{2 n}=M
$$

with $f_{n}^{+}: S^{n} \leftrightarrow S^{2 n}$ the single double point immersion of (7.12) with $\mu_{1}\left(f_{n}^{+}\right)=1$. (ii) For any immersion $g: S^{n} \rightarrow M$ let $\widetilde{g}: S^{n} \rightarrow \widetilde{M}$ be the prescribed lift to the universal cover $\widetilde{M}$, and let $g^{e m b}: S^{n} \uparrow M$ be any immersion in the regular homotopy class

$$
g^{e m b}=\left(-\mu_{1}(g), g\right) \in I_{n}(M)=Q_{(-1)^{n}}(\mathbb{Z}) \times_{\lambda_{1}} \pi_{n}(M)
$$

of immersions which are homotopic to $g$ and lift to an embedding $\widetilde{g}^{\text {emb }}: S^{n} \hookrightarrow \widetilde{M}$ in the universal cover $\widetilde{M}$. The reduced geometric self-intersection and the normal bundle define functions

$$
\begin{aligned}
& \widetilde{\mu}: \pi_{n}(M) \rightarrow \widetilde{Q}_{(-1)^{n}}\left(\mathbb{Z}\left[\pi_{1}(M)\right]\right) ;\left(g: S^{n} \rightarrow M\right) \mapsto \widetilde{\mu}(g), \\
& \nu: I_{n}(M) \rightarrow \pi_{n}(B S O(n)) ; g \mapsto \nu \widetilde{g}, \\
& \nu^{e m b}: \pi_{n}(M) \rightarrow \pi_{n}(B S O(n)) ; g \mapsto \nu_{\widetilde{g}^{e m b}}
\end{aligned}
$$

such that

$$
\begin{aligned}
& \widetilde{\mu}(x+y)=\widetilde{\mu}(x)+\widetilde{\mu}(y)+\widetilde{\lambda}(x, y) \in \widetilde{Q}_{(-1)^{n}}\left(\mathbb{Z}\left[\pi_{1}(M)\right]\right), \\
& \nu(x+y)=\nu(x)+\nu(y), \\
& \nu^{e m b}(x+y)=\nu^{e m b}(x)+\nu^{e m b}(y)+\lambda_{1}(x, y) \tau_{S^{n}} \in \pi_{n}(B S O(n)), \\
& \chi\left(\nu^{e m b}(x)\right)=\lambda_{1}(x, x) \in Q^{(-1)^{n}}(\mathbb{Z}) .
\end{aligned}
$$

The various functions fit into a commutative diagram

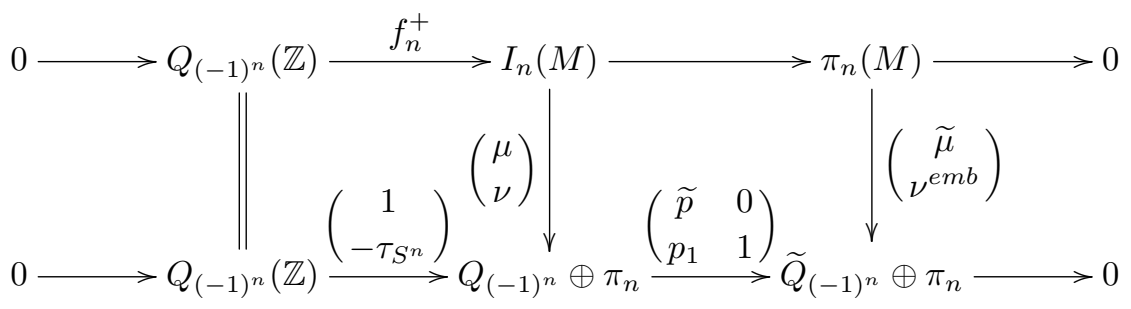

with 


$$
\begin{aligned}
\widetilde{p}= & \text { proj. }: Q_{(-1)^{n}}=Q_{(-1)^{n}}\left(\mathbb{Z}\left[\pi_{1}(M)\right]\right) \rightarrow \widetilde{Q}_{(-1)^{n}}=\widetilde{Q}_{(-1)^{n}}\left(\mathbb{Z}\left[\pi_{1}(M)\right]\right), \\
p_{1}: & Q_{(-1)^{n}}=Q_{(-1)^{n}}\left(\mathbb{Z}\left[\pi_{1}(M)\right]\right) \rightarrow Q_{(-1)^{n}}(\mathbb{Z}) \rightarrow \pi_{n}=\pi_{n}(B S O(n)), \\
\mu: & I_{n}(M)=Q_{(-1)^{n}}(\mathbb{Z}) \times_{\lambda_{1}} \pi_{n}(M) \\
& \rightarrow Q_{(-1)^{n}}\left(\mathbb{Z}\left[\pi_{1}(M)\right]\right)=Q_{(-1)^{n}}(\mathbb{Z}) \oplus \widetilde{Q}_{(-1)^{n}}\left(\mathbb{Z}\left[\pi_{1}(M)\right]\right) ; \\
& \left(g: S^{n} \rightarrow M\right)=\left(\mu_{1}(g), g\right) \mapsto \mu(g)=\left(\mu_{1}(g), \widetilde{\mu}(g)\right) .
\end{aligned}
$$

Proof (i) The morphism

$$
Q_{(-1)^{n}}(\mathbb{Z})=I_{n}\left(S^{2 n}\right) \rightarrow I_{n}(M) ;\left(f: S^{n} \rightarrow S^{2 n}\right) \mapsto\left(f: S^{n} \rightarrow M \# S^{2 n}=M\right)
$$

in the exact sequence of 7.39

$$
\begin{aligned}
\ldots \rightarrow \pi_{n+1}(M) & \rightarrow \pi_{n+1}(B O, B O(n))=Q_{(-1)^{n}}(\mathbb{Z}) \\
& \rightarrow I_{n}(M) \rightarrow \pi_{n}(M) \rightarrow \pi_{n}(B O, B O(n))=0
\end{aligned}
$$

is split by the self-intersection function $\mu: I_{n}(M) \rightarrow Q_{(-1)^{n}}(\mathbb{Z})$. The stated formulae for the addition and $\mathbb{Z}\left[\pi_{1}(M)\right]$-action in $I_{n}(M)$ are direct consequences of 11.24 (iv) and (v).

(ii) Combine Propositions 11.24 and 11.30.

Example 11.35 Wall [89] proved that for $n \geqslant 3$ the diffeomorphism classes of $(n-1)$-dimensional $2 n$-dimensional manifolds $(M, \partial M)$ with homotopy sphere boundary $\partial M=\Sigma^{2 n-1}$ are in one-one correspondence with the isomorphism classes of ' $n$-spaces' $(H, \lambda, \nu)$ with $(H, \lambda: H \times H \rightarrow \mathbb{Z})$ a nonsingular $(-1)^{n}$ symmetric form over $\mathbb{Z}$ and $\nu: H \rightarrow \pi_{n}(B S O(n))$ a function such that

$$
\begin{aligned}
& \nu(a x)=a^{2} \nu(x), \nu(x+y)=\nu(x)+\nu(y)+\lambda(x, y) \tau_{S^{n}} \in \pi_{n}(B S O(n)) \\
& \lambda(x, x)=\chi(\nu(x)) \in Q^{(-1)^{n}}(\mathbb{Z}) \quad(x, y \in H, a \in \mathbb{Z}) .
\end{aligned}
$$

The triple associated to $(M, \partial M)$ is given by

$$
(H, \lambda, \nu)=\left(H_{n}(M), \lambda_{1}, \nu^{e m b}\right)
$$

with $\nu^{\text {emb }}$ as in 11.34 .

Proposition 11.36 For any 2n-dimensional normal map $(f, b): M^{2 n} \rightarrow X$ the intersections and self-intersections of b-framed $n$-immersions in $f$ define a $(-1)^{n}$-quadratic form $\left(\pi_{n+1}(f), \lambda, \mu\right)$ over $\mathbb{Z}\left[\pi_{1}(X)\right]$ such that if $x$ can be killed by surgery on $(f, b)$ then

$$
\lambda(x, x)=0 \in \mathbb{Z}\left[\pi_{1}(X)\right], \mu(x)=0 \in Q_{(-1)^{n}}\left(\mathbb{Z}\left[\pi_{1}(X)\right]\right) .
$$


Proof Define $\lambda$ to be the composite

$$
\lambda: \pi_{n+1}(f) \times \pi_{n+1}(f) \longrightarrow H_{n}(\widetilde{M}) \times H_{n}(\widetilde{M}) \stackrel{\lambda}{\longrightarrow} \mathbb{Z}\left[\pi_{1}(X)\right]
$$

with $\widetilde{M}=f^{*} \widetilde{X}$ the pullback of the universal cover $\widetilde{X}$ of $X$. By 10.16 the bundle map $b: \nu_{M} \rightarrow \eta$ determines a splitting of the forgetful map $I_{n+1}(f) \rightarrow \pi_{n+1}(f)$

$$
s_{b}: \pi_{n+1}(f) \rightarrow I_{n+1}(f) ; \phi \mapsto \phi_{b}
$$

sending the homotopy class of a commutative square

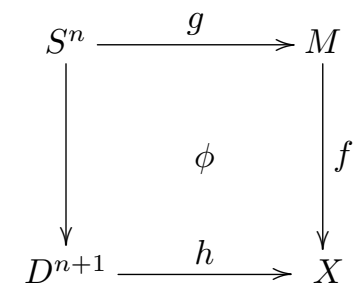

to the regular homotopy class of a $b$-framed $n$-immersion $\phi_{b}$ in $f$ with $g_{b}: S^{n} \rightarrow$ $M$ homotopic to $g$, which differ by the $b$-framing obstruction (10.8)

$$
\mu\left(g_{b}\right)-\mu(g)=\nu_{b}(\phi) \in Q_{(-1)^{n}}(\mathbb{Z}) \subseteq Q_{(-1)^{n}}\left(\mathbb{Z}\left[\pi_{1}(M)\right]\right) .
$$

The self-intersection defines a function

$$
\mu: I_{n+1}(f) \rightarrow Q_{(-1)^{n}}\left(\mathbb{Z}\left[\pi_{1}(X)\right]\right) ; \phi \mapsto \mu(g)
$$

sending the regular homotopy class of an $n$-immersion $\phi$ in $f$ to the self-intersection of the immersion $g: S^{n} \rightarrow M$ with

$$
\nu_{g}=\epsilon^{n}: S^{n} \rightarrow B S O(n), \chi\left(\nu_{g}\right)=Q^{(-1)^{n}}(\mathbb{Z}) .
$$

Define $\mu$ on $\pi_{n+1}(f)$ to be the composite

$$
\mu: \pi_{n+1}(f) \stackrel{s_{b}}{\longrightarrow} I_{n+1}(f) \stackrel{\partial}{\longrightarrow} I_{n}(M) \stackrel{\mu}{\longrightarrow} Q_{(-1)^{n}}\left(\mathbb{Z}\left[\pi_{1}(X)\right]\right) .
$$

Properties (i),(ii),(iii),(iv) follow from 11.24.

Definition 11.37 The kernel form of a $2 n$-dimensional degree 1 normal map $(f, b): M^{2 n} \rightarrow X$ is the geometric (intersection, self-intersection) $(-1)^{n}$-quadratic form $\left(\pi_{n+1}(f), \lambda, \mu\right)$ over $\mathbb{Z}\left[\pi_{1}(X)\right]$ of 11.36 .

Proposition 11.38 The kernel form of an n-connected $2 n$-dimensional degree 1 normal map $(f, b): M^{2 n} \rightarrow X$ is a nonsingular $(-1)^{n}$-quadratic form $\left(K_{n}(M), \lambda\right.$, $\mu$ ) on the stably f.g. free $\mathbb{Z}\left[\pi_{1}(X)\right]$-module $K_{n}(M)=\pi_{n+1}(f)$. 
Proof Let $\widetilde{X}$ be the universal cover of $X$, and let $\widetilde{M}=f^{*} \widetilde{X}$ be the pullback cover of $M$. By Proposition 10.21 the Poincaré duality isomorphisms of $\widetilde{M}$ split as

$$
\begin{aligned}
& {[M] \cap-=([M] \cap-) \oplus([X] \cap-):} \\
& H^{n}(\widetilde{M})=K^{n}(M) \oplus H^{n}(\widetilde{X}) \rightarrow H_{n}(\widetilde{M})=K_{n}(M) \oplus H_{n}(\widetilde{X}) .
\end{aligned}
$$

The natural $\mathbb{Z}\left[\pi_{1}(X)\right]$-module morphism $K^{n}(M) \rightarrow K_{n}(M)^{*}$ is an isomorphism and $K_{n}(M)$ is stably f.g. free by Corollary 10.29. The kernel Poincaré duality isomorphism $[M] \cap-: K^{n}(M) \cong K_{n}(M)$ is the inverse of the homology intersection pairing

$$
\lambda: K_{n}(M) \rightarrow K_{n}(M)^{*}=K^{n}(M)
$$

so that $\lambda$ is an isomorphism.

The nonsingular kernel form of 11.38 will be used in Section 11.4 below to represent the surgery obstruction

$$
\sigma_{*}(f, b)=\left(K_{n}(M), \lambda, \mu\right) \in L_{2 n}\left(\mathbb{Z}\left[\pi_{1}(X)\right]\right) .
$$

Example 11.39 For any $m$-dimensional degree 1 normal map $(f, b): M \rightarrow X$ and any $n$-immersion

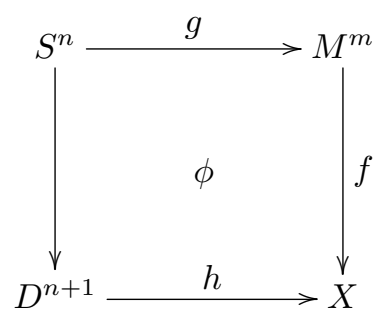

there is a $b$-framing obstruction

$$
\nu_{b}(\phi)=\left(\delta \nu_{h, b}, \nu_{g}\right) \in \pi_{n+1}(B O, B O(m-n))
$$

as in 10.8. See Ranicki [69] for the homotopy-theoretic construction of a $(-1)^{n_{-}}$ quadratic function in the case $m=2 n$

$$
\mu_{b}: K_{n}(M) \rightarrow Q_{(-1)^{n}}\left(\mathbb{Z}\left[\pi_{1}(X)\right]\right)
$$

using a stable $\pi_{1}(X)$-equivariant geometric Umkehr map $F: \Sigma^{\infty} \widetilde{X}_{+} \rightarrow \Sigma^{\infty} \widetilde{M}_{+}$ inducing the $\mathbb{Z}\left[\pi_{1}(X)\right]$-module chain Umkehr $f^{!}: C(\widetilde{X}) \rightarrow C(\widetilde{M})$. The composite

$$
\mu_{b}: \pi_{n+1}(f) \longrightarrow K_{n}(M) \stackrel{\mu_{b}}{\longrightarrow} Q_{(-1)^{n}}\left(\mathbb{Z}\left[\pi_{1}(X)\right]\right)
$$

sends the homotopy class of an $n$-immersion $\phi$ to the sum of the self-intersection $\mu(g)$ and the $b$-framing obstruction $\nu_{b}(\phi) \in \pi_{n+1}(B O, B O(n))=Q_{(-1)^{n}}(\mathbb{Z})$

$$
\mu_{b}(\phi)=\mu(g)+\nu_{b}(\phi) \in Q_{(-1)^{n}}\left(\mathbb{Z}\left[\pi_{1}(X)\right]\right) .
$$

In particular, for a $b$-framed $n$-immersion $\mu_{b}(\phi)=\mu(g)$ is the self-intersection, while for an $n$-embedding $\mu_{b}(\phi)=\nu_{b}(\phi)$ is the $b$-framing obstruction. For $\pi_{1}(X)=$ 
$\{1\} \mu_{b}$ is the form over $\mathbb{Z}$ originally constructed by Browder [14] (III.4, IV.4) using functional Steenrod squares.

Next, consider the kernel form of a $2 n$-dimensional degree 1 normal map which is the boundary of a $(2 n+1)$-dimensional degree 1 normal map of pairs.

Proposition 11.40 Let $(f, b):\left(M^{2 n+1}, \partial M\right) \rightarrow(X, \partial X)$ be a $(2 n+1)$-dimensional degree 1 normal map of pairs, such that $\pi_{1}(\partial X) \cong \pi_{1}(X)$.

(i) The kernel form $\left(\pi_{n+1}(\partial f), \lambda, \mu\right)$ of 11.37 is such that

$$
\lambda\left(x, x^{\prime}\right)=0 \in \mathbb{Z}\left[\pi_{1}(X)\right], \mu(x)=0 \in Q_{(-1)^{n}}\left(\mathbb{Z}\left[\pi_{1}(X)\right]\right)
$$

for any

$$
x, x^{\prime} \in \operatorname{im}\left(\partial: \pi_{n+2}(f, \partial f) \rightarrow \pi_{n+1}(\partial f)\right) \subseteq \pi_{n+1}(\partial f) .
$$

(ii) If $(f, b)$ is n-connected then the kernel form $\left(K_{n}(\partial M), \lambda, \mu\right)$ is such that

$$
\lambda\left(x, x^{\prime}\right)=0 \in \mathbb{Z}\left[\pi_{1}(X)\right], \mu(x)=0 \in Q_{(-1)^{n}}\left(\mathbb{Z}\left[\pi_{1}(X)\right]\right)
$$

for any

$$
x, x^{\prime} \in \operatorname{im}\left(K_{n+1}(M, \partial M) \rightarrow K_{n}(\partial M)\right)=\operatorname{im}\left(\pi_{n+2}(f, \partial f) \rightarrow \pi_{n+1}(\partial f)\right) .
$$

Proof (i) The intersection pairing $\lambda$ on $\pi_{n+1}(\partial f)$ factors through the homological intersection pairing

$$
\lambda: K_{n}(\partial M) \times K_{n}(\partial M) \rightarrow \mathbb{Z}\left[\pi_{1}(X)\right]
$$

adjoint to the Poincaré duality isomorphism

$$
\lambda=[\partial M] \cap-: K^{n}(\partial M) \rightarrow K_{n}(\partial M) .
$$

It is immediate from the isomorphism of exact sequences

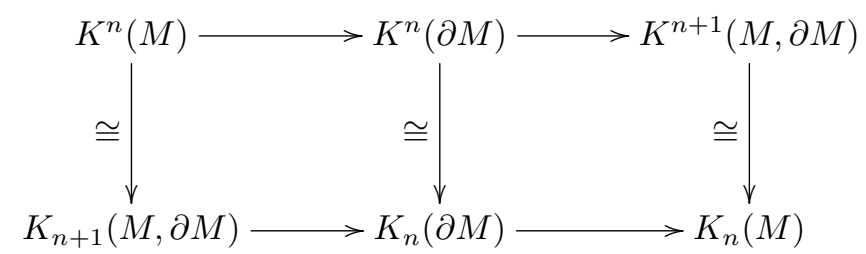

that the composite

$$
K^{n}(M) \rightarrow K^{n}(\partial M) \rightarrow K_{n}(\partial M) \rightarrow K_{n}(M)
$$

is 0 , so that $\lambda$ vanishes on

$$
\operatorname{ker}\left(K_{n}(\partial M) \rightarrow K_{n}(M)\right)=\operatorname{im}\left(K_{n+1}(M, \partial M) \rightarrow K_{n}(\partial M)\right)
$$


and hence also on $\operatorname{im}\left(\pi_{n+2}(f, \partial f) \rightarrow \pi_{n+1}(f)\right)$.

Every element $x \in \pi_{n+2}(f, \partial f)$ is represented by an relative $n$-immersion $(h, g)$ in $f$

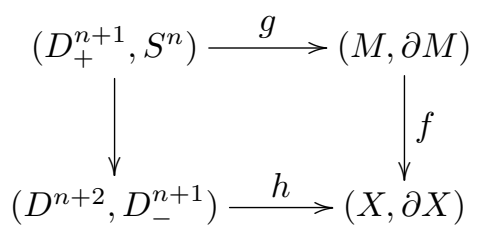

with the immersion of pairs

$$
(g, \partial g):\left(D^{n+1}, S^{n}\right) \hookrightarrow\left(M^{2 n+1}, \partial M\right)
$$

intersecting transversely. By definition

$$
\mu(\partial x)=\mu(\partial g)+\nu_{\partial b}(\partial h, \partial g) \in Q_{(-1)^{n}}\left(\mathbb{Z}\left[\pi_{1}(M)\right]\right) .
$$

The extension of $(\partial h, \partial g)$ to $(h, g)$ determines a null-homotopy of the $\partial b$-framing obstruction, so that

$$
\nu_{\partial b}(\partial h, \partial g)=0 \in Q_{(-1)^{n}}(\mathbb{Z}) \subseteq Q_{(-1)^{n}}\left(\mathbb{Z}\left[\pi_{1}(X)\right]\right) .
$$

The unordered double point sets $\left(S_{2}[g], S_{2}[\partial g]\right)$ define a 1-dimensional manifold with boundary. The components are either closed circles in $S_{2}[g]$, or else paths in $S_{2}[g]$ with endpoints in $S_{2}[\partial g]$. The paths match up the elements $[y] \in S_{2}[\partial g]$ in pairs $[y],[z]$ with lifts to unordered double points $y, z \in S_{2}(\partial g)$ such that

$$
I(y)=-I(z) \in \mathbb{Z}\left[\pi_{1}(M)\right]
$$

(exactly as in the proof of 11.25 ), so that

$$
\mu(\partial g)=\sum_{y \in S_{2}[\partial g]} I[y]=0 \in Q_{(-1)^{n}}\left(\mathbb{Z}\left[\pi_{1}(X)\right]\right) .
$$

(ii) Immediate from (i).

The kernel form is also defined on degree 1 normal maps of pairs:

Definition 11.41 The kernel form of an $n$-connected $2 n$-dimensional degree 1 normal map of pairs $(f, b):\left(M^{2 n}, \partial M\right) \rightarrow(X, \partial X)$ is the $(-1)^{n}$-quadratic form

$$
\left(K_{n}(M), \lambda: K_{n}(M) \times K_{n}(M) \rightarrow \mathbb{Z}\left[\pi_{1}(X)\right], \mu: K_{n}(M) \rightarrow Q_{(-1)^{n}}\left(\mathbb{Z}\left[\pi_{1}(X)\right]\right)\right)
$$

on the stably f.g. free $\mathbb{Z}\left[\pi_{1}(X)\right]$-module $\pi_{n+1}(f)=K_{n}(M)$ given by the construction of 11.36 on $K_{n}(M)=\pi_{n+1}(f)$. 
The various kernel $\mathbb{Z}\left[\pi_{1}(X)\right]$-modules are related by an exact sequence

$$
\ldots \rightarrow K_{r}(\partial M) \rightarrow K_{r}(M) \rightarrow K_{r}(M, \partial M) \rightarrow K_{r-1}(\partial M) \rightarrow \ldots,
$$

with $K_{r}(M)=0$ for $r \neq n$. By Poincaré duality and the universal coefficient theorem

$$
\begin{aligned}
K_{r}(M, \partial M) \cong K^{2 n-r}(M) & \cong K_{2 n-r}(M)^{*} \\
& =0 \text { for } r \neq n,
\end{aligned}
$$

so that $K_{r}(\partial M)=0$ for $r \neq n-1, n$. The $\mathbb{Z}\left[\pi_{1}(X)\right]$-module morphism

$$
\lambda: K_{n}(M) \rightarrow K_{n}(M)^{*} \cong K^{n}(M) \cong K_{n}(M, \partial M)
$$

thus fits into the exact sequence

$$
0 \rightarrow K_{n}(\partial M) \rightarrow K_{n}(M) \stackrel{\lambda}{\rightarrow} K_{n}(M, \partial M) \rightarrow K_{n-1}(\partial M) \rightarrow 0
$$

and the kernel form $\left(K_{n}(M), \lambda, \mu\right)$ is nonsingular if and only if $\partial f: \partial M \rightarrow \partial X$ is a $\mathbb{Z}\left[\pi_{1}(X)\right]$-homology equivalence: if $\pi_{1}(\partial M)=\pi_{1}(\partial X)=\pi_{1}(X)$ this is the same as homotopy equivalence.

Proposition 11.42 (Realization of forms)

Let $N^{2 n-1}$ be a $(2 n-1)$-dimensional manifold with fundamental group $\pi_{1}(N)=$ $\pi$, and $n \geqslant 3$.

Every $(-1)^{n}$-quadratic form $(K, \lambda, \mu)$ over $\mathbb{Z}[\pi]$ with $K$ f.g. free is realized as the kernel form of an $n$-connected $2 n$-dimensional degree 1 normal bordism

$$
(f, b):\left(M^{2 n} ; N^{2 n-1}, N^{\prime 2 n-1}\right) \rightarrow N \times(I ;\{0\},\{1\})
$$

with $K_{n}(M)=K$ and $(f, b) \mid=$ identity $: N \rightarrow N$. The restriction $(f, b) \mid: N^{\prime} \rightarrow$ $N$ is $(n-1)$-connected, with

$$
K_{n-1}\left(N^{\prime}\right)=\operatorname{coker}\left(\lambda: K \rightarrow K^{*}\right), K_{n}\left(N^{\prime}\right)=\operatorname{ker}\left(\lambda: K \rightarrow K^{*}\right) .
$$

The form $(K, \lambda, \mu)$ is nonsingular if and only if $f \mid: N^{\prime} \rightarrow N$ is a homotopy equivalence.

Proof Let $K=\mathbb{Z}[\pi]^{k}$ be of rank $k$. Construct $M$ by attaching $k n$-handles to $N \times I$

$$
M^{2 n}=N \times I \cup_{e_{1}} \bigcup_{k} D^{n} \times D^{n}
$$

exactly as in Theorem 5.8 of Wall [92] : starting with a null-homotopic unlinked embedding

$$
e_{0}: \bigcup_{k} S^{n-1} \times D^{n} \hookrightarrow N
$$

construct a regular homotopy with track an immersion 


$$
e: \bigcup_{k} S^{n-1} \times D^{n} \times[0,1] \rightarrow N \times[0,1]
$$

with (intersection, self-intersection) data $(\lambda, \mu)$, and so obtain a linked embedding

$$
e_{1}: \bigcup_{k} S^{n-1} \times D^{n} \hookrightarrow N ; x \mapsto e(x, 1) .
$$

Thus $N^{\prime 2 n-1}$ is the effect of $k(n-1)$-surgeries on the restrictions

$$
e_{1} \mid: S^{n-1} \times D^{n} \hookrightarrow N^{2 n-1} .
$$

Remark 11.43 The realization of forms by even-dimensional normal maps is a non-simply-connected generalisation of the plumbing construction of Milnor [52]. Let $(K, \lambda)$ be a $(-1)^{n}$-symmetric form over $\mathbb{Z}$ with $K$ a f.g. free $\mathbb{Z}$-module, together with a function $\nu: K \rightarrow \pi_{n}(B S O(n))$ such that

$$
\begin{aligned}
& \nu(a x)=a^{2} \nu(x), \nu(x+y)=\nu(x)+\nu(y)+\lambda(x, y) \tau_{S^{n}} \in \pi_{n}(B S O(n)) \\
& \lambda(x, x)=\chi(\nu(x)) \in Q^{(-1)^{n}}(\mathbb{Z}) \quad(x, y \in K, a \in \mathbb{Z}) .
\end{aligned}
$$

For any basis $\left(b_{1}, b_{2}, \ldots, b_{k}\right)$ of $K$ the functions $\lambda, \nu$ are determined by the values

$$
\lambda_{i j}=\lambda\left(b_{i}, b_{j}\right) \in \mathbb{Z}(i \neq j), \nu_{i}=\nu\left(b_{i}\right) \in \pi_{n}(B S O(n)) .
$$

Let $G$ be the graph with $k$ vertices $v_{1}, v_{2}, \ldots, v_{k}$ and $\left|\lambda_{i j}\right|$ edges joining $v_{i}$ to $v_{j}$ $(i \neq j)$, so that $\left(\lambda_{i j}\right)$ is the incidence matrix, and give each vertex $v_{i}$ the weight $\nu_{i}$. Assuming $(K, \lambda, \mu)$ cannot be decomposed as a nontrivial direct sum, $G$ is connected with the homotopy type of a wedge of circles

$$
G \simeq \bigvee_{g} S^{1}
$$

with $g=1-\chi(G)$. (Terminology: the wedge of pointed spaces $X, Y$ is the one-point union

$$
X \vee Y=X \times\left\{y_{0}\right\} \cup\left\{x_{0}\right\} \times Y \subseteq X \times Y
$$

with $x_{0} \in X, y_{0} \in Y$ the base points.) The disk bundles

$$
\left(D^{n}, S^{n-1}\right) \rightarrow\left(E\left(\nu_{i}\right), S\left(\nu_{i}\right)\right) \rightarrow S^{n} \quad(1 \leqslant i \leqslant k)
$$

can be spliced together using $\lambda_{i j}, \nu_{i}$ to obtain a connected framed $2 n$-dimensional manifold with boundary

$$
P=\left(\coprod_{i=1}^{k} E\left(\nu_{i}\right)\right) / \sim
$$


a thickening of the space obtained from $k$ copies of $S^{n}$ by identifying $\left|\lambda_{i j}\right|$ points in the $i$ th and $j$ th copies $(1 \leqslant i<j \leqslant k)$ such that

$$
\begin{aligned}
& P=h^{0} \cup \bigcup_{g} h^{1} \cup \bigcup_{k} h^{n} \simeq \bigvee_{g} S^{1} \cup \bigcup_{k} D^{n}, \\
& H_{r}(P)= \begin{cases}\mathbb{Z} & \text { if } r=0 \\
\mathbb{Z}^{g} & \text { if } r=1 \\
K & \text { if } r=n \\
0 & \text { otherwise }\end{cases}
\end{aligned}
$$

with an exact sequence

$$
0 \rightarrow H_{n}(\partial P) \rightarrow H_{n}(P)=K \stackrel{\lambda}{\longrightarrow} H_{n}(P, \partial P)=K^{*} \rightarrow H_{n-1}(\partial P) \rightarrow 0
$$

For $n \geqslant 3 \pi_{1}(P)=*_{g} \mathbb{Z}$ can be killed by $g$ 1-surgeries $S^{1} \times D^{2 n-1} \hookrightarrow P$ and removing an interior $D^{2 n} \hookrightarrow P$ there is obtained an $(n-1)$-connected $2 n$-dimensional cobordism $\left(M ; S^{2 n-1}, \partial P\right)$. If the function $\nu$ is actually determined by a $(-1)^{n}-$ quadratic function $\mu$ for $\lambda$

$$
\nu: K \stackrel{\mu}{\longrightarrow} Q_{(-1)^{n}}(\mathbb{Z})=\pi_{n+1}(B S O, B S O(n)) \rightarrow \pi_{n}(B S O(n))
$$

plumbing gives an $n$-connected $2 n$-dimensional normal map

$$
(f, b):\left(M^{2 n} ; S^{2 n-1}, \partial P\right) \rightarrow S^{2 n-1} \times(I ;\{0\},\{1\})
$$

such that $(f, b) \mid=$ identity $: S^{2 n-1} \rightarrow S^{2 n-1}$, with kernel form $\left(K_{n}(M)=\right.$ $K, \lambda, \mu)$, i.e. the realization of the form as in 11.42 . The $(n-1)$-connected $(2 n-1)$ dimensional normal map $(f, b) \mid: \partial P \rightarrow S^{2 n-1}$ has kernel homology

$$
K_{*}(\partial P)=H_{*}(\partial P) \quad(* \neq 0,2 n-1)
$$

so that it is a homotopy equivalence if and only if $(K, \lambda)$ is nonsingular. Here is an illustration of plumbing in the case $k=2$

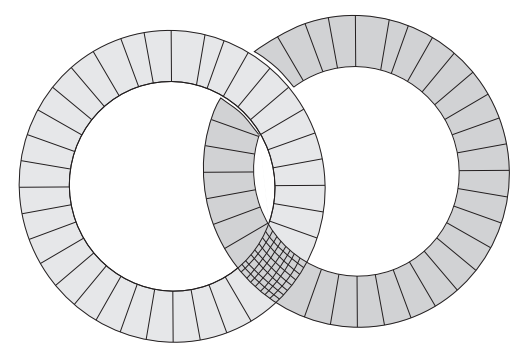

See Chapter V of Browder [14] for a detailed account of plumbing. 
Example 11.44 Let $N^{2 n-1}=S^{2 n-1}$ in the construction of 11.42.

(i) For the singular $(-1)^{n}$-quadratic form $(K, \lambda, \mu)=\left(\mathbb{Z}^{k}, 0,0\right)$ over $\mathbb{Z}$

$$
\begin{aligned}
\left(M^{2 n} ; N^{2 n-1}, N^{\prime 2 n-1}\right) & \\
= & \begin{cases}\#\left(\operatorname{cl} .\left(S^{n} \times D^{n} \backslash D^{2 n}\right) ; S^{2 n-1}, S^{n} \times S^{n-1}\right) & \text { if } n \geqslant 2 \\
\left(\operatorname{cl} .\left(D^{2} \backslash \cup_{k+2} D^{2}\right) ; S^{1}, \cup_{k+1} S^{1}\right) & \text { if } n=1\end{cases}
\end{aligned}
$$

is the trace of surgeries on an unlinked embedding $\cup_{k} S^{n-1} \times D^{n} \hookrightarrow S^{2 n-1}$. Here is an illustration of the case $k=2, n=1$.

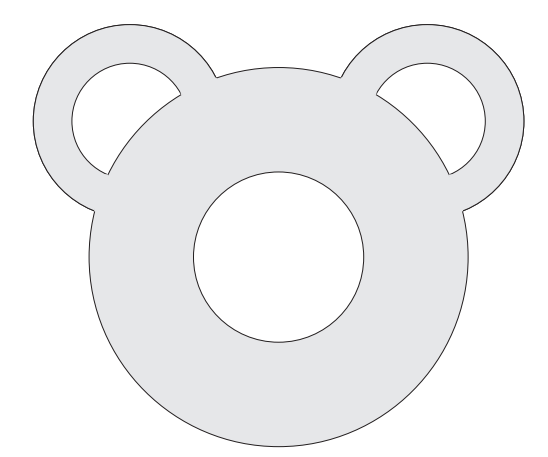

(ii) For the nonsingular hyperbolic $(-1)^{n}$-quadratic form over $\mathbb{Z}(K, \lambda, \mu)=$ $H_{(-1)^{n}}\left(\mathbb{Z}^{k}\right)$

$$
\left(M^{2 n} ; N^{2 n-1}, N^{2 n-1}\right)=\underset{k}{\#}\left(\operatorname{cl} .\left(S^{n} \times S^{n} \backslash\left(D^{2 n} \cup D^{2 n}\right)\right) ; S^{2 n-1}, S^{2 n-1}\right)
$$

is the trace of surgeries on a linked embedding $\cup_{2 k} S^{n-1} \times D^{n} \hookrightarrow S^{2 n-1}$. Here is an illustration of the case $k=2, n=1$.

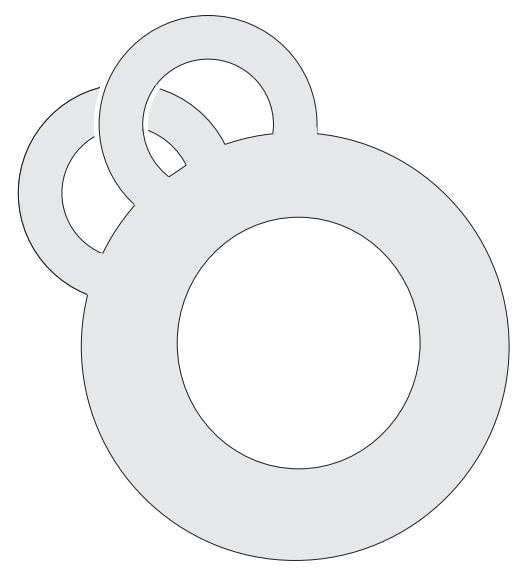

(Exercise : follow the boundary round to see that there are just two circles). 
Example 11.45 An element

$$
x=(\delta \omega, \omega) \in \pi_{n}(S O / S O(n))=\pi_{n}(S O, S O(n))
$$

classifies a map $\omega: S^{n-1} \rightarrow S O(n)$ with a null-homotopy $\delta \omega: \omega \simeq *: S^{n-1} \rightarrow$ $S O$. As in 5.68 twist the embedding

$$
e: S^{n-1} \times D^{n} \hookrightarrow S^{n-1} \times D^{n} \cup D^{n} \times S^{n-1}=S^{2 n-1}
$$

by $\omega$, to define an $n$-surgery

$g_{\omega}: S^{n-1} \times D^{n} \hookrightarrow S^{2 n-1}=S^{n-1} \times D^{n} \cup D^{n} \times S^{n-1} ;(x, y) \mapsto e(x, \omega(x)(y))$

with effect the $(n-1)$-sphere bundle over $S^{n}$

$$
S(\omega)^{2 n-1}=D^{n} \times S^{n-1} \cup_{\omega} D^{n} \times S^{n-1}
$$

classified by $\omega \in \pi_{n-1}(S O(n))=\pi_{n}(B S O(n))$. Use $\delta \omega$ to extend the degree 1 map $S(\omega) \rightarrow S^{2 n-1}$ to a normal bordism

$$
(f, b):\left(M^{2 n} ; S^{2 n-1}, S(\omega)^{2 n-1}\right) \rightarrow S^{2 n-1} \times(I ;\{0\},\{1\})
$$

with $(f, b) \mid=\mathrm{id}$ : $S^{2 n-1} \rightarrow S^{2 n-1}$. This is just the normal bordism of 11.42 for

$$
N^{2 n-1}=S^{2 n-1}, N^{\prime 2 n-1}=S(\omega)^{2 n-1},(K, \lambda, \mu)=(\mathbb{Z}, x),
$$

using the isomorphism of 5.83

$$
\pi_{n}(S O / S O(n)) \cong Q_{(-1)^{n}}(\mathbb{Z})
$$

to identify $x$ with a $(-1)^{n}$-quadratic structure $(\lambda, \mu)$ on $K=\mathbb{Z}$. Under this identification

$$
\lambda=\chi(\omega): \mathbb{Z} \rightarrow \mathbb{Z}^{*}=\mathbb{Z} .
$$

In particular, for the generator $x=1 \in \pi_{n}(S O / S O(n)) \omega$ is the tangent bundle of the $n$-sphere

$$
\omega=\tau_{S^{n}}: S^{n} \rightarrow B S O(n),
$$

and the framing $\delta \omega: \omega \simeq *: S^{n} \rightarrow B S O$ is determined by an embedding $S^{n} \hookrightarrow S^{n+k}$ for $k \geqslant 1$ (as in 10.8). In this case

$$
(K, \lambda, \mu)=\left(\mathbb{Z}, 1+(-1)^{n}, 1\right)
$$

and $N^{\prime 2 n-1}=S(\omega)$ is the $(2 n-1)$-dimensional Stiefel manifold

$$
S(\omega)^{2 n-1}=S O(n+1) / S O(n-1)=V_{n+1,2}
$$

of orthonormal 2-frames in $\mathbb{R}^{n+1}$ (cf. 5.83).

The kernel form $\left(K_{n}(M), \lambda, \mu\right)$ of an $n$-connected $2 n$-dimensional degree 1 normal map $(f, b): M^{2 n} \rightarrow X$ can also be obtained as follows. 
Remark 11.46 Define a presentation of $(f, b)$ to be the $n$-connected $(2 n+1)$ dimensional normal bordism

$$
\left((F, B) ;(f, b),\left(f^{\prime}, b^{\prime}\right)\right):\left(W^{2 n+1} ; M^{2 n}, M^{\prime 2 n}\right) \rightarrow X \times(I ;\{0\},\{1\})
$$

obtained from the product

$$
(f, b) \times \text { id }: M \times(I ;\{0\},\{1\}) \rightarrow X \times(I ;\{0\},\{1\})
$$

by surgery on a basis of the (stably) f.g. free $\mathbb{Z}\left[\pi_{1}(X)\right]$-module

$$
K_{n}(M \times I)=K_{n}(M)
$$

so that $\left(f^{\prime}, b^{\prime}\right)$ is a copy of $(f, b)$. The identification $(2 n+1)$-dimensional degree 1 normal map

$$
(F, B) /\left((f, b)=\left(f^{\prime}, b^{\prime}\right)\right): W /\left(M=M^{\prime}\right) \rightarrow X \times(I / 0 \sim 1)=X \times S^{1}
$$

is an $n$-connected $\mathbb{Z}\left[\pi_{1}(X)\right]$-homology equivalence. The $\mathbb{Z}\left[\pi_{1}(X)\right]$-module morphisms induced by the inclusions $M \hookrightarrow W, M^{\prime} \hookrightarrow W$

$$
\begin{aligned}
& h=\text { inclusion }_{*}: K_{n}(M) \rightarrow K_{n}(W), \\
& h^{\prime}=\text { inclusion }_{*}: K_{n}(M)=K_{n}\left(M^{\prime}\right) \rightarrow K_{n}(W)
\end{aligned}
$$

are such that $h-h^{\prime}: K_{n}(M) \rightarrow K_{n}(W)$ is a $\mathbb{Z}\left[\pi_{1}(X)\right]$-module isomorphism. The $\mathbb{Z}\left[\pi_{1}(X)\right]$-module morphism

$$
\psi: K_{n}(M) \stackrel{\left(h-h^{\prime}\right)^{-1} h}{\longrightarrow} K_{n}(M) \stackrel{\lambda}{\longrightarrow} K_{n}(M)^{*}
$$

defines a split $(-1)^{n}$-quadratic form $\left(K_{n}(M), \psi\right)$ which corresponds to $\left(K_{n}(M)\right.$, $\lambda, \mu)$ under the one-one correspondence of 11.9 (ii).

Example 11.47 Presentations as in 11.46 arise naturally in knot theory. A knot $k: S^{2 n-1} \hookrightarrow S^{2 n+1}$ is simple if $S^{2 n+1} \backslash k\left(S^{2 n-1}\right)$ is $(n-1)$-connected. Such a knot admits a simple Seifert surface which is an $(n-1)$-connected framed codimension 1 submanifold $M^{2 n} \hookrightarrow S^{2 n+1}$ with boundary $\partial M=k\left(S^{2 n-1}\right)$. (The simple Seifert terminology has nothing to do with Whitehead torsion). The inclusion defines an $n$-connected degree 1 normal map

$$
(f, b):\left(M^{2 n}, \partial M\right) \rightarrow\left(D^{2 n+2}, k\left(S^{2 n-1}\right)\right)
$$

which is the identity on the boundary. The knot complement is the $(2 n+1)$ dimensional manifold with boundary defined by

$$
\left(N^{2 n+1}, \partial N\right)=\left(\operatorname{cl} .\left(S^{2 n+1} \backslash\left(k\left(S^{2 n-1}\right) \times D^{2}\right)\right), S^{2 n-1} \times S^{1}\right),
$$

with an $n$-connected $(2 n+1)$-dimensional normal map 


$$
(e, a):\left(N^{2 n+1}, \partial N\right) \rightarrow\left(D^{2 n+2}, k\left(S^{2 n-1}\right)\right) \times S^{1}
$$

which is the identity on the boundary. Cutting $(e, a)$ along $(f, b)$ results in a presentation

$$
\left((F, B) ;(f, b),\left(f^{\prime}, b^{\prime}\right):\left(W^{2 n+1} ; M^{2 n}, M^{\prime 2 n}\right) \rightarrow D^{2 n+2} \times(I ;\{0\},\{1\}) .\right.
$$

A Seifert matrix of the knot is the matrix of the $\mathbb{Z}$-module morphism $\psi$ : $K_{n}(M) \rightarrow K_{n}(M)^{*}$ given by 11.46 , with respect to any basis of the f.g. free $\mathbb{Z}$-module $K_{n}(M)=H_{n}(M)$.

See Ranicki [70, 8.9], [74] for accounts of high-dimensional knot theory from the surgery theoretic point of view.

\subsection{Surgery on forms}

This section develops algebraic surgery on forms. The effect of a geometric $n$ surgery on an $n$-connected $2 n$-dimensional degree 1 normal map is an algebraic surgery on the kernel $(-1)^{n}$-quadratic form. Moreover, geometric surgery is possible if and only if algebraic surgery is possible.

Given an $\epsilon$-quadratic form $(K, \lambda, \mu)$ over a ring with involution $A$ it is possible to kill an element $x \in K$ by algebraic surgery if and only if $x$ is isotropic

$$
\mu(x)=0 \in Q_{\epsilon}(A)
$$

and $x$ generates a direct summand $\langle x\rangle=A x \subset K$, with

$$
\langle x\rangle \subseteq\langle x\rangle^{\perp}=\{y \in K \mid \lambda(x, y)=0\}
$$

The effect of the algebraic surgery is the $\epsilon$-quadratic form

$$
\left(K^{\prime}, \lambda^{\prime}, \mu^{\prime}\right)=\left(\langle x\rangle^{\perp} /\langle x\rangle,[\lambda],[\mu]\right)
$$

More generally, algebraic surgery can be defined for any 'sublagrangian' of $(K, \lambda, \mu)$ :

Definition 11.48 (i) Given an $\epsilon$-symmetric form $(K, \lambda)$ and a submodule $L \subseteq$ $K$ define the orthogonal submodule

$$
\begin{aligned}
L^{\perp} & =\{y \in K \mid \lambda(x, y)=0 \in A \text { for all } x \in L\} \\
& =\operatorname{ker}\left(i^{*} \lambda: K \rightarrow L^{*}\right) \subseteq K
\end{aligned}
$$

with $i: L \rightarrow K$ the inclusion. If $(K, \lambda)$ is nonsingular and $L$ is a direct summand of $K$ then so is $L^{\perp}$. 
(ii) An algebraic surgery on an $\epsilon$-quadratic form $(K, \lambda, \mu)$ over $A$ kills a submodule $L \subseteq K$ such that

$$
\mu(L)=\{0\} \subseteq Q_{\epsilon}(A)
$$

so that

$$
\lambda(L \times L)=\{0\} \subseteq A, L \subseteq L^{\perp} .
$$

The effect of the algebraic surgery is the $\epsilon$-quadratic form

$$
\left(K^{\prime}, \lambda^{\prime}, \mu^{\prime}\right)=\left(L^{\perp} / L,[\lambda],[\mu]\right)
$$

(iii) A sublagrangian of an $\epsilon$-quadratic form $(K, \lambda, \mu)$ over $A$ is a direct summand $L \subseteq K$ such that $\mu(L)=\{0\}$.

(iv) A lagrangian of $(K, \lambda, \mu)$ is a sublagrangian $L$ such that

$$
L^{\perp}=L
$$

so that the effect of the corresponding algebraic surgery is $\left(K^{\prime}, \lambda^{\prime}, \mu^{\prime}\right)=(0,0,0)$.

The main result of this section is that the inclusion of a sublagrangian in a nonsingular $\epsilon$-quadratic form

$$
i:(L, 0,0) \rightarrow(K, \lambda, \mu)
$$

extends to an isomorphism

$$
f: H_{\epsilon}(L) \oplus\left(L^{\perp} / L,[\lambda],[\mu]\right) \rightarrow(K, \lambda, \mu)
$$

with $H_{\epsilon}(L)$ the hyperbolic $\epsilon$-quadratic form (11.6). In particular, it follows that the effect of the algebraic surgery $\left(L^{\perp} / L,[\lambda],[\mu]\right)$ is also nonsingular.

In the classical theory of quadratic forms over fields a lagrangian is a "maximal isotropic subspace". Wall [92] called hyperbolic forms "kernels" and the lagrangians "subkernels". Novikov [64] called hyperbolic forms "hamiltonian", and introduced the name "lagrangian", because of the analogy with the hamiltonian formulation of physics in which the lagrangian expresses a minimum condition.

The algebraic effect of an $n$-surgery on an $n$-connected $2 n$-dimensional degree 1 normal map is an algebraic surgery on the kernel $(-1)^{n}$-quadratic form, and for $n \geqslant 3$ algebraic surgeries can be realized geometrically:

Proposition 11.49 Let $(f, b): M^{2 n} \rightarrow X$ be an $n$-connected $2 n$-dimensional degree 1 normal map, with kernel $(-1)^{n}$-quadratic form $\left(K_{n}(M), \lambda, \mu\right)$ over $\mathbb{Z}\left[\pi_{1}(X)\right]$. (i) An element $x \in K_{n}(M)$ is such that $\mu(x)=0$ if (and for $n \geqslant 3$ only if ) $x$ 
can be killed by an n-surgery on $(f, b)$.

(ii) A degree 1 normal bordism

$$
\left((F, B) ;(f, b),\left(f^{\prime}, b^{\prime}\right)\right):\left(W^{2 n+1} ; M^{2 n}, M^{\prime 2 n}\right) \rightarrow X \times(I ;\{0\},\{1\})
$$

is such that $K_{*}(W, M)=0$ for $* \neq n+1$ if and only if it is the trace of $\ell$ n-surgeries on $(f, b)$ killing $x_{1}, x_{2}, \ldots, x_{\ell} \in K_{n}(M)$, with $K_{n+1}(W, M)=$ $\mathbb{Z}\left[\pi_{1}(X)\right]^{\ell}$. Given such a degree 1 normal bordism let

$$
L=\operatorname{im}\left(K_{n+1}(W, M) \rightarrow K_{n}(M)\right)=\left\langle x_{1}, x_{2}, \ldots, x_{\ell}\right\rangle \subseteq K_{n}(M) .
$$

The map $F: W \rightarrow X \times I$ is n-connected, the map $f^{\prime}: M^{\prime} \rightarrow X$ is $(n-1)$ connected, and the kernel $\mathbb{Z}\left[\pi_{1}(X)\right]$-modules fit into a commutative braid of exact sequences

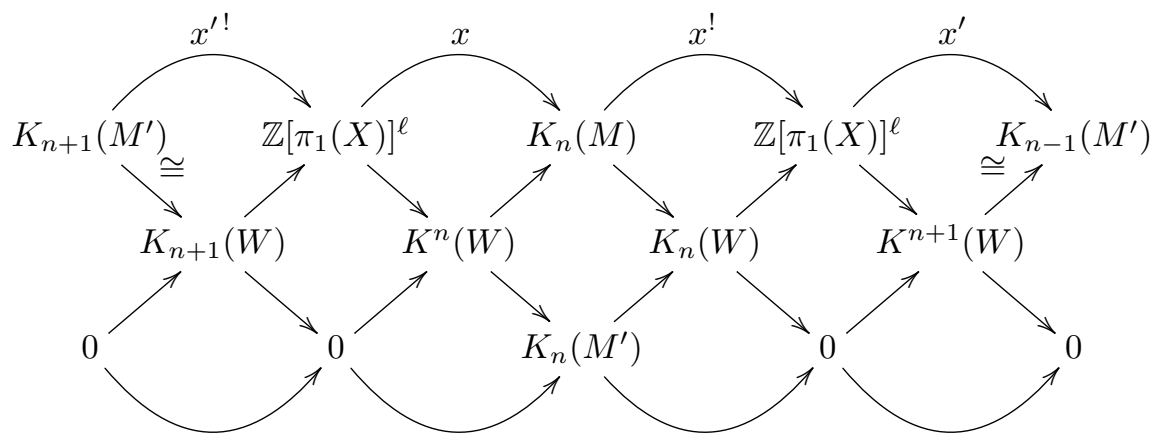

with

$$
\begin{aligned}
& x^{!}: K_{n}(M) \rightarrow \mathbb{Z}\left[\pi_{1}(X)\right]^{\ell} ; y \mapsto\left(\lambda\left(x_{1}, y\right), \lambda\left(x_{2}, y\right), \ldots, \lambda\left(x_{\ell}, y\right)\right), \\
& K_{n-1}\left(M^{\prime}\right)=\operatorname{coker}\left(x^{!}: K_{n}(M) \rightarrow \mathbb{Z}\left[\pi_{1}(X)\right]^{\ell}\right), \\
& \left(K_{n}\left(M^{\prime}\right), \lambda^{\prime}, \mu^{\prime}\right)=\left(L^{\perp} / L,[\lambda],[\mu]\right), \\
& K_{n+1}\left(M^{\prime}\right)=\operatorname{ker}\left(x: \mathbb{Z}\left[\pi_{1}(X)\right]^{\ell} \rightarrow K_{n}(M)\right), \\
& K_{n}(W)=K_{n}(M) / L, K^{n}(W)=L^{\perp} .
\end{aligned}
$$

$L$ is a sublagrangian of $\left(K_{n}(M), \lambda, \mu\right)$ if and only if $\left(f^{\prime}, b^{\prime}\right)$ is $n$-connected, in which case

$$
K_{n+1}(W, M)=\mathbb{Z}\left[\pi_{1}(X)\right]^{\ell}=L, K_{n+1}\left(M^{\prime}\right)=K_{n-1}\left(M^{\prime}\right)=0 .
$$

$L$ is a lagrangian of $\left(K_{n}(M), \lambda, \mu\right)$ if and only if $\left(f^{\prime}, b^{\prime}\right)$ is a homotopy equivalence.

(iii) The kernel form $\left(K_{n}(M), \lambda, \mu\right)$ admits a lagrangian if (and for $n \geqslant 3$ only if ) there exists a normal bordism $(F, B): W \rightarrow X \times I$ of $(f, b)$ to a homotopy equivalence $\left(f^{\prime}, b^{\prime}\right)$ with $K_{*}(W, M)=0$ for $* \neq n+1$. 
Proof (i) Immediate from the Wall Embedding Theorem 11.25.

(ii) This is a special case of Proposition 10.23, noting that $K_{*}(W, M)=0$ for $* \neq n+1$ if and only if $W$ has a handle decomposition on $M$ of the type

$$
W=M \times I \cup \bigcup_{\ell}(n+1) \text {-handles } D^{n+1} \times D^{n},
$$

with $K_{n+1}(W, M)=\mathbb{Z}\left[\pi_{1}(X)\right]^{\ell}$.

(iii) Combine (i) and (ii).

Example 11.50 The effect of an $n$-surgery on an $n$-connected $2 n$-dimensional degree 1 normal map $(f, b): M^{2 n} \rightarrow X$ killing $0 \in K_{n}(M)$ is the $(n-1)$-connected degree 1 normal map

$$
\left(f^{\prime}, b^{\prime}\right)=(f, b) \# 1: M^{\prime 2 n}=M \#\left(S^{n+1} \times S^{n-1}\right) \rightarrow X
$$

with

$$
K_{n-1}\left(M^{\prime}\right)=K_{n+1}\left(M^{\prime}\right)=\mathbb{Z}\left[\pi_{1}(X)\right], K_{n}\left(M^{\prime}\right)=K_{n}(M)
$$

Theorem 11.51 A nonsingular $\epsilon$-quadratic form $(K, \lambda, \mu)$ admits a lagrangian $L$ if and only if it is isomorphic to the hyperbolic form $H_{\epsilon}(L)$.

Proof An isomorphism of forms sends lagrangians to lagrangians, so any form isomorphic to a hyperbolic form has at least one lagrangian. In proving the converse, it is convenient to use the language of split $\epsilon$-quadratic forms of 11.1 replacing $(\lambda, \mu)$ by $\psi \in Q_{\epsilon}(K)$ (as in 11.9) and choosing a representative $\psi \in$ $S(K)$. Suppose that

$$
(i, \theta):(L, 0) \rightarrow(K, \psi)
$$

is the inclusion of a lagrangian of $(K, \lambda, \mu)$. An extension of $(i, \theta)$ to an isomorphism

$$
(f, \chi): H_{\epsilon}(L) \rightarrow(K, \psi)
$$

determines a lagrangian $f\left(L^{*}\right) \subset K$ complementary to $L$. Construct an isomorphism $(f, \chi)$ by first choosing a complementary lagrangian to $L$ in $(K, \psi)$. Let $i \in \operatorname{Hom}_{A}(L, K)$ be the inclusion, and choose a splitting $j^{\prime} \in \operatorname{Hom}_{A}\left(L^{*}, K\right)$ of $i^{*}\left(\psi+\epsilon \psi^{*}\right) \in \operatorname{Hom}_{A}\left(K, L^{*}\right)$, so that

$$
i^{*}\left(\psi+\epsilon \psi^{*}\right) j^{\prime}=1 \in \operatorname{Hom}_{A}\left(L^{*}, L^{*}\right) .
$$

In general, $j^{\prime}: L^{*} \rightarrow K$ is not the inclusion of a lagrangian, with $j^{\prime *} \psi j^{\prime} \neq 0 \in$ $Q_{\epsilon}\left(L^{*}\right)$. Given any $k \in \operatorname{Hom}_{A}\left(L^{*}, L\right)$ there is defined another splitting

$$
j=j^{\prime}+i k: L^{*} \rightarrow K
$$


such that

$$
\begin{aligned}
j^{*} \psi j & =j^{\prime *} \psi j^{\prime}+k^{*} i^{*} \psi i k+k^{*} i^{*} \psi j^{\prime}+j^{\prime *} \psi i k \\
& =j^{\prime *} \psi j^{\prime}+k \in Q_{\epsilon}\left(L^{*}\right) .
\end{aligned}
$$

Choose a representative $\psi \in \operatorname{Hom}_{A}\left(K, K^{*}\right)$ of $\psi \in Q_{\epsilon}(K)$ and set

$$
k=-j^{*} \psi j^{\prime}: L^{*} \rightarrow L .
$$

The corresponding splitting $j: L^{*} \rightarrow K$ is the inclusion of a lagrangian

$$
(j, \nu):\left(L^{*}, 0\right) \rightarrow(K, \psi)
$$

which extends to an isomorphism of split $\epsilon$-quadratic forms

$$
\left((i j),\left(\begin{array}{cc}
\theta & 0 \\
j^{*} \psi i & \nu
\end{array}\right)\right): H_{\epsilon}(L) \rightarrow(K, \psi) .
$$

Theorem 11.51 is a generalisation of Witt's theorem on the extension to isomorphism of an isometry of quadratic forms over fields. The procedure for modifying the choice of complement to be a lagrangian is a generalisation of the Gram-Schmidt method of constructing orthonormal bases in an inner product space.

Corollary 11.52 The inclusion of a lagrangian in a nonsingular $\epsilon$-quadratic form

$$
i:(L, 0,0) \rightarrow(M, \lambda, \mu)
$$

extends to an isomorphism of forms

$$
f: H_{\epsilon}(L) \rightarrow(M, \lambda, \mu) .
$$

Proof The proof of 11.51 gives an explicit extension.

Proposition 11.53 The inclusion $i:(L, 0,0) \rightarrow(K, \lambda, \mu)$ of a sublagrangian in a nonsingular $\epsilon$-quadratic form $(K, \psi)$ extends to an isomorphism of forms

$$
f: H_{\epsilon}(L) \oplus\left(L^{\perp} / L,[\lambda],[\mu]\right) \rightarrow(K, \lambda, \mu) .
$$

Proof Again, use the language of split $\epsilon$-quadratic forms, writing the inclusion of the sublagrangian as

$$
(i, \theta):(L, 0) \rightarrow(K, \psi)
$$

For any direct complement $L_{1}$ to $L^{\perp}$ in $K$ there is defined an $A$-module isomorphism 


$$
h: L_{1} \rightarrow L^{*} ; x \mapsto\left(y \mapsto\left(1+T_{\epsilon}\right) \psi(x)(y)\right) .
$$

Define an $A$-module morphism

$$
j: L^{*} \stackrel{h^{-1}}{\longrightarrow} L_{1} \stackrel{\text { inclusion }}{\longrightarrow} K .
$$

The nonsingular split $\epsilon$-quadratic form defined by

$$
(H, \phi)=\left(L \oplus L^{*},\left(\begin{array}{cc}
0 & 1 \\
0 & j^{*} \psi j
\end{array}\right)\right)
$$

has lagrangian $L$, so that it is isomorphic to the hyperbolic form $H_{\epsilon}(L)$ by 11.52 . Also, there is defined a morphism of split $\epsilon$-quadratic forms

$$
\left(g=(i j),\left(\begin{array}{cc}
\theta & i^{*} \psi j \\
0 & 0
\end{array}\right)\right):(H, \phi) \rightarrow(K, \psi)
$$

with $g: H \rightarrow K$ an injection split by

$$
h=\left(\left(1+T_{\epsilon}\right) \phi\right)^{-1} g^{*}\left(1+T_{\epsilon}\right) \psi: K \rightarrow H .
$$

The direct summand of $K$ defined by

$$
\begin{aligned}
H^{\perp} & =\left\{x \in K \mid\left(1+T_{\epsilon}\right) \psi(x)(g y)=0 \text { for all } y \in H\right\} \\
& =\operatorname{ker}\left(g^{*}\left(1+T_{\epsilon}\right) \psi: K \rightarrow H^{*}\right) \\
& =\operatorname{ker}(h: K \rightarrow H)
\end{aligned}
$$

is such that

$$
K=g(H) \oplus H^{\perp} .
$$

It follows from the factorisation

$$
i^{*}\left(1+T_{\epsilon}\right) \psi: K \stackrel{h}{\longrightarrow} H=L \oplus L^{*} \stackrel{\text { projection }}{\longrightarrow} L^{*}
$$

that

$$
L^{\perp}=\operatorname{ker}\left(i^{*}\left(1+T_{\epsilon}\right) \psi: K \rightarrow L^{*}\right)=L \oplus H^{\perp} .
$$

The restriction of $\psi \in S(K)$ to $H^{\perp}$ defines a nonsingular split $\epsilon$-quadratic form $\left(H^{\perp}, \phi^{\perp}\right)$. Define an extension of $g$ to an isomorphism of split forms

$$
\left(f=\left(g g^{\perp}\right), \chi\right):(H, \phi) \oplus\left(H^{\perp}, \phi^{\perp}\right) \rightarrow(K, \psi)
$$

with $g^{\perp}: H^{\perp} \rightarrow K$ the inclusion, and with $\left(H^{\perp}, \phi^{\perp}\right)$ isomorphic to $\left(L^{\perp} / L,[\psi]\right)$. 
Example 11.54 An $n$-connected $(2 n+1)$-dimensional degree 1 normal bordism

$$
\left((F, B) ;(f, b),\left(f^{\prime}, b^{\prime}\right)\right):\left(W^{2 n+1} ; M^{2 n}, M^{\prime 2 n}\right) \rightarrow X \times(I ;\{0\},\{1\})
$$

is such that $W$ has a handle decomposition on $M$ of the type

$$
W=M \times I \cup \bigcup_{k} n \text {-handles } D^{n} \times D^{n+1} \cup \bigcup_{\ell}(n+1) \text {-handles } D^{n+1} \times D^{n} .
$$

Let

$$
\left(W ; M, M^{\prime}\right)=\left(W^{\prime} ; M, N\right) \cup_{N}\left(W^{\prime \prime} ; N, M^{\prime}\right)
$$

with

$$
\begin{aligned}
& W^{\prime}=M \times I \cup \bigcup_{k} n \text {-handles } D^{n} \times D^{n+1}, \\
& W^{\prime \prime}=N \times I \cup \bigcup_{\ell}(n+1) \text {-handles } D^{n+1} \times D^{n} .
\end{aligned}
$$

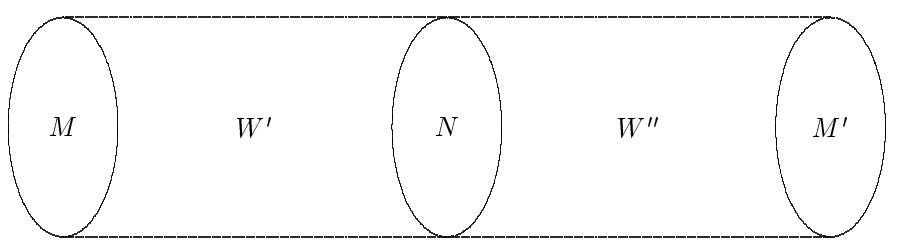

W

The restriction of $(F, B)$ to $N$ is an $n$-connected $2 n$-dimensional degree 1 normal map

$$
\left.\left.\left(f_{N}, b_{N}\right): N \cong M \underset{k}{\#} \underset{k}{\#} S^{n} \times S^{n}\right) \cong \underset{\ell}{\#} M^{\prime} \underset{S^{n}}{\#} S^{n}\right) \rightarrow X
$$

with kernel $(-1)^{n}$-quadratic form

$$
\begin{aligned}
\left(K_{n}(N), \lambda_{N}, \mu_{N}\right) & \cong\left(K_{n}(M), \lambda, \mu\right) \oplus H_{(-1)^{n}}\left(\mathbb{Z}\left[\pi_{1}(X)\right]^{k}\right) \\
& \cong\left(K_{n}\left(M^{\prime}\right), \lambda^{\prime}, \mu^{\prime}\right) \oplus H_{(-1)^{n}}\left(\mathbb{Z}\left[\pi_{1}(X)\right]^{\ell}\right)
\end{aligned}
$$

Thus $\left(K_{n}(N), \lambda_{N}, \mu_{N}\right)$ has sublagrangians

$$
\begin{aligned}
& L=\operatorname{im}\left(K_{n+1}\left(W^{\prime}, N\right) \rightarrow K_{n}(N)\right) \cong \mathbb{Z}\left[\pi_{1}(X)\right]^{k}, \\
& L^{\prime}=\operatorname{im}\left(K_{n+1}\left(W^{\prime \prime}, N\right) \rightarrow K_{n}(N)\right) \cong \mathbb{Z}\left[\pi_{1}(X)\right]^{\ell} \subseteq K_{n}(N)
\end{aligned}
$$

such that

$$
\begin{aligned}
& \left(L^{\perp} / L,\left[\lambda_{N}\right],\left[\mu_{N}\right]\right) \cong\left(K_{n}(M), \lambda, \mu\right), \\
& \left(L^{\prime \perp} / L^{\prime},\left[\lambda_{N}\right]^{\prime},\left[\mu_{N}\right]^{\prime}\right) \cong\left(K_{n}\left(M^{\prime}\right), \lambda^{\prime}, \mu^{\prime}\right) .
\end{aligned}
$$

Note that $L$ is a lagrangian if and only if $(f, b): M \rightarrow X$ is a homotopy equivalence. Similarly for $L^{\prime}$ and $\left(f^{\prime}, b^{\prime}\right): M^{\prime} \rightarrow X$. 
Definition 11.55 The nonsingular $\epsilon$-quadratic forms $(K, \lambda, \mu),\left(K^{\prime}, \lambda^{\prime}, \mu^{\prime}\right)$ are cobordant if there exists an isomorphism

$$
(K, \lambda, \mu) \oplus H_{\epsilon}(F) \cong\left(K^{\prime}, \lambda^{\prime}, \mu^{\prime}\right) \oplus H_{\epsilon}\left(F^{\prime}\right)
$$

for some f.g. free $A$-modules $F, F^{\prime}$.

Proposition 11.56 (i) Cobordism is an equivalence relation on nonsingular $\epsilon$ quadratic forms $(K, \lambda, \mu)$ over $A$.

(ii) $(K, \lambda, \mu) \oplus(K,-\lambda,-\mu)$ is cobordant to 0 , for any nonsingular $\epsilon$-quadratic form $(K, \lambda, \mu)$ over $A$ with $K$ f.g. free.

Proof (i) Clear.

(ii) The diagonal is a lagrangian

$$
\Delta=\{(x, x) \in K \oplus K \mid x \in K\}
$$

of $(K \oplus K, \lambda \oplus-\lambda, \mu \oplus-\mu)$. For any split $\epsilon$-quadratic structure $\psi \in S(K)$ representing $(\lambda, \mu)$ apply 11.52 to obtain an extension of the inclusion $\Delta \hookrightarrow$ $K \oplus K$ to an isomorphism of $\epsilon$-quadratic forms

$$
\left(\begin{array}{cc}
1 & -\epsilon \psi^{*} \\
1 & \psi
\end{array}\right): H_{\epsilon}(K) \rightarrow(K, \lambda, \mu) \oplus(K,-\lambda,-\mu) .
$$

\subsection{The even-dimensional $L$-groups}

The even-dimensional surgery obstruction groups $L_{2 *}(A)$ are now defined using forms. The surgery obstruction of an even-dimensional normal map will be defined in Section 11.5 using the kernel form of Section 11.2.

Definition 11.57 The $2 n$-dimensional $L$-group $L_{2 n}(A)$ of a ring with involution $A$ is the group of cobordism classes of nonsingular $(-1)^{n}$-quadratic forms $(K, \lambda, \mu)$ on f.g. free $A$-modules. The addition and inverses in $L_{2 n}(A)$ are given by

$$
\begin{gathered}
\left(K_{1}, \lambda_{1}, \mu_{1}\right)+\left(K_{2}, \lambda_{2}, \mu_{2}\right)=\left(K_{1} \oplus K_{2}, \lambda_{1} \oplus \lambda_{2}, \mu_{1} \oplus \mu_{2}\right) \\
-(K, \lambda, \mu)=(K,-\lambda,-\mu) \in L_{2 n}(A) .
\end{gathered}
$$

It should be clear that $L_{2 n}(A)$ depends only on the residue $n(\bmod 2)$, so that only two $L$-groups have actually been defined, $L_{0}(A)$ and $L_{2}(A)$. 
The surgery obstruction of an $n$-connected $2 n$-dimensional degree 1 normal map $(f, b): M^{2 n} \rightarrow X$ will be defined in Section 11.5 to be the element

$$
\sigma_{*}(f, b)=\left(K_{n}(M), \lambda, \mu\right) \in L_{2 n}\left(\mathbb{Z}\left[\pi_{1}(X)\right]\right)
$$

represented by the kernel form of 11.37, such that $\sigma_{*}(f, b)=0$ if and only if $(f, b)$ is bordant to a homotopy equivalence.

Proposition $\mathbf{1 1 . 5 8}$ The even-dimensional L-groups of $\mathbb{R}$ are given by

$$
L_{2 n}(\mathbb{R})= \begin{cases}\mathbb{Z} & \text { if } n \equiv 0(\bmod 2) \\ 0 & \text { if } n \equiv 1(\bmod 2) .\end{cases}
$$

Proof Since $1 / 2 \in \mathbb{R}$ there is no difference between symmetric and quadratic forms over $\mathbb{R}$. If $(K, \lambda)$ is a nonsingular $(-1)^{n}$-symmetric form over $\mathbb{R}$ then for any $x \neq 0 \in K$ with $\lambda(x, x)=0 \in \mathbb{R}$ there exists $y \in K$ with $\lambda(y, y)=0$, $\lambda(x, y)=1$, so that

$$
(K, \lambda)=H_{(-1)^{n}}(\mathbb{R}) \oplus\left(K^{\prime}, \lambda^{\prime}\right)
$$

with $K^{\prime}=\langle x, y\rangle^{\perp}$.

Consider first the case $n \equiv 0(\bmod 2)$. The signature of a nonsingular symmetric form $(K, \lambda)$ over $\mathbb{R}(6.30)$ is defined by

$$
\begin{aligned}
& \sigma(K, \lambda) \\
& =\text { no. of positive eigenvalues of } \lambda-\text { no. of negative eigenvalues of } \lambda \in \mathbb{Z} .
\end{aligned}
$$

In particular, $\sigma(\mathbb{R}, 1)=1$. The symmetric form $\lambda \in Q^{+}(K)$ is identified with the symmetric $k \times k$ matrix $\left(\lambda\left(x_{i}, x_{j}\right)_{1 \leqslant i, j \leqslant k}\right) \in M_{k, k}(\mathbb{R})$ determined by any choice of basis $x_{1}, x_{2}, \ldots, x_{k}$ for $K$. By Sylvester's Law of Inertia (6.33) the rank of $K$ and the signature define a complete set of invariants for the isomorphism classification of nonsingular symmetric forms $(K, \lambda)$ on finite-dimensional vector spaces $K$ over $\mathbb{R}$, meaning that two forms are isomorphic if and only if they have the same rank and signature. A nonsingular symmetric form $(K, \lambda)$ over $\mathbb{R}$ is isomorphic to a hyperbolic form if and only if it has signature 0 . Nonsingular symmetric forms $(K, \lambda),\left(K^{\prime}, \lambda^{\prime}\right)$ over $\mathbb{R}$ are related by an isomorphism

$$
(K, \lambda) \oplus H_{+}\left(\mathbb{R}^{d}\right) \cong\left(K^{\prime}, \lambda^{\prime}\right) \oplus H_{+}\left(\mathbb{R}^{d^{\prime}}\right)
$$

for some $d, d^{\prime} \geqslant 0$ if and only if they have the same signature

$$
\sigma(K, \lambda)=\sigma\left(K^{\prime}, \lambda^{\prime}\right) \in \mathbb{Z},
$$

so that the signature defines an isomorphism

$$
L_{0}(\mathbb{R}) \rightarrow \mathbb{Z} ;(K, \lambda) \mapsto \sigma(K, \lambda)
$$

For $n \equiv 1(\bmod 2)$ we have $L_{2}(\mathbb{R})=0$ because every nonsingular skewsymmetric (alias symplectic) form over $\mathbb{R}$ admits a lagrangian, and is thus isomorphic to a hyperbolic form. 
See Chapter II of Milnor and Husemoller [60] for what is known about the isomorphism classification of nonsingular symmetric and quadratic forms over $\mathbb{Z}$. For the applications to surgery it suffices to know if two forms become isomorphic after adding hyperbolics.

Definition 11.59 The signature of a nonsingular symmetric form $(K, \lambda)$ over $\mathbb{Z}$ is the signature of the induced nonsingular symmetric form over $\mathbb{R}$

$$
\sigma(K, \lambda)=\sigma(\mathbb{R} \otimes K, 1 \otimes \lambda) \in \mathbb{Z}
$$

In particular, $\sigma(\mathbb{Z}, 1)=1$.

Definition 11.60 The Arf invariant of a nonsingular quadratic form over $\mathbb{Z}_{2}$ $(K, \lambda, \mu)$ is given by

$$
c(K, \lambda, \mu)=\sum_{i=1}^{m} \mu\left(x_{i}\right) \mu\left(x_{i+m}\right) \in \mathbb{Z}_{2}
$$

for any symplectic basis $x_{1}, \ldots, x_{2 m}$ for $K$, i.e. a basis such that

$$
\lambda\left(x_{i}, x_{j}\right)= \begin{cases}1 & \text { if }|i-j|=m \\ 0 & \text { otherwise }\end{cases}
$$

The original reference is Arf [4].

Proposition 11.61 The even-dimensional L-groups of $\mathbb{Z}$ are given by

$$
L_{2 n}(\mathbb{Z})= \begin{cases}\mathbb{Z} & \text { if } n \equiv 0(\bmod 2) \\ \mathbb{Z}_{2} & \text { if } n \equiv 1(\bmod 2) .\end{cases}
$$

Proof Consider first the case $n \equiv 0(\bmod 2)$. By Serre $[79](\S 1$, Chapter V) the signature of a nonsingular even symmetric form $(K, \lambda)$ is divisible by 8

$$
\sigma(K, \lambda) \equiv 0(\bmod 8)
$$

Two such forms $(K, \lambda),\left(K^{\prime} \lambda^{\prime}\right)$ are related by an isomorphism

$$
(K, \lambda) \oplus H_{+}\left(\mathbb{Z}^{k}\right) \equiv\left(K^{\prime}, \lambda^{\prime}\right) \oplus H_{+}\left(\mathbb{Z}^{\ell}\right)
$$

if and only if they have the same signature

$$
\sigma(K, \lambda)=\sigma\left(K^{\prime}, \lambda^{\prime}\right) \in 8 \mathbb{Z} \subset \mathbb{Z} .
$$

The nonsingular even symmetric form $\left(\mathbb{Z}^{8}, E_{8}\right)$ associated to the exceptional Lie group $E_{8}$ 


$$
E_{8}=\left(\begin{array}{llllllll}
2 & 0 & 0 & 1 & 0 & 0 & 0 & 0 \\
0 & 2 & 1 & 0 & 0 & 0 & 0 & 0 \\
0 & 1 & 2 & 1 & 0 & 0 & 0 & 0 \\
1 & 0 & 1 & 2 & 1 & 0 & 0 & 0 \\
0 & 0 & 0 & 1 & 2 & 1 & 0 & 0 \\
0 & 0 & 0 & 0 & 1 & 2 & 1 & 0 \\
0 & 0 & 0 & 0 & 0 & 1 & 2 & 1 \\
0 & 0 & 0 & 0 & 0 & 0 & 1 & 2
\end{array}\right): \mathbb{Z}^{8} \longrightarrow\left(\mathbb{Z}^{8}\right)^{*}
$$

has signature

$$
\sigma\left(\mathbb{Z}^{8}, E_{8}\right)=8 \in \mathbb{Z} .
$$

Thus $L_{0}(\mathbb{Z})=\mathbb{Z}$, with an isomorphism

$$
L_{0}(\mathbb{Z}) \rightarrow \mathbb{Z} ;(K, \lambda) \mapsto \sigma(K, \lambda) / 8
$$

See Chapter III.1 of Browder [14] for the classification of quadratic forms over $\mathbb{Z}_{2}$ which shows that the Arf invariant defines an isomorphism

$$
L_{2}\left(\mathbb{Z}_{2}\right) \rightarrow \mathbb{Z}_{2} ;(K, \lambda, \mu) \mapsto \operatorname{Arf}(K, \lambda, \mu)
$$

and also that the natural map

$$
L_{2}(\mathbb{Z}) \rightarrow L_{2}\left(\mathbb{Z}_{2}\right)=\mathbb{Z}_{2} ;(K, \lambda, \mu) \mapsto \mathbb{Z}_{2} \otimes_{\mathbb{Z}}(K, \lambda, \mu)
$$

defines an isomorphism. Thus $L_{2}(\mathbb{Z})$ is generated by the nonsingular skewquadratic form $(\mathbb{Z} \oplus \mathbb{Z}, \lambda, \mu)$ over $\mathbb{Z}$ defined by

$$
\begin{aligned}
& \lambda((a, b),(c, d))=a d-b c \in \mathbb{Z}, \\
& \mu(a, b)=a+b+a b \in Q_{-1}(\mathbb{Z})=\mathbb{Z}_{2}
\end{aligned}
$$

such that the induced form over $\mathbb{Z}_{2}$ has Arf invariant 1.

Example 11.62 For $k \geqslant 2$ let

$$
(F, B):\left(W^{4 k} ; S^{4 k-1}, \Sigma^{4 k-1}\right) \rightarrow S^{4 k-1} \times(I ;\{0\},\{1\})
$$

be the $4 k$-dimensional normal bordism realizing the nonsingular quadratic form $(K, \lambda, \mu)=\left(\mathbb{Z}^{8}, E_{8}\right)$ over $\mathbb{Z}$ generating $L_{4 k}(\mathbb{Z})=\mathbb{Z}$. This can be obtained by plumbing together (11.43) 8 copies of the tangent bundle $\tau_{S^{2 k}}: S^{2 k} \rightarrow B S O(2 k)$ of the $2 k$-sphere $S^{2 k}$, using the $E_{8}$-graph

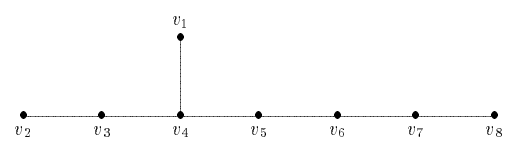

with incidence matrix $E_{8}$, weighted at each vertex by

$$
1 \in \pi_{2 k+1}(B S O, B S O(2 k))=Q_{+}(\mathbb{Z})=\mathbb{Z} .
$$

The effect of the corresponding $8(2 k-1)$-surgeries on $(F, B) \mid=$ id. $: S^{4 k-1} \rightarrow$ $S^{4 k-1}$ is a homotopy equivalence $(F, B) \mid: \Sigma^{4 k-1} \rightarrow S^{4 k-1}$ with $\Sigma^{4 k-1}$ an exotic 
$(4 k-1)$-dimensional sphere generating the cyclic subgroup $b P_{4 k} \subseteq \Theta^{4 k-1}$ (13.22) of the exotic spheres which bound framed $4 k$-manifolds. In particular, for $k=2$ this gives one of the exotic 7 -spheres $\Sigma^{7}$ originally constructed by Milnor [49] (cf. Section 13.3 below and also Example 11.44).

Example 11.63 As in 5.72 for any map $\omega: S^{4} \rightarrow S O(5)$ surgery on $S^{9}$ removing the embedding

$$
g_{\omega}: S^{4} \times D^{5} \hookrightarrow S^{9}=S^{4} \times D^{5} \cup D^{5} \times S^{4} ;(x, y) \mapsto(x, \omega(x)(y))
$$

is a 10-dimensional cobordism $\left(W^{10} ; S^{9}, S(\omega)\right)$ with $S(\omega)$ the sphere bundle of $\omega: S^{5} \rightarrow B S O(5)$. For the unique non-zero element

$$
\omega \in \operatorname{ker}\left(\pi_{4}(S O(5)) \rightarrow \pi_{4}(S O)\right)=\mathbb{Z}_{2}
$$

and the trace of framed 4-surgeries removing two disjoint linked embeddings

$$
g_{\omega}, g_{\omega}^{\prime}: S^{4} \times D^{5} \hookrightarrow S^{9}
$$

the standard framing of $S^{9}$ extends to a framing $b$ of $W^{10}$ such that

$$
\left(H_{5}\left(W ; \mathbb{Z}_{2}\right), \lambda, \mu_{b}\right)=\left(\mathbb{Z}_{2} \oplus \mathbb{Z}_{2},\left(\begin{array}{ll}
0 & 1 \\
1 & 0
\end{array}\right), \text { Arf form }\right)
$$

The sphere bundle $S(\omega)=\Sigma^{9}$ is the exotic sphere of Kervaire [36], such that $D^{10} \cup W \cup D^{10}$ is a closed 10-dimensional $P L$ manifold without differentiable structure.

Definition 11.64 Let $\mathbb{C}$ be the ring of complex numbers, with the complex conjugation involution

$$
\overline{x+i y}=x-i y \quad(x, y \in \mathbb{R}) .
$$

The signature of a $(-1)^{n}$-symmetric form $(K, \lambda)$ over $\mathbb{C}$ is defined by $\sigma(K, \lambda)=$

no. of positive eigenvalues of $i^{n} \lambda$ - no. of negative eigenvalues of $i^{n} \lambda \in \mathbb{Z}$ with $n=0,1$.

Proposition 11.65 The even-dimensional L-groups of $\mathbb{C}$ with the complex conjugation involution $\bar{i}=-i$ are given by

$$
L_{2 n}(\mathbb{C})=\mathbb{Z}
$$

Proof As for the real case (11.58), with the signature defining isomorphisms

$$
L_{2 n}(\mathbb{C}) \rightarrow \mathbb{Z} ;(K, \lambda, \mu) \mapsto \sigma(K, \lambda) .
$$


The rest of this section is devoted to a brief introduction to the even-dimensional $L$-groups of finite group rings.

For a finite group $\pi$ and $F=\mathbb{R}, \mathbb{C}, \mathbb{H}$ let $\alpha_{F}(\pi)$ be the number of irreducible real representations of $\pi$ which correspond to a type $F$ summand in the Wedderburn decomposition of $\mathbb{R}[\pi]$ as a product of matrix algebras

$$
\mathbb{R}[\pi]=\bigoplus_{i=1}^{\alpha_{\mathbb{R}}(\pi)} M_{d_{i}(\mathbb{R}, \pi)}(\mathbb{R}) \oplus \bigoplus_{j=1}^{\alpha_{\mathbb{C}}(\pi)} M_{d_{j}(\mathbb{C}, \pi)}(\mathbb{C}) \oplus \bigoplus_{k=1}^{\alpha_{\mathbb{H}}(\pi)} M_{d_{k}(\mathbb{H}, \pi)}(\mathbb{H})
$$

with

$$
|\pi|=\sum_{i=1}^{\alpha_{\mathbb{R}}(\pi)} d_{i}(\mathbb{R}, \pi)^{2}+2 \sum_{j=1}^{\alpha_{\mathbb{C}}(\pi)} d_{j}(\mathbb{C}, \pi)^{2}+4 \sum_{k=1}^{\alpha_{\mathbb{H}}(\pi)} d_{k}(\mathbb{H}, \pi)^{2} .
$$

The sum

$$
\begin{aligned}
r(\pi) & =\alpha_{\mathbb{R}}(\pi)+\alpha_{\mathbb{C}}(\pi)+\alpha_{\mathbb{H}}(\pi) \\
& =\text { no. of conjugacy classes of unordered pairs }\left\{g, g^{-1}\right\} \text { in } \pi
\end{aligned}
$$

is the number of irreducible real representations of $\pi$ (as in Example 8.8). Every $\epsilon$-quadratic form $(K, \lambda, \mu)$ over $\mathbb{R}[\pi]$ with the oriented involution $\bar{g}=g^{-1}(g \in \pi)$ has a corresponding splitting. For each summand $M_{d}(F) \subset \mathbb{R}[\pi]$ there is defined an $\epsilon$-quadratic form $F^{d} \otimes_{R[\pi]}(K, \lambda, \mu)$ over $F$, regarding $F^{d}$ as an $\left(F, M_{d}(F)\right)$ bimodule (Morita theory). For $F=\mathbb{R}$ (with $\epsilon=1$ ) and $F=\mathbb{C}(\epsilon= \pm 1$ ) this gives a signature $\sigma \in \mathbb{Z}$ as in Definitions 6.30, 11.64.

Definition 11.66 For $\epsilon=(-1)^{n}$ the multisignature of a nonsingular $\epsilon$-quadratic form $(K, \lambda, \mu)$ over $\mathbb{R}[\pi]$ is the collection of signatures

$$
\sigma_{*}(K, \lambda, \mu)=\left\{\begin{array}{cl}
\left(\sum_{i=1}^{\alpha_{\mathbb{R}}(\pi)} \sigma_{i}(K, \lambda, \mu), \sum_{j=1}^{\alpha_{\mathbb{C}}(\pi)} \sigma_{j}(K, \lambda, \mu)\right) & \\
\in \bigoplus_{\alpha_{\mathbb{R}}(\pi)} \mathbb{Z} \oplus \bigoplus_{\alpha_{\mathbb{C}}(\pi)} \mathbb{Z} & \text { if } n \equiv 0(\bmod 2) \\
\sum_{j=1}^{\alpha_{\mathbb{C}}(\pi)} \sigma_{j}(K, \lambda, \mu) \in \bigoplus_{\alpha_{\mathbb{C}}(\pi)} \mathbb{Z} & \text { if } n \equiv 1(\bmod 2)
\end{array}\right.
$$

one for each of the $\alpha_{\mathbb{R}}(\pi)$ summands $M_{d_{i}}(\mathbb{R}) \subset \mathbb{R}[\pi](\epsilon=+1)$ and one for each of the $\alpha_{\mathbb{C}}(\pi)$ summands $M_{d_{j}}(\mathbb{C}) \subset \mathbb{R}[\pi](\epsilon= \pm 1)$.

Proposition 11.67 (Wall [92, Chap.13A], [93]) The even-dimensional surgery obstruction groups of a finite group $\pi$ with the oriented involution are such that

$$
L_{2 n}(\mathbb{Z}[\pi])= \begin{cases}\bigoplus{ }^{\oplus} \mathbb{Z} \oplus(2 \text {-primary torsion }) & \text { if } n \equiv 0(\bmod 2) \\ \bigoplus_{\mathbb{R}}(\pi)+\alpha_{\mathbb{C}}(\pi) & \text { if } n \equiv 1(\bmod 2)\end{cases}
$$

with the $\mathbb{Z}$-summands detected by the multisignature. 
Proof The original references may be supplemented by Chapter 22 of Ranicki [71] for the (easy) torsion-free part.

Remark 11.68 See Hambleton and Taylor [30] for a survey of the computations of the $L$-groups of finite groups.

Example 11.69 (i) The even-dimensional $L$-groups of $\mathbb{Z}\left[\mathbb{Z}_{2}\right]$ with the oriented involution $\bar{T}=T$ are given by

$$
L_{2 n}\left(\mathbb{Z}\left[\mathbb{Z}_{2}\right]\right)= \begin{cases}\mathbb{Z} \oplus \mathbb{Z} & \text { if } n \equiv 0(\bmod 2) \\ \mathbb{Z}_{2} & \text { if } n \equiv 1(\bmod 2)\end{cases}
$$

detected by the multisignature and Arf invariant. The multisignature isomorphism

$$
\begin{aligned}
& L_{0}\left(\mathbb{Z}\left[\mathbb{Z}_{2}\right]\right) \rightarrow L_{0}(\mathbb{Z}) \oplus L_{0}(\mathbb{Z})=\mathbb{Z} \oplus \mathbb{Z} ; \\
& (K, \lambda, \mu) \mapsto\left(\sigma\left(\epsilon_{+}(K, \lambda, \mu)\right) / 8, \sigma\left(\epsilon_{-}(K, \lambda, \mu)\right) / 8\right)
\end{aligned}
$$

has components induced by the morphisms of rings with involution

$$
\epsilon_{ \pm}: \mathbb{Z}\left[\mathbb{Z}_{2}\right] \rightarrow \mathbb{Z} ; a+b T \mapsto a \pm b
$$

The Arf invariant isomorphism is given by

$$
L_{2}\left(\mathbb{Z}\left[\mathbb{Z}_{2}\right]\right) \rightarrow L_{2}(\mathbb{Z})=\mathbb{Z}_{2} ;(K, \lambda, \mu) \mapsto c\left(\epsilon_{+}(K, \lambda, \mu)\right)=c\left(\epsilon_{-}(K, \lambda, \mu)\right)
$$

(ii) The irreducible complex representaton of $\mathbb{Z}_{m}$

$$
\rho_{j}: \mathbb{Z}_{m} \rightarrow \mathbb{C} ; t \mapsto e^{2 \pi i j / m} \quad(0 \leqslant j<m)
$$

give the real group ring of $\mathbb{Z}_{m}$ to be

$$
\mathbb{R}\left[\mathbb{Z}_{m}\right]= \begin{cases}\mathbb{R}^{2} \oplus \underset{(m-2) / 2}{\bigoplus} \mathbb{C} & \text { if } m \equiv 0(\bmod 2) \\ \mathbb{R} \oplus \underset{(m-1) / 2}{\bigoplus} \mathbb{C} & \text { if } m \equiv 1(\bmod 2)\end{cases}
$$

so that

$$
\begin{aligned}
r\left(\mathbb{Z}_{m}\right) & =\alpha_{\mathbb{R}}\left(\mathbb{Z}_{m}\right)+\alpha_{\mathbb{C}}\left(\mathbb{Z}_{m}\right) \\
& = \begin{cases}2+(m-2) / 2 & \text { if } m \equiv 0(\bmod 2) \\
1+(m-1) / 2 & \text { if } m \equiv 1(\bmod 2) .\end{cases}
\end{aligned}
$$

The even-dimensional $L$-groups of

$$
\mathbb{Z}\left[\mathbb{Z}_{m}\right]=\mathbb{Z}[t] /\left(t^{m}-1\right)
$$

with the oriented involution $\bar{t}=t^{-1}$ are such that 


$$
\begin{aligned}
& L_{0}\left(\mathbb{Z}\left[\mathbb{Z}_{m}\right]\right)= \begin{cases}\underset{(m+2) / 2}{\bigoplus} \mathbb{Z} \oplus(2 \text {-primary torsion }) & \text { if } m \equiv 0(\bmod 2) \\
\bigoplus_{(m+1) / 2} \mathbb{Z} \oplus(2 \text {-primary torsion }) & \text { if } m \equiv 1(\bmod 2)\end{cases} \\
& L_{2}\left(\mathbb{Z}\left[\mathbb{Z}_{m}\right]\right)= \begin{cases}\bigoplus_{(m-2) / 2} \mathbb{Z} \oplus(2 \text {-primary torsion }) & \text { if } m \equiv 0(\bmod 2) \\
\bigoplus_{(m-1) / 2} \mathbb{Z} \oplus(2 \text {-primary torsion }) & \text { if } m \equiv 1(\bmod 2) .\end{cases}
\end{aligned}
$$

The multisignature components are given by the morphisms of rings with involution $\rho_{j}: \mathbb{Z}\left[\mathbb{Z}_{m}\right] \rightarrow \mathbb{C}$

$$
\rho_{j}: L_{2 n}\left(\mathbb{Z}\left[\mathbb{Z}_{m}\right]\right) \rightarrow L_{2 n}(\mathbb{C})=\mathbb{Z}(0 \leqslant j \leqslant m / 2) .
$$

\subsection{The even-dimensional surgery obstruction}

It was shown in Section 10.4 that every $2 n$-dimensional degree 1 normal map is bordant to an $n$-connected degree 1 normal map. In general, there is an obstruction to the existence of a further bordism to an $(n+1)$-connected degree 1 normal map (= homotopy equivalence), which is defined as follows.

Definition 11.70 The surgery obstruction of an $n$-connected $2 n$-dimensional degree 1 normal map $(f, b): M^{2 n} \rightarrow X$ is the class of the kernel $(-1)^{n}$-quadratic form over $\mathbb{Z}\left[\pi_{1}(X)\right]$

$$
\sigma_{*}(f, b)=\left(K_{n}(M), \lambda, \mu\right) \in L_{2 n}\left(\mathbb{Z}\left[\pi_{1}(X)\right]\right) .
$$

The main result of this section is that $\sigma_{*}(f, b)=0$ if (and for $n \geqslant 3$ only if) $(f, b)$ is bordant to a homotopy equivalence. It is clear that if $(f, b)$ is a homotopy equivalence then $\sigma_{*}(f, b)=0$, for then $K_{n}(M)=0$.

Proposition 11.71 The surgery obstructions of bordant n-connected $2 n$-dimensional degree 1 normal maps $(f, b): M^{2 n} \rightarrow X,\left(f^{\prime}, b^{\prime}\right): M^{2 n} \rightarrow X$ are the same

$$
\sigma_{*}(f, b)=\sigma_{*}\left(f^{\prime}, b^{\prime}\right) \in L_{2 n}\left(\mathbb{Z}\left[\pi_{1}(X)\right]\right) .
$$

Proof Given a $(2 n+1)$-dimensional degree 1 normal bordism

$$
\left((F, B) ;(f, b),\left(f^{\prime}, b^{\prime}\right)\right):\left(W^{2 n+1} ; M^{2 n}, M^{\prime 2 n}\right) \rightarrow X \times(I ;\{0\},\{1\})
$$

with $(f, b)$ and $\left(f^{\prime}, b^{\prime}\right) n$-connected use the rel $\partial$ version of 10.30 to kill the kernel $\mathbb{Z}\left[\pi_{1}(X)\right]$-modules $K_{i}(W)$ for $i \leqslant n-1$ by $i$-surgeries on the interior of $W$ keeping $f$ and $f^{\prime}$ fixed. Thus it may be assumed that $F$ is $n$-connected, such that 


$$
W=M \times I \cup \bigcup_{k} n \text {-handles } D^{n} \times D^{n+1} \cup \bigcup_{\ell}(n+1) \text {-handles } D^{n+1} \times D^{n},
$$

and by Example 11.54

$$
\begin{aligned}
\left(K_{n}(N), \lambda_{N}, \mu_{N}\right) & \cong\left(K_{n}(M), \lambda, \mu\right) \oplus H_{(-1)^{n}}\left(\mathbb{Z}\left[\pi_{1}(X)\right]^{k}\right) \\
& \cong\left(K_{n}\left(M^{\prime}\right), \lambda^{\prime}, \mu^{\prime}\right) \oplus H_{(-1)^{n}}\left(\mathbb{Z}\left[\pi_{1}(X)\right]^{\ell}\right) .
\end{aligned}
$$

It follows that

$$
\sigma_{*}(f, b)=\left(K_{n}(M), \lambda, \mu\right)=\left(K_{n}\left(M^{\prime}\right), \lambda^{\prime}, \mu^{\prime}\right)=\sigma_{*}\left(f^{\prime}, b^{\prime}\right) \in L_{2 n}\left(\mathbb{Z}\left[\pi_{1}(X)\right]\right) .
$$

In view of the invariance of the surgery obstruction under normal bordism (11.71) and the result of Section 10.4 that every $2 n$-dimensional degree 1 normal map is bordant to an $n$-connected degree 1 normal map the surgery obstruction can be defined in general:

Definition $\mathbf{1 1 . 7 2}$ The surgery obstruction of a $2 n$-dimensional degree 1 normal map $(f, b): M^{2 n} \rightarrow X$ is defined to be the class of the kernel $(-1)^{n}$-quadratic form over $\mathbb{Z}\left[\pi_{1}(X)\right]$

$$
\sigma_{*}(f, b)=\left(K_{n}\left(M^{\prime}\right), \lambda^{\prime}, \mu^{\prime}\right) \in L_{2 n}\left(\mathbb{Z}\left[\pi_{1}(X)\right]\right)
$$

of any bordant $n$-connected $2 n$-dimensional degree 1 normal map $\left(f^{\prime}, b^{\prime}\right): M^{\prime 2 n} \rightarrow$ $X$.

Proposition 11.73 Let $n \geqslant 3$. Given an $n$-connected $2 n$-dimensional degree 1 normal map $(f, b): M^{2 n} \rightarrow X$ such that

$$
\sigma_{*}(f, b)=0 \in L_{2 n}\left(\mathbb{Z}\left[\pi_{1}(X)\right]\right)
$$

there exists an $n$-connected $(2 n+1)$-dimensional degree 1 normal bordism

$$
\left((F, B) ;(f, b),\left(f^{\prime}, b^{\prime}\right)\right):\left(W^{2 n+1} ; M^{2 n}, M^{\prime 2 n}\right) \rightarrow X \times(I ;\{0\},\{1\})
$$

to a homotopy equivalence $\left(f^{\prime}, b^{\prime}\right): M^{\prime} \rightarrow X$.

Proof For any embedding $D^{2 n} \hookrightarrow M$ set

$$
M_{0}=\operatorname{cl} .\left(M \backslash D^{2 n}\right) .
$$

By the Poincaré Disc Theorem (9.14) it may be assumed that

$$
X=X_{0} \cup D^{2 n}
$$


for a $2 n$-dimensional Poincaré pair $\left(X_{0}, S^{2 n-1}\right)$, with

$$
(f, b)=\left(f_{0}, b_{0}\right) \cup 1: M=M_{0} \cup D^{2 n} \rightarrow X=X_{0} \cup D^{2 n} .
$$

By hypothesis there exists an isomorphism of forms

$$
\left(K_{n}(M), \lambda, \mu\right) \oplus H_{(-1)^{n}}\left(\mathbb{Z}\left[\pi_{1}(X)\right]^{k}\right) \cong H_{(-1)^{n}}\left(\mathbb{Z}\left[\pi_{1}(X)\right]^{\ell}\right) .
$$

The effect of $k(n-1)$-surgeries on $(f, b)$ is the bordant degree 1 map

$$
\left(f_{1}, b_{1}\right)=(f, b) \# \text { std. }: M_{1}=M \underset{k}{\#} \underset{S^{n}}{ } \times S^{n} \rightarrow X=X \# S^{2 n}
$$

(using $X_{0}$ and $\left(f_{0}, b_{0}\right)$ to define the connected sums \#), with hyperbolic kernel form

$\left(K_{n}\left(M_{1}\right), \lambda_{1}, \mu_{1}\right)=\left(K_{n}(M), \lambda, \mu\right) \oplus H_{(-1)^{n}}\left(\mathbb{Z}\left[\pi_{1}(X)\right]^{k}\right)=H_{(-)^{n}}\left(\mathbb{Z}\left[\pi_{1}(X)\right]\right)^{\ell}$.

The effect of $\ell n$-surgeries on $\left(f_{1}, b_{1}\right)$ killing a basis $x_{1}, x_{2}, \ldots, x_{\ell} \in K_{n}\left(M_{1}\right)$ for a lagrangian is a homotopy equivalence $\left(f^{\prime}, b^{\prime}\right): M^{\prime 2 n} \rightarrow X$ bordant to $\left(f_{1}, b_{1}\right)$, which is also bordant to $(f, b)$.

Theorem 11.74 Let $n \geqslant 3$. A $2 n$-dimensional degree 1 normal map of pairs

$$
(f, b):\left(M^{2 n}, \partial M\right) \rightarrow(X, \partial X)
$$

with $\partial f: \partial M \rightarrow \partial X$ a homotopy equivalence has a rel $\partial$ surgery obstruction

$$
\sigma_{*}(f, b) \in L_{2 n}\left(\mathbb{Z}\left[\pi_{1}(X)\right]\right)
$$

such that $\sigma_{*}(f, b)=0$ if and only if $(f, b)$ is bordant rel $\partial$ to a homotopy equivalence of pairs.

Proof The surgery obstruction of $(f, b)$ is defined by

$$
\sigma_{*}(f, b)=\left(K_{n}\left(M^{\prime}\right), \lambda^{\prime}, \mu^{\prime}\right) \in L_{2 n}\left(\mathbb{Z}\left[\pi_{1}(X)\right]\right)
$$

for any $n$-connected degree 1 normal map $\left(f^{\prime}, b^{\prime}\right):\left(M^{\prime}, \partial M\right) \rightarrow(X, \partial X)$ bordant to $(f, b)$ relative to the boundary, with $\partial f^{\prime}=\partial f$, exactly as in the closed case $\partial M=\partial X=\emptyset$ in 11.72. The rel $\partial$ version of 11.71 shows that the surgery obstruction is a normal bordism invariant, which is 0 for a homotopy equivalence. Also, if $n \geqslant 3$ and $\sigma_{*}(f, b)=0$ then $(f, b)$ is bordant rel $\partial$ to a homotopy equivalence of pairs, proved exactly as in the closed case in 11.73.

Proposition 11.75 Let $\pi$ be a finitely presented group with an orientation character $w: \pi \rightarrow \mathbb{Z}_{2}$. For any $m \geqslant 4$ there exists a closed $m$-dimensional manifold $N$ with

$$
\left(\pi_{1}(N), w(N)\right)=(\pi, w) .
$$


Proof Given a finite presentation $\pi=\left\{g_{1}, g_{2}, \ldots, g_{k} \mid r_{1}, r_{2}, \ldots, r_{\ell}\right\}$ realize the orientation characters

$$
\omega_{i}=w\left(g_{i}\right) \in \pi_{1}(B O(m))=\mathbb{Z}_{2} \quad(1 \leqslant i \leqslant k)
$$

by $m$-plane bundles over $S^{1}$. The sphere bundles $S^{m-1} \rightarrow S\left(\omega_{i}\right) \rightarrow S^{1}$ are such that $S\left(\omega_{i}\right)=S^{m-1} \times S^{1}$ in the orientable case, and

$$
S\left(\omega_{i}\right)=S^{m-1} \times I /\{(x, 0) \sim(T x, 1)\}
$$

in the nonorientable case, with $T: S^{m-1} \rightarrow S^{m-1} ;\left(x_{1}, \ldots, x_{m}\right) \mapsto\left(-x_{1}, \ldots, x_{m}\right)$ of degree -1 . The connected sum is a closed $m$-dimensional manifold $M=$ $\stackrel{k}{\#} S\left(\omega_{i}\right)$ with $\pi_{1}(M)=\left\{g_{1}, g_{2}, \ldots, g_{k}\right\}=*_{k} \mathbb{Z}, w(M)\left(g_{i}\right)=\omega_{i}$. The relations $r_{j} \in \pi_{1}(M)$ are realized by disjoint framed 1-embeddings $S^{1} \times D^{m-1} \subset M$ such that the effect of the corresponding 1-surgeries on $M$

$$
N=\left(M \backslash\left(\bigcup_{j=1}^{\ell} S^{1} \times D^{m-1}\right)\right) \cup \bigcup_{j=1}^{\ell} D^{2} \times S^{m-2}
$$

is a closed $m$-dimensional manifold with $\pi_{1}(N)=\pi, w(N)=w$.

Corollary 11.76 Let $\pi$ be a finitely presented group with an orientation character $w: \pi \rightarrow \mathbb{Z}_{2}$, and let $n \geqslant 3$. Every element $x \in L_{2 n}(\mathbb{Z}[\pi])$ is the rel $\partial$ surgery obstruction $x=\sigma_{*}(f, b)$ of an $n$-connected $2 n$-dimensional degree 1 normal bor$\operatorname{dism}(f, b): M^{2 n} \rightarrow X$ with $\left(\pi_{1}(X), w(X)\right)=(\pi, w)$.

Proof By Proposition 11.75 there exists a closed $(2 n-1)$-dimensional manifold $N$ with $\left(\pi_{1}(N), w(N)\right)=(\pi, w)$. By Proposition 11.42 every $(-1)^{n}$-quadratic form $(K, \lambda, \mu)$ representing $x$ is realized as the kernel form of an $n$-connected $2 n$-dimensional degree 1 normal bordism

$$
(f, b):\left(M^{2 n} ; N^{2 n-1}, N^{\prime 2 n-1}\right) \rightarrow N \times(I ;\{0\},\{1\})
$$

with $(f, b) \mid=1: N \rightarrow N$ and $(f, b) \mid: N^{\prime} \rightarrow N$ a homotopy equivalence. The rel $\partial$ surgery obstruction is

$$
\sigma_{*}(f, b)=(K, \lambda, \mu)=x \in L_{2 n}(\mathbb{Z}[\pi])
$$

Example 11.77 (i) The surgery obstruction of an $n$-connected $2 n$-dimensional degree 1 normal map $(f, b): M^{2 n} \rightarrow X$ with $\pi_{1}(X)=\{1\}$ is given in the case $n=2 k$ by

$$
\sigma_{*}(f, b)=\frac{1}{8}\left(\operatorname{signature}\left(H^{2 k}(M), \lambda\right)-\operatorname{signature}\left(H^{2 k}(X), \lambda\right)\right) \in L_{4 k}(\mathbb{Z})=\mathbb{Z},
$$


and in the case $n=2 k+1$ by

$$
\sigma_{*}(f, b)=\operatorname{Arf} \text { invariant }\left(K_{2 k+1}\left(M ; \mathbb{Z}_{2}\right), \lambda, \mu\right) \in L_{4 k+2}(\mathbb{Z})=\mathbb{Z}_{2} .
$$

(ii) A framed $m$-dimensional manifold $\left(M^{m}, b\right)$ determines an $m$-dimensional degree 1 normal map $(f, b): M^{m} \rightarrow S^{m}$ with

$$
f: M \rightarrow M / \operatorname{cl} .\left(M \backslash D^{m}\right)=S^{m}, \eta=\epsilon^{\infty}: S^{m} \rightarrow B O .
$$

The $m$-dimensional surgery obstruction defines morphisms

$$
\sigma_{*}^{f r}: \Omega_{m}^{f r}=\pi_{m}^{S} \rightarrow L_{m}(\mathbb{Z}) ;\left(M^{m}, b\right) \mapsto \sigma_{*}\left((f, b): M^{m} \rightarrow S^{m}\right) .
$$

If $\left(M^{4 k}, b\right)$ is a framed $4 k$-dimensional manifold then for $k \geqslant 1$

$$
\sigma_{*}^{f r}(M, b)=\text { signature of }\left(H_{2 k}(M), \lambda\right) / 8=0 \in L_{4 k}(\mathbb{Z})=\mathbb{Z}
$$

by the Hirzebruch Signature Theorem $(6.41)$. If $\left(M^{4 k+2}, b\right)$ is a framed $(4 k+2)$ dimensional manifold then

$$
\sigma_{*}^{f r}(M, b)=\operatorname{Arf} \text { invariant }\left(H_{2 k+1}\left(M ; \mathbb{Z}_{2}\right), \lambda, \mu\right) \in L_{4 k+2}(\mathbb{Z})=\mathbb{Z}_{2}
$$

For $k=0,1,3,7,15$ there exists a framed $(4 k+2)$-dimensional manifold with Arf invariant 1 , so that $\sigma_{*}^{f r}$ is onto. In particular, the Arf invariant map

$$
\sigma_{*}^{f r}: \Omega_{2}^{f r}=\pi_{2}^{S} \rightarrow \mathbb{Z}_{2}
$$

is an isomorphism, with $\Omega_{2}^{f r}$ generated by a non-standard framing $b$ on $T^{2}=$ $S^{1} \times S^{1}$, corresponding to the essential map $\eta^{2}: S^{4} \rightarrow S^{2}$ obtained by composing the Hopf map $\eta: S^{3} \rightarrow S^{2}$ and its suspension $\eta: S^{4} \rightarrow S^{3}$. Browder [13] showed that $\sigma_{*}^{f r}: \Omega_{4 k+2}^{f r} \rightarrow L_{4 k+2}(\mathbb{Z})=\mathbb{Z}_{2}$ has image 0 for $k \neq 2^{i}-1$, using the Adams spectral sequence. The existence or otherwise of framed $(4 k+2)$-dimensional manifolds with Arf invariant 1 with $k=2^{i}-1$ for $i \geqslant 5$ is the subject of much speculation in homotopy theory.

Example 11.78 (i) As in Example 11.47, given a knot $k: S^{2 n-1} \hookrightarrow S^{2 n+1}$ and a Seifert surface $M^{2 n} \subset S^{2 n+1}$ inclusion defines a degree 1 normal map

$$
(f, b):\left(M^{2 n}, \partial M\right) \rightarrow\left(D^{2 n+2}, k\left(S^{2 n-1}\right)\right)
$$

which is the identity on the boundary. The surgery obstruction

$$
\sigma_{*}(f, b) \in L_{2 n}(\mathbb{Z})
$$

is the signature (resp. Arf invariant) of $k$ for $n$ even (resp. odd).

(ii) The unknot $k: S^{1} \hookrightarrow S^{3}$ has a contractible Seifert surface $D^{2} \hookrightarrow S^{3}$ with Seifert matrix (11.47) 0 . 


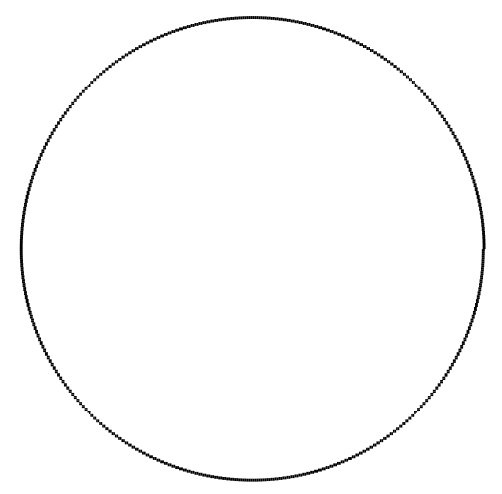

The 2-dimensional degree 1 normal map $(f, b):\left(D^{2}, S^{1}\right) \rightarrow\left(D^{4}, k\left(S^{1}\right)\right)$ is a homotopy equivalence, with surgery obstruction

$$
\sigma_{*}(f, b)=0 \in L_{2}(\mathbb{Z})=\mathbb{Z}_{2} .
$$

(iii) The trefoil knot $k: S^{1} \hookrightarrow S^{3}$ has a Seifert surface which is a punctured torus

$$
M^{2}=\operatorname{cl} .\left(T^{2} \backslash D^{2}\right) \subset S^{3} .
$$

with Seifert matrix $\left(\begin{array}{ll}1 & 1 \\ 0 & 1\end{array}\right)$.

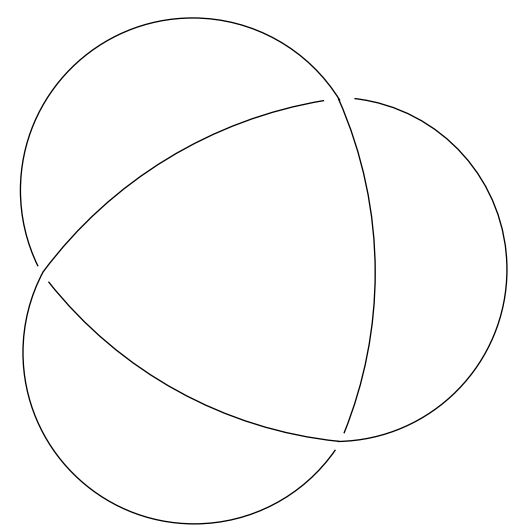

The 2-dimensional degree 1 normal map $(f, b):\left(M, S^{1}\right) \rightarrow\left(D^{4}, k\left(S^{1}\right)\right)$ has surgery obstruction

$$
\sigma_{*}(f, b)=\left(\mathbb{Z} \oplus \mathbb{Z},\left(\begin{array}{ll}
1 & 1 \\
0 & 1
\end{array}\right)\right)=1 \in L_{2}(\mathbb{Z})=\mathbb{Z}_{2} .
$$

Thus the trefoil knot has Arf invariant 1, and cannot be unknotted.

Remark 11.79 See Section 13.4 below for a brief account of the surgery theory of topological manifolds. The surgery obstructions $\sigma_{*}(f, b) \in L_{2 n}\left(\mathbb{Z}\left[\pi_{1}(X)\right]\right)$ of normal maps $(f, b): M \rightarrow X$ of closed $2 n$-dimensional topological manifolds with 
$\pi_{1}(X)=\pi$ constitute a subgroup of $L_{2 n}(\mathbb{Z}[\pi])$. For finite $\pi$ the multisignature (11.66) of such $(f, b)$ is constrained to have equal components, by Proposition 13B.1 of Wall [92]. See Hambleton, Milgram, Taylor and Williams [29] and Milgram [48] for the solution of the 'oozing conjecture' concerning this subgroup for finite $\pi$ (in both the even and odd dimensions). 


\section{THE ODD-DIMENSIONAL SURGERY OBSTRUCTION}

This chapter defines the Wall surgery obstruction of a $(2 n+1)$-dimensional degree 1 map $(f, b): M \rightarrow X$

$$
\sigma_{*}(f, b) \in L_{2 n+1}\left(\mathbb{Z}\left[\pi_{1}(X)\right]\right) .
$$

The main result is that $\sigma_{*}(f, b)=0$ if (and for $n \geqslant 2$ only if) $(f, b)$ is normal bordant to a homotopy equivalence.

Section 12.1 introduces the notion of an $\epsilon$-quadratic formation $(K, \lambda, \mu ; F, G)$, which is a nonsingular $\epsilon$-quadratic form $(K, \lambda, \mu)$ with lagrangians $F, G$. Section 12.2 constructs a kernel $(-1)^{n}$-quadratic formation over $\mathbb{Z}\left[\pi_{1}(X)\right]$ for an $n$-connected $(2 n+1)$-dimensional degree 1 normal map $(f, b)$. The $(2 n+1)$ dimensional surgery obstruction group $L_{2 n+1}(A)$ of cobordism classes of nonsingular $(-1)^{n}$-quadratic formations $(K, \lambda, \mu ; F, G)$ over $A$ is defined in Section 12.3. The odd-dimensional surgery obstruction is defined in Section 12.4 to be the cobordism class of a kernel formation. Section 12.5 describes the algebraic effect on a kernel formation of a geometric surgery on $(f, b)$. Finally, Section 12.6 is a brief account of linking forms, the original approach to odd-dimensional surgery obstruction theory.

Odd-dimensional surgery obstruction theory is technically more complicated than the even-dimensional case. Specifically, an $n$-connected $2 n$-dimensional normal map has a unique kernel form, whereas an $n$-connected $(2 n+1)$-dimensional normal map has many kernel formations.

\subsection{Quadratic formations}

The surgery obstruction of an even-dimensional degree 1 normal map was expressed in Section 11.5 as the equivalence class of the kernel nonsingular $\epsilon$ quadratic form, with the zero class represented by the forms which admit a lagrangian. The different lagrangians admitted by the kernel form correspond to different ways of solving the even-dimensional surgery problem by a normal bordism to a homotopy equivalence. The odd-dimensional surgery obstruction will be expressed in Section 12.4 as an equivalence class of $\epsilon$-quadratic 'formations', which are nonsingular $\epsilon$-quadratic forms with ordered pairs of lagrangians, corresponding to two solutions of an even-dimensional surgery problem in codimension 1. 
The fundamental algebraic structure determined by a closed $(n-1)$-connected $2 n$-dimensional manifold $N^{2 n}$ is the nonsingular $(-1)^{n}$-symmetric form $\left(H_{n}(N), \lambda\right)$ over $\mathbb{Z}$. The fundamental algebraic structure determined by an $(n-1)$-connected $(2 n+1)$-dimensional manifold with boundary $\left(M^{2 n+1}, \partial M\right)$ with $H_{n}(M, \partial M)=$ 0 is the lagrangian of the $(-1)^{n}$-symmetric form $\left(H_{n}(\partial M), \lambda\right)$ defined by

$$
L=\operatorname{im}\left(H_{n+1}(M, \partial M) \rightarrow H_{n}(\partial M)\right) \subset H_{n}(\partial M) .
$$

Now suppose that $M$ is a closed $(2 n+1)$-dimensional manifold which is expressed as a union of two $(2 n+1)$-dimensional manifolds $\left(M_{+}^{2 n+1}, \partial M_{+}\right),\left(M_{-}^{2 n+1}, \partial M_{-}\right)$ with the same $(n-1)$-connected boundary $N^{2 n}=\partial M_{+}=\partial M_{-}$

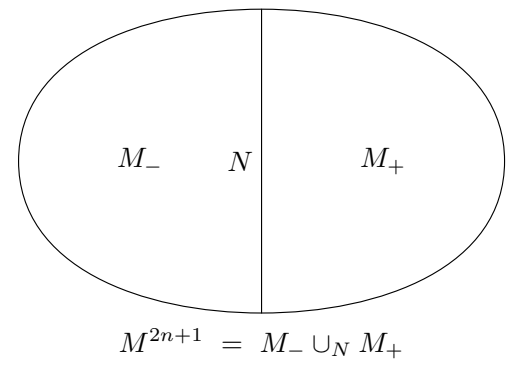

If $M, M_{+}, M_{-}, N$ are $(n-1)$-connected and $H_{n}\left(M_{+}, N\right)=H_{n}\left(M_{-}, N\right)=0$ the $(-1)^{n}$-symmetric form on $K=H_{n}(N)$ has lagrangians

$$
L_{ \pm}=\operatorname{im}\left(H_{n+1}\left(M_{ \pm}, N\right) \rightarrow H_{n}(N)\right)
$$

giving a $(-1)^{n}$-symmetric formation $\left(K, \lambda ; L_{+}, L_{-}\right)$. Such splittings were first used by Heegaard (in 1898) in the study of 3-dimensional manifolds : every connected 3-dimensional manifold $M^{3}$ can be expressed as a union

$$
M=M_{-} \cup_{N} M_{+}
$$

of solid tori along a genus $g$ surface, so that

$$
\begin{aligned}
& M_{+}=M_{-}=\#_{g} S^{1} \times D^{2}, \\
& M_{+} \cap M_{-}=N=M(g)=\#_{g} S^{1} \times S^{1}
\end{aligned}
$$

corresponding to a handle decomposition

$$
M=\left(h^{0} \cup \bigcup_{g} h^{1}\right) \cup\left(\bigcup_{g} h^{2} \cup h^{3}\right) .
$$

It should be clear that such expressions are not unique, since forming the connected sum of $M$ with

$$
S^{3}=\partial\left(D^{2} \times D^{2}\right)=S^{1} \times D^{2} \cup D^{2} \times S^{1}
$$

increases the genus $g$ by 1 without affecting the diffeomorphism type. 
The remainder of this section will only consider the algebraic properties of formations. As before, let $A$ be a ring with involution, and let $\epsilon= \pm 1$.

Definition 12.1 An $\epsilon$-quadratic formation over $A(K, \lambda, \mu ; F, G)$ is a nonsingular $\epsilon$-quadratic form $(K, \lambda, \mu)$ together with an ordered pair of lagrangians $F, G$.

Strictly speaking, Definition 12.1 defines a "nonsingular formation". In the general theory a formation $(K, \lambda, \mu ; F, G)$ is a nonsingular form $(K, \lambda, \mu)$ together with a lagrangian $F$ and a sublagrangian $G$, with $F, G$ and $K$ f.g. projective. For basic odd-dimensional surgery obstruction theory only nonsingular formations with $F, G$ and $K$ f.g. free need be considered. Also, $A$ can be assumed to be such that the rank of f.g. free $A$-modules is well-defined (e.g. $A=\mathbb{Z}[\pi]$ ), with $A^{k}$ isomorphic to $A^{\ell}$ if and only if $k=\ell$. This hypothesis ensures that for every formation $(K, \lambda, \mu ; F, G)$ over $A$ there exists an automorphism $\alpha:(K, \lambda, \mu) \rightarrow(K, \lambda, \mu)$ such that $\alpha(F)=G$ (Proposition 12.3 below). In the original work of Wall [92, Chapter 6] the odd-dimensional surgery obstruction was defined in terms of such automorphisms. Novikov [64] proposed the use of pairs of lagrangians instead, and the expression of odd-dimensional surgery obstructions in terms of formations was worked out in Ranicki [68].

Definition 12.2 An isomorphism of $\epsilon$-quadratic formations over $A$

$$
f:(K, \lambda, \mu ; F, G) \rightarrow\left(K^{\prime}, \lambda^{\prime}, \mu^{\prime} ; F^{\prime}, G^{\prime}\right)
$$

is an isomorphism of forms $f:(K, \lambda, \mu) \rightarrow\left(K^{\prime}, \lambda^{\prime}, \mu^{\prime}\right)$ such that

$$
f(F)=F^{\prime}, f(G)=G^{\prime} \text {. }
$$

Proposition 12.3 (i) Every $\epsilon$-quadratic formation $(K, \lambda, \mu ; F, G)$ is isomorphic to one of the type $\left(H_{\epsilon}(F) ; F, G\right)$.

(ii) Every $\epsilon$-quadratic formation $(K, \lambda, \mu ; F, G)$ is isomorphic to one of the type $\left(H_{\epsilon}(F) ; F, \alpha(F)\right)$ for some automorphism $\alpha: H_{\epsilon}(F) \rightarrow H_{\epsilon}(F)$.

Proof (i) By Theorem 11.51 the inclusion of the lagrangian $F \rightarrow K$ extends to an isomorphism of $\epsilon$-quadratic forms

$$
f: H_{\epsilon}(F) \rightarrow(K, \lambda, \mu),
$$

defining an isomorphism of $\epsilon$-quadratic formations

$$
f:\left(H_{\epsilon}(F) ; F, f^{-1}(G)\right) \rightarrow(K, \lambda, \mu ; F, G) .
$$

(ii) As in (i) extend the inclusions of the lagrangians $F \rightarrow K, G \rightarrow K$ to isomorphisms of forms 


$$
f: H_{\epsilon}(F) \rightarrow(K, \lambda, \mu) \quad, \quad g: H_{\epsilon}(G) \rightarrow(K, \lambda, \mu) .
$$

Then

$$
\operatorname{rank}_{A}(F)=\operatorname{rank}_{A}(K) / 2=\operatorname{rank}_{A}(G),
$$

so that $F$ is isomorphic to $G$. Choosing an $A$-module isomorphism $\beta: F \rightarrow G$ there is defined an automorphism of $H_{\epsilon}(F)$

$$
\alpha: H_{\epsilon}(F) \stackrel{\left(\begin{array}{cc}
\beta & 0 \\
0 & \beta^{*-1}
\end{array}\right)}{\longrightarrow} H_{\epsilon}(G) \stackrel{g}{\longrightarrow}(K, \lambda, \mu) \stackrel{f^{-1}}{\longrightarrow} H_{\epsilon}(F)
$$

such that there is defined an isomorphism of formations

$$
f:\left(H_{\epsilon}(F) ; F, \alpha(F)\right) \longrightarrow(K, \lambda, \mu ; F, G) .
$$

In Section 12.2 there is associated to an $n$-connected $(2 n+1)$-dimensional degree 1 normal map $(f, b): M^{2 n+1} \rightarrow X$ a 'stable isomorphism' class of kernel $(-1)^{n}$-quadratic formations $(K, \lambda, \mu ; F, G)$ over $\mathbb{Z}\left[\pi_{1}(X)\right]$ such that

$$
K_{n}(M)=K /(F+G), K_{n+1}(M)=F \cap G .
$$

Stable isomorphism is defined as follows:

Definition 12.4 (i) An $\epsilon$-quadratic formation $T=(K, \lambda, \mu ; F, G)$ is trivial if it is isomorphic to $\left(H_{\epsilon}(F) ; F, F^{*}\right)$.

(ii) A stable isomorphism of $\epsilon$-quadratic formations

$$
[f]:(K, \lambda, \mu ; F, G) \rightarrow\left(K^{\prime}, \lambda^{\prime}, \mu^{\prime} ; F^{\prime}, G^{\prime}\right)
$$

is an isomorphism of $\epsilon$-quadratic formations of the type

$$
f:(K, \lambda, \mu ; F, G) \oplus T \rightarrow\left(K^{\prime}, \lambda^{\prime}, \mu^{\prime} ; F^{\prime}, G^{\prime}\right) \oplus T^{\prime}
$$

with $T, T^{\prime}$ trivial.

Proposition 12.5 (i) An $\epsilon$-quadratic formation $(K, \lambda, \mu ; F, G)$ is trivial if and only if the lagrangians $F$ and $G$ are direct complements in $K$

$$
F \cap G=\{0\}, F+G=K .
$$

(ii) A stable isomorphism $[f]:(K, \lambda, \mu ; F, G) \rightarrow\left(K^{\prime}, \lambda^{\prime}, \mu^{\prime} ; F^{\prime}, G^{\prime}\right)$ of $\epsilon$-quadratic formations over $A$ induces $A$-module isomorphisms

$$
F \cap G \rightarrow F^{\prime} \cap G^{\prime} \quad, \quad K /(F+G) \rightarrow K^{\prime} /\left(F^{\prime}+G^{\prime}\right) .
$$

(iii) For any $\epsilon$-quadratic formation $(K, \lambda, \mu ; F, G)$ there is defined a stable isomorphism

$$
[f]:(K,-\lambda,-\mu ; G, F) \rightarrow(K, \lambda, \mu ; F, G) \text {. }
$$


Proof (i) If $F$ and $G$ are direct complements in $K$ represent $(\lambda, \mu)$ by a split $\epsilon$-quadratic form $(K, \psi \in S(K))$ with

$$
\psi=\left(\begin{array}{cc}
\alpha-\epsilon \alpha^{*} & \gamma \\
\delta & \beta-\epsilon \beta^{*}
\end{array}\right): K=F \oplus G \rightarrow K^{*}=F^{*} \oplus G^{*} .
$$

Then $\gamma+\epsilon \delta^{*} \in \operatorname{Hom}_{A}\left(G, F^{*}\right)$ is an $A$-module isomorphism, and there is defined an isomorphism of formations

$$
\left(\begin{array}{cc}
1 & 0 \\
0 & \left(\gamma+\epsilon \delta^{*}\right)^{-1}
\end{array}\right):\left(H_{\epsilon}(F) ; F, F^{*}\right) \rightarrow(K, \lambda, \mu ; F, G) .
$$

(ii) By (i) an $\epsilon$-quadratic formation $(K, \lambda, \mu ; F, G)$ is trivial if and only if

$$
F \cap G=0, K /(F+G)=0
$$

(iii) By Proposition 12.3 we can take

$$
(K, \lambda, \mu ; F, G)=\left(H_{\epsilon}(F) ; F, \alpha(G)\right)
$$

with

$$
\alpha=\left(\begin{array}{ll}
\gamma & \widetilde{\delta} \\
\delta & \widetilde{\gamma}
\end{array}\right): H_{\epsilon}(G) \rightarrow H_{\epsilon}(F)
$$

an isomorphism of hyperbolic $\epsilon$-quadratic forms, which defines an isomorphism of $\epsilon$-quadratic formations

$$
\alpha:\left(H_{\epsilon}(G) ; G, \alpha^{-1}(F)\right) \rightarrow(K, \lambda, \mu ; G, F) .
$$

The $A$-module isomorphism

$$
f=\left(\begin{array}{cccc}
\gamma & \widetilde{\delta} & 0 & \gamma \\
\delta & \widetilde{\gamma} & -\epsilon \widetilde{\gamma} & 0 \\
0 & 0 & -\widetilde{\gamma} & -\epsilon \delta \\
0 & 0 & \widetilde{\delta} & \gamma
\end{array}\right): G \oplus G^{*} \oplus G^{*} \oplus G \rightarrow F \oplus F^{*} \oplus F^{*} \oplus F
$$

defines an isomorphism of $\epsilon$-quadratic formations

$f:\left(H_{\epsilon}(G) ; G, \alpha^{-1}(F)\right) \oplus\left(H_{\epsilon}\left(G^{*}\right) ; G^{*}, G\right) \rightarrow\left(-H_{\epsilon}(F) ; F, \alpha(G)\right) \oplus\left(H_{\epsilon}\left(F^{*}\right) ; F^{*}, F\right)$

giving a stable isomorphism

$$
[f]:(K, \lambda, \mu ; G, F) \rightarrow(K,-\lambda,-\mu ; F, G) .
$$

\subsection{The kernel formation}

An $n$-connected $(2 n+1)$-dimensional degree 1 normal map $(f, b): M^{2 n+1} \rightarrow X$ is such that $K_{i}(M)=0$ for $i \neq n, n+1$. We shall now construct a stable isomorphism class of kernel $(-1)^{n}$-quadratic formations $(K, \lambda, \mu ; F, G)$ over $\mathbb{Z}\left[\pi_{1}(X)\right]$ such that

$$
K_{n}(M)=K /(F+G), K_{n+1}(M)=F \cap G,
$$

using the following generalisation of the Heegaard splitting of a 3-dimensional manifold as a union of solid tori. 
The kernel $\mathbb{Z}\left[\pi_{1}(X)\right]$-module $K_{n}(M)$ is finitely generated, by Corollary 10.29. Every finite set $\left\{x_{1}, x_{2}, \ldots, x_{k}\right\}$ of generators is realized by disjoint $n$-surgeries

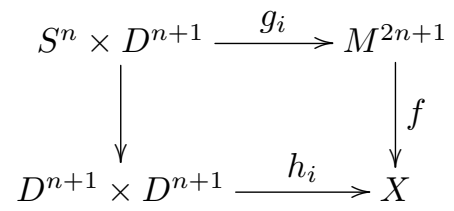

for $i=1,2, \ldots, k$.

By the Poincaré Disc Theorem (9.14) it may be assumed that the target $(2 n+1)$-dimensional geometric Poincaré complex $X$ is obtained from a $(2 n+1)$ dimensional geometric Poincaré pair $\left(X_{0}, S^{2 n}\right)$ by attaching a $(2 n+1)$-cell

$$
X=X_{0} \cup D^{2 n+1} .
$$

Any choice of generators $x_{1}, x_{2}, \ldots, x_{k} \in K_{n}(M)$ is realized by framed embeddings $g_{i}: S^{n} \times D^{n+1} \subset M^{2 n+1}$ together with null-homotopies $h_{i}: f g_{i} \simeq\{*\}$ : $S^{n} \rightarrow X$.

Definition 12.6 A Heegaard splitting of an $n$-connected (2n+1)-dimensional degree 1 normal map $(f, b): M^{2 n+1} \rightarrow X$ is an expression as a union

$$
(f, b)=\left(f_{0}, b_{0}\right) \cup(e, a): M^{2 n+1}=M_{0} \cup U \rightarrow X=X_{0} \cup D^{2 n+1}
$$

with

$$
\begin{aligned}
& U^{2 n+1}=\#_{i=1}^{k} g_{i}\left(S^{n} \times D^{n+1}\right) \subset M^{2 n+1}, M_{0}=\operatorname{cl} .(M \backslash U), \\
& M_{0} \cap U=\partial M_{0}=\partial U=\#_{i=1}^{k} g_{i}\left(S^{n} \times S^{n}\right)
\end{aligned}
$$

associated to a set of framed $n$-embeddings

$$
g_{i}: S^{n} \times D^{n+1} \hookrightarrow M(1 \leqslant i \leqslant k)
$$

with null-homotopies in $X$ representing a set $\left\{x_{1}, x_{2}, \ldots, x_{k}\right\} \subset K_{n}(M)$ of $\mathbb{Z}\left[\pi_{1}(X)\right]$-module generators.

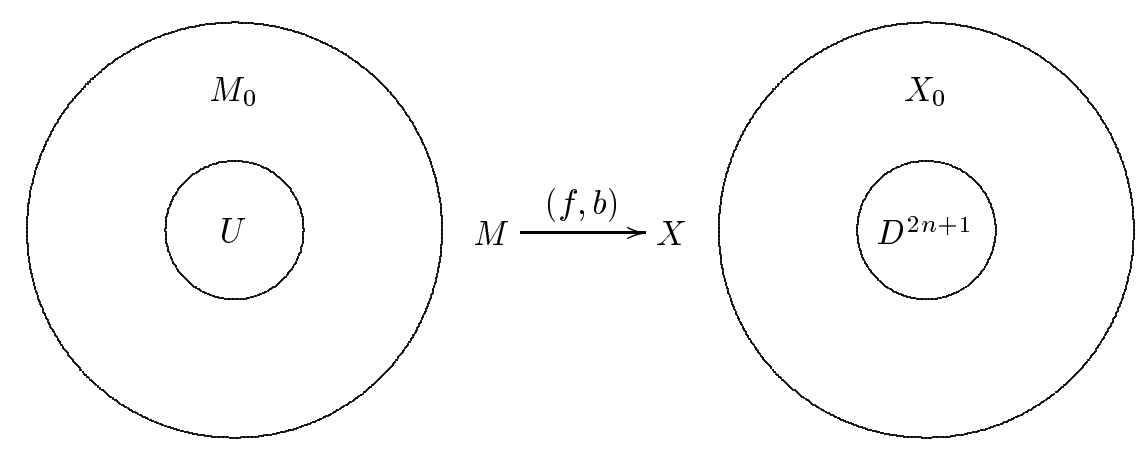

Every finite set of generators of $K_{n}(M)$ is realized by a Heegaard splitting. 
Definition 12.7 The kernel formation of an $n$-connected $(2 n+1)$-dimensional degree 1 normal map $(f, b): M^{2 n+1} \rightarrow X$ with respect to a Heegaard splitting is the $(-1)^{n}$-quadratic formation over $\mathbb{Z}\left[\pi_{1}(X)\right]$

$$
(K, \lambda, \mu ; F, G)=\left(K_{n}(\partial U), \lambda, \mu ; K_{n+1}(U, \partial U), K_{n+1}\left(M_{0}, \partial U\right)\right)
$$

with $\left(K_{n}(\partial U), \lambda, \mu\right)=H_{(-1)^{n}}\left(K_{n+1}(U, \partial U)\right)$ the hyperbolic $(-1)^{n}$-quadratic kernel form over $\mathbb{Z}\left[\pi_{1}(X)\right]$ of the $n$-connected $2 n$-dimensional degree 1 normal map $\partial U \rightarrow S^{2 n}$. The lagrangians $F, G$ are determined by the $n$-connected $(2 n+1)$-dimensional degree 1 normal maps of pairs

$$
(e, a):(U, \partial U) \rightarrow\left(D^{2 n+1}, S^{2 n}\right) \quad, \quad\left(f_{0}, b_{0}\right):\left(M_{0}, \partial U\right) \rightarrow\left(X_{0}, S^{2 n}\right),
$$

with

$$
\begin{aligned}
& F=\operatorname{im}\left(\partial: K_{n+1}(U, \partial U) \rightarrow K_{n}(\partial U)\right) \cong \mathbb{Z}\left[\pi_{1}(X)\right]^{k} \\
& G=\operatorname{im}\left(\partial: K_{n+1}\left(M_{0}, \partial U\right) \rightarrow K_{n}(\partial U)\right) \cong \mathbb{Z}\left[\pi_{1}(X)\right]^{k}
\end{aligned}
$$

The Heegaard splittings and kernel formations of an $n$-connected $(2 n+1)$ dimensional degree 1 normal map $(f, b): M^{2 n+1} \rightarrow X$ are highly non-unique.

Proposition 12.8 For any kernel formation $(K, \lambda, \mu ; F, G)$ of an $n$-connected $(2 n+1)$-dimensional degree 1 normal map $(f, b): M^{2 n+1} \rightarrow X$ there are natural identifications of $\mathbb{Z}\left[\pi_{1}(X)\right]$-modules

$$
K_{n}(M)=K /(F+G), K_{n+1}(M)=F \cap G .
$$

Proof Immediate from the exact sequence

$$
0 \rightarrow K_{n+1}(M) \rightarrow K_{n+1}(M, U) \rightarrow K_{n}(U) \rightarrow K_{n}(M) \rightarrow 0
$$

and the identification of the map in the middle with the natural $\mathbb{Z}\left[\pi_{1}(X)\right]$-module morphism

$$
K_{n+1}(M, U)=K_{n+1}\left(M_{0}, \partial U\right)=G \rightarrow K_{n}(U)=K / F .
$$

The kernel formations will be used in Section 12.4 below to represent the surgery obstruction $\sigma_{*}(f, b)=(K, \lambda, \mu ; F, G) \in L_{2 n+1}\left(\mathbb{Z}\left[\pi_{1}(X)\right]\right)$.

Example 12.9 The kernel formation of the 1-connected degree 1 normal map on the 3-dimensional lens space (8.39)

$$
(f, b): L(m, n)^{3} \rightarrow S^{3}
$$

is the $(-1)$-quadratic formation over $\mathbb{Z}$ given by 


$$
\left(H_{-}(\mathbb{Z}) ; \mathbb{Z}, \operatorname{im}\left(\left(\begin{array}{c}
n \\
m
\end{array}\right): \mathbb{Z} \rightarrow \mathbb{Z} \oplus \mathbb{Z}^{*}\right)\right)
$$

In particular,

(i) $L(1,0)=S^{3}$ with trivial formation $\left(H_{-}(\mathbb{Z}) ; \mathbb{Z},\left(\begin{array}{l}0 \\ 1\end{array}\right) \mathbb{Z}\right)$,

(ii) $L(0,1)=S^{1} \times S^{2}$ with boundary formation $\left(H_{-}(\mathbb{Z}) ; \mathbb{Z},\left(\begin{array}{l}1 \\ 0\end{array}\right) \mathbb{Z}\right)$,

(iii) $L(1,2)=S^{3}$ with trivial formation $\left(H_{-}(\mathbb{Z}) ; \mathbb{Z},\left(\begin{array}{l}2 \\ 1\end{array}\right) \mathbb{Z}\right)$,

(iv) $L(2,1)=\mathbb{R P}^{3}$ with boundary formation $\left(H_{-}(\mathbb{Z}) ; \mathbb{Z},\left(\begin{array}{l}1 \\ 2\end{array}\right) \mathbb{Z}\right)$.

Proposition 12.10 Let $(f, b): M^{2 n+1} \rightarrow X$ be an $n$-connected $(2 n+1)$-dimensional normal map.

(i) The kernel formations associated to all the Heegaard splittings of $(f, b)$ are stably isomorphic.

(ii) For $n \geqslant 2$ every formation in the stable isomorphism class is realized by a Heegaard splitting of $(f, b)$.

(iii) A kernel formation of $(f, b)$ is trivial if (and for $n \geqslant 2$ only if ) $(f, b)$ is a homotopy equivalence.

Proof (i) Consider first the effect on the kernel formation of changing the framed $n$-embeddings $g_{i}: S^{n} \times D^{n+1} \hookrightarrow M^{2 n+1}$ representing a set $\left\{x_{1}, x_{2}, \ldots, x_{k}\right\}$ of $\mathbb{Z}\left[\pi_{1}(X)\right]$-module generators for $K_{n}(M)$. Proposition 10.13 gives

$$
K_{n}(M)=\pi_{n+1}(f)=I_{n+1}(f)
$$

so that the framed $n$-embeddings $g_{i}$ are unique up to regular homotopy. Any two sets of framed $n$-embeddings representing $x_{i}$

$$
g_{i}, g_{i}^{\prime}: S^{n} \times D^{n+1} \hookrightarrow M^{2 n+1} \quad(1 \leqslant i \leqslant k)
$$

are thus related by regular homotopies

$$
d_{i}: g_{i} \simeq g_{i}^{\prime}: S^{n} \times D^{n+1} \rightarrow M^{2 n+1}
$$

with null-homotopies in $X$. Write the inclusions of the lagrangians associated to the two Heegaard splittings

$$
\begin{aligned}
& (f, b)=\left(f_{0}, b_{0}\right) \cup(e, a): M^{2 n+1}=M_{0} \cup U \rightarrow X, \\
& (f, b)=\left(f_{0}^{\prime}, b_{0}^{\prime}\right) \cup\left(e^{\prime}, a^{\prime}\right): M^{2 n+1}=M_{0}^{\prime} \cup U^{\prime} \rightarrow X
\end{aligned}
$$

as 


$$
\begin{aligned}
\left(\begin{array}{l}
\gamma \\
\delta
\end{array}\right): G & =K_{n+1}\left(M_{0}, \partial U\right) \\
\rightarrow & K_{n}(\partial U)=K_{n+1}(U, \partial U) \oplus K_{n}(U)=F \oplus F^{*} \\
\left(\begin{array}{l}
\gamma^{\prime} \\
\delta^{\prime}
\end{array}\right): G^{\prime} & =K_{n+1}\left(M_{0}^{\prime}, \partial U^{\prime}\right) \\
\rightarrow & K_{n}\left(\partial U^{\prime}\right)=K_{n+1}\left(U^{\prime}, \partial U^{\prime}\right) \oplus K_{n}\left(U^{\prime}\right)=F^{\prime} \oplus F^{\prime *}
\end{aligned}
$$

Let

$$
\alpha: F \rightarrow F^{\prime}, \beta: G \rightarrow G^{\prime}
$$

be the $\mathbb{Z}\left[\pi_{1}(X)\right]$-module isomorphisms determined by the given basis elements, and let $\left(F^{*}, \psi\right)$ be a kernel split $(-)^{n+1}$-quadratic form for the immersion

$$
\bigcup_{k} S^{n} \times D^{n+1} \times I \leftrightarrow M \times I ;\left(y_{i}, z_{i}, t\right) \mapsto\left(d_{i}\left(y_{i}, z_{i}, t\right), t\right)
$$

(defined as in the proof of Proposition 11.42). The commutative diagram

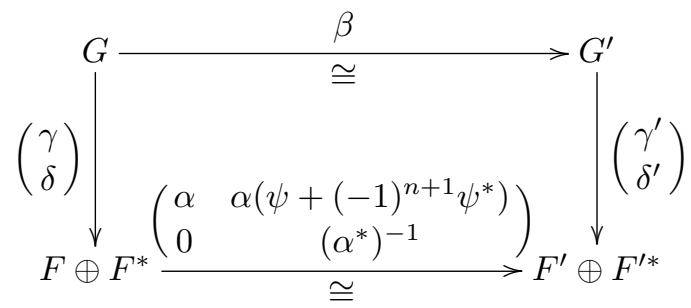

defines an isomorphism of the kernel $(-1)^{n}$-quadratic formations

$$
\begin{aligned}
& \left(K_{n}(\partial U), \lambda, \mu ; K_{n+1}(U, \partial U), K_{n+1}\left(M_{0}, U\right)\right) \\
& =\left(H_{(-1)^{n}}(F) ; F, \operatorname{im}\left(\left(\begin{array}{l}
\gamma \\
\delta
\end{array}\right): G \rightarrow F \oplus F^{*}\right)\right) \\
& \rightarrow\left(K_{n}\left(\partial U^{\prime}\right), \lambda^{\prime}, \mu^{\prime} ; K_{n+1}\left(U^{\prime}, \partial U^{\prime}\right), K_{n+1}\left(M_{0}^{\prime}, U^{\prime}\right)\right) \\
& =\left(H_{(-1)^{n}}\left(F^{\prime}\right) ; F^{\prime}, \operatorname{im}\left(\left(\begin{array}{c}
\gamma^{\prime} \\
\delta^{\prime}
\end{array}\right): G^{\prime} \rightarrow F^{\prime} \oplus F^{\prime *}\right)\right) .
\end{aligned}
$$

Next, consider the relationship between the kernel formations associated to two different sets of generators of $K_{n}(M)$. Proceed as in Wall [92, Chapter 6]. Any two sets $\left\{x_{1}, x_{2}, \ldots, x_{k}\right\},\left\{y_{1}, y_{2}, \ldots, y_{\ell}\right\}$ of $\mathbb{Z}\left[\pi_{1}(X)\right]$-module generators for $K_{n}(M)$ are related by a sequence of elementary operations

$$
\begin{aligned}
\left\{x_{1}, x_{2}, \ldots, x_{k}\right\} & \rightarrow\left\{x_{1}, x_{2}, \ldots, x_{k}, 0\right\} \rightarrow\left\{x_{1}, x_{2}, \ldots, x_{k}, y_{1}\right\} \\
& \rightarrow\left\{x_{1}, x_{2}, \ldots, x_{k}, y_{1}, 0\right\} \rightarrow\left\{x_{1}, x_{2}, \ldots, x_{k}, y_{1}, y_{2}\right\} \\
& \rightarrow \ldots \rightarrow\left\{x_{1}, x_{2}, \ldots, x_{k}, y_{1}, y_{2}, \ldots, y_{\ell}\right\} \\
& \rightarrow\left\{y_{1}, y_{2}, \ldots, y_{\ell}, x_{1}, x_{2}, \ldots, x_{k}\right\} \\
& \rightarrow \ldots \rightarrow\left\{y_{1}, y_{2}, \ldots, y_{\ell}\right\}
\end{aligned}
$$

with $y_{i}=\sum_{j} a_{i j} x_{j}$ for some $a_{i j} \in \mathbb{Z}\left[\pi_{1}(X)\right]$. Each of these operations has one of the following types : 
(1) adjoin (or delete) a zero,

(2) permute the elements,

(3) add to the last element a $\mathbb{Z}\left[\pi_{1}(X)\right]$-linear combination of the others.

The effect of (1) on the kernel formation of $(f, b)$ is to add (or delete) a trivial formation

$$
\left(H_{(-1)^{n}}\left(\mathbb{Z}\left[\pi_{1}(X)\right]\right) ; \mathbb{Z}\left[\pi_{1}(X)\right], \mathbb{Z}\left[\pi_{1}(X)\right]^{*}\right),
$$

while (2) and (3) do not change it.

(ii) For $n \geqslant 2$ it is possible to realize every elementary operation geometrically. (iii) A kernel formation for $(f, b)$ is trivial if and only if $K_{*}(M)=0$. Now $K_{*}(M)=0$ if (and for $n \geqslant 2$ only if) $(f, b)$ is a homotopy equivalence.

The kernel formations of $(f, b): M^{2 n+1} \rightarrow X$ were obtained in 12.7 by working inside $M$, using Heegaard splittings. However, as described in Example 6.3 of Ranicki [75] (and pp. 71-72 of the second edition of Wall [92]) there is an alternative construction, working outside of $M$ as follows.

Definition 12.11 A presentation of an $n$-connected $(2 n+1)$-dimensional degree 1 normal map $(f, b): M^{2 n+1} \rightarrow X$ is a degree 1 normal bordism

$$
\left((e, a) ;(f, b),\left(f^{\prime}, b^{\prime}\right)\right):\left(W^{2 n+2} ; M^{2 n+1}, M^{2 n+1}\right) \rightarrow X \times(I ;\{0\},\{1\})
$$

such that $f^{\prime}: M^{\prime} \rightarrow X^{\prime}=X \times\{1\}$ is $n$-connected and $e: W \rightarrow X \times I$ is $(n+1)$-connected.

The $(2 n+2)$-dimensional manifold with boundary $W$ in a presentation has a handle decomposition on $M$ of the type

$$
W=M \times I \cup \bigcup_{k}(n+1) \text {-handles } D^{n+1} \times D^{n+1} .
$$

The kernels $K_{n+1}(W), K_{n+1}(W, M), K_{n+1}\left(W, M^{\prime}\right)$ are f.g. free $\mathbb{Z}\left[\pi_{1}(X)\right]$-modules of rank $k$. The cobordism $\left(W ; M, M^{\prime}\right)$ is the trace of $n$-surgeries

$$
g_{i}: S^{n} \times D^{n+1} \hookrightarrow M \quad(1 \leqslant i \leqslant k)
$$

with null-homotopies in $X$ representing a set $\left\{x_{1}, x_{2}, \ldots, x_{k}\right\}$ of $\mathbb{Z}\left[\pi_{1}(X)\right]$-module generators of $K_{n}(M)$. The kernel modules $K_{*}(M), K_{*}\left(M^{\prime}\right)$ fit into exact sequences

$$
\begin{aligned}
& 0 \rightarrow K_{n+1}(M) \rightarrow K_{n+1}(W) \rightarrow K_{n+1}(W, M) \rightarrow K_{n}(M) \rightarrow 0 \\
& 0 \rightarrow K_{n+1}\left(M^{\prime}\right) \rightarrow K_{n+1}(W) \rightarrow K_{n+1}\left(W, M^{\prime}\right) \rightarrow K_{n}\left(M^{\prime}\right) \rightarrow 0
\end{aligned}
$$


Proposition 12.12 Let $(f, b): M^{2 n+1} \rightarrow X$ be an $n$-connected $(2 n+1)$-dimensional normal map.

(i) There exist presentations $(e, a):\left(W ; M, M^{\prime}\right) \rightarrow X \times(I ;\{0\},\{1\})$.

(ii) A presentation $(e, a)$ determines a kernel $(-1)^{n}$-quadratic formation for $(f, b)$

$$
\left(H_{(-1)^{n}}(F) ; F, G\right)=\left(H_{(-1)^{n}}\left(K_{n+1}\left(W, M^{\prime}\right)\right) ; K_{n+1}\left(W, M^{\prime}\right), K_{n+1}(W)\right) .
$$

The inclusion of the lagrangian

$$
\left(\begin{array}{l}
\gamma \\
\delta
\end{array}\right): G=K_{n+1}(W) \rightarrow F \oplus F^{*}=K_{n+1}\left(W, M^{\prime}\right) \oplus K_{n+1}\left(W, M^{\prime}\right)^{*}
$$

has components

$$
\begin{aligned}
\gamma: G= & K_{n+1}(W) \stackrel{\text { inclusion }_{*}}{\longrightarrow} K_{n+1}\left(W, M^{\prime}\right)=F \\
\delta: G= & K_{n+1}(W) \stackrel{\text { inclusion }_{*}}{\longrightarrow} \\
& K_{n+1}(W, M) \cong K^{n+1}\left(W, M^{\prime}\right) \cong K_{n+1}\left(W, M^{\prime}\right)^{*}=F^{*} .
\end{aligned}
$$

(iii) For $n \geqslant 2$ every formation in the stable isomorphism class is realized by a presentation of $(f, b)$.

Proof (i) A Heegaard splitting

$$
(f, b)=\left(f_{0}, b_{0}\right) \cup(e, a): M^{2 n+1}=M_{0} \cup U \rightarrow X=X_{0} \cup D^{2 n+1}
$$

determines $k n$-surgeries $U=\underset{k}{\#} S^{n} \times D^{n+1} \hookrightarrow M$ on $(f, b)$.

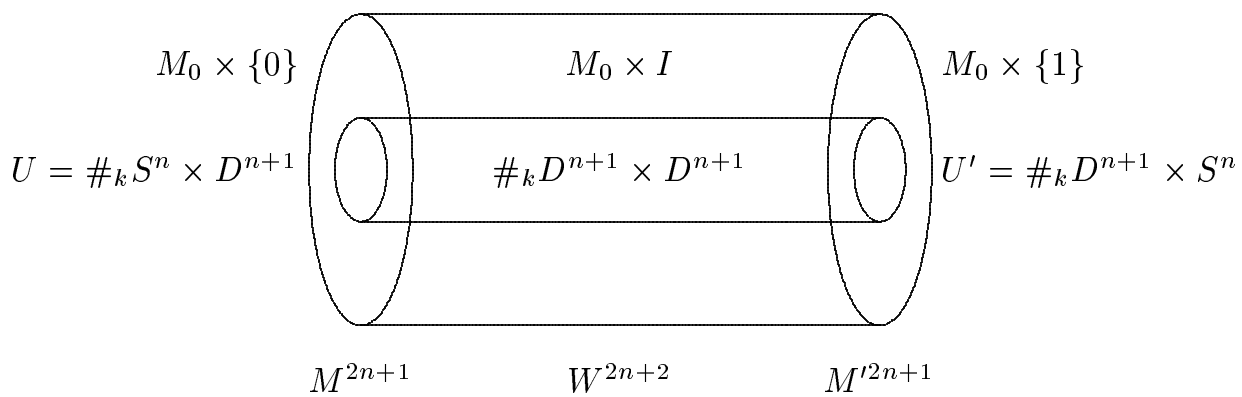

The trace of the surgeries is a presentation

$$
\left((e, a) ;(f, b),\left(f^{\prime}, b^{\prime}\right)\right):\left(W^{2 n+2} ; M^{2 n+1}, M^{\prime 2 n+1}\right) \rightarrow X \times(I ;\{0\},\{1\}) .
$$

The kernel formation of $(f, b)$ given by 12.7 is

$$
\left(H_{(-1)^{n}}\left(K_{n+1}(U, \partial U)\right) ; K_{n+1}(U, \partial U), K_{n+1}\left(M_{0}, \partial U\right)\right)=\left(H_{(-1)^{n}}(F) ; F, G\right) .
$$

(ii) Any presentation arises from a Heegaard splitting as in (i).

(iii) Combine (i), (ii) and 12.10. 
Note that turning a presentation of $(f, b)$ around, and viewing it as a presentation of $\left(f^{\prime}, b^{\prime}\right)$ gives the kernel formation $\left(H_{(-1)^{n}}(F) ; F^{*}, G\right)$ for $\left(f^{\prime}, b^{\prime}\right)$, with

$$
\begin{aligned}
& \gamma^{\prime}=(-1)^{n+1} \delta: G^{\prime}=G \rightarrow F^{\prime}=F^{*}, \\
& \delta^{\prime}=\gamma: G^{\prime}=G \rightarrow F^{*}=F .
\end{aligned}
$$

Proposition 12.13 A kernel $(-1)^{n}$-quadratic formation of an $n$-connected $(2 n+$ 1)-dimensional degree 1 normal map $(f, b): M^{2 n+1} \rightarrow X$ is stably isomorphic to the boundary of a $(-1)^{n+1}$-quadratic form if (and for $n \geqslant 2$ only if ) $(f, b)$ is bordant to a homotopy equivalence.

Proof Given a normal bordism

$$
\left((e, a) ;(f, b),\left(f^{\prime}, b^{\prime}\right)\right):\left(W^{2 n+2} ; M^{2 n+1}, M^{2 n+1}\right) \rightarrow X \times(I ;\{0\},\{1\})
$$

with $\left(f^{\prime}, b^{\prime}\right): M^{\prime} \rightarrow X$ a homotopy equivalence make $(e, a)(n+1)$-connected by surgery below the middle dimension, with kernel $(-1)^{n+1}$-quadratic form $\left(K_{n+1}(W), \lambda_{W}, \mu_{W}\right)$. This defines a presentation of $(f, b)$ with

$$
\gamma: K_{n+1}(W) \rightarrow K_{n+1}\left(W, M^{\prime}\right)
$$

an isomorphism which is used to identify

$$
K_{n+1}(W)=K_{n+1}\left(W, M^{\prime}\right)=K^{n+1}(W, M)=K_{n+1}(W, M)^{*},
$$

and

$$
\delta=\lambda_{W}: K_{n+1}(W) \rightarrow K_{n+1}(W, M)=K_{n+1}(W)^{*},
$$

so that the kernel formation of $(f, b)$ is the boundary

$$
\left(H_{(-1)^{n}}(F) ; F, G\right)=\partial\left(K_{n+1}(W), \lambda_{W}, \mu_{W}\right) .
$$

Conversely, suppose that $n \geqslant 2$ and that $(f, b): M^{2 n+1} \rightarrow X$ has a kernel formation which is stably isomorphic to the boundary of a $(-1)^{n+1}$-quadratic form. By 12.12 it is possible to realize this boundary by a presentation

$$
\left((e, a) ;(f, b),\left(f^{\prime}, b^{\prime}\right)\right):\left(W^{2 n+2} ; M^{2 n+1}, M^{2 n+1}\right) \rightarrow X \times(I ;\{0\},\{1\})
$$

with $\left(f^{\prime}, b^{\prime}\right): M^{\prime} \rightarrow X$ a homotopy equivalence.

Example 12.14 An element

$$
(\delta \omega, \omega) \in \pi_{n+1}(S O, S O(n+1))=\pi_{n+2}(B S O, B S O(n+1))=Q_{(-1)^{n+1}}(\mathbb{Z})
$$

classifies an oriented $(n+1)$-plane bundle $\omega: S^{n+1} \rightarrow B S O(n+1)$ with a stable trivialisation $\delta \omega: \omega \simeq\{*\}: S^{n+1} \rightarrow B S O$. As in 5.68 use $(\delta \omega, \omega)$ to define an 
$n$-surgery $\left(g_{\delta \omega}, g_{\omega}\right)$ on the identity degree 1 normal map $1: S^{2 n+1} \rightarrow S^{2 n+1}$, with trace

$$
((e, a) ; 1,(f, b)):\left(W^{2 n+2} ; S^{2 n+1}, S(\omega)^{2 n+1}\right) \rightarrow S^{2 n+1} \times(I ;\{0\},\{1\}) .
$$

The $n$-connected $(2 n+1)$-dimensional degree 1 normal map $(f, b): S(\omega) \rightarrow$ $S^{2 n+1}$ has kernel $(-1)^{n}$-quadratic formation over $\mathbb{Z}$

$$
\left(H_{(-1)^{n}}(\mathbb{Z}) ; \mathbb{Z}, \operatorname{im}\left(\left(\begin{array}{c}
1 \\
\chi(\omega)
\end{array}\right): \mathbb{Z} \rightarrow \mathbb{Z} \oplus \mathbb{Z}^{*}\right)\right)=\partial(\mathbb{Z},(\delta \omega, \omega)),
$$

with $\chi(\omega)=\left(1+(-1)^{n+1}\right) \chi(\delta \omega)$.

\section{Proposition 12.15 Let}

$$
\left((e, a) ;(f, b),\left(f^{\prime}, b^{\prime}\right)\right):\left(W^{2 n+2} ; M^{2 n+1}, M^{2 n+1}\right) \rightarrow X \times(I ;\{0\},\{1\})
$$

be an $(n+1)$-connected $(2 n+2)$-dimensional normal bordism between $n$-connected $(2 n+1)$-dimensional degree 1 normal maps $(f, b),\left(f^{\prime}, b^{\prime}\right)$. The kernel formations $(K, \lambda, \mu ; F, G),\left(K^{\prime}, \lambda^{\prime}, \mu^{\prime} ; F^{\prime}, G^{\prime}\right)$ of $(f, b),\left(f^{\prime}, b^{\prime}\right)$ are related by a stable isomorphism

$$
(K, \lambda, \mu ; F, G) \oplus\left(K^{\prime},-\lambda^{\prime},-\mu^{\prime} ; F^{\prime}, G^{\prime}\right) \rightarrow \partial\left(K_{n+1}(W), \lambda_{W}, \mu_{W}\right) .
$$

Proof The disjoint union

$$
(f, b) \cup\left(f^{\prime},-b^{\prime}\right): M \cup-M^{\prime} \rightarrow X \cup-X
$$

is a degree 1 normal map, where - reverses orientations. The stable isomorphism of formations is determined by the degree 1 normal map of pairs

$$
\left((e, a),(f, b) \cup\left(f^{\prime},-b^{\prime}\right)\right):\left(W^{2 n+2}, M \cup-M^{\prime}\right) \rightarrow X \times(I,\{0,1\}),
$$

working as in the proof of 12.13 .

The kernel formation is also defined for an $n$-connected $(2 n+1)$-dimensional degree 1 normal map

$$
(f, b):(M, \partial M) \rightarrow(X, \partial X)
$$

which is a homotopy equivalence on the boundary, realizing a set $\left\{x_{1}, x_{2}, \ldots, x_{k}\right\}$ of $\mathbb{Z}\left[\pi_{1}(X)\right]$-module generators of $K_{n}(M)$ as in 12.6 by a decomposition

$$
\begin{aligned}
& (f, b)=\left(f_{0}, b_{0}\right) \cup(e, a): \\
& \begin{aligned}
(M, \partial M)=\left(M_{0} ; \partial M, \partial U\right) & \cup(U, \partial U) \\
& \rightarrow(X, \partial X)=\left(X_{0} ; \partial X, S^{2 n}\right) \cup\left(D^{2 n+1}, S^{2 n}\right)
\end{aligned}
\end{aligned}
$$

with

$$
\begin{aligned}
& U^{2 n+1}=\#_{i=1}^{k} g_{i}\left(S^{n} \times D^{n+1}\right) \subset M \backslash \partial M, M_{0}=\operatorname{cl} .(M \backslash U), \\
& M_{0} \cap U=\partial U=\#_{i=1}^{k} g_{i}\left(S^{n} \times S^{n}\right) .
\end{aligned}
$$

There is also a version for normal bordisms, as follows. 
Definition 12.16 Suppose given an $n$-connected $(2 n+1)$-dimensional degree 1 normal bordism

$$
(f, b):\left(M^{2 n+1} ; N, N^{\prime}\right) \rightarrow N \times(I ;\{0\},\{1\})
$$

such that $f \mid=1: N \rightarrow N$ and $f \mid: N^{\prime} \rightarrow N$ is a homotopy equivalence. The Umkehr maps in this case are just

$$
f^{!}=\operatorname{inclusion}_{*}: H_{*}(\widetilde{N}) \rightarrow H_{*}(\widetilde{M}),
$$

the kernel $\mathbb{Z}\left[\pi_{1}(N)\right]$-modules are such that

$$
K_{i}(M)=H_{i}(\widetilde{M}, \tilde{N})=0 \text { for } i \neq n, n+1,
$$

and the cobordism $\left(M ; N, N^{\prime}\right)$ has an $(n, n+1)$-index handle decomposition $(8.23)$

$$
M=N \times I \cup \bigcup_{k} D^{n} \times D^{n+1} \cup \bigcup_{k} D^{n+1} \times D^{n} .
$$

(i) A Heegaard splitting for $(f, b)$ is an expression as a union

$$
\begin{aligned}
& (f, b)=(e, a) \cup\left(f_{0}, b_{0}\right): \\
& \begin{array}{l}
\left(M ; N, N^{\prime}\right)=\left(U ; N, \partial_{+} U\right) \cup\left(M_{0} ; \partial_{+} U, N^{\prime}\right) \\
\quad \rightarrow(N \times[0,1 / 2] ;\{0\},\{1 / 2\}) \cup(N \times[1 / 2,1] ;\{1 / 2\},\{1\})
\end{array}
\end{aligned}
$$

determined by a choice of handle decomposition as above, with

$$
\begin{aligned}
& U^{2 n+1}=N \times I \cup \bigcup_{k} D^{n} \times D^{n+1}, \\
& M_{0} \cap U=\partial_{+} U=N \# \underset{k}{\#}\left(S^{n} \times S^{n}\right), \\
& M_{0}=\partial_{+} U \times I \cup \bigcup_{k} D^{n+1} \times D^{n} .
\end{aligned}
$$

The $i$ th handle represents $x_{i} \in K_{n}(M)=H_{n}(\widetilde{M}, \widetilde{N})$, with $\left\{x_{1}, x_{2}, \ldots, x_{k}\right\} \subset$ $K_{n}(M)$ a set of $\mathbb{Z}\left[\pi_{1}(N)\right]$-module generators.

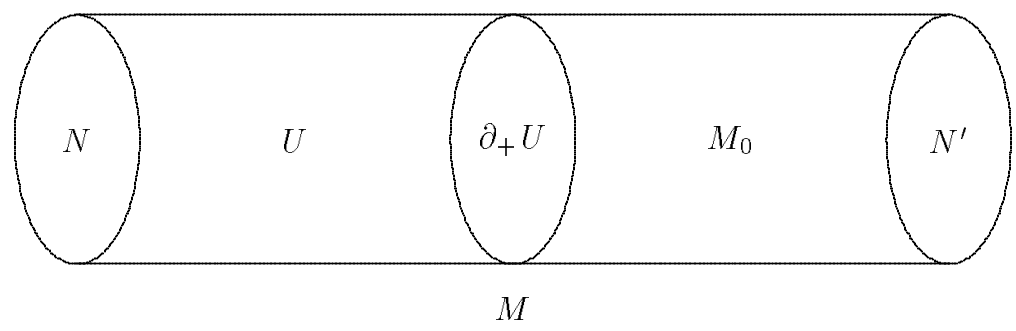

(ii) The kernel formation associated to a Heegaard splitting as in (i) is

$$
(K, \lambda, \mu ; F, G)=\left(K_{n}(\partial U), \lambda, \mu ; K_{n+1}(U, \partial U), K_{n+1}\left(M_{0}, \partial U\right)\right)
$$

with $\left(K_{n}(\partial U), \lambda, \mu\right)=H_{(-1)^{n}}\left(K_{n+1}(U, \partial U)\right)$. 
As in the closed case (12.6) every finite set of generators of $K_{n}(M)$ is realized by a Heegaard splitting of $(f, b)$, and so determines a kernel formation $(K, \lambda, \mu ; F, G)$.

Proposition 12.17 (Realization of formations)

Let $N^{2 n}$ be a $2 n$-dimensional manifold with fundamental group $\pi_{1}(N)=\pi$, with $n \geqslant 2$. Every $(-1)^{n}$-quadratic formation $(K, \lambda, \mu ; F, G)$ over $\mathbb{Z}[\pi]$ is realized as a kernel formation (12.16) of an $n$-connected $(2 n+1)$-dimensional degree 1 normal bordism

$$
(f, b):\left(M^{2 n+1} ; N^{2 n}, N^{\prime 2 n}\right) \rightarrow N \times(I ;\{0\},\{1\})
$$

with $(f, b) \mid=1: N \rightarrow N$ and $(f, b) \mid: N^{\prime} \rightarrow N$ a homotopy equivalence.

Proof By 11.51 the form $(K, \lambda, \mu)$ is isomorphic to the hyperbolic form $H_{(-1)^{n}}(F)$, so there is no loss of generality in taking $(K, \lambda, \mu)=H_{(-1)^{n}}(F)$. Let $k \geqslant 0$ be the rank of the f.g. free $\mathbb{Z}[\pi]$-modules $F, G$, so that

$$
F \cong G \cong \mathbb{Z}[\pi]^{k}
$$

Let

$$
\left(f^{\prime}, b^{\prime}\right):\left(U^{2 n+1} ; N, \partial_{+} U\right) \rightarrow N \times([0,1 / 2] ;\{0\},\{1 / 2\})
$$

be the $(2 n+1)$-dimensional normal bordism defined by the trace of $k$ trivial $(n-1)$-surgeries on $(f, b)$, with

$$
\begin{aligned}
& U=N \times I \cup \underset{k}{\bigcup} n \text {-handles } D^{n} \times D^{n+1}, \\
& \left(f^{\prime}, b^{\prime}\right) \mid=1 \text { \#std. }: \partial_{+} U=N \underset{k}{\#} \underset{k}{\#} \times S^{n} \rightarrow N .
\end{aligned}
$$

Realize the lagrangian of the kernel form

$$
G \subset\left(K_{n}\left(\partial_{+} U\right), \lambda, \mu\right)=H_{(-1)^{n}}(F)
$$

by a $(2 n+1)$-dimensional normal bordism

$$
\left(f^{\prime \prime}, b^{\prime \prime}\right):\left(M_{0}^{2 n+1} ; \partial_{+} U, N^{\prime}\right) \rightarrow N \times([1 / 2,1] ;\{1 / 2\},\{1\})
$$

defined by the trace of $k n$-surgeries on $\left(f^{\prime}, b^{\prime}\right) \mid$, with

$$
\begin{aligned}
& M_{0}=\partial_{+} U \times I \cup \bigcup_{k}(n+1) \text {-handles } D^{n+1} \times D^{n}, \\
& G=\operatorname{im}\left(K_{n+1}\left(M_{0}, \partial_{+} U\right) \rightarrow K_{n}\left(\partial_{+} U\right)\right) \subset K_{n}\left(\partial_{+} U\right) .
\end{aligned}
$$

The effect is a homotopy equivalence $\left(f^{\prime}, b^{\prime}\right) \mid: N^{\prime} \rightarrow N$, since the kernel form is

$$
\left(K_{n}\left(N^{\prime}\right), \lambda^{\prime}, \mu^{\prime}\right)=\left(G^{\perp} / G,[\lambda],[\mu]\right)=0 .
$$

The $n$-connected $(2 n+1)$-dimensional degree 1 normal map of pairs

$$
\begin{aligned}
& (f, b)=\left(f^{\prime}, b^{\prime}\right) \cup\left(f^{\prime \prime}, b^{\prime \prime}\right): \\
& \left(M^{2 n+1} ; N, N^{\prime}\right)=\left(U ; N, \partial_{+} U\right) \cup\left(M_{0} ; \partial_{+} U, N^{\prime}\right) \rightarrow N \times(I ;\{0\},\{1\})
\end{aligned}
$$

realizes the formation $(K, \lambda, \mu ; F, G)$. 


\subsection{The odd-dimensional $L$-groups}

The odd-dimensional surgery obstruction groups $L_{2 *+1}(A)$ are now defined using formations. The surgery obstruction of an odd-dimensional normal map will be defined in Section 12.4 using the kernel formation of Section 12.2, and it will be proved that for $n \geqslant 2$ an $n$-connected $(2 n+1)$-dimensional degree 1 normal map $(f, b): M^{2 n+1} \rightarrow X$ is bordant to a homotopy equivalence if and only if the stable isomorphism class of kernel $(-1)^{n}$-quadratic formations contains the 'boundary' of a $(-1)^{n+1}$-quadratic form (= the kernel form of the $(2 n+2)$-dimensional trace), in the following sense :

Definition 12.18 Let $(K, \lambda, \mu)$ be a $(-\epsilon)$-quadratic form.

(i) The graph lagrangian of $(K, \lambda, \mu)$ is the lagrangian

$$
\Gamma_{(K, \lambda)}=\left\{(x, \lambda(x)) \in K \oplus K^{*} \mid x \in K\right\}
$$

in the hyperbolic $\epsilon$-quadratic form $H_{\epsilon}(K)$

(ii) The boundary of $(K, \lambda, \mu)$ is the graph $\epsilon$-quadratic formation

$$
\partial(K, \lambda, \mu)=\left(H_{\epsilon}(K) ; K, \Gamma_{(K, \lambda)}\right) \text {. }
$$

The graph lagrangian $\Gamma_{(K, \lambda)}$ and formation $\partial(K, \lambda, \mu)$ depend only on the even $\epsilon$-symmetric form $(K, \lambda)$, and not on the $\epsilon$-quadratic function $\mu$. Note that the form $(K, \lambda, \mu)$ may be singular, that is the $A$-module morphism $\lambda: K \rightarrow K^{*}$ need not be an isomorphism.

Proposition 12.19 (i) The graphs $\Gamma_{(K, \lambda)}$ of $(-\epsilon)$-quadratic forms $(K, \lambda, \mu)$ are precisely the lagrangians of $H_{\epsilon}(K)$ which are the direct complements of $K^{*}$.

(ii) An $\epsilon$-quadratic formation $(K, \lambda, \mu ; F, G)$ is isomorphic to a boundary if and only if $(K, \lambda, \mu)$ has a lagrangian $H$ which is a direct complement of both the lagrangians $F, G$.

Proof (i) The direct complements of $K^{*}$ in $K \oplus K^{*}$ are the graphs

$$
L=\left\{(x, \lambda(x)) \in K \oplus K^{*} \mid x \in K\right\}
$$

of $A$-module morphisms $\lambda: K \rightarrow K^{*}$, with

$$
L^{\perp}=\left\{\left(y,-\epsilon \lambda^{*}(y)\right) \in K \oplus K^{*} \mid y \in K\right\} .
$$

Thus $L=L^{\perp}$ if and only if $\lambda=-\epsilon \lambda^{*}$, with $\mu_{H_{\epsilon}(K)}(L)=0$ if and only if $\lambda$ admits a $(-\epsilon)$-quadratic refinement $\mu$.

(ii) For the boundary $\partial(F, \phi, \theta)$ of a $(-\epsilon)$-quadratic form $(F, \phi, \theta)$ the lagrangian $F^{*}$ of $H_{\epsilon}(F)$ is a direct complement of both the lagrangians $F, \Gamma_{(F, \phi)}$. Conversely, 
suppose that $(K, \lambda, \mu ; F, G)$ is such that there exists a lagrangian $H$ in $(K, \lambda, \mu)$ which is a direct complement to both $F$ and $G$. By the proof of Proposition 12.5 (i) there exists an isomorphism of formations

$$
f:\left(H_{\epsilon}(F) ; F, F^{*}\right) \rightarrow(K, \lambda, \mu ; F, H)
$$

which is the identity on $F$. Now $f^{-1}(G)$ is a lagrangian of $H_{\epsilon}(F)$ which is a direct complement of $F^{*}$, so that it is the graph $\Gamma_{(F, \phi)}$ of a $(-\epsilon)$-quadratic form $(F, \phi, \theta)$, and $f$ defines an isomorphism of $\epsilon$-quadratic formations

$$
f: \partial(F, \phi, \theta)=\left(H_{\epsilon}(F) ; F, \Gamma_{(F, \phi)}\right) \rightarrow(K, \lambda, \mu ; F, G) .
$$

Definition 12.20 The $\epsilon$-quadratic formations $(K, \lambda, \mu ; F, G),\left(K^{\prime}, \lambda^{\prime}, \mu^{\prime} ; F^{\prime}, G^{\prime}\right)$ over $A$ are cobordant

$$
(K, \lambda, \mu ; F, G) \sim\left(K^{\prime}, \lambda^{\prime}, \mu^{\prime} ; F^{\prime}, G^{\prime}\right)
$$

if there exists a stable isomorphism

$$
[f]:(K, \lambda, \mu ; F, G) \oplus B \rightarrow\left(K^{\prime}, \lambda^{\prime}, \mu^{\prime} ; F^{\prime}, G^{\prime}\right) \oplus B^{\prime}
$$

with $B, B^{\prime}$ boundaries.

Proposition 12.21 (i) Cobordism is an equivalence relation on $\epsilon$-quadratic formations over $A$.

(ii) For any lagrangians $F, G, H$ in a nonsingular $\epsilon$-quadratic form $(K, \lambda, \mu)$

$$
(K, \lambda, \mu ; F, G) \oplus(K, \lambda, \mu ; G, H) \sim(K, \lambda, \mu ; F, H) .
$$

(iii) For any $\epsilon$-quadratic formation $(K, \lambda, \mu ; F, G)$

$$
\begin{aligned}
& (K, \lambda, \mu ; F, G) \oplus(K, \lambda, \mu ; G, F) \sim 0, \\
& (K, \lambda, \mu ; F, G) \oplus(K,-\lambda,-\mu ; F, G) \sim 0 .
\end{aligned}
$$

Proof (i) Clear.

(ii) (Taken from Proposition 9.14 of [69]). Choose lagrangians $F^{*}, G^{*}, H^{*}$ in $(K, \lambda, \mu)$ complementary to $F, G, H$ respectively. The $\epsilon$-quadratic formations $\left(K_{i}, \lambda_{i}, \mu_{i} ; F_{i}, G_{i}\right)(1 \leqslant i \leqslant 4)$ defined by

$$
\begin{aligned}
\left(K_{1}, \lambda_{1}, \mu_{1} ; F_{1}, G_{1}\right)= & \left(K,-\lambda,-\mu ; G^{*}, G^{*}\right), \\
\left(K_{2}, \lambda_{2}, \mu_{2} ; F_{2}, G_{2}\right)= & \left(K \oplus K, \lambda \oplus-\lambda, \mu \oplus-\mu ; F \oplus F^{*}, H \oplus G^{*}\right) \\
& \oplus\left(K \oplus K,-\lambda \oplus \lambda,-\mu \oplus \mu ; \Delta_{K}, H^{*} \oplus G\right), \\
\left(K_{3}, \lambda_{3}, \mu_{3} ; F_{3}, G_{3}\right)= & \left(K \oplus K, \lambda \oplus-\lambda, \mu \oplus-\mu ; F \oplus F^{*}, G \oplus G^{*}\right), \\
\left(K_{4}, \lambda_{4}, \mu_{4} ; F_{4}, G_{4}\right)= & \left(K \oplus K, \lambda \oplus-\lambda, \mu \oplus-\mu ; G \oplus G^{*}, H \oplus G^{*}\right) \\
& \oplus\left(K \oplus K,-\lambda \oplus \lambda,-\mu \oplus \mu ; \Delta_{K}, H^{*} \oplus G\right)
\end{aligned}
$$


are such that

$$
\begin{aligned}
(K, \lambda, \mu ; F, G) & \oplus(K, \lambda, \mu ; G, H) \oplus\left(K_{1}, \lambda_{1}, \mu_{1} ; F_{1}, G_{1}\right) \oplus\left(K_{2}, \lambda_{2}, \mu_{2} ; F_{2}, G_{2}\right) \\
& =(K, \lambda, \mu ; F, H) \oplus\left(K_{3}, \lambda_{3}, \mu_{3} ; F_{3}, G_{3}\right) \oplus\left(K_{4}, \lambda_{4}, \mu_{4} ; F_{4}, G_{4}\right) .
\end{aligned}
$$

Each of $\left(K_{i}, \lambda_{i}, \mu_{i} ; F_{i}, G_{i}\right)(1 \leqslant i \leqslant 4)$ is isomorphic to a boundary, since there exists a lagrangian $H_{i}$ in $\left(K_{i}, \lambda_{i}, \mu_{i}\right)$ complementary to both $F_{i}$ and $G_{i}$, so that 12.19 (ii) applies and $\left(K_{i}, \lambda_{i}, \mu_{i} ; F_{i}, G_{i}\right) \sim 0$. Explicitly, take

$$
\begin{aligned}
& H_{1}=G \subset K_{1}=K, \\
& H_{2}=\Delta_{K \oplus K} \subset K_{2}=(K \oplus K) \oplus(K \oplus K), \\
& H_{3}=\Delta_{K} \subset K_{3}=K \oplus K, \\
& H_{4}=\Delta_{K \oplus K} \subset K_{4}=(K \oplus K) \oplus(K \oplus K) .
\end{aligned}
$$

(iii) By (ii)

$$
(K, \lambda, \mu ; F, G) \oplus(K, \lambda, \mu ; G, F) \sim(K, \lambda, \mu ; F, F)=\partial(F, 0,0) \sim 0,
$$

and by Proposition 12.5 (iii)

$$
(K, \lambda, \mu ; G, F) \sim(K,-\lambda,-\mu ; F, G)
$$

Remark 12.22 The identity of 12.21 (ii)

$$
(K, \lambda, \mu ; F, G) \oplus(K, \lambda, \mu ; G, H) \sim(K, \lambda, \mu ; F, H)
$$

is the $L$-theoretic analogue of the Whitehead Lemma 8.2. See Lemma 6.2 of Wall [92] and the commentary on pp. 72-73 of [92] for the geometric motivation.

Definition 12.23 The $(2 n+1)$-dimensional $L$-group $L_{2 n+1}(A)$ of a ring with involution $A$ is the group of cobordism classes of $(-1)^{n}$-quadratic formations $(K, \lambda, \mu ; F, G)$ over $A$, with addition and inverses given by

$$
\begin{aligned}
\left(K_{1}, \lambda_{1}, \mu_{1} ; F_{1}, G_{1}\right) & +\left(K_{2}, \lambda_{2}, \mu_{2} ; F_{2}, G_{2}\right) \\
= & \left(K_{1} \oplus K_{2}, \lambda_{1} \oplus \lambda_{2}, \mu_{1} \oplus \mu_{2} ; F_{1} \oplus F_{2}, G_{1} \oplus G_{2}\right), \\
-(K, \lambda, \mu ; F, G)= & (K,-\lambda,-\mu ; F, G) \in L_{2 n+1}(A) .
\end{aligned}
$$

Since $L_{2 n+1}(A)$ depends on the residue $n(\bmod 2)$, only two $L$-groups have actually been defined, $L_{1}(A)$ and $L_{3}(A)$. 
Example 12.24 Kervaire and Milnor [38] proved that the odd-dimensional $L$ groups of $\mathbb{Z}$ are trivial

$$
L_{2 n+1}(\mathbb{Z})=0
$$

See Example 12.44 below for an outline of the computation.

Remark 12.25 Chapter 22 of Ranicki [71] is an introduction to the computation of the odd-dimensional surgery obstruction groups of finite groups $\pi$, with

$$
L_{2 n+1}(\mathbb{Z}[\pi])=(2 \text {-primary torsion }) .
$$

See Hambleton and Taylor [30] for a considerably more complete account.

Example 12.26 The odd-dimensional $L$-groups of $\mathbb{Z}\left[\mathbb{Z}_{2}\right]$ with the oriented involution $\bar{T}=T$ are given by

$$
L_{2 n+1}\left(\mathbb{Z}\left[\mathbb{Z}_{2}\right]\right)= \begin{cases}0 & \text { if } n \equiv 0(\bmod 2) \\ \mathbb{Z}_{2} & \text { if } n \equiv 1(\bmod 2)\end{cases}
$$

\subsection{The odd-dimensional surgery obstruction}

It was shown in Section 10.4 that every $(2 n+1)$-dimensional degree 1 normal map is bordant to an $n$-connected degree 1 normal map. As in the even-dimensional case considered in Section 11.5 there is an obstruction to the existence of a further bordism to an $(n+1)$-connected degree 1 normal map (= homotopy equivalence), which is defined as follows.

Definition 12.27 The surgery obstruction of an $n$-connected $(2 n+1)$-dimensional degree 1 normal map $(f, b): M^{2 n+1} \rightarrow X$ is the cobordism class of a kernel $(-1)^{n}$-quadratic formation over $\mathbb{Z}\left[\pi_{1}(X)\right]$

$$
\sigma_{*}(f, b)=(K, \lambda, \mu ; F, G) \in L_{2 n+1}\left(\mathbb{Z}\left[\pi_{1}(X)\right]\right) .
$$

The main result of this section is that $\sigma_{*}(f, b)=0$ if (and for $n \geqslant 2$ only if) $(f, b)$ is bordant to a homotopy equivalence. It is clear that if $(f, b)$ is a homotopy equivalence then $\sigma_{*}(f, b)=0$, for then $(K, \lambda, \mu ; F, G)$ is a trivial formation.

Proposition 12.28 The surgery obstructions of bordant $n$-connected $(2 n+1)$ dimensional degree 1 normal maps $(f, b): M^{2 n+1} \rightarrow X,\left(f^{\prime}, b^{\prime}\right): M^{2 n+1} \rightarrow X$ are the same

$$
\sigma_{*}(f, b)=\sigma_{*}\left(f^{\prime}, b^{\prime}\right) \in L_{2 n+1}\left(\mathbb{Z}\left[\pi_{1}(X)\right]\right) .
$$


Proof By 12.15 the kernel formations $(K, \lambda, \mu ; F, G),\left(K^{\prime}, \lambda^{\prime}, \mu^{\prime} ; F^{\prime}, G^{\prime}\right)$ of $(f, b)$, $\left(f^{\prime}, b^{\prime}\right)$ are related by a stable isomorphism

$$
(K, \lambda, \mu ; F, G) \oplus\left(K^{\prime},-\lambda^{\prime},-\mu^{\prime} ; F^{\prime}, G^{\prime}\right) \rightarrow \partial\left(K_{n+1}(W), \lambda_{W}, \mu_{W}\right)
$$

with $\left(K_{n+1}(W), \lambda_{W}, \mu_{W}\right)$ the kernel $(-1)^{n+1}$-quadratic form of an $(n+1)$ connected normal bordism

$$
\left((e, a) ;(f, b),\left(f^{\prime}, b^{\prime}\right)\right):\left(W^{2 n+2} ; M^{2 n+1}, M^{\prime 2 n+1}\right) \rightarrow X \times(I ;\{0\},\{1\}) .
$$

By Proposition 12.21 (iii)

$$
(K, \lambda, \mu ; F, G) \oplus(K,-\lambda,-\mu ; F, G)=0 \in L_{2 n+1}\left(\mathbb{Z}\left[\pi_{1}(X)\right]\right) .
$$

(This can also be proved geometrically, by considering the $(n+1)$-connected normal bordism $(e, a)$ obtained from

$$
(f, b) \times 1: M \times(I ;\{0\},\{1\}) \rightarrow X \times(I ;\{0\},\{1\})
$$

by $n$-surgeries on the interior killing $K_{n}(M \times I)=K_{n}(M)$, with a stable isomorphism

$$
(K, \lambda, \mu ; F, G) \oplus(K,-\lambda,-\mu ; F, G) \rightarrow \partial\left(K_{n+1}(W), \lambda_{W}, \mu_{W}\right)
$$

as above). The surgery obstructions are such that

$$
\begin{aligned}
\sigma_{*}(f, b) & =(K, \lambda, \mu ; F, G)=-(K,-\lambda,-\mu ; F, G) \\
& =\left(K^{\prime}, \lambda^{\prime}, \mu^{\prime} ; F^{\prime}, G^{\prime}\right)=\sigma_{*}\left(f^{\prime}, b^{\prime}\right) \in L_{2 n+1}\left(\mathbb{Z}\left[\pi_{1}(X)\right]\right) .
\end{aligned}
$$

Theorem 12.29 A $(2 n+1)$-dimensional degree 1 normal map of pairs

$$
(f, b):\left(M^{2 n+1}, \partial M\right) \rightarrow(X, \partial X)
$$

with $\partial f: \partial M \rightarrow \partial X$ a homotopy equivalence has a rel $\partial$ surgery obstruction

$$
\sigma_{*}(f, b) \in L_{2 n+1}\left(\mathbb{Z}\left[\pi_{1}(X)\right]\right)
$$

such that $\sigma_{*}(f, b)=0$ if (and for $n \geqslant 2$ only if) $(f, b)$ is bordant rel $\partial$ to $a$ homotopy equivalence of pairs.

Proof The surgery obstruction of $(f, b)$ is defined by

$$
\sigma_{*}(f, b)=\left(K^{\prime}, \lambda^{\prime}, \mu^{\prime} ; F^{\prime}, G^{\prime}\right) \in L_{2 n+1}\left(\mathbb{Z}\left[\pi_{1}(X)\right]\right)
$$

with $\left(K^{\prime}, \lambda^{\prime}, \mu^{\prime} ; F^{\prime}, G^{\prime}\right)$ a kernel $(-1)^{n}$-quadratic formation for any $n$-connected degree 1 normal map $\left(f^{\prime}, b^{\prime}\right):\left(M^{\prime}, \partial M\right) \rightarrow(X, \partial X)$ bordant to $(f, b)$ relative to the boundary, with $\partial f^{\prime}=\partial f$, exactly as in the closed case $\partial M=\partial X=\emptyset$ in 
12.27. The rel $\partial$ version of 12.28 shows that the surgery obstruction is a normal bordism invariant, which is 0 for a homotopy equivalence. Conversely, assume that $n \geqslant 2$ and $\sigma_{*}(f, b)=0 \in L_{2 n+1}\left(\mathbb{Z}\left[\pi_{1}(X)\right]\right)$, so that $(f, b)$ has a kernel $(-1)^{n}$-quadratic formation $(K, \lambda, \mu ; F, G)$ with a stable isomorphism

$$
(K, \lambda, \mu ; F, G) \oplus B \rightarrow B^{\prime}
$$

for some boundary formations $B=\partial(H, \phi, \theta), B^{\prime}=\partial\left(H^{\prime}, \phi^{\prime}, \theta^{\prime}\right)$, with $H, H^{\prime}$ f.g. free $\mathbb{Z}\left[\pi_{1}(X)\right]$-modules of ranks $k, k^{\prime}$ (say). As in the proof of Proposition 11.42 use the $(-1)^{n+1}$-quadratic form $(H, \phi, \theta)$ to perform $k n$-surgeries on $(f, b)$ : $M \rightarrow X$ killing $0 \in K_{n}(M)$, such that the trace

$$
\left((e, a) ;(f, b),\left(f^{\prime}, b^{\prime}\right)\right):\left(W^{2 n+2} ; M^{2 n+1}, M^{\prime 2 n+1}\right) \rightarrow X \times(I ;\{0\},\{1\})
$$

is $(n+1)$-connected with kernel $(-1)^{n+1}$-quadratic form

$$
\left(K_{n+1}(W), \lambda_{W}, \mu_{W}\right)=(H, \phi, \theta) .
$$

The effect is an $n$-connected degree 1 normal map $\left(f^{\prime}, b^{\prime}\right): M^{\prime} \rightarrow X$ with kernel formation $(K, \lambda, \mu ; F, G) \oplus B$ stably isomorphic to the boundary $B^{\prime}$. By Proposition $12.13 K_{n}\left(M^{\prime}\right)$ can be killed by $k^{\prime} n$-surgeries on $\left(f^{\prime}, b^{\prime}\right)$, so that $(f, b)$ is bordant to a homotopy equivalence.

Corollary 12.30 Let $\pi$ be a finitely presented group with an orientation character $w: \pi \rightarrow \mathbb{Z}_{2}$, and let $n \geqslant 2$. Every element $x \in L_{2 n+1}(\mathbb{Z}[\pi])$ is the rel $\partial$ surgery obstruction $x=\sigma_{*}(f, b)$ of an $n$-connected $(2 n+1)$-dimensional degree 1 normal bordism $(f, b): M^{2 n+1} \rightarrow X$ with $\left(\pi_{1}(X), w(X)\right)=(\pi, w)$.

Proof By Proposition 11.75 there exists a closed $2 n$-dimensional manifold $N$ with $\left(\pi_{1}(N), w(N)\right)=(\pi, w)$. By Proposition 12.17 every $(-1)^{n}$-quadratic formation $(K, \lambda, \mu ; F, G)$ representing $x$ is realized as the kernel formation of an $n$-connected $(2 n+1)$-dimensional degree 1 normal bordism

$$
(f, b):\left(M^{2 n+1} ; N^{2 n}, N^{\prime 2 n}\right) \rightarrow N \times(I ;\{0\},\{1\})
$$

with $(f, b) \mid=1: N \rightarrow N$ and $(f, b) \mid: N^{\prime} \rightarrow N$ a homotopy equivalence. The rel $\partial$ surgery obstruction is

$$
\sigma_{*}(f, b)=(K, \lambda, \mu ; F, G)=x \in L_{2 n+1}(\mathbb{Z}[\pi]) .
$$

Remark 12.31 An $\epsilon$-quadratic formation over a ring with involution $A$ is nullcobordant if and only if it is stably isomorphic to the boundary of a $(-\epsilon)$ quadratic form (Corollary 9.12 of Ranicki [75]). A $(-1)^{n}$-quadratic formation $(K, \lambda, \mu ; F, G)$ is thus such that 


$$
(K, \lambda, \mu ; F, G)=0 \in L_{2 n+1}(A)
$$

if and only if $(K, \lambda, \mu ; F, G)$ is stably isomorphic to the boundary $\partial(H, \phi, \theta)$ of a $(-1)^{n+1}$-quadratic form $(H, \phi, \theta)$. For a group $\operatorname{ring} A=\mathbb{Z}[\pi]$ this can be proved geometrically, using Theorem 12.29 and Corollary 12.30 .

\subsection{Surgery on formations}

The odd-dimensional surgery obstruction theory developed in Section 12.4 is somewhat indirect - it is hard to follow through the algebraic effect of geometric surgeries. This will now be made easier, using algebraic surgery on formations.

If $(f, b): M \rightarrow X,\left(f^{\prime}, b^{\prime}\right): M^{\prime} \rightarrow X$ are $n$-connected $(2 n+1)$-dimensional degree 1 normal maps such that $\left(f^{\prime}, b^{\prime}\right)$ is obtained from $(f, b)$ by an $n$-surgery then a kernel $(-1)^{n}$-quadratic formation for $\left(f^{\prime}, b^{\prime}\right)$ can be obtained by an algebraic surgery on a kernel $(-1)^{n}$-quadratic formation for $(f, b)$. The geometric surgeries on $(f, b)$ correspond to algebraic surgeries on a kernel formation, as in the evendimensional case considered in Section 11.3. However, odd-dimensional surgery behaves somewhat differently from even-dimensional surgery. In both cases, the aim of performing surgery is to make the kernel modules as small as possible. Given an $\epsilon$-quadratic form $(K, \lambda, \mu)$ over $A$ it is possible to kill an element $x \in K$ if and only if $\mu(x)=0$, with unique effect : if $x \neq 0$ generates a direct summand $\langle x\rangle \subset K$ the effect of the surgery is a cobordant form $\left(K^{\prime}, \lambda^{\prime}, \mu^{\prime}\right)$ with

$$
\begin{aligned}
& \left(K^{\prime}, \lambda^{\prime}, \mu^{\prime}\right)=\left(\langle x\rangle^{\perp} /\langle x\rangle,[\lambda],[\mu]\right) \\
& (K, \lambda, \mu) \cong\left(K^{\prime}, \lambda^{\prime}, \mu^{\prime}\right) \oplus H_{\epsilon}(A) \\
& \operatorname{rank}_{A}\left(K^{\prime}\right)=\operatorname{rank}_{A}(K)-2<\operatorname{rank}_{A}(K) .
\end{aligned}
$$

Given an $\epsilon$-quadratic formation $(K, \lambda, \mu ; F, G)$ it is possible to kill every element in the kernel module $x \in K /(F+G)$ by algebraic surgery, but there are many choices in carrying out such a surgery, and the effect of any such surgery may result in a formation $\left(K^{\prime}, \lambda^{\prime}, \mu^{\prime} ; F^{\prime}, G^{\prime}\right)$ with kernel module $K^{\prime} /\left(F^{\prime}+G^{\prime}\right)$ bigger than $K /(F+G)$. In the context of geometric surgery consider the trace of $k$ $n$-surgeries on an $n$-connected $(2 n+1)$-dimensional normal map $(f, b): M \rightarrow X$ killing $x_{1}, x_{2}, \ldots, x_{k} \in K_{n}(M)$

$$
\left((F, B) ;(f, b),\left(f^{\prime}, b^{\prime}\right)\right):\left(W^{2 n+2} ; M^{2 n+1}, M^{\prime 2 n+1}\right) \rightarrow X \times(I ;\{0\},\{1\})
$$

and let $(K, \lambda, \mu ; F, G),\left(K^{\prime}, \lambda^{\prime}, \mu^{\prime} ; F^{\prime}, G^{\prime}\right)$ be kernel $(-1)^{n}$-quadratic formations for $(f, b),\left(f^{\prime}, b^{\prime}\right)$. Proposition 10.25 (iii) gives a commutative braid of exact sequences 


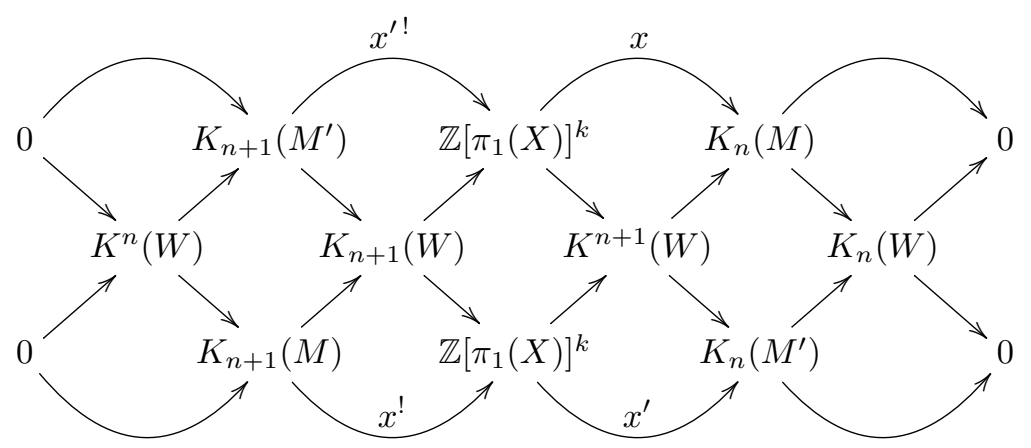

and a set of $\mathbb{Z}\left[\pi_{1}(X)\right]$-module generators $\left\{x_{1}^{\prime}, x_{2}^{\prime}, \ldots, x_{k}^{\prime}\right\} \subset \operatorname{ker}\left(K_{n}\left(M^{\prime}\right) \rightarrow\right.$ $\left.K_{n}(W)\right)$ with

$$
\begin{aligned}
& K_{n+1}(M)=F \cap G, K_{n}(M)=K /(F+G), \\
& K_{n+1}\left(M^{\prime}\right)=F^{\prime} \cap G^{\prime}, K_{n}\left(M^{\prime}\right)=K^{\prime} /\left(F^{\prime}+G^{\prime}\right), \\
& K_{n}(W)=K_{n}(M) /\left\langle x_{1}, x_{2}, \ldots, x_{k}\right\rangle=K_{n}\left(M^{\prime}\right) /\left\langle x_{1}^{\prime}, x_{2}^{\prime}, \ldots, x_{k}^{\prime}\right\rangle .
\end{aligned}
$$

The different effects of killing $x_{1}, x_{2}, \ldots, x_{k}$ correspond to the different ways of framing $n$-embeddings $g_{i}: S^{n} \hookrightarrow M^{2 n+1}$ representing $x_{i}$, or equivalently to the different extensions of $g_{i}$ to framed $n$-embeddings $\bar{g}_{i}: S^{n} \times D^{n+1} \hookrightarrow M^{2 n+1}$. Every set of $\mathbb{Z}\left[\pi_{1}(X)\right]$-module generators $\left\{x_{1}, x_{2}, \ldots, x_{k}\right\} \subset K_{n}(M)$ can be killed by $n$-surgeries with $(n+1)$-connected trace (i.e. $K_{n}(W)=0$ ) but in general the effect $\left(f^{\prime}, b^{\prime}\right): M^{\prime} \rightarrow X$ will not be a homotopy equivalence, with $K_{n}\left(M^{\prime}\right) \neq 0$.

In order to keep track of algebraic surgeries on formations it is convenient to work with the following refinement of the notion of a formation.

Definition 12.32 (i) A split $\epsilon$-quadratic formation over $A$

$$
(F, G)=\left(F,\left(\left(\begin{array}{l}
\gamma \\
\delta
\end{array}\right), \theta\right) G\right)
$$

is given by f.g. free $A$-modules $F, G$, morphisms $\gamma: G \rightarrow F, \delta: G \rightarrow F^{*}$ and a $(-\epsilon)$-quadratic form $(G, \theta)$ such that

(a) $\gamma^{*} \delta=\theta-\epsilon \theta^{*}: G \rightarrow G^{*}$,

(b) the sequence

$$
0 \longrightarrow G \stackrel{\left(\begin{array}{l}
\gamma \\
\delta
\end{array}\right)}{\longrightarrow} F \oplus F^{*} \stackrel{\left(\delta^{*} \quad \epsilon \gamma^{*}\right)}{\longrightarrow} G^{*} \longrightarrow 0
$$

is exact. 
Equivalently,

$$
\left(\left(\begin{array}{l}
\gamma \\
\delta
\end{array}\right), \theta\right):(G, 0) \rightarrow H_{\epsilon}(F)
$$

is a morphism of split $\epsilon$-quadratic forms which is the inclusion of a lagrangian.

(ii) An isomorphism of split $\epsilon$-quadratic formations over $A$

$$
(\alpha, \beta, \chi):(F, G) \rightarrow\left(F^{\prime}, G^{\prime}\right)
$$

is given by isomorphisms $\alpha: F \rightarrow F^{\prime}, \beta: G \rightarrow G^{\prime}$ and a $(-\epsilon)$-quadratic form $\left(F^{*}, \chi\right)$ such that the diagram

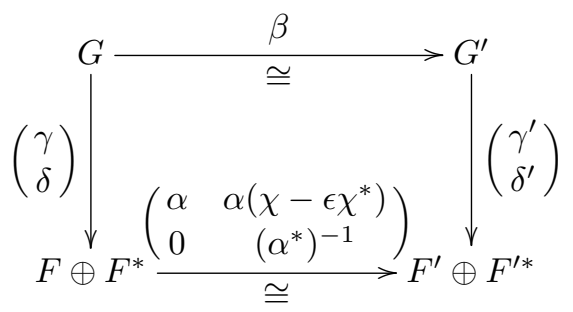

commutes. Thus

$$
f=\left(\begin{array}{cc}
\alpha & \alpha\left(\chi-\epsilon \chi^{*}\right) \\
0 & \left(\alpha^{*}\right)^{-1}
\end{array}\right): H_{\epsilon}(F) \rightarrow H_{\epsilon}\left(F^{\prime}\right)
$$

is an isomorphism of hyperbolic $\epsilon$-quadratic forms with $f(F)=F^{\prime}, f(G)=G^{\prime}$.

(iii) A split $\epsilon$-quadratic formation $(F, G)$ is trivial if it is isomorphic to

$$
\left(F, F^{*}\right)=\left(F,\left(\left(\begin{array}{l}
0 \\
1
\end{array}\right), 0\right) F^{*}\right) .
$$

(iv) A stable isomorphism of split $\epsilon$-quadratic formations over $A$

$$
[\alpha, \beta, \chi]:(F, G) \rightarrow\left(F^{\prime}, G^{\prime}\right)
$$

is an isomorphism of the type

$$
(\alpha, \beta, \chi):(F, G) \oplus\left(H, H^{*}\right) \rightarrow\left(F^{\prime}, G^{\prime}\right) \oplus\left(H^{\prime}, H^{* *}\right)
$$

with $\left(H, H^{*}\right),\left(H^{\prime}, H^{*}\right)$ trivial split formations.

(v) The boundary of a split $(-\epsilon)$-quadratic form $(K, \psi)$ is the graph split $\epsilon$-quadratic formation

$$
\partial(K, \psi)=\left(K,\left(\left(\begin{array}{c}
1 \\
\psi-\epsilon \psi^{*}
\end{array}\right), \psi\right) K\right) .
$$

(vi) Split $\epsilon$-quadratic formations $(F, G),\left(F^{\prime}, G^{\prime}\right)$ are cobordant if there exists a stable isomorphism

$$
[\alpha, \beta, \chi]:(F, G) \oplus \partial(K, \psi) \rightarrow\left(F^{\prime}, G^{\prime}\right) \oplus \partial\left(K^{\prime}, \psi^{\prime}\right)
$$

for some split $(-\epsilon)$-quadratic forms $(K, \psi),\left(K^{\prime}, \psi^{\prime}\right)$. 
Proposition 12.33 (i) A split $\epsilon$-quadratic formation $(F, G)$ is isomorphic to a boundary if and only if there exists a split $(-\epsilon)$-quadratic form $\left(F^{*}, \chi\right)$ such that $\gamma+\left(\chi-\epsilon \chi^{*}\right) \delta: G \rightarrow F$ is an isomorphism.

(ii) A split formation $(F, G)$ is stably isomorphic to 0 if and only if $\delta: G \rightarrow F^{*}$ is an isomorphism.

(iii) Cobordism is an equivalence relation on split $\epsilon$-quadratic formations over $A$.

(iv) The cobordism group of split $(-1)^{n}$-quadratic formations over $A$ is isomorphic to $L_{2 n+1}(A)$.

(v) For any split $(-1)^{n}$-quadratic formation $(F, G)=\left(F,\left(\left(\begin{array}{l}\gamma \\ \delta\end{array}\right), \theta\right) G\right)$ over $A$

$$
\begin{aligned}
-(F, G) & =\left(F,\left(\left(\begin{array}{c}
-\gamma \\
\delta
\end{array}\right),-\theta\right) G\right) \\
(F, G) & =\left(F^{*},\left(\left(\begin{array}{c}
\delta \\
(-1)^{n} \gamma
\end{array}\right),-\theta\right) G\right) \in L_{2 n+1}(A) .
\end{aligned}
$$

Proof (i) If $(\alpha, \beta, \chi):(F, G) \rightarrow \partial(K, \psi)$ is an isomorphism of split formations then

$$
\alpha^{-1} \beta=\gamma+\left(\chi-\epsilon \chi^{*}\right) \delta: G \rightarrow F
$$

is an $A$-module isomorphism.

For the converse, consider first the special case when $\gamma$ is an isomorphism. There is defined an isomorphism of split formations

$$
(\gamma, 1, \chi): \partial(G, \theta) \rightarrow(F, G)
$$

More generally, if $\gamma^{\prime}=\gamma+\left(\chi-\epsilon \chi^{*}\right) \delta: G \rightarrow F$ is an isomorphism there is defined an isomorphism of split formations

$$
(1,1, \chi):(F, G) \rightarrow\left(F^{\prime}, G^{\prime}\right)=\left(F,\left(\left(\begin{array}{c}
\gamma^{\prime} \\
\delta
\end{array}\right), \theta+\delta^{*} \chi \delta\right) G\right)
$$

and $\left(F^{\prime}, G^{\prime}\right)$ is isomorphic to a boundary by the special case.

(ii) If $(\alpha, \beta, \chi):(F, G) \rightarrow\left(K, K^{*}\right)$ is an isomorphism of split formations then

$$
\delta=\alpha^{*} \beta: G \rightarrow F^{*}
$$

is an $A$-module isomorphism.

Conversely, if $\delta$ is an isomorphism there is defined an isomorphism of split formations

$$
\left(1, \delta,-\left(\delta^{*}\right)^{-1} \theta \delta^{-1}\right):(F, G) \rightarrow\left(F, F^{*}\right)
$$

(iii) Clear.

(iv) A split $\epsilon$-quadratic formation $(F, G)$ determines an $\epsilon$-quadratic formation

$$
\left(H_{\epsilon}(F) ; F, \operatorname{im}\left(\left(\begin{array}{l}
\gamma \\
\delta
\end{array}\right): G \rightarrow F \oplus F^{*}\right)\right)
$$


and an isomorphism of split formations determines an isomorphism of formations. Every $\epsilon$-quadratic formation $(K, \lambda, \mu ; F, G)$ is isomorphic to one of this type, by 12.3 , and a (stable) isomorphism of formations

$$
f:(K, \lambda, \mu ; F, G) \rightarrow\left(K^{\prime}, \lambda^{\prime}, \mu^{\prime} ; F^{\prime}, G^{\prime}\right)
$$

lifts to a (stable) isomorphism of split formations

$$
(\alpha, \beta, \gamma):(F, G) \rightarrow\left(F^{\prime}, G^{\prime}\right)
$$

So the only essential difference between a formation and a split formation is the choice of 'Hessian' form $\theta$. Suppose given split $\epsilon$-quadratic formations

$$
\Phi=\left(F,\left(\left(\begin{array}{l}
\gamma \\
\delta
\end{array}\right), \theta\right) G\right), \Phi^{\prime}=\left(F,\left(\left(\begin{array}{l}
\gamma \\
\delta
\end{array}\right), \theta^{\prime}\right) G\right)
$$

with different $\theta, \theta^{\prime}$ such that

$$
\gamma^{*} \delta=\theta-\epsilon \theta^{*}=\theta^{\prime}-\epsilon \theta^{*}: G \rightarrow G^{*}
$$

Let

$$
\widetilde{\Phi}=\left(F^{*},\left(\left(\begin{array}{c}
\widetilde{\gamma} \\
-\epsilon
\end{array}\right), \widetilde{\theta}\right) G^{*}\right)
$$

be the split $\epsilon$-quadratic formation given by an extension (provided by 11.51) of the inclusion of the lagrangian

$$
\left(\left(\begin{array}{l}
\gamma \\
\delta
\end{array}\right), \theta\right):(G, 0) \rightarrow H_{\epsilon}(F)=\left(F \oplus F^{*},\left(\begin{array}{ll}
0 & 1 \\
0 & 0
\end{array}\right)\right)
$$

to an isomorphism of hyperbolic split $\epsilon$-quadratic forms

$$
\begin{aligned}
& \left(\left(\begin{array}{cc}
\gamma & \widetilde{\delta} \\
\delta & \widetilde{\gamma}
\end{array}\right),\left(\begin{array}{cc}
\theta & 0 \\
\widetilde{\gamma}^{*} \delta & \widetilde{\theta}
\end{array}\right)\right): \\
& H_{\epsilon}(G)=\left(G \oplus G^{*},\left(\begin{array}{ll}
0 & 1 \\
0 & 0
\end{array}\right)\right) \rightarrow H_{\epsilon}(F)=\left(F \oplus F^{*},\left(\begin{array}{ll}
0 & 1 \\
0 & 0
\end{array}\right)\right) .
\end{aligned}
$$

The split $\epsilon$-quadratic formation

$$
\Phi \oplus \widetilde{\Phi}=\left(F \oplus F^{*},\left(\left(\begin{array}{c}
\left(\begin{array}{cc}
\gamma & 0 \\
0 & \widetilde{\gamma}
\end{array}\right) \\
\left(\begin{array}{cc}
\delta & 0 \\
0 & -\epsilon \widetilde{\delta}
\end{array}\right)
\end{array}\right),\left(\begin{array}{cc}
\theta & 0 \\
0 & \widetilde{\theta}
\end{array}\right)\right) G \oplus G^{*}\right)
$$

is isomorphic to a boundary by (i), since

$$
\left(\begin{array}{cc}
\gamma & 0 \\
0 & \widetilde{\gamma}
\end{array}\right)+\left(\begin{array}{cc}
0 & -\epsilon \\
1 & 0
\end{array}\right)\left(\begin{array}{cc}
\delta & 0 \\
0 & -\epsilon \widetilde{\delta}
\end{array}\right)=\left(\begin{array}{cc}
\gamma & \widetilde{\delta} \\
\delta & \widetilde{\gamma}
\end{array}\right): G \oplus G^{*} \rightarrow F \oplus F^{*}
$$

is an isomorphism. Similarly for the split formation $\Phi^{\prime} \oplus \widetilde{\Phi}$. The split formation $\Phi \oplus \Phi^{\prime} \oplus \widetilde{\Phi}$ is cobordant to both $\Phi$ and $\Phi^{\prime}$, which are thus cobordant to each 
other.

(v) These identities follow from 12.21 (ii) + (iii).

(Alternatively, note that there exist stable isomorphisms

$$
\begin{aligned}
& \left(F,\left(\left(\begin{array}{l}
\gamma \\
\delta
\end{array}\right), \theta\right) G\right) \oplus\left(F,\left(\left(\begin{array}{c}
-\gamma \\
\delta
\end{array}\right),-\theta\right) G\right) \rightarrow \partial\left(G \oplus F,\left(\begin{array}{ll}
\theta & 0 \\
\delta & 0
\end{array}\right)\right) \\
& \left(F,\left(\left(\begin{array}{l}
\gamma \\
\delta
\end{array}\right), \theta\right) G\right) \oplus\left(F^{*},\left(\left(\begin{array}{c}
\delta \\
(-1)^{n+1} \gamma
\end{array}\right), \theta\right) G\right) \rightarrow \partial(G, \theta) .
\end{aligned}
$$

The construction of such stable isomorphisms are exercises for the reader.)

Definition 12.34 The data $(H, \chi, j)$ for an algebraic surgery on a split $\epsilon$ quadratic formation $(F, G)$ is a split $(-\epsilon)$-quadratic form $(H, \chi)$ together with a morphism $j: F \rightarrow H^{*}$. The effect of the algebraic surgery is the split $\epsilon$-quadratic formation $\left(F^{\prime}, G^{\prime}\right)$ with

$$
\begin{aligned}
\gamma^{\prime} & =\left(\begin{array}{ll}
\gamma & 0 \\
0 & 1
\end{array}\right): G^{\prime}=G \oplus H \rightarrow F^{\prime}=F \oplus H \\
\delta^{\prime} & =\left(\begin{array}{cc}
\delta & -\epsilon j^{*} \\
j \gamma & \chi-\epsilon \chi^{*}
\end{array}\right): G^{\prime}=G \oplus H \rightarrow F^{*}=F^{*} \oplus H^{*} \\
\theta^{\prime} & =\left(\begin{array}{ll}
\theta & 0 \\
j \gamma & \chi
\end{array}\right): G^{\prime}=G \oplus H \rightarrow G^{*}=G^{*} \oplus H^{*} .
\end{aligned}
$$

Proposition 12.35 (i) If $\left(F_{1}, G_{1}\right),\left(F_{2}, G_{2}\right),\left(F_{3}, G_{3}\right)$ are split $\epsilon$-quadratic formations such that $\left(F_{i+1}, G_{i+1}\right)$ is stably isomorphic to the effect of an algebraic surgery on $\left(F_{i}, G_{i}\right)(i=1,2)$ then $\left(F_{3}, G_{3}\right)$ is stably isomorphic to the effect of an algebraic surgery on $\left(F_{1}, G_{1}\right)$.

(ii) Split $\epsilon$-quadratic formations $(F, G),\left(F^{\prime}, G^{\prime}\right)$ are cobordant if and only if $\left(F^{\prime}, G^{\prime}\right)$ is stably isomorphic to the effect of an algebraic surgery on $(F, G)$.

(iii) A split $(-1)^{n}$-quadratic formation $(F, G)$ is such that $(F, G)=0 \in L_{2 n+1}(A)$ if and only if there exist algebraic surgery data $(H, \chi, j)$ such that

$$
\delta^{\prime}=\left(\begin{array}{cc}
\delta & (-1)^{n+1} j^{*} \\
j \gamma & \chi+(-1)^{n+1} \chi^{*}
\end{array}\right): G^{\prime}=G \oplus H \rightarrow F^{*}=F^{*} \oplus H^{*}
$$

is an isomorphism, in which case $(F, G)$ is stably isomorphic to the boundary $\partial\left(G \oplus H,\left(\begin{array}{cc}\theta & 0 \\ j \gamma & \chi\end{array}\right)\right)$.

Proof (i) Exercise for the reader!

(ii) Suppose first that $(F, G),\left(F^{\prime}, G^{\prime}\right)$ are cobordant, so that there exists stable isomorphism

$$
(F, G) \oplus \partial(H, \chi) \rightarrow\left(F^{\prime}, G^{\prime}\right) \oplus \partial\left(H^{\prime}, \chi^{\prime}\right)
$$

for some $(-\epsilon)$-quadratic forms $(H, \chi),\left(H^{\prime}, \chi^{\prime}\right)$. Now $(F, G) \oplus \partial(H, \chi)$ is the effect of the algebraic surgery on $(F, G)$ with data $(H, \chi, 0)$, and $\left(F^{\prime}, G^{\prime}\right)$ is stably 
isomorphic to the effect of the algebraic surgery on $\left(F^{\prime}, G^{\prime}\right) \oplus \partial\left(H^{\prime}, \chi^{\prime}\right)$ with data $\left(H^{\prime}, \chi^{\prime}, j^{\prime}=\left(\begin{array}{l}0 \\ 1\end{array}\right): F^{\prime} \oplus H^{\prime *} \rightarrow H^{\prime *}\right)$. It now follows from (i) that $\left(F^{\prime}, G^{\prime}\right)$ is stably isomorphic to the effect of an algebraic surgery on $(F, G)$.

Conversely, suppose that $\left(F^{\prime}, G^{\prime}\right)$ is the effect of an algebraic surgery on $(F, G)$ with data $(H, \chi, j)$. By $12.33(\mathrm{v})\left(F^{\prime}, G^{\prime}\right)$ is cobordant to the split formation

$$
\left(F^{*}, G^{\prime}\right)=\left(F^{* *},\left(\left(\begin{array}{c}
\delta^{\prime} \\
-\epsilon \gamma^{\prime}
\end{array}\right), \theta^{\prime}\right) G^{\prime}\right) .
$$

Now $\left(F^{* *}, G^{\prime}\right)$ is isomorphic to $\left(F^{*}, G\right) \oplus\left(H^{*}, H\right)$, and $\left(F^{*}, G\right)$ is cobordant to $(F, G)$ (by $12.33(\mathrm{v}))$, so that $\left(F^{\prime}, G^{\prime}\right)$ is cobordant to $(F, G)$.

(iii) By (ii) a split $(-1)^{n}$-quadratic formation $(F, G)$ is null-cobordant if and only if there exists data $(H, \chi, j)$ such that the effect of the algebraic surgery $\left(F^{\prime}, G^{\prime}\right)$ is trivial.

All this can now be applied to surgery on highly-connected odd-dimensional normal maps.

Proposition 12.36 Let $(f, b): M^{2 n+1} \rightarrow X$ be an $n$-connected $(2 n+1)$-dimensional degree 1 normal map.

(i) A presentation (12.11) of $(f, b)$

$$
((e, a) ;(f, b),(\widehat{f}, \widehat{b})):\left(W^{2 n+2} ; M^{2 n+1}, \widehat{M}^{2 n+1}\right) \rightarrow X \times(I ;\{0\},\{1\})
$$

determines a kernel split $(-1)^{n}$-quadratic formation over $\mathbb{Z}\left[\pi_{1}(X)\right]$

$$
\left(F,\left(\left(\begin{array}{l}
\gamma \\
\delta
\end{array}\right), \theta\right) G\right)=\left(K_{n+1}(W, \widehat{M}), K_{n+1}(W)\right)
$$

with

$$
\begin{aligned}
& \gamma=\text { inclusion }_{*}: G=K_{n+1}(W) \rightarrow F=K_{n+1}(W, \widehat{M}), \\
& \delta=\text { inclusion }_{*}: G=K_{n+1}(W) \rightarrow F^{*}=K_{n+1}(W, M), \\
& \theta=\mu_{W}: G=K_{n+1}(W) \rightarrow G^{*}=K_{n+1}(W, \partial W), \\
& \gamma^{*} \delta=\theta+(-1)^{n+1} \theta^{*}=\lambda_{W}: G=K_{n+1}(W) \rightarrow G^{*}=K_{n+1}(W, \partial W),
\end{aligned}
$$

and exact sequences

$$
\begin{aligned}
& 0 \longrightarrow K_{n+1}(\widehat{M}) \longrightarrow G \stackrel{\gamma}{\longrightarrow} F \longrightarrow K_{n}(\widehat{M}) \longrightarrow 0 \\
& 0 \longrightarrow K_{n+1}(M) \longrightarrow G \stackrel{\delta}{\longrightarrow} F^{*} \longrightarrow K_{n}(M) \longrightarrow 0 .
\end{aligned}
$$

(ii) The surgery obstruction of $(f, b)$ is the cobordism class

$$
\sigma_{*}(f, b)=(F, G) \in L_{2 n+1}\left(\mathbb{Z}\left[\pi_{1}(X)\right]\right)
$$

of the kernel split formation $(F, G)$ constructed in (i) from any presentation of $(f, b)$. 
(iii) The effect of $\ell$ simultaneous geometric $n$-surgeries on $(f, b)$ killing $x_{1}, x_{2}, \ldots, x_{\ell} \in$ $K_{n}(M)$ is a bordant $n$-connected $(2 n+1)$-dimensional normal map $\left(f^{\prime}, b^{\prime}\right): M^{\prime} \rightarrow$ $X$ with kernel split formation $\left(F^{\prime}, G^{\prime}\right)$ obtained by algebraic surgery on $(F, G)$ with data $\left(H, \chi, j: F \rightarrow H^{*}\right)$ such that

$$
\left[j^{*}\right]=\left(\begin{array}{llll}
x_{1} & x_{2} & \ldots & x_{\ell}
\end{array}\right): H=\mathbb{Z}\left[\pi_{1}(X)\right]^{\ell} \rightarrow K_{n}(M)=\operatorname{coker}\left(\delta: G \rightarrow F^{*}\right) .
$$

(iv) For $n \geqslant 2$ algebraic surgeries on $(F, G)$ are realized by geometric surgeries on $(f, b)$.

(v) Let $x_{1}, x_{2}, \ldots, x_{\ell} \in K_{n}(M)$ be as in (iii) (or (iv)). If there exist $y_{1}, y_{2}, \ldots, y_{\ell} \in$ $K_{n+1}(M)$ such that

$$
\lambda\left(x_{i}, y_{i}\right)=1 \in \mathbb{Z}\left[\pi_{1}(X)\right] \quad(1 \leqslant i \leqslant \ell)
$$

with $\lambda: K_{n}(M) \times K_{n+1}(M) \rightarrow \mathbb{Z}\left[\pi_{1}(X)\right]$ the homology intersection pairing (10.22) then

$$
K_{n}\left(M^{\prime}\right)=K_{n}(M) /\left\langle x_{1}, x_{2}, \ldots, x_{\ell}\right\rangle
$$

Proof (i) The split formation $(F, G)$ is just the split version of the kernel formation 12.12 (ii).

(ii) Immediate from (i) and 12.29.

(iii) Let $\left(f^{\prime}, b^{\prime}\right): M^{\prime 2 n+1} \rightarrow X$ be the effect of $\ell n$-surgeries on $(f, b)$ killing $x_{1}, x_{2}, \ldots, x_{\ell} \in K_{n}(M)$. The trace degree 1 normal bordism

$$
\left((g, c) ;(f, b),\left(f^{\prime}, b^{\prime}\right)\right):\left(N^{2 n+2} ; M, M^{\prime}\right) \rightarrow X \times(I ;\{0\},\{1\})
$$

is $n$-connected, and such that

$$
(f, b)\left|=\left(f^{\prime}, b^{\prime}\right)\right|: M_{0}=\operatorname{cl} .\left(M \backslash \bigcup_{\ell} S^{n} \times D^{n+1}\right) \rightarrow X_{0}, X=X_{0} \cup D^{2 n+1} .
$$

Given a presentation $(e, a)$ of $(f, b)$ as in (i) define a presentation of $\left(f^{\prime}, b^{\prime}\right)$

$$
\begin{aligned}
& \left(e^{\prime}, a^{\prime}\right)=-(g, c) \cup(e, a): \\
& \left(W^{\prime} ; M^{\prime}, \widehat{M}\right)=\left(-N ; M^{\prime}, M\right) \cup(W ; M, \widehat{M}) \rightarrow X \times(I ;\{0\},\{1\}) .
\end{aligned}
$$

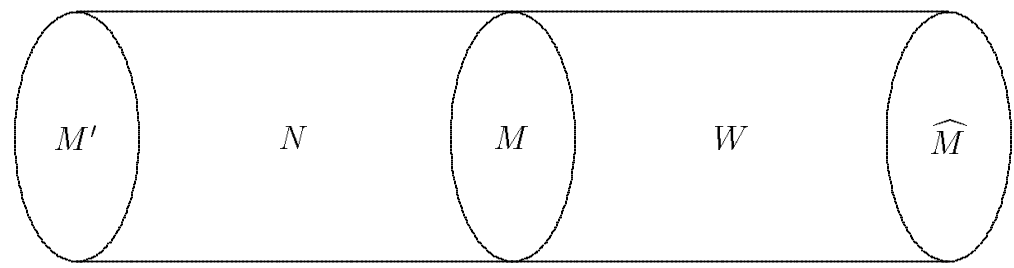

$W^{\prime}$

The corresponding kernel split $(-1)^{n}$-quadratic formation $\left(F^{\prime}, G^{\prime}\right)$ for $\left(f^{\prime}, b^{\prime}\right)$ is the effect of an algebraic surgery on the kernel $(F, G)$ in (i) with data $(H, \chi, j$ : $\left.F \rightarrow H^{*}\right)$ such that 


$$
\begin{aligned}
& H=K_{n+1}(N, M)=\mathbb{Z}\left[\pi_{1}(X)\right]^{\ell}, \\
& j^{*}(H)=\left\langle x_{1}, x_{2}, \ldots, x_{\ell}\right\rangle \subseteq \operatorname{coker}\left(\delta: G \rightarrow F^{*}\right)=K_{n}(M), \\
& \mu_{W^{\prime}}=\left(\begin{array}{cc}
\theta & 0 \\
j \gamma & \chi
\end{array}\right): K_{n+1}\left(W^{\prime}\right)=G \oplus H \rightarrow K_{n+1}\left(W^{\prime}\right)^{*}=G^{*} \oplus H^{*}
\end{aligned}
$$

with exact sequences of $\mathbb{Z}\left[\pi_{1}(X)\right]$-modules

$$
\begin{gathered}
0 \longrightarrow K_{n+1}(N) \longrightarrow K_{n+1}\left(W^{\prime}\right)=G \oplus H \stackrel{\left(\delta \quad(-1)^{n+1} j^{*}\right)}{\longrightarrow} \\
K_{n+1}\left(W^{\prime}, N\right)=K_{n+1}(W, M)=F^{*} \longrightarrow K_{n}(N) \longrightarrow 0, \\
0 \longrightarrow K_{n+1}\left(M^{\prime}\right) \longrightarrow K_{n+1}\left(W^{\prime}\right)=G \oplus H \stackrel{\left(\begin{array}{cc}
\delta & (-1)^{n+1} j^{*} \\
j \gamma & \chi+(-1)^{n+1} \chi^{*}
\end{array}\right)}{\longrightarrow} \\
K_{n+1}\left(W^{\prime}, M^{\prime}\right)=F^{*} \oplus H^{*} \longrightarrow K_{n}\left(M^{\prime}\right) \longrightarrow 0 \\
0 \longrightarrow K_{n+1}\left(M_{0}\right) \longrightarrow K_{n+1}(W)=G \stackrel{\left(\begin{array}{c}
\delta \\
j \gamma
\end{array}\right)}{\longrightarrow} \\
K_{n+1}\left(W^{\prime}, M^{\prime}\right)=F^{*} \oplus H^{*} \longrightarrow K_{n}\left(M_{0}\right) \longrightarrow 0
\end{gathered}
$$

and a commutative braid of exact sequences

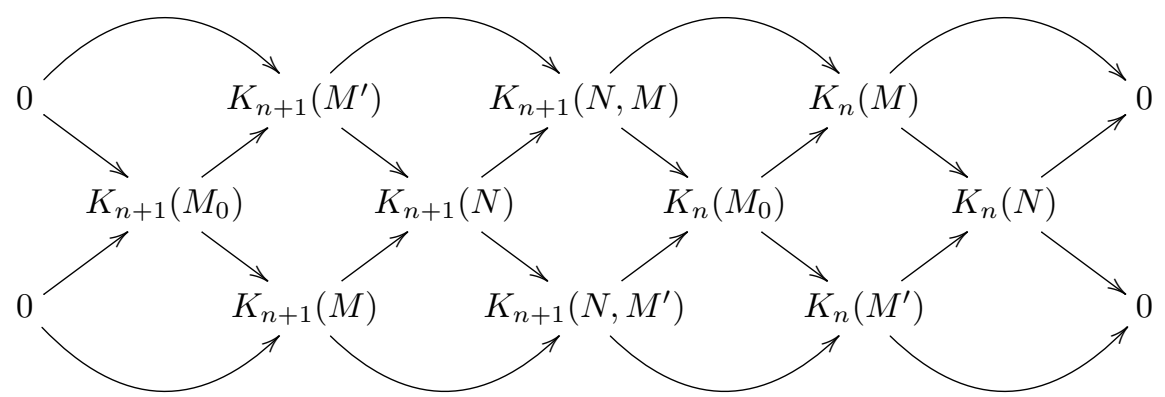

The normal map $(e, a): N \rightarrow X \times I$ is $(n+1)$-connected if and only if the $\mathbb{Z}\left[\pi_{1}(X)\right]$-module morphism

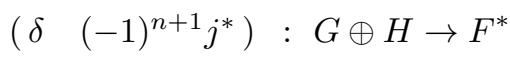

is onto, in which case $K_{n+1}(N)$ is a stably f.g. free $\mathbb{Z}\left[\pi_{1}(X)\right]$-module and the kernel $(-1)^{n+1}$-quadratic form is given by

$$
\begin{aligned}
& \mu_{N}=\left(\begin{array}{cc}
\theta & 0 \\
j \gamma & \chi
\end{array}\right) \mid: \\
& K_{n+1}(N)=\operatorname{ker}\left(\left(\delta \quad(-1)^{n+1} j^{*}\right): G \oplus H \rightarrow F^{*}\right) \rightarrow K_{n+1}(N)^{*} .
\end{aligned}
$$

(iv) Suppose given a presentation $(e, a)$ of $(f, b)$ as in (i) and data $(H, \chi, j)$ for algebraic surgery on the kernel split $(-1)^{n}$-quadratic formation $(F, G)$ with 
$H=\mathbb{Z}\left[\pi_{1}(X)\right]^{\ell}$ and effect $\left(F^{\prime}, G^{\prime}\right)$. Let $x_{1}, x_{2}, \ldots, x_{\ell} \in K_{n}(M)$ be the images of the basis elements $(0, \ldots, 0,1,0, \ldots, 0) \in H$ under the composite

$$
H \stackrel{j^{*}}{\longrightarrow} F^{*}=K_{n+1}(W, M) \stackrel{\partial}{\longrightarrow} K_{n}(M) .
$$

Use the $b$-framing section $s_{b}^{f r}: I_{n+1}(f) \rightarrow I_{n+1}^{f r}(f)(10.14)$ to identify each

$$
x_{i} \in K_{n}(M)=s_{b}^{f r}\left(I_{n+1}(f)\right) \subseteq I_{n+1}^{f r}(f)
$$

with a regular homotopy class of framed $n$-immersions in $(f, b)$. As in the proof of Proposition $10.25 x_{i}$ contains framed $n$-embeddings. For $n \geqslant 2$ the framed $n$-embeddings can be varied within the regular homotopy class by arbitrary elements of $Q_{(-1)^{n+1}}\left(\mathbb{Z}\left[\pi_{1}(X)\right]\right)$, as in the proof of Proposition 11.42. It is therefore possible to kill $x_{1}, x_{2}, \ldots, x_{\ell} \in K_{n}(M)$ by $n$-surgeries on $(f, b)$ such that $\left(F^{\prime}, G^{\prime}\right)$ is the kernel split $(-1)^{n}$-quadratic formation for $\left(f^{\prime}, b^{\prime}\right)$ obtained as in (iii).

(v) The $\mathbb{Z}\left[\pi_{1}(X)\right]$-module morphism

$$
\begin{aligned}
K_{n+1}(M) \rightarrow K_{n+1}\left(N, M^{\prime}\right) & =H^{*}=\mathbb{Z}\left[\pi_{1}(X)\right]^{\ell} \\
y & \mapsto\left(\lambda\left(x_{1}, y\right), \lambda\left(x_{2}, y\right), \ldots, \lambda\left(x_{\ell}, y\right)\right)
\end{aligned}
$$

is onto, so that the braid in (iii) identifies $K_{n}\left(M^{\prime}\right)$ with the cokernel of the $\mathbb{Z}\left[\pi_{1}(X)\right]$-module morphism

$$
\begin{aligned}
K_{n+1}(N, M)=H= & \mathbb{Z}\left[\pi_{1}(X)\right]^{\ell} \rightarrow K_{n}(M)=K_{n}\left(M_{0}\right) ; \\
& \left(a_{1}, a_{2}, \ldots, a_{\ell}\right) \mapsto a_{1} x_{1}+a_{2} x_{2}+\ldots+a_{\ell} x_{\ell} .
\end{aligned}
$$

Remark 12.37 For any normal bordism between $n$-connected $(2 n+1)$-dimensional normal maps $(f, b),\left(f^{\prime}, b^{\prime}\right)$

$$
\left((g, c) ;(f, b),\left(f^{\prime}, b^{\prime}\right)\right):\left(N^{2 n+2} ; M^{2 n+1}, M^{2 n+1}\right) \rightarrow X \times(I ;\{0\},\{1\})
$$

it is possible to kill the kernel $\mathbb{Z}\left[\pi_{1}(X)\right]$-modules $K_{i}(N)(i \leqslant n)$ by surgery below the middle dimension. Thus $(g, c): N \rightarrow X \times I$ can be made $(n+1)$-connected, with $K_{n+1}(N, M)$ a f.g. free $\mathbb{Z}\left[\pi_{1}(X)\right]$-module of rank $\ell \geqslant 0$ (say). The cobordism $\left(N ; M, M^{\prime}\right)$ is the trace of $\ell n$-surgeries on $(f, b)$ with geometric effect $\left(f^{\prime}, b^{\prime}\right)$, and with algebraic effect given by 12.36 .

Algebraic surgery on formations can also be used for purely algebraic computations :

Proposition 12.38 Let $A$ be a principal ideal domain with involution, with quotient field $K$.

(i) Every split $\epsilon$-quadratic formation $(F, G)$ over $A$ is cobordant to a formation $\left(F^{\prime}, G^{\prime}\right)$ with $\delta^{\prime}: G^{\prime} \rightarrow F^{*}$ injective.

(ii) $L_{2 n+1}(K)=0$. 
Proof (i) The $A$-module $\operatorname{coker}\left(\delta: G \rightarrow F^{*}\right)$ is finitely generated, so that it can be expressed as a direct sum of a f.g. free $A$-module $S$ and a f.g. torsion A-module $T$

$$
\operatorname{coker}(\delta)=S \oplus T .
$$

(Here, torsion means that $a T=0$ for some $a \neq 0 \in A$.) The sesquilinear pairing

$$
\lambda: \operatorname{coker}(\delta) \times \operatorname{ker}(\delta) \rightarrow A ;(x, y) \mapsto x(\gamma(y))
$$

is such that for any $x \in S$ there exists $y \in \operatorname{ker}(\delta)$ such that $\lambda(x, y)=1 \in A$. The abstract version of 12.36 (iv) shows that for any algebraic surgery on $(F, G)$ with data $(H, \chi, j)$ such that

$$
j^{*}: H=A^{\ell} \rightarrow \operatorname{coker}(\delta) ;\left(a_{1}, a_{2}, \ldots, a_{\ell}\right) \mapsto a_{1} x_{1}+a_{2} x_{2}+\ldots+a_{\ell} x_{\ell}
$$

the effect of the algebraic surgery $\left(F^{\prime}, G^{\prime}\right)$ has $\delta^{\prime}: G^{\prime} \rightarrow F^{\prime *}$ injective with

$$
\operatorname{coker}\left(\delta^{\prime}\right)=T
$$

(ii) Take $A=K, \epsilon=(-1)^{n}$ in (i). The only torsion $A$-module is 0 , so $\delta^{\prime}$ is an isomorphism, and $\left(F^{\prime}, G^{\prime}\right)$ is a trivial split $(-1)^{n}$-quadratic formation.

\subsection{Linking forms}

Odd-dimensional surgery obstructions were originally formulated by Kervaire and Milnor [38] and Wall [90] in terms of linking forms, but the method only applies to finite fundamental groups $\pi$. However, linking forms remain useful tools in surgery theory. This section is a brief introduction to linking forms and their use in the computation $L_{2 *+1}(\mathbb{Z})=0$. See Chapter 3 of Ranicki [70] for a considerably more complete account.

Let $A$ be a ring with involution, and let $S \subset A$ be a subset such that

(i) each $s \in S$ is a central non-zero divisor, with $\bar{s} \in S$,

(ii) if $s, t \in S$ then $s t \in S$,

(iii) $1 \in S$.

Definition 12.39 (i) The localization of $A$ is the ring of fractions

$$
S^{-1} A=A \times S /\{(a, s) \sim(b, t) \mid a t=b s \in A\}
$$

with elements denoted $\frac{a}{s}$. The natural map

$$
A \rightarrow S^{-1} A ; a \mapsto \frac{a}{1}
$$


is an injective morphism of rings with involution.

(ii) An $(A, S)$-module $T$ is a f.g. $A$-module such that $s T=0$ for some $s \in S$, and which admits a f.g. free $A$-module resolution of the type

$$
0 \longrightarrow F_{1} \stackrel{d}{\longrightarrow} F_{0} \longrightarrow T \longrightarrow 0 .
$$

The dual of $T=\operatorname{coker}(d)$ is the $(A, S)$-module

$$
\widehat{T}=\operatorname{Hom}_{A}\left(T, S^{-1} A / A\right)=\operatorname{coker}\left(d^{*}:\left(F_{0}\right)^{*} \rightarrow\left(F_{1}\right)^{*}\right) .
$$

(iii) A nonsingular $\epsilon$-symmetric linking form $(T, \lambda)$ over $(A, S)$ is an $(A, S)$ module $T$ together with a sesquilinear pairing

$$
\lambda: T \times T \rightarrow S^{-1} A / A
$$

such that for all $x, y, z \in T, a, b \in A$

(a) $\lambda(x, y+z)=\lambda(x, y)+\lambda(x, z) \in S^{-1} A / A$,

(b) $\lambda(a x, b y)=b \lambda(x, y) \bar{a} \in S^{-1} A / A$,

(c) $\lambda(y, x)=\epsilon \overline{\lambda(x, y)} \in S^{-1} A / A$,

(d) the adjoint $A$-module morphism

$$
\lambda: T \rightarrow \widehat{T} ; x \mapsto(y \mapsto \lambda(x, y))
$$

is an $A$-module isomorphism.

(iv) A nonsingular split $\epsilon$-quadratic linking form $(T, \lambda, \nu)$ over $(A, S)$ is an $\epsilon$-quadratic linking form $(T, \lambda)$ together with a function

$$
\nu: T \rightarrow Q_{\epsilon}\left(S^{-1} A / A\right)=\left(S^{-1} A / A\right) /\left\{b-\epsilon \bar{b} \mid b \in S^{-1} A / A\right\}
$$

such that

(a) $\nu(a x)=a \nu(x) \bar{a} \in Q_{\epsilon}\left(S^{-1} A / A\right) \quad(x \in T, a \in A)$,

(b) $\nu(x+y)-\nu(x)-\nu(y)=\lambda(x, y) \in Q_{\epsilon}\left(S^{-1} A / A\right)(x, y \in T)$,

(c) $\lambda(x, x)=\nu(x)+\epsilon \overline{\nu(x)} \in S^{-1} A / A \quad(x \in T)$.

Example 12.40 Let $A$ be an integral domain, and let $S=A \backslash\{0\} \subset A$.

(i) The localization $S^{-1} A=K$ is the quotient field of $A$.

(ii) If $A$ is a principal ideal domain an $(A, S)$-module $T$ is a f.g. $A$-module such that $s T=0$ for some $s \in S$. For any f.g. $A$-module $H$ the torsion submodule $T H \subseteq H$ is an $(A, S)$-module, and $H / T H$ is a f.g. free $A$-module.

There is a close connection between formations and linking forms: 
Proposition 12.41 The isomorphism classes of nonsingular split $\epsilon$-quadratic linking forms $(T, \lambda, \nu)$ over $(A, S)$ are in one-one correspondence with the stable isomorphism classes of split $(-\epsilon)$-quadratic formations $\left(F,\left(\left(\begin{array}{l}\gamma \\ \delta\end{array}\right), \theta\right) G\right)$ over $A$ such that $\delta: S^{-1} G \rightarrow S^{-1} F^{*}$ is an $S^{-1} A$-module isomorphism.

Proof Given such a split formation $(F, G)$ define a linking form $(T, \lambda, \nu)$ by

$$
\begin{aligned}
& T=\operatorname{coker}\left(\delta: G \rightarrow F^{*}\right), \\
& \lambda: T \times T \rightarrow S^{-1} A / A ;(x, y) \mapsto \frac{y(\gamma(z))}{s}, \\
& \nu: T \rightarrow Q_{\epsilon}\left(S^{-1} A / A\right) ; x \mapsto \frac{\theta(z)(z)}{s \bar{s}}, \\
& \left(x, y \in F^{*}, z \in G, s \in S, s x=\delta(z)\right) .
\end{aligned}
$$

For further details of the one-one correspondence see Proposition 3.4.3 of [70].

Definition 12.42 (i) A lagrangian of a split $\epsilon$-quadratic linking form $(K, \lambda, \nu)$ is an $(A, S)$-submodule $L \subseteq K$ such that

$$
\lambda(L \times L)=\{0\} \subset S^{-1} A / A, \nu(L)=\{0\} \subset Q_{\epsilon}\left(S^{-1} A / A\right)
$$

and such that the sequence

$$
0 \longrightarrow L \stackrel{i}{\longrightarrow} T \stackrel{\widehat{i} \lambda}{\longrightarrow} \widehat{L} \longrightarrow 0
$$

is exact, with $i: L \rightarrow T$ the inclusion.

(ii) Let $(K, \psi)$ be a split $\epsilon$-quadratic form over $A$ which is $S^{-1} A$-nonsingular, meaning that $\psi+\epsilon \psi^{*}: S^{-1} K \rightarrow S^{-1} K^{*}$ is an $S^{-1} A$-module isomorphism. The boundary of $(K, \psi)$ is the split $\epsilon$-quadratic linking form over $(A, S)$

$$
\partial(K, \psi)=(T, \lambda, \mu)
$$

with

$$
\begin{aligned}
& T=\operatorname{coker}\left(\psi+\epsilon \psi^{*}: K \rightarrow K^{*}\right) \\
& \lambda: T \times T \rightarrow S^{-1} A / A ;([x],[y]) \mapsto \frac{y(z)}{s}, \\
& \nu: T \rightarrow Q_{\epsilon}\left(S^{-1} A / A\right) ;[x] \mapsto \frac{\mu(z)}{s \bar{s}} \\
& \left(x, y \in K^{*}, z \in K, s \in S, s x=\lambda(z)\right) .
\end{aligned}
$$

The localization exact sequence in algebraic $L$-theory is a useful computational tool: 
Theorem 12.43 The L-groups of $A$ and $S^{-1} A$ are related by an exact sequence $\ldots \longrightarrow L_{m}(A) \longrightarrow L_{m}\left(S^{-1} A\right) \stackrel{\partial}{\longrightarrow} L_{m}(A, S) \longrightarrow L_{m-1}(A) \longrightarrow \ldots$

The $2 n$-dimensional $S$-torsion L-group $L_{2 n}(A, S)$ is the Witt group of split $(-1)^{n}$ quadratic linking forms over $(A, S)$, and

$$
\partial \quad L_{2 n}\left(S^{-1} A\right) \rightarrow L_{2 n}(A, S) ; S^{-1}(K, \psi) \mapsto \partial(K, \psi)
$$

The $(2 n+1)$-dimensional S-torsion L-group $L_{2 n+1}(A, S)$ is the Witt group of split $(-1)^{n}$-quadratic linking formations over $(A, S)$.

Proof See Chapter 3 of [70] (which includes references to the many authors who contributed to the development of the sequence).

\section{Example 12.44 Let}

$$
S=\mathbb{Z} \backslash\{0\} \subset A=\mathbb{Z}
$$

so that $S^{-1} A=\mathbb{Q}$, and an $(A, S)$-module is just a finite abelian group.

(i) Given an abelian group $A$ write the torsion subgroup and the torsion-free quotient as

$$
T A=\{x \in A \mid s x=0 \text { for some } s \neq 0 \in \mathbb{Z}\}, F A=A / T A .
$$

The Universal Coefficient Theorem (3.17) gives for any space $M$ natural isomorphisms

$$
\begin{gathered}
T H^{n}(M) \rightarrow \operatorname{Hom}_{\mathbb{Z}}\left(T H_{n-1}(M), \mathbb{Q} / \mathbb{Z}\right) ; x \mapsto\left(y \mapsto \frac{z(y)}{s}\right) \\
\left(s x=d z, z \in C^{n-1}(M)\right) .
\end{gathered}
$$

If $M$ is an oriented $m$-dimensional manifold then cap product with fundamental class $[M] \in H_{m}(M)$ defines an isomorphism of exact sequences

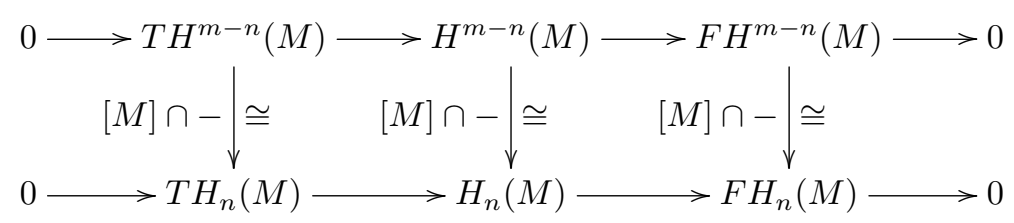

The torsion homology groups $T H_{*}(M)$ are finite abelian groups with a bilinear homology linking pairing

$$
\begin{aligned}
\lambda: & T H_{n}(M) \times T H_{m-n-1}(M) \rightarrow \mathbb{Q} / \mathbb{Z} ;(x, y) \mapsto \frac{z(y)}{s} \\
& \left(z \in C^{m-n-1}(M), s x=d([M] \cap z) \in C_{n}(M)\right)
\end{aligned}
$$

such that the adjoint morphisms 


$$
\lambda: T H_{n}(M) \rightarrow \operatorname{Hom}_{\mathbb{Z}}\left(T H_{m-n-1}(M), \mathbb{Q} / \mathbb{Z}\right) ; x \mapsto(y \mapsto \lambda(x, y))
$$

are isomorphisms, and

$$
\lambda(y, x)=(-1)^{(n+1)(m-n)} \lambda(x, y) \in \mathbb{Q} / \mathbb{Z} .
$$

In particular, if $m=2 n+1$ there is defined a nonsingular $(-1)^{n+1}$-symmetric linking form $\left(T H_{n}(M), \lambda\right)$.

(ii) Let $(f, b): M \rightarrow X$ be an $n$-connected $(2 n+1)$-dimensional degree 1 normal map with $\pi_{1}(X)=\{1\}$. The torsion subgroup $T K_{n}(M) \subseteq K_{n}(M)$ is finite, and the quotient $F K_{n}(M)=K_{n}(M) / T K_{n}(M)=\mathbb{Z}^{k}$ is a f.g. free $\mathbb{Z}$-module. Lift a basis for $F K_{n}(M)$ to elements $\left\{x_{1}, x_{2}, \ldots, x_{k}\right\} \subset K_{n}(M)$. Killing these elements by $n$-surgeries on $(f, b)$ results in a normal bordant $n$-connected map (also denoted by $(f, b))$ such that

$$
K_{n}(M)=T K_{n}(M), K_{n+1}(M)=0 .
$$

A kernel split $(-1)^{n}$-quadratic formation $\left(F,\left(\left(\begin{array}{l}\gamma \\ \delta\end{array}\right), \theta\right) G\right)$ for $(f, b)$ determines a split $(-1)^{n+1}$-quadratic linking pairing $\left(K_{n}(M), \lambda, \nu\right)$ over $(\mathbb{Z}, S)$ as in 12.41 , with

$$
K_{n}(M)=\operatorname{coker}\left(\delta: G \rightarrow F^{*}\right)
$$

a finite abelian group. The proof of $L_{2 n+1}(\mathbb{Z})=0$ in Kervaire and Milnor [38] analyzes the effects that $n$-surgeries on $(f, b)$ have on the kernel linking form $\left(K_{n}(M), \lambda, \nu\right)$. It is shown that $(f, b)$ is normal bordant to a homotopy equivalence by essentially first proving algebraically that the linking form is isomorphic to the boundary $\partial\left(\mathbb{Z}^{\ell}, \psi\right)$ of a $\mathbb{Q}$-nonsingular split $(-1)^{n+1}$-quadratic form $\left(\mathbb{Z}^{\ell}, \psi\right)$ over $\mathbb{Z}$, and then killing $K_{n}(M)$ by the corresponding $\ell n$-surgeries on $(f, b)$. (In [38] only the rel $\partial$ case with $X=D^{2 n+1}$ was considered. However, the algebra is the same for any $X$ with $\pi_{1}(X)=\{1\}$ - see Chapter IV.3 of Browder [14]). (iii) The computation $L_{2 n+1}(\mathbb{Z})=0$ corresponds to the surjectivity of the map $\partial: L_{2 n+2}(\mathbb{Q}) \rightarrow L_{2 n+2}(\mathbb{Z}, S)$ in the $L$-theory localization exact sequence (12.43)

$$
\ldots \longrightarrow L_{m}(\mathbb{Z}) \longrightarrow L_{m}(\mathbb{Q}) \stackrel{\partial}{\longrightarrow} L_{m}(\mathbb{Z}, S) \longrightarrow L_{m-1}(\mathbb{Z}) \longrightarrow \ldots
$$

The decomposition of finite abelian groups into $p$-primary components extends to linking forms, and 


$$
\begin{aligned}
& L_{m}(\mathbb{Z}, S)= \begin{cases}\mathbb{Z}_{8} \oplus \underset{p \neq 2 \text { prime }}{\bigoplus_{0}} L_{0}\left(\mathbb{F}_{p}\right) & \text { if } m \equiv 0(\bmod 4) \\
0 & \text { if } m \equiv 1,2(\bmod 4) \\
\mathbb{Z}_{2} & \text { if } m \equiv 3(\bmod 4)\end{cases} \\
& L_{m}(\mathbb{Z})= \begin{cases}\mathbb{Z} & \text { if } m \equiv 0(\bmod 4) \\
\mathbb{Z}_{2} & \text { if } m \equiv 2(\bmod 4) \\
0 & \text { if } m \equiv 1,3(\bmod 4)\end{cases} \\
& L_{m}(\mathbb{Q})= \begin{cases}\mathbb{Z} \oplus \underset{p \neq 2 \text { prime }}{\bigoplus_{0}} L_{0}\left(\mathbb{F}_{p}\right) & \text { if } m \equiv 0(\bmod 4) \\
0 & \text { if } m \equiv 1,2,3(\bmod 4)\end{cases} \\
& L_{0}\left(\mathbb{F}_{p}\right)= \begin{cases}\mathbb{Z}_{2} \oplus \mathbb{Z}_{2} & \text { if } p \equiv 1(\bmod 4) \\
\mathbb{Z}_{4} & \text { if } p \equiv 3(\bmod 4)\end{cases}
\end{aligned}
$$

with $\mathbb{F}_{p}$ the finite field with $p$ elements and

$$
L_{0}(\mathbb{Q}) \rightarrow \mathbb{Z} ;(V, \lambda) \mapsto \sigma(V, \lambda)
$$

the signature map. See Chapter IV.3 of Milnor and Husemoller [60] for a more detailed account of $L_{0}(\mathbb{Q})$. 


\section{THE STRUCTURE SET}

We are now in a position to prove the results on the structure set $\mathscr{S}(X)$ (1.14) which were stated in Chapter 1.

\subsection{The structure set}

Manifold Existence Theorem 13.1 (Browder, Novikov, Sullivan, Wall)

Let $X$ be an $m$-dimensional geometric Poincaré complex with $m \geqslant 5$.

The structure set $\mathscr{S}(X)$ is non-empty (i.e. $X$ is homotopy equivalent to an $m$ dimensional manifold) if and only if the Spivak normal fibration $\nu_{X}: X \rightarrow B G$ has a vector bundle reduction $\widetilde{\nu}_{X}: X \rightarrow B O$ for which the corresponding normal map $(f, b): M \rightarrow X$ has surgery obstruction

$$
\sigma_{*}(f, b)=0 \in L_{m}\left(\mathbb{Z}\left[\pi_{1}(X)\right]\right) .
$$

Proof Combine the criterion of Proposition 9.38 with the surgery obstructions of Chapters 11,12.

A choice of homotopy equivalence $M \simeq X$ determines an identification

$$
\mathscr{S}(X)=\mathscr{S}(M)
$$

We are now also in a position to prove the surgery exact sequence (1.18) of pointed sets

$$
\ldots \rightarrow L_{m+1}\left(\mathbb{Z}\left[\pi_{1}(M)\right]\right) \rightarrow \mathscr{S}(M) \rightarrow[M, G / O] \stackrel{A}{\rightarrow} L_{m}\left(\mathbb{Z}\left[\pi_{1}(M)\right]\right)
$$

stated in Chapter 1.

An element of the normal structure set (9.40)

$$
x=(\eta, h) \in \mathscr{T}(M)=[M, G / O]
$$

is classified by a stable vector bundle $\eta: M \rightarrow B O$ with a fibre homotopy trivialisation $h: J \eta \simeq\{*\}: M \rightarrow B G$, corresponding to a normal map $(f, b)$ : 
$N \rightarrow M$ with $b: \nu_{N} \rightarrow \nu_{M} \oplus \eta$. The function $A$ in the surgery exact sequence is the surgery obstruction

$$
A(x)=\sigma_{*}(f, b) \in L_{m}\left(\mathbb{Z}\left[\pi_{1}(M)\right]\right) .
$$

The study of normal maps and surgery obstruction theory in Chapters 10, 11 and 12 establishes the exactness of the sequence, made precise as follows.

Manifold Uniqueness Theorem 13.2 (Browder, Novikov, Sullivan, Wall) Let $M$ be an $m$-dimensional manifold with $m \geqslant 5$.

(i) An m-dimensional degree 1 normal map $(f, b): N \rightarrow M$ is such that

$$
\sigma_{*}(f, b)=0 \in L_{m}\left(\mathbb{Z}\left[\pi_{1}(M)\right]\right)
$$

if and only if $(f, b)$ is bordant to a homotopy equivalence $\left(f^{\prime}, b^{\prime}\right): N^{\prime} \rightarrow M$, i.e. if and only if there exists a manifold structure $\left(N^{\prime}, f^{\prime}\right) \in \mathscr{S}(M)$ with image

$$
\left[N^{\prime}, f^{\prime}\right]=(f, b) \in \mathscr{T}(M)=[M, G / O] .
$$

(ii) The group $L_{m+1}\left(\mathbb{Z}\left[\pi_{1}(M)\right]\right)$ acts on the manifold structure set $\mathscr{S}(M)$ by

$$
L_{m+1}\left(\mathbb{Z}\left[\pi_{1}(M)\right]\right) \times \mathscr{S}(M) \rightarrow \mathscr{S}(M) ;\left(x,\left(N_{1}, f_{1}\right)\right) \mapsto x\left(N_{1}, f_{1}\right)=\left(N_{2}, f_{2}\right)
$$

if there exists a degree 1 normal bordism

$$
(F, B):\left(W ; N_{1}, N_{2}\right) \rightarrow M \times(I ;\{0\},\{1\})
$$

such that

$$
\begin{aligned}
& \left.(F, B)\right|_{N_{i}}=\left(f_{i}, b_{i}\right): N_{i} \rightarrow M \quad(i=1,2), \\
& \sigma_{*}(F, B)=x \in L_{m+1}\left(\mathbb{Z}\left[\pi_{1}(M)\right]\right) .
\end{aligned}
$$

(iii) Manifold structures $\left(N_{1}, f_{1}\right),\left(N_{2}, f_{2}\right)$ on $M$ are such that

$$
\left[N_{1}, f_{1}\right]=\left[N_{2}, f_{2}\right] \in \mathscr{T}(M)=[M, G / O]
$$

if and only if there exists an element $x \in L_{m+1}\left(\mathbb{Z}\left[\pi_{1}(M)\right]\right)$ such that

$$
x\left(N_{1}, f_{1}\right)=\left(N_{2}, f_{2}\right) \in L_{m+1}\left(\mathbb{Z}\left[\pi_{1}(M)\right]\right),
$$

i.e. if and only if there exists a degree 1 normal bordism

$$
(F, B):\left(W ; N_{1}, N_{2}\right) \rightarrow M \times(I ;\{0\},\{1\})
$$

such that

$$
\begin{aligned}
& \left.(F, B)\right|_{N_{i}}=\left(f_{i}, b_{i}\right): N_{i} \rightarrow M \quad(i=1,2), \\
& \sigma_{*}(F, B)=x \in L_{m+1}\left(\mathbb{Z}\left[\pi_{1}(M)\right]\right) .
\end{aligned}
$$

Proof Combine the criterion of Proposition 9.48 with the rel $\partial$ surgery obstructions of Chapters 11, 12. 
Example 13.3 Let $M$ be an $m$-dimensional manifold (with $m \geqslant 5$ ) which is simply-connected, so that $\pi_{1}(M)=\{1\}$. The surgery exact sequence and the computation of $L_{*}(\mathbb{Z})$

\begin{tabular}{|c|c|c|c|c|}
\hline$m(\bmod 4)$ & 0 & 1 & 2 & 3 \\
\hline$L_{m}(\mathbb{Z})$ & $\mathbb{Z}$ & 0 & $\mathbb{Z}_{2}$ & 0 \\
\hline
\end{tabular}

give the structure set $\mathscr{S}(M)$ according to the residue $m(\bmod 4)$, as follows.

(i) $m=4 k$. The exact sequence of pointed sets is

$$
0 \rightarrow \mathscr{S}(M) \rightarrow[M, G / O] \stackrel{A}{\rightarrow} L_{4 k}(\mathbb{Z})
$$

with

$$
\begin{aligned}
A(\eta, h) & =\sigma_{*}((f, b): N \rightarrow M) \\
& =\frac{1}{8}(\sigma(N)-\sigma(M)) \\
& =\frac{1}{8}(\langle\mathscr{L}(N),[N]\rangle-\langle\mathscr{L}(M),[M]\rangle) \\
& =\frac{1}{8}\langle\mathscr{L}(M) \cup(\mathscr{L}(-\eta)-1),[M]\rangle \\
& =\frac{1}{8} \sum_{j=1}^{k}\left\langle\mathscr{L}_{k-j}(M) \cup \mathscr{L}_{j}(-\eta),[M]\right\rangle \in L_{4 k}(\mathbb{Z})=\mathbb{Z}
\end{aligned}
$$

by the Hirzebruch signature theorem $(6.41)$, where $\mathscr{L}_{j} \in H^{4 j}(M ; \mathbb{Q})$ is the $4 j$ dimensional component of the $\mathscr{L}$-genus, $[M]=1 \in H_{4 k}(M ; \mathbb{Q})=\mathbb{Q}$ is the fundamental class and $\mathscr{L}_{0}=1 \in H^{0}(M ; \mathbb{Q})=\mathbb{Q}$ (assuming $M$ is connected). Note the non-additivity of $A$ :

$$
\begin{aligned}
A\left(\left(\eta_{1}, h_{1}\right) \oplus\left(\eta_{2}, h_{2}\right)\right) & =\frac{1}{8}\left\langle\mathscr{L}(M) \cup\left(\mathscr{L}\left(-\eta_{1} \oplus-\eta_{2}\right)-1\right),[M]\right\rangle \\
& =\frac{1}{8}\left\langle\mathscr{L}(M) \cup\left(\mathscr{L}\left(-\eta_{1}\right) \cup \mathscr{L}\left(-\eta_{2}\right)-1\right),[M]\right\rangle \\
& =\frac{1}{8} \sum_{i+j=1}^{k}\left\langle\mathscr{L}_{k-i-j}(M) \cup \mathscr{L}_{i}\left(-\eta_{1}\right) \cup \mathscr{L}_{j}\left(-\eta_{2}\right),[M]\right\rangle \\
& \neq A\left(\eta_{1}, h_{1}\right)+A\left(\eta_{2}, h_{2}\right) \in L_{4 k}(\mathbb{Z})=\mathbb{Z} \text { (in general). }
\end{aligned}
$$

For an actual example, consider $M^{4 k}=\mathbb{C} \mathbb{P}^{2 k}$ and use 6.42. Products of spheres also give examples (13.26).

(ii) $m=4 k+2$. The exact sequence of pointed sets is

$$
0 \rightarrow \mathscr{S}(M) \rightarrow[M, G / O] \stackrel{A}{\rightarrow} L_{4 k+2}(\mathbb{Z})
$$

with $A:[M, G / O] \rightarrow L_{4 k+2}(\mathbb{Z})=\mathbb{Z}_{2}$ the Arf invariant map.

(iii) $m=4 k+1$. The exact sequence of pointed sets is 


$$
[M \times I, \partial ; G / O] \stackrel{A}{\rightarrow} L_{4 k+2}(\mathbb{Z}) \rightarrow \mathscr{S}(M) \rightarrow[M, G / O] \rightarrow 0
$$

with $A:[M \times I, \partial ; G / O] \rightarrow L_{4 k+2}(\mathbb{Z})=\mathbb{Z}_{2}$ the rel $\partial$ version of (ii). (iv) $m=4 k+3$. The exact sequence of pointed sets is

$$
[M \times I, \partial ; G / O] \stackrel{A}{\rightarrow} L_{4 k+4}(\mathbb{Z}) \rightarrow \mathscr{S}(M) \rightarrow[M, G / O] \rightarrow 0
$$

with $A:[M \times I, \partial ; G / O] \rightarrow L_{4 k+4}(\mathbb{Z})=\mathbb{Z}$ the rel $\partial$ version of (i).

See Morgan and Sullivan [62] and Madsen and Milgram [45] for the expression of $A:[M, G / O] \rightarrow L_{m}(\mathbb{Z})$ in the cases $m=4 k, 4 k+2$ in terms of characteristic classes.

Example 13.4 An element $\left(\Sigma^{m}, f\right)$ of the structure set $\mathscr{S}\left(S^{m}\right)$ is an equivalence class of pairs $\left(\Sigma^{m}, f\right)$ with $\Sigma^{m}$ an $m$-dimensional manifold and $f: \Sigma^{m} \rightarrow S^{m}$ a homotopy equivalence. The surgery obstruction functions

$$
A: \pi_{m}(G / O)=\left[S^{m}, G / O\right] \longrightarrow L_{m}(\mathbb{Z})
$$

are morphisms of abelian groups, with relative groups $\mathscr{S}_{*}$ fitting into a long exact sequence

$$
\begin{aligned}
\ldots \longrightarrow \pi_{m+1}(G / O) \stackrel{A}{\longrightarrow} L_{m+1}(\mathbb{Z}) \longrightarrow \\
\mathscr{S}_{m} \longrightarrow \pi_{m}(G / O) \stackrel{A}{\longrightarrow} L_{m}(\mathbb{Z}) \longrightarrow \ldots
\end{aligned}
$$

The structure sets of spheres were the first to be computed, with

$$
\mathscr{S}\left(S^{m}\right)=\mathscr{S}_{m} \text { for } m \geqslant 5 .
$$

See section 13.3 below for a more detailed discussion.

Example 13.5 The $m$-dimensional torus

$$
T^{m}=S^{1} \times S^{1} \times \ldots \times S^{1}
$$

has fundamental group $\pi_{1}\left(T^{m}\right)=\mathbb{Z}^{m}$ the free abelian group of rank $m$. The normal maps $(f, b): M^{m} \rightarrow T^{m}$ are classified by

$$
\mathscr{T}\left(T^{m}\right)=\left[T^{m}, G / O\right]=\sum_{k=0}^{m}\left(\begin{array}{c}
m \\
k
\end{array}\right) \pi_{m-k}(G / O) .
$$

The surgery obstruction groups of the group ring $\mathbb{Z}\left[\mathbb{Z}^{m}\right]$ are given by

$$
L_{n}\left(\mathbb{Z}\left[\mathbb{Z}^{m}\right]\right)=\sum_{k=0}^{m}\left(\begin{array}{c}
m \\
k
\end{array}\right) L_{n-k}(\mathbb{Z})
$$

(Shaneson [80], Wall [92, 13A.8], Novikov [64], Ranicki [68]), so that the structure set of $T^{m}$ for $m \geqslant 5$ is given by 


$$
\mathscr{S}\left(T^{m}\right)=\sum_{k=0}^{m}\left(\begin{array}{c}
m \\
k
\end{array}\right) \mathscr{S}_{m-k}
$$

with $\mathscr{S}_{*}$ as in 13.4. The surgery classification of manifolds homotopy equivalent to $T^{m}$ (the 'fake tori' of Wall $[92,15 \mathrm{~A}]$, Hsiang and Shaneson [35]) in the PL category was an essential ingredient of the structure theory of topological manifolds obtained by Kirby and Siebenmann [39]. (The actual result required is that for $m \geqslant 5$ every homotopy equivalence of $P L$ manifolds of the type $h: M^{m} \rightarrow T^{m}$ has a finite cover $\bar{h}: \bar{M}^{m} \rightarrow \bar{T}^{m}$ which is homotopic to a $P L$ homeomorphism).

\subsection{The simple structure set}

There is also a simple surgery theory for deciding if a simple geometric Poincaré complex $X$ is simple homotopy equivalent to a manifold, and if a simple homotopy equivalence $f: N^{m} \rightarrow M^{m}$ of manifolds is homotopic to a diffeomorphism.

As already noted in Chapter 8.1 the involution on the group ring

$$
\mathbb{Z}[\pi] \rightarrow \mathbb{Z}[\pi] ; a=\sum_{g \in \pi} n_{g} g \mapsto \bar{a}=\sum_{g \in \pi} n_{g} g^{-1}
$$

induces an involution on the Whitehead group

$$
*: W h(\pi) \rightarrow W h(\pi) ; \tau(\alpha) \mapsto \tau\left(\alpha^{*}\right)
$$

with $\alpha^{*}=\left(\bar{a}_{j i}\right)$ if $\alpha=\left(a_{i j}\right)$.

Definition 13.6 (i) The torsion of an $m$-dimensional geometric Poincaré complex $X$ is defined by

$$
\tau(X)=\tau\left([X] \cap-: C(\tilde{X})^{m-*} \rightarrow C(\tilde{X})\right) \in W h\left(\pi_{1}(X)\right),
$$

such that

$$
\tau(X)^{*}=(-1)^{m} \tau(X) \in W h\left(\pi_{1}(X)\right) .
$$

(ii) A geometric Poincaré complex $X$ is simple if $\tau(X)=0$.

Example 13.7 An $m$-dimensional manifold $M$ is a simple $m$-dimensional geometric Poincaré complex, and hence so is any finite $C W$ complex $X$ simple homotopy equivalent to $M$.

Definition 13.8 Let $X$ be a simple $m$-dimensional geometric Poincaré complex. (i) A simple manifold structure $(M, f)$ on $X$ is an $m$-dimensional manifold $M$ together with a simple homotopy equivalence $f: M \rightarrow X$. 
(ii) The simple manifold structure set $\mathscr{S}^{s}(X)$ is the set of equivalence classes of $m$-dimensional manifold structures $(M, f)$, subject to the equivalence relation :

$(M, f) \sim\left(M^{\prime}, f^{\prime}\right)$ if there exists a bordism

$$
\left(F ; f, f^{\prime}\right):\left(W ; M, M^{\prime}\right) \rightarrow X \times(I ;\{0\},\{1\})
$$

with $F: W \rightarrow X \times I$ a simple homotopy equivalence, so that $\left(W ; M, M^{\prime}\right)$

is an $s$-cobordism.

For $m \geqslant 5$ this is the equivalence relation

$(M, f) \sim\left(M^{\prime}, f^{\prime}\right)$ if there exist a diffeomorphism $h: M \rightarrow M^{\prime}$ and a

homotopy $f \simeq f^{\prime} h: M \rightarrow X$

by the $s$-Cobordism Theorem (1.11).

Definition 13.9 The simple surgery obstruction groups $L_{*}^{s}(\mathbb{Z}[\pi])$ are defined by analogy with $L_{*}(\mathbb{Z}[\pi])$, using quadratic forms and formations on based f.g. free $\mathbb{Z}[\pi]$-modules and simple isomorphisms.

Theorem 13.10 (Wall [92])

A degree 1 normal map $(f, b): M \rightarrow X$ from an $m$-dimensional manifold $M$ to a simple $m$-dimensional geometric Poincaré complex $X$ has a simple surgery obstruction

$$
\sigma_{*}^{s}(f, b) \in L_{m}^{s}\left(\mathbb{Z}\left[\pi_{1}(X)\right]\right)
$$

such that $\sigma_{*}^{s}(f, b)=0$ if (and for $m \geqslant 5$ only if) $(f, b)$ is bordant to a simple homotopy equivalence.

In fact, the obstruction groups $L_{*}^{s}(\mathbb{Z}[\pi])$ for surgery up to simple homotopy equivalence were the original $L$-groups of Wall [92].

Proposition 13.11 (Shaneson [80], Novikov [64], Ranicki [68])

The simple surgery obstruction groups $L_{*}^{s}(\mathbb{Z}[\pi])$ are related to the surgery obstruction groups $L_{*}(\mathbb{Z}[\pi])$ by the Rothenberg exact sequence

$$
\ldots \rightarrow L_{m}^{s}(\mathbb{Z}[\pi]) \rightarrow L_{m}(\mathbb{Z}[\pi]) \rightarrow \widehat{H}^{m}\left(\mathbb{Z}_{2} ; W h(\pi)\right) \rightarrow L_{m-1}^{s}(\mathbb{Z}[\pi]) \rightarrow \ldots
$$

with

$$
\widehat{H}^{m}\left(\mathbb{Z}_{2} ; W h(\pi)\right)=\frac{\left\{x \in W h(\pi) \mid x^{*}=(-1)^{m} x\right\}}{\left\{y+(-1)^{m} y^{*} \mid y \in W h(\pi)\right\}}
$$

The Tate $\mathbb{Z}_{2}$-cohomology groups $\widehat{H}^{*}\left(\mathbb{Z}_{2} ; W h(\pi)\right)$ are of exponent 2 , so the groups $L_{*}^{s}(\mathbb{Z}[\pi]), L_{*}(\mathbb{Z}[\pi])$ differ in 2-torsion only. 
Theorem 13.12 Let $m \geqslant 5$.

(i) A simple $m$-dimensional geometric Poincaré complex $X$ is simple homotopy equivalent to a manifold if and only if there exists a normal map $(f, b): M^{m} \rightarrow X$ with simple surgery obstruction

$$
\sigma_{*}^{s}(f, b)=0 \in L_{m}^{s}\left(\mathbb{Z}\left[\pi_{1}(X)\right]\right)
$$

(ii) The simple structure set $\mathscr{S}^{s}(M)$ of an m-dimensional manifold $M^{m}$ fits into an exact sequence of pointed sets

$$
\ldots \rightarrow L_{m+1}^{s}\left(\mathbb{Z}\left[\pi_{1}(M)\right]\right) \rightarrow \mathscr{S}^{s}(M) \rightarrow[M, G / O] \stackrel{A}{\rightarrow} L_{m}^{s}\left(\mathbb{Z}\left[\pi_{1}(M)\right]\right)
$$

A simple homotopy equivalence of m-dimensional manifolds $f: N \rightarrow M$ is homotopic to a diffeomorphism if and only if $(N, f)=(M, 1) \in \mathscr{S}^{s}(M)$.

Remark 13.13 The simple version of the surgery exact sequence is particularly important for manifolds with finite fundamental group. See Chapter 14E of Wall [92] for the application of the simple $L$-groups of cyclic groups to the highdimensional surgery classification in the $P L$ category of generalised lens spaces (= manifolds with cyclic fundamental group and universal cover a sphere).

\subsection{Exotic spheres}

This section outlines the surgery classification of manifolds homotopy equivalent to spheres. The high-dimensional homotopy spheres are simply-connected, so only the simply-connected surgery obstruction groups $L_{*}(\mathbb{Z})$ are required.

Definition 13.14 A homotopy $m$-sphere $\Sigma^{m}$ is an $m$-dimensional manifold which is homotopy equivalent to $S^{m}$.

Poincaré Conjecture 13.15 (1905)

Every homotopy 3-sphere $\Sigma^{3}$ is homeomorphic to $S^{3}$.

The Poincaré Conjecture is still unresolved, and is the main difficulty in the way of a complete classification of all 3-dimensional manifolds.

Milnor Exotic Sphere Theorem 13.16 ([49], 1956)

There exist exotic differentiable structures on $S^{7}$, i.e. there exist homotopy 7 spheres $\Sigma^{7}$ which are homeomorphic but not diffeomorphic to $S^{7}$. 
Proof The first 7 examples were constructed as the sphere bundles of certain 4-plane bundles over $S^{4}$, so we start by recalling the classification of oriented 4plane bundles over $S^{4}$. The isomorphism class of $\omega: S^{4} \rightarrow B S O(4)$ is determined by the Euler number and first Pontrjagin class

$$
\chi(\omega), p_{1}(\omega) \in H^{4}\left(S^{4}\right)=\mathbb{Z}
$$

subject to the congruence

$$
p_{1}(\omega) \equiv 2 \chi(\omega)(\bmod 4)
$$

with the function

$$
\pi_{4}(B S O(4)) \rightarrow \mathbb{Z} \oplus \mathbb{Z} ; \omega \mapsto\left(\frac{1}{4}\left(2 \chi(\omega)+p_{1}(\omega)\right), \frac{1}{4}\left(2 \chi(\omega)-p_{1}(\omega)\right)\right)
$$

defining an isomorphism. Let

$$
\left(D^{4}, S^{3}\right) \rightarrow(D(\omega), S(\omega)) \rightarrow S^{4}
$$

be the $\left(D^{4}, S^{3}\right)$-bundle over $S^{4}$ associated to $\omega$. The homology groups of the sphere bundle $S(\omega)$ are given by

$$
H_{i}(S(\omega))= \begin{cases}\mathbb{Z} & \text { if } i=0,7 \\ \operatorname{coker}(\chi(\omega): \mathbb{Z} \rightarrow \mathbb{Z}) & \text { if } i=3 \\ \operatorname{ker}(\chi: \mathbb{Z} \rightarrow \mathbb{Z}) & \text { if } i=4 \\ 0 & \text { otherwise }\end{cases}
$$

The Euler number of $\omega: S^{4} \rightarrow B S O(4)$ is the Hopf invariant (5.76) of $J(\omega) \in$ $\pi_{7}\left(S^{4}\right)$

$$
\chi(\omega)=H J(\omega) \in \mathbb{Z} .
$$

If the Hopf invariant is $\chi(\omega)=1 \in \mathbb{Z}$ then $S(\omega)$ is a homotopy 7-sphere which is the boundary of an oriented 8-dimensional manifold $D(\omega)$. For any odd integer $k$ let $\omega_{k}: S^{4} \rightarrow B S O(4)$ be the oriented 4-plane bundle with

$$
p_{1}\left(\omega_{k}\right)=2 k, \chi\left(\omega_{k}\right)=1 \in \mathbb{Z},
$$

and write

$$
\left(D\left(\omega_{k}\right)^{8}, S\left(\omega_{k}\right)^{7}\right)=\left(W_{k}, \Sigma_{k}\right) .
$$

There exists a Morse function $\Sigma_{k} \rightarrow \mathbb{R}$ with two critical points, so that $\Sigma_{k} \backslash$ pt. $\}$ is diffeomorphic to $\mathbb{R}^{7}$ and $\Sigma_{k}$ is homeomorphic to $S^{7}$ by a theorem of Reeb. The tangent bundle of $W_{k}$ is classified by

$$
\tau_{W_{k}}=\tau_{S^{4}} \oplus \omega_{k}: W_{k} \simeq S^{4} \rightarrow B S O(8),
$$

with Pontrjagin class

$$
p_{1}\left(W_{k}\right)=p_{1}\left(\omega_{k}\right)=2 k \in H^{4}\left(W_{k}\right)=\mathbb{Z} .
$$


If there exists a diffeomorphism $f: \Sigma_{k} \rightarrow S^{7}$ consider the closed oriented 8dimensional manifold

$$
M^{8}=W_{k} \cup_{f} D^{8}
$$

with intersection form and signature

$$
\left(H^{4}(M), \lambda\right)=(\mathbb{Z}, 1), \sigma(M)=\sigma\left(H^{4}(M), \lambda\right)=1 .
$$

By the Hirzebruch signature theorem (6.41)

$$
\sigma(M)=\left\langle\mathscr{L}_{2}(M),[M]\right\rangle=1 \in \mathbb{Z}
$$

with

$$
\begin{aligned}
& \mathscr{L}_{2}(M)=\frac{1}{45}\left(7 p_{2}(M)-p_{1}(M)^{2}\right)=1 \in H^{8}(M)=\mathbb{Z}, \\
& p_{1}(M)=p_{1}\left(\omega_{k}\right)=2 k
\end{aligned}
$$

so that

$$
p_{2}(M)=\frac{1}{7}\left(45+4 k^{2}\right)=7+\frac{4}{7}\left(k^{2}-1\right) \in H^{4}(M)=\mathbb{Z} .
$$

The Pontrjagin class $p_{2}(M)=p_{2}\left(\omega_{k}\right) \in H^{8}(M)=\mathbb{Z}$ of a vector bundle is integral, so the existence of a diffeomorphism $f: \Sigma_{k}^{7} \rightarrow S^{7}$ implies that $k^{2} \equiv$ $1(\bmod 7)$. Thus the homotopy 7 -spheres $\Sigma_{k}$ with $k^{2} \not \equiv 1(\bmod 7)$ have an exotic differentiable structure: they are homeomorphic but not diffeomorphic to $S^{7}$. See Milnor and Stasheff [61, pp. 242-248] for a detailed account of this and other applications of 4-plane bundles over $S^{4}$.

See Milnor [59] for a first-hand account of the discovery of the exotic differentiable structures.

Definition 13.17 An exotic sphere is a homotopy $m$-sphere $\Sigma^{m}$ which is not diffeomorphic to $S^{m}$.

Theorem 13.18 (Generalised Poincaré Conjecture) (Smale [82], 1961) For $m \geqslant 5$ every homotopy $m$-sphere $\Sigma^{m}$ is homeomorphic to $S^{m}$.

Proof For $m \geqslant 6$ this can be deduced from the $h$-Cobordism Theorem (1.9,8.34), although in fact it was the original proof of the Generalised Poincaré Conjecture which led to the $h$-Cobordism Theorem. Given a homotopy $m$-sphere $\Sigma^{m}$ define an $h$-cobordism $\left(W^{m} ; S^{m-1}, S^{m-1}\right)$ by punching out two disjoint $m$-disks

$$
W=\operatorname{cl} .\left(\Sigma^{m} \backslash\left(D^{m} \cup D^{m}\right)\right) .
$$

By the $h$-Cobordism Theorem there is a diffeomorphism

$$
(g ; 1, h):\left(W^{m} ; S^{m-1}, S^{m-1}\right) \cong S^{m-1} \times(I ;\{0\},\{1\})
$$


for some self-diffeomorphism $h: S^{m-1} \rightarrow S^{m-1}$, and hence a diffeomorphism

$$
\Sigma^{m} \cong D^{m} \cup_{h} D^{m}
$$

Thus $\Sigma^{m}$ has a handle decomposition with one 0 -handle and one $m$-handle

$$
\Sigma^{m}=h^{0} \cup h^{m} .
$$

Milnor [49] proved that every homotopy sphere $\Sigma^{m}$ of this type is homeomorphic to $S^{m}$, using a homotopy $h \simeq 1: S^{m-1} \rightarrow S^{m-1}$ which is a homeomorphism at each level (i.e. a topological isotopy), and which determines a homeomorphism

$$
\Sigma^{m}=D^{m} \cup_{h} D^{m} \rightarrow D^{m} \cup_{1} D^{m}=S^{m}
$$

The case $m=5$ requires a special argument. (See Chapter VIII of Kosinski [42] for another way of deducing the Generalised Poincaré Conjecture from the $h$-Cobordism Theorem, which applies for $m \geqslant 5$ ).

The 4-dimensional Poincaré conjecture holds by the 1982 result of Freedman [25]. The original 3-dimensional Poincaré conjecture holds by the 2002 work of Perelman.

The original exotic spheres depended on the existence of a map $S^{7} \rightarrow S^{4}$ of Hopf invariant 1, but the subsequent development of the surgery method provided a systematic classification of exotic spheres $\Sigma^{m}$ for all dimensions $m \geqslant$ 5. (There are no exotic spheres in dimensions 5,6, however). Every homotopy $m$-sphere $\Sigma^{m}$ is of the form

$$
\Sigma^{m}=D^{m} \cup W \cup D^{m}
$$

for an $h$-cobordism $\left(W ; S^{m-1}, S^{m-1}\right)$ which is the union of traces of a sequence of surgeries on $S^{m-1}$. In order to classify the $m$-dimensional homotopy spheres it is therefore enough to classify the possible sequences of surgeries on $S^{m-1}$ for which the union of traces is an $h$-cobordism.

Definition 13.19 Let $\Theta_{m}$ be the group of $h$-cobordism classes of $m$-dimensional homotopy spheres, with addition by connected sum (2.9).

Lemma 13.20 The function

$$
\mathscr{S}\left(S^{m}\right) \rightarrow \Theta_{m} ;\left(\Sigma^{m}, f\right) \mapsto\left[\Sigma^{m}\right]
$$

is a bijection, for any $m \geqslant 1$. 
Proof For any oriented homotopy $m$-sphere $\Sigma^{m}$ there is a unique homotopy class of orientation-preserving homotopy equivalences $f: \Sigma^{m} \rightarrow S^{m}$, allowing the definition of an inverse bijection

$$
\Theta_{m} \rightarrow \mathscr{S}\left(S^{m}\right) ;\left[\Sigma^{m}\right] \mapsto\left(\Sigma^{m}, f\right) .
$$

Note that by the $h$-Cobordism Theorem, for $m \geqslant 5$ an $m$-dimensional homotopy sphere $\Sigma^{m}$ is $h$-cobordant to $S^{m}$ if and only if $\Sigma^{m}$ is diffeomorphic to $S^{m}$.

For $m \geqslant 2$ an $m$-dimensional homotopy sphere $\Sigma^{m}$ is simply-connected

$$
\pi_{1}\left(\Sigma^{m}\right)=\pi_{1}\left(S^{m}\right)=\{1\},
$$

so that the associated surgery theory involves only the simply-connected surgery obstruction groups $L_{*}(\mathbb{Z})$. The surgery classification of homotopy spheres thus avoids the complications caused in the general theory by the fundamental group, and the groups of homotopy spheres $\mathscr{S}\left(S^{m}\right)=\Theta_{m}$ are computed for $m \geqslant 5$ by the surgery exact sequence

$$
\ldots \rightarrow \pi_{m+1}(G / O) \stackrel{A}{\rightarrow} L_{m+1}(\mathbb{Z}) \rightarrow \mathscr{S}\left(S^{m}\right) \rightarrow \pi_{m}(G / O) \stackrel{A}{\rightarrow} L_{m}(\mathbb{Z}) .
$$

Note that $\mathscr{S}\left(S^{m}\right)=\Theta_{m}$ is an abelian group, although the structure set $\mathscr{S}(M)$ of a manifold $M$ is not in general a group.

Example 13.21 (Milnor [49], Kervaire and Milnor [38])

(i) Every 7-dimensional homotopy sphere is the boundary $\Sigma^{7}=\partial W$ of an oriented 8-dimensional manifold $W$, and so can be obtained from $S^{7}$ by oriented surgery. The morphism of abelian groups

$$
\lambda: \Theta_{7} \rightarrow \mathbb{Z}_{7} ; \Sigma^{7} \mapsto \lambda\left(\Sigma^{7}\right)=45 \sigma(W)+p_{1}(W)^{2}
$$

is a surjection. For any odd integer $k=2 \ell+1$ the exotic sphere $\Sigma_{k}$ of 13.16 is obtained from $S^{7}$ by a single 3 -surgery removing

$$
g_{\omega_{k}}: S^{3} \times D^{4} \rightarrow S^{3} \times D^{4} \hookrightarrow S^{7}
$$

(terminology of 5.68, with $\omega_{k}: S^{3} \rightarrow S O(4)$ as in 13.16), but this surgery is not framed. The trace of the surgery is an oriented 8-dimensional cobordism $\left(V_{k} ; S^{7}, \Sigma_{k}^{7}\right)$. The oriented 8-dimensional manifold with boundary

$$
\left(W_{k}, \partial W_{k}\right)=\left(D^{8} \cup V_{k}, \Sigma_{k}^{7}\right)
$$

has signature 1 and $p_{1}\left(W_{k}\right)=2 k \in H^{4}\left(W_{k}\right)=\mathbb{Z}$, so that

$$
\lambda\left(\Sigma_{k}\right)=45+4 k^{2}=2 \ell(\ell+1) \in \mathbb{Z}_{7} .
$$

In fact, $\Theta_{7}$ is cyclic of order 28 .

(ii) Every 7-dimensional homotopy sphere is the boundary $\Sigma^{7}=\partial W^{\prime}$ of a framed 
8-dimensional manifold $W^{\prime}$. The signature of $W^{\prime}$ is divisible by 8 , and the function

$$
\lambda^{\prime}: \Theta_{7} \rightarrow \mathbb{Z}_{28} ; \Sigma^{7} \mapsto \frac{1}{8} \sigma\left(W^{\prime}\right)
$$

is an isomorphism such that

$$
\lambda\left(\Sigma^{7}\right)=3 \lambda^{\prime}\left(\Sigma^{7}\right) \in \mathbb{Z}_{7}
$$

It is possible to obtain $\Sigma_{k}^{7}$ from $S^{7}$ by $4 \ell(\ell+1)$ framed 3 -surgeries, and $\Sigma_{k}^{7}=\partial W_{k}^{\prime}$ is the boundary of a framed 8-dimensional manifold $W_{k}^{\prime}$ with

$$
\begin{aligned}
\sigma\left(W_{k}^{\prime}\right) & =4 \ell(\ell+1) \in 8 \mathbb{Z} \subset \mathbb{Z}, \\
\lambda^{\prime}\left(\Sigma_{k}^{7}\right) & =\frac{1}{8} \sigma\left(W_{k}^{\prime}\right)=\frac{1}{2} \ell(\ell+1) \in \mathbb{Z}_{28} .
\end{aligned}
$$

More precisely, let $\left(W^{8} ; S^{7}, \Sigma^{7}\right)$ be the 8-dimensional framed cobordism constructed by $E_{8}$-plumbing of 8 copies of $\tau_{S^{8}}$ as in 11.62 , and set

$$
\left(W_{k}^{\prime} ; S^{7}, \Sigma_{k}^{7}\right)=\#_{\ell(\ell+1) / 2}\left(W ; S^{7}, \Sigma^{7}\right)
$$

In particular, $\Sigma_{3}^{7}$ has $\lambda^{\prime}(\Sigma)=1$, so that $\Sigma_{3}^{7} \in \Theta_{7}=\mathbb{Z}_{28}$ is a generator.

In general, a homotopy sphere $\Sigma^{m}$ is not the boundary of a framed $(m+1)$ manifold.

Definition 13.22 The subgroup

$$
\begin{aligned}
b P_{m+1} & =\operatorname{ker}\left(\mathscr{S}\left(S^{m}\right) \rightarrow \pi_{m}(G / O)\right) \\
& =\operatorname{im}\left(L_{m+1}(\mathbb{Z}) \rightarrow \Theta_{m}\right) \subseteq \Theta_{m}=\mathscr{S}\left(S^{m}\right)
\end{aligned}
$$

consists of the homotopy $m$-spheres $\Sigma^{m}$ which arise in one of three equivalent ways :

(i) as the boundary $\partial W=\Sigma^{m}$ of a framed $(m+1)$-dimensional manifold $W^{m+1}$,

(ii) as the result of a sequence of framed surgeries on $S^{m}$,

(iii) as the domain of a boundary component of a degree 1 normal map

$$
(f, b):\left(W^{m+1} ; S^{m}, \Sigma^{m}\right) \rightarrow S^{m} \times(I ;\{0\},\{1\})
$$

with $f \mid: S^{m} \rightarrow S^{m} \times\{0\}$ the identity and $f \mid: \Sigma^{m} \rightarrow S^{m} \times\{1\}$ a homotopy equivalence. 
The $b P$-terminology dates from the time when the map $L_{m+1}(\mathbb{Z}) \rightarrow \mathscr{S}\left(S^{m}\right)$ in the surgery exact sequence was written $b: P_{m+1} \rightarrow \Theta_{m}$.

Here are some sample results :

Proposition 13.23 (Kervaire and Milnor [38])

(i) For $m \geqslant 5$ two homotopy $m$-spheres $\Sigma^{m}, \Sigma^{\prime m}$ are $h$-cobordant if and only if there exists an orientation-preserving diffeomorphism $\Sigma^{m} \rightarrow \Sigma^{\prime m}$.

(ii) The tangent bundle $\tau_{\Sigma^{m}}$ of a homotopy $m$-sphere $\Sigma^{m}$ is stably trivial, with $\tau_{\Sigma^{m}} \oplus \epsilon$ trivial. Sending $\Sigma^{m}$ to its framed cobordism class (modulo the indeterminacy from the choice of framing) defines an isomorphism

$$
\Theta_{m} / b P_{m+1} \rightarrow \operatorname{coker}\left(J: \pi_{m}(O) \rightarrow \pi_{m}(G)\right)=\operatorname{im}\left(\pi_{m}(G) \rightarrow \pi_{m}(G / O)\right)
$$

with

$$
J: \pi_{m}(O) \rightarrow \pi_{m}(G)=\pi_{m}^{S}=\Omega_{m}^{f r}
$$

the J-homomorphism (5.80).

(iii) The groups $\Theta_{m}$ are finite. The surgery exact sequence

$$
\ldots \rightarrow \pi_{m+1}(G / O) \stackrel{A}{\rightarrow} L_{m+1}(\mathbb{Z}) \rightarrow \Theta_{m} \rightarrow \pi_{m}(G / O) \stackrel{A}{\rightarrow} L_{m}(\mathbb{Z}) \rightarrow \ldots
$$

breaks up into exact sequences of abelian groups

$$
\begin{aligned}
& 0 \rightarrow \Theta_{2 n} \rightarrow \pi_{2 n}(G / O) \stackrel{A}{\rightarrow} L_{2 n}(\mathbb{Z}) \rightarrow b P_{2 n} \rightarrow 0 \\
& 0 \rightarrow b P_{2 n+2} \rightarrow \Theta_{2 n+1} \rightarrow \pi_{2 n+1}(G / O) \rightarrow 0
\end{aligned}
$$

with $b P_{2 n+1}=0$ and $b P_{2 n}, b P_{2 n+2}$ finite cyclic groups.

(iv) The order of $b P_{4 k}$ is

$$
t_{k}=a_{k} 2^{2 k-2}\left(2^{2 k-1}-1\right) \operatorname{num}\left(B_{k} / 4 k\right) .
$$

A generator of bP $P_{4 k}$ is represented by the homotopy $(4 k-1)$-sphere $\Sigma^{4 k-1}=M_{8}$ obtained from $S^{4 k-1}$ by 8 framed $(2 k-1)$-surgeries $S^{2 k-1} \times D^{2 k} \hookrightarrow S^{4 k-1}$, realizing the even symmetric form $\left(\mathbb{Z}^{8}, E_{8}\right)$ generating $L_{4 k}(\mathbb{Z})=\mathbb{Z}$. The trace of the surgeries is a framed $4 k$-dimensional manifold with boundary which is obtained by plumbing together 8 copies of the tangent bundle $\tau_{S^{2 k}}: S^{2 k} \rightarrow B S O(2 k)$ of the $2 k$-sphere $S^{2 k}$ and removing the interior of a $4 k$-disk $D^{4 k}$.

(v) The order of $b P_{4 k+2}$ is $\leqslant 2$. A generator of $b P_{4 k+2}$ is represented by the homotopy $(4 k+1)$-sphere $\Sigma^{4 k+1}$ obtained from $S^{4 k+1}$ by 2 framed surgeries on embeddings $S^{2 k} \times D^{2 k+1} \hookrightarrow S^{4 k+1}$, realizing the Arf (-1)-quadratic form $\left(\mathbb{Z}^{2}, \lambda, \mu\right)$ generating $L_{4 k+2}(\mathbb{Z})=\mathbb{Z}_{2}$. The trace of the surgeries is a framed $(4 k+2)$ dimensional manifold with boundary which is obtained by plumbing together 2 copies of the tangent bundle $\tau_{S^{2 k+1}}: S^{2 k+1} \rightarrow B S O(2 k+1)$ of the $(2 k+1)$ sphere $S^{2 k+1}$ and removing the interior of a $(4 k+2)$-disk $D^{4 k+2}$. 
Idea of proof (i) Immediate from the $h$-Cobordism Theorem (1.9).

(ii) The proof uses a hard result of Adams [3] on the image of the $J$-homomorphism.

(iii) $+($ iv $)+(\mathrm{v})$ The surgery method for computing $b P_{m+1}$ for $m \geqslant 4$ proceeds as follows. Let $\Sigma^{m}$ be an $m$-dimensional exotic sphere which bounds a framed $(m+1)$-dimensional manifold $W^{m+1}$, so that $\Sigma^{m}=\partial W$ represents an element of $b P_{m+1}$. The differentiable structure on $\Sigma^{m}$ is standard if (perhaps after a change of framing) it is possible to modify $W$ by a sequence of framed surgeries until $\pi_{*}(W)=0$; by Poincaré duality it suffices to have $\pi_{r}(W)=0$ for $2 r \leqslant m+1$. Suppose inductively that $\pi_{r}(W)=0$ for $r=1,2, \ldots, n-1$. If $m \geqslant 4$ and $n<m / 2$ it is possible to represent find a finite collection of framed embeddings $S^{n} \times D^{m+1-n} \hookrightarrow W$ representing a set of generators of $\pi_{n}(W)$, such that the effect of the corresponding surgeries is a cobordant framed manifold $W^{\prime}$ with $\partial W^{\prime}=\Sigma^{m}$ and $\pi_{r}\left(W^{\prime}\right)=0$ for $r=1,2, \ldots, n$. If $m=4 k-1$ (resp. $\left.4 k+1\right)$ the signature (resp. Arf invariant) of $W^{m+1}$ is the obstruction to also having $\pi_{2 k}(W)=0$ (resp. $\pi_{2 k+1}(W)=0$ ). If $m$ is even there is no obstruction to also having $\pi_{m / 2}(W)=0$.

Remark 13.24 The orders of the low-dimensional groups of exotic spheres $\Theta_{m}$, $b P_{m+1}$ and $\Theta_{m} / b P_{m+1}$ are given in the table

\begin{tabular}{|c|c|c|c|c|c|c|c|c|c|c|c|c|c|c|c|}
\hline$m$ & 1 & 2 & 3 & 4 & 5 & 6 & 7 & 8 & 9 & 10 & 11 & 12 & 13 & 14 & 15 \\
\hline$\Theta_{m}$ & 1 & 1 & 1 & 1 & 1 & 1 & 28 & 2 & 8 & 6 & 992 & 1 & 3 & 2 & 16256 \\
\hline$b P_{m+1}$ & 1 & 1 & 1 & 1 & 1 & 1 & 28 & 1 & 2 & 1 & 992 & 1 & 1 & 1 & 8128 \\
\hline$\Theta_{m} / b P_{m+1}$ & 1 & 1 & 1 & 1 & 1 & 1 & 1 & 2 & 4 & 6 & 1 & 1 & 3 & 2 & 2 \\
\hline
\end{tabular}

Remark 13.25 (i) An $m$-dimensional manifold $M$ is almost framed if

$$
M_{0}=\operatorname{cl} \cdot\left(M \backslash D^{m}\right)
$$

is framed, i.e. if there is given a trivialization of $\left.\nu_{M}\right|_{M_{0}}: M_{0} \rightarrow B O$. The obstruction to framing $M$ is the isomorphism class of a stable vector bundle over $S^{m}$

$$
\eta \in\left[M, M_{0} ; B O,\{*\}\right]=\pi_{m}(B O)
$$

which is equipped with a canonical fibre homotopy trivialization $h: \eta^{G} \simeq\{*\}$ : $S^{m} \rightarrow B G$. The cobordism group $A_{m}$ of almost framed $m$-dimensional manifolds is such that there is defined an isomorphism

$$
A_{m} \rightarrow \pi_{m}(G / O) ; M \mapsto(\eta, h) .
$$

The degree 1 Pontrjagin-Thom map

$$
f: M \rightarrow M / M_{0}=S^{m}
$$


is covered by a bundle map $b: \nu_{M} \rightarrow \eta$. The surgery obstruction of the normal map $(f, b): M \rightarrow S^{m}$ defines the morphism

$$
A: A_{m} \rightarrow L_{m}(\mathbb{Z}) ; M \mapsto \sigma_{*}(f, b)
$$

which fits into the exact sequence

$$
\ldots \rightarrow \Theta_{m} \rightarrow A_{m} \stackrel{A}{\rightarrow} L_{m}(\mathbb{Z}) \rightarrow \Theta_{m-1} \rightarrow \ldots .
$$

(ii) Let $B P L$ be the classifying space for $P L$ bundles. The fibre $P L / O$ of the forgetful map $B O \rightarrow B P L$ classifies stable vector bundles $\eta$ with a $P L$ bundle trivialization $h: \eta^{P L} \cong \epsilon^{\infty}$. The homotopy spheres measure the discrepancy between differentiable and $P L$ topology, with

$$
\pi_{m}(P L / O)=\Theta_{m}
$$

the relative homotopy groups in the homotopy exact sequence

$$
\ldots \rightarrow \pi_{m}(O) \rightarrow \pi_{m}(P L) \rightarrow \Theta_{m} \rightarrow \pi_{m}(B O) \rightarrow \pi_{m}(B P L) \rightarrow \ldots
$$

and $\pi_{m}(P L)=\Theta_{m}^{f r}$ the $h$-cobordism group of framed $m$-dimensional exotic spheres. The exact sequence breaks up into short exact sequences

$$
0 \rightarrow \pi_{m}(O) \rightarrow \pi_{m}(P L) \rightarrow \Theta_{m} \rightarrow 0 \text {. }
$$

The stable normal bundle $\nu_{\Sigma^{m}}: \Sigma^{m} \rightarrow B O$ of a homotopy sphere $\Sigma^{m}$ is trivial, and is equipped with a canonical $P L$ trivialization

$$
h: \nu_{\Sigma}^{P L} \simeq *: \Sigma^{m} \rightarrow B P L .
$$

The morphisms

$$
\Theta_{m} \rightarrow \pi_{m}(P L / O) ; \Sigma^{m} \mapsto\left(\nu_{\Sigma^{m}}, h\right)
$$

are isomorphisms. If $\eta: S^{m} \rightarrow B O(k)$ ( $k$ large) is a stable vector bundle with a $P L$ trivialization $h: \eta^{P L} \simeq *: S^{m} \rightarrow B P L(k)$ then by smoothing theory (M.Hirsch and B.Mazur, Smoothings of piecewise linear manifolds, Annals of Mathematics Studies 80, Princeton (1974)) $E(\eta)$ has a compatible differentiable structure of the form $\Sigma^{m} \times \mathbb{R}^{k}$, giving the inverse isomorphisms

$$
\pi_{m}(P L / O) \rightarrow \Theta_{m} ;(\eta, h) \mapsto \Sigma^{m} .
$$

(iii) The simply-connected surgery obstruction groups are the relative homotopy groups

$$
L_{m}(\mathbb{Z})=\pi_{m}(G / P L) \quad(m \neq 4)
$$

in the homotopy exact sequence

$$
\ldots \rightarrow \pi_{m}(P L) \rightarrow \pi_{m}(G) \rightarrow L_{m}(\mathbb{Z}) \rightarrow \pi_{m}(B P L) \rightarrow \pi_{m}(B G) \rightarrow \ldots
$$

with a commutative braid of exact sequences 


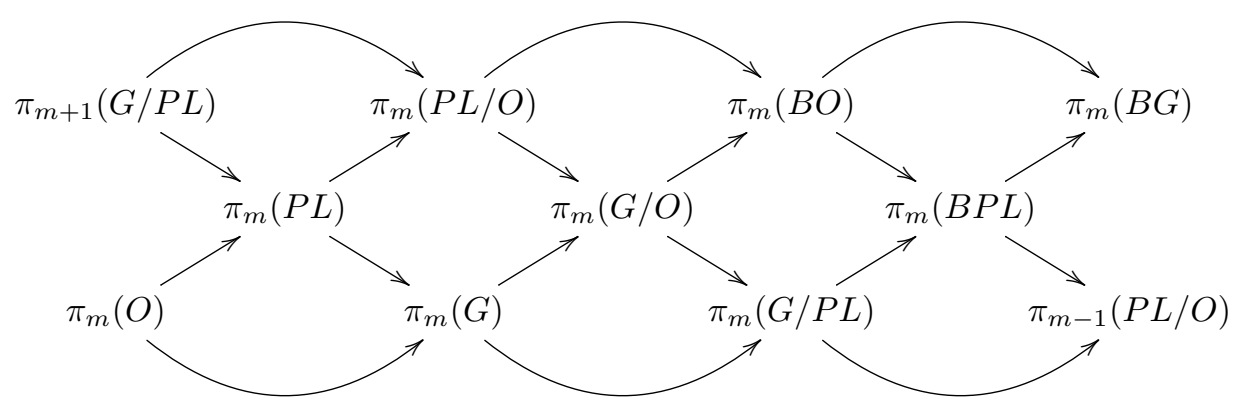

The braid can be expressed as

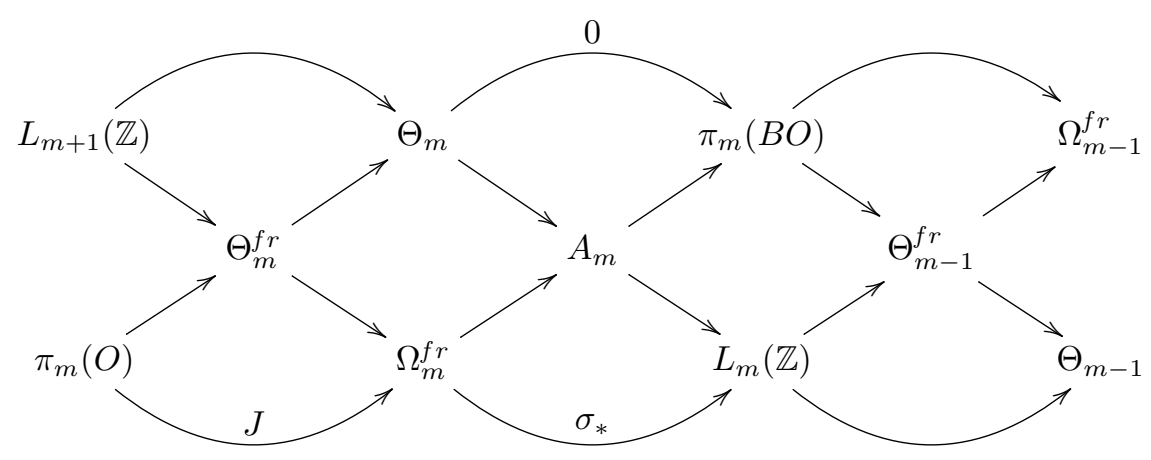

where

$$
\begin{aligned}
A_{m} & =\pi_{m}(G / O) \\
& =\text { cobordism group of almost framed } m \text {-dimensional manifolds }, \\
\Omega_{m}^{f r} & =\pi_{m}(G)=\pi_{m}^{S} \\
& =\text { cobordism group of framed } m \text {-dimensional manifolds }, \\
\Theta_{m}^{f r} & =\pi_{m}(P L) \\
& =h \text {-cobordism group of framed } m \text {-dimensional homotopy spheres }, \\
\Theta_{m} & =\pi_{m}(P L / O) \\
& =h \text {-cobordism group of } m \text {-dimensional homotopy spheres } .
\end{aligned}
$$

In dimensions 7,8 the braid is given by 


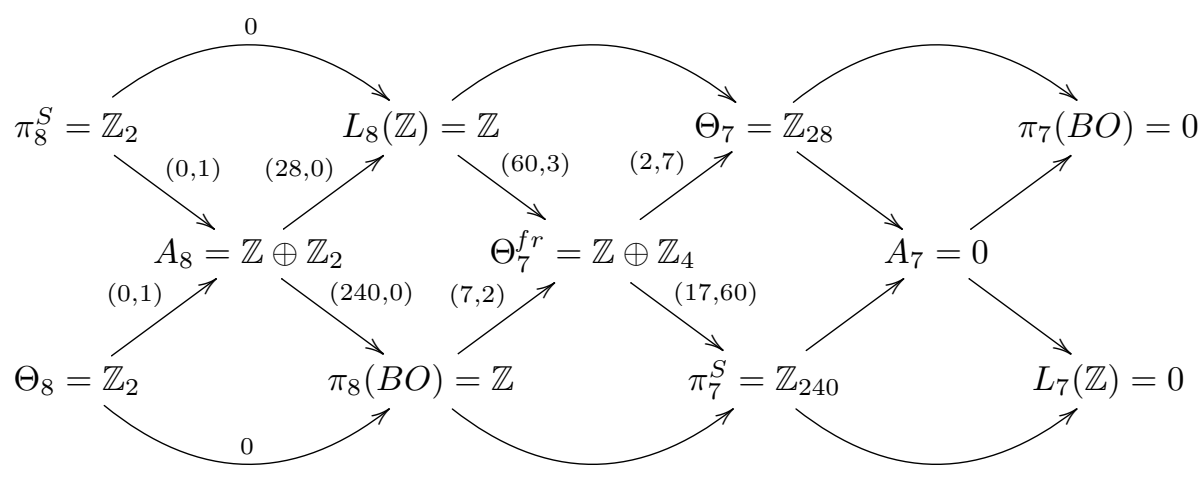

See Kosinski [42] and Levine [44] (which is Part II of the original Kervaire and Milnor [38]) for a more detailed exposition, as well as Lance [43] for a survey of differentiable structures on manifolds.

Example 13.26 For any $m, n \geqslant 1$ with $m+n \geqslant 5$ the structure set $\mathscr{S}\left(S^{m} \times S^{n}\right)$ of $S^{m} \times S^{n}$ fits into the surgery exact sequence of pointed sets

$$
\ldots \rightarrow L_{m+n}(\mathbb{Z}) \rightarrow \mathscr{S}\left(S^{m} \times S^{n}\right) \rightarrow\left[S^{m} \times S^{n}, G / O\right] \stackrel{A}{\rightarrow} L_{m+n}(\mathbb{Z})
$$

with the addition in $\left[S^{m} \times S^{n}, G / O\right]$ corresponding to the Whitney sum of fibre homotopy trivialized vector bundles over $S^{m} \times S^{n}$. As already noted in Example $13.3 A$ is not a homomorphism of abelian groups, and $\mathscr{S}\left(S^{m} \times S^{n}\right)$ may not have the structure of an abelian group. See the papers of A.R. A composition formula for manifold structures, http://arXiv.org/abs/math.AT/0608705, Pure and Applied Mathematics Quarterly 5 (Hirzebruch 80th birthday issue), 701-727 (2009) and Diarmuid Crowley The smooth structure set of $S^{p} \times S^{q}$ http://arXiv.org/abs/0904.1370 (2009) for explicit computations.

In particular, suppose $m=4 k$, so that $\pi_{m}(G / O)$ is a finitely generated abelian group of rank 1. Assume also that $k \geqslant 2$, and let $\left(W^{4 k}, \Sigma^{4 k-1}\right)$ be the framed $(2 k-1)$-connected $4 k$-dimensional manifold with homotopy $(4 k-1)$ sphere boundary obtained by the $E_{8}$-plumbing of 8 copies of $\tau_{S^{2 k}}: S^{2 k} \rightarrow$ $B S O(2 k)$. The exotic $(4 k-1)$-sphere $\Sigma^{4 k-1}$ is a generator of the cyclic subgroup $b P_{4 k} \subseteq \Theta_{4 k-1}$, with order

$$
t_{k}=a_{k} 2^{2 k-2}\left(2^{2 k-1}-1\right) \operatorname{num}\left(B_{k} / 4 k\right)
$$

where $a_{k}=$ g.c.d. $(2, k+1)$. Let

$$
Q^{4 k}=W^{4 k} \cup_{\Sigma^{4 k-1}} D^{4 k}
$$

be the framed $(2 k-1)$-connected $4 k$-dimensional $P L$ manifold with signature $\sigma(Q)=8$ obtained from $\left(W^{4 k}, \Sigma^{4 k-1}\right)$ by coning the boundary. The $t_{k}$-fold 
connected sum $\#_{t_{k}} Q^{4 k}$ has a differentiable structure. The topological $K$-group of isomorphism classes of stable vector bundles over $S^{4 k}$

$$
\widetilde{K O}\left(S^{4 k}\right)=\pi_{4 k}(B S O)=\pi_{4 k}(B S O(n+1))
$$

is such that there is defined an isomorphism

$$
\pi_{4 k}(B S O(n+1)) \rightarrow \mathbb{Z} ; \eta \mapsto p_{k}(\eta) / a_{k}(2 k-1) !
$$

by the Bott integrality theorem. The group of isomorphism classes of spherical fibrations over $S^{4 k}$

$$
\pi_{4 k}(B S G)=\pi_{4 k}(B S G(n))=\pi_{4 k-1}^{S}(n \geqslant 4 k+1)
$$

is finite, so there is only a finite number of distinct fibre homotopy classes of $(n-1)$-spherical fibrations over $S^{4 k}$. The subgroup

$$
\begin{aligned}
& \operatorname{im}\left(\pi_{4 k}(G / O) \rightarrow \pi_{4 k}(B S O(n+1))\right) \\
& \quad=\operatorname{ker}\left(J: \pi_{4 k}(B S O(n+1)) \rightarrow \pi_{4 k}(B S G(n+1))\right) \subseteq \pi_{4 k}(B S O(n+1))
\end{aligned}
$$

of fibre homotopy trivial bundles is the infinite cyclic subgroup of index

$$
j_{k}=\text { denominator }\left(B_{k} / 4 k\right)
$$

with the generator $\eta: S^{4 k} \rightarrow B S O(n+1)$ such that

$$
p_{k}(\eta)=a_{k} j_{k}(2 k-1) ! \in H^{4 k}\left(S^{4 k}\right)=\mathbb{Z} .
$$

For any fibre homotopy trivialisation

$$
h: J \eta \simeq J \epsilon^{n+1}: S^{4 k} \rightarrow B S G(n+1)
$$

the corresponding homotopy equivalence

$$
S(h): S(\eta) \rightarrow S\left(\epsilon^{n+1}\right)=S^{4 k} \times S^{n}
$$

is such that the inverse image of $S^{4 k} \times\{*\} \hookrightarrow S^{4 k} \times S^{n}$ is a submanifold of the type

$$
N^{4 k}=\#_{t_{k}} Q^{4 k} \hookrightarrow S(\eta)
$$

and $S(h)$ restricts to a degree 1 normal map

$$
(f, b)=S(h) \mid: N^{4 k} \rightarrow S^{4 k}
$$

with $b: \nu_{N} \rightarrow-\eta$. Moreover,

$$
\begin{aligned}
& \tau_{N}=f^{*}(\eta): N \rightarrow B S O(4 k), \\
& p_{k}(N)=f^{*} p_{k}(\eta)=a_{k} j_{k}(2 k-1) ! \in H^{4 k}(N)=\mathbb{Z}, \\
& \sigma(N)=s_{k} p_{k}(N)=s_{k} a_{k} j_{k}(2 k-1) !=8 t_{k} \in \mathbb{Z},
\end{aligned}
$$


with

$$
s_{k}=\frac{2^{2 k}\left(2^{2 k-1}-1\right)}{(2 k) !} B_{k}
$$

the coefficient of $p_{k}$ in $\mathscr{L}_{k}$. The homotopy equivalence $S(h): S(\eta) \rightarrow S^{4 k} \times S^{n}$ is not homotopic to a diffeomorphism (or indeed a homeomorphism) since the surgery obstruction of $(f, b)$ is

$$
\begin{aligned}
\sigma_{*}(f, b) & =\frac{1}{8}\left(\sigma(N)-\sigma\left(S^{4 k}\right)\right) \\
& =t_{k} \neq 0 \in L_{4 k}(\mathbb{Z})=\mathbb{Z} .
\end{aligned}
$$

The stable normal bundle of $S(\eta)$ is classified by

$$
\nu_{S(\eta)}: S(\eta) \rightarrow S^{4 k} \stackrel{\eta}{\rightarrow} B O .
$$

and $S(h)$ is a homotopy equivalence of $(n+4 k)$-dimensional manifolds which does not preserve Pontrjagin classes. This was the original surgery construction of a homotopy equivalence of simply-connected manifolds which is not homotopic to a diffeomorphism (Novikov [63]).

\subsection{Surgery obstruction theory}

This final section describes just a few of the developments in surgery theory since the main framework was set up by Wall [92]. The surveys listed in the Preface naturally give a broader picture!

The definition of the algebraic $L$-groups $L_{m}(A)$ separately for even and odd $m$ was unified by the chain complex theory of Ranicki [69], with $L_{m}(A)$ expressed as the cobordism group of chain complexes with $m$-dimensional Poincaré duality, as follows. Given a f.g. free $A$-module chain complex $C$ define the signed transposition involution on $\operatorname{Hom}_{A}\left(C^{*}, C\right)$ by

$$
T: \operatorname{Hom}_{A}\left(C^{p}, C_{q}\right) \rightarrow \operatorname{Hom}_{A}\left(C^{q}, C_{p}\right) ; \phi \mapsto(-1)^{p q} \phi^{*},
$$

with $C^{p}=C_{p}^{*}=\operatorname{Hom}_{A}\left(C_{p}, A\right)$. An $m$-dimensional quadratic Poincaré complex $(C, \psi)$ is an $m$-dimensional f.g. free $A$-module chain complex

$$
C: C_{m} \stackrel{d}{\rightarrow} C_{m-1} \rightarrow \ldots \rightarrow C_{1} \stackrel{d}{\rightarrow} C_{0}
$$

together with a $\mathbb{Z}_{2}$-hyperhomology class $\psi \in H_{m}\left(\mathbb{Z}_{2} ; \operatorname{Hom}_{A}\left(C^{*}, C\right)\right)$, as represented by $A$-module morphisms

$$
\psi_{s}: C^{m-r-s} \rightarrow C_{r} \quad(s \geqslant 0)
$$

such that 
$d \psi_{s}+(-1)^{r} \psi_{s} d^{*}+(-1)^{m-s-1}\left(\psi_{s+1}+(-1)^{s+1} T \psi_{s+1}\right)=0: C^{m-r-s-1} \rightarrow C_{r}$, with the $A$-module chain map

$$
(1+T) \psi_{0}: C^{m-*} \rightarrow C
$$

a chain equivalence. See [69] for the corresponding definition of an $(m+1)$ dimensional quadratic Poincaré pair $(f: C \rightarrow D,(\delta \psi, \psi))$, with a chain equivalence

$$
(1+T)(\delta \psi, \psi)_{0}: \mathscr{C}(f)^{m+1-*} \rightarrow D
$$

A quadratic Poincaré cobordism of $m$-dimensional quadratic Poincaré complexes $(C, \psi),\left(C^{\prime} \psi^{\prime}\right)$ is an $(m+1)$-dimensional quadratic Poincaré pair of the type

$$
\left(\left(f f^{\prime}\right): C \oplus C^{\prime} \rightarrow D,\left(\delta \psi, \psi \oplus-\psi^{\prime}\right)\right)
$$

Cobordism is an equivalence relation on $m$-dimensional quadratic Poincaré complexes over $A$. The set of cobordism classes is an abelian group, with addition and inverses by

$$
(C, \psi)+\left(C^{\prime}, \psi^{\prime}\right)=\left(C \oplus C^{\prime}, \psi \oplus \psi^{\prime}\right), \quad-(C, \psi)=(C,-\psi)
$$

If $m=2 n$ (resp. $2 n+1$ ) every $m$-dimensional quadratic Poincaré complex $(C, \psi)$ over $A$ is cobordant (by algebraic surgery below the middle dimension) to an $n$-connected complex, with

$$
\left.C_{r}=0 \text { for } r \neq n \quad \text { (resp. } n, n+1\right) \text {. }
$$

The cobordism group of $n$-connected $m$-dimensional quadratic Poincaré complexes over $A$ is isomorphic to the $L$-group $L_{m}(A)$ of stable isomorphism classes of nonsingular $(-1)^{n}$-quadratic forms (resp. formations) over $A$ defined in Chapter 11 (resp. 12), as well as to the cobordism group of all $m$-dimensional quadratic Poincaré complexes over $A$, so that

$$
L_{m}(A)=L_{m+4}(A)
$$

The surgery obstruction of an $m$-dimensional degree 1 normal map $(f, b): M \rightarrow$ $X$ is the cobordism class of an $m$-dimensional quadratic Poincaré complex $(C, \psi)$

$$
\sigma_{*}(f, b)=(C, \psi) \in L_{m}\left(\mathbb{Z}\left[\pi_{1}(X)\right]\right)
$$

with $C=\mathscr{C}\left(f^{!}\right)$the algebraic mapping cone of the Umkehr chain map $f^{!}$: $C(\widetilde{X}) \rightarrow C(\widetilde{M})$. The homology and cohomology of $C$ are given by

$$
H_{*}(C)=K_{*}(M), H^{*}(C)=K^{*}(M),
$$

and the quadratic structure $\psi$ is determined by a stable $\pi_{1}(X)$-equivariant geometric Umkehr map $F: \Sigma^{\infty} \widetilde{X}_{+} \rightarrow \Sigma^{\infty} \widetilde{M}_{+}$inducing $f$ ! The 'instant surgery obstruction' of [69] is a $(-1)^{n}$-quadratic form (resp. formation) if $m=2 n$ (resp. 
$m=2 n+1)$ representing the surgery obstruction $\sigma_{*}(f, b) \in L_{m}\left(\mathbb{Z}\left[\pi_{1}(X)\right]\right)$, which is constructed from $(C, \psi)$ by algebraic surgeries below the middle dimensions, rather than by geometric surgeries below the middle dimension as in Chapter 11 (resp. 12).

The expression of $L_{*}(A)$ as the cobordism groups of quadratic Poincaré complexes gives various useful properties of the algebraic $L$-groups, such as relative $L$-groups : a morphism of rings with involution $f: A \rightarrow B$ induces morphisms

$$
f_{*}: L_{m}(A) \rightarrow L_{m}(B) ;(C, \psi) \mapsto B \otimes_{A}(C, \psi)
$$

and the group $L_{m}(f)$ of cobordism classes of pairs

( $(m-1)$-dimensional quadratic Poincaré complex $(C, \psi)$ over $A$, $m$-dimensional quadratic Poincaré pair over $\left.B\left(g: B \otimes_{A} C \rightarrow D,(\delta \psi, 1 \otimes \psi)\right)\right)$

fits into an exact sequence

$$
\ldots \longrightarrow L_{m}(A) \stackrel{f_{*}}{\longrightarrow} L_{m}(B) \longrightarrow L_{m}(f) \longrightarrow L_{m-1}(A) \longrightarrow \ldots
$$

The localization exact sequence of Section 12.6 is the special case of the inclusion $f: A \rightarrow B=S^{-1} A$, with $L_{*}(f)=L_{*}(A, S)$.

The chain complex method is also useful for obtaining surgery product and composition formulae, and for the algebraic surgery classification of topological manifolds.

An extensive literature is devoted to the computation of $L_{*}(\mathbb{Z}[\pi]), L_{*}^{s}(\mathbb{Z}[\pi])$ for various groups $\pi$, using algebra for finite $\pi$ and geometry for infinite $\pi$. See Wall [93], Hambleton, Milgram, Taylor and Williams [29], Hambleton and Taylor [30] and Milgram [48] for general results for finite $\pi$. See Ferry, Ranicki and Rosenberg [24] for an account of the geometric methods used for infinite $\pi$, including the connections with the Novikov conjecture on the homotopy invariance of the higher signatures and the closely related Borel conjecture on the existence and uniqueness of a topological manifold structure in the homotopy type of an aspherical Poincaré complex $B \pi=K(\pi, 1)$. Ranicki [72] is an introduction to the Novikov conjecture from the surgery point of view.

The set $[M, G / O]$ of equivalence classes of fibre homotopy trivialised vector bundles over a manifold $M$ is an abelian group, with addition by Whitney sum. The computation of $[M, G / O]$ can be carried out in many cases using standard algebraic topology (modulo the homotopy groups of spheres) - see Chapter 9.2 for an exposition of some of the homotopy theoretic properties of $G / O$. The function $A:[M, G / O] \rightarrow L_{m}\left(\mathbb{Z}\left[\pi_{1}(M)\right]\right)$ sending a normal map to the surgery 
obstruction is not in general a homomorphism of abelian groups (cf. Example 13.3). The surgery exact sequence

$$
\ldots \rightarrow L_{m+1}\left(\mathbb{Z}\left[\pi_{1}(M)\right]\right) \rightarrow \mathscr{S}(M) \rightarrow[M, G / O] \stackrel{A}{\rightarrow} L_{m}\left(\mathbb{Z}\left[\pi_{1}(M)\right]\right)
$$

is not an exact sequence of abelian groups (cf. Example 13.26), so computations of $[M, G / O]$ and $L_{*}\left(\mathbb{Z}\left[\pi_{1}(M)\right]\right)$ may be quite difficult to match up.

See Ranicki [71] for the surgery exact sequence and the 'total surgery obstruction' in the topological category. For any space $X$ there is defined a long exact sequence of abelian groups

$$
\begin{aligned}
\ldots \rightarrow L_{m+1}\left(\mathbb{Z}\left[\pi_{1}(X)\right]\right) \rightarrow \mathscr{S}_{m+1}(X) & \rightarrow H_{m}\left(X ; \mathbb{L}_{\bullet}\right) \\
& \stackrel{A}{\rightarrow} L_{m}\left(\mathbb{Z}\left[\pi_{1}(X)\right]\right) \rightarrow \mathscr{S}_{m}(X) \rightarrow \ldots
\end{aligned}
$$

with $\mathbb{L}_{\bullet}$ a 1-connective spectrum of quadratic complexes over $\mathbb{Z}$ such that

$$
\mathbb{L}_{0} \simeq G / T O P, \pi_{*}\left(\mathbb{L}_{\bullet}\right)=\pi_{*}(G / T O P)=L_{*}(\mathbb{Z})
$$

and $A$ the algebraic $L$-theory version of the assembly map of Quinn [67]. The various classifying spaces fit into a commutative braid of fibrations

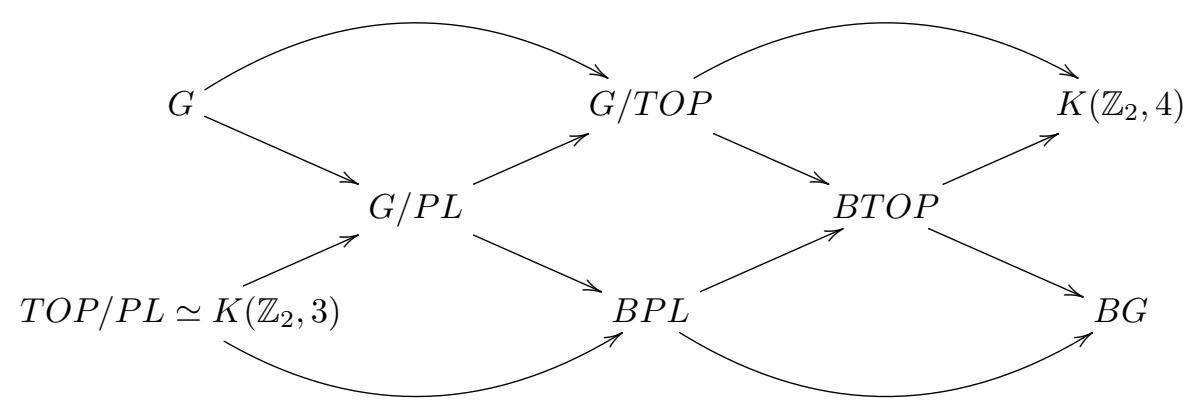

with $B T O P$ the classifying space for stable topological bundles (Kirby and Siebenmann [39], Ranicki et al. [73]). The topological structure set $\mathscr{S}^{T O P}(X)$ of a space $X$ is defined in the same way as $\mathscr{S}(X)(1.14)$, but using topological manifolds. The structure set of an $m$-dimensional topological manifold $M$ is given for $m \geqslant 5$ by

$$
\mathscr{S}^{T O P}(M)=\mathscr{S}_{m+1}(M) .
$$

The topological surgery exact sequence is an exact sequence of abelian groups

$$
\ldots \rightarrow L_{m+1}\left(\mathbb{Z}\left[\pi_{1}(M)\right]\right) \rightarrow \mathscr{S}^{T O P}(M) \rightarrow[M, G / T O P] \stackrel{A}{\rightarrow} L_{m}\left(\mathbb{Z}\left[\pi_{1}(M)\right]\right)
$$

with respect to the addition on $[M, G / T O P]$ determined by the direct sum in $\mathbb{L}_{0}$, and

$$
A:[M, G / T O P] \cong H_{m}\left(M ; \mathbb{L}_{\bullet}\right) \rightarrow L_{m}\left(\mathbb{Z}\left[\pi_{1}(M)\right]\right)
$$

is the assembly map. The image of $A: H_{m}\left(K ; \mathbb{L}_{\bullet}\right) \rightarrow L_{m}\left(\mathbb{Z}\left[\pi_{1}(K)\right]\right)$ for an Eilenberg-MacLane space $K=K(\pi, 1)$ is the subgroup of $L_{m}(\mathbb{Z}[\pi])$ consisting 
of the surgery obstructions of normal maps of closed $m$-dimensional manifolds (cf. Remark 11.79). The total surgery obstruction $s(X) \in \mathscr{S}_{m}(X)$ of an $m$ dimensional Poincaré complex $X$ is such that $s(X)=0$ if (and for $m \geqslant 5$ only if) $X$ is homotopy equivalent to an $m$-dimensional topological manifold. 


\section{REFERENCES}

[1] M. Adachi, Embeddings and immersions, Translations of Mathematical Monographs 124, A. M. S. (1993)

[2] J.F. Adams, On the non-existence of elements of Hopf invariant one, Ann. of Maths. 72, 20-104 (1960)

[3] --, On the groups $J(X)$. IV., Topology 5, 21-71 (1966)

[4] C. Arf, Untersuchungen über quadratische Formen in Körpern der Charakteristik 2, Crelles Math. J. 184, 148-168 (1941)

[5] M. Atiyah, Thom complexes, Proc. L. M. S. 11, 291-310 (1961)

[6] H. Bass, The Dirichlet unit theorem, induced characters and Whitehead groups of finite groups, Topology 4, 391-410 (1965)

[7] J. M. Boardman and R. M. Vogt, Homotopy-everything $H$-spaces, Bull. A.M.S. $74,1117-1122(1968)$

[8] R. Bott and L. Tu, Differential forms in algebraic topology, Graduate Texts in Mathematics 82, Springer (1982)

[9] -- and J. Milnor, On the parallelizability of the spheres, Bull. A.M.S. 64, 87-89 (1958)

[10] G. Bredon, Topology and geometry, Graduate Texts in Mathematics 139, Springer (1993)

[11] W. Browder, Torsion in H-spaces, Ann. of Maths. 74, 24-51 (1961)

[12] --, Homotopy type of differentiable manifolds, Proc. Århus Topology Symp. (1962)

reprinted in Proc. 1993 Oberwolfach conference on the Novikov conjecture, index theorems and rigidity, Vol. 1, L. M. S. Lecture Notes 226, 97-100 (1995)

[13] --, The Kervaire invariant of framed manifolds and its generalisations, Ann. of Maths. 90, 157-187 (1969)

[14] --, Surgery on simply connected manifolds, Ergebnisse der Mathematik und ihrer Grenzgebiete 65, Springer (1972)

[15] --, Poincaré spaces, their normal fibrations and surgery, Inv. Math. 17, 192-202 (1972)

[16] E. H. Brown, A remark concerning immersions of $S^{n}$ in $\mathbb{R}^{2 n}$, Quart. J. Math. Oxford (2) 24, 559-560 (1973)

[17] S. Cappell, A. A. Ranicki and J. Rosenberg (eds.), Surveys on surgery theory, Ann. of Maths. Studies 145 (vol.1), 149 (vol.2) Princeton University Press (2000) 
[18] - - and J. Shaneson, On 4-dimensional s-cobordisms, J. Diff. Geo. 22, 97115 (1985)

[19] M. Cohen, A course in simple homotopy theory, Graduate Texts in Mathematics 10, Springer (1973)

[20] G. deRham, M. Kervaire and S. Maumary, Torsion et type simple d'homotopie, Lecture Notes in Mathematics 48, Springer (1967)

[21] S. Donaldson, An application of gauge theory to the topology of 4-manifolds, J. Diff. Geo. 18, 279-315 (1983)

[22] B. Eckmann and P. Linnell, Poincaré duality groups of dimension two, II., Comm. Math. Helv. 58, 111-114 (1983)

[23] F. T. Farrell and W. Lück (eds.), Topology of High-Dimensional Manifolds, Volume 9 of ICTP Lecture Notes Series, ICTP, Trieste (2002)

[24] S. Ferry, A. A. Ranicki and J. Rosenberg (eds.), Novikov conjectures, rigidity and index theorems, Proceedings of 1993 Oberwolfach Conference, L. M. S. Lecture Notes 226, 227, Cambridge University Press (1995)

[25] M. Freedman, The topology of four-dimensional manifolds, J. Diff. Geo. 17, 357-453 (1982)

[26] - - and F. Quinn, The topology of 4-manifolds, Princeton University Press (1990)

[27] S. Gitler and J. Stasheff, The first exotic class of BF, Topology 4, 257-266 (1965)

[28] D. Gottlieb, Poincaré duality and fibrations, Proc. A. M. S. 76, 148-150 (1979)

[29] I. Hambleton, J. Milgram, L. Taylor and B. Williams, Surgery with finite fundamental group, Proc. L. M. S. 56 (3), 349-379 (1988)

[30] - - and L. Taylor, A guide to the calculation of the surgery obstruction groups for finite groups, in Surveys on surgery theory, Vol. 1, Ann. of Maths. Studies 145, 225-274, Princeton University Press (2000)

[31] A. Hatcher, Algebraic topology, Cambridge University Press (2002)

[32] M. Hirsch, Immersions of manifolds, Trans. A. M. S. 93, 242-276 (1959)

[33] --, Differential Topology, Graduate Texts in Mathematics 33, Springer (1976)

[34] F. Hirzebruch, Topological methods in algebraic geometry, Grundlehren der mathematischen Wissenschaften 131, Springer (1978)

[35] W. C. Hsiang and J. Shaneson, Fake tori, Topology of Manifolds, Proc. 1969 Georgia Topology Conference, Markham Press, 18-51 (1970)

[36] M. Kervaire, A manifold which does not admit a differentiable structure, Comm. Math. Helv. 34, 257-270 (1960)

[37] --, Le théorème de Barden-Mazur-Stallings, Comment. Math. Helv. 40, 31-42 (1965)

[38] - - and J. Milnor, Groups of homotopy spheres I., Ann. of Maths. 77, 504$537(1963)$ 
[39] R. Kirby and L. Siebenmann, Foundational essays on topological manifolds smoothings and triangulations, Ann. of Maths. Studies 88, Princeton University Press (1977)

[40] J. Klein, Poincaré duality spaces, in Surveys on surgery theory, Vol. 1, Ann. of Maths. Studies 145, 135-167, Princeton University Press (2000)

[41] U. Koschorke and B.Sanderson, Geometric interpretations of the generalised Hopf invariant, Math. Scand. 41, 199-217 (1977)

[42] A. Kosinski, Differential manifolds, Academic Press (1993)

[43] T. Lance, Differentiable structures on manifolds, in Surveys on surgery theory, Vol. 1, Ann. of Maths. Studies 145, 73-104, Princeton University Press (2000)

[44] J. Levine, Lectures on groups of homotopy spheres, Algebraic and Geometric Topology, Rutgers 1983, Lecture Notes in Mathematics 1126, 62-95, Springer (1983)

[45] I. Madsen and J. Milgram, The classifying spaces for surgery and cobordism of manifolds, Ann. of Maths. Studies 92, Princeton University Press (1979)

[46] --, C. B. Thomas and C. T. C. Wall, The topological space form problem, Topology 15, 375-382 (1976)

[47] B. Mazur, Stable equivalence of differentiable manifolds, Bull. A.M.S. 67, 377-384 (1961)

[48] J. Milgram, Surgery with finite fundamental group, Pacific J. Math. 151, I. 65-115, II. 117-150 (1991)

[49] J. Milnor, On manifolds homeomorphic to the 7-sphere, Ann. of Maths. 64, 399-405 (1956)

[50] --, On the Whitehead homomorphism J, Bull. A.M.S. 64, 79-82 (1958)

[51] --, Some consequences of a theorem of Bott, Ann. of Maths. 68, 444-449 (1958)

[52] --, Differentiable structures on spheres, Amer. J. of Math. 81, 962-972 (1959)

[53] --, A procedure for killing the homotopy groups of differentiable manifolds, Proc. Symp. on Pure Math. 3 (Differential Geometry), A. M. S. 39-55 (1961)

[54] --, Two complexes which are homeomorphic but combinatorially distinct, Ann. of Maths. 74, 575-590 (1961)

[55] --, Morse theory, Ann. of Maths. Studies 51, Princeton University Press (1963)

[56] --, Topology from the differentiable viewpoint, University Press of Virginia (1965)

[57] --, Lectures on the h-cobordism theorem, Mathematical Notes 1, Princeton University Press (1965)

http://www.maths.ed.ac.uk/ aar/surgery/hcobord.pdf

[58] --, Whitehead torsion, Bull. A. M. S. 72, 358-426 (1966) 
[59] --, Classification of $(n-1)$-connected $2 n$-manifolds and the discovery of exotic spheres, in Surveys on surgery theory, Vol. 1, Ann. of Maths. Studies 145, 25-30, Princeton University Press (2000)

[60] - - and D. Husemoller, Symmetric bilinear forms, Springer (1973)

[61] - - and J.Stasheff, Characteristic classes, Ann. of Maths. Studies 76, Princeton University Press (1974)

[62] J. Morgan and D.P.Sullivan, The transversality characteristic class and linking cycles in surgery theory, Ann. of Maths. 99, 463-544 (1974)

[63] S. P. Novikov, Homotopy equivalent smooth manifolds I., Izv. Akad. Nauk SSSR, ser. mat. 28, 365-474 (1965) English translation: A. M.S. Transl. (2) 48, 271-396 (1965)

[64] --, The algebraic construction and properties of hermitian analogues of $K$ theory for rings with involution, from the point of view of the hamiltonian formalism. Some applications to differential topology and the theory of characteristic classes, Izv. Akad. Nauk SSSR, ser. mat. 34, I. 253-288, II. 478-500 (1970) English translation: Math. USSR Izv. 4, 257-292, 479505 (1970)

[65] - -, Topology I, Encyclopedia of Mathematical Sciences 12, Springer (1996)

[66] L. S. Pontrjagin, Smooth manifolds and their applications in homotopy theory, A.M.S. Translations 11, 1-114 (1955)

[67] F. Quinn, A geometric formulation of surgery, in Topology of manifolds, Proceedings 1969 Georgia Topology Conference, Markham Press, 500-511 (1970)

[68] A. A. Ranicki, Algebraic L-theory I., II., Proc. L. M.S. (3) 27, 101-125, 126-158 (1973)

[69] --, The algebraic theory of surgery I., II., Proc. L. M.S. (3) 40, 87-193, 194-287 (1980)

[70] --, Exact sequences in the algebraic theory of surgery, Mathematical Notes 26, Princeton University Press (1981) http://www.maths.ed.ac.uk/ aar/surgery/exact.pdf

[71] --, Algebraic L-theory and topological manifolds, Tracts in Mathematics 102, Cambridge University Press (1992)

[72] --, On the Novikov conjecture, Proceedings of 1993 Oberwolfach Conference, Vol. 1, L. M. S. Lecture Notes 226, 272-337, Cambridge University Press (1995)

[73] - - (ed.), The Hauptvermutung book, A collection of papers in the topology of manifolds, by Casson, Sullivan, Armstrong, Rourke, Cooke and Ranicki, $K$-Monographs 1, Kluwer (1996)

[74] --, High dimensional knot theory, Springer Mathematical Monograph, Springer (1998)

[75] --, An introduction to algebraic surgery, in Surveys on surgery theory, Vol. 2, Ann. of Maths. Studies 149, 81-163, Princeton University Press 
(2001)

http://arXiv.org/abs/math.AT/0008070

[76] --, Algebraic Poincaré cobordism, in Topology, Geometry, and Algebra: Interactions and New Directions. Proc. Stanford conference for 60th birthday of R.J.Milgram, Contemp. Maths. 279, A.M.S., 213-255 (2001) http://arXiv.org/abs/math.AT/0008228

[77] E. G. Rees, Problems concerning embeddings of manifolds, Advances in Mathematics 19, Beijing, 72-79 (1990)

[78] J. Rosenberg, Algebraic K-theory and its applications, Graduate Texts in Mathematics 147, Springer (1994)

[79] J.-P.Serre, A course in arithmetic, Graduate Texts in Mathematics 7, Springer (1973)

[80] J. Shaneson, Wall's surgery obstruction groups for $G \times \mathbb{Z}$, Ann. of Maths. 90, 296-334 (1969)

[81] S. Smale, Classification of immersions of spheres in Euclidean space, Ann. of Maths. 69, 327-344 (1959)

[82] -- Generalized Poincaré's conjecture in dimensions greater than 4, Ann. of Maths. 74, 391-406 (1961)

[83] --, On the structure of manifolds, Am. J. of Math. 84, 387-399 (1962)

[84] M. Spivak, Spaces satisfying Poincaré duality, Topology 6, 77-101 (1967)

[85] J.Stasheff, A classification theorem for fibrations, Topology 2, 239-246 (1963)

[86] N. Steenrod, The topology of fibre bundles, Princeton University Press (1951)

[87] R. Thom, Sur une partition en cellules associée à une fonction sur une variété, Comptes Rendus Acad. Sc. 228, 973-975 (1949)

[88] --, Quelques propriétés globales des variétés differentiables, Comm. Math. Helv. 28, 17-86 (1954)

[89] C. T. C. Wall, Classification of $(n-1)$-connected $2 n$-manifolds, Ann. of Maths. 75, 163-189 (1962)

[90] --, Surgery of non-simply connected manifolds, Ann. of Maths. 84, 217276 (1966)

[91] --, Poincaré complexes, Ann. of Maths. 86, 213-245 (1967)

[92] --, Surgery on compact manifolds, Academic Press (1970). 2nd edition (ed. A. A. Ranicki), Mathematical Surveys and Monographs 69, A.M.S. (1999)

[93] --, On the classification of hermitian forms VI. Group rings, Ann. of Maths. 103, 1-80 (1976)

[94] S. Weinberger, The topological classification of stratified spaces, Chicago University Press (1994)

[95] M. Weiss, Visible L-theory, Forum Math. 4, 465-498 (1992)

[96] G. W. Whitehead, Elements of homotopy theory, Graduate Texts in Mathematics 61, Springer (1978)

[97] H. Whitney, Differentiable manifolds, Ann. of Maths. 37, 647-680 (1936) 
[98] --, The general type of singularity of a set of $2 n-1$ smooth functions of $n$ variables, Duke Math. J. 10, 161-173 (1943)

[99] --, The self-intersections of a smooth n-manifold in $2 n$-space, Ann. of Maths. 45, 220-246 (1944)

[100] --, The singularities of a smooth $n$-manifold in $(2 n-1)$-space, Ann. of Maths. 45, 247-293 (1944) 